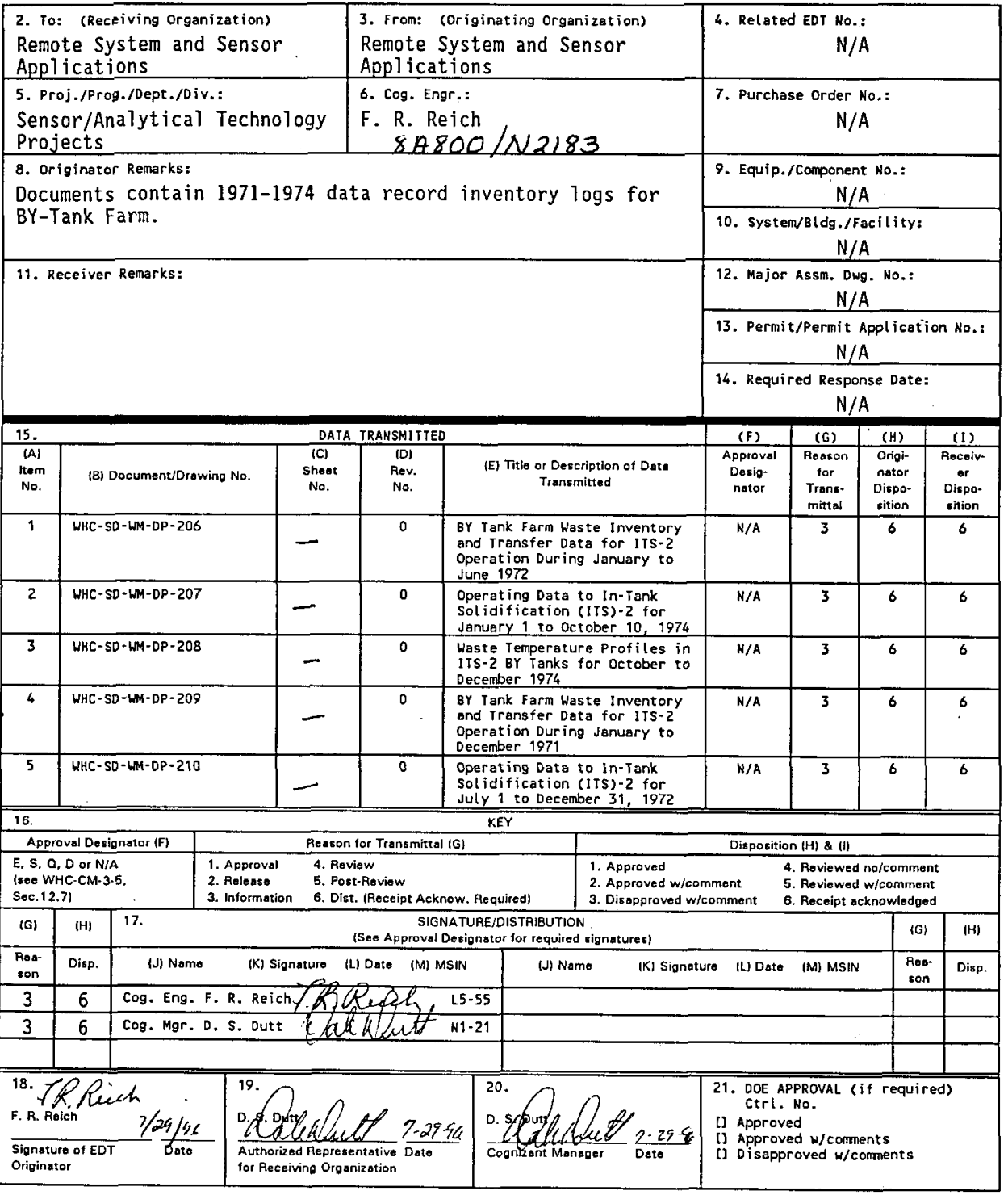




\section{BY Tank Farm Waste Inventory and Transfer Data for ITS-2 Operation during January to December 1971}

F. R. Reich

Westinghouse Hanford Company, Richland, WA 99352

U.S. Department of Energy Contract DE-AC06-87RL10930

\begin{tabular}{|c|c|c|}
\hline $\begin{array}{l}\text { EDT/ECN: } \\
\text { Org Code: } \\
\text { B\&R Code: }\end{array}$ & $\begin{array}{l}617410 \\
8 A 800 \\
E W 4010000\end{array}$ & $\begin{array}{l}\text { UC: } 721 \\
\text { Charge Code: } \\
\text { Total Pages: }\end{array}$ \\
\hline
\end{tabular}

Key Words: BY Tanks, Pump Activity, Pump Inventory, Daily Record Inventory, Waste Inventory, Transfer Data, ITS-2 Operation, Liquid Levels, Pumping

Abstract: Data record inventory of pumping activities and liquid level changes including occasional operations comments for the BY Tank Farm. Waste inventory and transfer data for ITS-2 operation during January to December 1971 .

\section{BEST AMAULBSE COPY}

TRADEMARK 0ISCLAIMER. Reference herein to any specific comnercial product, process, or service by trade name, trademark, manufacturer, or otherwise, does not necessarily constitute or imply its endorsement, recomnendation, of favoring by the United States Government or any agency thereof or its contractors or subcontractors.

Printed in the United states of America. To obtain copies of this document, contact: WhC/BCs Document Control Services, P.0. Box 1970, Mailstop H6-08, Richland WA 99352, Phone (509) 372-2420; Fax (509) 376-4989.
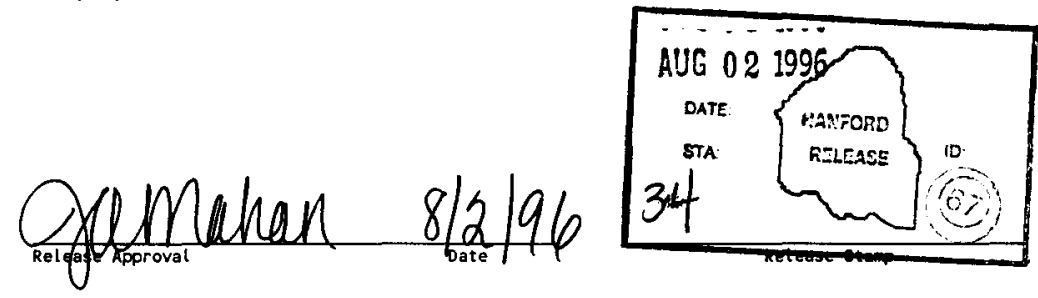

Approved for Public Release 
Document Date: 01-01-71

Document Number: None given

RHA Box Number: 58102

File Number: None given

Title:

ITS-2 DATA SHEETS 1971, INVENTORY AND PUHPING

Description:

365 PAGES / BOUND DATA SHEETS OF ITS-2 INVENTORY AND PUMPING

Comments:

DATA SHEETS CONTAIN PUMP NUMBER, L.L. START, L.L. END, PUMP AMPS, REMARKS.

Addressee, Company:

Author:

Company:

Tank $\#$ :

241-BX-111

241-BY FARM

Keywords:

INVENTORY

LIQUID LEVELS

PUMPING

References: N

* References: None given

Action Required: N

Topic: None given

Action Taken: None given

User: RM SCHWARZ

User Daté: 02-04-92 07:50:12

Reviewer: ECKSTEIN D

Review Date: 09-16-91

Checked By: SCHWARZ RM

Checked Date: 10-10-91

WTS Key: 960

Document Type: DS 
box $5 \gamma 1$ BOP

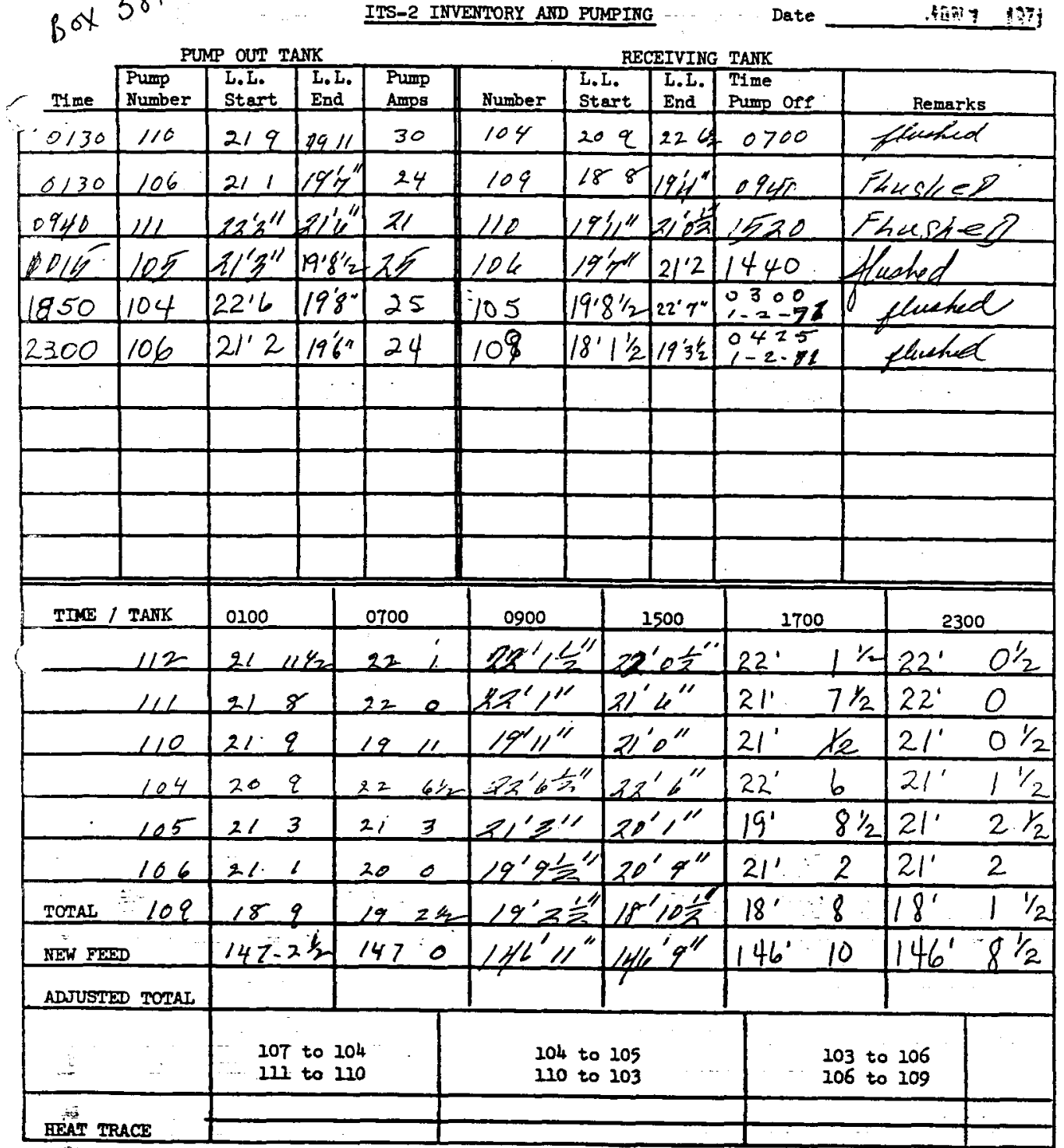

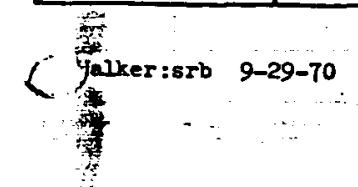


PUMP OUT TANK

RECEIVING TANK

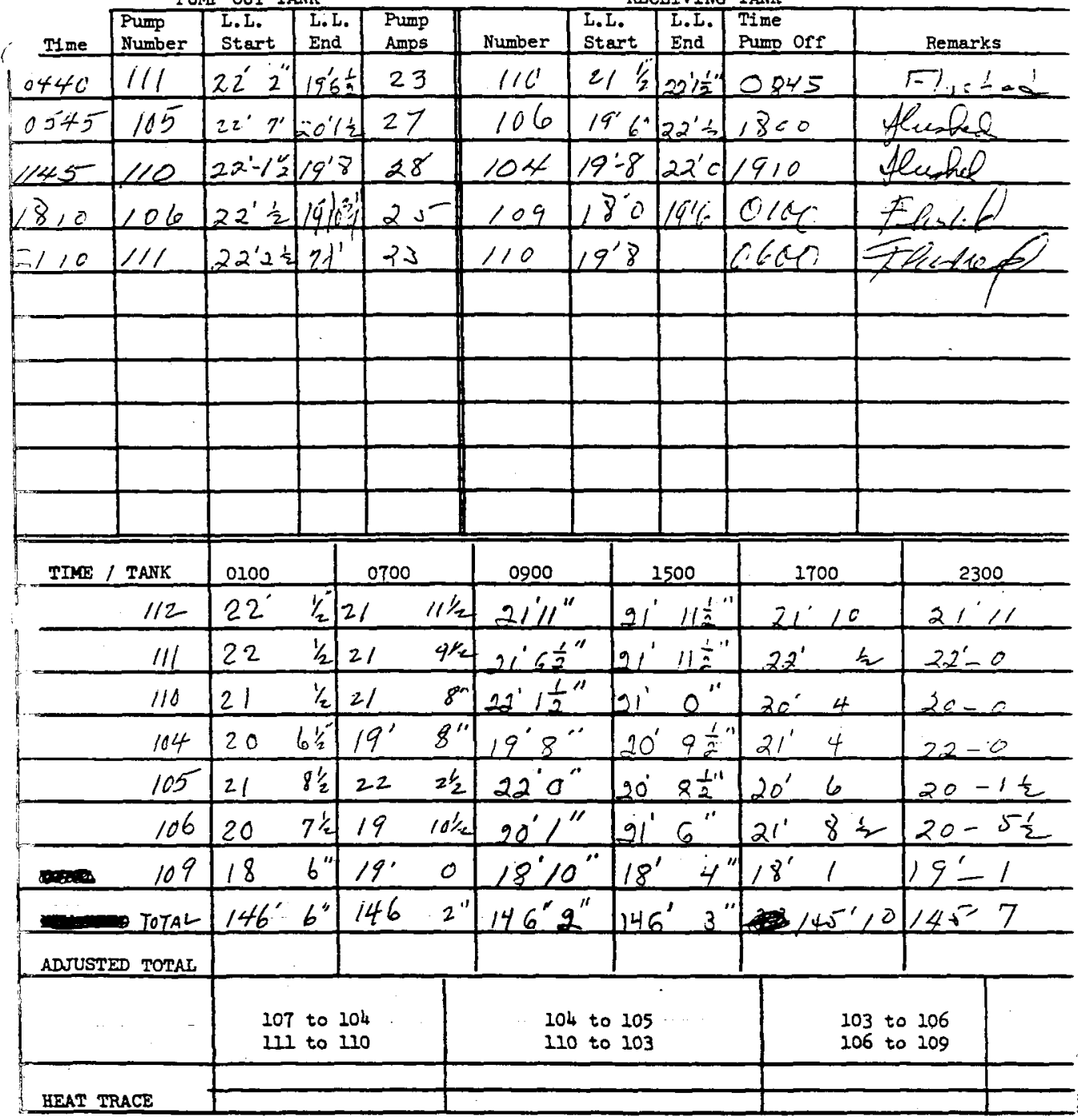

f. Walker:srb 9-29-70 
PUMP OUT TANK

RECEIVING TANK

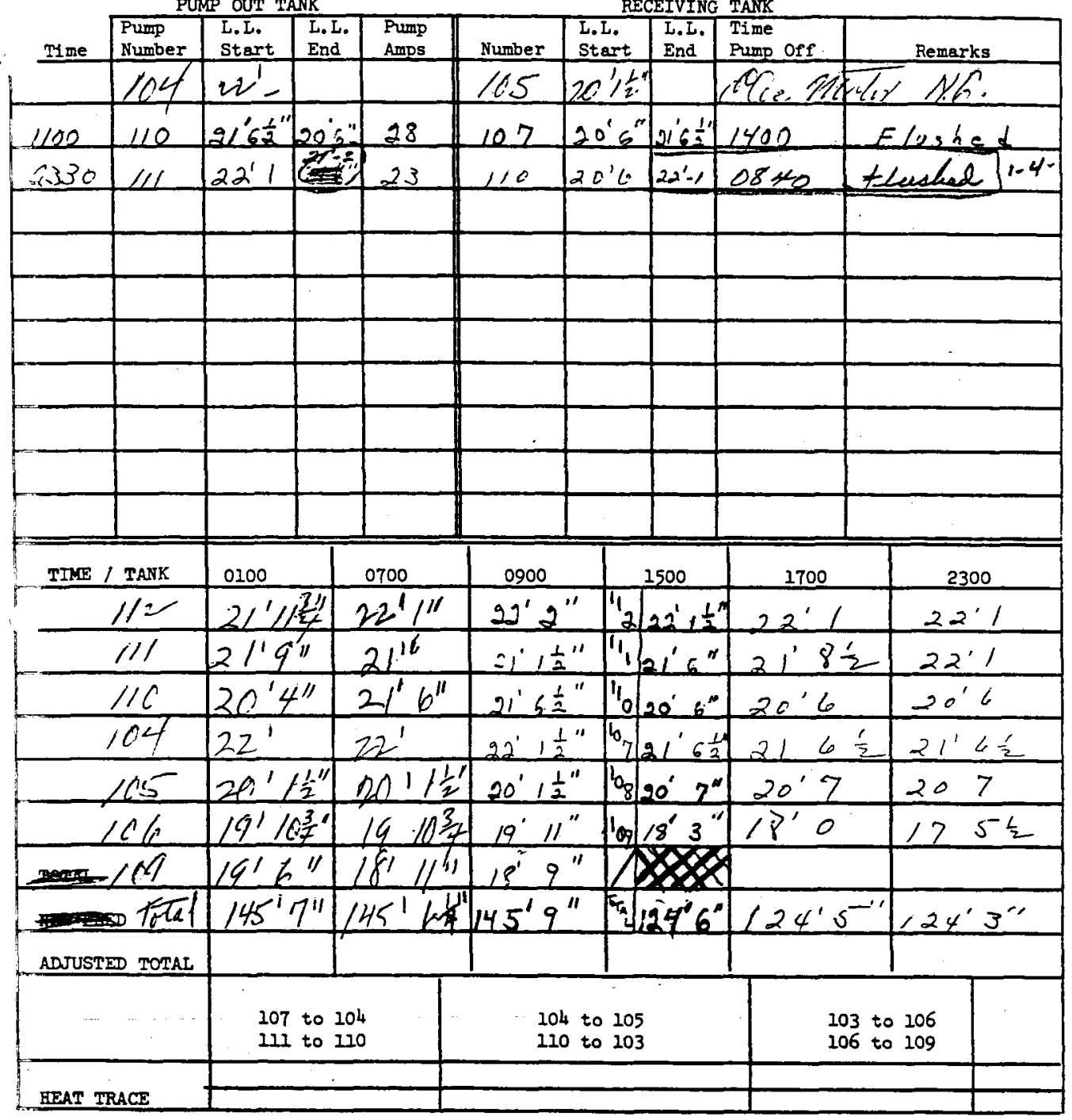

Walker:srb 9-29-70 
PUMP OUT TANK

RECEIVING TANK

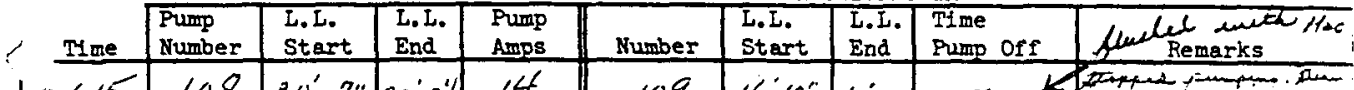

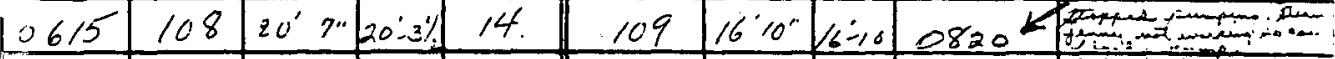

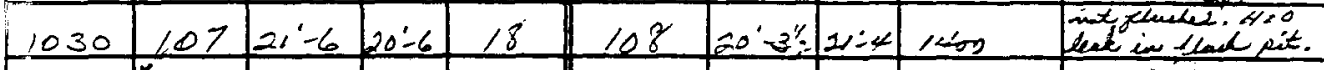

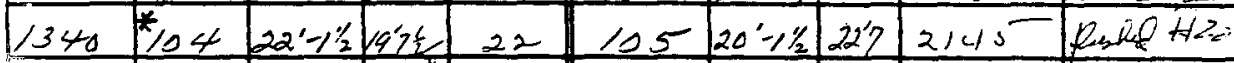

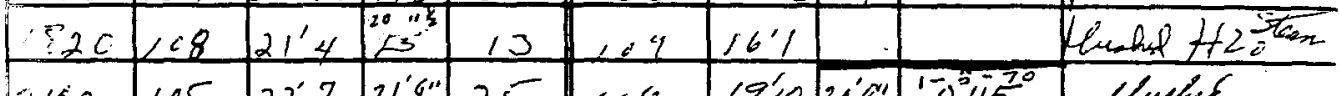

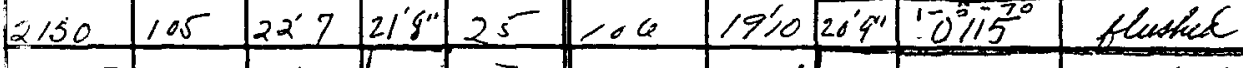

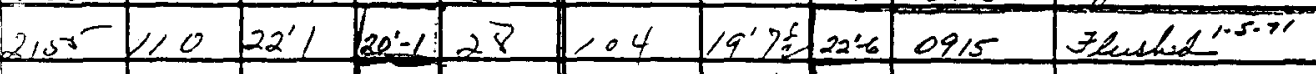
\begin{tabular}{|l|l|l|l|l|}
2310 & $21 / 22$ \\
\hline
\end{tabular} $1100.2180^{\circ}-1 / 243$ $7 \operatorname{loc} 1-5-71$

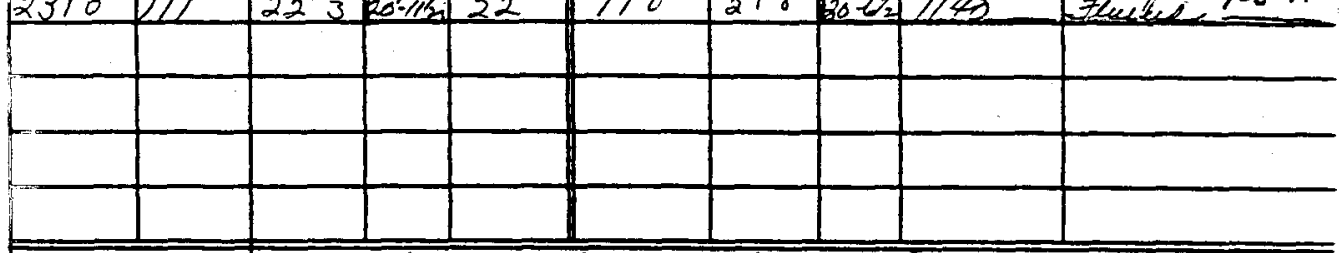

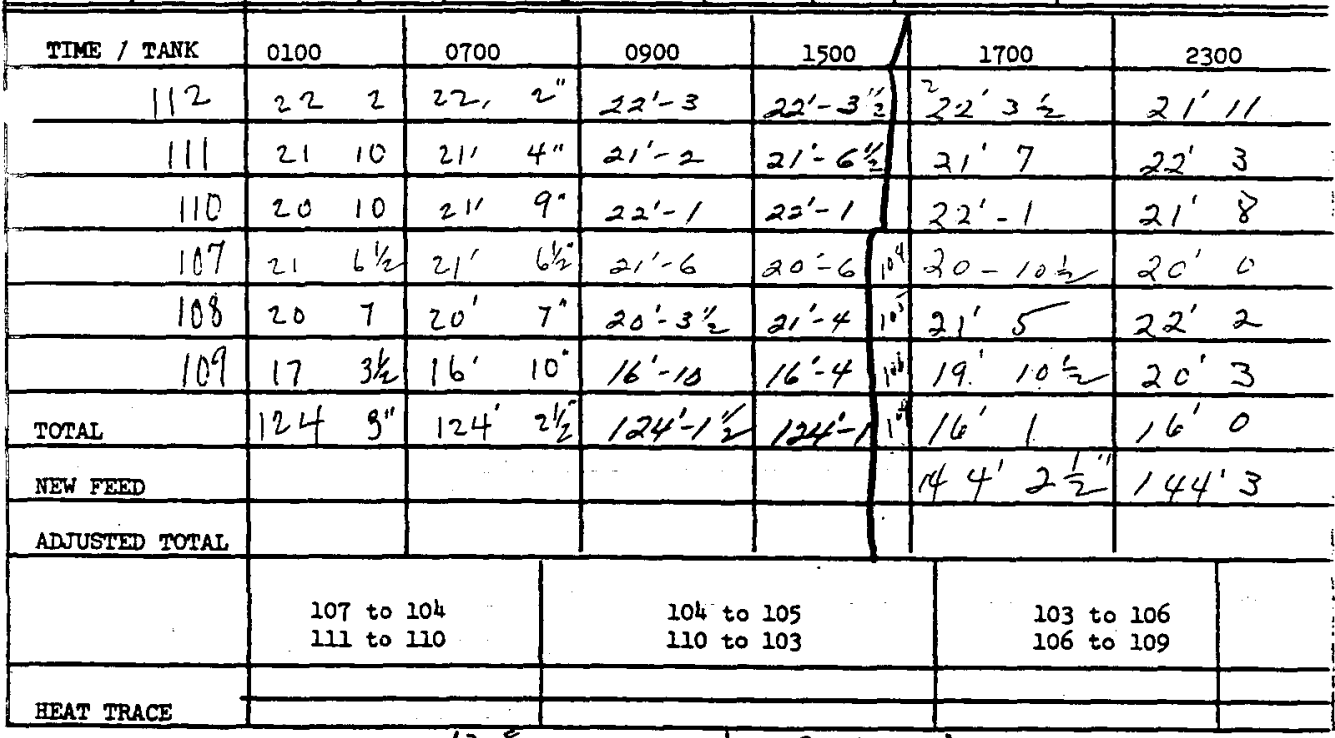

$\therefore$ Walker:srb 9-29-70 $\frac{12.8}{104-22 / 1 / 2} \mid \frac{8-48}{104-21: 8 \%}$ $105-2 c, 1 " 2 " 105-20: 7$ $106-1 q^{\prime} " 10$, $106-19: 10 \%$

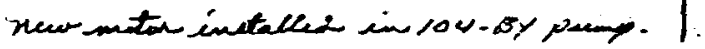


PURP OUT TANK

RECEIVING TANK

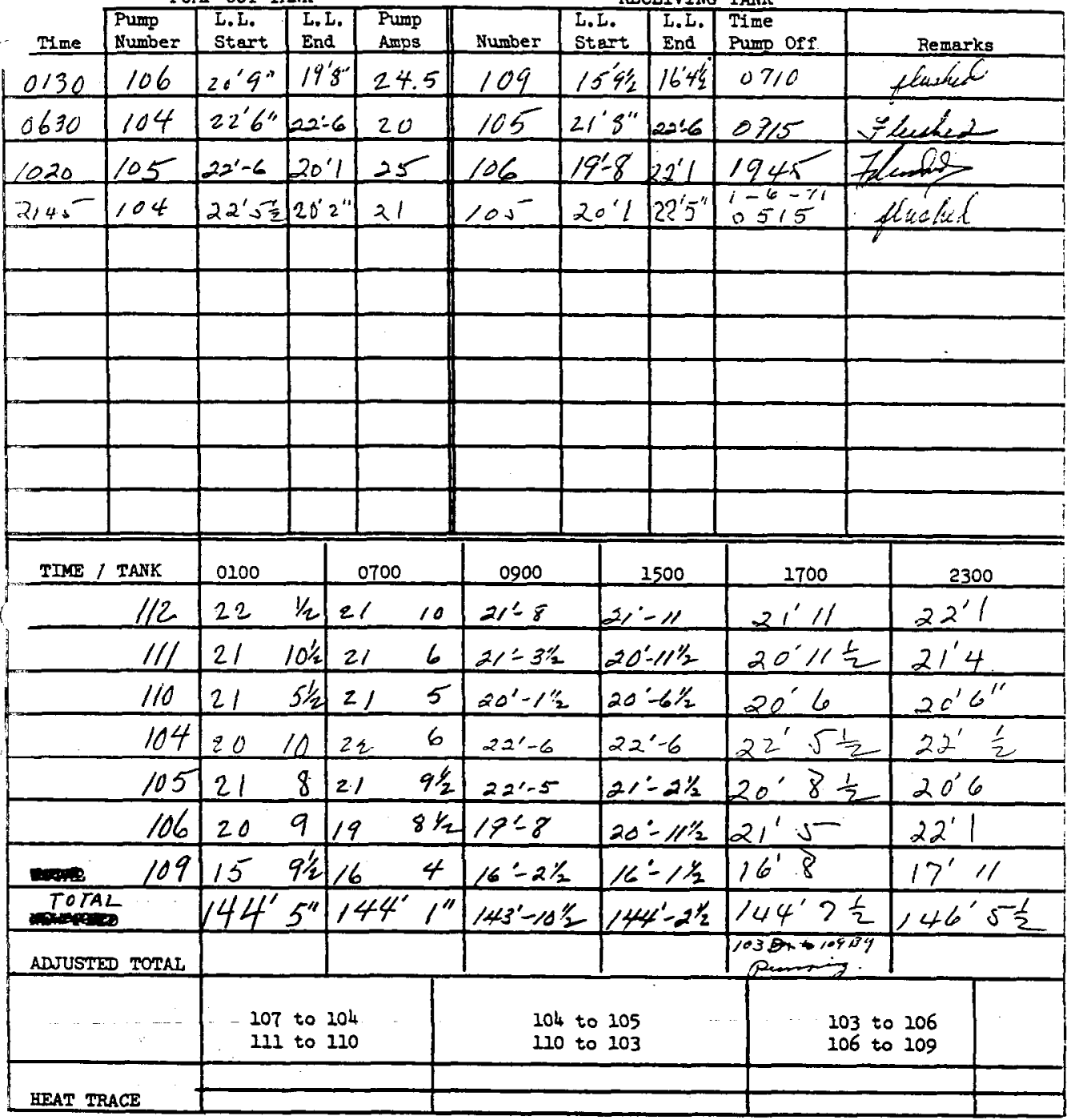

Walker:srb 9-29-70 
WHC-SD-LMYDP-209, ReV. O

ITS-2 INVENTORY AND PUMPING

Date JAN. 6 1971

PUMP OUT TANK

RECEIVING TANK

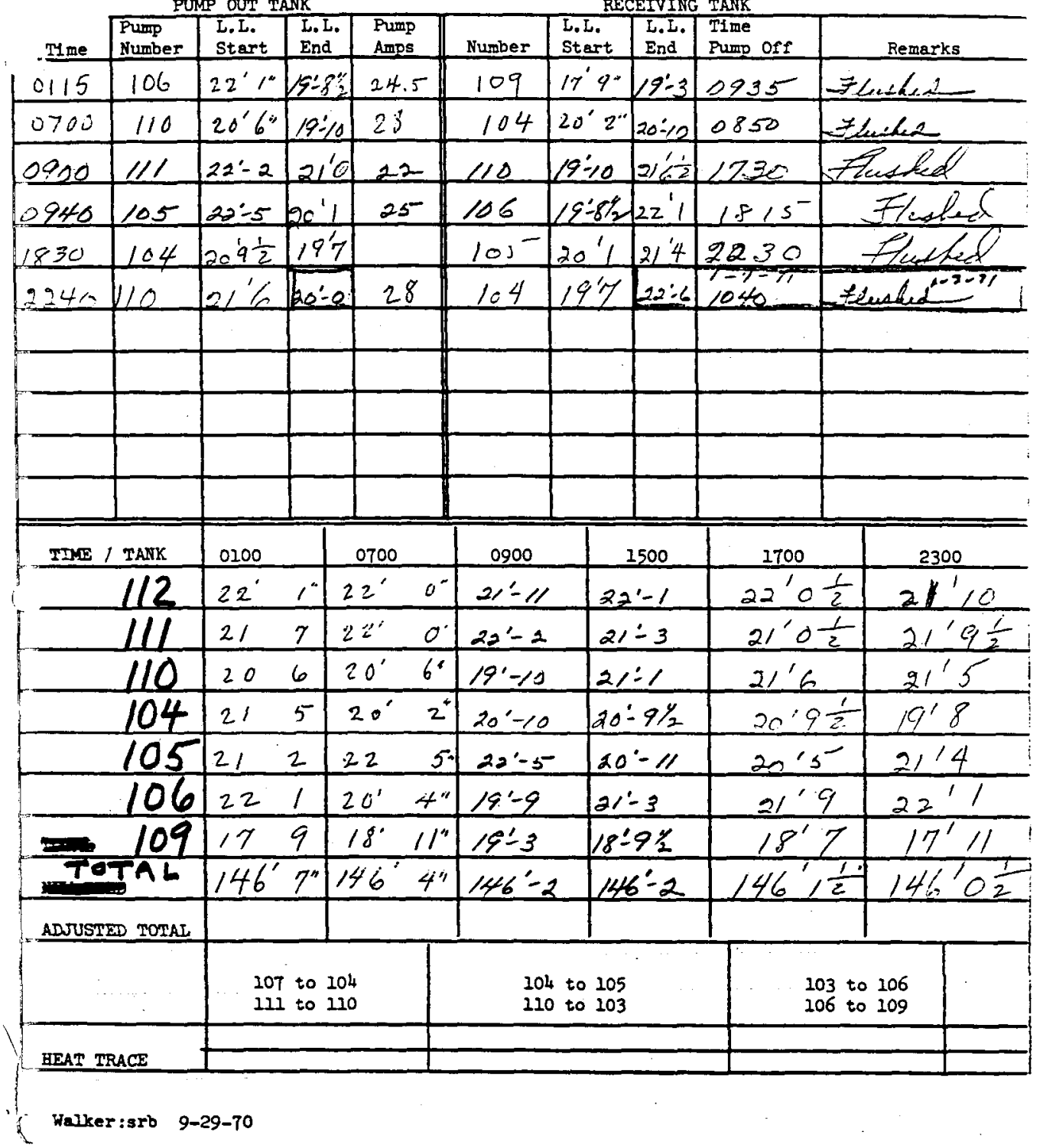

6 
PUMP OUT TANK

RECEIVING TANK

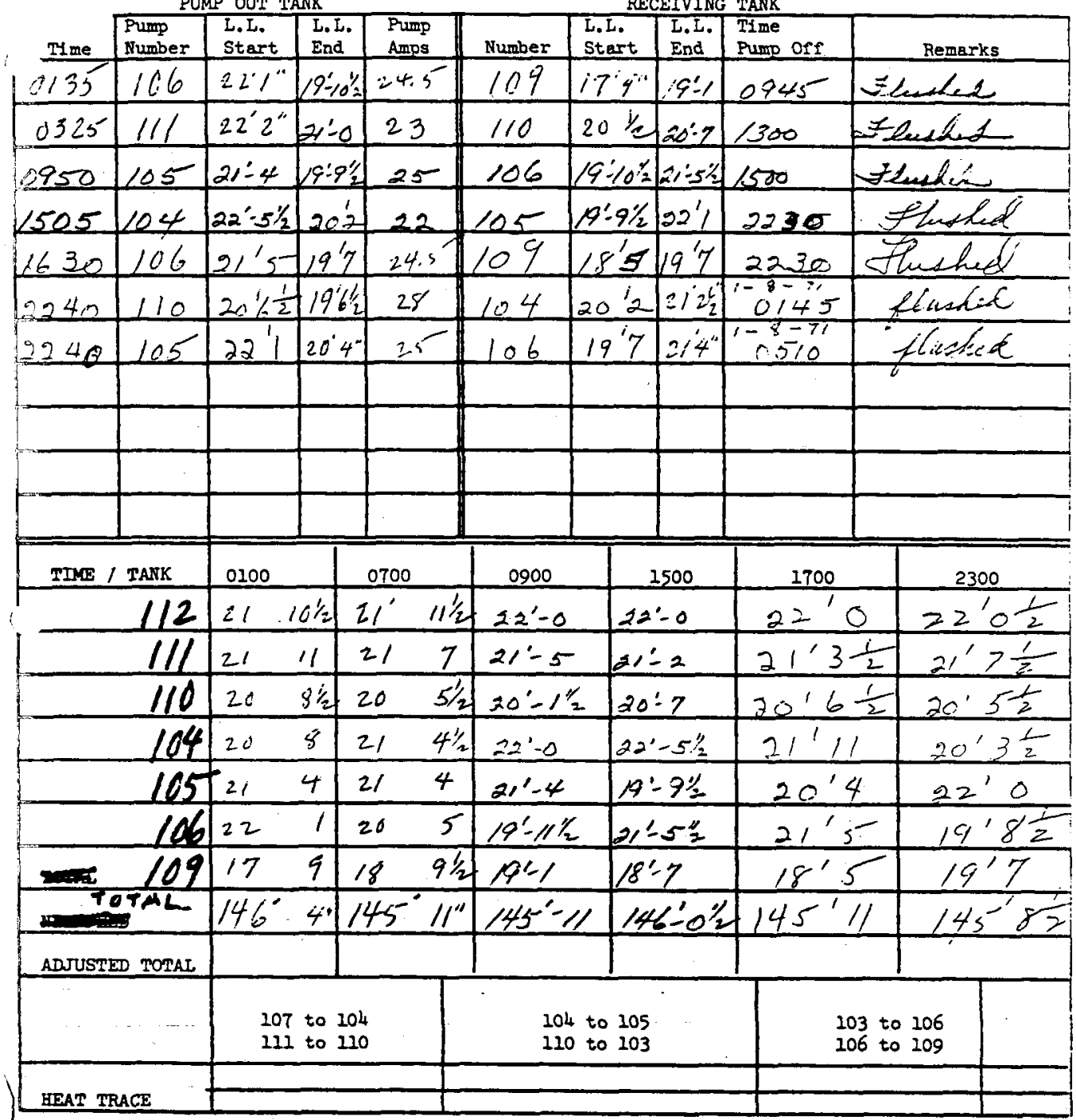

Wavker:srb 9-29-70 
PUMP OUT TANK

RECEIVING TANK

\begin{tabular}{l|l|l|l|l|l|l|l|l|}
\cline { 2 - 4 } & $\begin{array}{l}\text { Pump } \\
\text { Number }\end{array}$ & $\begin{array}{l}\text { L.L. } \\
\text { Start }\end{array}$ & $\begin{array}{l}\text { L.L. } \\
\text { End }\end{array}$ & $\begin{array}{l}\text { Pump } \\
\text { Amps }\end{array}$ & Number & $\begin{array}{l}\text { L.L. } \\
\text { Sttart, }\end{array}$ & $\begin{array}{l}\text { L.L. } \\
\text { End }\end{array}$ & $\begin{array}{l}\text { Time } \\
\text { Pump off }\end{array}$ \\
\hline
\end{tabular}

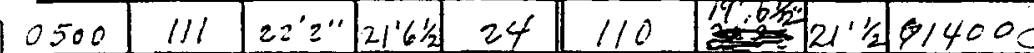

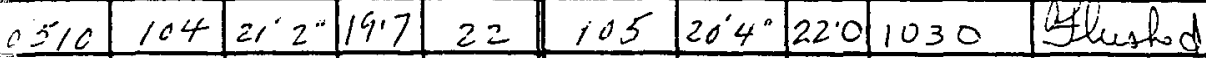

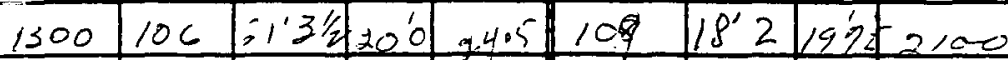

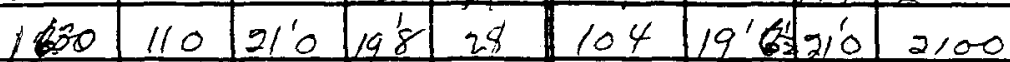

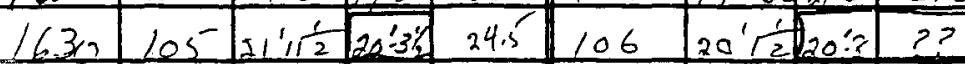

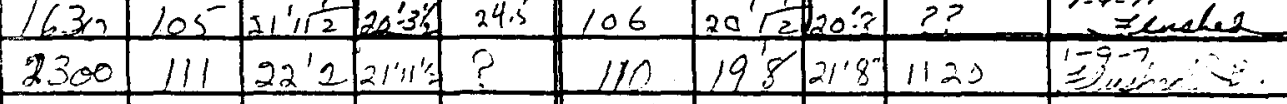

\begin{tabular}{|c|c|c|c|c|c|c|}
\hline TIYE $/$ TANK & 0100 & 0700 & 0900 & 1500 & 1700 & 2300 \\
\hline 112 & $22 \quad 2$ & $22^{\circ} 2^{\prime \prime}$ & $22^{\prime} 0$ & $22^{\prime}, " \prime$ & $22^{\prime} /$ & 222 \\
\hline lll & $21 \quad 10 \frac{1}{2}$ & 22 & $=2^{\prime} 11^{\prime \prime}$ & $21^{\prime \prime} 8^{\prime \prime}$ & 2119 & $22^{\prime} 2$ \\
\hline 110 & 198 & 19 & $\therefore 0^{\prime} 11 / 4$ & 21.0 & $21^{\prime} C^{-1}$ & $19^{\prime} E$ \\
\hline 104 & 21 & $20 \quad 91 / 2$ & $20 \% 1 \%$ & $19^{\prime} 6 \%$ & $19^{\prime} 7$ & $20^{\prime} / 1 \frac{1}{2}$ \\
\hline 105 & 212 & 209. & $21^{\prime} 5^{\prime \prime \prime}$ & $=1^{\prime} 11 \%$ & $2,11 \frac{1}{2}$ & $20^{\prime} 4$ \\
\hline & $20 \quad 6$ & $21^{\prime} 4^{\prime \prime}$ & $213 \frac{1}{2}$ & $207 \frac{1}{2}$ & $20^{\prime} 1 \frac{1}{2}$ & $20^{\prime} 6$ \\
\hline 169 & $19 \quad 4$ & $18^{\prime} \quad 9 \%$ & $18^{\prime} 7^{\prime \prime}$ & $18^{\circ} 7^{\prime \prime}$ & $18^{\prime} 11 \frac{1}{2}$ & $19^{\prime} 6 \frac{1}{2}$ \\
\hline$T \cdot 746$ & $145^{\prime} 8 \%$ & $14588^{\prime \prime}$ & $145^{17} \cdot \frac{1}{2}$ & $145+1, \frac{1}{2}$ & $145^{\prime} 5 \frac{1}{2}$ & $145^{-3} 3$ \\
\hline ADJUSTED TOTAL & & & & & & \\
\hline & $\begin{array}{l}107 \text { to } \\
111 \text { to }\end{array}$ & $\begin{array}{l}104 \\
110\end{array}$ & $\begin{array}{l}104 \text { to } \\
110 \text { to }\end{array}$ & $\begin{array}{l}105 \\
103\end{array}$ & $\begin{array}{l}103 \text { to } \\
106 \text { to }\end{array}$ & $\begin{array}{l}106 \\
109\end{array}$ \\
\hline HEAT TRACE & & & & & & \\
\hline
\end{tabular}

L Walker:srb 9-29-70

$\alpha$, Go100

$\dot{x}<-200^{\circ}-1 / H_{2}$

$365-20 \div 312$

$106-20: 8$ 
PUMP OUT TANK

RECEIVING TANK

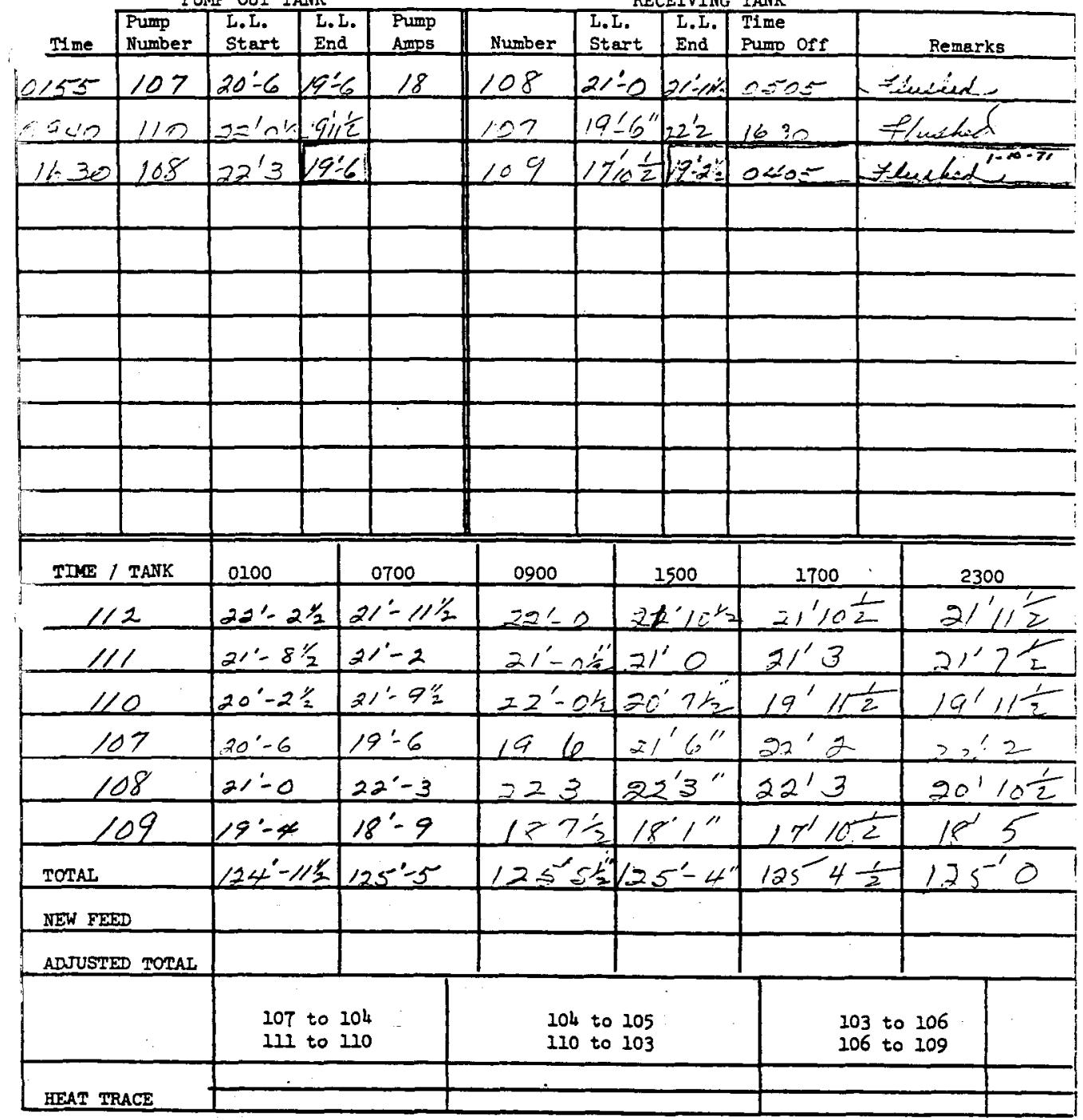

\section{if Walker:srb 9-29-70}

the ceodo

; $45-20011 \%$ 126

$5-20.5 \frac{5}{2}: 105$

$6-20^{\circ}-8$ : 
PUMP OUT TANK

RECEIVING TANK

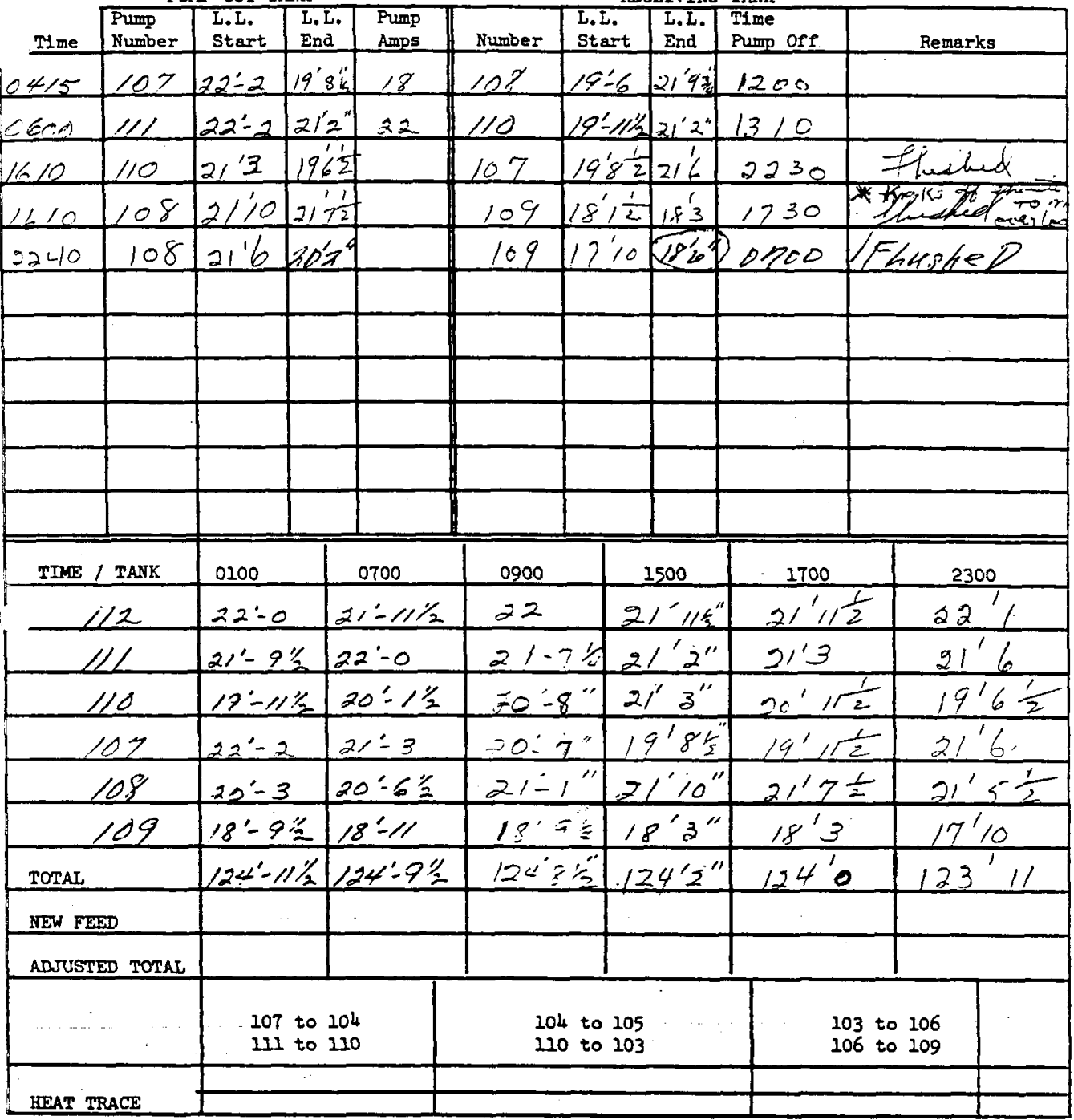

Walker:srb 9-29-70 
ITS-2 INVENTORY AND PUNPING Date $<-1 /$

PUMP OUT TANK RECEIVING TANK

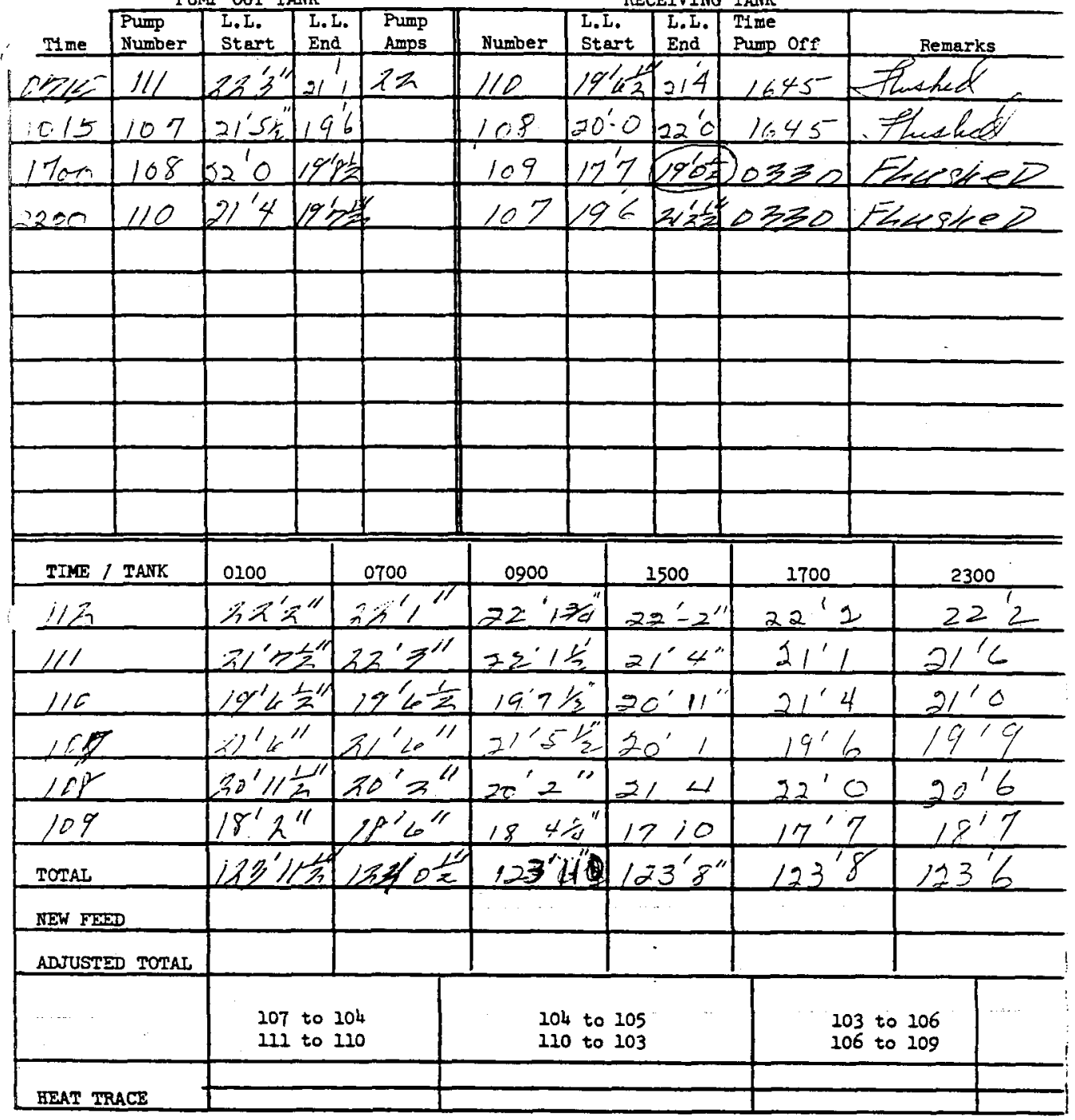

Wayker:srb 9-29-70 
PUMP OUT TANK

RECEIVING TANK

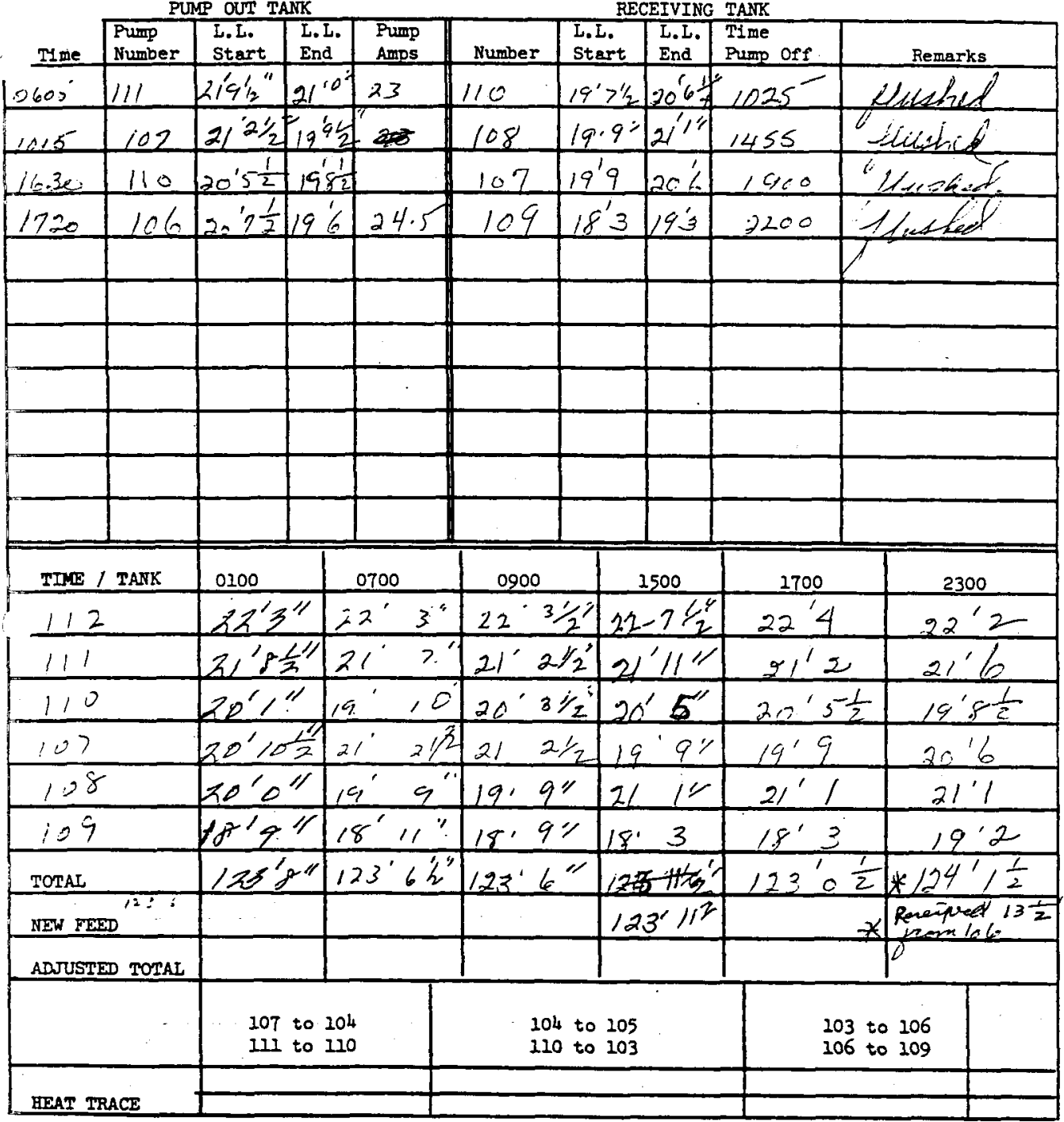

(Walker:srb 9-29-70 
PUMP OUT TANK

RECEIVING TANK

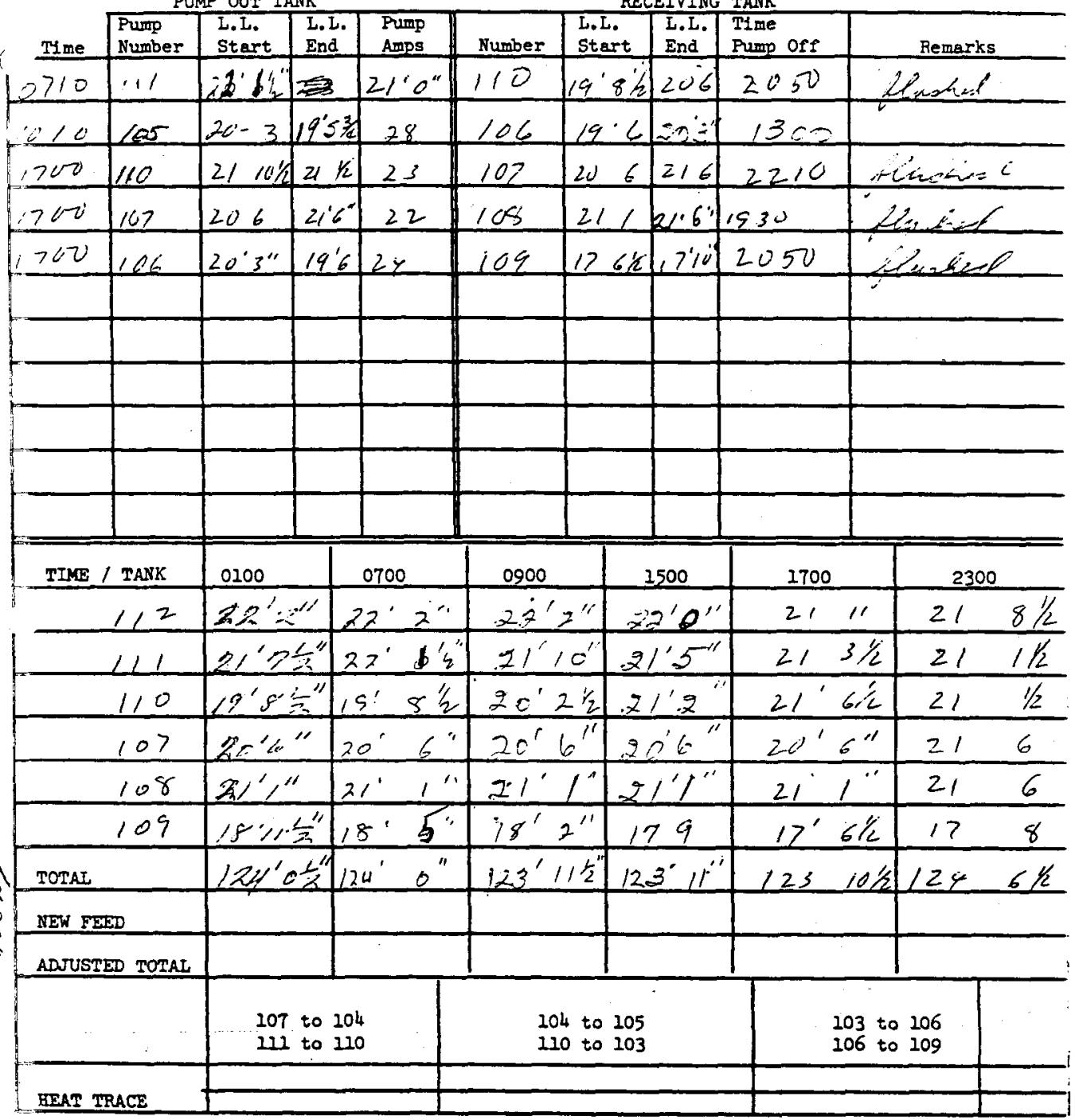

Walket:sxb 9-29-70 
RECEIVING TANK

PUMP OUT TANK

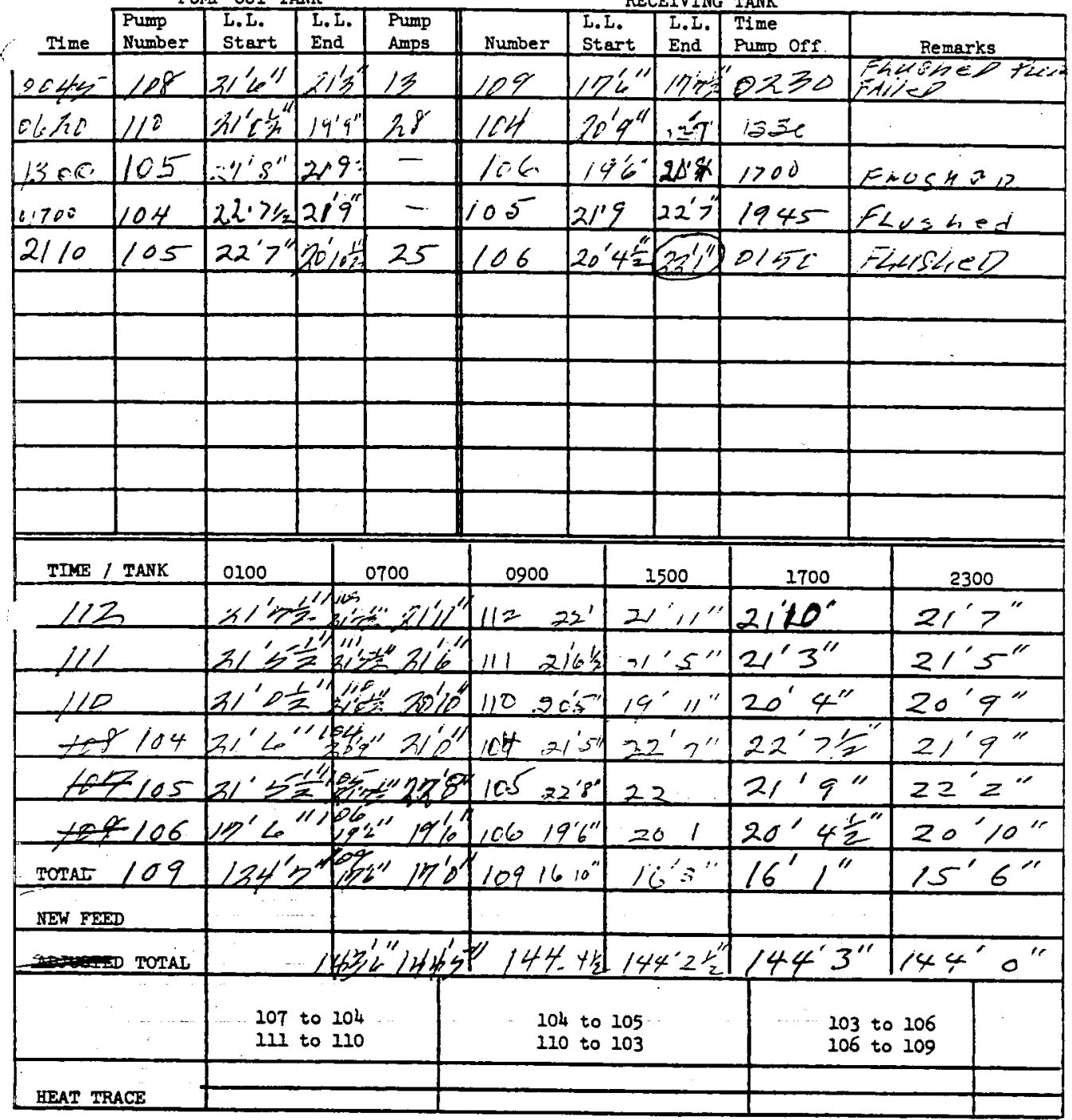

1. Walker:srb 9-29-70 
PUMP OUT TANK

RECEIVING TANK

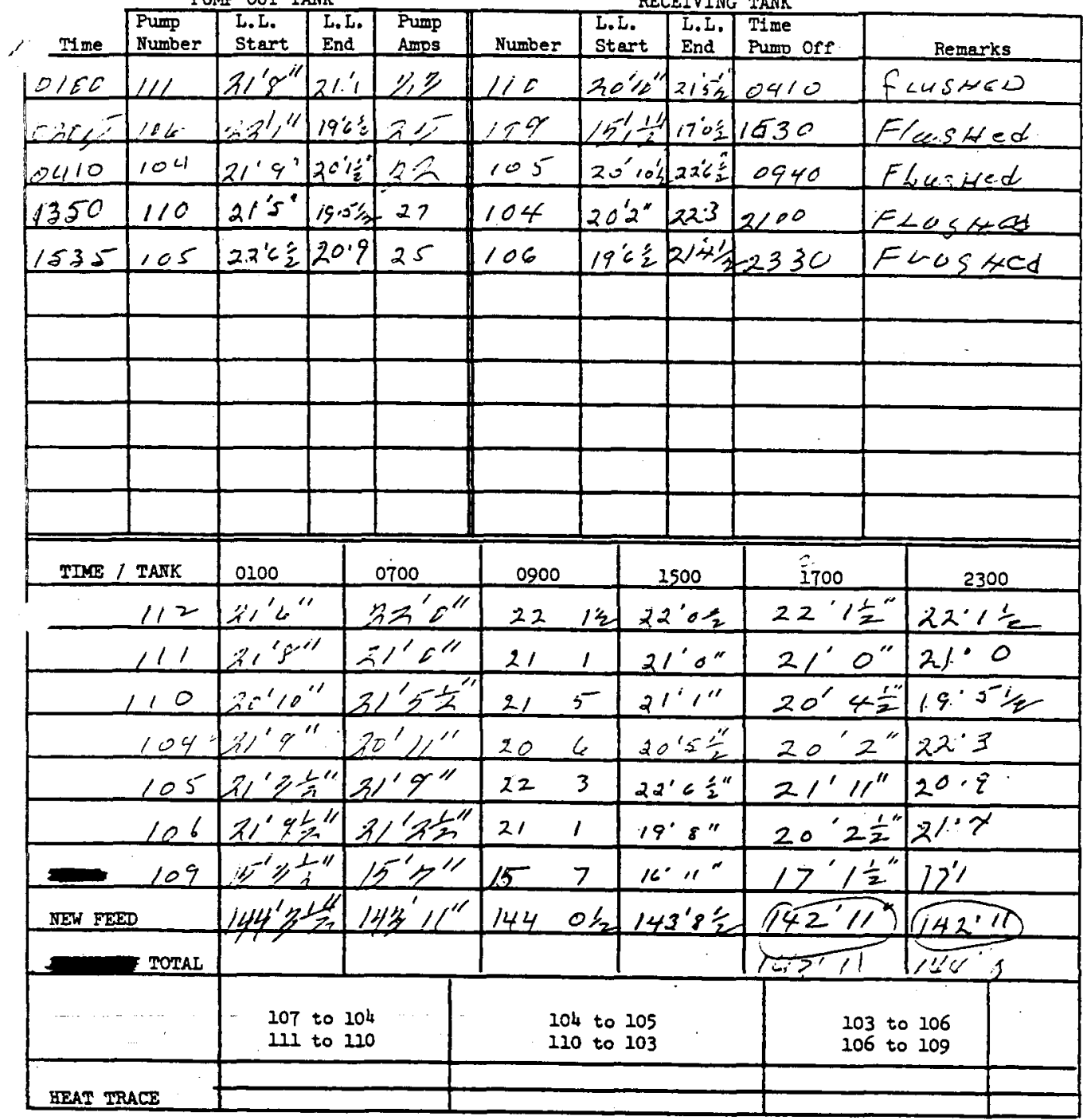

1 Walker: 9-29-70 
PUMP OUT TANK

RECEIVING TANK

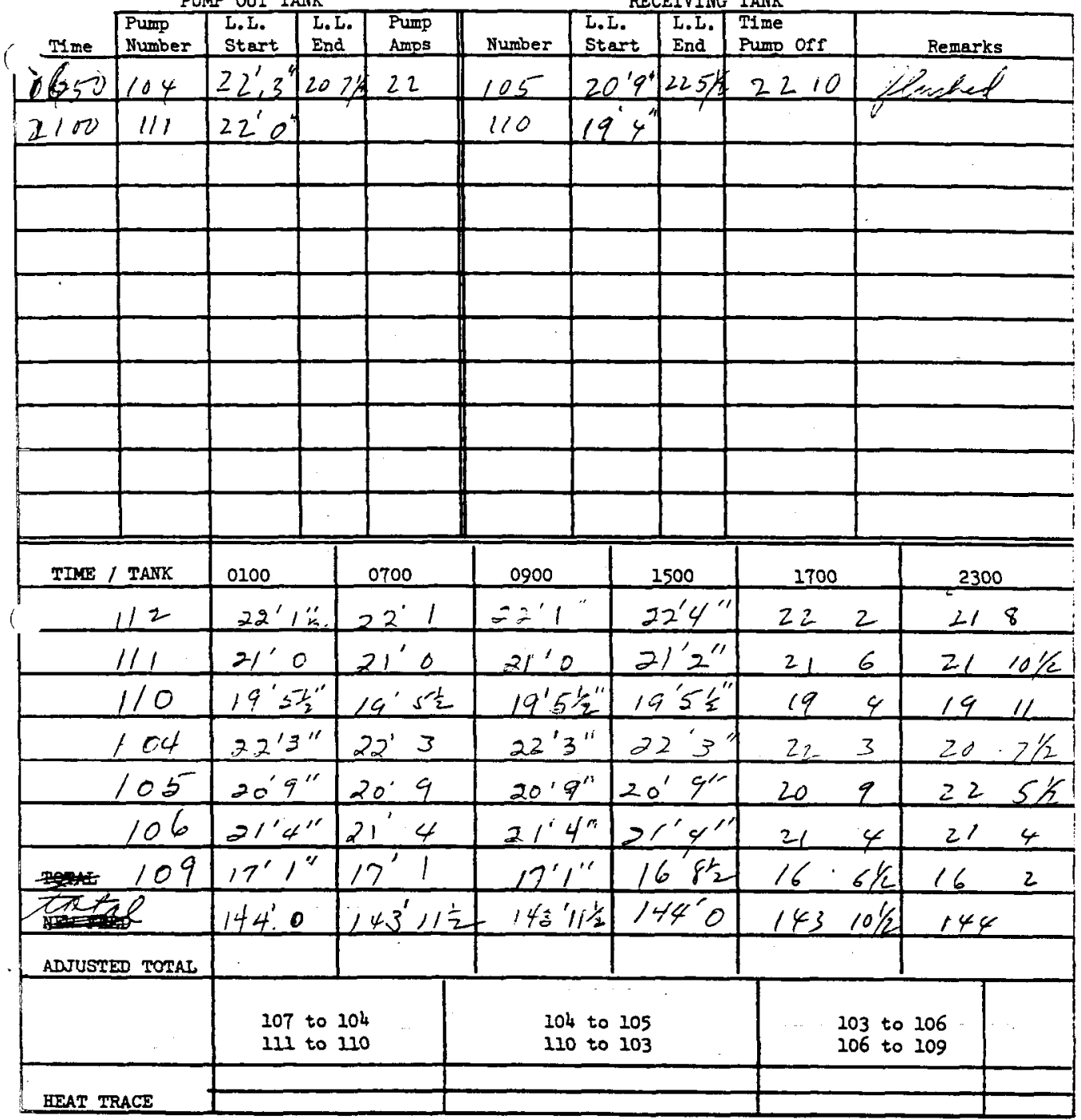

Halker:srb 9-29-70 
PUMP OUT TANK

RECEIVING TANK

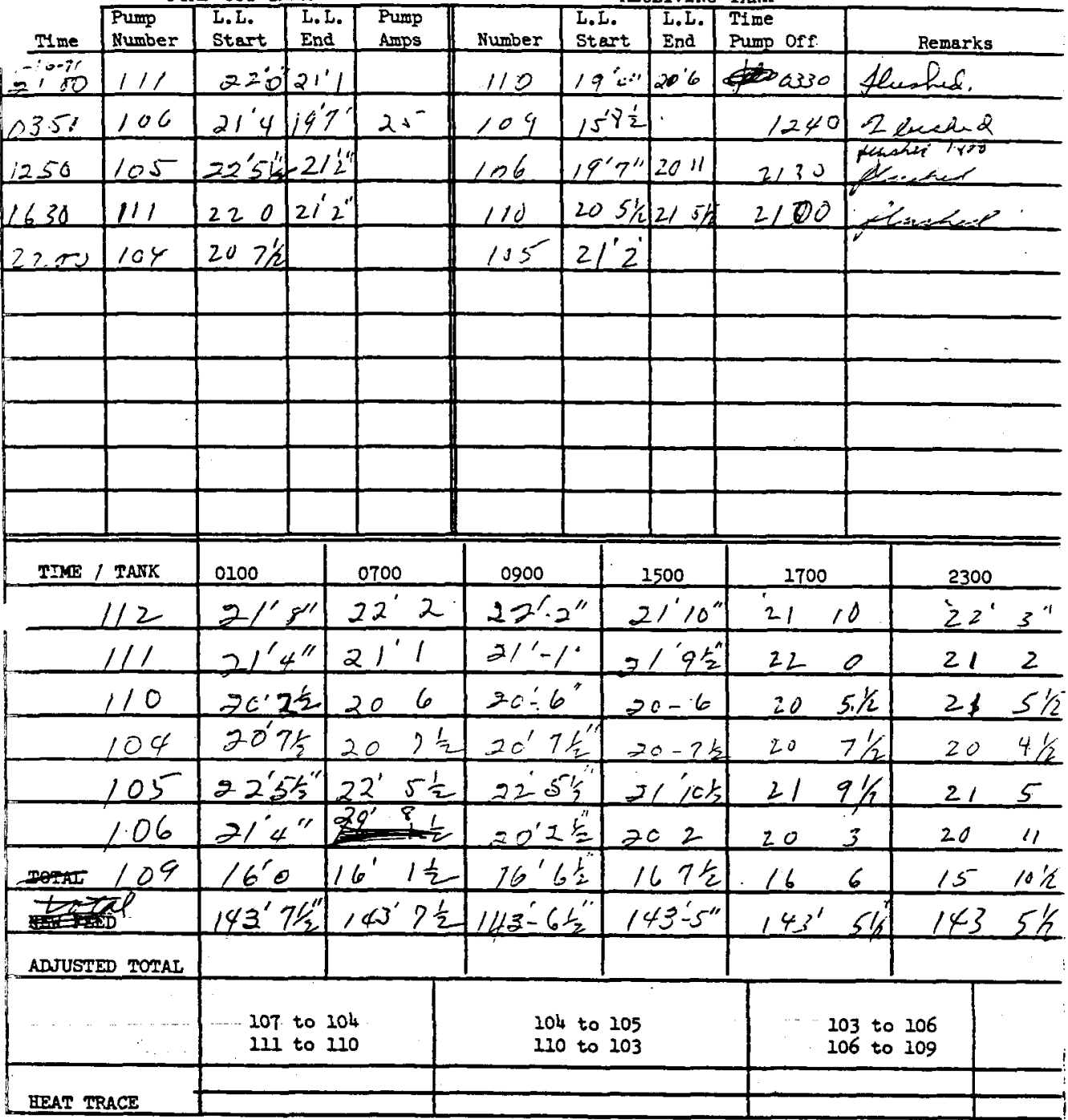

5 fo at 1045

Walker:srb 9-29-70 
PUMP OUT TANK

RECEIVING TANK

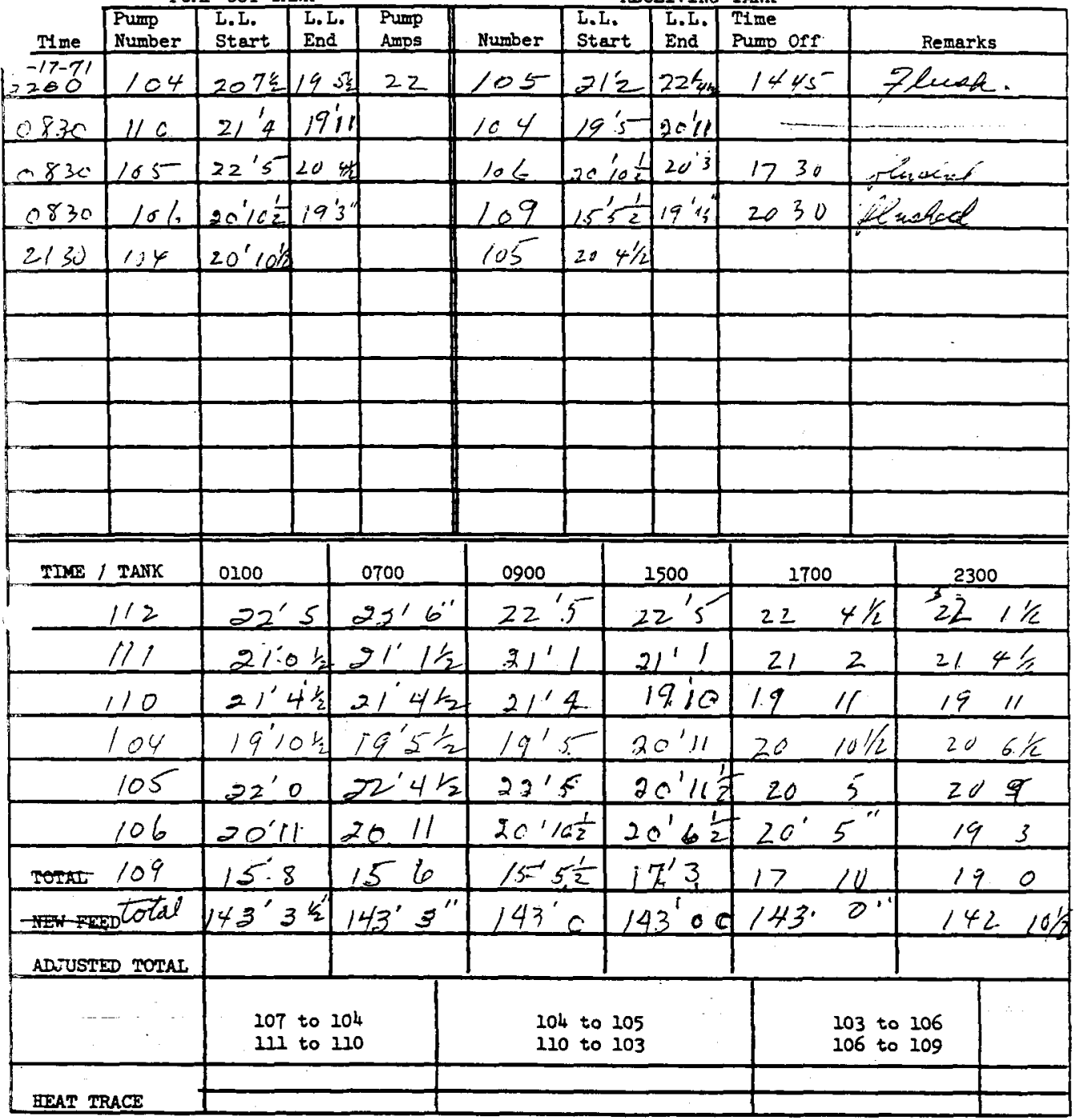

Walker :srb 9-29-70 
PUMP OUT TANK

RECEIVING TANK

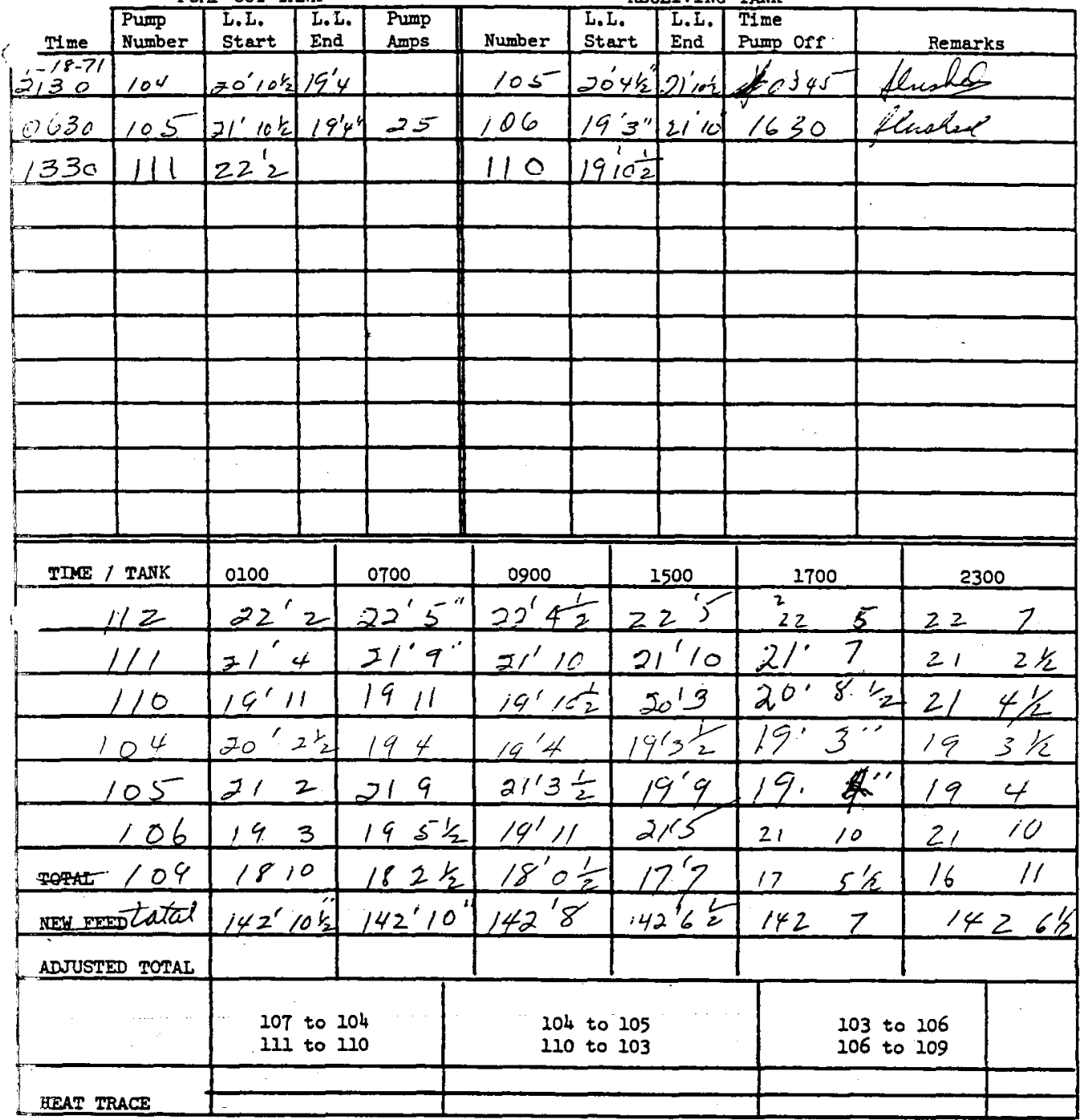

Wajker:srb 9-29-70 
PUMP OUT TANK RECEIVING TANK

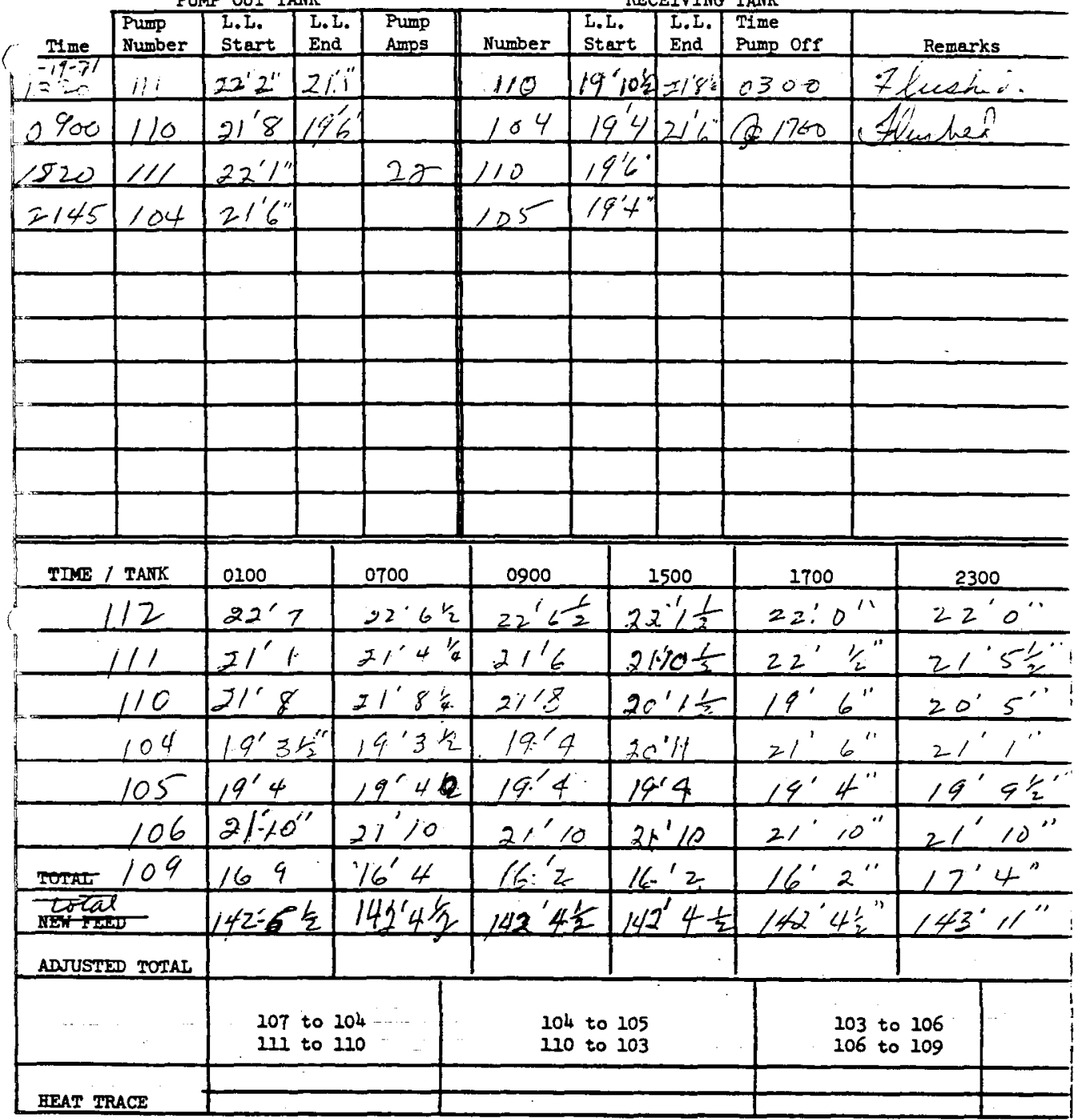

Walker:srb 9-29-70 
PUNP OUT TANK

RECEIVING TANK

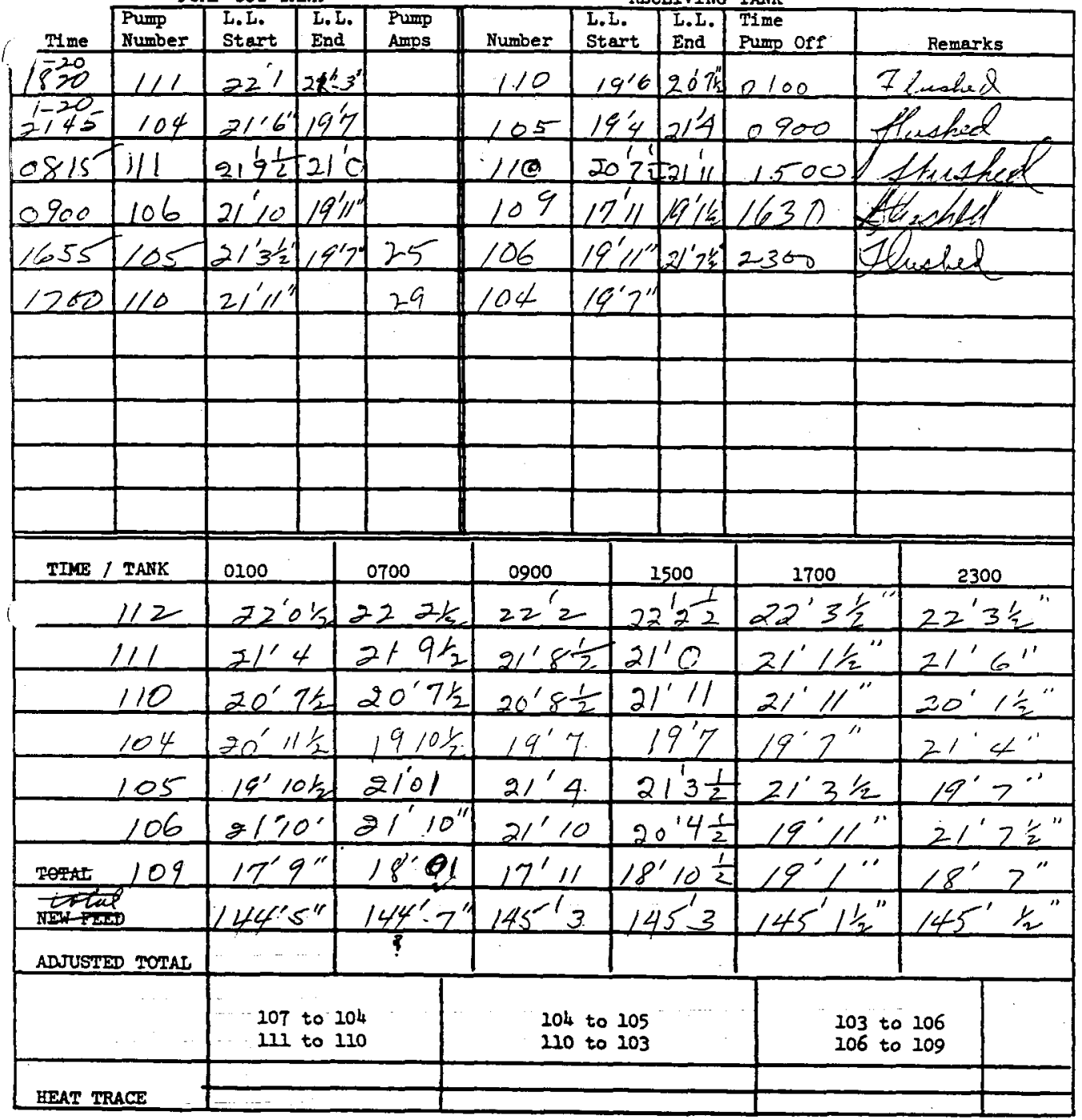

(Yauker:srb 9-29-70 
WHC-50-WM-OP-209, ReV. O

ITS-2 INVENTORY AND PUMPING

Date $1-\geq 2->0$

PUMP OUT TANK

RECEIVING TANK

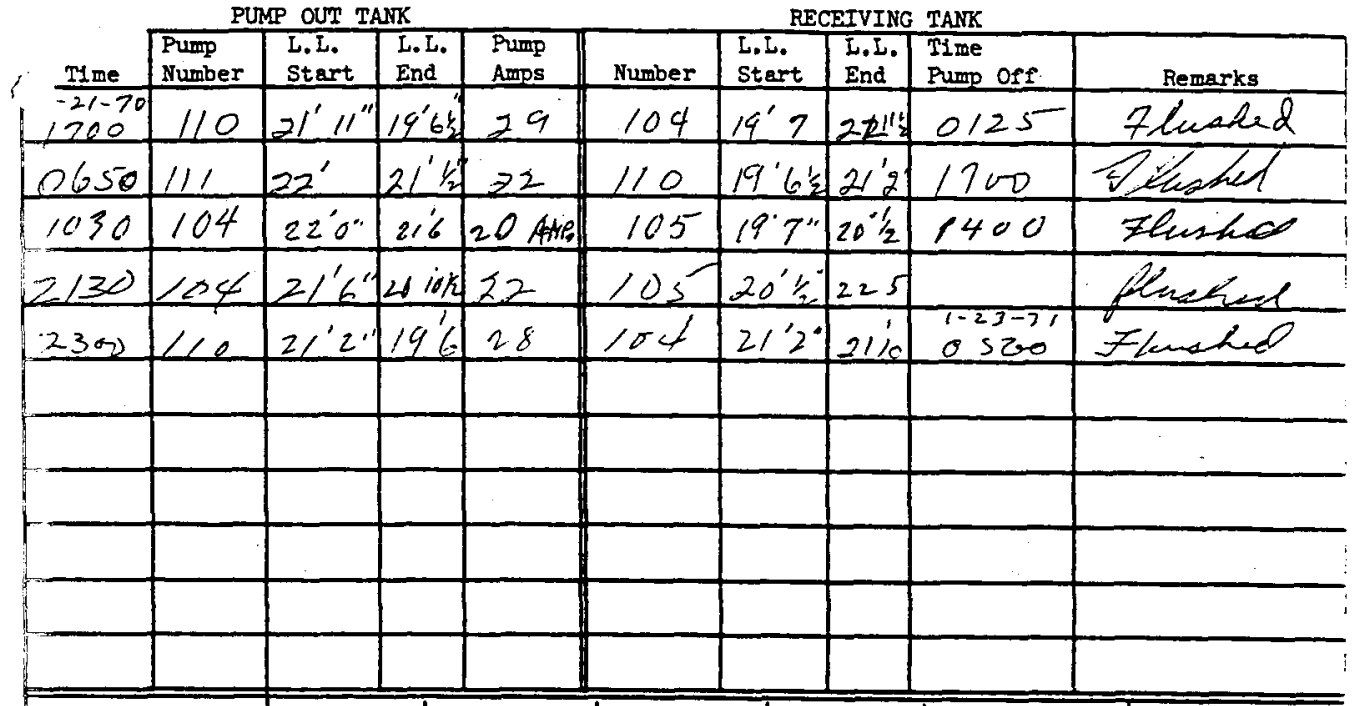

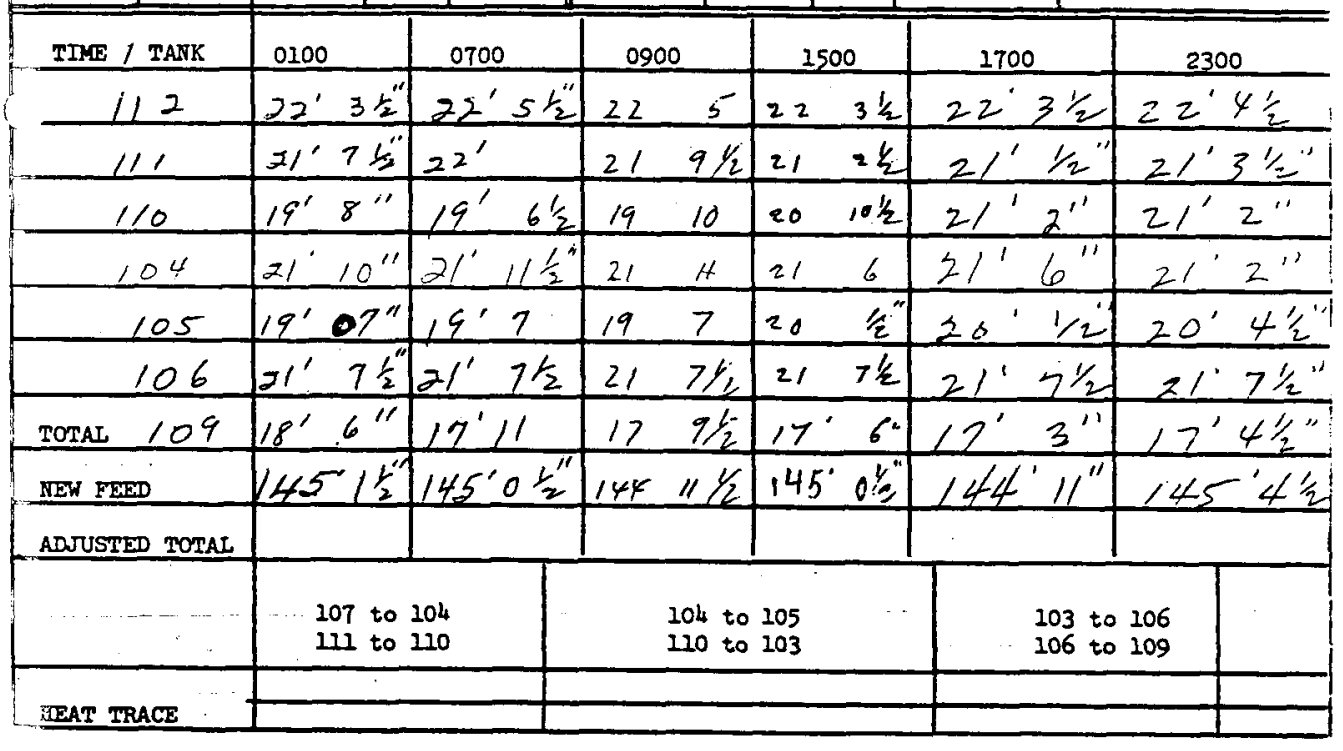

$f^{\circ}$ WaIker:srb 9:29-70

aver

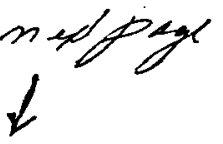

22 


$$
\text { ITS-2 INVENTORY AND PUMPING Date } 1-23-71
$$

PUMP OUT TANK RECEIVING TANK

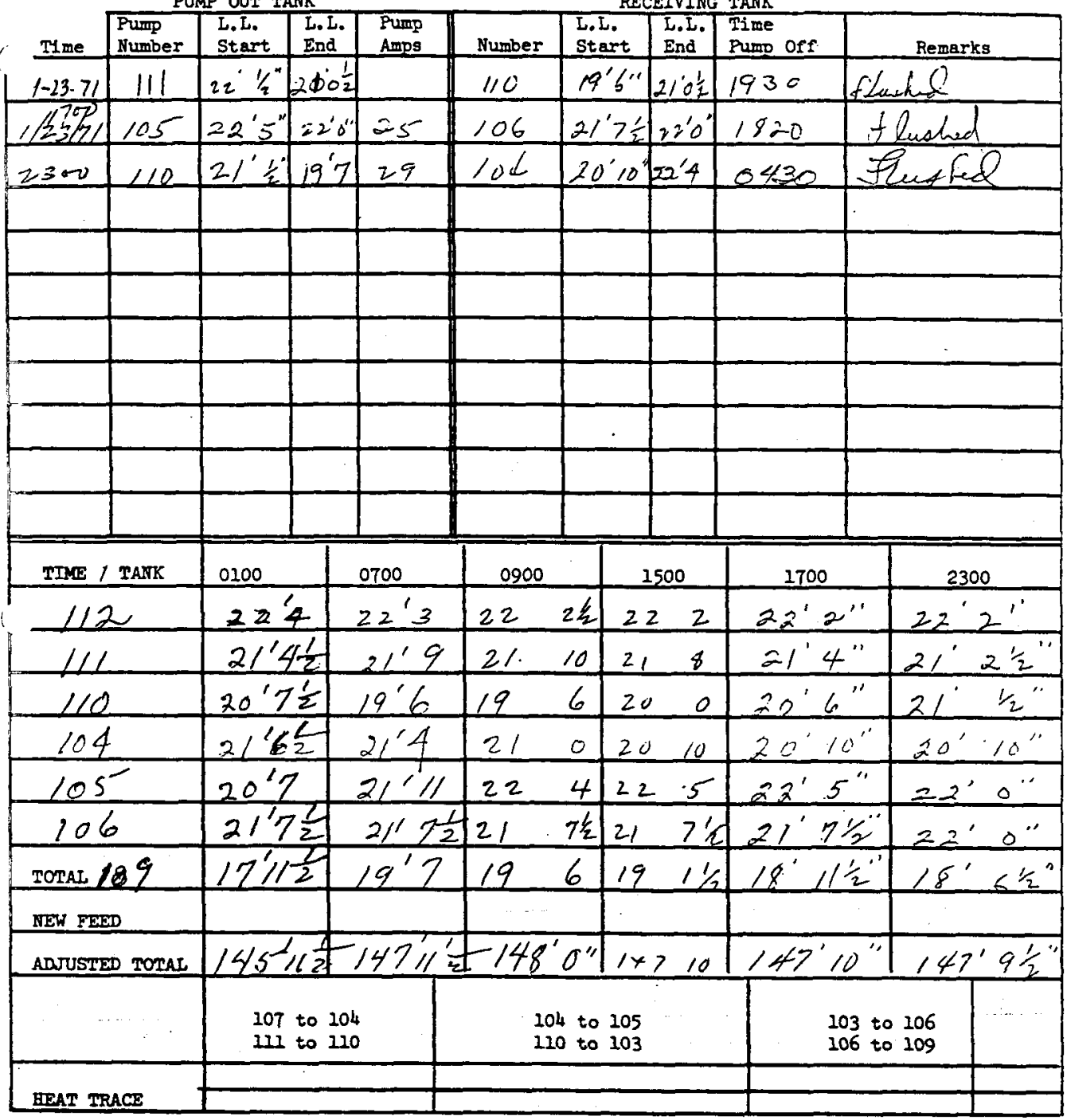

Walker:srb 9-29-70 
PUMP OUT TANK

RECEIVING TANK

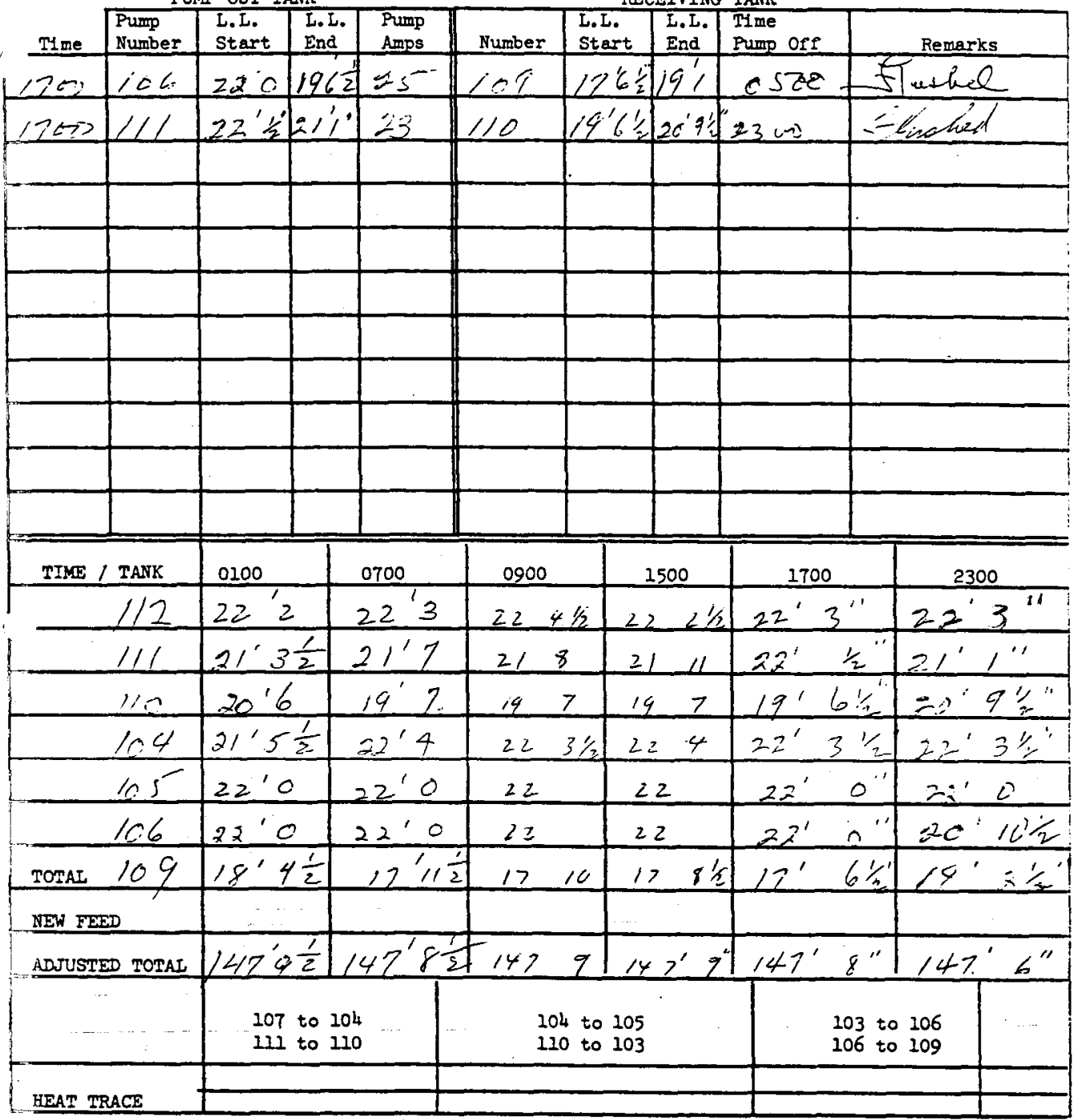

(2. Walker:srb 9-29-70 
PUMP OUT TANK

RECEIVING TANK

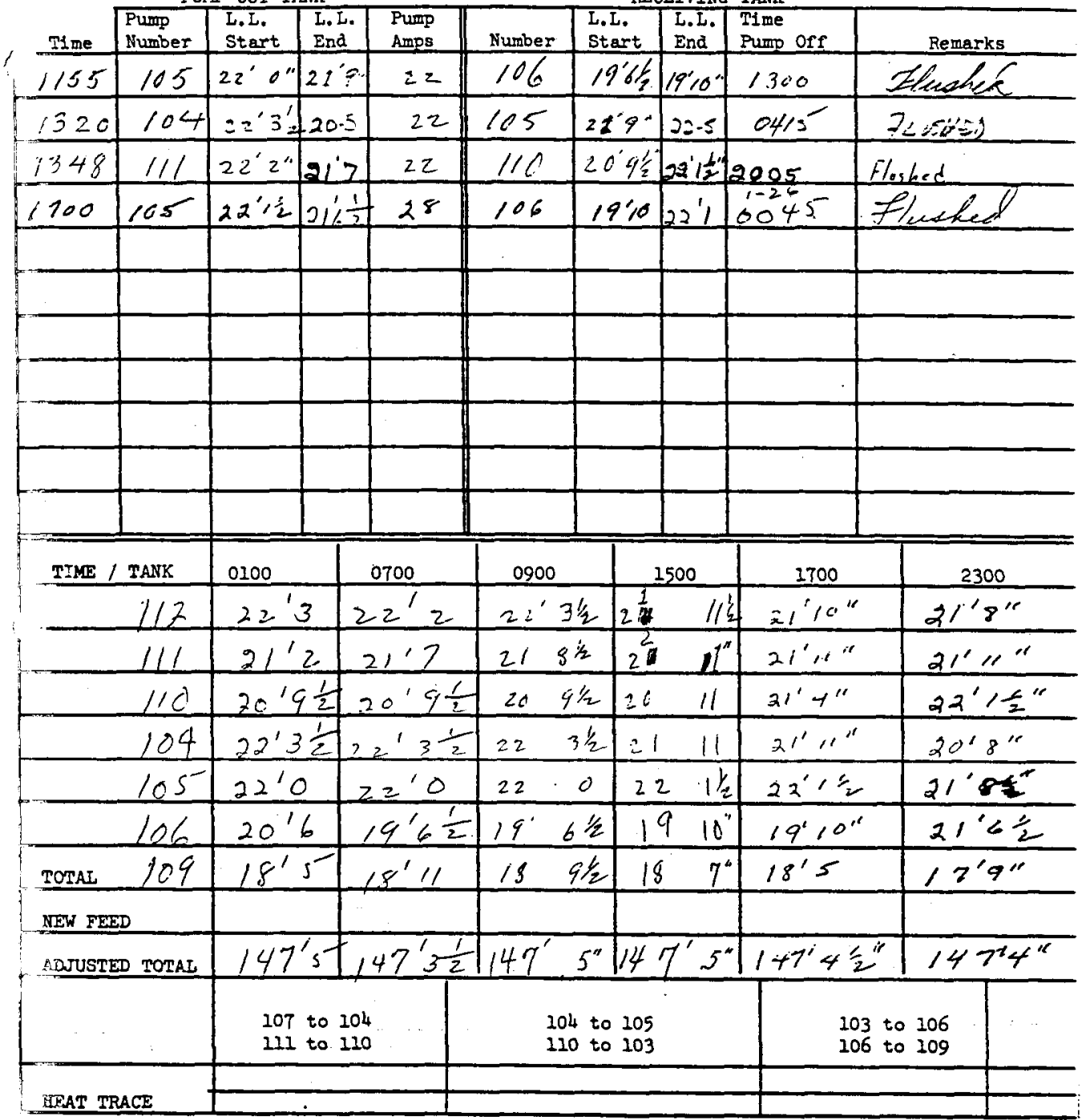

Walker:srb 9-29-70 
PUMP OUT TANK

RECEIVING TANK

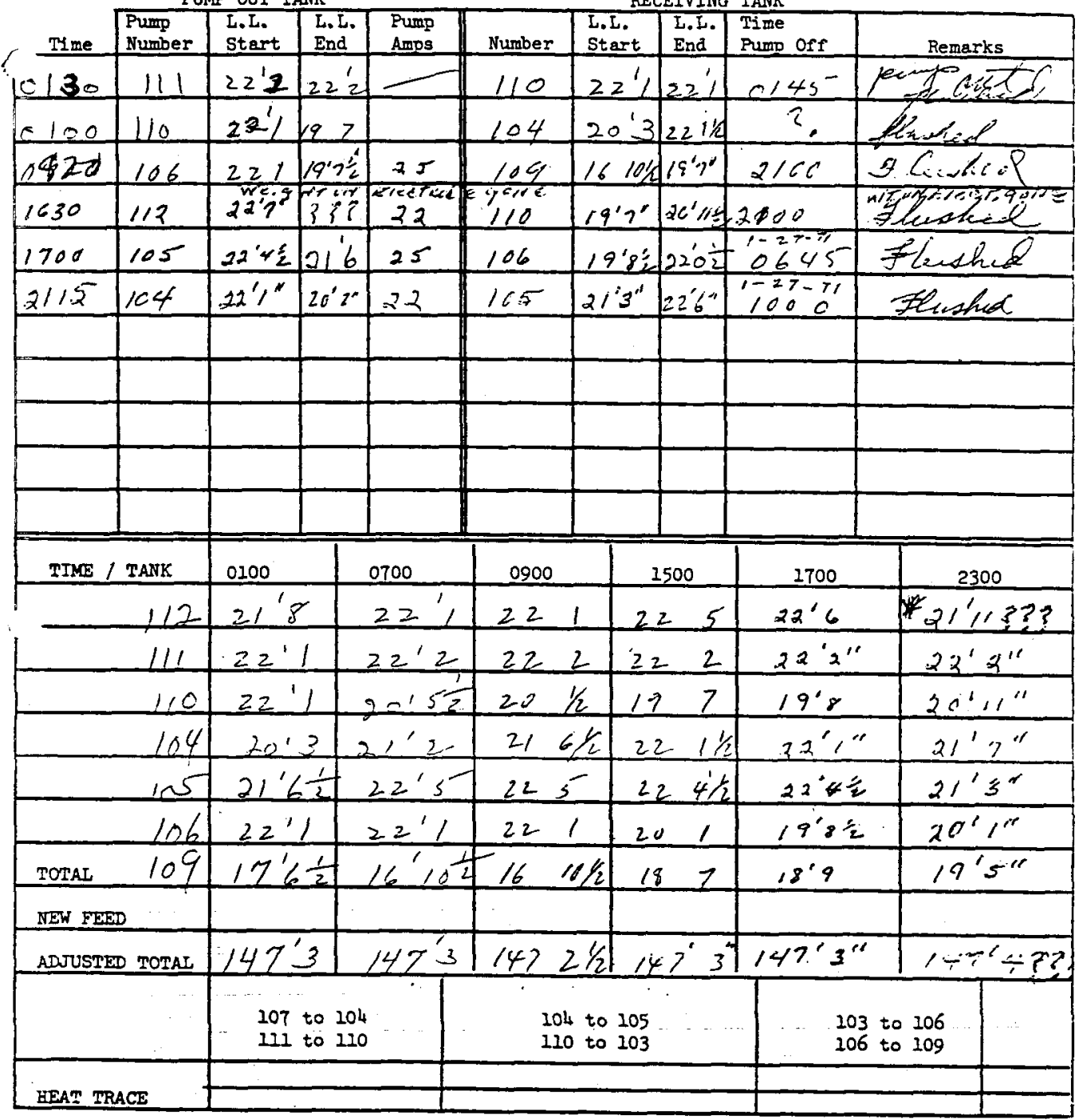

no Wayker:srb 9-29-70

* Electrode wost ENo i R Welght ?. APPROX 21'" 
PUMP OUT TANK

RECEIVING TANK

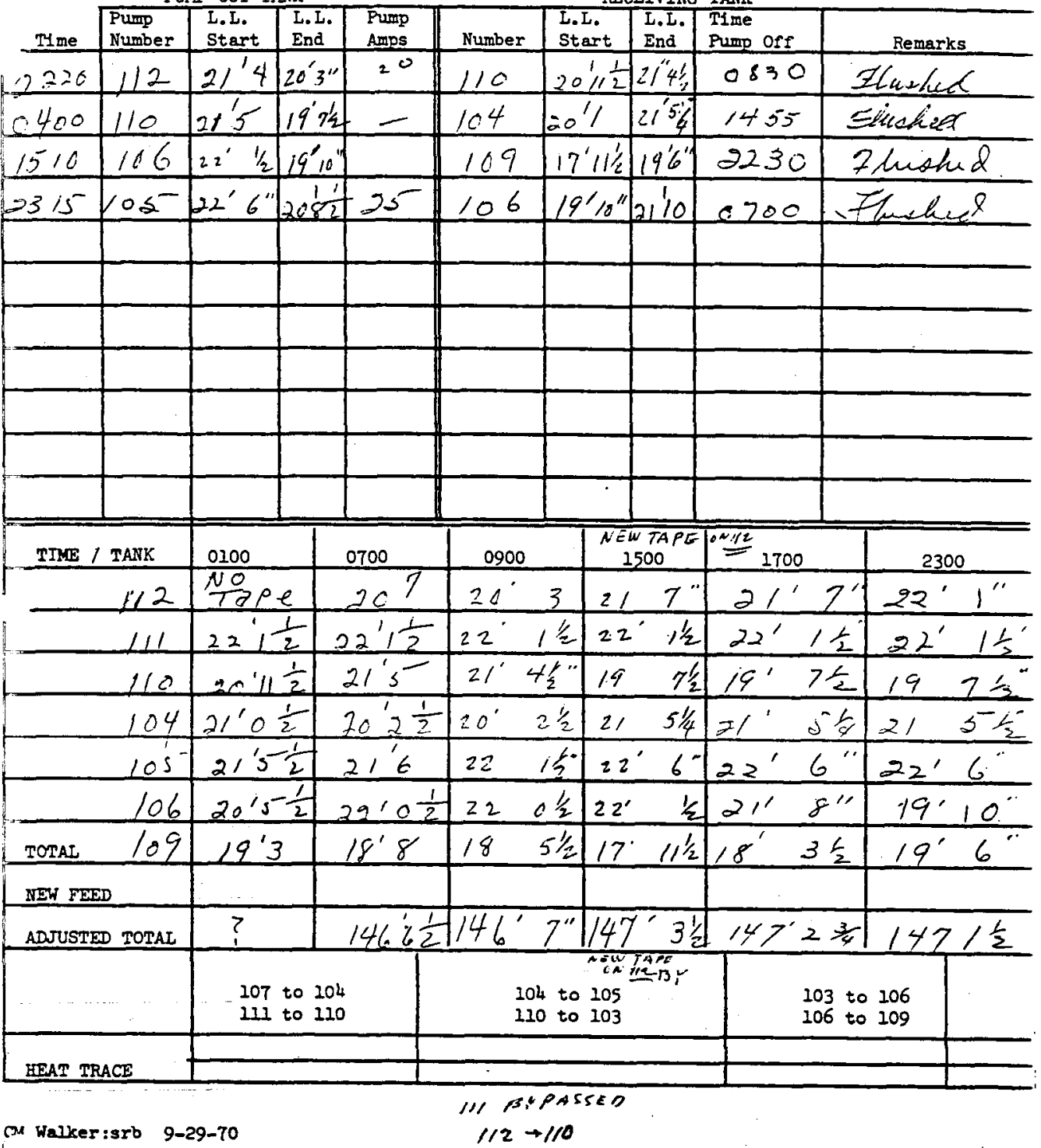


WHC-SD-LM-DP-209, REV. O

ITS-2 INVENTORY AND PUMPING

Date

IAS 2 8

PUMP OUT TANK

RECEIVING TANK

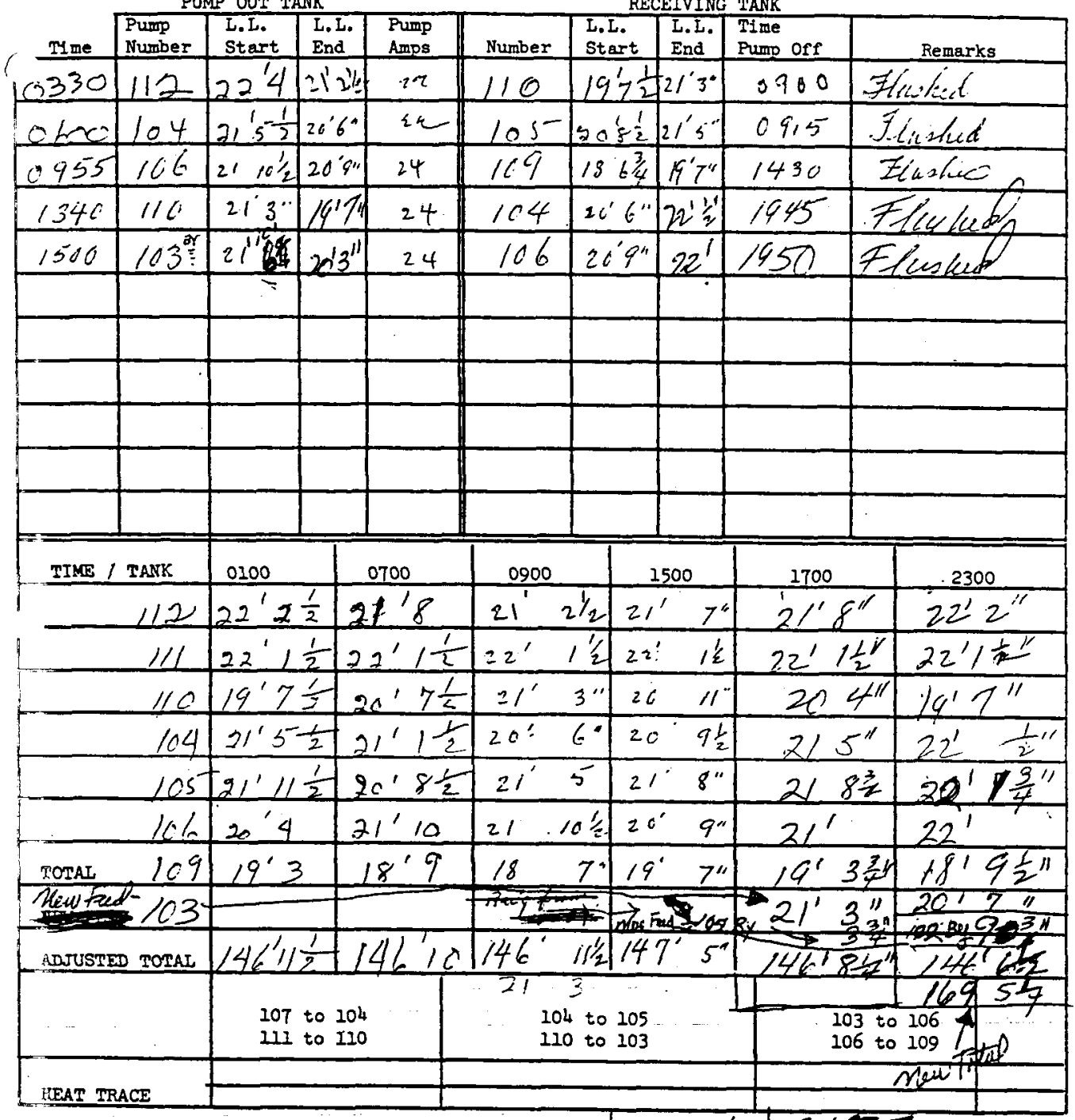

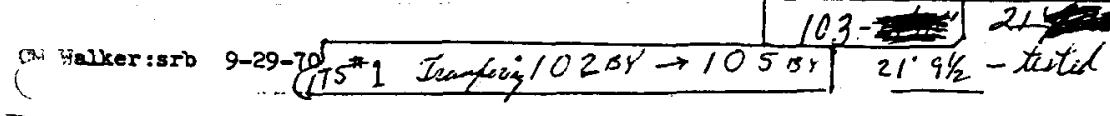

(*) 2300 Tot ip

28

Fer adelectrom 10218y 
WHC-SD-LM-DP-209, ReV. 0

ITS-2 INVENTORY AND PUMPING

Date JAI291971

PUMP OUT TANK

RECEIVING TANK

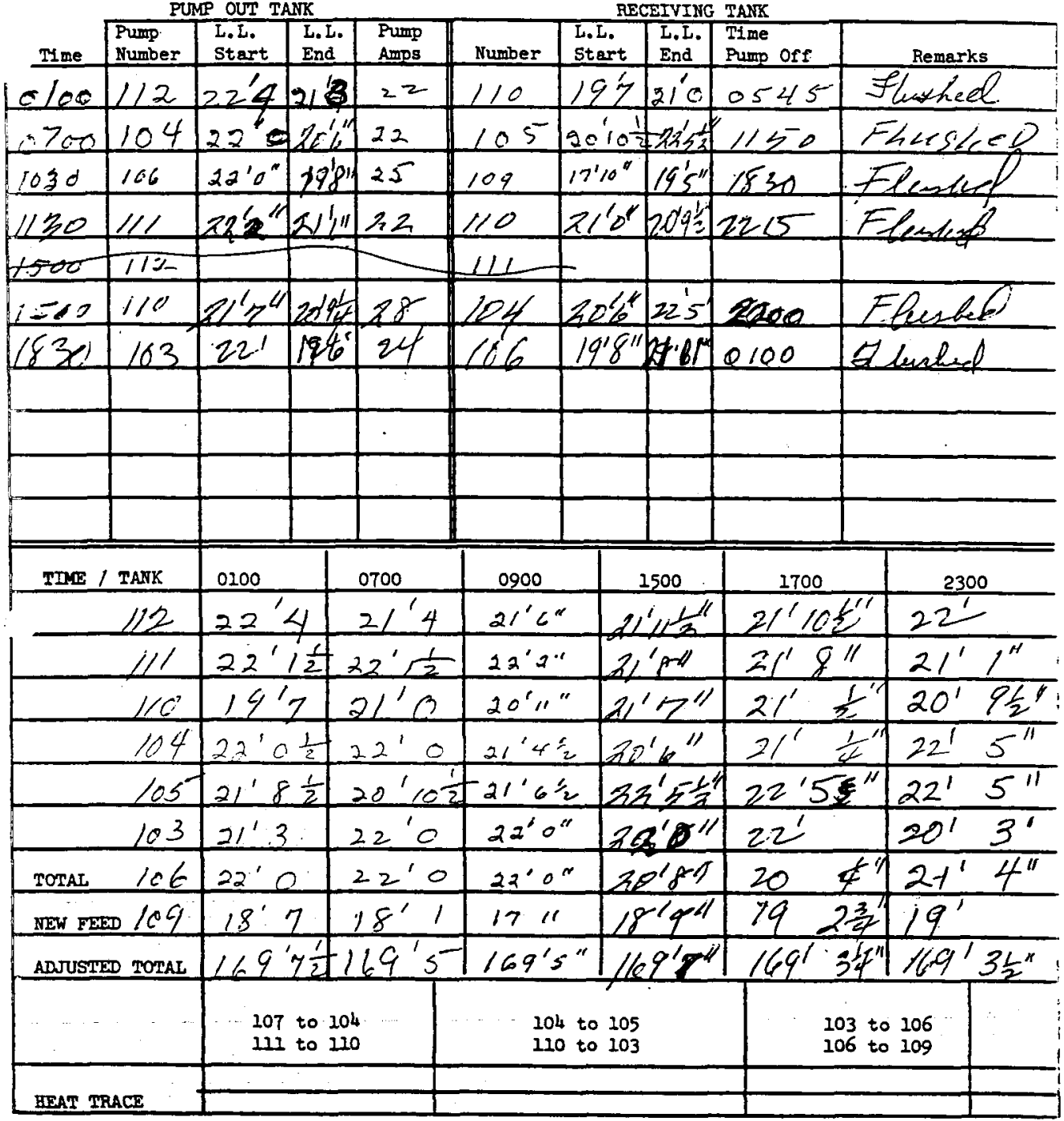

[Walker:srb 9-29-70

29 
WHC-SD-WM-DP-209, Rev. 0

ITS-2 INVENTORY AND PUMPING

Date 1-30-7L

PUMP OUT TANK

RECEIVING TANK

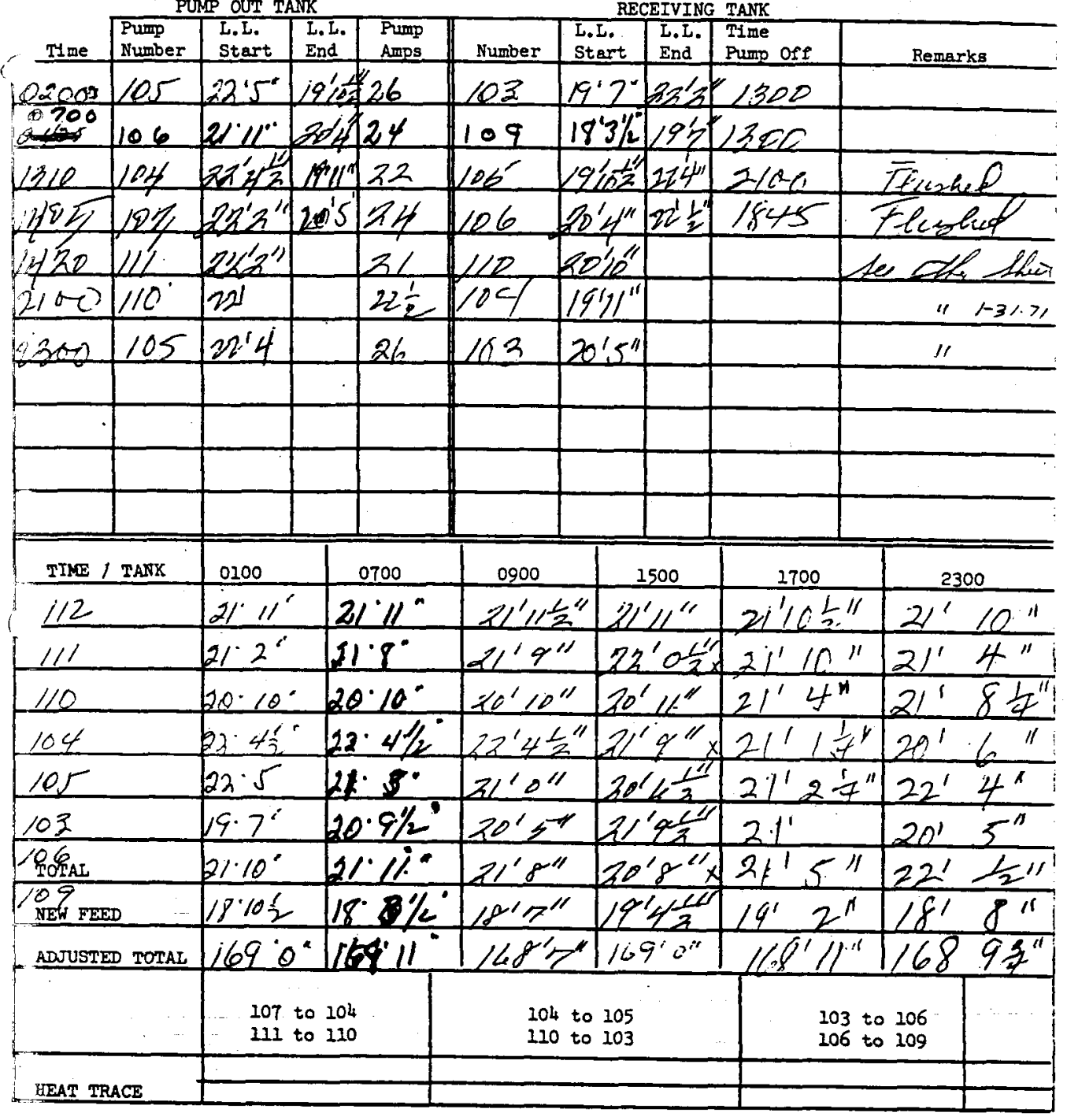

Walker:srb 9-29-70

$7=33$

30 
WHC-SD-LAM-DP-209, REV. 0

ITS-2 INVENTORY AND PUMPING

Date $|-3|-7 \mid$

PUMP OUT TANK

RECEIVING TANK

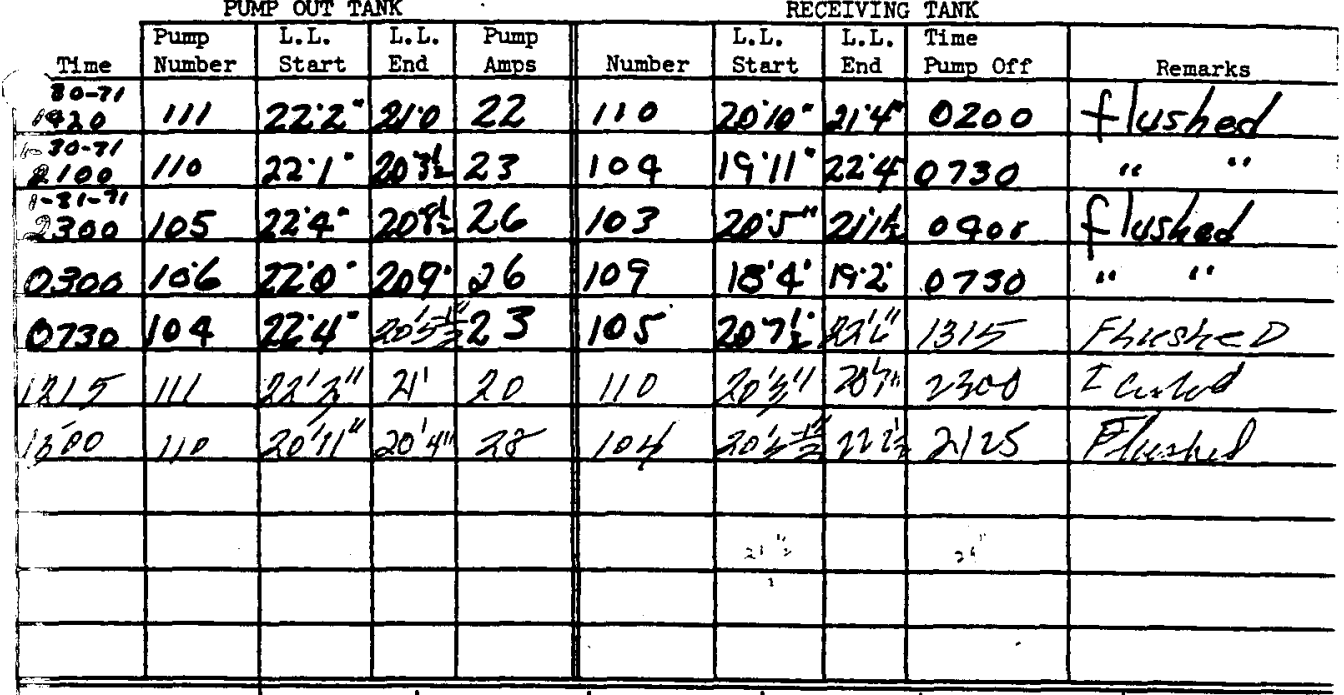

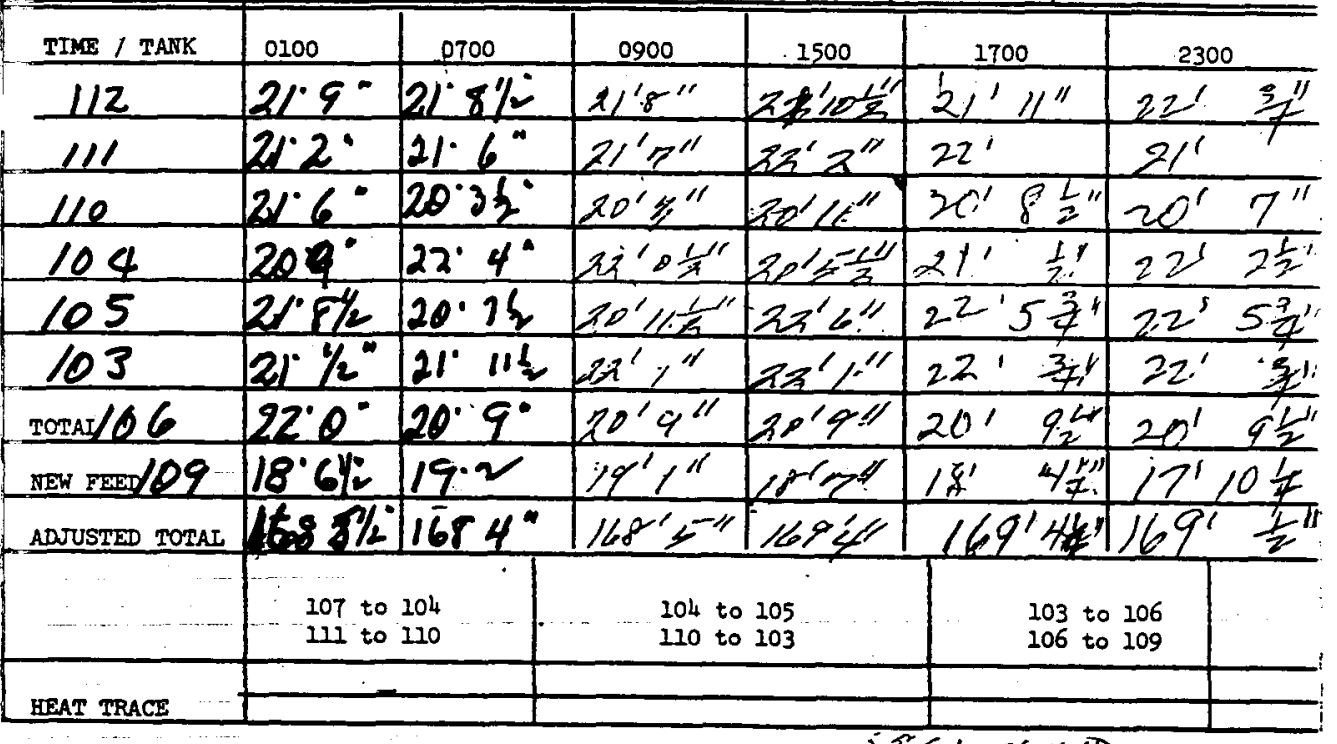

Palker:srb 9-29-70

$\left(a^{2} 198^{\prime} 4^{\prime}\right)$

31. 
PURP OUT TANK

RECEIVING TANK

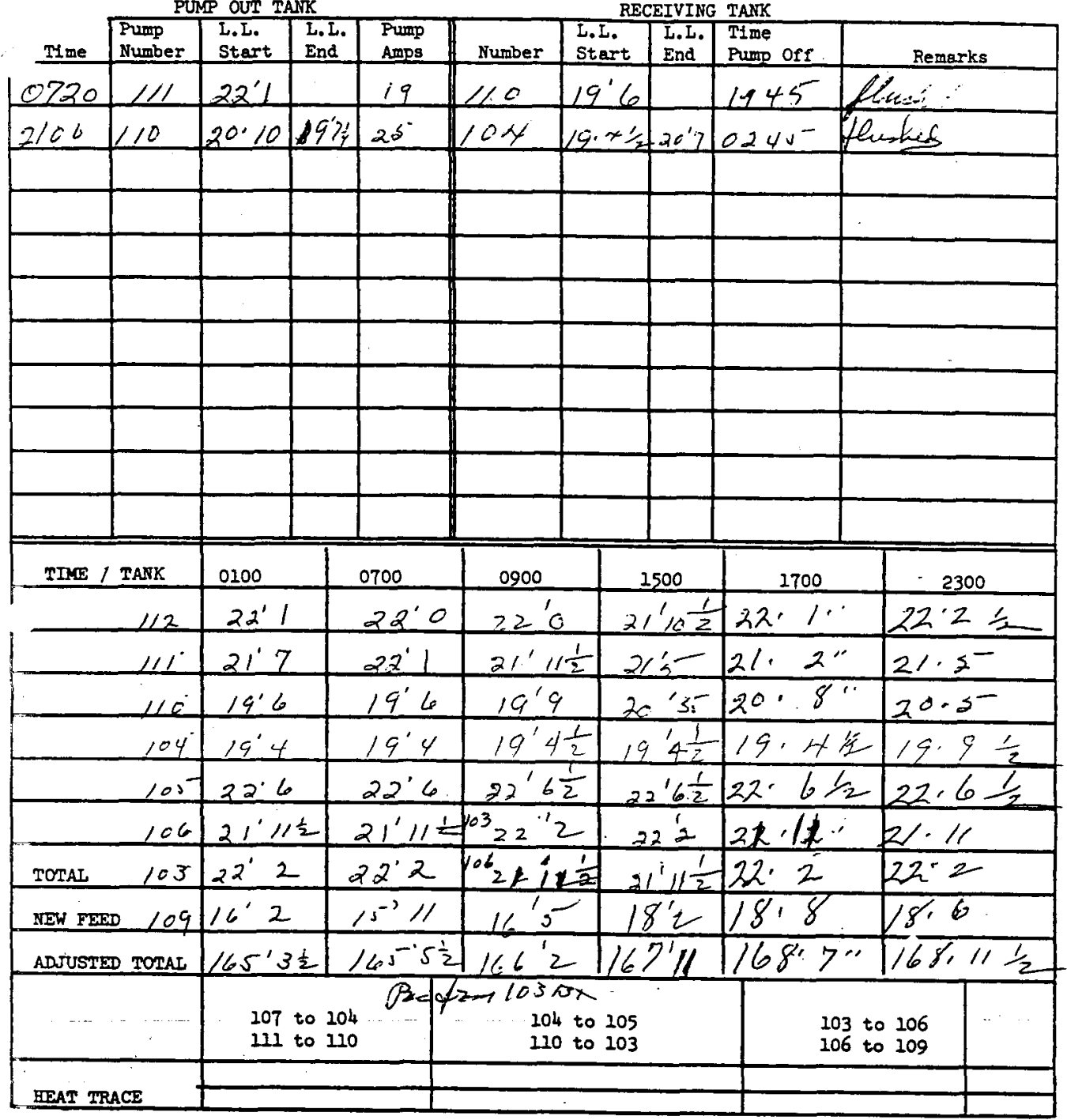

Walker:srb 9-29-70 
PUMP OUT TANK

RECEIVING TANK

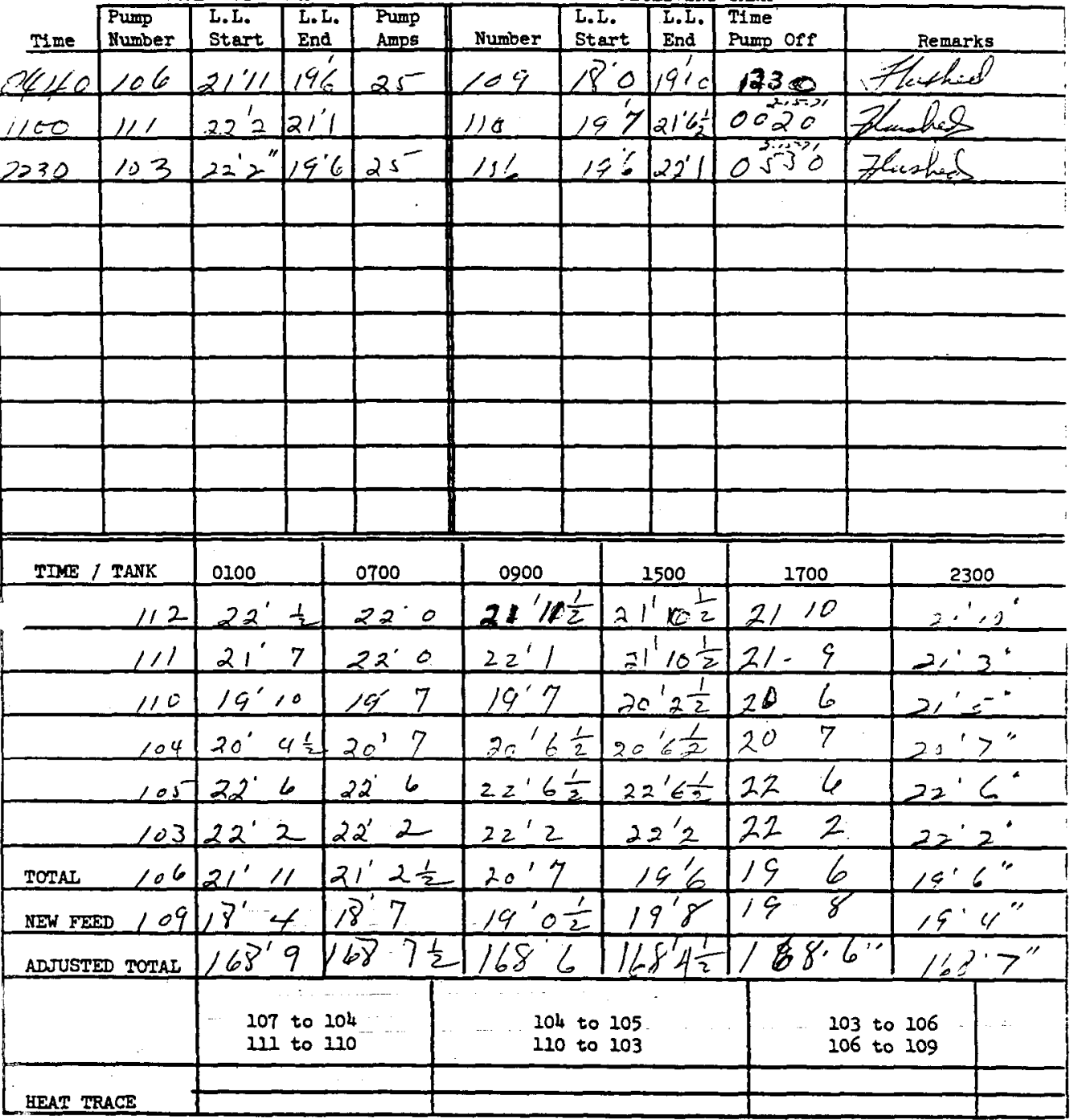

Walker:srb 9-29-70 
PUMP OUT TANK

RECEIVING TANK

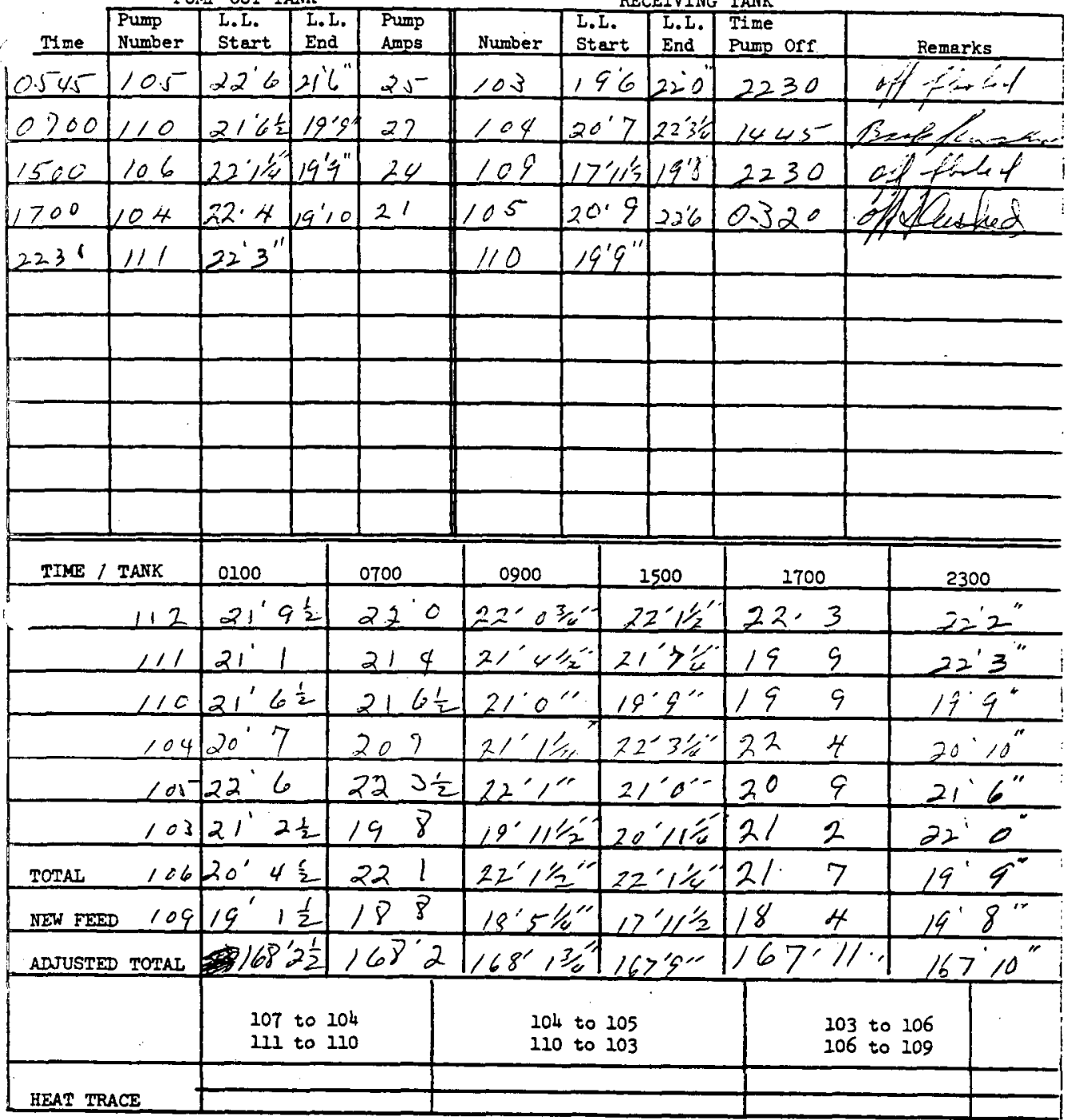

Walker:srb 9-29-70 
PUMP OUT TANK RECEIVING TANK

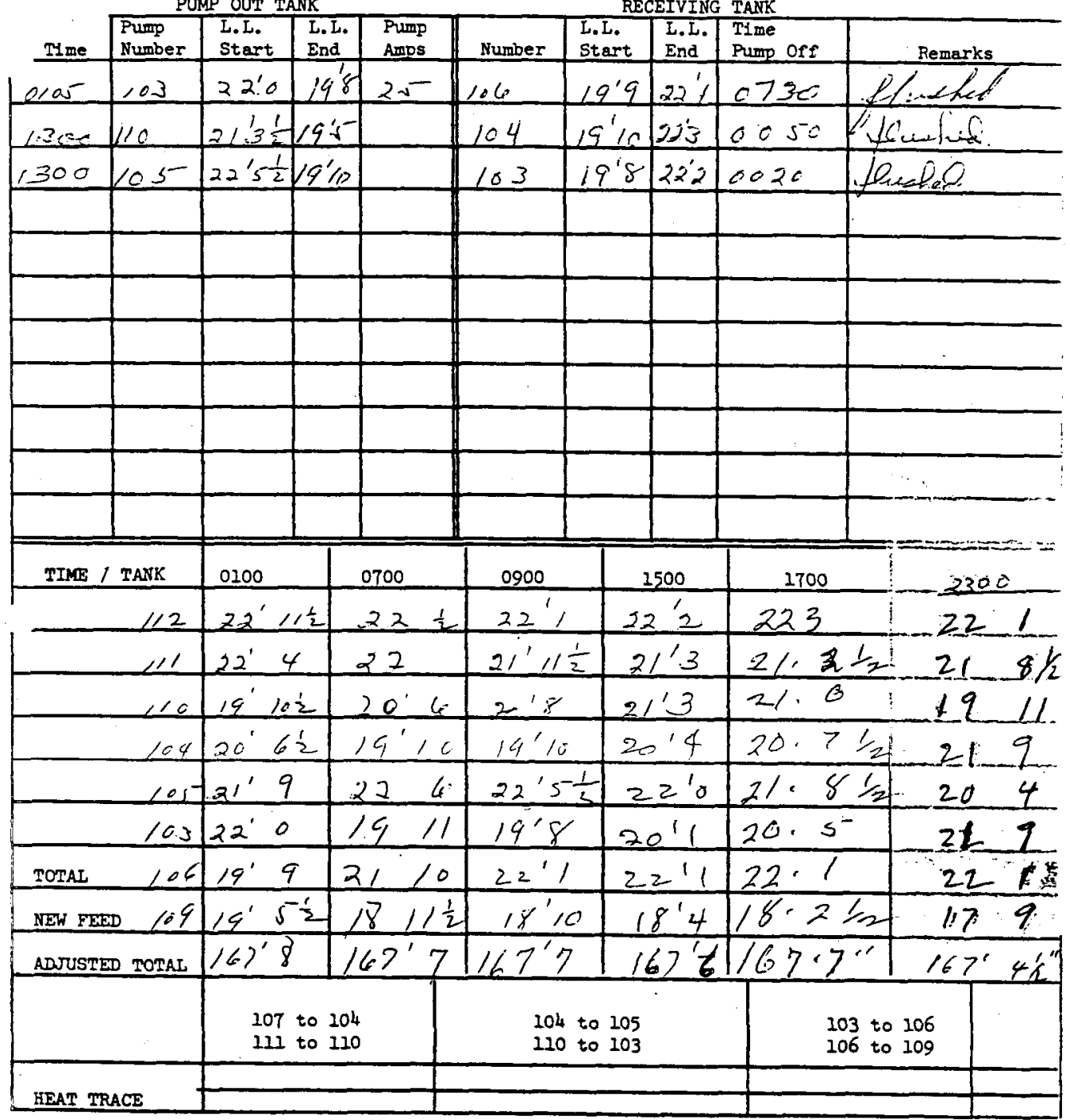

Walker:srb 9-29-70 
PUMP OUT TANK

RECEIVING TANK

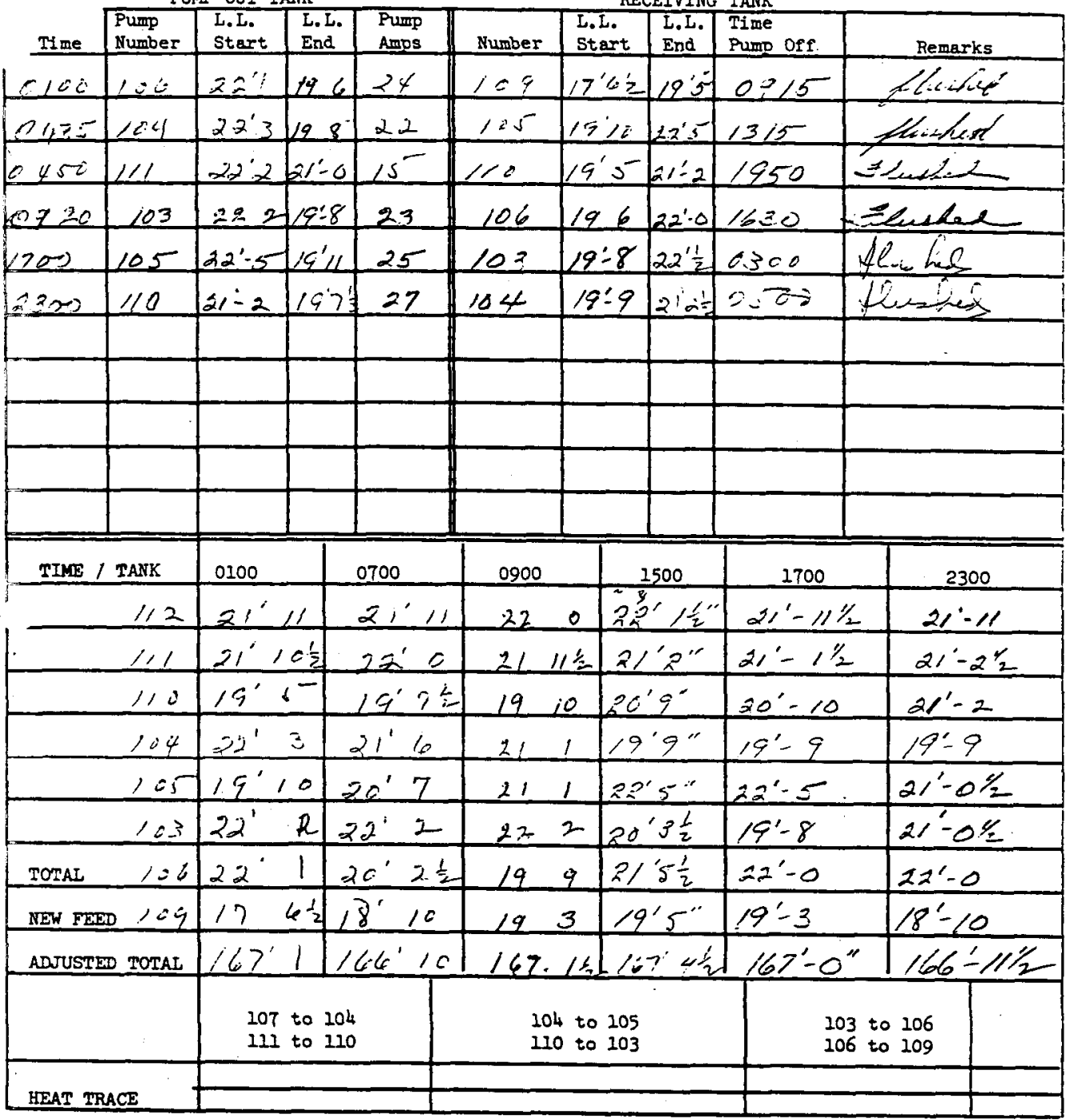

Walker:srb 9-29-70 
PUMP OUT TANK

RECEIVING TANK

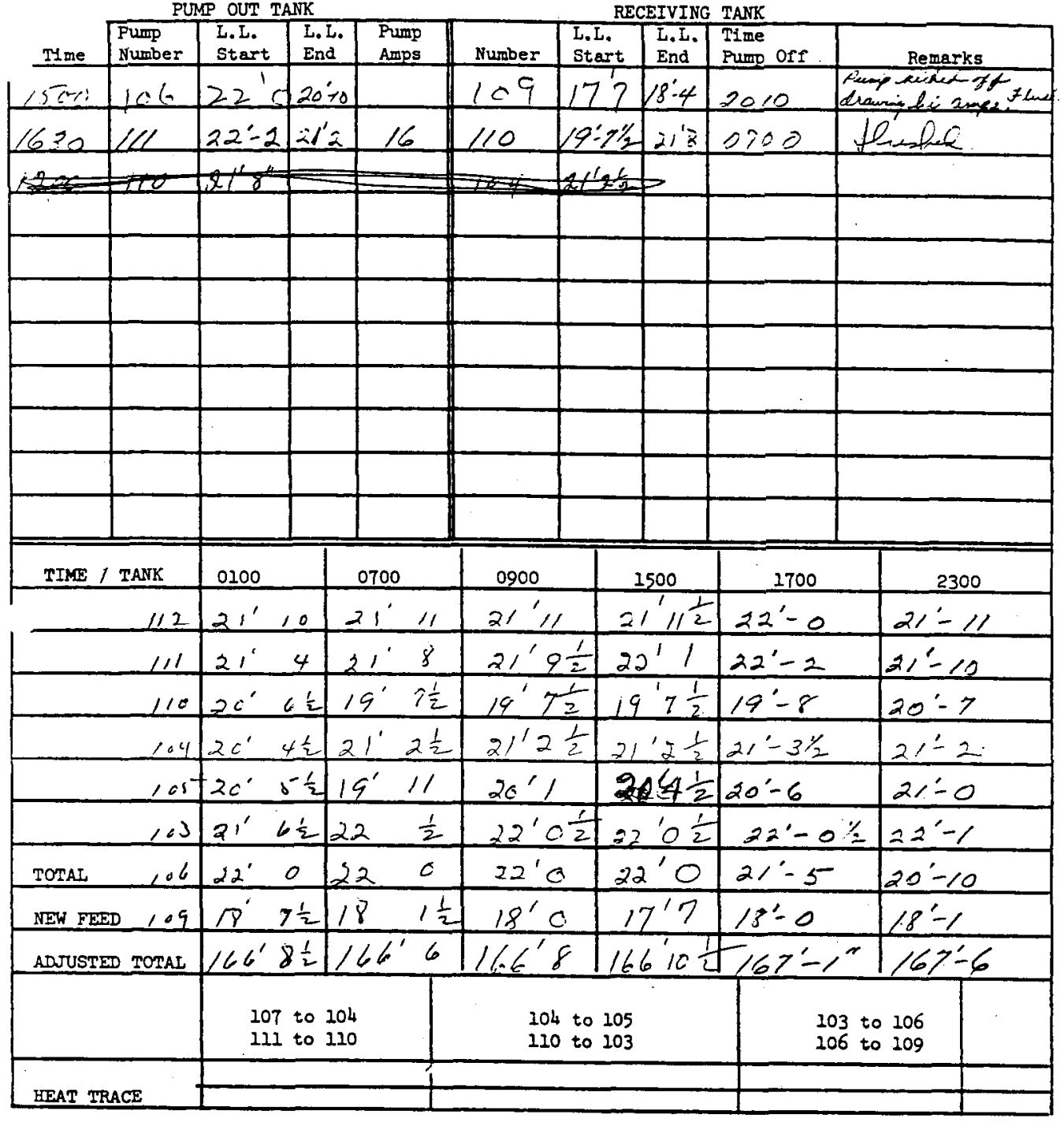

Walker:srb 9-29-70 
PUMP OUT TANK

RECEIVING TANK

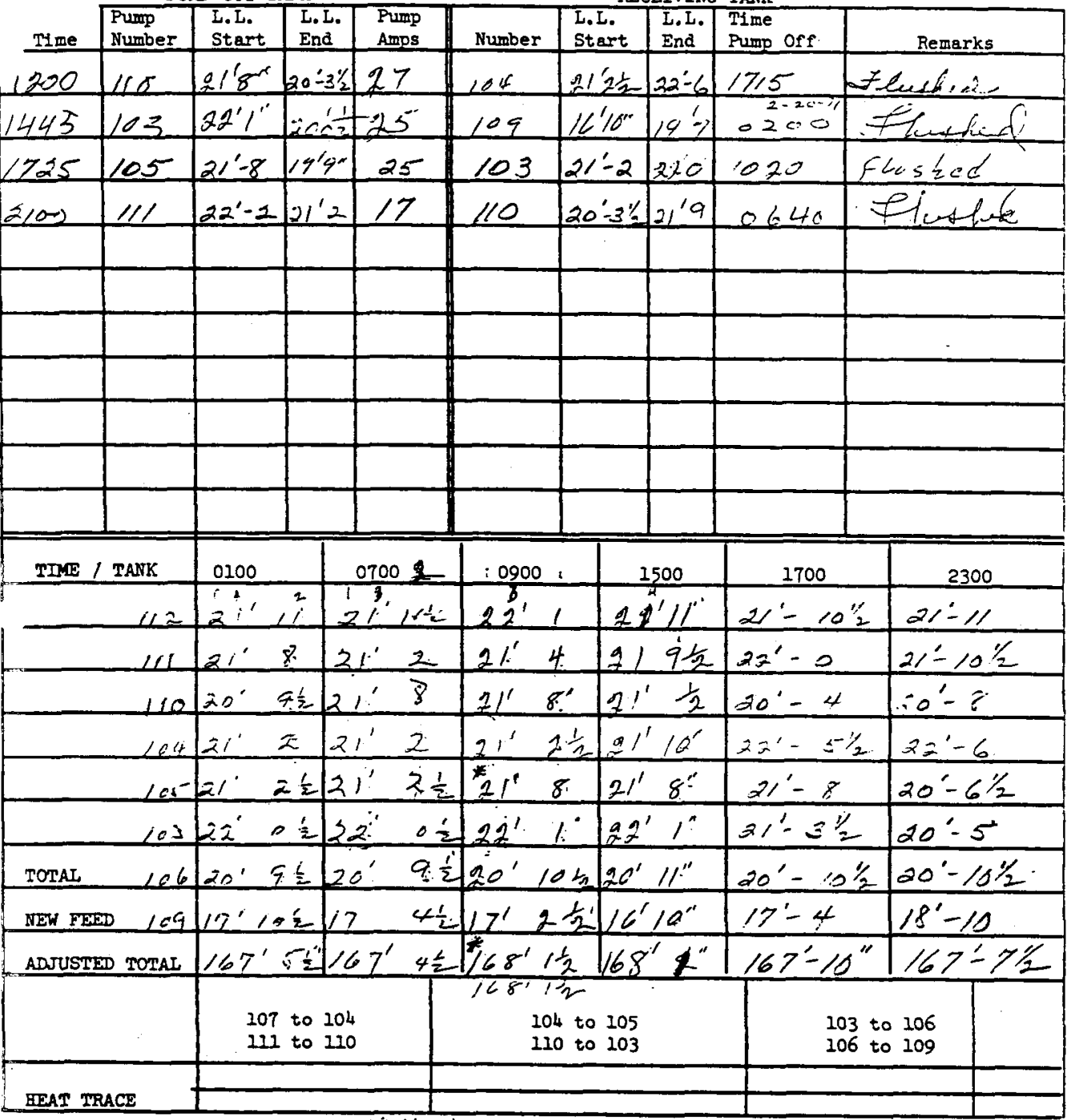

WaJker:srb 9-29-70

* Promped 'Fióm' 102 3y

Kió:. 
PUMP OUT TANK

RECEIVING TANK

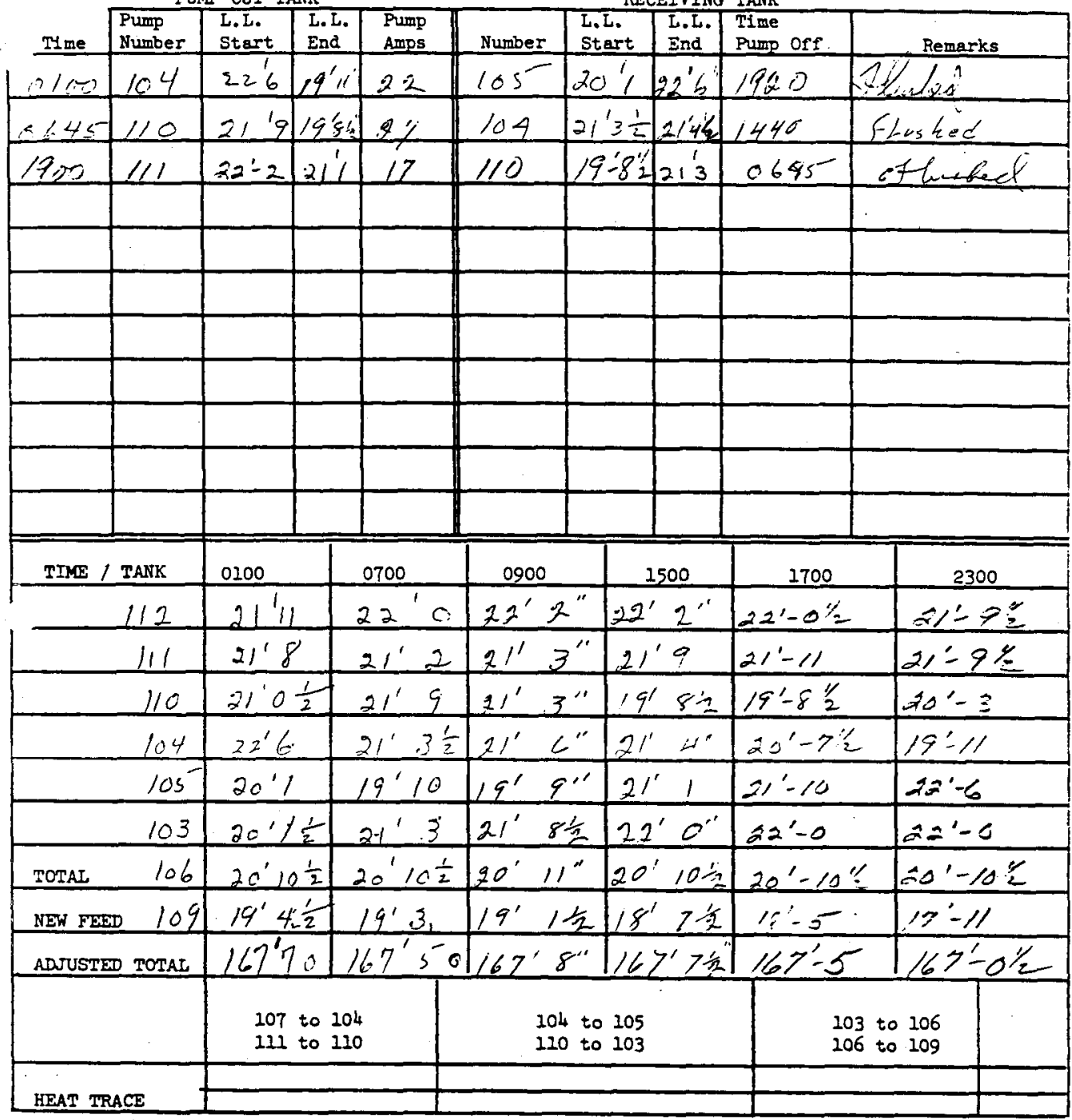

Walker :srb 9-29-70 
PURP OUT TAMK

RECEIVING TANK

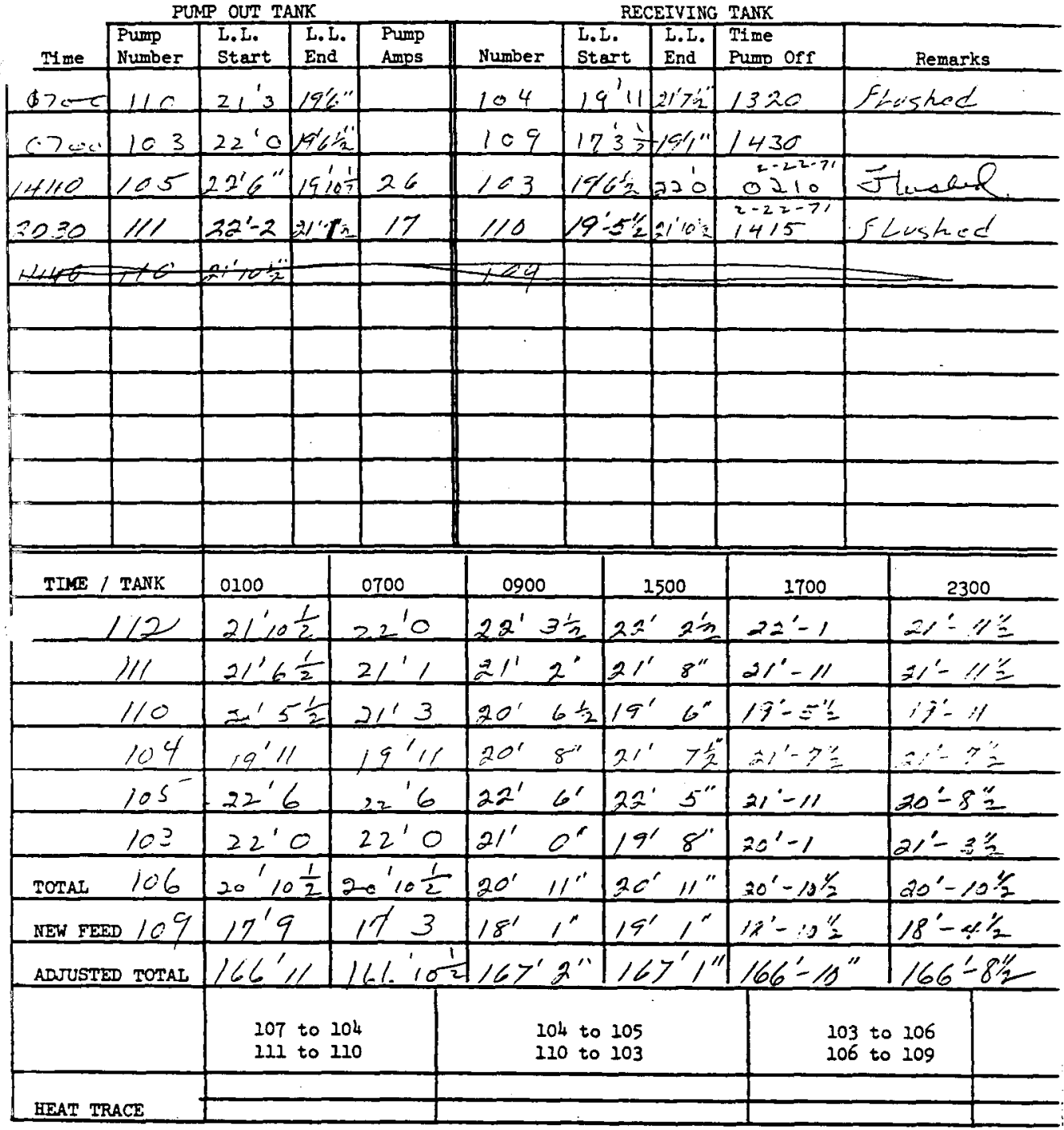

Walker :srb 9-29-70 
PUMP OUT TANK

RECEIVING TANK

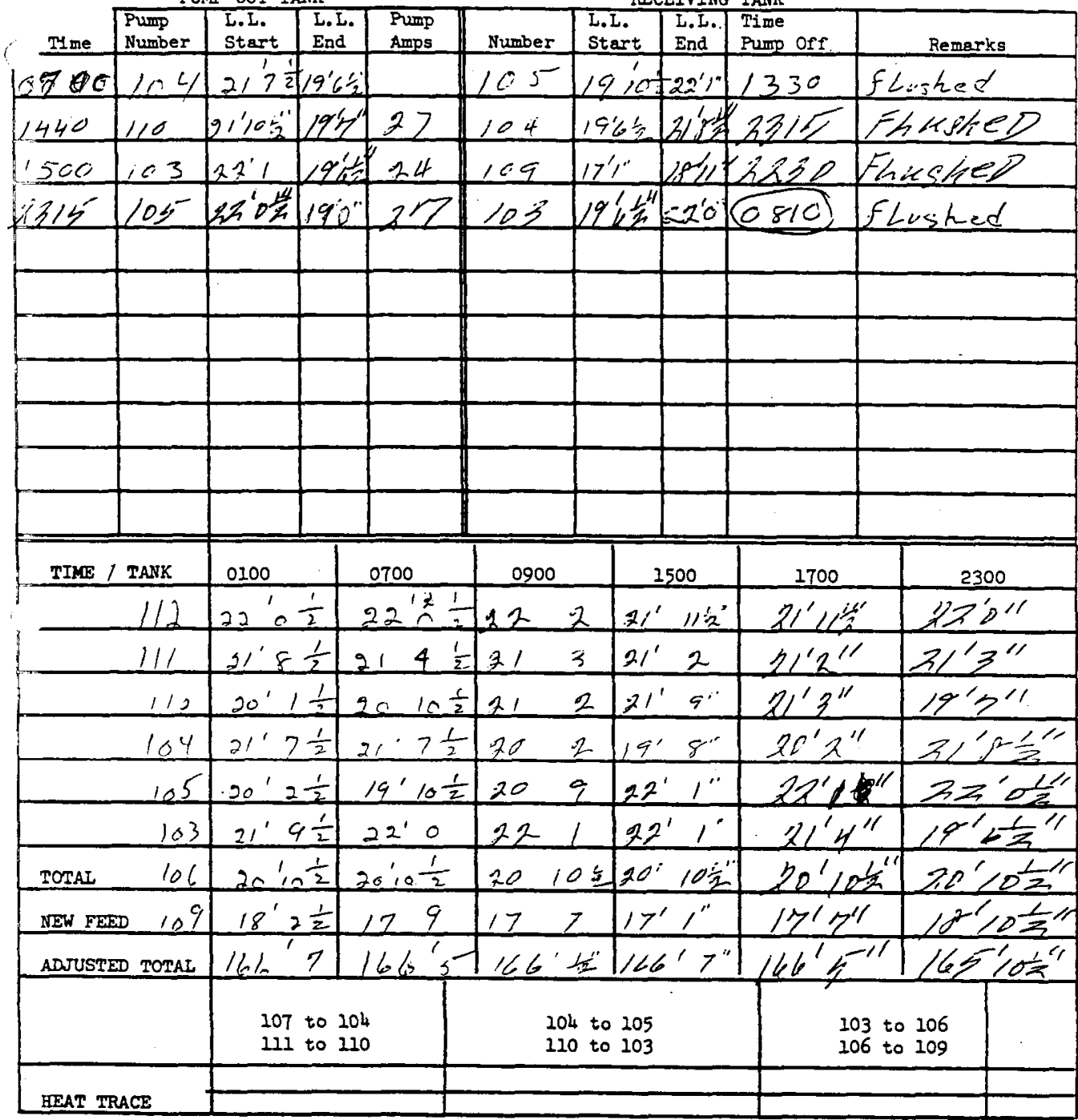

Walker:srb 9-29-70 
PUMP OUT TANK

RECEIVING TANK

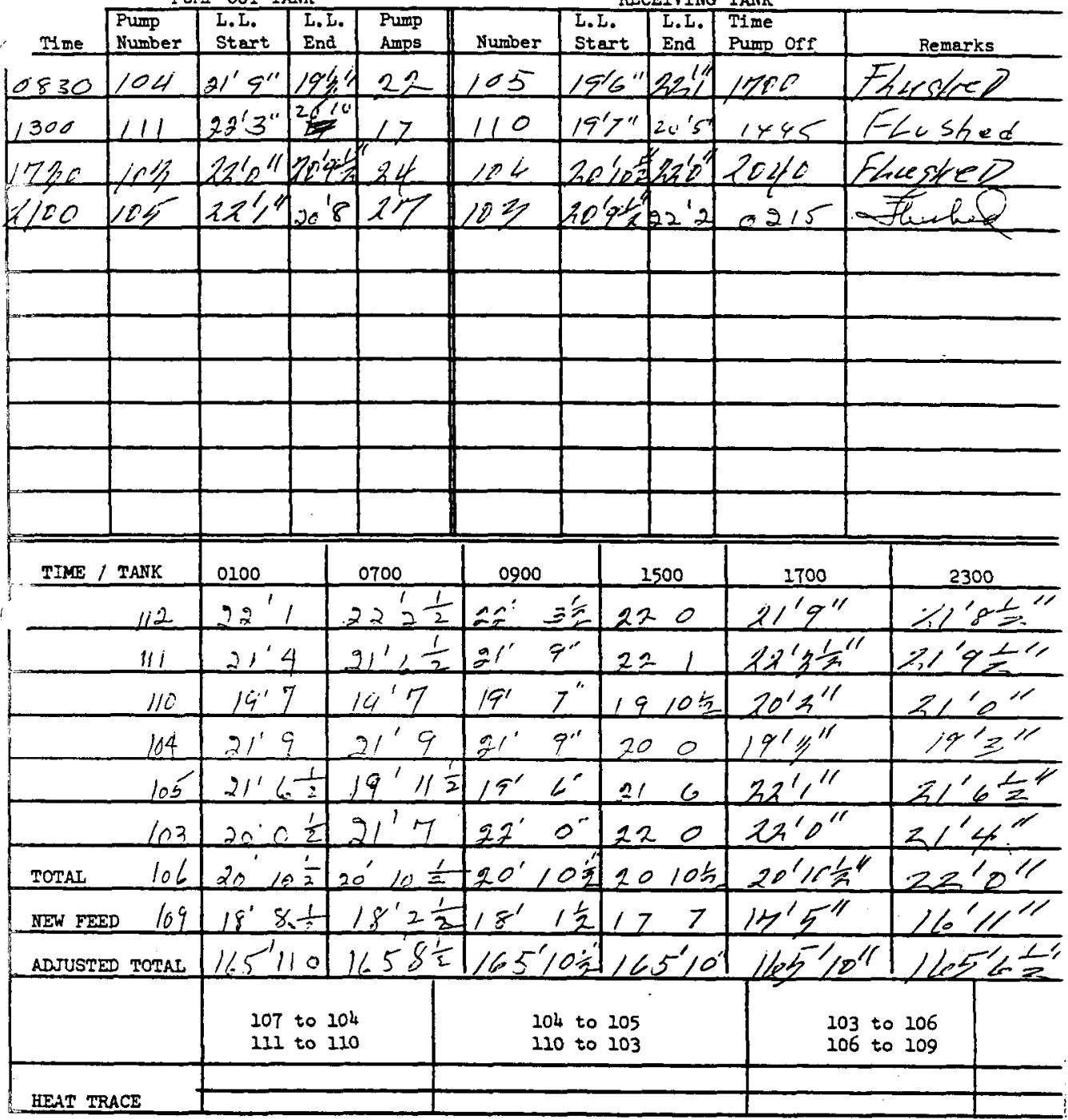

Walker:srb 9-29-70 
PUNP OUT TANK

RECEIVING TANK

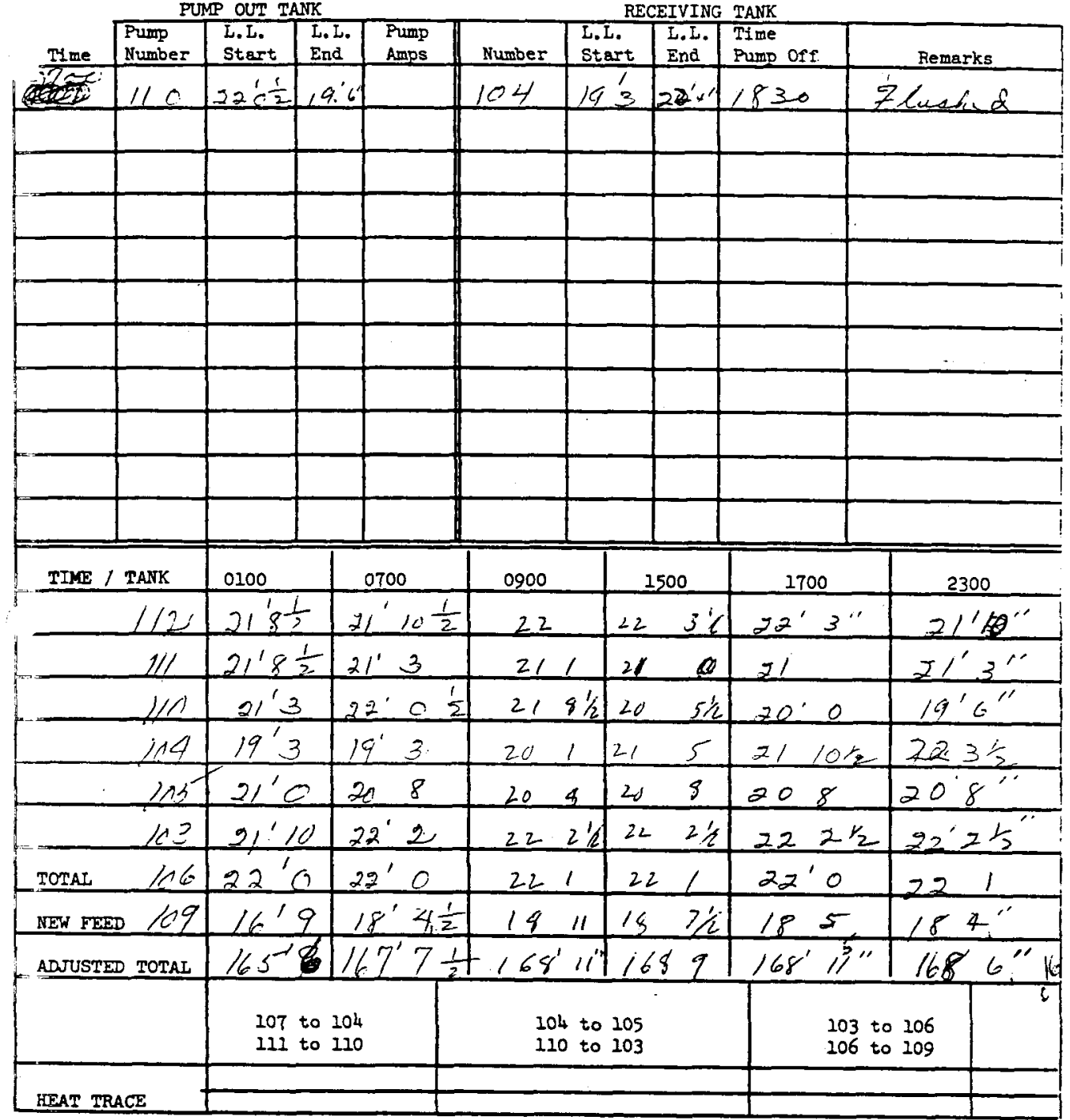

Walker:srb 9-29-70 
PUMP OUT TANK

RECEIVING TANK

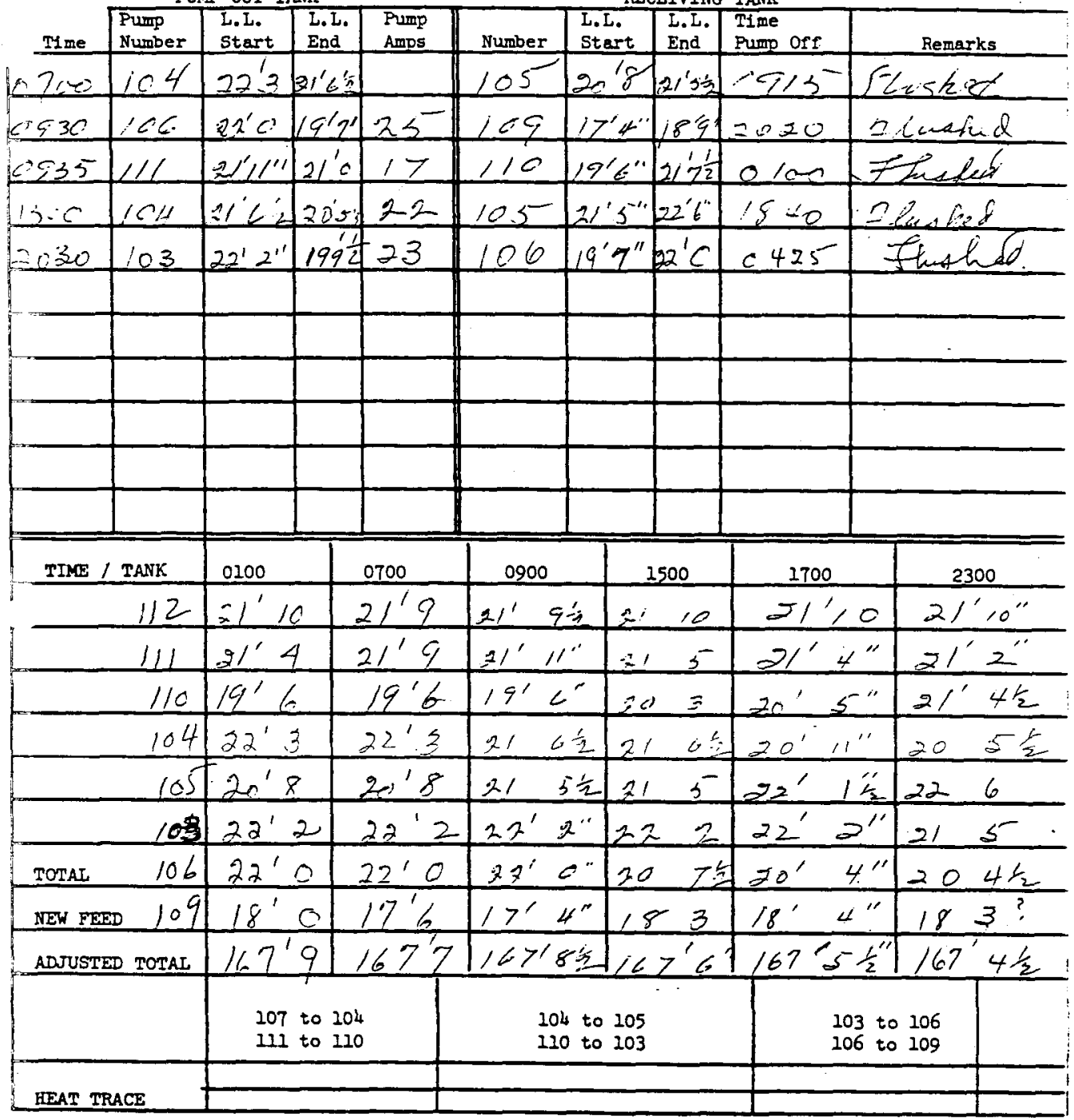

Walker:srb 9-29-70 
PUNP OUT TANK

RECEIVING TANK

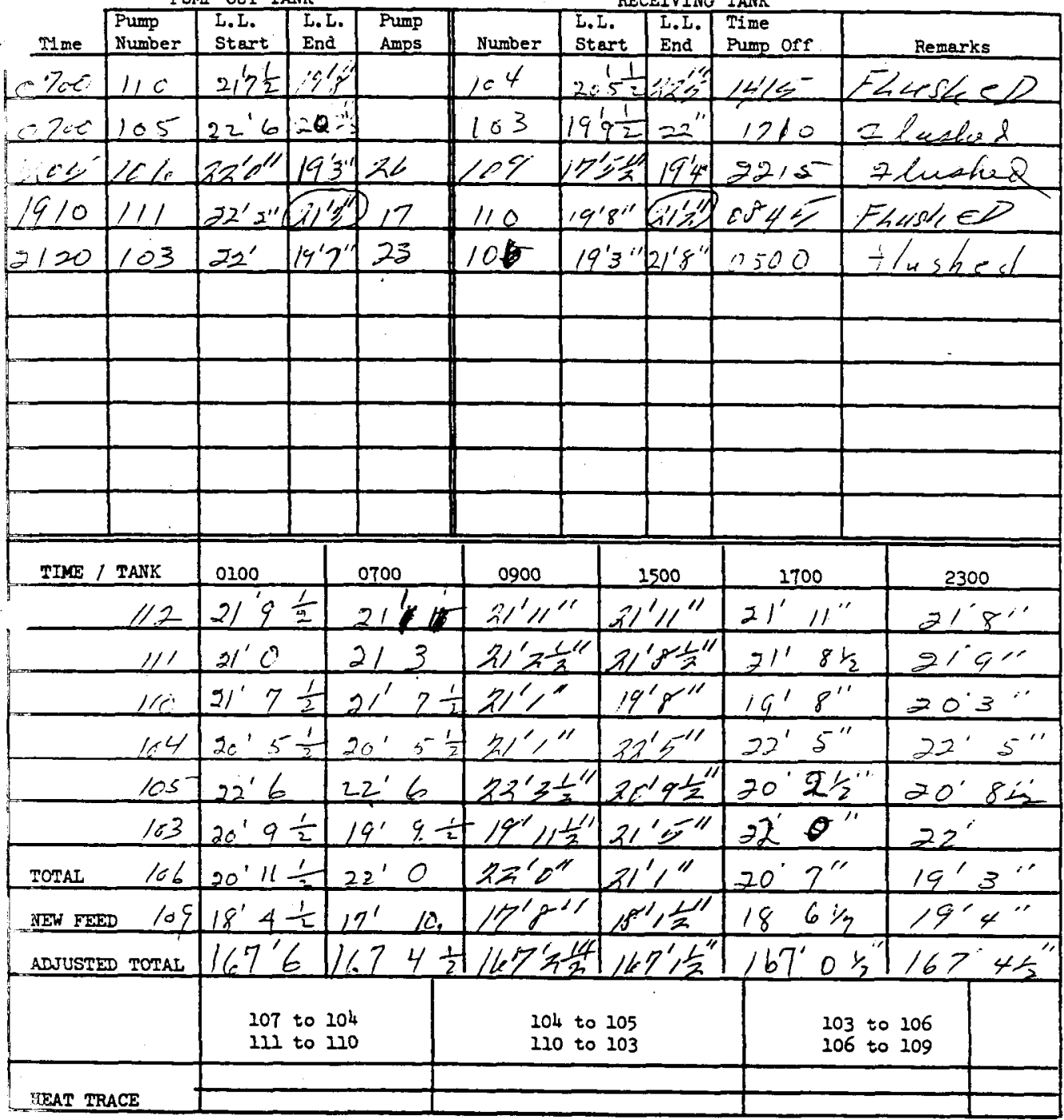

Halker:srb 9-29-70 


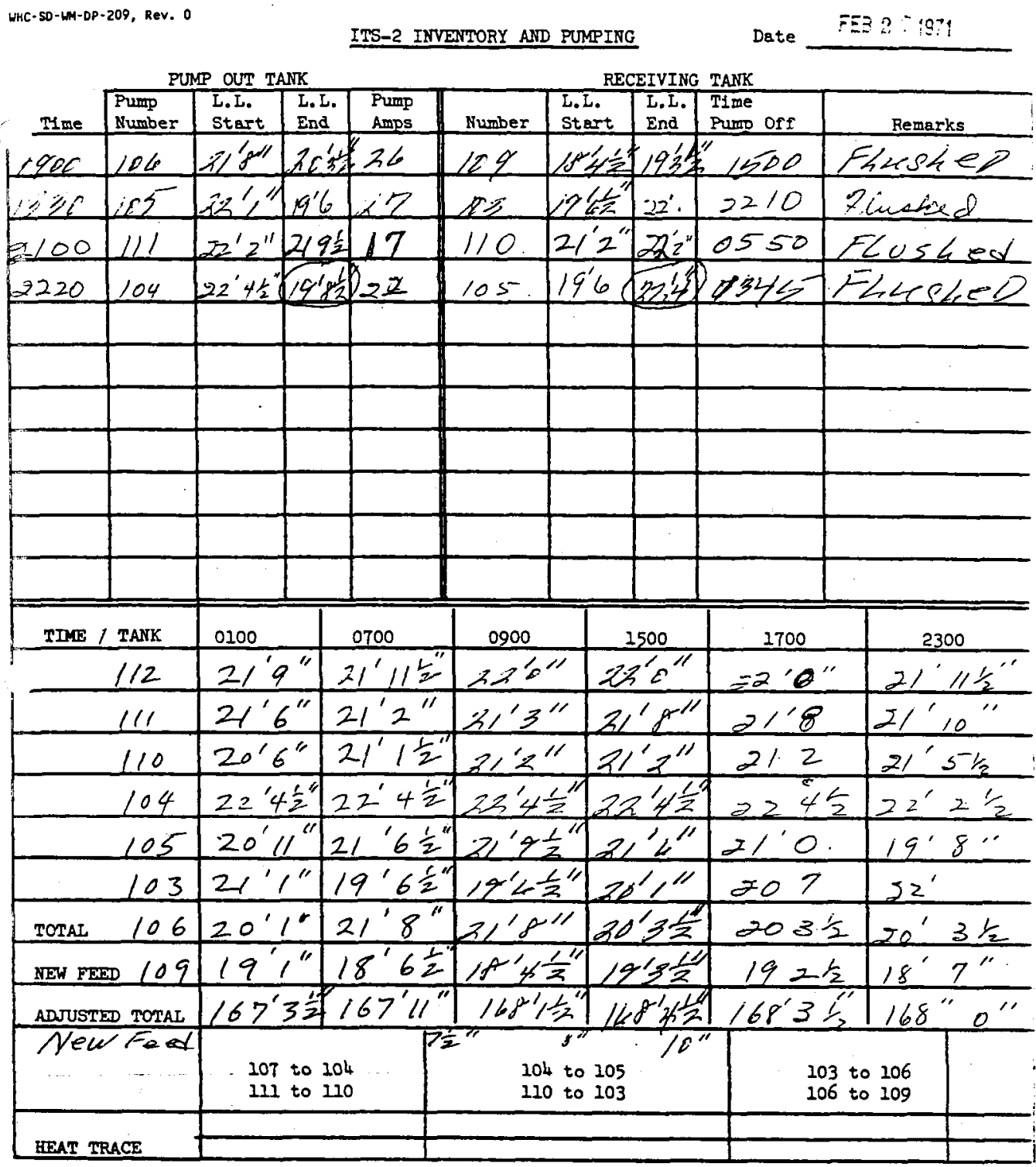

(Walker:srb 9-29-70

46 
WHC-50-WM-DP-209, Rev. 0

ITS-2 INVENTORY AND PUMPING

Date $\div 5281971$

PUMP OUT TANK

RECEIVING TANK

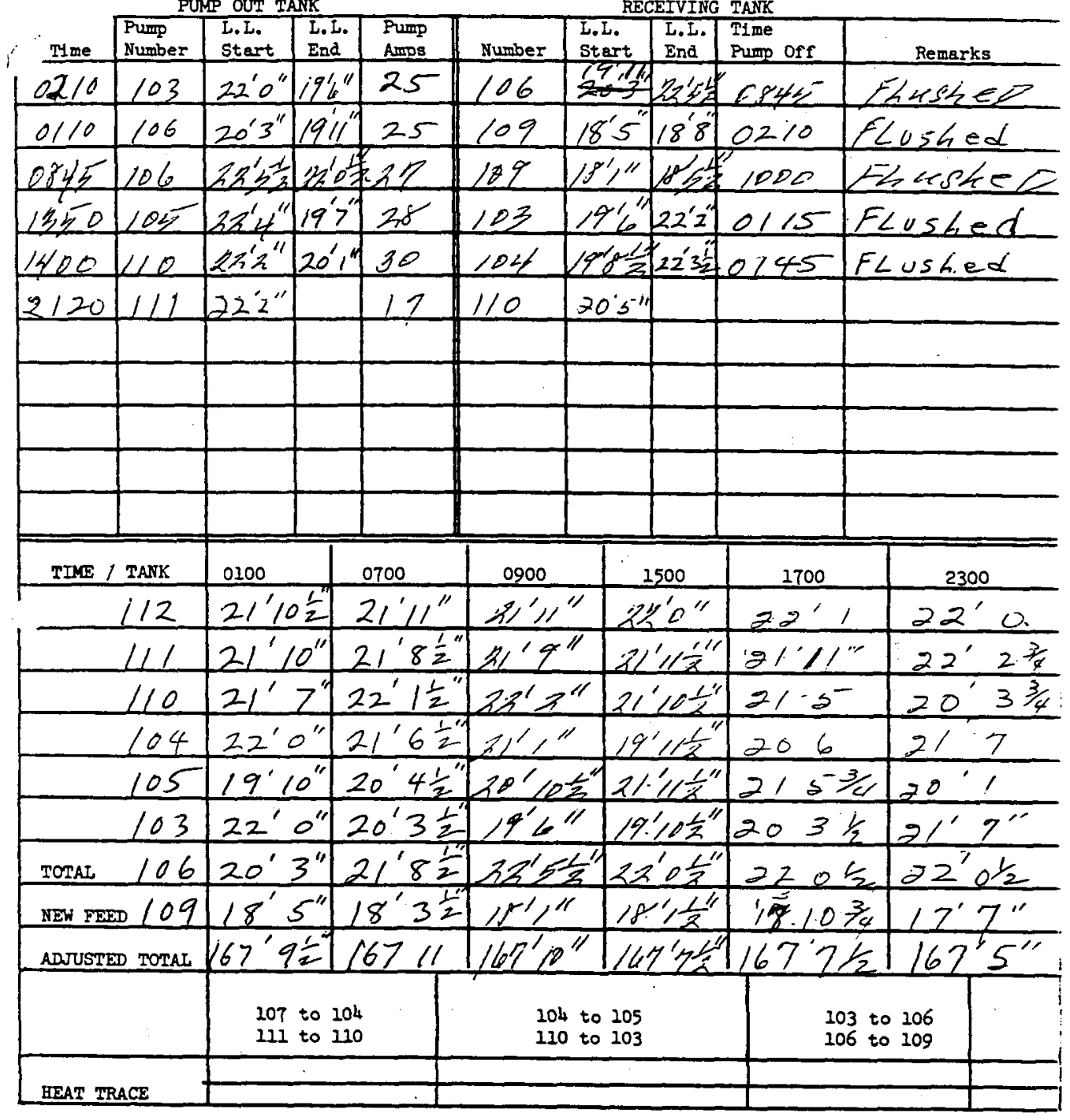

( Walker:srb 9-29-70 $)>20-\%$

47 
WHC-SD-LMA-DP-209, ReV, O

ITS-2 INVENTORY AND PUMPING

Date $2-|-7|$

PUMP OUT TANK

RECEIVING TANK

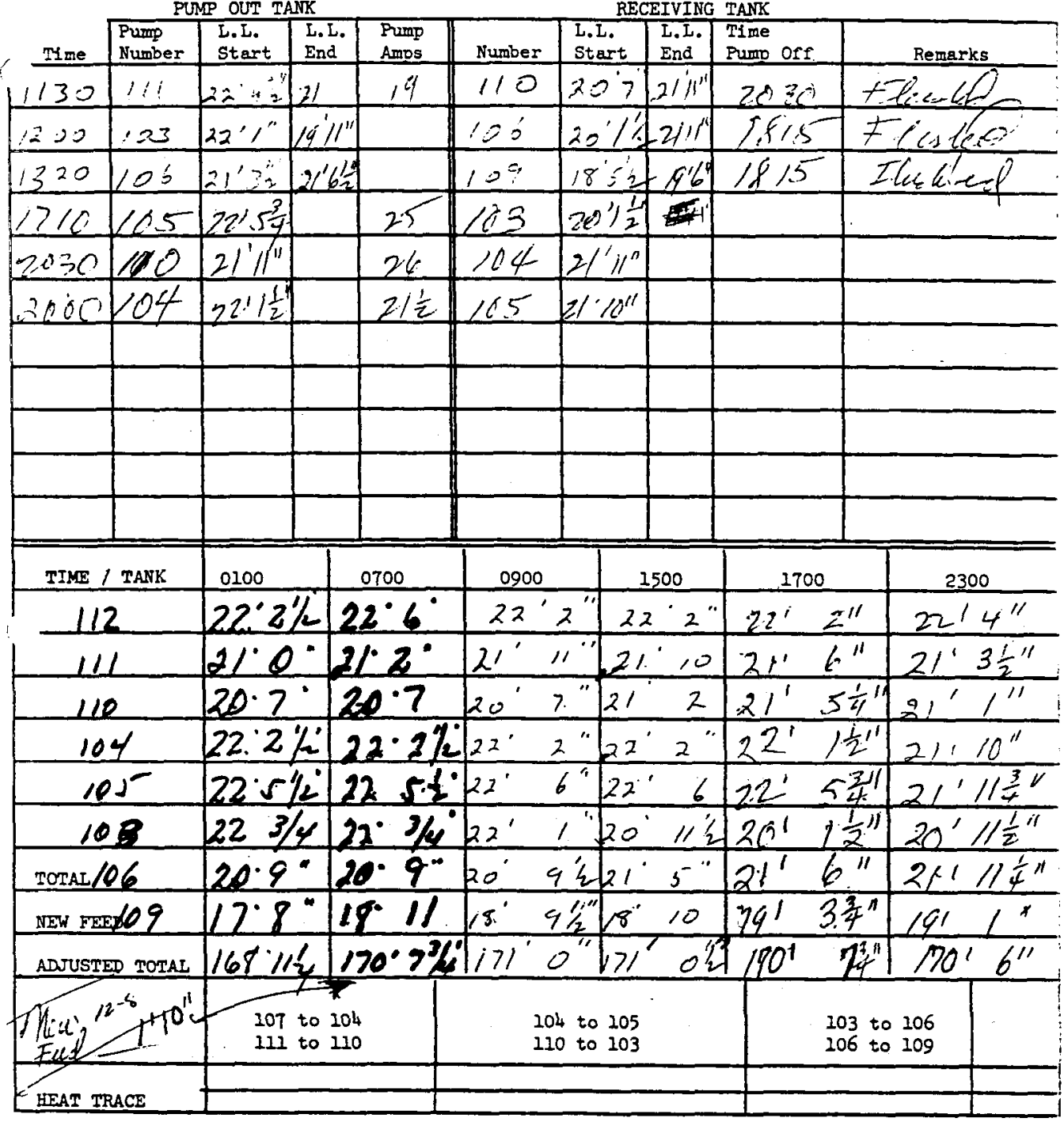

mi Walker:srb 9-29-70

48 
PUMP OUT TANK

RECEIVING TANK

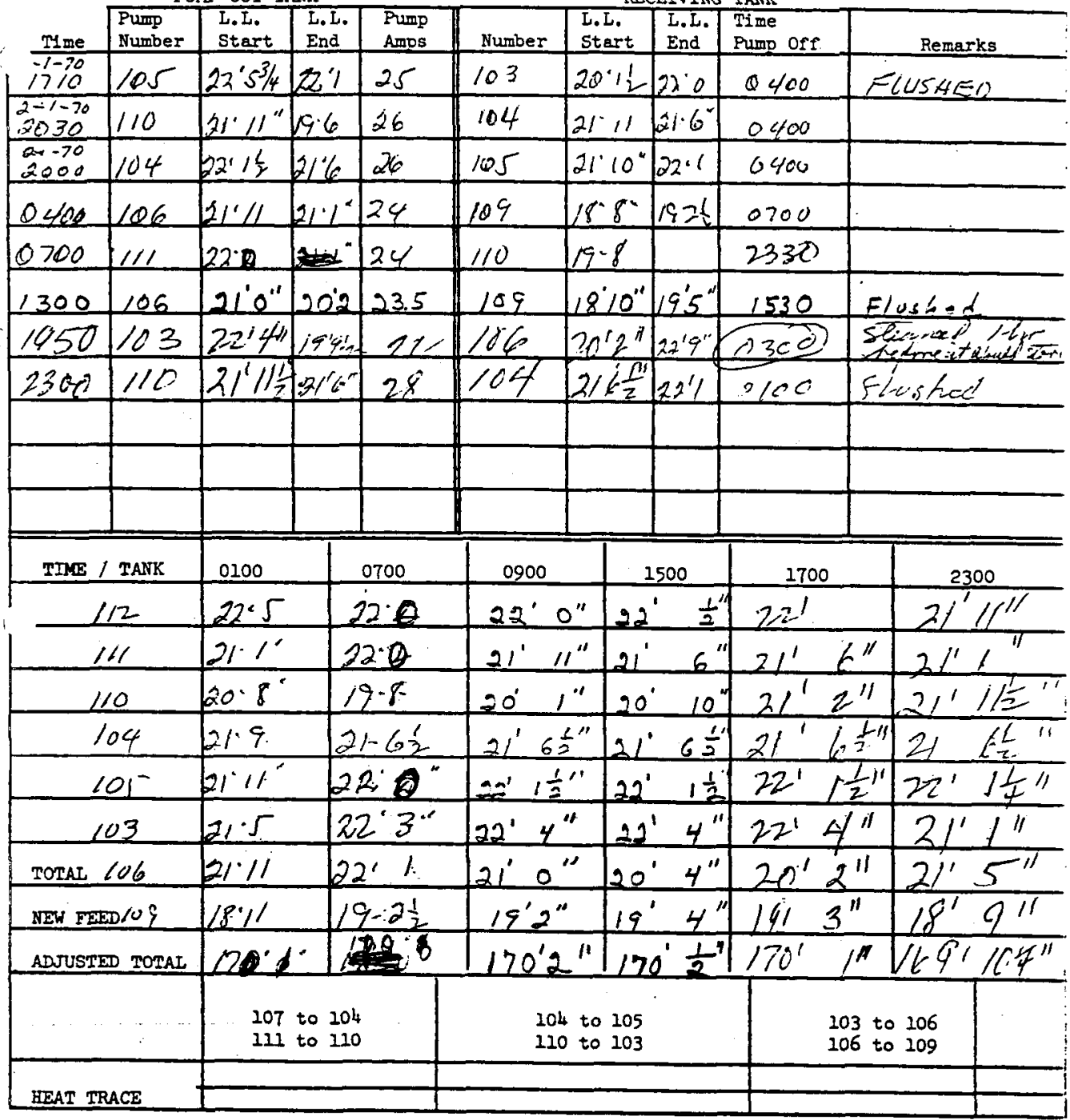

S. Walker:srb 9-29-70

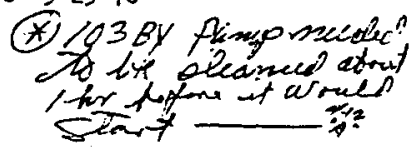




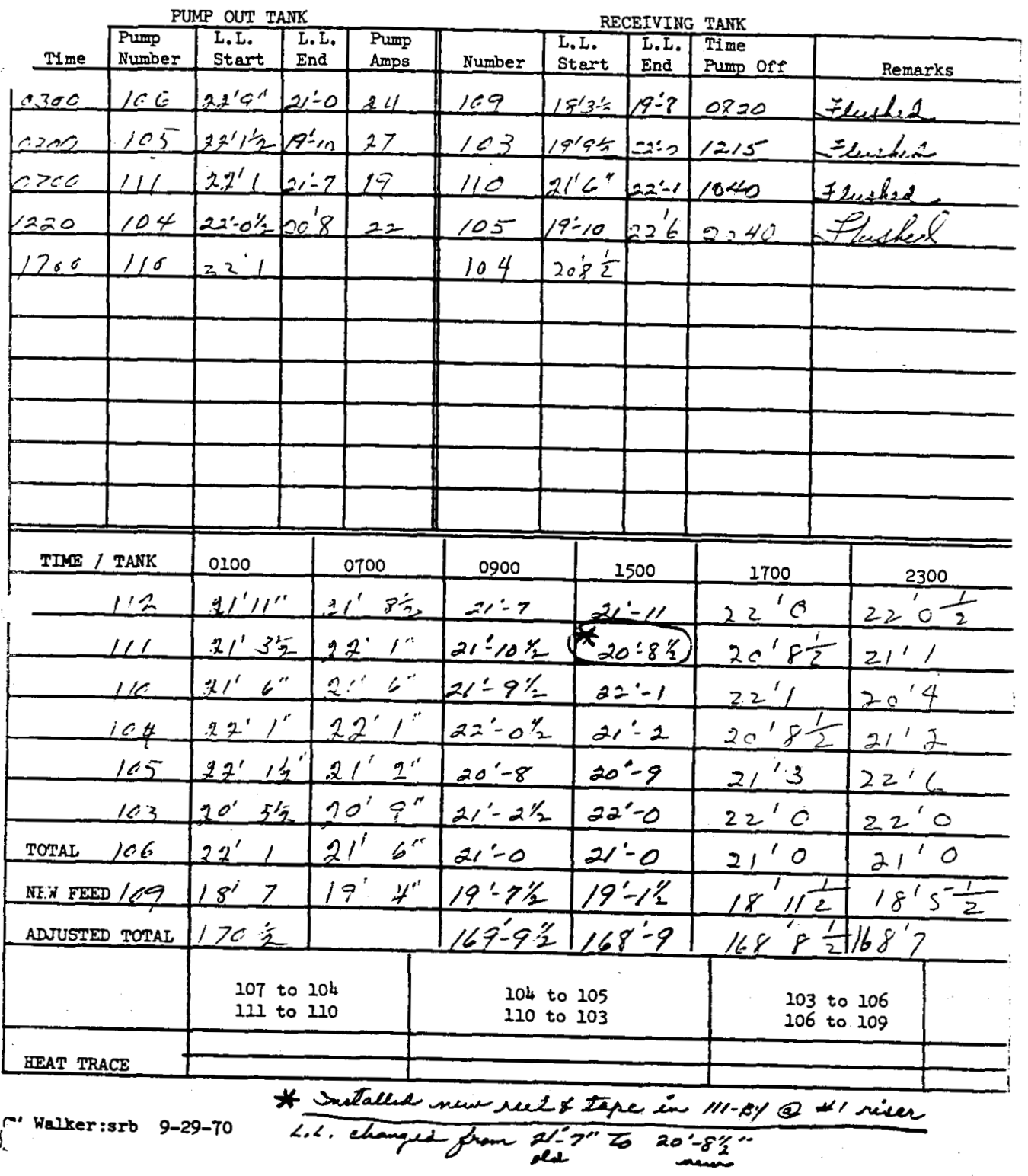


WHC-SO-WH-DP-209, ReV. 0

ITS-2 INVENTORY AND PUMPING

Date $2,1-7$

PUMP OUT TANK

RECEIVING TANK

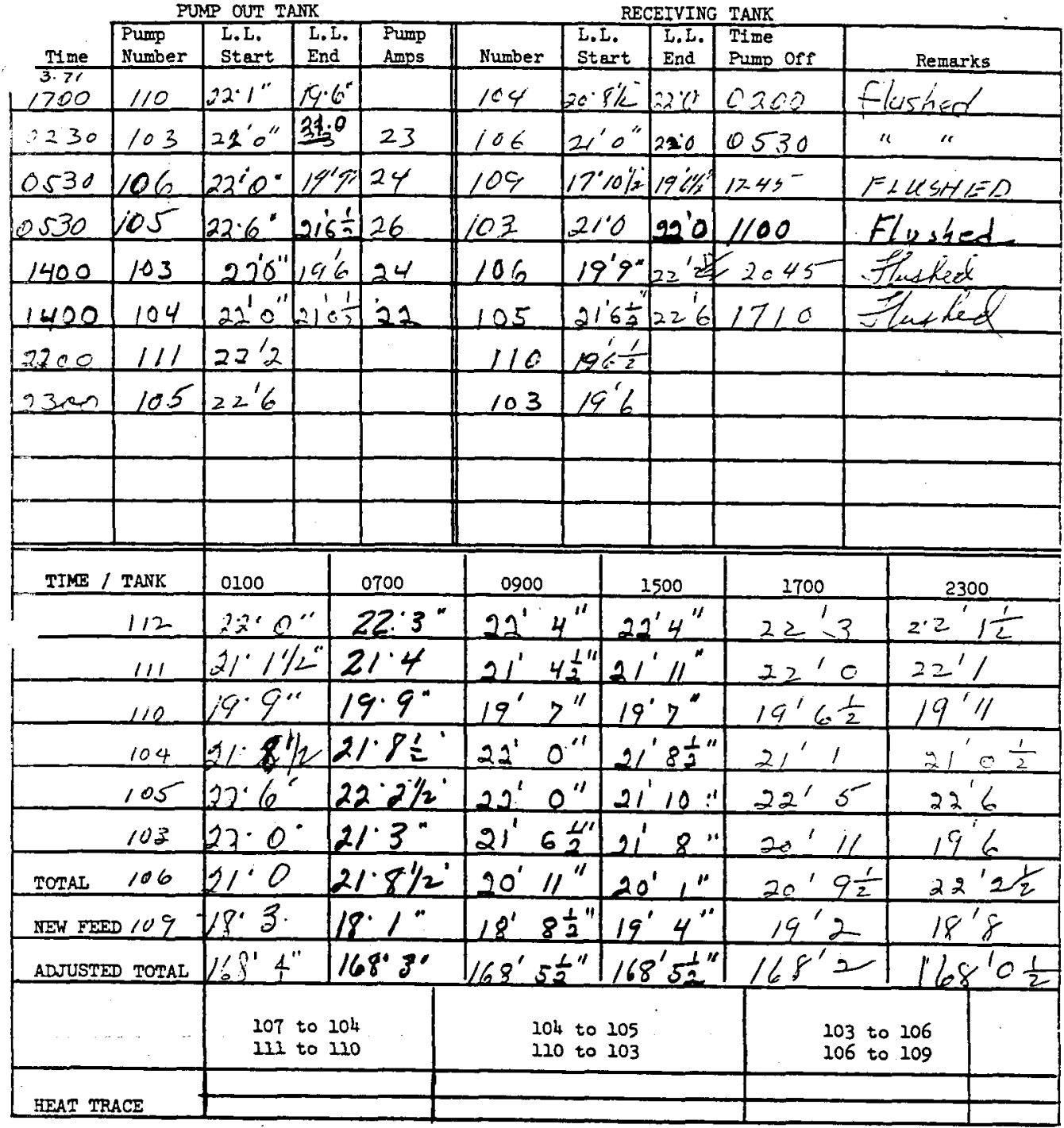

M Walker:srb 9-29-70

16

51 
WHC-SD-WH-DP-209, ReV. O

ITS-2 INVENTORY AND PUMPING

Date $2-\sqrt{-7}$

PUMP OUT TANK

RECEIVING TANK

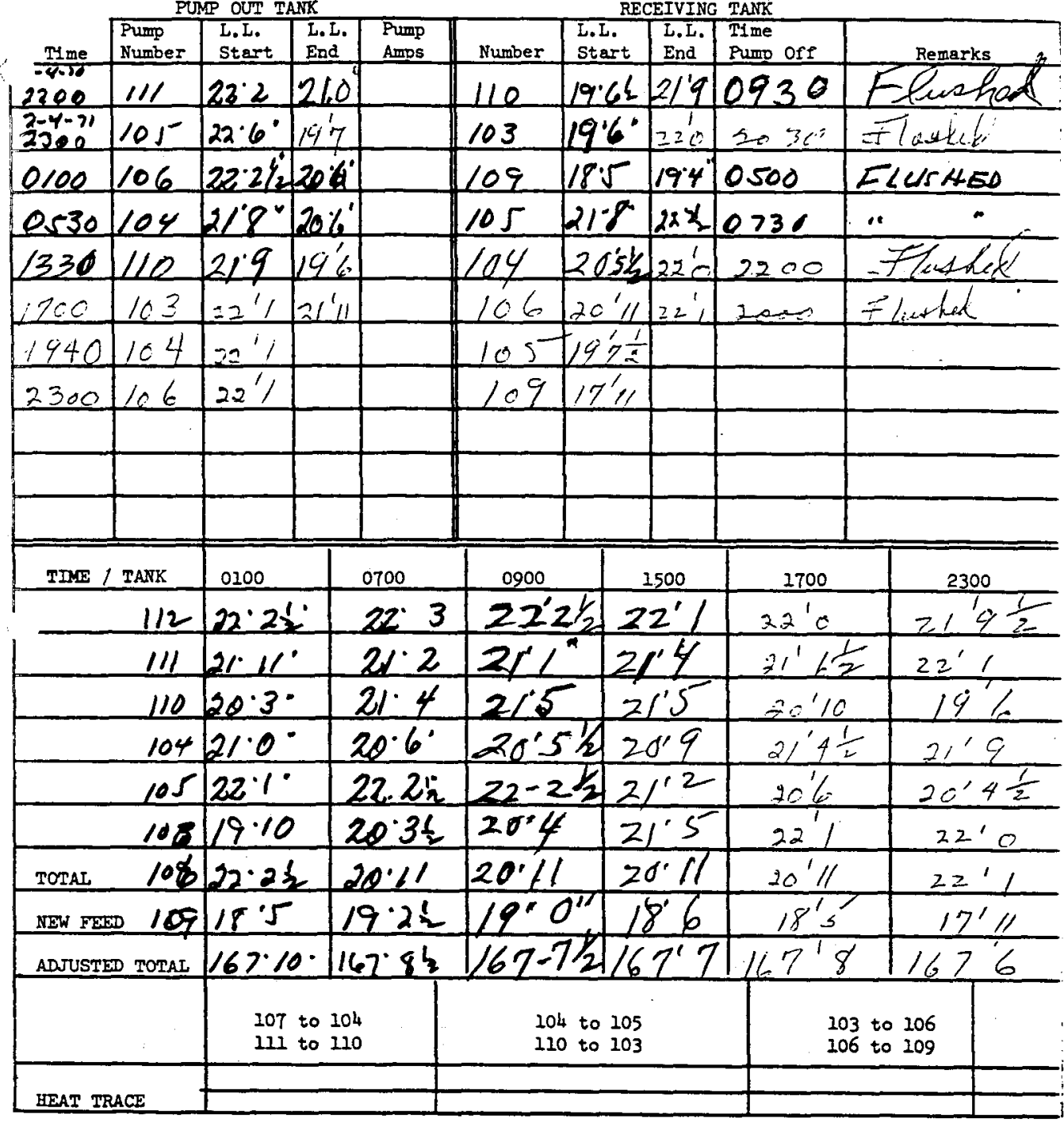

(N) Wauker:srb 9-29-70

52 
PUMP OUT TANK

RECEIVING TANK

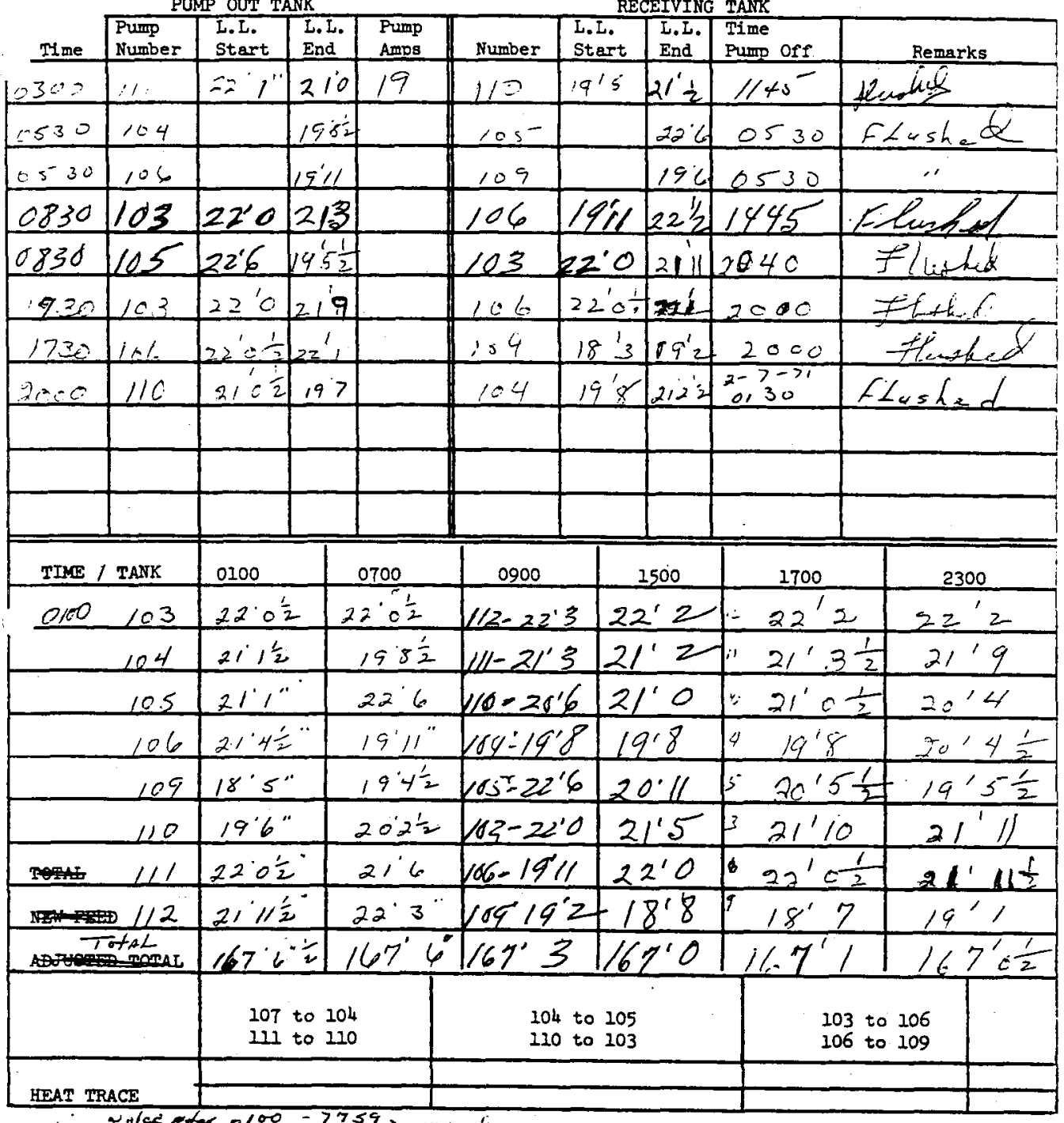

(i. WaIker:8rb 9-29-70

$-100-7759$
$0>00 \quad 7929>170 \mathrm{~g}^{2}$ 
PUMP OUT TANK

RECEIVING TANK

\begin{tabular}{|c|c|c|c|c|c|c|c|c|c|}
\hline Time & $\begin{array}{l}\text { Pump } \\
\text { Number }\end{array}$ & $\begin{array}{l}\text { L.I. } \\
\text { Start }\end{array}$ & $\begin{array}{l}\text { L.I. } \\
\text { End }\end{array}$ & $\begin{array}{l}\text { Fump } \\
\text { Amps }\end{array}$ & Number & $\begin{array}{l}\text { L.L. } \\
\text { Start }\end{array}$ & \begin{tabular}{|l|} 
L.L. L. \\
End \\
\end{tabular} & $\begin{array}{l}\text { Time } \\
\text { Pump off }\end{array}$ & Remarks \\
\hline 330 & 111 & $22^{\prime} 2$ & $2 / 2$ & 18 & 110 & $19^{\circ} 7$ & 210 & 1200 & Elushed \\
\hline$\angle 430$ & 106 & $2 / 11$ & $91 \frac{11}{62}$ & 24 & 109 & 179 & 191 & $12 \mathrm{rc}$ & Flukhe \\
\hline 1800 & 103 & $21^{\prime} 11$ & $19^{\prime} 8:$ & & 10,6 & $212 \frac{1}{2}$ & $x \frac{1}{6}$ & 2230 & Hewhit \\
\hline 1700 & 110 & $21^{\prime} 0$ & 198 & & 164 & $212 \frac{1}{2}$ & $226 \frac{1}{2}$ & $2 / 2=$ & 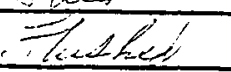 \\
\hline
\end{tabular}

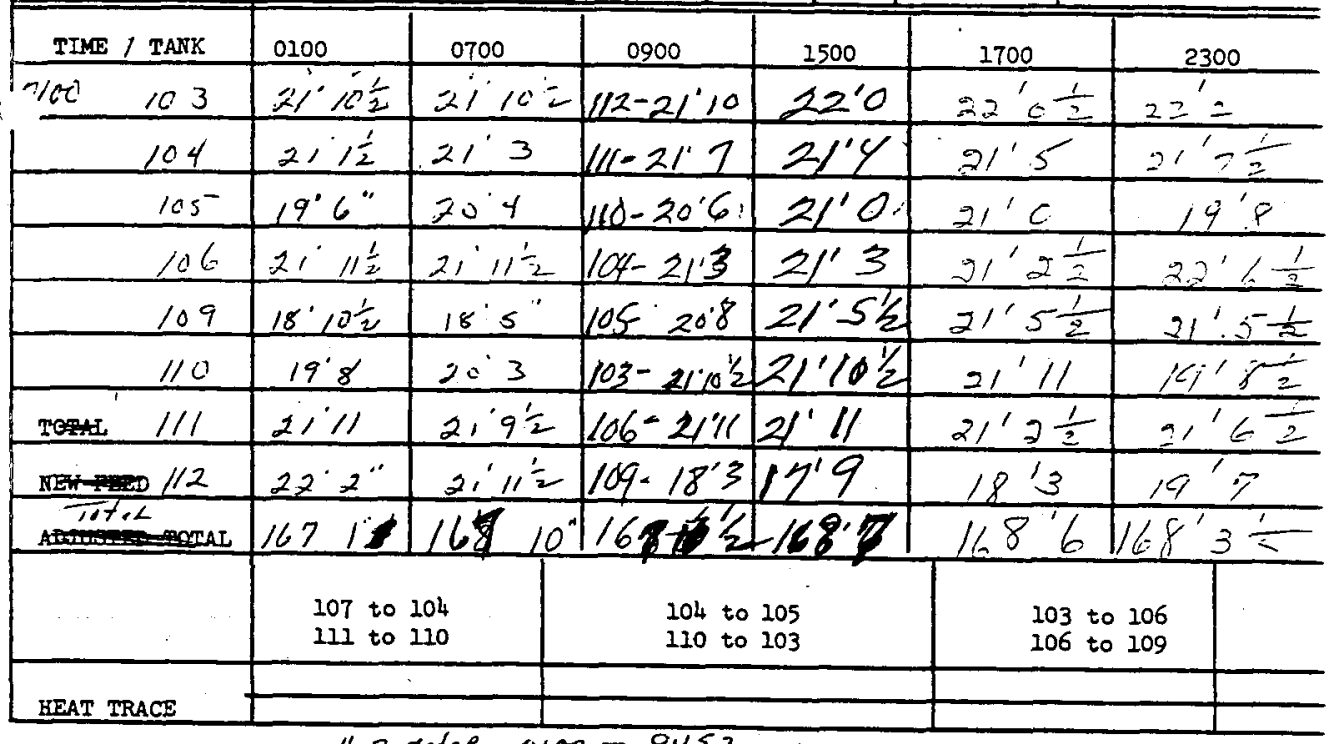

$\therefore$ Halker:srb 9-29-70 $14 \mathrm{rl} 0700-4530$

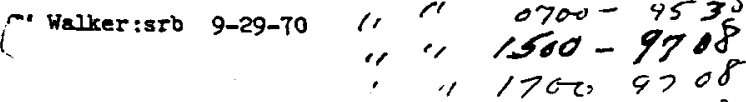

3309862 . 
PUMP OUT TANK

RECEIVING TANK

\begin{tabular}{|c|c|c|c|c|c|c|c|c|c|}
\hline Time & $\begin{array}{l}\text { Pump } \\
\text { Number }\end{array}$ & $\begin{array}{l}\text { L.I. } \\
\text { Start } \\
\end{array}$ & $\begin{array}{l}\text { L.L. } \\
\text { End }\end{array}$ & $\begin{array}{l}\text { Pump } \\
\text { Amps }\end{array}$ & Number & $\begin{array}{l}\text { L.L. } \\
\text { Start } \\
\end{array}$ & $\begin{array}{l}\text { L.I. } \\
\text { End }\end{array}$ & $\begin{array}{l}\text { Time } \\
\text { Pump off }\end{array}$ & Remariss \\
\hline 2200 & 105 & $2 / 5 \frac{1}{2}$ & $196^{\prime \prime}$ & 25 & 103 & $19^{\prime} 9^{\prime \prime}$ & $213 \frac{1}{2}$ & 0210 & $F\left(y_{2}{ }^{2}=a^{\prime}\right.$ \\
\hline 0430 & $\mu$ & $22^{\prime} 1 \frac{1}{2}$ & 213 & 20 & 112 & $19^{\prime} 8^{\prime \prime}$ & 215 & 1445 & Eluofied \\
\hline 095 & 104 & 226 & 197 & 22 & 105 & $19^{\prime} 6 \frac{1}{2}$ & $22^{\prime} 6^{\prime} \frac{1}{2}$ & 1840 & $F / 4+k i d$ \\
\hline$\therefore-\infty$ & 110 & 21.5 & $196 \frac{1}{2}$ & 26 & 104 & 197 & $x^{\prime} 6$ & 0300 & Elustod \\
\hline 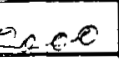 & 106 & $216 \frac{1}{2}$ & $196+6$ & 24 & 109 & $17^{\prime}, 1$ & $19^{\prime} y^{\prime \prime}$ & 0230 & Flushed \\
\hline & & & & & & & & & \\
\hline & & & & & & & & & \\
\hline & & & & & & & & & \\
\hline & & & & & & & & & \\
\hline & & & & & & & & & \\
\hline & & & & & & & & & \\
\hline
\end{tabular}

\begin{tabular}{l|l|l|l|l|l|l} 
TINE / TANK & 0100 & 0700 & 0900 & 1500 & 1700 & 2300
\end{tabular}

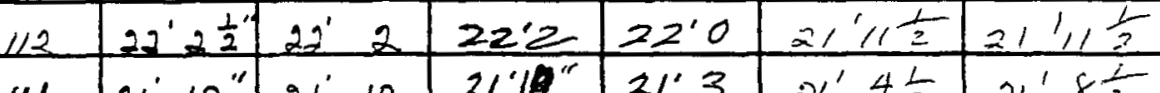

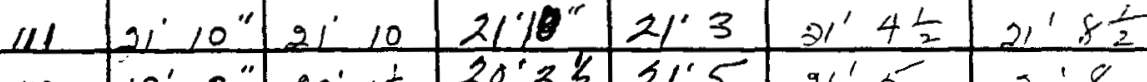
\begin{tabular}{l|ll|l|l|l|l|l}
110 & $19^{\prime} 8^{\prime \prime}$ & $20^{\prime} i \vdots$ & $20^{\prime} 3 \%$ & $21^{\prime} 5$ & $2^{\prime} 5$ & $20^{\prime}, 8$ \\
104 & $22^{\circ} 6^{\prime \prime}$ & $22^{\prime} 6^{\prime \prime}$ & $22^{\prime} 6$ & $20^{\circ} 92$ & $20^{\prime 2}$ & $20^{\prime} 3$
\end{tabular}

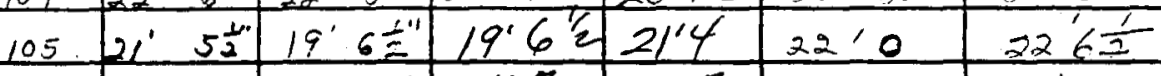

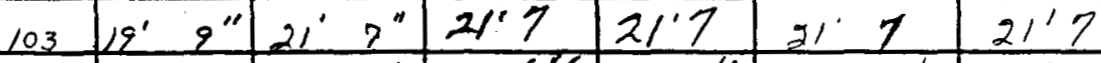

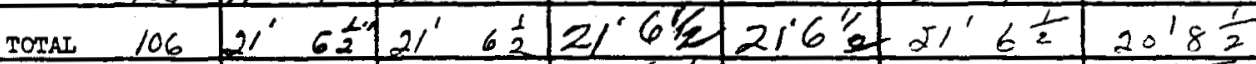

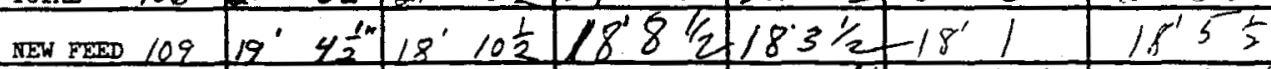

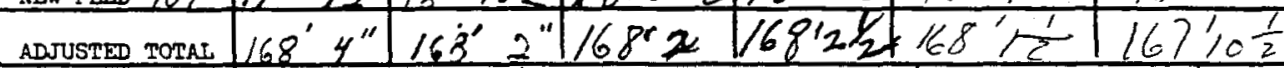

\begin{tabular}{|l|l|l|l|l|}
\hline & & & & \\
& 107 to 104 & 104 to 105 & 103 to 106 & \\
& 111 to 110 & to 103 & 106 to 109 & \\
\hline \multirow{2}{*}{ MEAT TRACE } & & & & \\
\hline
\end{tabular}

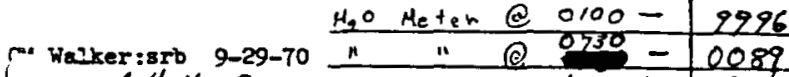

Secial 429

1O9BV-SAMPLE TAKETO144O

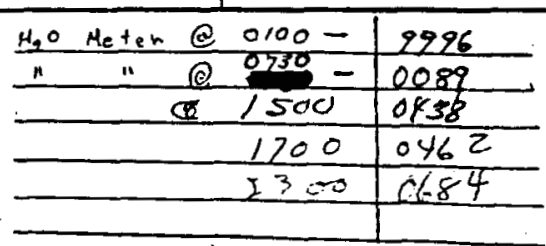


PUMP OUT TANK

RECEIVING TANK

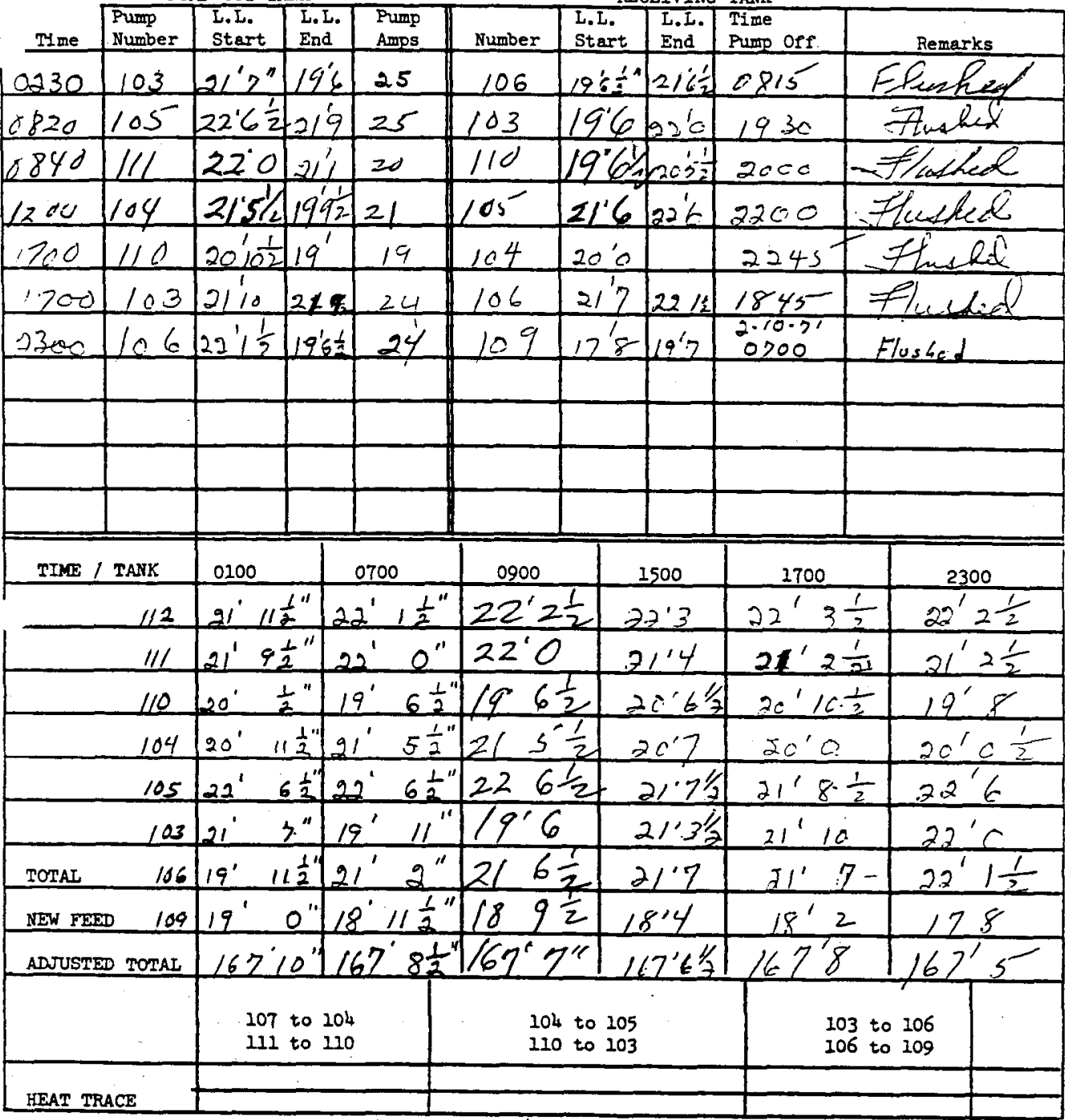

Walker:srb 9-29-70

$$
\text { Ho moter }
$$

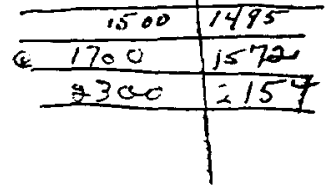


PUMP OUT TANK

RECEIVING TANK

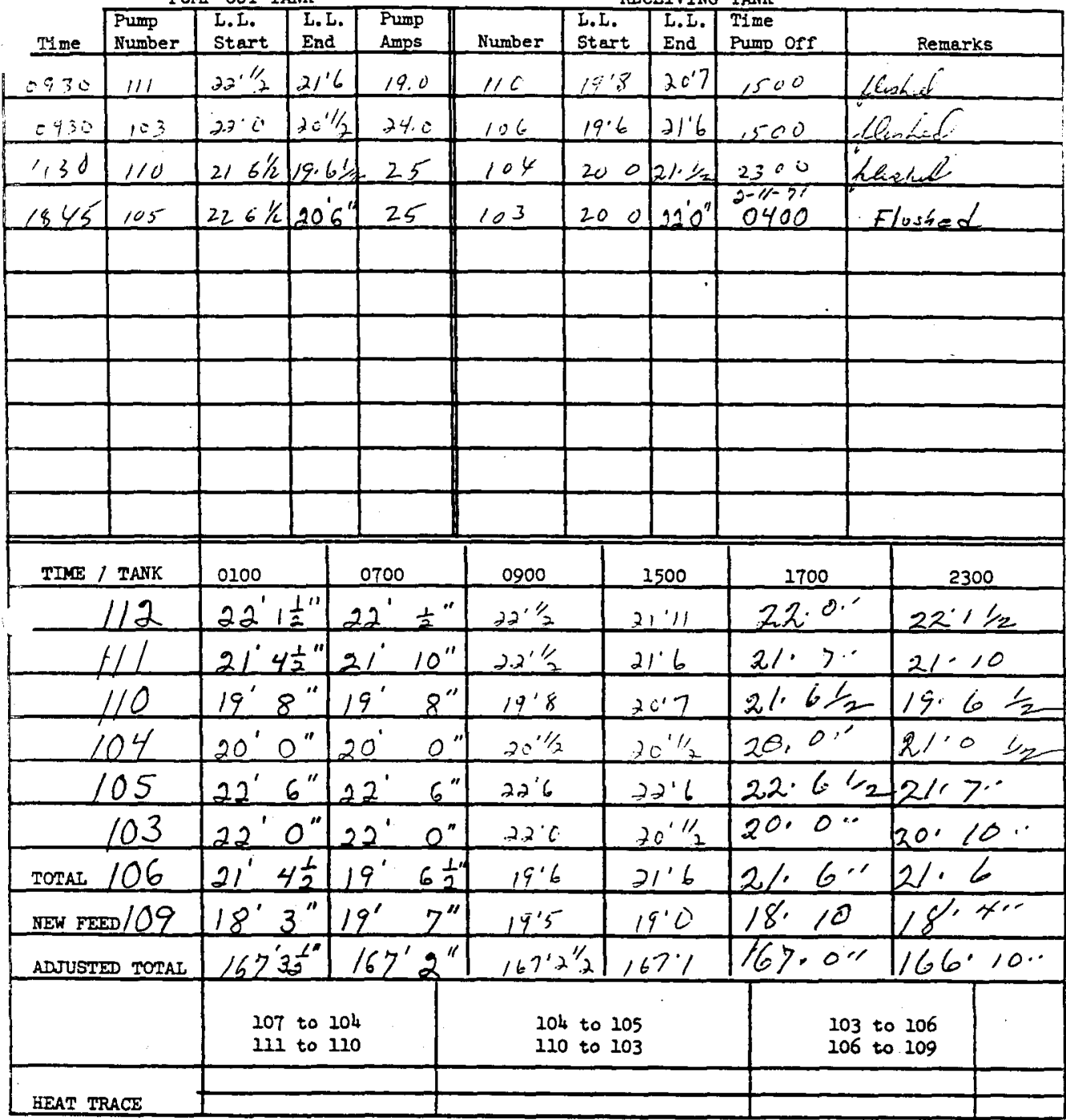

Walker:srb 9-29-70 
FUMP OUT TANK

RECEIVING TANK

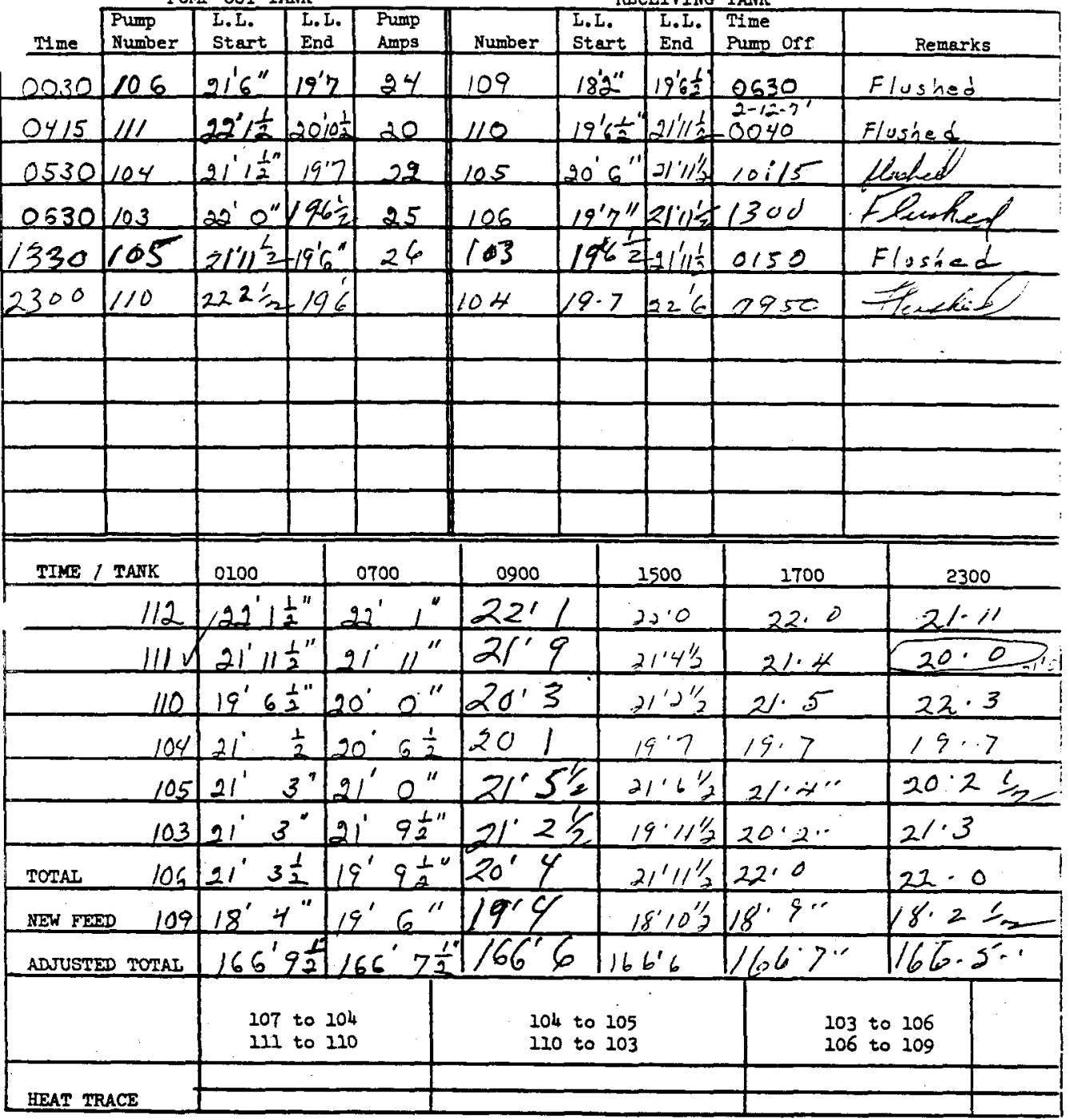

$i$

Wauker:s2b 9-29-70 
WHC-SD-LM-DP-209, ReV. 0

$$
\text { ITS-2 INVENTORY AND PUMPINC Date } 2-12.71
$$

PUMP OUT TANK RECEIVING TANK

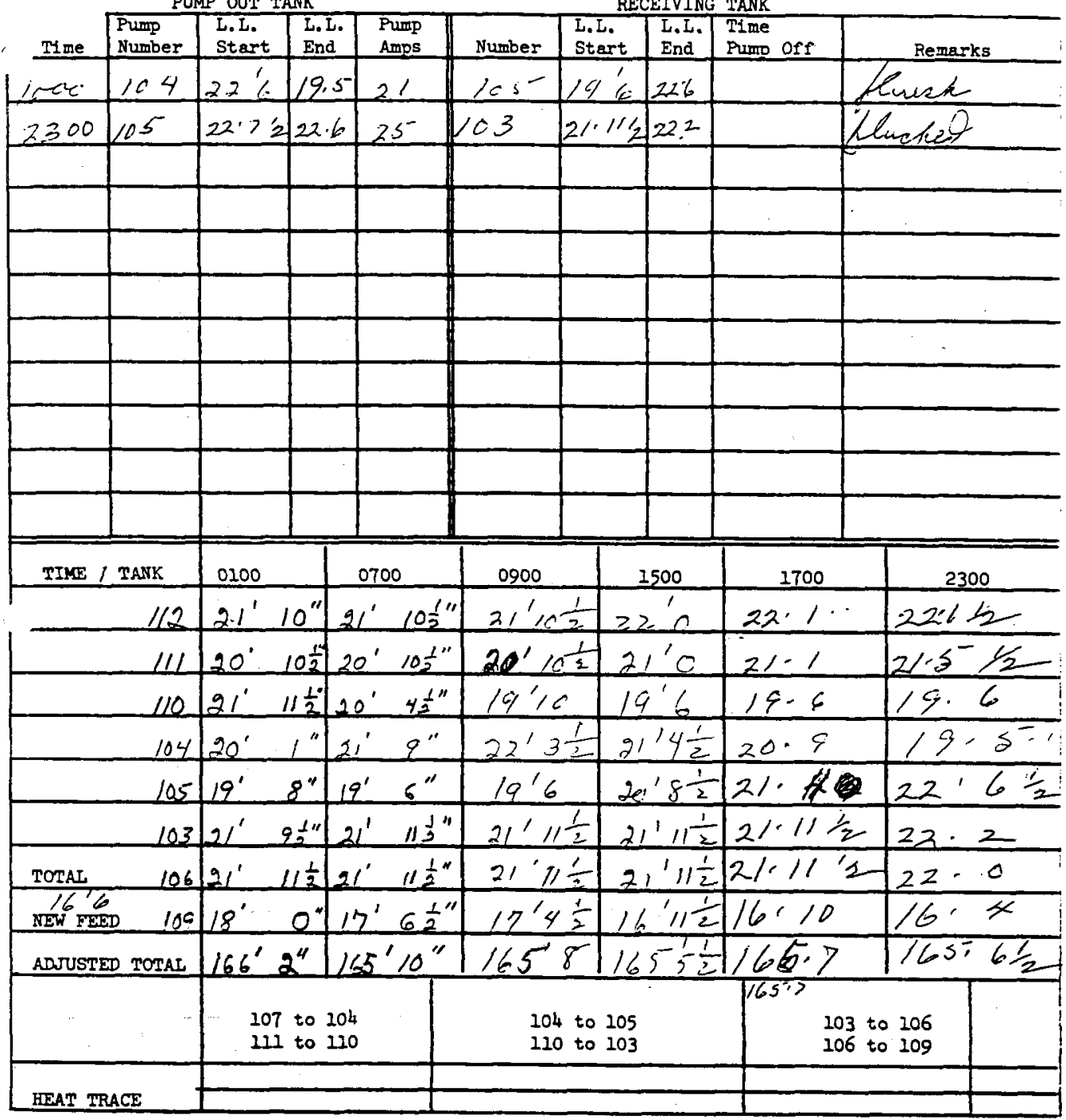

Walker:srb 9-29-70 
PUMP OUT TANK

RECEIVING TANK

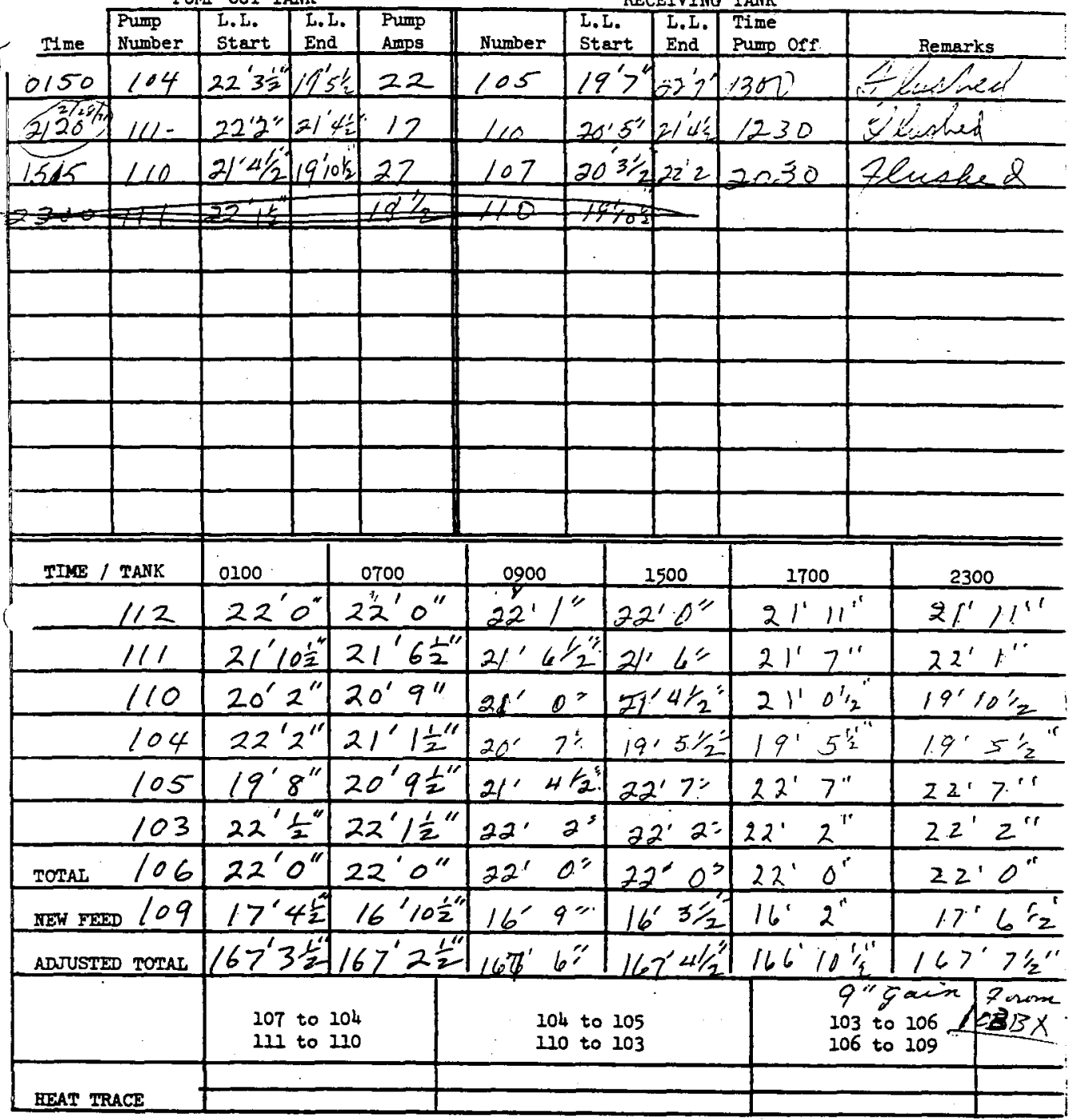

Falker:srb 9-29-70

(c) $/ 110 \frac{1}{2}$ m.to 107

( ) Rec 26' from 103Bx newreid 
PUMP OUT TANK

RECEIVING TANK

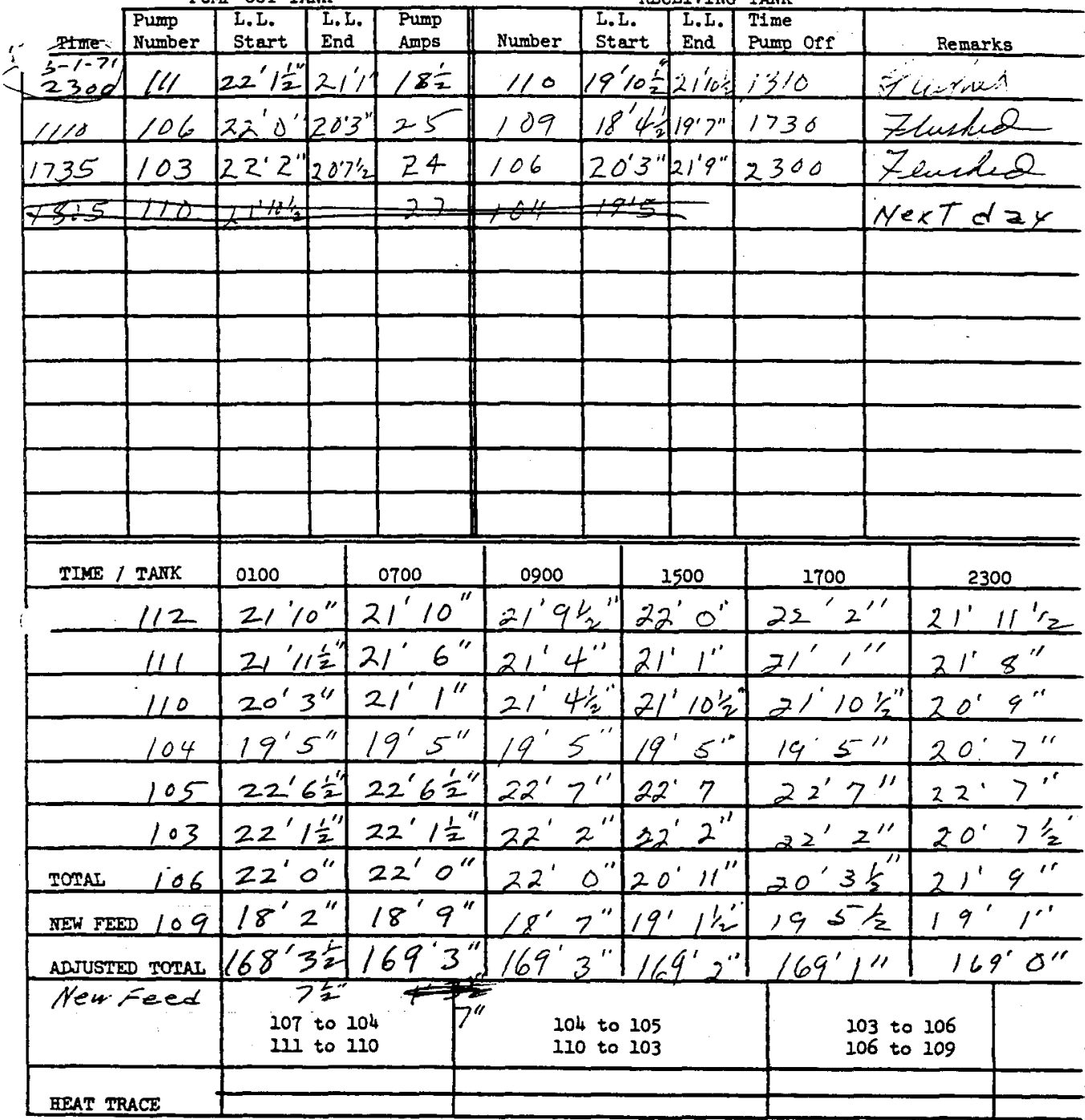

$f^{\prime}$ Walker:srb 9-29-70 
PUMP OUT TANK

RECEIVING TANK

\begin{tabular}{|c|c|c|c|c|c|c|c|c|c|}
\hline Tyme & \begin{tabular}{|l} 
Pump \\
Number
\end{tabular} & $\begin{array}{l}\text { L.I. } \\
\text { Start }\end{array}$ & $\begin{array}{l}\text { L.L. } \\
\text { End }\end{array}$ & $\begin{array}{l}\text { Pump } \\
\text { Amps }\end{array}$ & Number & $\begin{array}{l}\text { L.L. } \\
\text { Start }\end{array}$ & $\begin{array}{l}\text { L.L. } \\
\text { End }\end{array}$ & $\begin{array}{l}\text { Time } \\
\text { Pump off }\end{array}$ & Remerks \\
\hline $\begin{array}{l}-2-71 \\
1805\end{array}$ & 110 & 2110 & $199 \frac{1}{2}$ & 27 & 104 & 195 & $22^{\prime} 6 \frac{1}{2}$ & 0630 & \\
\hline 0045 & 105 & $22^{\prime} 7^{\prime \prime}$ & "' & 24 & 103 & $20^{\prime} 7 \frac{1}{2}^{\prime \prime}$ & $222 \frac{1}{2}$ & 0910 & 7 leaste \\
\hline $01: 00$ & 111 & $21^{\circ} 11$ & $21^{\prime} 3 \frac{1}{2}$ & 19 & 110 & $20^{\prime} 1 \dot{1}^{\prime \prime}$ & $19^{\prime} 9^{\prime}$ & 0630 & \\
\hline
\end{tabular}

\begin{tabular}{|l|l|l|l|l|l|l|l|l}
\hline 0605 & 106 & $21^{\prime} 9^{\prime \prime}$ & $203^{\prime \prime}$ & 25 & 109 & $18^{\prime} 5^{\prime \prime}$ & $19^{\prime} \prime^{\prime \prime}$ & 1140 \\
\hline
\end{tabular}

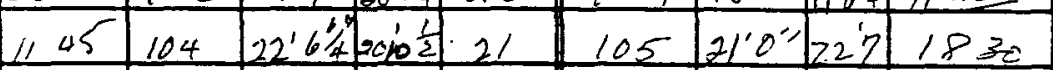

\begin{tabular}{|c|c|c|}
\hline 1150 & 103 & $22^{\prime} 2 / 210=4$ \\
\hline
\end{tabular}

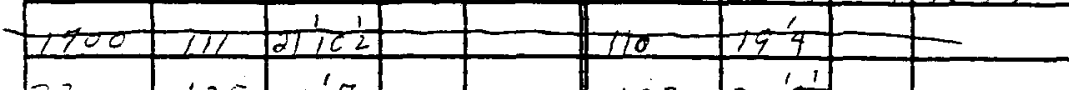

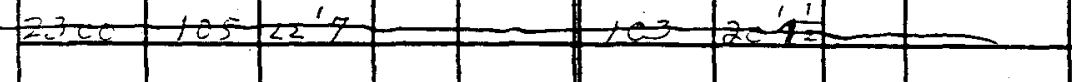
\begin{tabular}{|l|l|}
\hline & \\
\hline
\end{tabular}

\begin{tabular}{|l|l|l|l|l|l|l|}
\hline TINE / TANK & 0100 & 0700 & 0900 & 1500 & 1700 & 2300 \\
\hline
\end{tabular}

\begin{tabular}{|c|c|c|c|c|c|c|}
\hline 112 & $2 l^{\prime} \|^{\prime \prime}$ & 2192 & $21^{\prime} 110$ & $22^{\prime} 2 \frac{1}{2}$ & $22^{\prime} 1$ & 2111 \\
\hline 111 & $21^{\prime} 11^{\circ}$ & $21^{\prime} 3 \frac{1}{2}^{\prime \prime}$ & 21 & $21^{\prime} 7^{\prime \prime}$ & $2,10 \frac{1}{2}$ & $218 \frac{1}{2}$ \\
\hline 110 & $20^{\prime} 12^{\prime \prime}$ & $19^{\prime} 9 \frac{1}{2}^{\circ}$ & $19^{\prime} 10^{\prime \prime}$ & $19^{\circ} / 0^{\prime \prime}$ & $19^{\prime} 10$ & $20 \cdot 5=\frac{1}{2}$ \\
\hline 104 & $21^{\prime} 2^{\prime \prime}$ & $22^{\prime} 62^{\prime \prime}$ & $226 \frac{1}{2}$ & $21^{\prime} 10 \frac{2}{2}$ & $2,4 \frac{1}{2}$ & $20.10 \frac{1}{2}$ \\
\hline 105 & $22^{\prime} 7^{\prime \prime}$ & $2 r^{\prime} 5^{\prime \prime}$ & $21^{\prime} 0^{\prime \prime}$ & $21^{\prime} 7 \frac{2^{\prime}}{}$ & $22,1 \frac{1}{2}$ & $22^{\circ} 7$ \\
\hline 103 & $20^{\prime} 7 \frac{1}{2}$ & $21^{\prime} 9^{\prime \prime}$ & $22^{\prime} 2 \frac{1}{2}$ & $21^{\prime} e^{\prime \prime}$ & $20^{\prime} 4 \frac{1}{2}$ & $20^{\prime} 4 \frac{1}{2}$ \\
\hline TOTAL $\quad 106$ & $21^{\prime} 9^{\prime \prime}$ & $21^{\prime} 6^{\prime \prime}$ & $20^{\prime} 11^{\prime \prime}$ & $21^{\prime} 5 \frac{1}{2}$ & $22^{\prime} 1$ & 221 \\
\hline NEW FEED 109 & $18^{\prime} 10^{\frac{1}{2}}$ & $18^{\prime} 7 \frac{14}{2}$ & $19^{\prime} 0^{\circ}$ & $19^{\prime} 2 \frac{1}{2}$ & $19^{\prime} 0^{\prime}$ & $18^{\prime} 6 \frac{1}{3}$ \\
\hline ADUUSTED TOTAL & $168^{\prime} 112^{\prime \prime}$ & $168^{\prime} 8 \div$ & $168^{\prime} 9^{\prime \prime}$ & $1689 \frac{1}{2}$ & $165^{\prime} 92$ & $168^{\prime} 62^{\prime}$ \\
\hline & $\begin{array}{l}107 \text { to } \\
111 \text { to }\end{array}$ & & $\begin{array}{l}104 \text { to } \\
110 \text { to }\end{array}$ & $\begin{array}{l}105 \\
103\end{array}$ & $\begin{array}{l}103 \text { to } \\
106 \text { to }\end{array}$ & $\begin{array}{l}106 \\
109\end{array}$ \\
\hline HEAT TRACE & & & & & & \\
\hline
\end{tabular}

Walker:srb 9-29-70 
WHC-SD-LMH-DP-209, REV. O

ITS-2 INVENTORY AND PUMPING

Date

WAR $1 \quad 1971$

PUMP OUT TANK

RECEIVING TANK

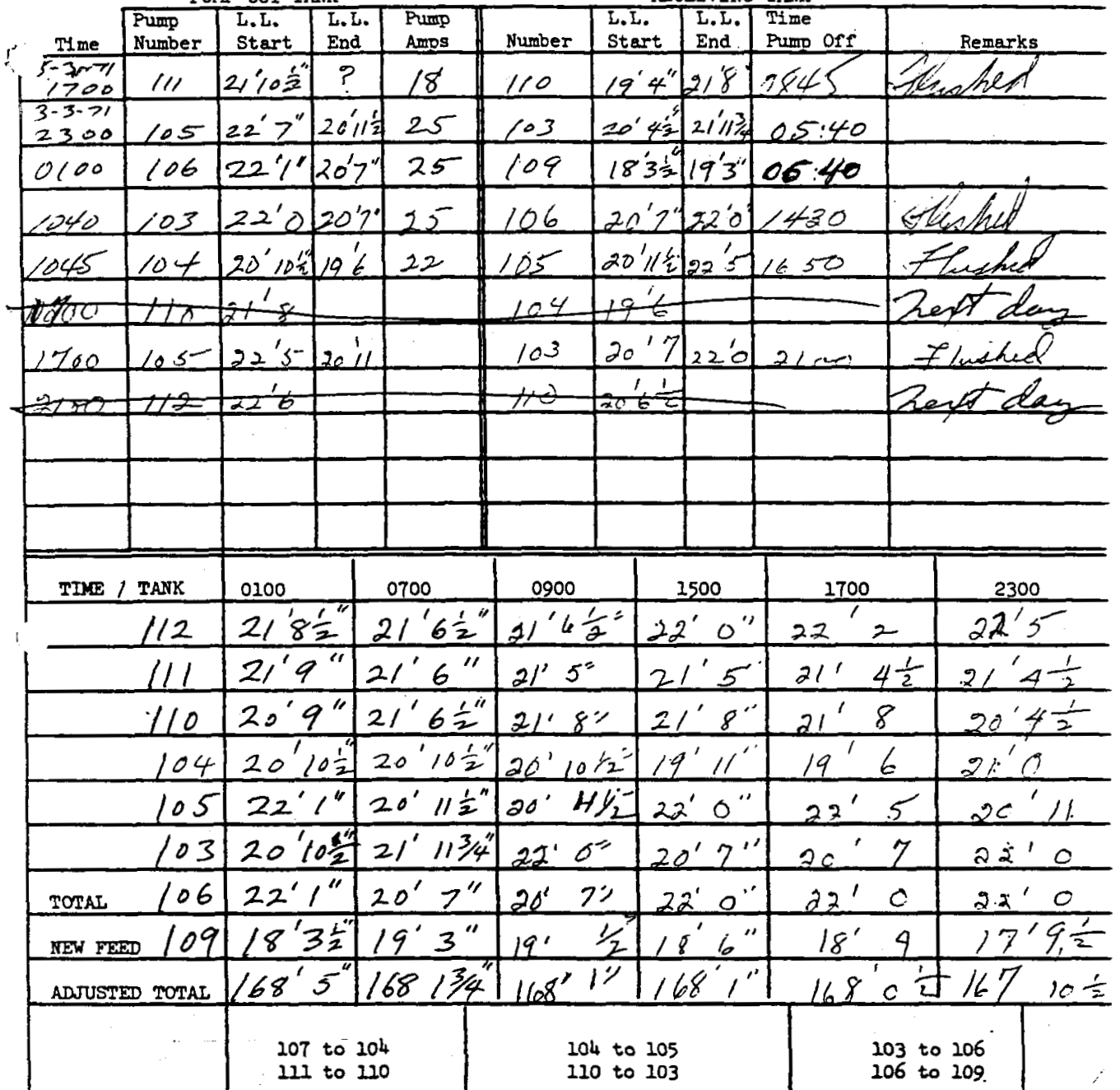

HEAT TRACE --

Wavker:srb 9-29-70 
PUMP OUT TANK

RECEIVING TANK

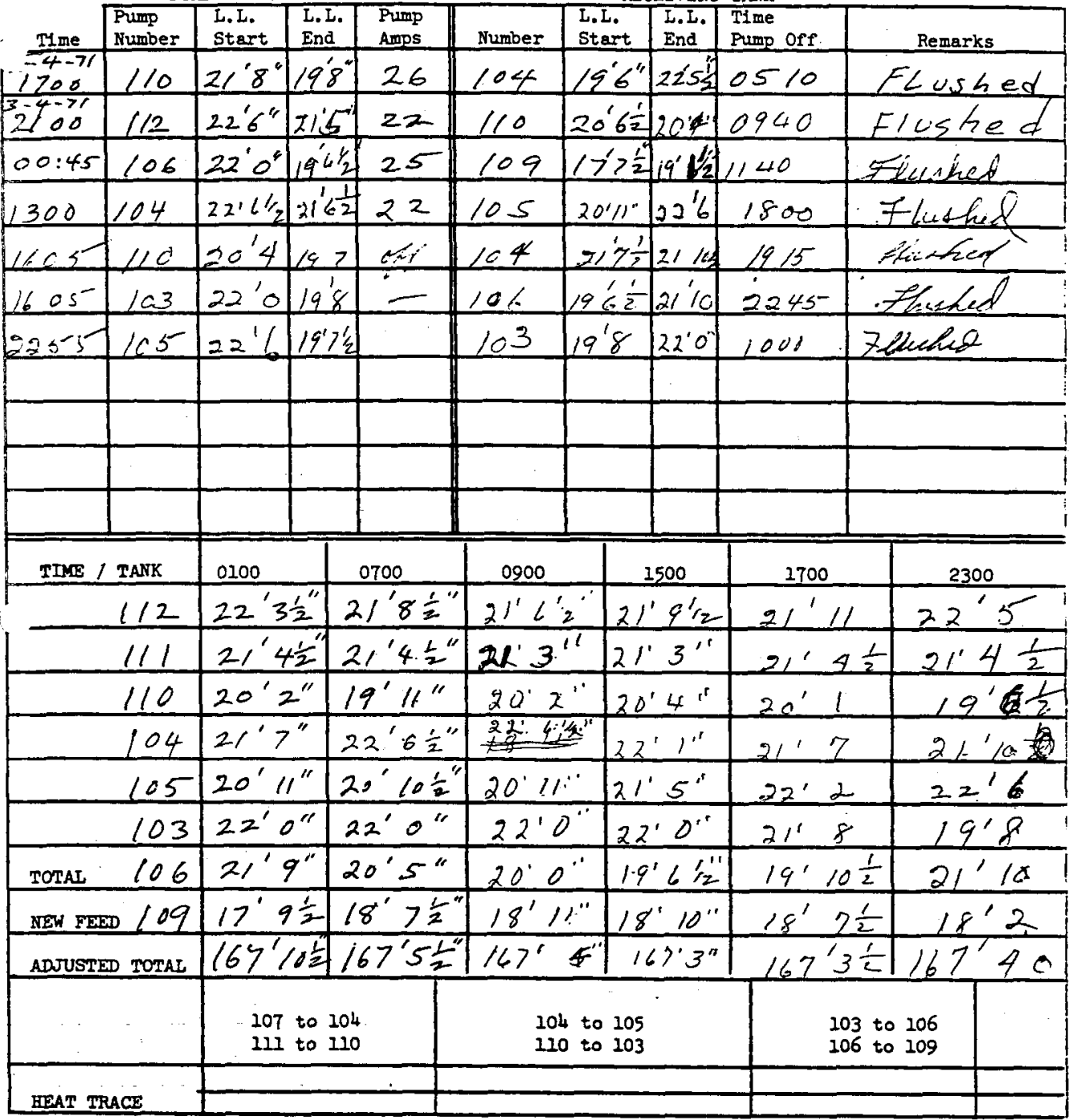

$f^{-1 \text { Walker:stb } 9-29-70}$ 
PUMP OUT TANK

RECEIVING TANK

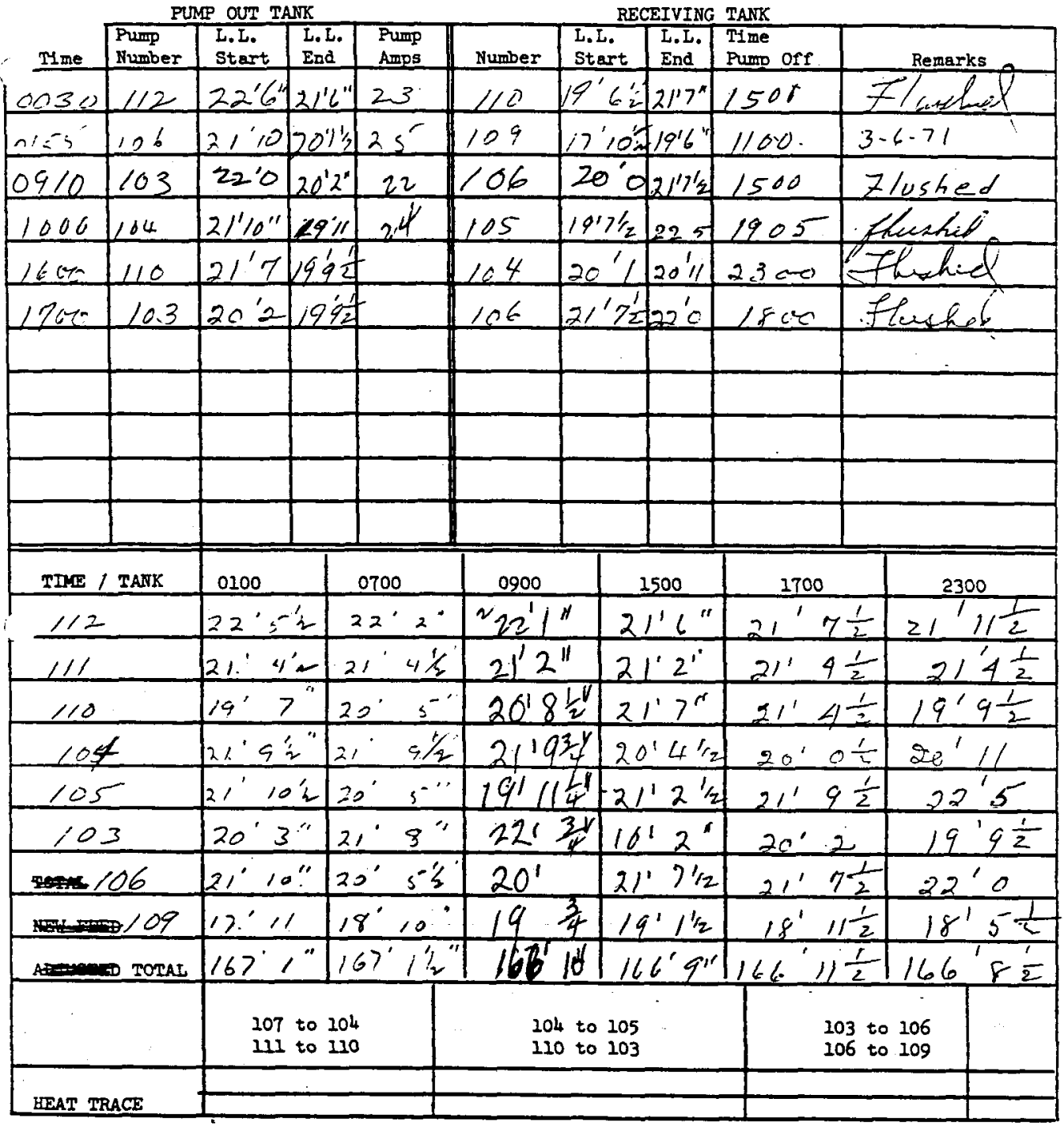

$f^{-\cdots}$ Walker:srb 9-29-70 


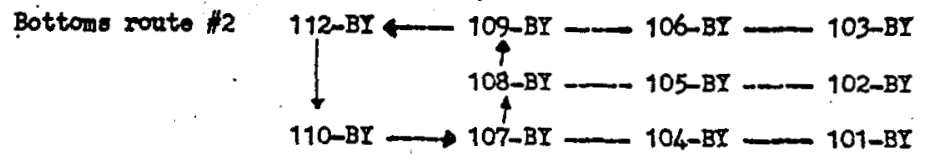

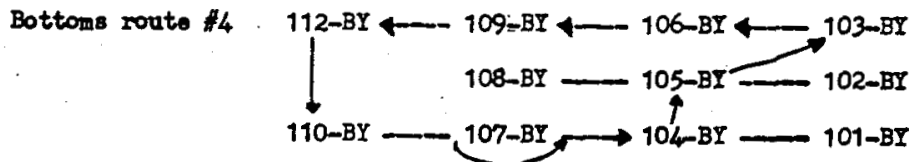

ITS-2 INVENTORY AND POMPING

DATE $3-7-71$

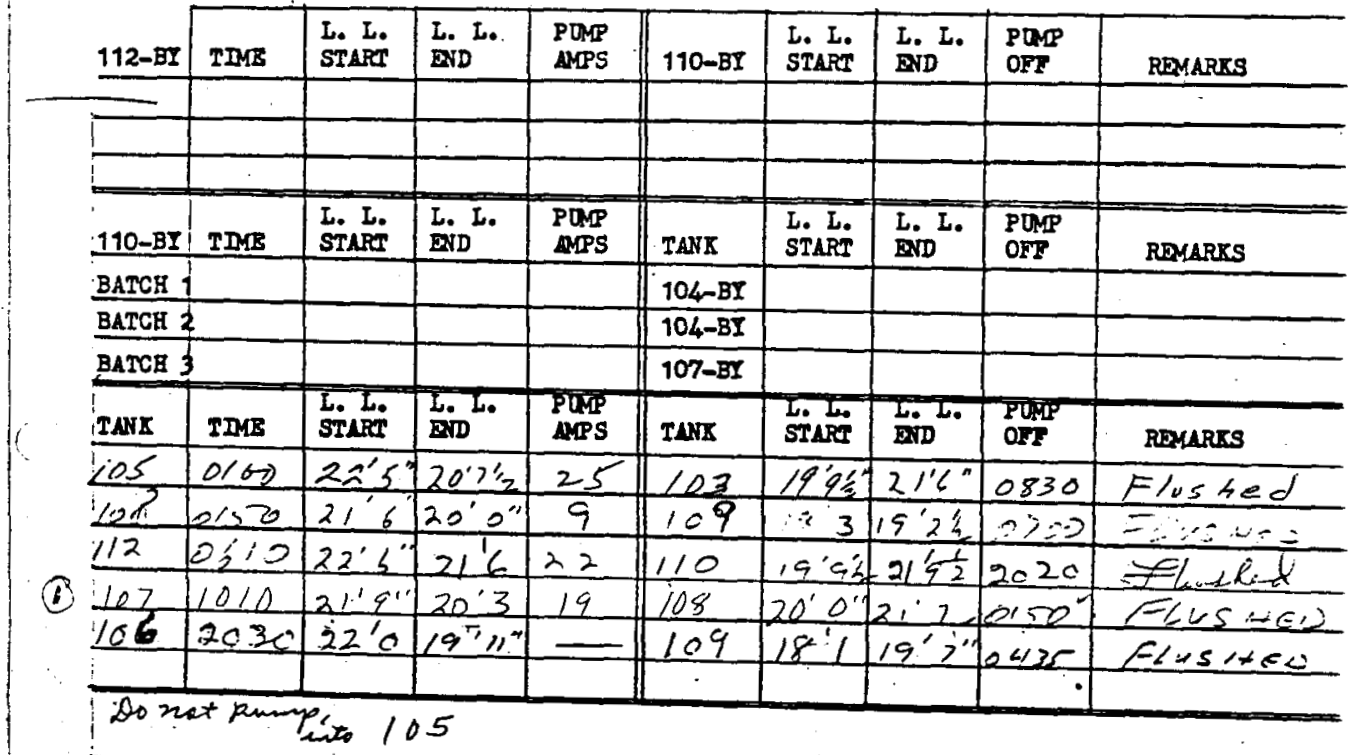

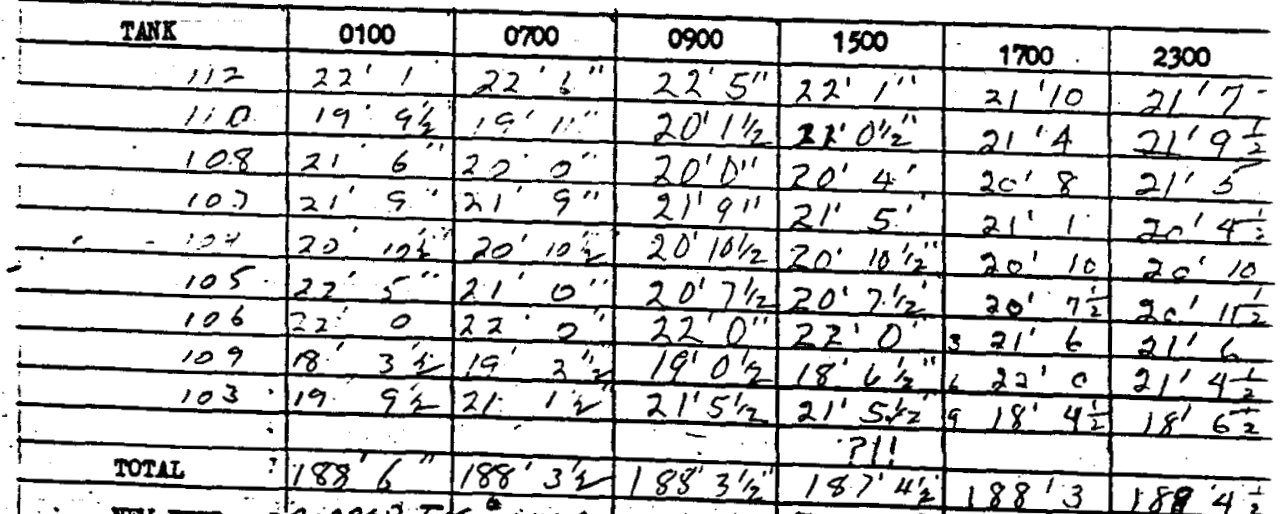

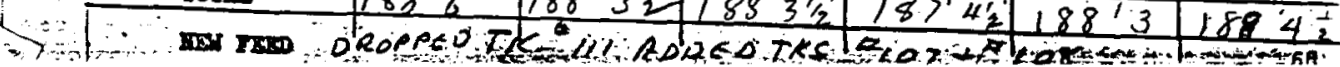


Boittone route $/ 2$ 112-BI 109-BI 106-BY -BY

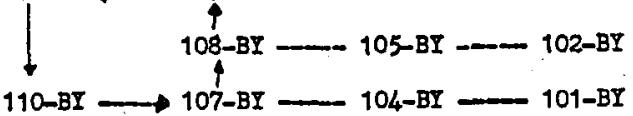

Bottoms route 4

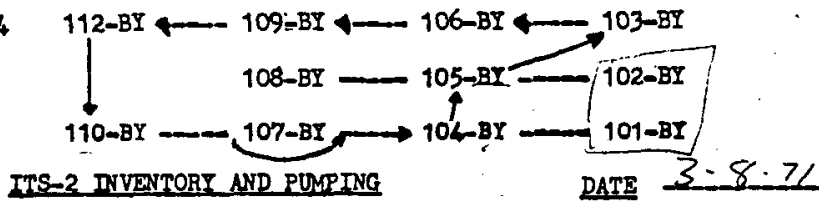

\begin{tabular}{|c|c|c|c|c|c|c|c|c|c|}
\hline 112-BI & TIMS & $\begin{array}{l}\text { L. L. } \\
\text { STAFT }\end{array}$ & $\begin{array}{l}\text { I. L. } \\
\text { END }\end{array}$ & $\begin{array}{l}\text { POMP } \\
\text { AMPS }\end{array}$ & $110-\mathrm{BY}$ & $\begin{array}{l}\text { L. L. } \\
\text { START }\end{array}$ & $\begin{array}{l}\text { L. I. } \\
\text { DND }\end{array}$ & $\begin{array}{l}\text { POMP } \\
\text { OFP }\end{array}$ & REMARKS \\
\hline & 1130 & $22^{\prime} 6^{\prime \prime}$ & 215 & & & $20^{\prime} 2^{\prime \prime}$ & 2182 & 2300 & \\
\hline & & & & & & & & & \\
\hline & & & & & & & & & \\
\hline $110-B I$ & $\operatorname{TDM}$ & $\begin{array}{l}\text { I. I. } \\
\text { STARI }\end{array}$ & $\begin{array}{l}\text { L. L. } \\
\text { END }\end{array}$ & $\begin{array}{l}\text { PQMP } \\
\text { AMPS }\end{array}$ & TANK & $\begin{array}{l}\text { L. I. } \\
\text { START }\end{array}$ & $\begin{array}{l}\text { L. I. } \\
\text { BND }\end{array}$ & $\begin{array}{l}\text { POMP } \\
\text { OFP }\end{array}$ & RDMARKS \\
\hline BATCH & & & & & 104-BI & & & & \\
\hline BATCH 2 & 20245 & $21^{\circ} 9^{\prime}$ & $20^{\prime} 2$ & 25 & 104-BI & $20 \%:$ & $225^{\prime \prime}$ & 0210 & Flased \\
\hline Barch 3 & & & & & 107-BI & & & & \\
\hline TANK & $\operatorname{TAg}$ & $\begin{array}{l}\text { L. I. } \\
\text { STARE }\end{array}$ & $\begin{array}{l}\text { L. } \\
\text { BDD }\end{array}$ & $\begin{array}{l}\text { PQP } \\
\text { MPS }\end{array}$ & TANK & $\begin{array}{l}\text { L. La } \\
\text { STARE }\end{array}$ & L. L. & $\begin{array}{l}\text { PUFir } \\
\text { OrT }\end{array}$ & REMARKS \\
\hline 103 & 0530 & $21^{\prime} z^{\prime \prime}$ & $196^{\prime}$ & 21 & 106 & $1911^{\prime}$ & $21^{1} 10^{2} 5$ & $\operatorname{lon}$ & 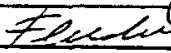 \\
\hline 205 & 1400 & $22^{\prime} 4 \frac{1}{2}$ & $18^{\prime}+1$ & 24 & 103 & $19^{\prime} 6$ & $22^{\prime} \cdot 2^{4}$ & 033.5 & $12 \sec 4$ \\
\hline 106 & 230 & 2111 & 2652 & 39 & 109 & $18^{\prime} 42$ & $19^{\prime} 7^{\prime}$ & 0340 & $72 \times 2$ \\
\hline . & & & & & & & & & \\
\hline & & & & & & & 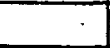 & & \\
\hline & & & & & & & & & \\
\hline
\end{tabular}

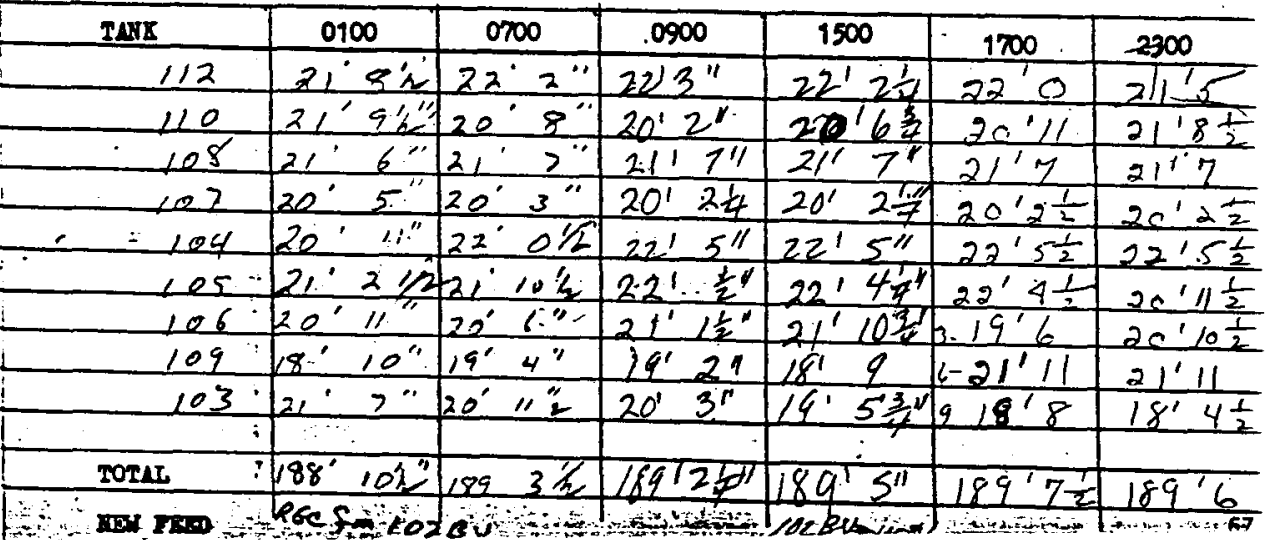




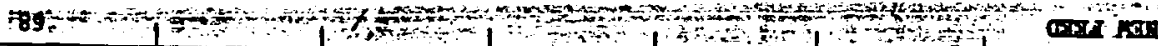

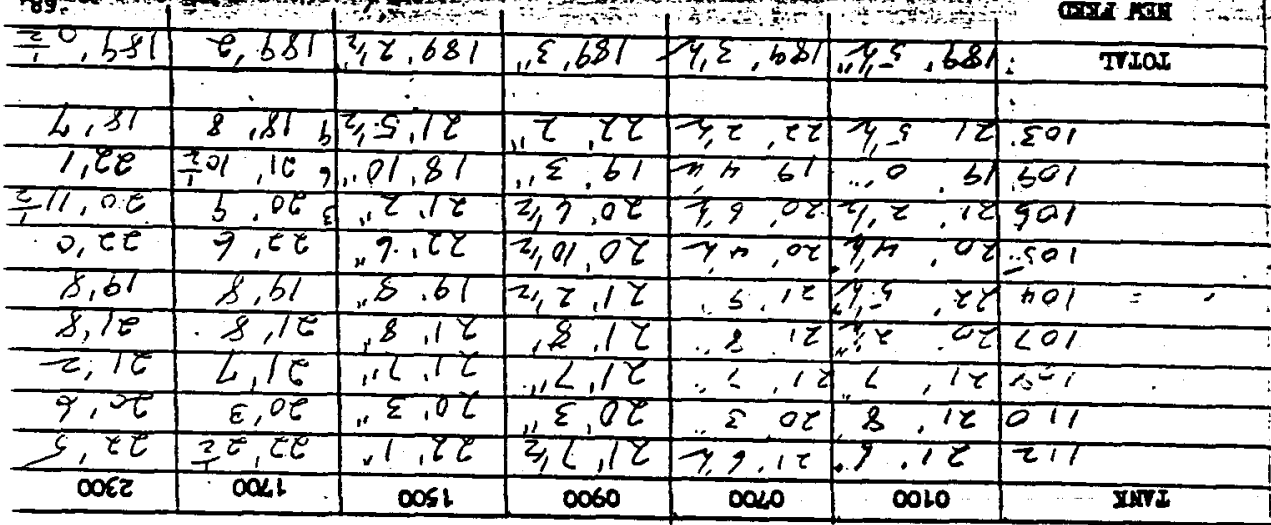

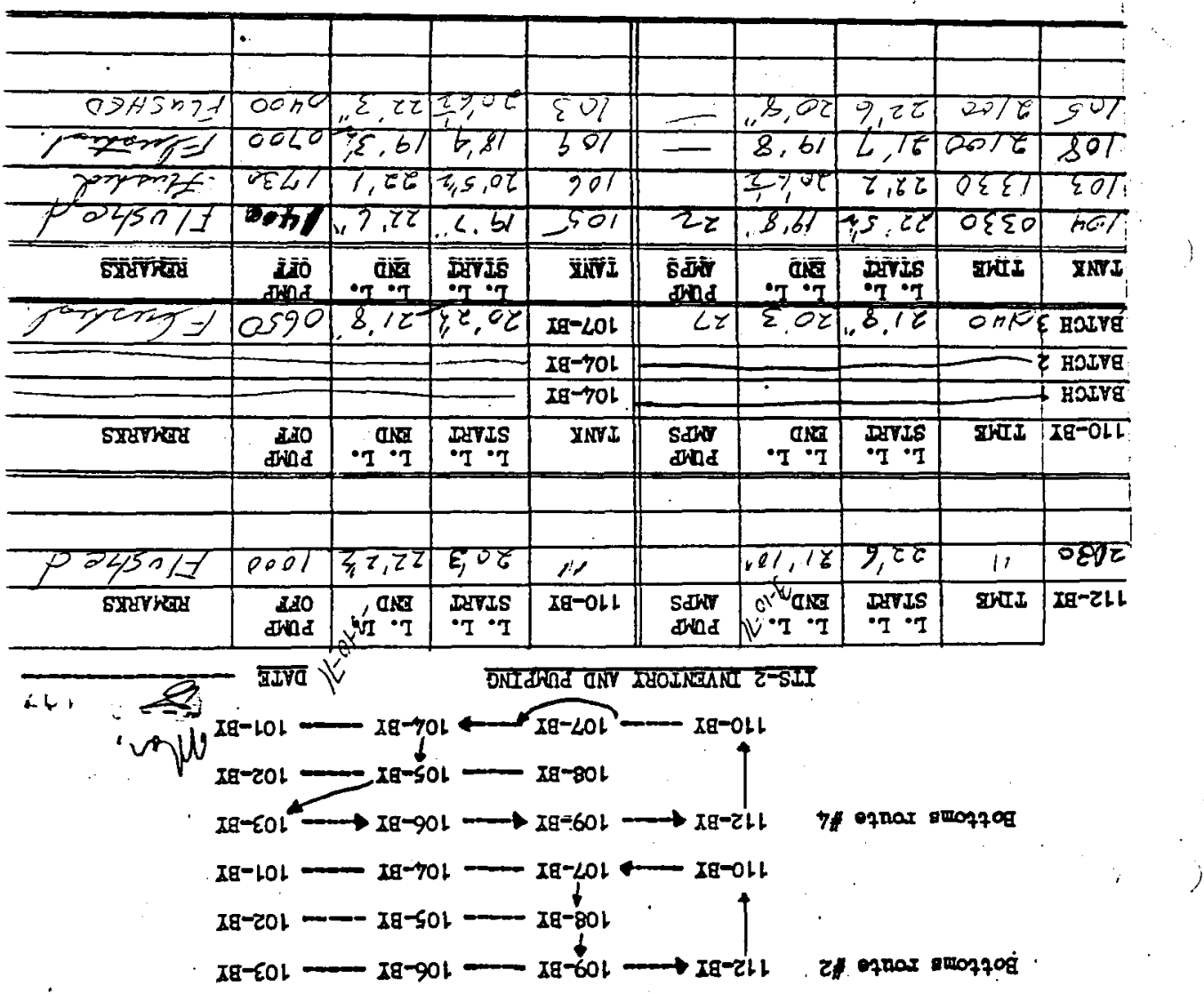




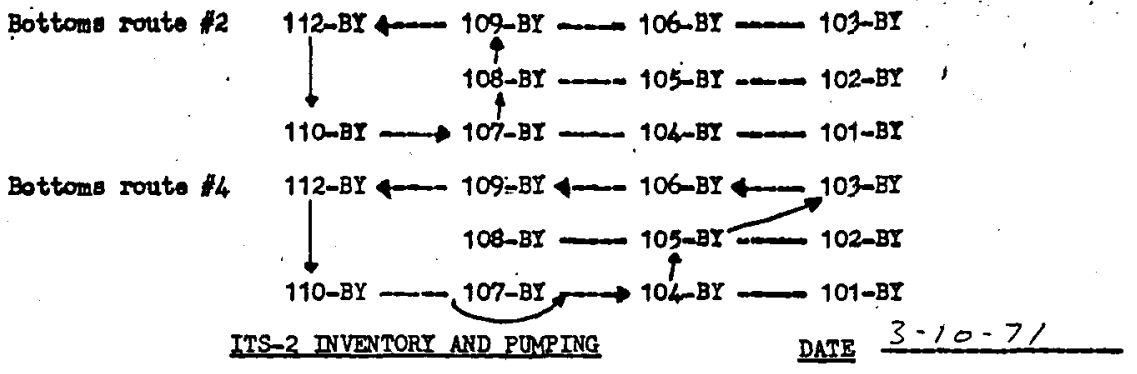

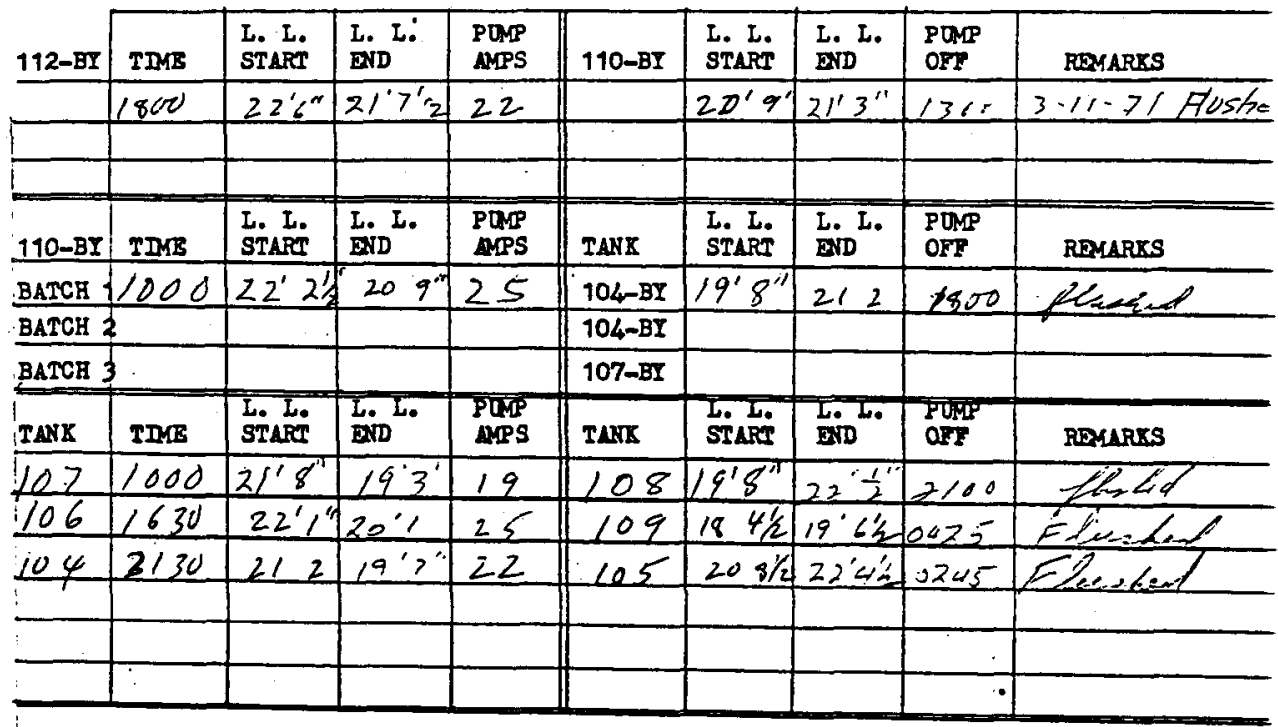

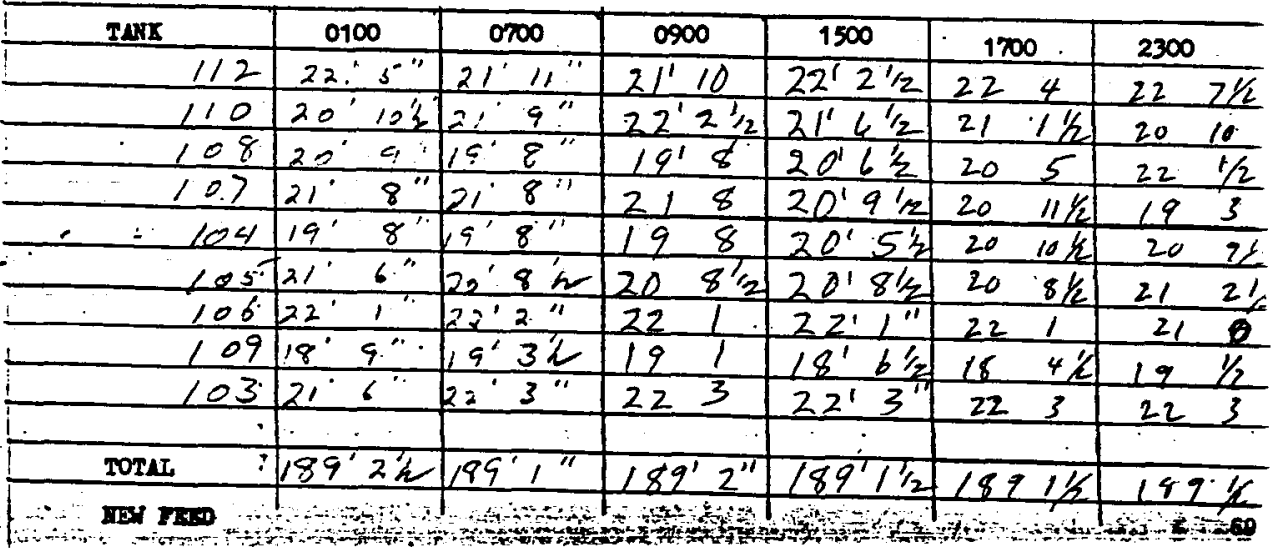


Boittoms route $42 \quad 106-\mathrm{BI}-103-\mathrm{BI}$

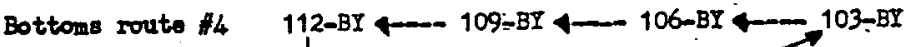

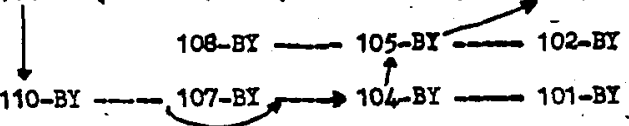

ITS-2 DNVENTORY AND PUMPING

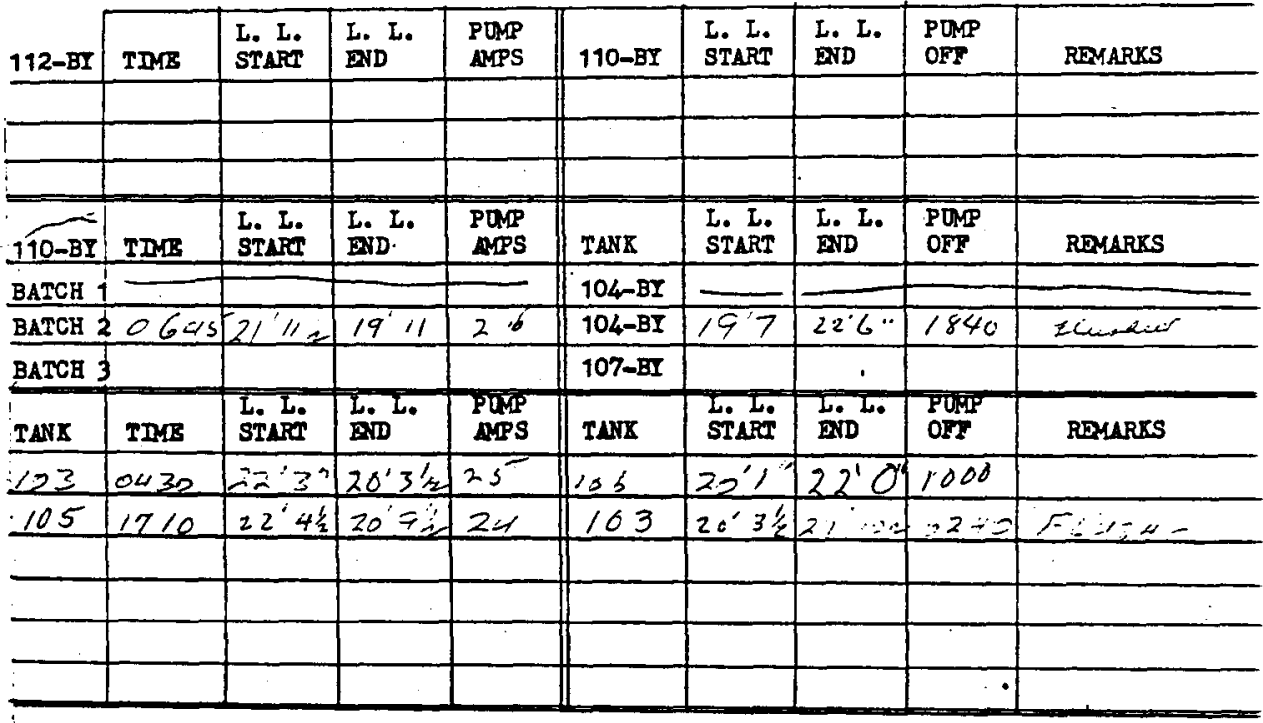

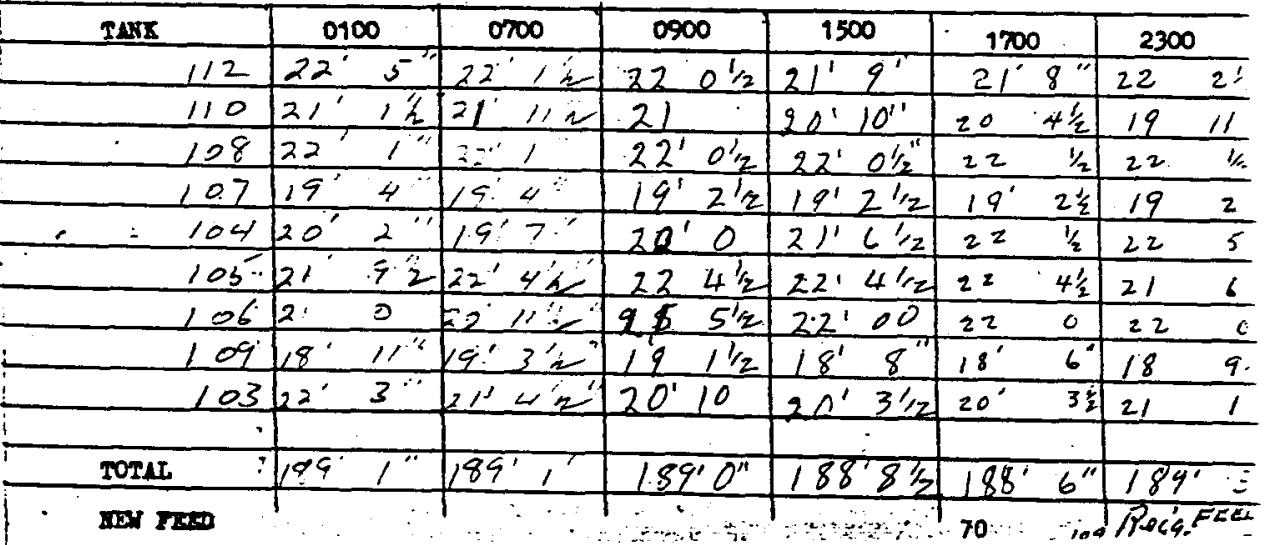


Bottome route 2 112-BI 4 109-BY $-106-\mathrm{BI}-103-\mathrm{BY}$

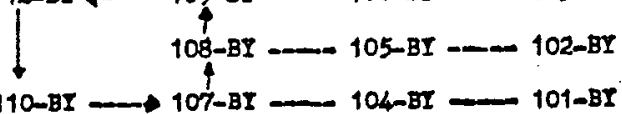

Bottoms route f4 112-BI 10 109\%-BI 106-BI 103-BI

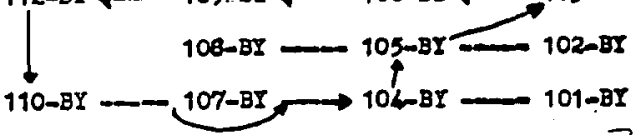

ITS-2 DVVETORI AND PUMPING

DATE $3-12-7$

\begin{tabular}{|c|c|c|c|c|c|c|c|c|c|c|c|}
\hline 112-BY & TMM & \multicolumn{2}{|c|}{$\begin{array}{l}\text { L. L. } \\
\text { START }\end{array}$} & $\begin{array}{l}\text { L. L. } \\
\text { END }\end{array}$ & $\begin{array}{l}\text { POMP } \\
\text { AMPS }\end{array}$ & 110-BI & $\begin{array}{l}\text { I. I. } \\
\text { START }\end{array}$ & \begin{tabular}{|l} 
L. L. \\
DND
\end{tabular} & $\begin{array}{l}\text { PONP } \\
\text { OFF }\end{array}$ & \\
\hline & 0155 & \multicolumn{2}{|c|}{226} & $22^{\prime}$ & 22 & 110-81 & 19 bi & & 1245 & \multicolumn{2}{|c|}{ Destest } \\
\hline & & \multirow{2}{*}{\multicolumn{2}{|c|}{ 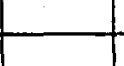 }} & & & & & & \multirow[b]{2}{*}{ 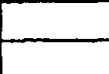 } & \multicolumn{2}{|c|}{ tes $x>2$} \\
\hline & & & & & & & & & & & \\
\hline $110-\mathrm{BI}$ & $\operatorname{Tn} s$ & \multicolumn{2}{|c|}{$\begin{array}{l}\text { L. L. } \\
\text { START }\end{array}$} & $\begin{array}{l}\text { L. I. } \\
\text { END }\end{array}$ & $\begin{array}{l}\text { PQMP } \\
\text { MPS }\end{array}$ & TANK & $\begin{array}{l}\text { I. L. } \\
\text { START }\end{array}$ & $\begin{array}{l}\text { L. L. } \\
\text { BND }\end{array}$ & $\begin{array}{l}\text { POMP } \\
\text { OFF } \\
\end{array}$ & \multicolumn{2}{|c|}{ RaMaRXS } \\
\hline \multicolumn{2}{|c|}{ BATCH $1-$} & \multicolumn{2}{|c|}{$\square-\square$} & & & $104=8 \%$ & & & 2 & \multicolumn{2}{|c|}{-2} \\
\hline BATCH 2 & $2 \ldots$ & \multicolumn{2}{|c|}{$=$} & 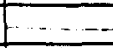 & $\cdots$ & $104-B I$ & $\ldots$ & & $=$ & $=$ & 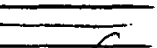 \\
\hline BATCH 3 & 1645 & 21 & $7 / 2$ & $14^{\prime} x^{\prime \prime}$ & 25 & 107-BI & $19^{\circ} 5^{\prime \prime}$ & $22^{\prime} 8^{\prime \prime}$ & 6645 & Ifes & Send \\
\hline TANS & TDMS & $\begin{array}{l}\text { L. } \\
\text { STA }\end{array}$ & & $\begin{array}{l}\text { L. } \\
\text { BND }\end{array}$ & $\begin{array}{l}\text { PUNP } \\
\text { DMPS }\end{array}$ & TANX & $\begin{array}{l}\text { L. L. } \\
\text { STAPT }\end{array}$ & LED & OrF & RaPS & LRKS \\
\hline 104 & $04=0$ & $22^{\prime}$ & 54 & $20^{\prime} 9$ & 22 & 105 & $20^{\prime} 46$ & 226 & 0945 & Fins & Ledi \\
\hline 106 & 1630 & $22^{\circ}$ & 0.1 & 2973 & 24 & 109 & $18^{\prime} 3^{\prime \prime}$ & $196^{\prime \prime} 0$ & $2<22$ & \pm & $-x+2$ \\
\hline & & & & & & & & & & & \\
\hline & & & & & & & & & & & \\
\hline & & & & & & & & & - & & \\
\hline & & & & & & & & & & & \\
\hline & & & & & & & & & & & \\
\hline & $\operatorname{sens}$ & & & 0100 & 0700 & & 900 & 1500 & & $\infty$ & 2300 \\
\hline & & 12 & $2=$ & $2^{\prime} 3{ }^{\prime}$ & $22^{\circ} 4$ & & $2^{\prime} 4$ & $224 \frac{1}{2}$ & 22 & $4 / 2$ & 22 \\
\hline & & 19 & 15 & $10 \%$ & $20^{\prime}$ & & $110 \frac{1}{2}$ & $21^{\prime} 4$ & 21 & $r / 2$ & 20 \\
\hline & & 08 & $\pi i$ & 20 & $22^{\circ}$ ats & & $2^{\prime}=\frac{1}{2}$ & $22^{\prime} c \frac{1}{2}$ & $2 q$ & 畜 & 22 \\
\hline & & $Q 7$ & $19^{\prime}$ & 32 & $\mathrm{ig}^{\circ}$ & 19 & $2 \frac{1}{2}$ & $19^{\circ} 2 \frac{1}{2}$ & 19 & 3 & 20 \\
\hline- & $=$ & 04 & $22^{\prime}$ & $55^{-1}$ & $21^{\prime} 7^{\circ}$ & & 0.11 & $20^{\circ} 9$ & 20 & $9 \frac{1}{2}$ & 20 \\
\hline & & $8 ;$ & & 2 a & $218 \%$ & & $7^{\prime} 4$ & $22^{\prime} 6$ & 22 & 6 & 22 \\
\hline & & 06 & $22^{\circ}$ & 1 & $22^{\circ} 1$ & 32 & 1.10 & $21^{\prime} 10$ & $2 z$ & 0 & 20 \\
\hline & & 10? & $19^{\circ}$ & $4 "$ & 45 & 62 & $x^{\prime} 0$ & $2 z^{\prime} c$ & 18 & 3 & $19^{\circ}$ \\
\hline & & 23 & 21 & $s^{-12}$ & 132 & $i 1$ & 810 & $18^{\prime}, 5$ & 21 & 10 & 21 \\
\hline & & & & & & & & & & & \\
\hline$\alpha$ & TOTAL & & 190 & $2^{\circ} 2$ & $190^{\circ} 8^{\prime \prime}$ & 190 & $4 \frac{1}{2}$ & $1905 \frac{1}{2}$ & 190 & $5^{\circ} 8^{\prime \prime}$ & $190^{\prime}$ \\
\hline
\end{tabular}




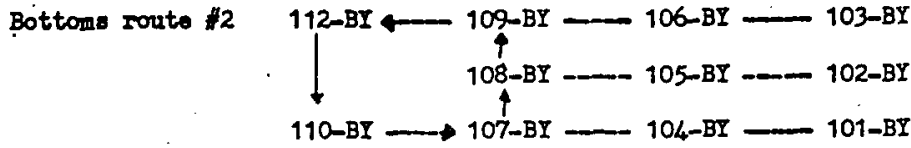

Bottoms route "4 112-BY $4-109=\mathrm{BY}-106-\mathrm{BY}+103-\mathrm{BY}$

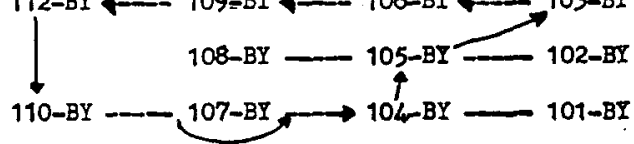

ITS-2 DNVENTORY AND PUMPING

DATE $3=|3-5|$

\begin{tabular}{|c|c|c|c|c|c|c|c|c|c|}
\hline ११२-BY & TIMB & $\begin{array}{l}\text { L. L. } \\
\text { START }\end{array}$ & $\begin{array}{l}\text { L. I. } \\
\text { DND }\end{array}$ & $\begin{array}{l}\text { PUMP } \\
\text { AMPS }\end{array}$ & $110-81$ & $\begin{array}{l}\text { L. L. } \\
\text { STARE }\end{array}$ & $\begin{array}{l}\text { L. I. } \\
\text { DND }\end{array}$ & $\begin{array}{l}\text { POMP } \\
\text { OFT }\end{array}$ & RDMARKS \\
\hline 112 & 0700 & $226^{\prime}$ & $320 "$ & 21 & $110 D^{Y}$ & $19^{6} 7^{\prime \prime}$ & 2015 & 6125 & $3-15-71$ \\
\hline & & & & & & & & & - \\
\hline & & & & & & & & & \\
\hline 110-BI & TMK & $\begin{array}{l}\text { L. I. } \\
\text { START }\end{array}$ & $\begin{array}{l}\text { L. L. } \\
\text { END }\end{array}$ & $\begin{array}{l}\text { PQMP } \\
\text { AMPS }\end{array}$ & TANK & $\begin{array}{l}\text { L. L. } \\
\text { START }\end{array}$ & $\begin{array}{l}\text { L. L. } \\
\text { END }\end{array}$ & $\begin{array}{l}\text { PQNP } \\
\text { OFY }\end{array}$ & RPMARXS \\
\hline BATCA 1 & 2150 & $21^{\prime} 6 \prime$ & $20^{\circ} 11$ & & 104-BI & $20^{\circ} 9$ & $22^{\prime} 51$ & $\therefore 860$ & \\
\hline BATCH 2 & - & & & & $104-B I$ & & & & 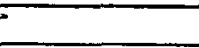 \\
\hline BATCH 3 & 3 & & & & 107-BY & & & & $=$ \\
\hline TANK & TIMS & $\begin{array}{l}\text { L. L. } \\
\text { START }\end{array}$ & $\begin{array}{l}\text { Le L } \\
\text { END }\end{array}$ & $\begin{array}{l}\text { PUMP } \\
\text { AMPS }\end{array}$ & TANK & $\begin{array}{l}\text { L. I. } \\
\text { START }\end{array}$ & END & $\begin{array}{l}\text { PUMIP } \\
\text { OFP }\end{array}$ & RDSARKS \\
\hline 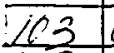 & $0 / 25$ & $21^{\prime} / 0^{i \prime}$ & 1993 & 22 & 106 & $\varphi^{\prime} Z^{\prime \prime}$ & $218 \frac{1}{2}$ & $?$ & \\
\hline $10 \div$ & 6715 & $221 \frac{1}{2}$ & $21^{\circ} 67 / 2$ & 20 & 109 & $190 \div 1$ & $18^{\circ} 2$ & 1815 & teariat \\
\hline 105 & 10730 & $22^{\prime} 6^{\prime \prime}$ & $19^{\prime} 1 / 2$ & 26 & 103 & $149 \frac{3}{4}$ & $22^{\prime} 3^{\prime \prime}=$ & 1630 & Fiushod \\
\hline 167 & 1000 & 2218 &. & & 108 & $=1 / 2 \frac{1}{2}$ & & $0 / 1$ & Leive lengd \\
\hline 103 & 2130 & $22^{\prime} 3$ & $21^{\circ} 7$ & 25 & 106 & $2181 / 2$ & $22^{\circ} 4$ & $23 / 5$ & Zinelues \\
\hline & & & & & & & & & \\
\hline
\end{tabular}

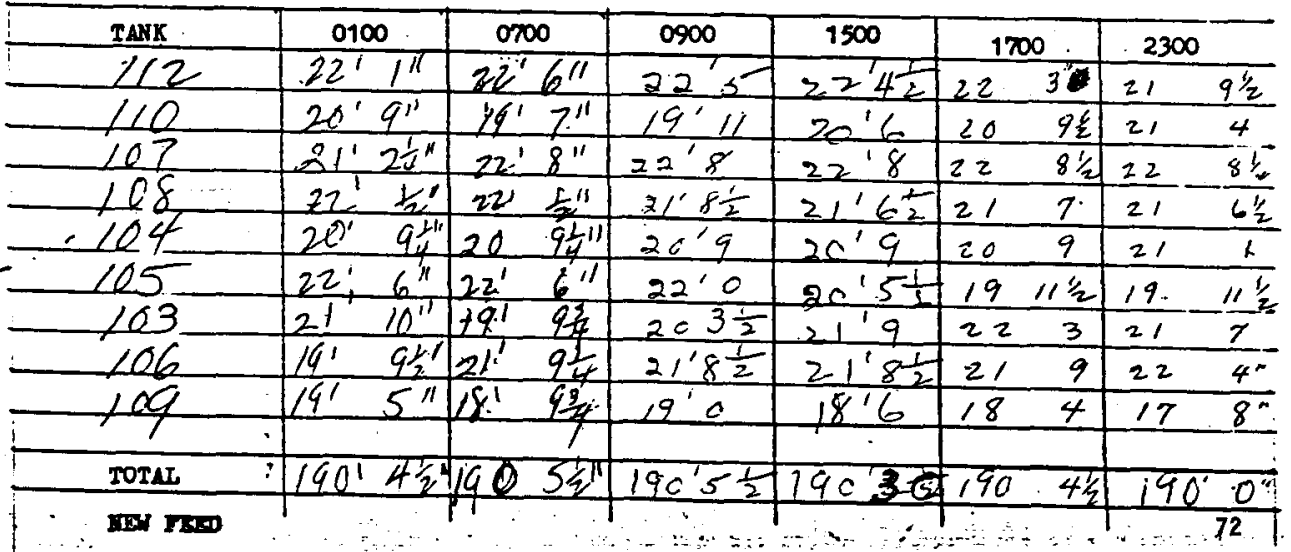



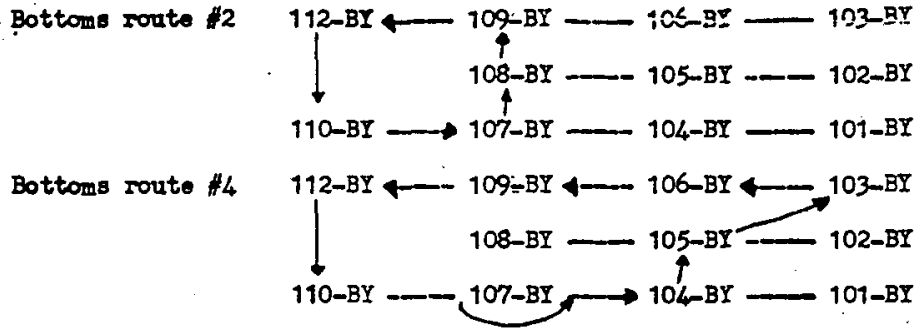

ITS-2 INVENTORY AND POMPING

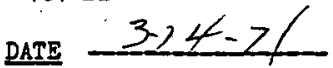

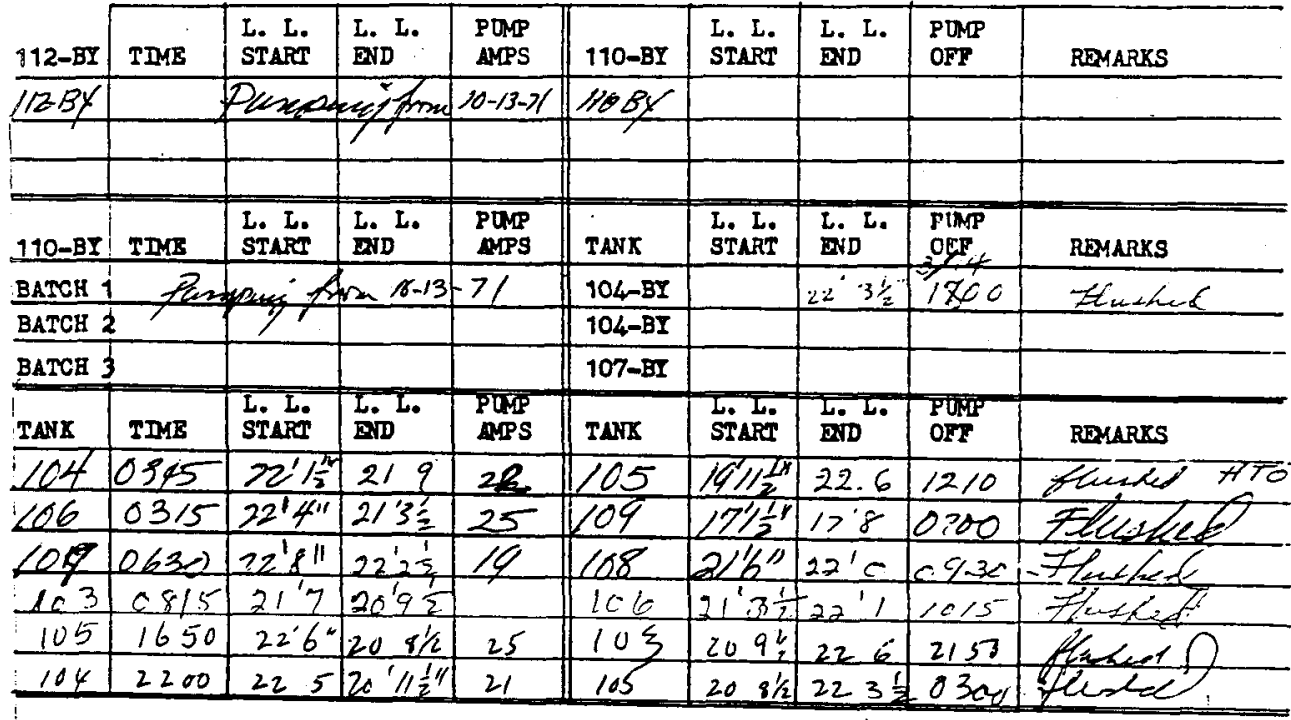

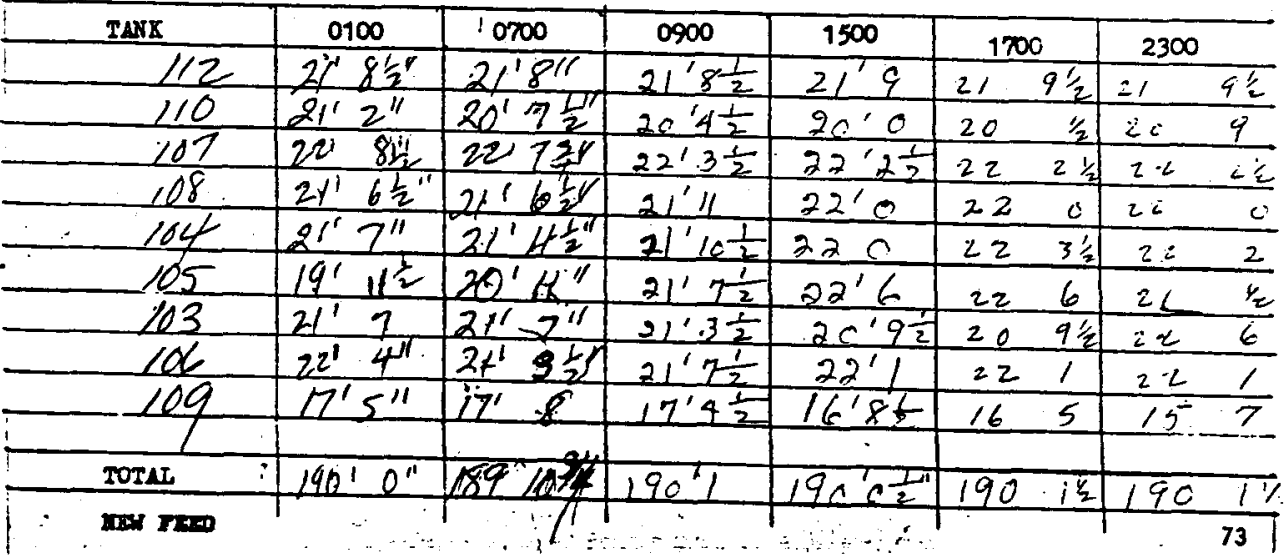




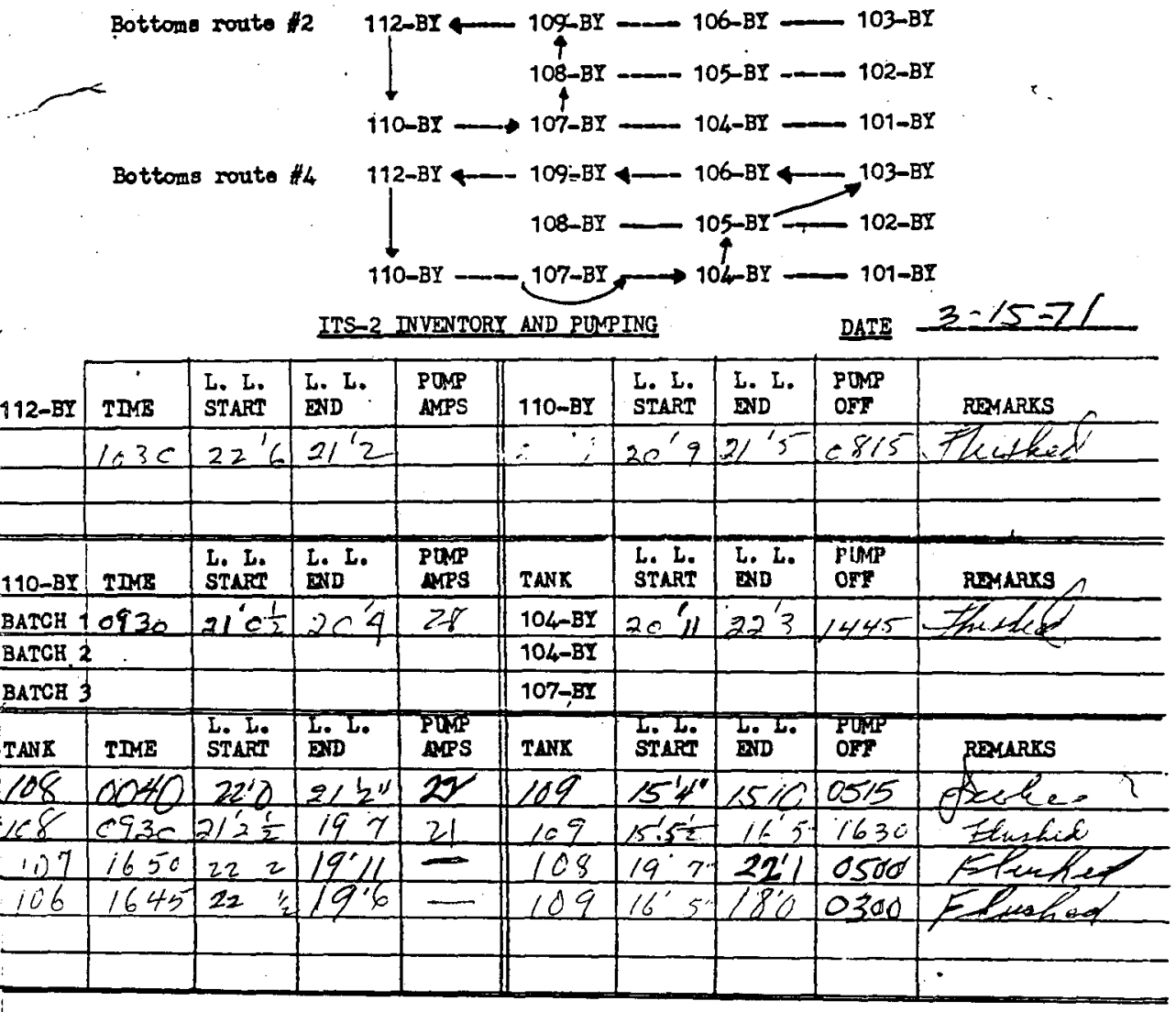

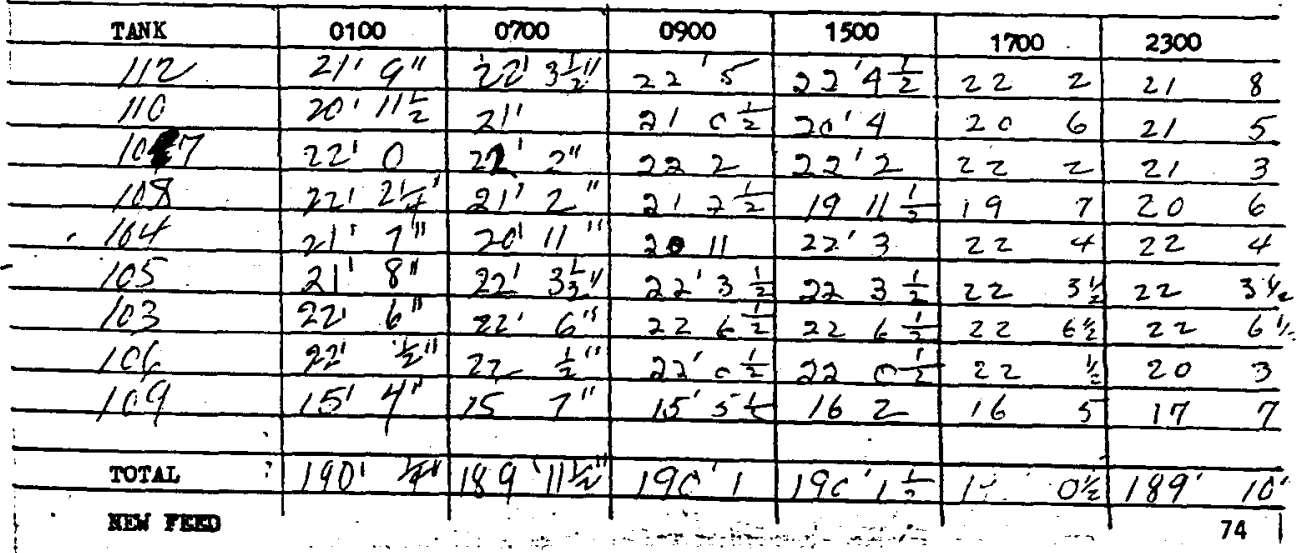


ITS-2 BOTTONS ROUTE - DEF DNITION
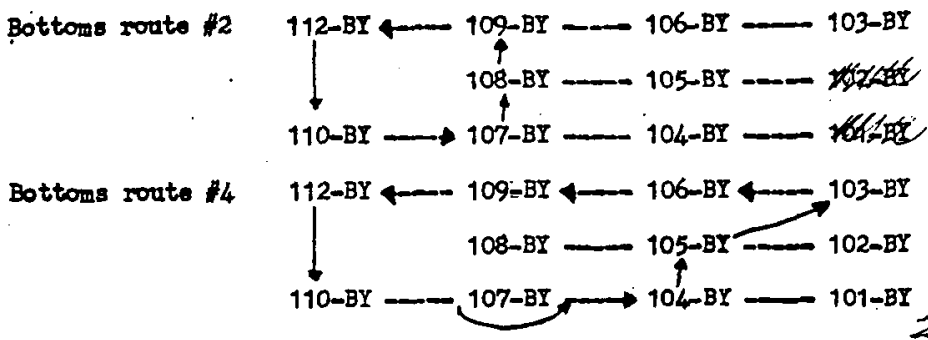

ITS-2 DNVENTORI AND PUMPING

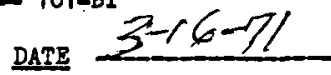

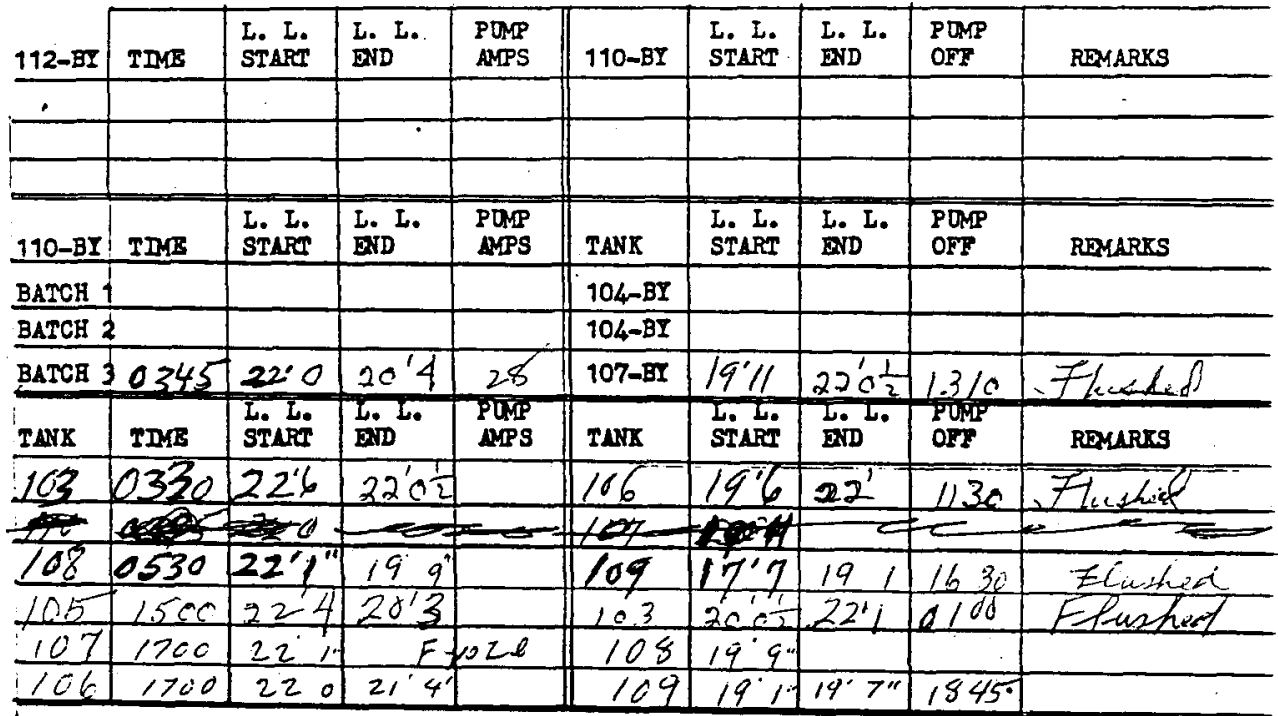

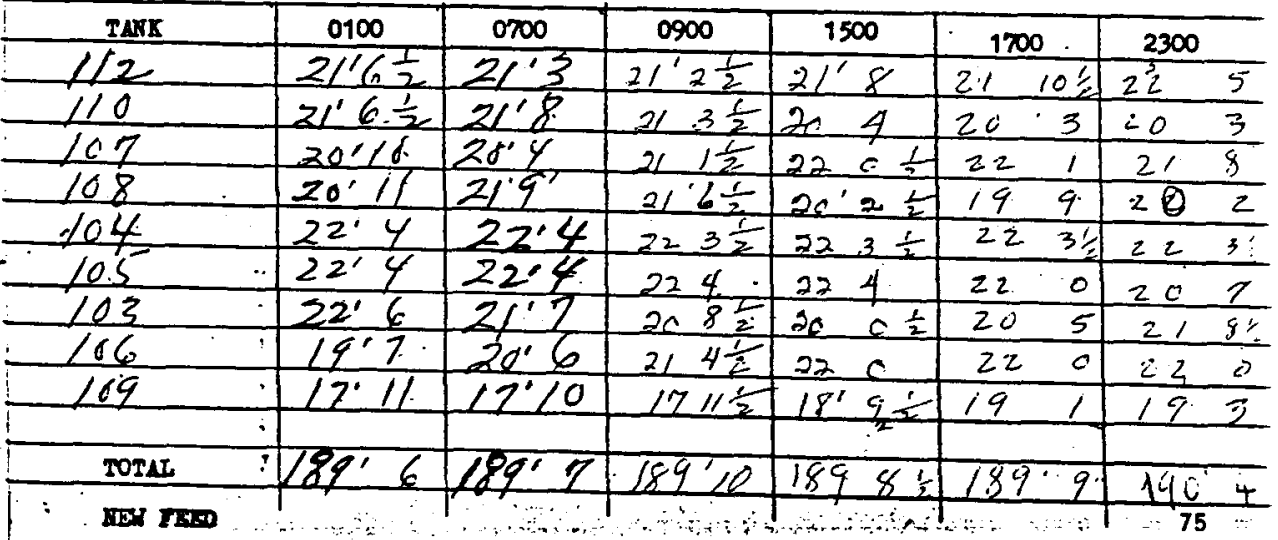


ITS-2 BOTTONS ROUTS - DEPINITION
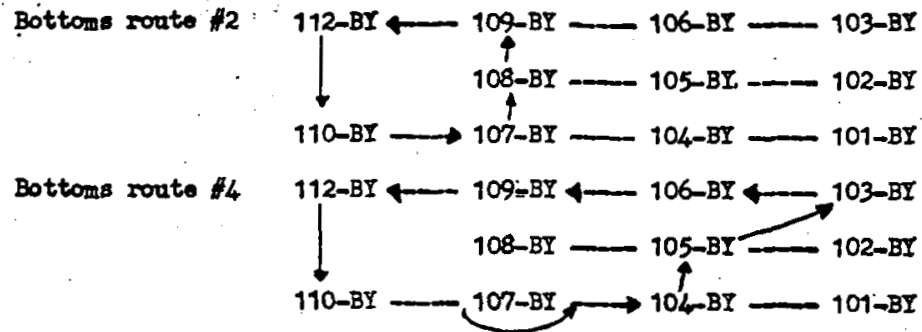

ITS-2 MIVENTORY AND POMPING DATE $3-\mid 7-11$

\begin{tabular}{|c|c|c|c|c|c|c|c|c|c|}
\hline 112-BI & $\tan$ & $\begin{array}{l}\text { L. I. } \\
\text { STAFT }\end{array}$ & $\begin{array}{l}\text { L. L. } \\
\text { END }\end{array}$ & $\begin{array}{l}\text { POMP } \\
\text { MMPS }\end{array}$ & $110-\mathrm{BY}$ & $\begin{array}{l}\text { L. L. } \\
\text { START }\end{array}$ & $\begin{array}{l}\text { L. L. } \\
\text { BND }\end{array}$ & $\begin{array}{l}\text { Panp } \\
\text { OEY }\end{array}$ & RPMARRS \\
\hline & 0100 & $22^{\prime} 6$ & $218 \div$ & 21 & $20^{\prime} 3$ & $2=1$ & 323 & 1420 & Ztantiak \\
\hline $1 / 28 Y$ & 2230 & $22^{\prime} 6^{\prime \prime}$ & $21 \div 6$ & 21 & $100 \mathrm{BY}$ & $20^{\prime} \prime \prime \prime$ & 196 & 0945 & \\
\hline & & & & & & & & & \\
\hline $110-B I$ & TIR & $\begin{array}{l}\text { I. I. } \\
\text { START }\end{array}$ & $\begin{array}{l}\text { L. L. } \\
\text { EID }\end{array}$ & $\begin{array}{l}\text { PQMP } \\
\text { MPS } \\
\end{array}$ & TANK & $\begin{array}{l}\text { L. I. } \\
\text { START }\end{array}$ & $\begin{array}{l}\text { L. I. } \\
\text { END }\end{array}$ & $\begin{array}{l}\text { POMP } \\
\text { OFP }\end{array}$ & REMARKS \\
\hline BATCH 1 & $12=0$ & 2012 & $20^{\prime} 6$ & 25 & 104-BI & $2 C^{\prime} \mathrm{C}$ & $2-2 \%$ & 0505 & \\
\hline BATCH 2 & & & & & $104-8 \mathrm{I}$ & & & & \\
\hline BATCB 3 & & & & & $107-\mathrm{EI}$ & & & & \\
\hline TANR & TME & $\begin{array}{l}\text { L. L. } \\
\text { START }\end{array}$ & $\begin{array}{l}\text { L. I. } \\
\text { EN }\end{array}$ & $\begin{array}{l}\text { PITP } \\
\text { APS }\end{array}$ & Turx & $\begin{array}{l}\text { L. L. } \\
\text { STAPS }\end{array}$ & Liv 1 & $\begin{array}{l}\text { Pugp } \\
\text { of }\end{array}$ & RPMARES \\
\hline 104 & 0145 & $22^{\prime} 3 \frac{1}{2}$ & $20 \div 2$ & 24 & 125 & $20^{\prime} 3$ & $226 \frac{1}{2}$ & 090 & Fleweled \\
\hline 106 & 1527 & 214 & $19^{\prime} x^{\prime}=$ & 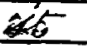 & $10 \mathrm{C}$ & $179 \frac{1}{2}$ & $19^{\prime} 0^{\prime \prime}$ & 305 & 7 biat 0 \\
\hline 107 & 1650 & $21^{\prime} 3$ & $19^{\circ} 8 \frac{1}{2}$ & 14 & 108 & 3020 & 221 & $0.30 i$ & 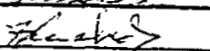 \\
\hline 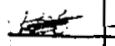 & $+2=$ & 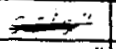 &. & & $4=$ & 20 & & & \\
\hline 103 & $21^{\prime} / 5$ & $22 \% "$ & $19^{\prime} 6$ & $23^{-}$ & 106 & $19^{\prime}=\frac{1}{2}$ & $22^{\prime} \mathrm{C}$ & 0130 & $16 \operatorname{lic}_{2}$ \\
\hline & & & & & & & & & \\
\hline
\end{tabular}

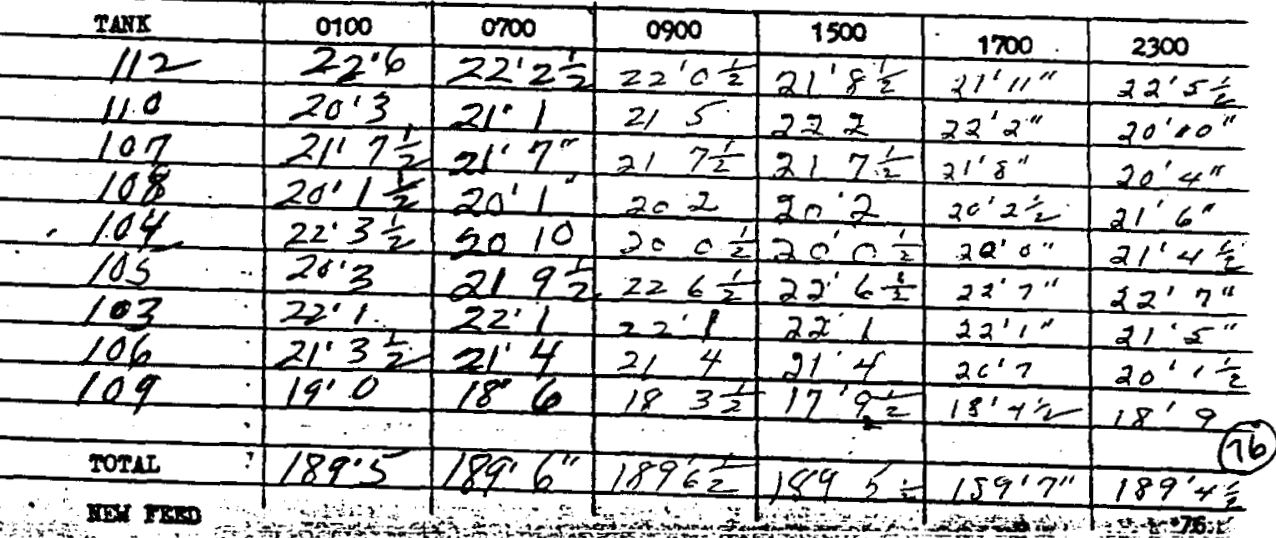




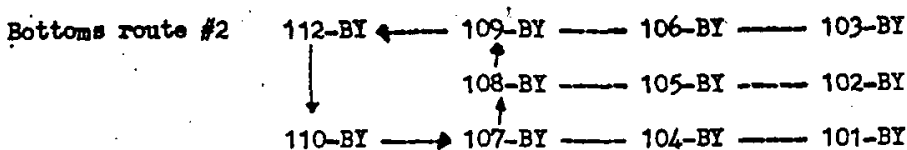

Bottoms route $: 4 \quad 109-\mathrm{BY}+106-\mathrm{BY}+10 \mathrm{BY}+103-\mathrm{BY}$

ITS-2 INVENTORI AND PUMPING

DATE $3=18-21$

\begin{tabular}{|c|c|c|c|c|c|c|c|c|c|}
\hline १12-BY & TIME & $\begin{array}{l}\text { L. L. } \\
\text { START }\end{array}$ & $\begin{array}{l}\text { L. I. } \\
\text { END }\end{array}$ & $\begin{array}{l}\text { POMP } \\
\text { AMPS }\end{array}$ & $110-B Y$ & $\begin{array}{l}\text { L. L. } \\
\text { START }\end{array}$ & $\begin{array}{l}\text { L. I. } \\
\text { DND }\end{array}$ & $\begin{array}{l}\text { POMP } \\
\text { OFE }\end{array}$ & RDMARKS \\
\hline & & & & & & & & & \\
\hline & & & & & & & & & \\
\hline & & & & & & & & & \\
\hline 110-BI! & TIME & $\begin{array}{l}\text { L. L. } \\
\text { START }\end{array}$ & $\begin{array}{l}\text { L. I. } \\
\text { DND }\end{array}$ & $\begin{array}{l}\text { PDMP } \\
\text { AMPS }\end{array}$ & TANK & $\begin{array}{l}\text { L. L. } \\
\text { STARI }\end{array}$ & $\begin{array}{l}\text { L. I. } \\
\text { END }\end{array}$ & $\begin{array}{l}\text { PUMP } \\
\text { OFP }\end{array}$ & RDMARKS \\
\hline BATCH 1 & & & & & 104-BI & & & & \\
\hline BATCH 2 & & & & & $104-B I$ & & & & \\
\hline BATCA 3 & 30510 & $20^{\circ} 6$ & & 25 & $107-\mathrm{EI}$ & $19^{\circ} 8 \frac{1}{2}$ & & & \\
\hline TANK & TIME & $\begin{array}{l}\text { L. I. } \\
\text { STAPR }\end{array}$ & $\begin{array}{l}\text { Lid } \\
\text { BND }\end{array}$ & $\begin{array}{l}\text { PUMP } \\
\text { AMPS }\end{array}$ & TANR & STARA & E. & $\begin{array}{l}\text { PUMP } \\
\text { Orf }\end{array}$ & RDMARRS \\
\hline$\angle 6,5$ & 0435 & $22^{\prime} 7$ & $20^{\prime} 1$ & $2+7$ & 10.3 & 196 & 2111 & 1500 & Mualael \\
\hline $10 \overline{3}$ & 0440 & 221 & 193 & 20 & 109 & $18^{\prime} 1$ & $19^{\prime} 8$ & 1300 & "leobef \\
\hline 2117 & 1520 & $22^{\prime} \mathrm{C}$ & 214 & 14 & 148 & $19^{\prime} 5^{\prime \prime}$ & $22^{\circ} \mathrm{C}$ & $\begin{array}{l}3-1507 \\
0630\end{array}$ & Lleshel \\
\hline 110 & 063.0 & $29-10 \frac{1}{2}$ & $19^{\circ} 6^{\circ}$ & & 102 & $214^{\prime}$ & $21 \cdot 10$ & 0950 & \\
\hline \multicolumn{10}{|l|}{ 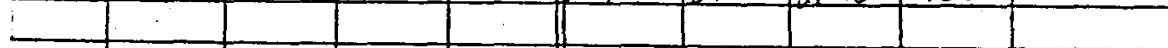 } \\
\hline & & & & & & & . & & : \\
\hline
\end{tabular}

.

\begin{tabular}{|c|c|c|c|c|c|c|}
\hline TANx & 0100 & 0700 & 0900 & 1500 & 1700 & 2300 \\
\hline $1<2$ & $22^{\prime} 5^{-}$ & $22^{\prime} 3$ & 223 & $22 \cdot 0$ & $22^{\prime} 0 \frac{1}{2}$ & $2 / 10 \frac{1}{2}^{\prime \prime}$ \\
\hline$\angle 10$ & $20^{\prime} 9$ & $205 \frac{1}{2}$ & $20 \quad 5$ & 20.41 & $20^{\prime} 3 \div 1$ & $19^{\prime} 82^{\circ}$ \\
\hline 107 & $20^{\prime} 1$ & 21.4 & 202 & $21 \cdot 21 / 2$ & $21 \cdot 8 \div 4$ & $21^{\prime 11} 1$ \\
\hline 108 & $21^{\prime} 81$ & $20^{\circ} 0$ & 210 & $19: 5$ & $19^{\prime} 5^{*}$ & $20^{\prime} 5^{\prime \prime}$ \\
\hline 104 & $21^{\prime} 9$ & $22^{\prime} c$ & 226 & $22 \cdot 5^{\prime \prime} 2$ & $22^{\prime} 5^{\prime \prime}$ & $22^{\prime} 5^{\prime \prime}$ \\
\hline 105 & $22^{\prime 7}$ & $21: 10$ & 266 & $20^{\circ} 1$ & $20^{\circ} 111$ & $20^{\prime} 5^{\prime \prime}$ \\
\hline 103 & $20^{\prime} 6 \frac{1}{2}$ & $20^{\prime} 2 \frac{1}{2}$ & 207 & 2111 & $21^{\prime \prime \prime \prime \prime}$ & $21^{\prime}+12^{x}$ \\
\hline 106 & $20^{\circ} 11 \frac{1}{2}$ & $22^{\prime} 0$ & 220 & $23 \cdot 0$ & $22 \circ 0$ & $22^{\prime} 0$ \\
\hline 109 & $28^{\prime} 6$ & $18^{\circ} 6$ & 18911 & $19 \cdot 6$ & $19^{\prime} 2 \frac{6}{2}$ & $18^{\prime} 5 \frac{2}{2}$ \\
\hline$\therefore$ & & & & & & 1 \\
\hline TOTAL & $189^{\circ} 3 \frac{1}{2}$ & $\angle 89^{\prime} 1$ & $189.3 / 2$ & $189^{\circ} 0$ & $189^{\circ} 17$ & $189^{\prime}$ \\
\hline
\end{tabular}




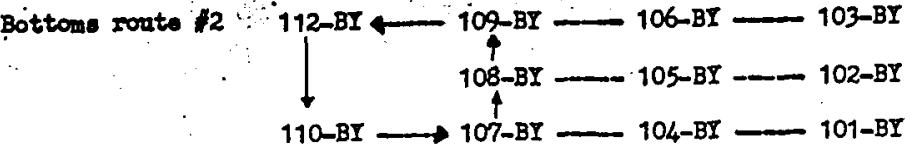

Bottoms route "4

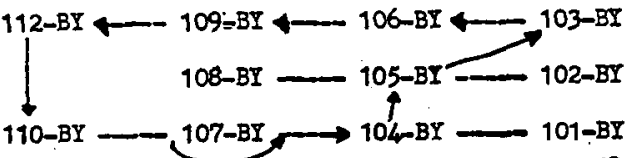

ITS-2 NVENTORY AND PIMPDNG

DATE $3-19-71$

\begin{tabular}{|c|c|c|c|c|c|c|c|c|c|}
\hline 112-BY & TAM & $\begin{array}{l}\text { I. I. } \\
\text { START }\end{array}$ & $\begin{array}{l}\text { L. Le. } \\
\text { END }\end{array}$ & $\begin{array}{l}\text { POMP } \\
\text { AMPS }\end{array}$ & $110-B I$ & $\begin{array}{l}\text { I. L. } \\
\text { START }\end{array}$ & $\begin{array}{l}\text { L. L. } \\
\text { END }\end{array}$ & $\begin{array}{l}\text { PAMP } \\
\text { OFF }\end{array}$ & RDMARKS \\
\hline $128 y$ & 1730 & $22^{\prime} 6^{\prime \prime}$ & $21^{\prime} 6^{\prime}$ & 20 & $1108 Y$ & $19^{\prime} 9^{\prime}$ & $219^{\circ}$ & $\begin{array}{l}3-x=4 \\
084,\end{array}$ & Gelesese \\
\hline
\end{tabular}

\begin{tabular}{|c|c|c|c|c|c|c|c|c|c|}
\hline $110-B I$ & Thes & $\begin{array}{l}\text { L. I. } \\
\text { STAPS }\end{array}$ & $\begin{array}{l}\text { L. I. } \\
\text { BND }\end{array}$ & $\begin{array}{l}\text { PUMP } \\
\text { AMPS }\end{array}$ & TANR & $\begin{array}{l}\text { L. L. } \\
\text { STARI }\end{array}$ & L. I. & $\begin{array}{l}\text { PUMP } \\
\text { OFT }\end{array}$ & RDMARKS \\
\hline BATCA 1 & & & & & $104-\mathrm{BI}$ & & & & . \\
\hline BATCH 2 & & & & & $104-B Y$ & & & & \\
\hline BATCH 3 & & & & & 107-EI & & & & 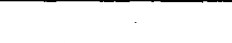 \\
\hline TANX & TDRS & $\begin{array}{l}\text { I. L. L. } \\
\text { STLPS }\end{array}$ & L. L & $\begin{array}{l}\text { PLPP } \\
\text { NQPS }\end{array}$ & SLMX & $\begin{array}{l}\text { L. Lo: } \\
\text { STuRer }\end{array}$ & Lid & $\begin{array}{l}\text { PuTP } \\
\text { Ort }\end{array}$ & RDMURES \\
\hline 106 & 0930 & $21 \% 1 / 2$ & $21^{\prime} 7^{\prime \prime}$ & & 109 & $17^{\prime} /{ }^{\circ}$ & $17 \% \frac{1}{2}$ & 1630 & Thuakeal \\
\hline 103 & 1115 & $211 \% k$ & $20^{\circ} 7^{\circ}$ & & 106 & $216^{\circ}$ & $221 / 2$ & 4500 & Alushe \\
\hline 108 & 1645 & $2111 \div$ & 116 & 31 & 109 & $12 \% \div$ & $196 \frac{1}{2}$ & $0 / 00$ & Fendhes \\
\hline$\angle 12$ & 1730 & $22^{\prime} 6$ & $21^{\circ} 6^{\prime \prime}$ & 20 & 110 & $19^{\prime} 9^{\prime \prime}$ & 2110 & 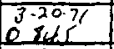 & Aluebor \\
\hline 163 & $\log 35$ & $20^{\prime} 7 \frac{1}{2}$ & $2 a^{\prime} 2$ & 24 & 106 & $21^{\prime} 7^{\prime \prime}$ & $22^{\prime} 0^{\circ}$ & $18+0$ & 3 custed \\
\hline 105 & 1900 & $22^{\prime}+\frac{6}{2}$ & $19 \%$ & 25 & 103 & 2212 & $22 \%$ & $0200^{\circ}$ & \\
\hline
\end{tabular}

\begin{tabular}{|c|c|c|c|c|c|c|c|}
\hline & $\cdots$ T员 & 0100 & 0700 & 0900 & 1500 & 1700 & 2300 \\
\hline & 103 & $21^{\prime} 11$ & 2111 & $21^{\prime} 10 \%$ & $20.7^{\circ}$ & $20 \cdot 7 \frac{1}{2}$ & 2113 \\
\hline & 104 & $22 \%$ & $22^{\circ} 6^{\prime \prime}$ & $12.5 / 2$ & $22: 5 / 2$ & $22^{\prime} 5 \frac{6}{2}$ & 223 \\
\hline & 105 & $20^{\circ} 6 \frac{1}{2}$ & $21^{\circ} 0^{\circ}$ & $11^{\circ}$ & $21^{\circ} 7^{\prime}$ & $21^{\prime} 10^{\prime \prime}$ & $21^{\prime} 3$ \\
\hline & 106 & $22^{\circ} 0^{\prime \prime}$ & $22^{\circ} 0^{\prime \prime}$ & $22^{\circ} 0^{\circ}$ & $22: 1 / 2$ & $21^{\prime} 7^{\prime \prime}:$ & $23^{\prime} 0$ \\
\hline & 107 & $21: 10$ & $21^{\circ} 4 \frac{1}{2}$ & $21.8 \div 2$ & $21 \cdot 10 \%$ & 2111 & 21111 \\
\hline & 108. & 2011 & $22^{\circ} 0^{\circ}$ & $2200 \div \cdots$ & $21 \cdot 11: \cdots$ & $21, ; 1 \div 2$ & $20 \%$ \\
\hline & 109 & $18^{\prime} 2^{\circ}$ & $17 \cdot 5 \frac{1}{2}$ & $17 \cdot 2^{*}$ & $17 \cdot 7^{\circ}$ & $17^{\prime} 10 \frac{1}{2}$ & $21: 11 \pi$ \\
\hline & 110 & $198^{\circ}$ & $19^{\prime} 102$ & 19.81. & $F 9^{\circ}$ & $19^{\prime} 9^{\prime \prime}$ & $20^{\prime} 6 \frac{1}{2}$ \\
\hline & 112 & $2 \cdot 11$ & $219^{\circ} \frac{1}{2}$ & $11^{\circ} q^{\circ}$ & $62.31=$ & $22^{\prime} 5^{\prime \prime}$ & $22^{\prime} x^{\prime \prime}$ \\
\hline & 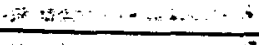 & $\therefore+\cdots+2=0$ & 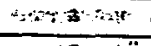 & $2 x: x_{0}+4+2$ & 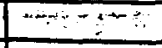 & & \\
\hline & $\therefore$ Torer & $189^{\circ}-5 \frac{1}{2}$ & $18^{\circ} 9^{\circ} 11^{\prime \prime}$ & $189.10^{\prime \prime}$ & $190^{\circ} 2^{\circ}$ & $190^{\prime} 5^{\circ}$ & $90^{\circ}$ \\
\hline
\end{tabular}




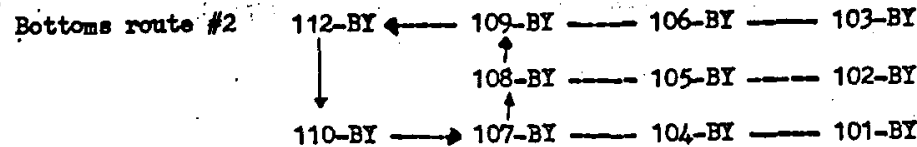

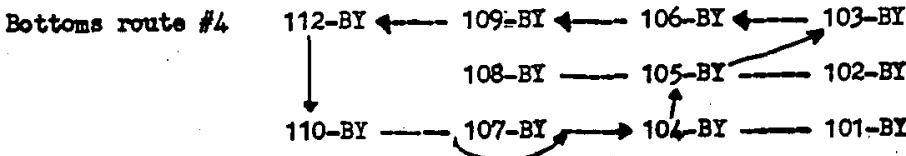
ITS-2 INVENTORY AND PUNPTNG DATE $3-20-71$

\begin{tabular}{|c|c|c|c|c|c|c|c|c|c|}
\hline 112-BY & TIME & $\begin{array}{l}\text { I. L. L. } \\
\text { STARI }\end{array}$ & $\begin{array}{l}\text { L. L. } \\
\text { DXD }\end{array}$ & $\begin{array}{l}\text { POMP } \\
\text { LNPS }\end{array}$ & 110-BI & $\begin{array}{l}\text { L. I. } \\
\text { START }\end{array}$ & $\begin{array}{l}\text { L. I. } \\
\text { QND }\end{array}$ & $\begin{array}{l}\text { POMP } \\
\text { OFP }\end{array}$ & REMARKS \\
\hline & & & & . & & & & & - \\
\hline $112 B Y$ & 2300 & $2 x^{\prime} 5-2$ & $28 \cdot 6$ & 20 & $1108 Y$ & $20^{\prime} 4^{\prime \prime}$ & $2 / 4^{\prime}$ & $133 \gamma_{<}=0-$ & Blevehos \\
\hline 110-BY & TDM & $\begin{array}{l}\text { L: L. } \\
\text { START }\end{array}$ & $\begin{array}{l}\text { L. L. } \\
\text { END }\end{array}$ & $\begin{array}{l}\text { PQMP } \\
\text { APS }\end{array}$ & TANR & $\begin{array}{l}\text { L. L. } \\
\text { START }\end{array}$ & $\begin{array}{l}\text { L. I. } \\
\text { END }\end{array}$ & $\begin{array}{l}\text { POMP } \\
\text { OFP }\end{array}$ & RDMARES \\
\hline BATCH 1 & 1200 & $21 \cdot 16$ & $20 \%$ & 25 & $104 \mathrm{mI}$ & $20^{\circ} 11^{\circ}$ & $22.5 \frac{1}{2}$ & $0.3 c c$ & 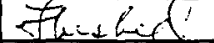 \\
\hline BATCH 2 & & & & & $104-8 I$ & & & & \\
\hline BATCE 3 & & & & & 107-BI & & & & . \\
\hline TANK & TIR & $\begin{array}{l}\text { Lo Lo } \\
\text { STARP }\end{array}$ & ED & $\begin{array}{l}\text { PUTP } \\
\text { APS }\end{array}$ & TAKK & $\begin{array}{l}\text { T. L. } \\
\text { sTaper }\end{array}$ & ER & $\begin{array}{l}\text { Fupr } \\
\text { art }\end{array}$ & RDYARES \\
\hline 1at & 0600 & $22^{\prime} 10$ & $19^{\prime} 6^{\circ}$ & 1.5 & 108 & 196 & 2200 & 1800 & Flecoked \\
\hline 104 & $c .2 \times$ & $225^{\prime}-\frac{1}{2}$ & $20 \% 1 \mathrm{i}$ & & 105 & 1911 & $22 \sqrt{2}$ & 1500 & tiluaber \\
\hline 1012 & 1200 & $220^{\circ}$ & 212 & & 109 & $18^{\circ} 712^{2}$ & $93^{\circ}$ & 1500 & It leube \\
\hline 103 & 2.208 & $22 \%$ & $31^{\prime} 2 x^{\prime \prime}$ & & 106 & $21^{\prime \prime} 2^{\circ}$ & $23^{\prime} 0^{\prime}$ & 1735 & oluaken \\
\hline & & & & & & & & • & . \\
\hline
\end{tabular}

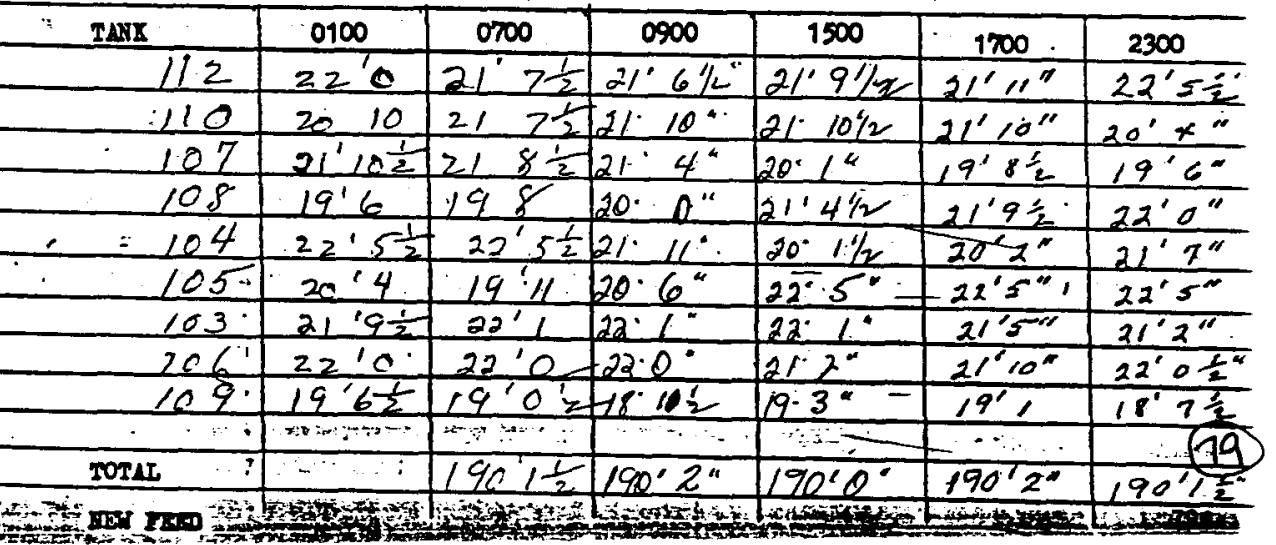


ITS-2 BOTSOMS ROOTE - DEF DNITIOA

Bottome route ig 112-BI 109-BI 103-BI

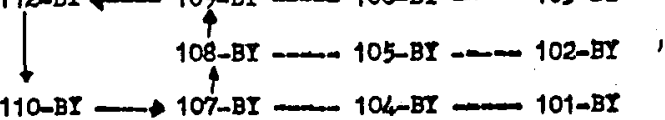

Bottons route 4 112-BI 109-BY 106-BY 103-BI

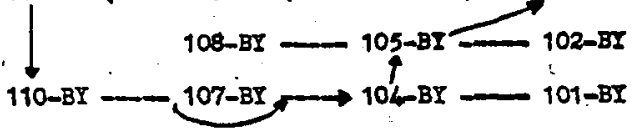

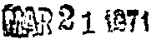
ITS-2 TNVENTORI AND PUNPTNG

\begin{tabular}{|c|c|c|c|c|c|c|c|c|c|}
\hline $112-E I$ & TIME & $\begin{array}{l}\text { L. L. } \\
\text { STAPC }\end{array}$ & L. L. & $\begin{array}{l}\text { PDMP } \\
\text { AMPS }\end{array}$ & 110-BI & $\begin{array}{l}\text { L. I. } \\
\text { START }\end{array}$ & $\begin{array}{l}\text { L. I. } \\
\text { BND }\end{array}$ & $\begin{array}{l}\text { PDMP } \\
\text { OPY }\end{array}$ & RDMARKS \\
\hline & & & & & & & & & $=$ \\
\hline & & & & & & & & & 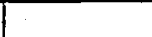 \\
\hline & & & & & & & & & \\
\hline $110-B I$ & IPAS & $\begin{array}{l}\text { L. I. } \\
\text { START }\end{array}$ & $\begin{array}{l}\text { L. L. } \\
\text { END }\end{array}$ & $\begin{array}{l}\text { PQMP } \\
\text { AMS }\end{array}$ & TANK & $\begin{array}{l}\text { I0. In } \\
\text { START }\end{array}$ & $\begin{array}{l}\text { L. I. } \\
\text { END }\end{array}$ & $\begin{array}{l}\text { PUMP } \\
\text { OFP }\end{array}$ & RBMARXS \\
\hline BATCH 1 & & & & & $104-B I$ & & & & \\
\hline BATCR 2 & 1750 & $21^{\prime} 5^{\prime \prime}$ & 196 & 25 & $104-B I$ & $21^{\prime} 8^{\prime \prime}$ & 21 & $2 !$ & - \\
\hline BATCA 3 & & & & & $107-\mathrm{BI}$ & & & & \\
\hline $\operatorname{TAN}$ & IngE & $\begin{array}{l}\text { L. Lo } \\
\text { STARA }\end{array}$ & LED & $\begin{array}{l}\text { PUPP } \\
\text { AMPS }\end{array}$ & THX & SI. & Did & $\begin{array}{l}\text { PUip } \\
\text { OTP }\end{array}$ & RIMUARS \\
\hline 1106 & 620 & 226 & $21 \times 1 / 2$ & & 109 & 1211 & $1 / 9^{\prime} 3^{\prime}$ & 1500 & Trluadel \\
\hline 105 & 1130 & $22 \cdot \sqrt{7^{\prime}}$ & $21^{\prime} 2^{x}$ & 25 & 103 & $21 \cdot 2^{\prime \prime}$ & $21^{\prime} 11^{\prime \prime}$ & 2020 & sterskad \\
\hline 103 & $113 \%$ & $21 \cdot 2^{\circ}$ & $21 \%$ & 25 & 106 & $206^{\circ}$ & $22^{\prime} 0^{\circ}$ & 1755 & 7 \\
\hline 105 & 1000 & $22512^{\circ}$ & $21^{\prime} 4$ & & 105 & $21 \cdot 4 \frac{1}{2}$ & $22 ' 6$ & $0,3-22$ & \\
\hline 110 & 1750 & $21^{\prime} 5^{\prime \prime}$ & 196 & 22 & 104 & $21^{\prime} 8^{\prime \prime}$ & $21^{\prime} 6$ & $0 / 20$ & \\
\hline & & & & & & & & & \\
\hline
\end{tabular}

\begin{tabular}{|c|c|c|c|c|c|c|}
\hline TAIS & 0100 & 0700 & 0900 & 1500 & $\therefore \quad 1700$ & 2300 \\
\hline 112 & 22.14 & 21112 & $21^{\circ} 10^{\circ}$ & $21^{\prime} 8^{\circ}$ & $31^{\prime} 10^{\prime \prime}$ & $27^{\prime} 3^{\prime}$ \\
\hline$\angle 10$ & 202 & $20^{\prime} 6 \frac{1}{2}$ & $20 \cdot 9 \%$ & $214 \%$ & $21^{\prime} E^{\prime \prime}$ & $20^{\prime} x^{\prime \prime}$ \\
\hline 107 & $19^{\prime} 6$ & $19^{\prime} 6$ & $19^{\circ} 6^{\prime \prime}$ & $19 \cdot 6^{\prime}$ & $19^{\prime} 6$ & $19^{\prime} 6^{\circ}$ \\
\hline 108 & $22^{\prime} 0$ & 220 & $220^{\circ}$ & $22 \cdot 0^{\circ}$ & $2 x^{\prime} 0$ & 2210 \\
\hline$=104$ & $22^{\prime} 0$ & $22^{\prime} 5^{-\frac{1}{2}}$ & $22 \cdot 5 / 2$ & $22^{2} \sqrt{1 / 2}$ & $21 \% \div$ & $215 \%$ \\
\hline 105 & 22 & $22^{\prime} 5$ & $22 \cdot 5^{\prime \prime}$ & $21 \cdot 4 / 2$ & $21^{\prime} 5 \pi$ & $31^{\circ} 11^{\circ}$ \\
\hline $103^{\circ}$ & 212 & $21 \div 2$ & $21^{\prime} 2^{\circ}$ & $21.0^{\circ}$ & $21^{\prime}, \cdots$ & $2111 / 2$ \\
\hline $106^{\circ}$ & $220^{\circ}$ & $22^{\prime} 0$ & $11^{\circ} 6 \%$ & $21 \% 1 \%$ & $21 \cdot 7 \div$ & $21^{\prime} 0^{\prime}$ \\
\hline 109. & 1815 & $17^{\prime} / 1$ & $10^{\circ} 21 / 2$ & $19 \cdot 3$ & $19^{\circ}, \circ$ & $18^{\prime} 7^{\circ}$ \\
\hline$\because \ldots+\cdots$ & 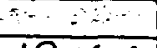 & $\because-n+\cdots$ & $=$ & $\because \cdots$ & $\therefore \because \cdots 4$ & \\
\hline 20TLI & $190^{\circ} 0$ & $188^{7} / 12$ & $189^{\circ} 1^{\prime \prime}$ & $189 \cdot 9^{4}$ & $189^{\prime} 11^{\prime \prime}$ & $189^{2} 10$ \\
\hline$A$ & & & & & 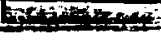 & $\operatorname{logh}=2$ \\
\hline
\end{tabular}




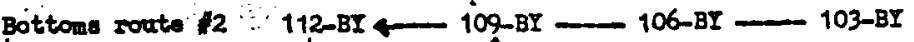

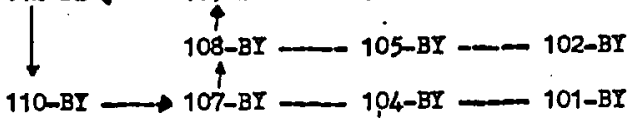

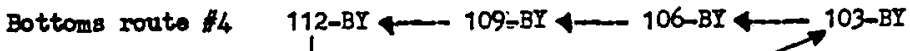

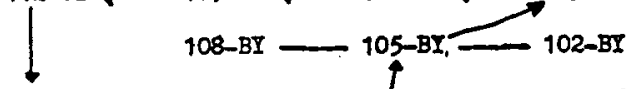

$110-\mathrm{BY} \longrightarrow 107-\mathrm{BY} \longrightarrow 104-\mathrm{BY} \longrightarrow 101-\mathrm{BY}$

ITS-2 INVENTORY AND PUMPING

DATE LAR 221971

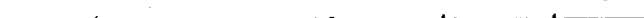

\begin{tabular}{|c|c|c|c|c|c|c|c|c|c|}
\hline 112-EI & TIMB & $\begin{array}{l}\text { I. I. } \\
\text { START }\end{array}$ & $\begin{array}{l}\text { I. I. } \\
\text { DND }\end{array}$ & $\begin{array}{l}\text { POMP } \\
\text { AMPS }\end{array}$ & $110-\mathrm{BY}$ & $\begin{array}{l}\text { L. I. } \\
\text { STARI }\end{array}$ & $\begin{array}{l}\text { L. L. } \\
\text { END, }{ }^{\circ}\end{array}$ & $\begin{array}{l}\text { POMP } \\
\text { OFF }\end{array}$ & RRAARKS \\
\hline & 0230 & $22 \%$ & $\therefore=-2$ & & Et & 196 & $2=2$ & & $2 \geqslant \geqslant=2$ 6lut \\
\hline & & & & & & & & & \\
\hline & & & & & & & & & \\
\hline $110-B I$ & Taxs & $\begin{array}{l}\text { I. L. } \\
\text { STARE }\end{array}$ & $\begin{array}{l}\text { L. L. } \\
\text { END }\end{array}$ & $\begin{array}{l}\text { PAMP } \\
\text { MPPS }\end{array}$ & TANK & $\begin{array}{l}\text { I. L. } \\
\text { BTART }\end{array}$ & $\begin{array}{l}\text { L. I. } \\
\text { END }\end{array}$ & $\begin{array}{l}\text { PUMP } \\
\text { OFP }\end{array}$ & RHMARKS \\
\hline BATCH 1 & & & & & $: 04-51$ & & & & \\
\hline BATCH 2 & & & & & 104-EI & & & & \\
\hline BATCH 3 & & & & & $\therefore C 7-B I$ & & & & \\
\hline TAX & TINE & $\begin{array}{l}\text { L. Lo } \\
\text { STLRS }\end{array}$ & L. & $\begin{array}{l}\text { Pl } \\
0\end{array}$ & $\mathbf{x}$ & $\begin{array}{l}\text { L. L. } \\
\text { START }\end{array}$ & END & $\begin{array}{l}\text { Fump } \\
\text { OrI }\end{array}$ & REMARTS \\
\hline $\log 8$ & n20 & $211 \frac{1}{2}$ & $199^{2}$ & & .9 & $17 \%$ & $195 \%$ & 1400 & Itended \\
\hline & & & & & & & & & $\therefore \quad$ \\
\hline & & & & & & & & & \\
\hline & & & & & & & & & \\
\hline & & & & & & & & & \\
\hline$i$ & & & & & & & & • & \\
\hline
\end{tabular}

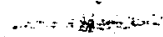

5

$=2 \frac{2}{8}$

Tux

\begin{tabular}{l|l}
0100 \\
\hline
\end{tabular}

\begin{tabular}{|c|c|c|c|c|c|c|}
\hline 110 & 1975 & 201 & $20.4 \%$ & $2112 \%$ & $11^{\circ} 5$ & $20^{\circ}$ \\
\hline 147 & 19 & $19^{\prime} \mathrm{C}$ & $19^{\circ} 6^{\prime}$ & $19^{\prime} 6^{\circ}$ & $19^{\circ} 6$ & \\
\hline
\end{tabular}

108

$21+1 \frac{1}{2}$

$19^{\prime} 69^{\circ} 6^{\prime} \cdot 19^{\prime} 6^{\circ}$

$2115 \frac{1}{2} 214 \%$

$219^{\circ} q^{\circ}$

$19^{\prime} 6$

$19^{\circ} 6$

$r$

$=104$

214

105.226

216

$21 \cdot 6$

$21 \cdot 6^{\circ}$

$19^{\prime} 9$

226

$2 \lambda^{\prime} 6^{\circ}$

103

211

$2116.211^{\prime \prime}$

$22 \cdot 6^{\circ}$

106

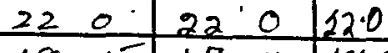

$21 \cdot 11^{\circ}$

$21^{\circ} \mathrm{C}$

$19^{\prime} 9^{\circ}$

is

109

$1711.18^{\circ} 31 / \%$

$12: 0^{\circ}$

$22^{\circ} \mathrm{C}$ $21^{\prime} 6$

$\therefore+$

185

$19^{\circ} 41 / 2$

$211 \%$

$22^{\circ} \mathrm{C}$. $2111 \frac{1}{2}$ $22^{\circ} 0$ $12^{\circ} \circ$

TOTAL

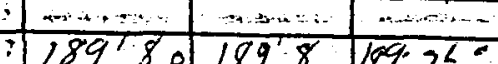

$187 \cdot 6$ $19 \div 2$ $18^{\circ} \frac{1}{2}$ 


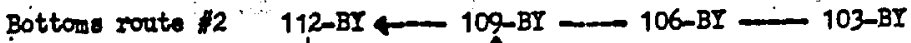

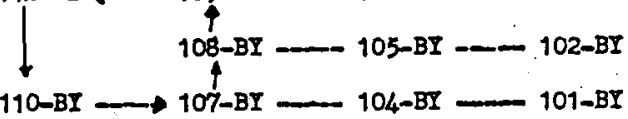

Bottoms route t/4 112-BY 4 109:-BY 4 106-BY $4-103-B Y$

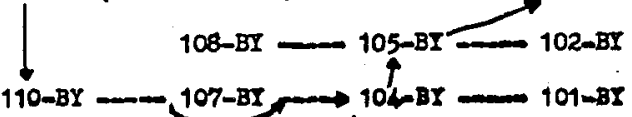

ITS-2 THVENTORY AND POMPTNG

DATE CIR 23104

\begin{tabular}{|c|c|c|c|c|c|c|c|c|c|}
\hline $112-B I$ & TDMS & $\begin{array}{l}\text { L. L. } \\
\text { START }\end{array}$ & L. L. & $\begin{array}{l}\text { POMP } \\
\text { AMPS }\end{array}$ & $110-\mathrm{BI}$ & $\begin{array}{l}\text { L. L. } \\
\text { START }\end{array}$ & $\begin{array}{l}\text { L. L. } \\
\text { END }\end{array}$ & $\begin{array}{l}\text { POMP } \\
\text { OFF }\end{array}$ & RPMARKS \\
\hline & 1155 & $22 \%$ & 218 & 20 & & 2023 & 221 & $0 \longdiv { 6 8 }$ & Mlatided \\
\hline & & & & & & & & & 4 \\
\hline & & & & & & & & & \\
\hline $110-B Y$ & TDMS & $\begin{array}{l}\text { L. I. } \\
\text { START }\end{array}$ & L. L. & $\begin{array}{l}\text { PQMP } \\
\text { AMPS }\end{array}$ & TANK & $\begin{array}{l}\text { L. L. L. } \\
\text { START }\end{array}$ & $\begin{array}{l}\text { L. }{ }^{2} \\
\text { BND }\end{array}$ & $\begin{array}{l}\text { PLMP } \\
\text { OFY }\end{array}$ & RPMARKS \\
\hline BATCR & & & & & 104-BI & & & & \\
\hline BATCE 2 & & & & & 104-BI & & & & \\
\hline BATCH & 0100 & $22^{\prime} 0$ & $20.2 \%$ & & $107-B I$ & $19^{\prime} 6$ & $21^{\prime} 3^{\prime \prime}$ & 1250 & Alwhed \\
\hline TANS & TDME & $\begin{array}{l}\text { L. L. } \\
\text { START }\end{array}$ & LED & $\begin{array}{l}\text { PUip } \\
\text { DPPS }\end{array}$ & TARS & STARt & END & $\begin{array}{l}\text { PUP } \\
\text { OFF }\end{array}$ & RDULRS \\
\hline 106 & c.70s & 220 & $926 \%$ & & 109 & $\angle 8^{\prime} 0$ & $7.6 \%$ & is0o & 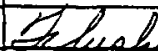 \\
\hline 103 & 1100 & $21^{\prime \prime} / 1^{*}$ & $21^{\prime} 7 \frac{1}{2}$ & & 106 & $20^{\circ} 9^{\prime}$ & $2111 \frac{1}{2}$ & 1800 & \\
\hline 105 & 1300 & $22^{\prime} 5 / 2$ & $215 \frac{1}{2}$ & & 103 & $214^{\prime \prime}$ & $2111 \frac{1}{2}$ & 1910 & \\
\hline 202 & 1500 & $2 / 3^{\prime}$ & 196 & & 108 & $19.8^{\circ}$ & $21^{\prime} 2^{\prime}$ & 2315 & \\
\hline 1021 & 1500 & $21.5 \%$ & 196 & & $105^{-}$ & 政 & 224 & 2200 & \\
\hline+6 & & & & & & & & & \\
\hline
\end{tabular}

\begin{tabular}{|c|c|c|c|c|c|c|}
\hline TANS & 0100 & 0700 & 0900 & 1500 & 1780 & 2300 \\
\hline 112 & $21^{\prime} 1 \frac{1}{2}$ & 221 & $32 \cdot 3^{2}$ & $28^{\prime \prime} 4^{\circ}$ & $22 \cdot 3$ & 2110 \\
\hline 110 & $22^{\prime} 0$ & 2016 & $10^{\circ} 6^{-}$ & $20^{*} 8 \frac{1}{2}$ & $20^{\circ} / 1 \frac{1}{2}$ & 2,10 \\
\hline 107 & $19^{\prime} 6$ & $207 \frac{1}{2}$ & $20^{\circ} 11 \frac{1}{2}$ & $21 \cdot 8$ & $20^{\circ} 10$ & $19^{\prime 6}$ \\
\hline 10.8 & $19^{\prime 9}$ & 199 & $19 \cdot q^{\circ}$ & 19.8 & $20^{\prime} 2 \frac{1}{2}$ & 217 \\
\hline 104 & $21 \quad 5 \frac{1}{2}$ & $21^{\prime} 5 \frac{10}{2}$ & $21.5 \%:$ & $21^{\circ} 61 / 2$ & $20 \% 10 \frac{1}{2}$ & 196 \\
\hline 105 & $22^{\circ} 6$ & $226 \ldots$ & $2 x-5 \frac{1}{x} \cdots$ & $21.72^{\circ}$ & $21.6 \frac{1}{2}$ & 224 \\
\hline 103 & 2111 & 2111 & $z^{\prime} \|^{\prime \prime}$ & $21 \cdot 51 / 2$ & 217 & $2111 \frac{1}{2}$ \\
\hline 166 & 22 & 22 & $21 \cdot 4 k$ & $21 \cdot 0^{*}$ & $21 \cdot 8 \frac{1}{2}$ & $21 / 1 \frac{1}{2}$ \\
\hline 109 & $186 \frac{1}{2}$ & $18^{\circ} 0$ & $18: 6^{\circ}$ & $19: 6 \%$ & 195 & $18 \% 10^{\frac{1}{2}}$ \\
\hline & $\Leftrightarrow$ & -100 & $46 \%$ & & & \\
\hline 20Tur & $189 \cdot 3 \frac{1}{2}$ & 18920 & $189^{\prime} 1^{\prime \prime}$ & $189^{\circ} 0^{\circ}$ & & \\
\hline
\end{tabular}


ITS-2 BOTTONS ROOTS - DEF IIITION

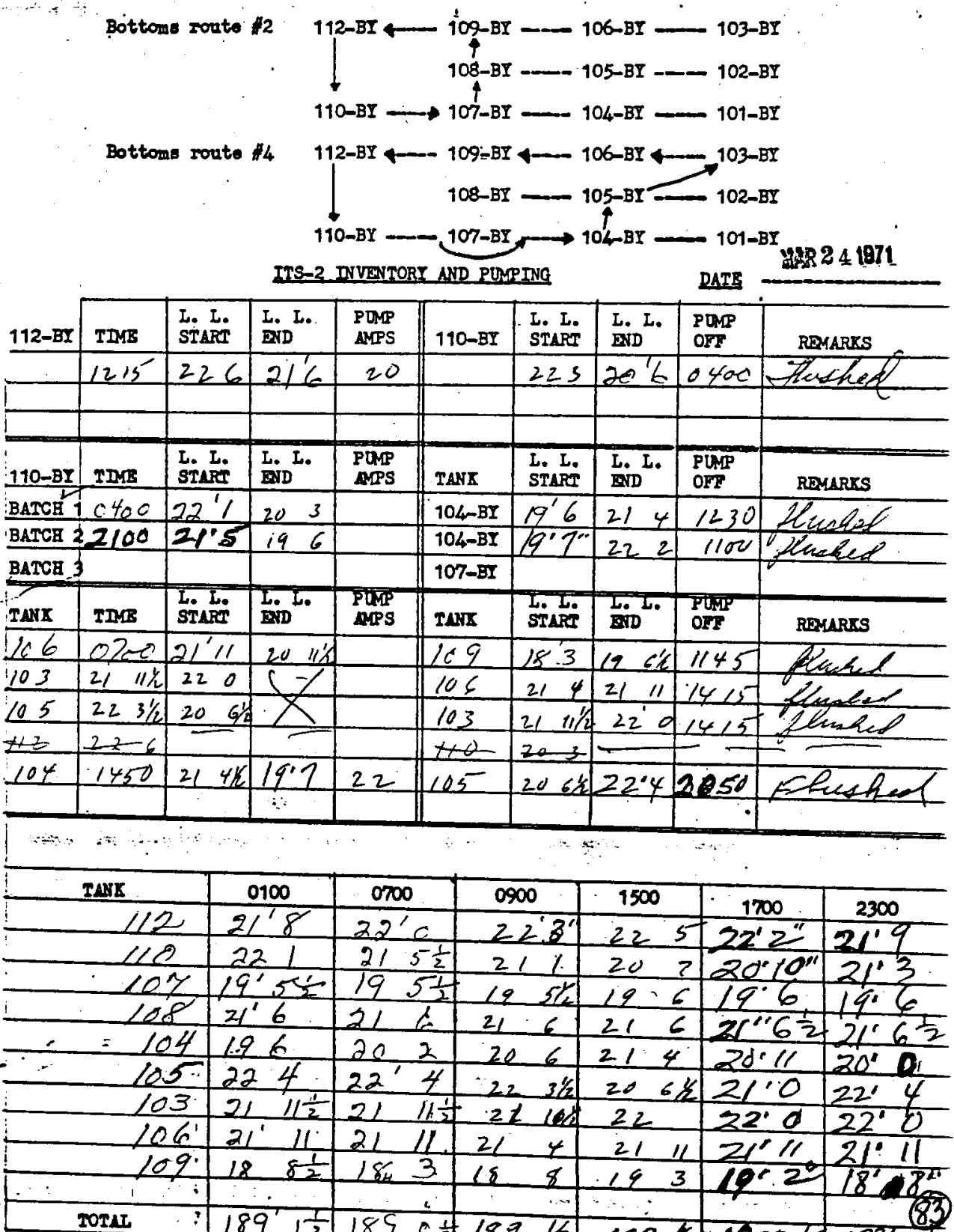




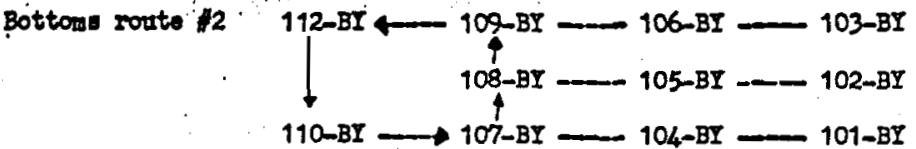

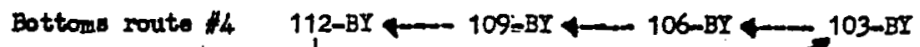

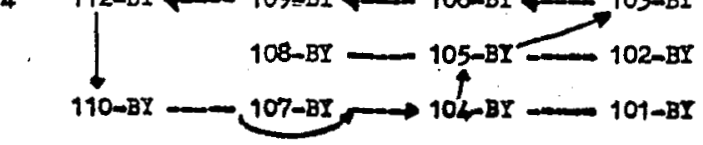

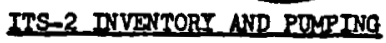

DATS

254974

\begin{tabular}{|c|c|c|c|c|c|c|c|c|c|}
\hline 112-BI & $\operatorname{Tan}$ & $\begin{array}{l}\text { L. I. } \\
\text { START }\end{array}$ & $\begin{array}{l}\text { L. L. } \\
\text { DND }\end{array}$ & $\begin{array}{l}\text { POMP } \\
\text { MMPS }\end{array}$ & $110-B I$ & $\begin{array}{l}\text { L. I. } \\
\text { STAPR }\end{array}$ & $\begin{array}{l}\text { L. L。 } \\
\text { BND }\end{array}$ & $\begin{array}{l}\text { POAP } \\
\text { ORI }\end{array}$ & RAMARKS \\
\hline & 1700 & $22^{\prime} 6$ & $96^{\prime \prime}$ & 20 & Fos & $19 \%$ & $218 \frac{1}{2}$ & $08 / 5$ & off of Elosho \\
\hline & & & & & & & & & \\
\hline & & & & & & & & & \\
\hline 110-BI & TMR & $\begin{array}{l}\text { L. I. } \\
\text { STARE }\end{array}$ & L. I. & $\begin{array}{l}\text { POMP } \\
\text { MPSS }\end{array}$ & TANK & $\begin{array}{l}\text { L. I. } \\
\text { STLPT }\end{array}$ & E. I. & $\begin{array}{l}\text { PIMP } \\
\text { OFF }\end{array}$ & REMARKS \\
\hline BATCH 1 & & & & & 104-BY & & & & \\
\hline BATCH 2 & & & & & 104-BI & & & & \\
\hline BATCA & & & & & 107-EY & $195 \frac{1}{2}$ & & & \\
\hline Tax & InRS & $\begin{array}{l}\text { L. I. } \\
\text { STARer }\end{array}$ & Lim & $\begin{array}{l}\text { PU⿴囗十 } \\
\text { MPS }\end{array}$ & TAX & STLER & Did & PUTP & RDYARKS \\
\hline 106 & 870 & $2110 \%$ & 1910 & $\nabla$ & 104 & $1711 /$ & $19^{\prime} 5$ & 1330 & $2 e_{4} \leq$ \\
\hline 103 & 1330 & 220 & 19.7 & $=$ & rog & 1910 & $22^{\prime} 3$ & 2130 & \\
\hline 105 & $2 / 35$ & $22^{\circ} 4$ & $20^{\prime} /$ & 25 & 103 & $19^{\prime 7}$ & $2+10$ & 0600 & Tlewest \\
\hline$\therefore$ & & & & & & & & & \\
\hline & & & & & & & & . & \\
\hline & & & & & & & & $\bullet$ & \\
\hline
\end{tabular}

THK

\begin{tabular}{|c|c|c|c|c|c|c|}
\hline & 0100 & 0700 & 0900 & 1500 & 1700 & 2300 \\
\hline 112 & 218 & $21^{\prime} 82$ & & 22 & $22 \cdot 6$ & $22^{\prime 2}$ \\
\hline & 2010 & so $3 \frac{1}{2}$ & 1910 & 10 & & \\
\hline
\end{tabular}

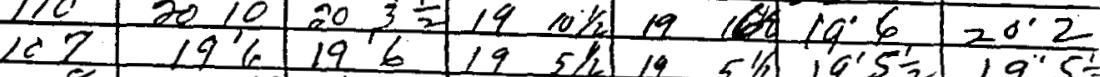

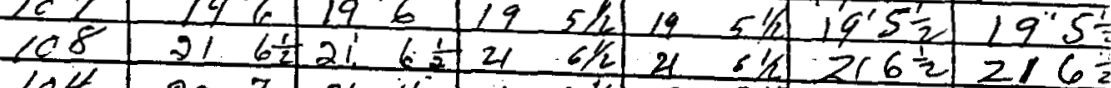

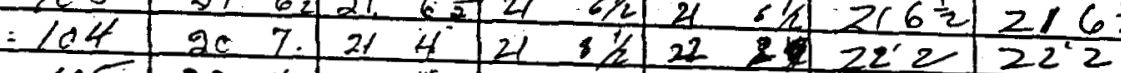
\begin{tabular}{l|ll|l|l|l|l|l}
105 & 224 & 224 & 22.4 & 22 & 4 & $22^{\prime} 4^{\prime \prime}$ & $21^{\prime} 11 !$
\end{tabular}

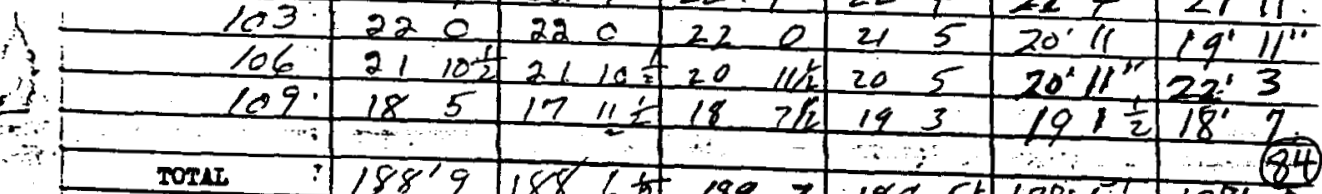

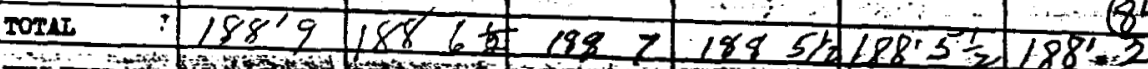

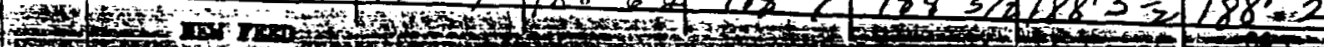


ITS-2 BOTTOMS ROUTE - DEFINITION

Boittoms route $/ 2 \quad 112-\mathrm{BI} \leftarrow$ 109-BY $=106-\mathrm{BI}-103-\mathrm{BI}$

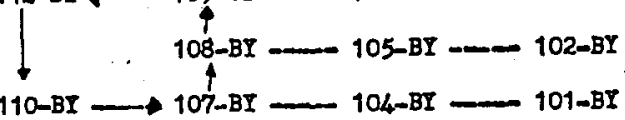

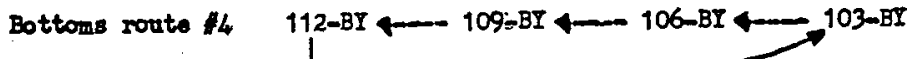

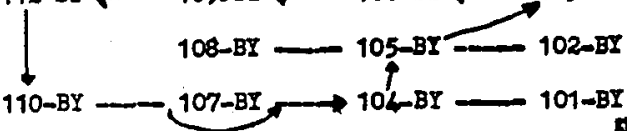

ITS-2 DVVENTORY AND PUMPING

\begin{tabular}{|c|c|c|c|c|c|c|c|c|c|}
\hline $112-B I$ & $\operatorname{TMR}$ & $\begin{array}{l}\text { L. L. } \\
\text { START }\end{array}$ & $\begin{array}{l}\text { L. I. } \\
\text { DND }\end{array}$ & $\begin{array}{l}\text { POMP } \\
\text { AMPS }\end{array}$ & 110-BY & $\begin{array}{l}\text { L. L. } \\
\text { START }\end{array}$ & $\begin{array}{l}\text { L. I. } \\
\text { DND }\end{array}$ & $\begin{array}{l}\text { PQMP } \\
\text { OFF }\end{array}$ & RRMARKS \\
\hline & $23 / 5$ & 2214 & & $z$ & $19 \cdot 9$ & & & & \\
\hline & & & & & & & & & \\
\hline & & & & & & & & & \\
\hline $110-B I$ & TIM & $\begin{array}{l}\text { L. L. } \\
\text { START }\end{array}$ & $\begin{array}{l}\text { L. L. } \\
\text { END }\end{array}$ & $\begin{array}{l}\text { PQMP } \\
\text { MPS }\end{array}$ & TANK & $\begin{array}{l}\text { L. I. } \\
\text { START }\end{array}$ & $\begin{array}{l}\text { I. I. } \\
\text { END }\end{array}$ & $\begin{array}{l}\text { PONP } \\
\text { OFE }\end{array}$ & REMARTS \\
\hline BATCH 1 & & & . & & 104-EI & & & & \\
\hline BATCH 2 & & & & & 104-BY & & & & \\
\hline BATCH 3 & 1245 & 2,9 & $19+9$ & 26 & 107-BI & $19^{\prime} 6$ & 215 & 2230 & \\
\hline TANK & $\operatorname{Tng}$ & $\begin{array}{l}\text { L. Lo, } \\
\text { STLRT }\end{array}$ & $\begin{array}{l}\text { L. Lo } \\
\text { END }\end{array}$ & $\begin{array}{l}\text { Ptip } \\
\text { APS }\end{array}$ & TAKR & $\begin{array}{l}\text { Lo } 1.0 \\
\text { srente }\end{array}$ & Did & oft & RPYARS \\
\hline 184 & 070 & 228 & $21^{\circ} 7 a^{\prime}$ & . & 105 & $20 \%$ & $20^{\prime} 8 \frac{1}{2}{ }^{\prime \prime}$ & 0830 & \& I Ilished \\
\hline 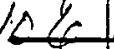 & 020 & 2713 & $202 \%$ & & 109 & $17 \%$ & $19^{\circ} 6^{\circ}$ & 1230 & Huskl \\
\hline 603 & $12+5=$ & 2110 & $19^{\prime} 10$ & & 66 & $20: 1 \frac{1}{2}$ & $22^{\prime} z$ & 2100 & \\
\hline & & & . & & & & & . & . \\
\hline & & & & & & & & $\bullet$ & \\
\hline
\end{tabular}

\begin{tabular}{|c|c|c|c|c|c|c|}
\hline Tux & 0100 & 0700 & 0900 & 1500 & 1700 & 2300 \\
\hline 112 & $22^{\prime} c$ & 218 & $2)^{\prime} 6^{\prime \prime}$ & $21^{\circ} 10 \frac{1}{2}$ & 211 & \\
\hline 110 & & 216 & $21^{\prime} 8 \frac{1}{2}$ & $21^{\circ} 1 \frac{1}{2}$ & 20 & \\
\hline 107 & $19 \sqrt{2}$ & $19.5 \frac{1}{2}$ & $19^{\prime} 6^{\prime \prime}$ & $20.1=$ & 20 & \\
\hline 108 & $216 \frac{1}{2}$ & $21^{\prime} 6 \pm 1$ & $21.6 \frac{1}{2}$ & $216 \frac{1}{2}$ & 21 & \\
\hline$\because \quad 104$ & 222 & 22.2 & $217 \frac{1}{2}$ & 218 & 21 & 218 \\
\hline$\cdots 105=$ & $21-1$ & 201 & $20^{\prime} 8 \frac{1}{x^{\prime \prime}}$ & $2 i 1$ & 21 & 21.8 \\
\hline 10.3 & 2010 & 2110 & $21^{\prime} 10^{\prime \prime}$ & $21^{\prime} 0^{\prime \prime}$ & 20 & 19110 \\
\hline 106 & 223 & $22^{\prime} .3$ & $21^{\prime} 4 \frac{1}{2}^{\prime \prime}$ & $210 \frac{1}{2}$ & 21 & $22^{\prime 2}$ \\
\hline $10 \%$ & 18 & 1711 & $18^{\prime} 7^{\prime \prime}$ & $19 \cdot 3$. & & \\
\hline & $\therefore \therefore$ & $\therefore \quad 1$ & $\because:=$ & $\therefore:$ & & \\
\hline TONAI & 188 & 188.50 & $\left.188^{\prime} 4^{\prime}\right)^{\circ}$ & & & \\
\hline
\end{tabular}

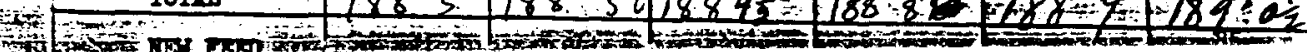


Bottoms soute 2 112-BI 103-BI

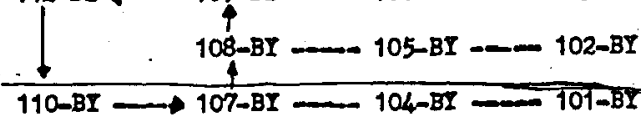

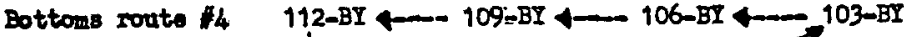

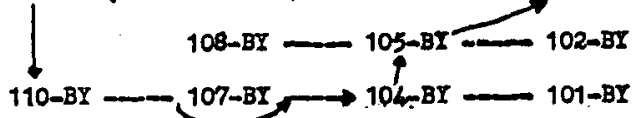

ITS-2 INVENTORY AND POMPING

DATE MAR? 7197

\begin{tabular}{|c|c|c|c|c|c|c|c|c|c|}
\hline 112-BI & TAMB & $\begin{array}{l}\text { L. L. } \\
\text { START }\end{array}$ & $\begin{array}{l}\text { I. L. } \\
\text { BND }\end{array}$ & $\begin{array}{l}\text { POMP } \\
\text { AMPS }\end{array}$ & $110-B I$ & $\begin{array}{l}\text { L. I. } \\
\text { START }\end{array}$ & $\begin{array}{l}\text { L. L. } \\
\text { END }\end{array}$ & $\begin{array}{l}\text { POMP } \\
\text { OFF }\end{array}$ & RDMARKS \\
\hline & $c / 30$ & $22 \cdot 3$ & $21^{\prime} 6$ & 21 & $20 \cdot 1$ & & 21.9 & 1400 & Learke $C$ \\
\hline & & & & & $\therefore$ & & & & \\
\hline & & & & & & & & & \\
\hline $110-8 x$ & TRE & $\begin{array}{l}\text { L. I. } \\
\text { STIEN }\end{array}$ & $\begin{array}{l}\text { L. I. } \\
\text { END }\end{array}$ & $\begin{array}{l}\text { POMP } \\
\text { MPS }\end{array}$ & TANR & $\begin{array}{l}\text { L. I. } \\
\text { STARI }\end{array}$ & L. I. & $\begin{array}{l}\text { PUMP } \\
\text { OFF }\end{array}$ & BDMARS \\
\hline BATCH 1 & 1815 & 219 & . & & $104 \mathrm{mI}$ & $19: 9$ & & & \\
\hline BATCH 2 & & & & & $104-8 \mathrm{I}$ & & & & \\
\hline BATCR 3 & & & & & 107-BI & & & & \\
\hline IMX & TIR & $\begin{array}{l}\text { L. L. } \\
\text { START }\end{array}$ & E. L & $\begin{array}{l}\text { PQPP } \\
\text { APS }\end{array}$ & TAKX & $\begin{array}{l}\text { L. LE. } \\
\text { START }\end{array}$ & E. I. & $\begin{array}{l}\text { Plup } \\
\text { Orf }\end{array}$ & BDMARKS \\
\hline 205 & 2400 & $22^{\prime} 1^{\prime \prime}$ & 1962 & 27 & 103 & $19^{\prime} 10^{\prime}$ & 220 & 1145 & \\
\hline 106 & 0630 & $22: 21 / 2$ & $20^{\circ} 2^{\circ}$ & 25 & 109 & 180 & $19 \cdot 7$ & 1300 & \\
\hline 204 & $1145^{\circ}$ & 217 & $19^{\prime} 8$ & & 105 & $198 \frac{1}{2}$ & 225 & 2130 & \\
\hline 10.3 & 1500 & $22^{\circ} 0^{\circ}$ & 20.0 & 23 & 106 & 2012 & 221 & 2200 & \\
\hline 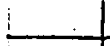 & & & & & & & & $\dot{.}$ & • \\
\hline & & & & & & & & $\bullet$ & \\
\hline
\end{tabular}

$\therefore$ TMr

Turr

$0100 \quad 0700$

112

$22: 3$

$21^{\circ} 16$

\begin{tabular}{l|l}
0900 & 1500 \\
\hline
\end{tabular}

\begin{tabular}{l|l}
1700 & 2300 \\
\hline $2 / 8 "$ & $22^{\prime}$ \\
\hline $21^{\prime} 9$ & $20^{\prime} 8:$ \\
\hline $21^{\prime} 5$ & 215 \\
\hline $21^{\prime} 6 \frac{5}{2}$ & $21^{\prime} 6 \frac{1}{2}$
\end{tabular}

$-1.04$

107

$20^{\circ}$

$21^{\prime} \%$

$21 \cdot 5 \frac{1}{2}$

\begin{tabular}{l|l}
$20^{\prime} 5^{\prime}$ & $21^{\prime} 1^{2}=$ \\
$21^{\prime} s^{\prime}$ & $21^{\circ} 5^{\frac{1}{2}}$
\end{tabular}

$21 \% \frac{1}{2}$

$216^{\prime} \div$

$21^{\prime} 65$

$21 \cdot 9$

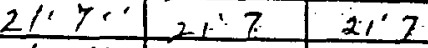

$2,5 \frac{1}{2}$

$20^{\circ} 6 \frac{1}{2}$

$20^{\prime} 8^{\prime} 2$

$20^{\circ} 1$

$20^{\prime} 1$

$=2105-21.11$

11103

$19 \cdot 10$

$21 " 4$

$22 \cdot 2 \%$

20 '6"

?

106

187

$18 \times 6$

$2 i^{\prime} 21$.

$21^{\circ} 2 \frac{1}{2}$

$10^{\prime} 9$

$21 \cdot 4 \frac{1}{2}+22 \cdot 5$

1

$189^{\prime} 4^{2}-189^{\prime} 8 \frac{1}{1}$

$21 ; 3$

TOTLI

1810

$20^{\circ} 2^{\prime}$

$21^{\prime} 3$

$19^{\prime} 5^{\frac{1}{2}}$

$\frac{20^{\prime} / 1}{19^{\circ} 3}$

$2 a^{\circ} 0$.

$22^{\circ}$ $1 p^{\circ} / C$ 


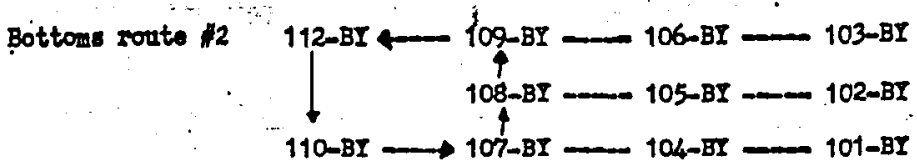

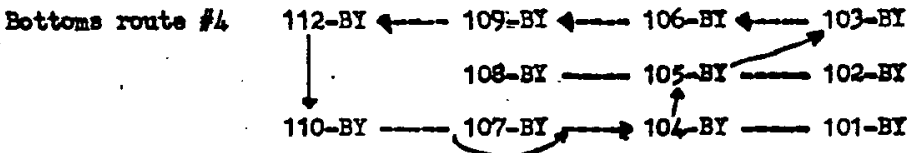
ITS-2 TNVENTORY AND PONRING DATE $3-28-31$

\begin{tabular}{|c|c|c|c|c|c|c|c|c|c|}
\hline $112-\mathrm{BI}$ & $\operatorname{TIE}$ & $\begin{array}{l}\text { L. L. } \\
\text { START }\end{array}$ & $\begin{array}{l}\text { I. I. } \\
\text { DND }\end{array}$ & $\begin{array}{l}\text { PQMP } \\
\text { AMPS }\end{array}$ & $110 \mathrm{mBY}$ & $\begin{array}{l}\text { L. I. } \\
\text { START }\end{array}$ & $\begin{array}{l}\text { L. L. } \\
\text { BND }\end{array}$ & $\begin{array}{l}\text { PONP } \\
\text { OPF }\end{array}$ & BPMARKS \\
\hline & $0 / 30$ & $22 \cdot 3 \cdot 1$ & $21^{\prime} 5 \frac{1}{2}$ & 21 & & $201 \frac{1}{3}$ & 211 & 1230 & lasihe \\
\hline & & & & & & & & & \\
\hline & & & & & & & & & \\
\hline $110-B I$ & $\operatorname{Tn} S$ & $\begin{array}{l}\text { L. I. } \\
\text { STupr }\end{array}$ & $\begin{array}{l}\text { L. I. } \\
\text { BuD. }\end{array}$ & $\begin{array}{l}\text { POMP } \\
\text { MPPS }\end{array}$ & TANK & $\begin{array}{l}\text { L. L.. } \\
\text { START }\end{array}$ & L. 1. & $\begin{array}{l}\text { POMP } \\
\text { OFF }\end{array}$ & RPMARKS \\
\hline BATCH & $1815^{\circ}$ & $21 \cdot 9 \cdot 1$ & 20.2 & & $104-B I$ & 19.9. & $21 \cdot 2$ & & $F+6 c 40$ \\
\hline BATCH 2 & & & & & $104-B I$ & & & & \\
\hline BATCE 3 & & & & . & $107-B I$ & & . & & \\
\hline TANX & TIMB & $\begin{array}{l}\text { L. L. } \\
\text { STLARS }\end{array}$ & I. L. & $\begin{array}{l}\text { PLiP } \\
\text { MPS }\end{array}$ & TANX & $\begin{array}{l}\text { Lolog } \\
\text { STARer }\end{array}$ & E. 1. & $\begin{array}{l}\text { FuMP } \\
\text { OLS }\end{array}$ & REYURTS \\
\hline 106 & 0630 & $22-1$ & 203 & $25^{\circ}$ & 105 & $18 \cdot 3$ & $19^{\prime} 7$ & 1330 & \\
\hline $105^{2}$ & 0630 & $22-4$ & 1911 & 26 & $\cos 3$ & 2010 & 224 & 1230 & \\
\hline 104 & 1230 & $21^{\circ} 3$ & $19^{\prime} 6$ & & Aat & $19 \cdot 11$ & $2118 \frac{1}{2}$ & 2430 & \\
\hline 103 & 1500 & 224 & $20^{\circ} 7$ & & 106 & 2013 & $22 \%$ & 3500 & Eluofes \\
\hline & & & & & & & & $\therefore$ & \\
\hline & & & & & & & . & - & \\
\hline
\end{tabular}

\begin{tabular}{|c|c|c|c|c|c|c|c|}
\hline TUIX & & 0100 & 0700 & 0900 & 1500 & 1700 & 2300 \\
\hline$\angle 12$ & & $22^{\prime} 3^{\prime \prime}$ & $22-0$. & 2110 & 216 & $216 \frac{1}{2}$ & $22 \cdot 0$ \\
\hline 110 & & $20^{\circ} 11 / 2$ & $20^{\circ} 2$ & $20^{\circ} 6 \frac{1}{2}$ & 211 & 211 & $21^{\prime} 1$ \\
\hline 107 & & $2 \cdot 5$ & $26^{\circ} 5$ & 215 & 215 & $z^{\prime} 5$ & \\
\hline 1108 & & $2161 / 2$ & $2 r 6 \% 2$ & $21^{\circ} 6 \frac{1}{2}$ & $2,6 \frac{1}{2}$ & $216 \frac{1}{2}$ & $6 \frac{1}{2}$ \\
\hline 10.4 & & $20^{\circ} 7^{\prime \prime}$ & $22^{\prime \prime}$ & $21^{\prime} 3$ & $20.6 \frac{1}{2}$ & $20^{\prime} 3$ & $19^{\circ} 6$ \\
\hline 205 & $\approx$ & $22^{\prime}$ ' $1 / 2$ & $22 \cdot 4:$ & $21^{\prime} 3 \frac{1}{2}$ & $20^{\circ} 8$ & 310 & $26^{\prime} \cdot 8 \frac{1}{2}$ \\
\hline 103 & & 200 & 20.0 & $21^{\prime} 1$ & 224 & 2111 & $20^{\circ} 7$ \\
\hline 106 & 1 & $22 \%$ & $22 \cdot 1$ & $215 \frac{1}{2}$ & $20^{\circ} 3$ & $20^{\prime} 8$ & 22.0 \\
\hline 109 & - & $18^{\circ} 8$ & $18 \times 3$ & 1899 & $.19 \cdot 5 \frac{1}{2}$ & $19^{\prime} 8$ & \\
\hline & & $189: 1 / 2$ & 18510 & $\therefore \ldots$ & $\cdots y$ & 7001 & \\
\hline TOTLL & $\because$ & & & $189^{\circ} 2^{\circ}$ & $1889 \frac{1}{2}$ & $188^{\circ} \cdot 9$ & \\
\hline
\end{tabular}


WHC-SD-LM-DP-209, Rev. 0

ITS-2 BOTTONS ROUTE - DERTIITION

Bottoms route $/ 2 \quad 106-\mathrm{BI}=103-\mathrm{BI}$

Bottom ronte $14 \quad 112-\mathrm{BI}+109=\mathrm{BI}+106-\mathrm{BI}+103-\mathrm{BI}$

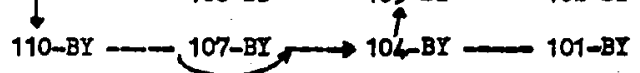

ITS-2 INVWNTORY AND POMPING DATE $3-29-11$

\begin{tabular}{|c|c|c|c|c|c|c|c|c|c|}
\hline $112-B I$ & TIMB & $\begin{array}{l}\text { L. L. } \\
\text { START }\end{array}$ & $\begin{array}{l}\text { L. I. } \\
\text { END }\end{array}$ & $\begin{array}{l}\text { POMP } \\
\text { AMPS }\end{array}$ & 110-BY & $\begin{array}{l}\text { L. L. } \\
\text { START }\end{array}$ & $\begin{array}{l}\text { L. L. } \\
\text { END }\end{array}$ & $\begin{array}{l}\text { PQRP } \\
\text { OFP }\end{array}$ & PRMARKS \\
\hline & & & & & & & & & - \\
\hline & & & & & & & & & \\
\hline & & & & & & & & & \\
\hline $110-B I$ & Tnas & $\begin{array}{l}\text { L. I. } \\
\text { STAFT }\end{array}$ & $\begin{array}{l}\text { L. I. } \\
\text { END }\end{array}$ & $\begin{array}{l}\text { PQMP } \\
\text { MPS }\end{array}$ & TANK & $\begin{array}{l}\text { L. L. } \\
\text { START }\end{array}$ & $\begin{array}{l}\text { L. I. } \\
\text { END }\end{array}$ & $\begin{array}{l}\text { POMP } \\
\text { OHF }\end{array}$ & RDMARKS \\
\hline BATCH 1 & & & & & $104-B Y$ & & & & \\
\hline BATCE 2 & & & & & $104-B Y$ & & & & \\
\hline BATCE 3 & & & & & $107-\mathrm{BI}$ & & & & \\
\hline TANK & TINS & $\begin{array}{l}\text { Lo L, } \\
\text { STuRs }\end{array}$ & E. Lo" & $\begin{array}{l}\text { PUTP } \\
\text { APS }\end{array}$ & TAKX & $\begin{array}{l}\text { Lo to } \\
\text { STHPI }\end{array}$ & Lid & $\begin{array}{l}\text { Puip } \\
\text { OF }\end{array}$ & RDAARKS \\
\hline
\end{tabular}

\begin{tabular}{|c|c|c|c|c|c|c|c|}
\hline TAIX & & 0100. & 0700 & 0900 & 1500 & 1700 & 2300 \\
\hline 112 & & $22^{\prime} 7$ & 22.7 & $22^{\prime}-8^{\prime \prime}$ & $22^{\prime} 8^{\frac{1}{2}}$ & $22^{\prime} 8^{\frac{1}{2}}$ & 22 \\
\hline 110 & & $20 \cdot 7$ & $20 \cdot 7$ & $20^{\prime} 2^{\prime \prime}$ & $20^{\prime} 7^{\prime \prime}$ & 2817 & 2017 \\
\hline 107 & & 21.5 & 21.5 & $21^{\prime} 4 \frac{1}{2}$ & $214 !^{\prime \prime}$ & 245 & 215 \\
\hline 108 & & 1919 & $19^{\prime} 9$ & $19^{\circ} 9^{\prime \prime}$ & $19^{\circ} 9^{\prime \prime}$ & $19 \cdot 9$ & 19.9 \\
\hline 104 & & $21 \cdot 3$ & $21 \cdot 3$ & $21^{\prime} 3{ }^{\prime \prime}$ & $21^{\prime} 3^{n}$ & 213 & $21 \cdot 3$ \\
\hline 105 & $\therefore$ & $20 \cdot 0$ & $20 \cdot 0$ & $20^{\prime}: 0^{\prime \prime}$ & $20^{\prime} 0^{\prime \prime}$ & 200 & 2000 \\
\hline 103 & & $22 \cdot 2$ & $22^{\prime} 2$ & $22^{\prime} 2^{\prime \prime}$ & $22^{\prime} 2^{\prime}$ & 2212 & $22^{\circ} 2$ \\
\hline 106 & .1 & $2^{\prime} 0$ & $22 ! 0$ & $22^{\prime} 0^{\prime \prime}$ & $22^{\prime} 0$ & $22^{\prime} 0$ & $22^{\prime} \sigma$ \\
\hline 109 & & $18 \cdot 9$ & 18.9 & $18^{\prime} 9^{\prime \prime}$ & $18^{\prime}$ & $18^{\circ} 83$ & \\
\hline & - & 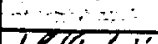 & $\therefore$ & $x_{z:}-{ }_{1}$ & 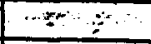 & & \\
\hline TOTAL & $\therefore$ & $16816^{\prime \prime}$ & 188.6 & $188^{\prime} 6 \frac{1}{5}=$ & $18812^{\prime \prime}$ & & \\
\hline
\end{tabular}


ITS-2 BOMTONS ROUTE - DEPINITTON

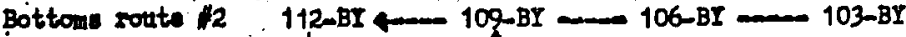

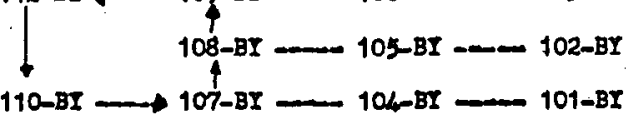

Bottoms route /4 112-BI 109-BI $106-\mathrm{BI}-103-\mathrm{BI}$

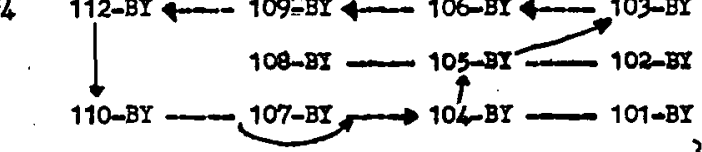

ITS-2 TNVENTORY AND POMPTNG

DATE $3-30-71$

\begin{tabular}{|c|c|c|c|c|c|c|c|c|c|}
\hline 112-EI & TIMB & $\begin{array}{l}\text { L. L. } \\
\text { START }\end{array}$ & $\begin{array}{l}\text { L. L. } \\
\text { DND }\end{array}$ & $\begin{array}{l}\text { PORP } \\
\text { AMPS }\end{array}$ & $110-B I$ & $\begin{array}{l}\text { L. I. } \\
\text { sTARA }\end{array}$ & $\begin{array}{l}\text { L. L. } \\
\text { END }\end{array}$ & $\begin{array}{l}\text { PQPP } \\
\text { OFP }\end{array}$ & REMARKS \\
\hline & $0 / 245$ & $22 \cdot 2 \cdot 1$ & $21: 9^{\prime \prime}$ & & $1 / 10$ & $2 / 1 \%$ & $29^{\prime}-1 \%^{\prime \prime}$ & 0830 & \\
\hline & 1730 & $22 \div 5$ & $22^{\prime} 3$ & & 110 & $21^{\prime} a$ & $20^{\prime 7}$ & 1900 & \\
\hline & & & & & & & & & \\
\hline $110-B I$ & InRE & $\begin{array}{l}\text { L. L. } \\
\text { STAFr }\end{array}$ & $\begin{array}{l}\text { L. L. } \\
\text { EXVD }\end{array}$ & $\begin{array}{l}\text { POPP } \\
\text { APS }\end{array}$ & TANK & $\begin{array}{l}\text { I. I. } \\
\text { START }\end{array}$ & $\begin{array}{l}\text { L. I. } \\
\text { END }\end{array}$ & $\begin{array}{l}\text { PUMP } \\
\text { OFF }\end{array}$ & RDMARKS \\
\hline BATCH 1 & $1 / 200$ & $22^{\prime}-1 / 2$ & 2017 & 25 & $104-B Y$ & $19^{\prime}-7^{4}$ & $2 \times 3$ & 1900 & \\
\hline BATCE 2 & & & & & $104-B I$ & & & & \\
\hline BATCB & & & & & $107-B I$ & & & & \\
\hline Tux & PrR: & $\begin{array}{l}\text { L. ID. } \\
\text { STLPU }\end{array}$ & EN & $\begin{array}{l}\text { PLPP } \\
\text { MPS }\end{array}$ & TAK & $\begin{array}{l}\text { L. Lo } \\
\text { sturar }\end{array}$ & LaD & $\begin{array}{l}\text { PUPP } \\
\text { OFP }\end{array}$ & RDXARKS \\
\hline 105 & 9600 & $2 / 6 \frac{1}{x}$ & $-19^{\prime} 8 \frac{1}{2}$ & 20 & 109 & $18 \cdot 3$ & $19^{\prime} 4$ & 1800 & \\
\hline 105 & 0630 & $21.8 \frac{1}{2}$ & $20^{\prime}-0^{\prime \prime}$ & 26 & 1103 & $20^{\prime} 7$ & $22^{\prime}-2^{\prime}$ & 1145 & Fluike 2 \\
\hline & & & & & & & & & . \\
\hline & & & . & & & & & . & \\
\hline 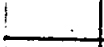 & & . & & & & & & . & \\
\hline & & & & & & & & & \\
\hline
\end{tabular}

\begin{tabular}{|c|c|c|c|c|c|c|}
\hline$\therefore$ TUSX & 0100 & 0700 & 0900 & 1500 & & 2300 \\
\hline 112 & $22: 2$ & $21.11 \cdot$ & $2^{\prime}-9$ & $22-2 y_{2}$ & 22.4 & $22^{\prime} 6$ \\
\hline$\angle 1.0$ & 3110 & 21.80 & $22^{\prime}+1 / y_{2}$ & $2^{\prime}-5$ & 210 & \\
\hline 107 & $21.5=$ & 21,5 & $21^{\prime}-5$ & $2,-5$ & 21.5 & \\
\hline 104 & $2 / .64 / 2$ & $21.61 / 2$ & $2 x^{\prime}-3$ & $20^{\prime}-2$ & $19^{\prime} 8 \frac{1}{2}$ & 141 \\
\hline 1.04 & 19.7 & 19.7 & $19^{\prime}-7$. & $20^{\prime}-4$ & $20^{\prime} 8 \frac{1}{2}$ & $21^{\prime} 3$ \\
\hline 105 & $21+8$ & $21.8: 1 / 2$ & $14-9 \%$ & $20^{\prime}-0$ & $20^{\circ} 0$ & 200 \\
\hline 103 & 20 & 202 & $2^{\prime}=5 s_{2}$ & $22^{\prime}-2$ & $22^{\prime 2}$ & $22 \cdot 2$ \\
\hline 106 & 70 & $20 \div 0$ & $22-0$ & $22^{\prime}-0$ & 220 & $22^{\circ} 0$ \\
\hline 104 & 78 & 18.3 & $18^{\prime}-4$ & $19^{\prime}-0$ & $19^{\prime} 3 \frac{1}{2}$ & $18^{\circ} 10$ \\
\hline-1 & & & & $x^{2}$ & & \\
\hline
\end{tabular}


ITS-2 BOTTOYS ROUTE - DER INITION

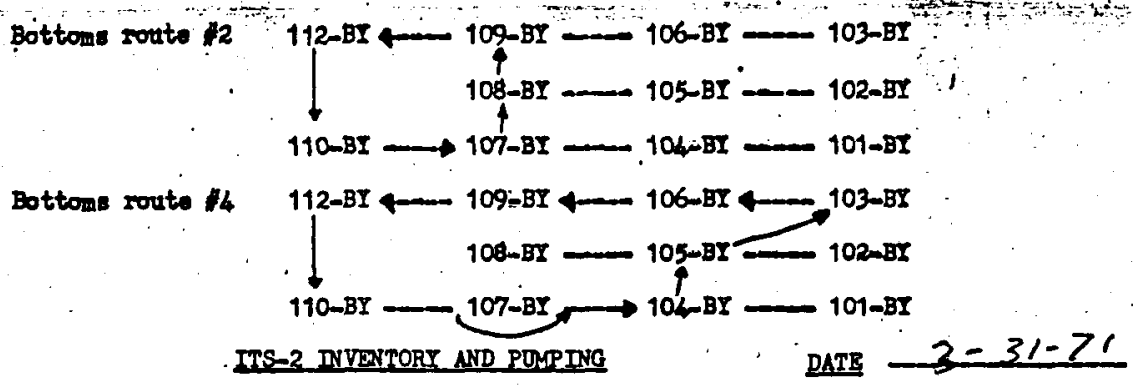

\begin{tabular}{|c|c|c|c|c|c|c|c|c|c|}
\hline $112-\mathrm{BI}$ & TIMS & $\begin{array}{l}\text { L. L. } \\
\text { START }\end{array}$ & L. L. & $\begin{array}{l}\text { POMP } \\
\text { MMPS }\end{array}$ & 110-BI & $\begin{array}{l}\text { L. L. } \\
\text { STIRT }\end{array}$ & $\begin{array}{l}\text { L. L. } \\
\text { BND }\end{array}$ & $\begin{array}{l}\text { PQMP } \\
\text { OFT }\end{array}$ & RRPARKS \\
\hline 112 & 1315 & $22^{\prime} 6 \frac{1}{2}$ & $21 \%$ & 23 & 110 & $20^{\prime} 7^{\prime}$ & $2111 \frac{1}{2}$ & 1815 & $21 \mathrm{ec} \leq \mathrm{kel}$ \\
\hline $11 n$ & $\cdots$ & $\therefore 1$ & & & $\therefore$ & & $\ldots$ & - & b $\ldots$ \\
\hline & & & & & & & & & \\
\hline $110-B Y$ & TIME & $\begin{array}{l}\text { L. L. } \\
\text { STuRT }\end{array}$ & L. L. & $\begin{array}{l}\text { POMP } \\
\text { AMPS }\end{array}$ & TANK & $\begin{array}{l}\text { L. L. } \\
\text { START }\end{array}$ & L. L. & $\begin{array}{l}\text { POMP } \\
\text { OFP }\end{array}$ & RPUARES \\
\hline BATCR 1 & 2110 & $2111 \frac{1}{2}$ & $19^{\prime} 5^{\prime \prime}$ & & 104-EI & 195 & $21^{\prime} \frac{1}{2}$ & 0820 & Flosted \\
\hline BATCH 2 & & & & & 104-BI & & & & \\
\hline BATCB 3 & & & & & 107-BI & & & & . \\
\hline TANS & TDRE & $\begin{array}{l}\text { L. Lo } \\
\text { START }\end{array}$ & L. Lo & $\begin{array}{l}\text { PupP } \\
\text { DPS }\end{array}$ & TAK & T.L. & D. LD. & $\begin{array}{l}\text { FuTp } \\
\text { Ofr }\end{array}$ & HGALRS \\
\hline 107 & 1400 & $2^{\prime} 4 \frac{1}{2}$ & 1962 & 12 & 108 & $19^{\prime \prime}$ & $2^{\prime 7}$ & 2200 & Hened \\
\hline 204 & 1400 & $2 i 3^{\prime \prime}$ & $19^{\prime} 5$ & $22^{\circ}$ & 105 & $20^{\prime} 0^{\prime \prime}$ & $221 \frac{1}{2}$ & 2100 & Iisutad \\
\hline$\therefore$ & & & & & & & & & \\
\hline 106 & 2300 & 21112 & $19^{\prime} 8 \frac{1}{2}=$ & & 109 & $18 \div 1$ & $19^{\prime} 2^{\prime \prime}$ & 0,20 & Elostead \\
\hline & & & & & & & & & \\
\hline & & & & & & $\therefore$ & $=$ & $\ldots$ & 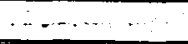 \\
\hline
\end{tabular}

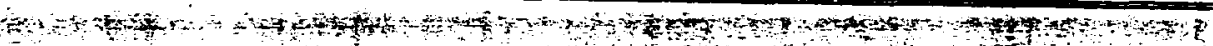

\begin{tabular}{|c|c|c|c|c|c|c|}
\hline ISIS & 0100 & 0700 & $0900 \div$ & $1500 \div$ & 1700 & \\
\hline 112 & $22110^{\prime \prime}$ & $22+9 \frac{1}{2}$ & $22^{\prime} q^{\prime \prime}$ & $22^{\prime} i^{\prime \prime}$ & $218 \frac{7}{2}$ & \\
\hline 110 & 2017 & $20 \cdots 7 \cdots$ & $20^{\prime} 2^{\prime \prime}$ & $1^{\prime} 1^{\prime \prime}$ & 218 & 21 \\
\hline 107 & 21.5 & $2 \%=$ & $214 \frac{1}{2}$ & $21^{\prime} \cdot 2^{\frac{1}{2}}$ & 209 & $19^{\circ}$ \\
\hline 1108 & 1.51 .5 & 19.5 & $19^{\prime} 9^{\prime \prime}$ & $19^{\circ} 11^{\circ}$ & 205 & 24 \\
\hline$\frac{10,4}{10}=$ & $21 \cdot 3$ & $2 / \cdot 3$ & $21^{\prime \prime}$ & $2 i^{\prime} 0^{\prime \prime}$ & $205 \frac{1}{2}$ & $19^{\prime}$ \\
\hline 105 & 2000 & 2000 & $20^{6} \sigma$ & $10^{11} 3^{\prime \prime}$ & $20 \%$ & $221 \frac{1}{2}$ \\
\hline 110.3 & $22 \cdot 2$ & $22^{\prime} 2$ & $22^{\circ} 2$ & $22^{\prime} 2^{\prime \prime}$ & 22 & $22^{12}$ \\
\hline$\frac{106}{105}$ & $\frac{22.0}{14.4}$ & $\frac{22,0}{414}$ & $\frac{22^{\prime} 6}{10^{\prime} 0}$ & $22^{\prime \prime} 0^{\prime \prime}$ & $11 \frac{1}{2}$ & \\
\hline 107 & $188: 84$ & $\frac{18 ! 87}{188.8 \cdot}$ & $\frac{189}{188.72^{11}}$ & $\frac{18^{\prime} 8^{\prime \prime}}{108^{\circ}}$ & 18 & \\
\hline TOTAL & $2+x+x_{y}-1+\cdots$ & 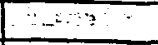 & & & 877 & \\
\hline
\end{tabular}




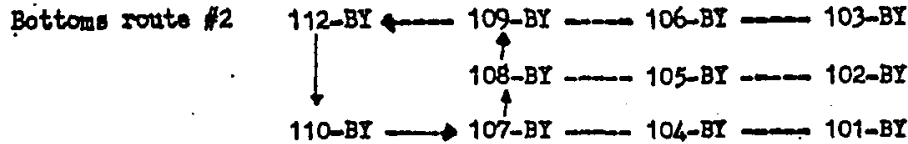

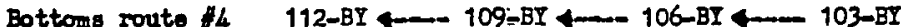

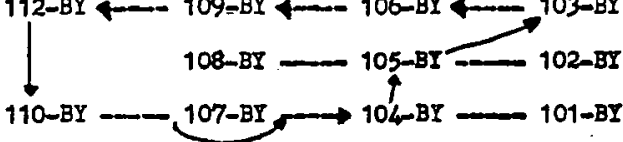

ITS $=2$ DNVENTORY AND POMPING

DATE $4-21$

\begin{tabular}{|c|c|c|c|c|c|c|c|c|c|}
\hline 112-BI & TMM & $\begin{array}{l}\text { L. L. } \\
\text { START }\end{array}$ & $\begin{array}{l}\text { L. L. } \\
\text { END }\end{array}$ & $\begin{array}{l}\text { POMP } \\
\text { AMPS }\end{array}$ & $110-\mathrm{BI}$ & $\begin{array}{l}\text { L. L. } \\
\text { START }\end{array}$ & $\begin{array}{l}\text { L. I. } \\
\text { END }\end{array}$ & $\begin{array}{l}\text { POMP } \\
\text { OFT }\end{array}$ & RDMARTS \\
\hline 112 & 0950 & $22^{\prime} 6^{\prime \prime}$ & 216 & 22 & 110 & $19^{\prime} 5^{\prime \prime}$ & & 115 & $71 \mathrm{sh}$ \\
\hline & & & & & & & & & \\
\hline$1 \longdiv { 1 0 - B Y }$ & TIME & $\begin{array}{l}\text { L. L. } \\
\text { START }\end{array}$ & $\begin{array}{l}\text { I. L. } \\
\text { END. }\end{array}$ & $\begin{array}{l}\text { POMP } \\
\text { AMPS }\end{array}$ & TANK & $\begin{array}{l}\text { L. L. } \\
\text { START }\end{array}$ & I. L. & $\begin{array}{l}\text { POMP } \\
\text { OFP }\end{array}$ & REMARKS \\
\hline BATCH 1 & & & & & $104-B Y$ & & & & \\
\hline BATCH 2 & 180 & $21,1 \frac{1}{2}$ & $19 \cdot 6 \cdot$ & & $104-\mathrm{BI}$ & 218 & 213 & $y=0$ & 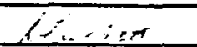 \\
\hline BATCR 3 & & & & & 107-BI & & . & & 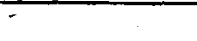 \\
\hline TAN & TDAB & $\begin{array}{l}\text { L. Lo } \\
\text { STLPR }\end{array}$ & END & $\begin{array}{l}\text { PUNIP } \\
\text { MPS }\end{array}$ & TAXX & $\begin{array}{l}\text { L. L: } \\
\text { STLPU }\end{array}$ & LED & $\begin{array}{l}\text { PUDP } \\
\text { ORT }\end{array}$ & RPYARKS \\
\hline 103 & 1010 & $22^{\prime} 2^{\prime \prime}$ & $20 \% \frac{1}{2}$ & 25 & 106 & $19^{\prime} 8 \frac{1}{2}$ & 723 & $0 / 00$ & Exhen \\
\hline 204 & 17 ar & 219 & $20^{\circ} 6^{\circ}$ & & 10.5 & $22^{\prime}, \frac{1}{2}$ & $22 \cdot 7$ & 2302 & \\
\hline 105 & 1200 & $221, \frac{1}{2}$ & 22,2 & & 10.3 & $20^{\prime} 7$ & $22^{\prime} 0$ & 0130 & 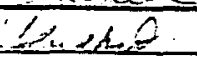 \\
\hline & & & & & & & & & 2 \\
\hline & & & & & & & & . & \\
\hline & & & & & & & & $\bullet$ & \\
\hline
\end{tabular}

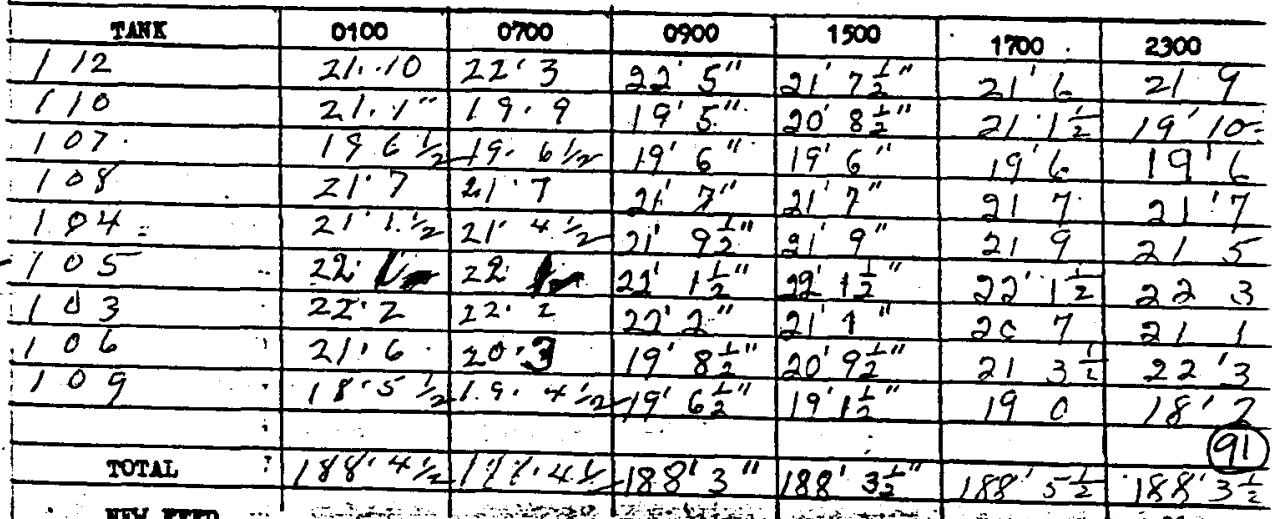


Bottoms rote $\mathrm{A2}$ 112-BI 4 109-BI $106-\mathrm{BY}-103-\mathrm{BY}$

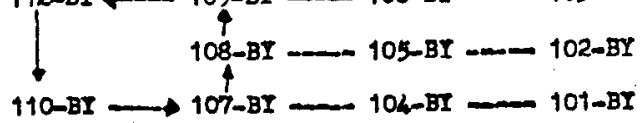

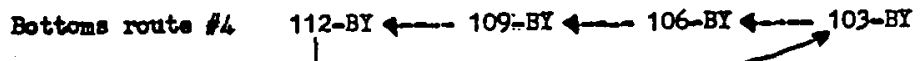

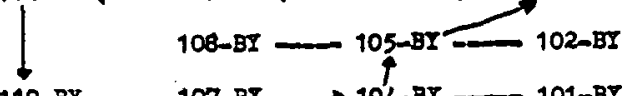

$110-\mathrm{BY} \longrightarrow 106 \mathrm{BY}-101-\mathrm{BI}$

ITS-2 TNVENTORI AND POMPDNG

Date $4-2 \cdot 21$

\begin{tabular}{|c|c|c|c|c|c|c|c|c|c|}
\hline $112-B I$ & TDMS & $\begin{array}{l}\text { L. L. } \\
\text { START }\end{array}$ & $\begin{array}{l}\text { L. I. } \\
\text { DND }\end{array}$ & $\begin{array}{l}\text { POMP } \\
\text { MMPS }\end{array}$ & $110-B I$ & $\begin{array}{l}\text { L. L. } \\
\text { STAPT }\end{array}$ & $\begin{array}{l}\text { L. I. } \\
\text { BND }\end{array}$ & $\begin{array}{l}\text { POMP } \\
\text { OFP }\end{array}$ & REMARTSS \\
\hline & $\log 30$ & $22^{\prime} 6$ & $21^{\prime 6}$ & 23 & táp & $19^{\prime} 4$ & 20.9 & 1430 & Huske \\
\hline & & & & & & & & & \\
\hline & & & & & & & & & \\
\hline 110-BI & $\mathrm{TMKS}$ & $\begin{array}{l}\text { I. I. } \\
\text { STuRT }\end{array}$ & $\begin{array}{l}\text { L. I. } \\
\text { END }\end{array}$ & $\begin{array}{l}\text { POPP } \\
\text { AMPS }\end{array}$ & TANK & $\begin{array}{l}\text { L. L. } \\
\text { START }\end{array}$ & $\begin{array}{l}\text { L. I. } \\
\text { END }\end{array}$ & $\begin{array}{l}\text { POMP } \\
\text { OFP }\end{array}$ & RPMARKS \\
\hline BATCR 1 & & & & & 104-BY & & & & \\
\hline BATCH 2 & & & & & 104-BI & & & & \\
\hline BATCH 3 & 32100 & $20^{\prime} 9$ & $19^{\prime} 6^{\prime \prime}$ & & 107-BI & 1916 & $2010 \frac{1}{2}$ & 0230 & Flosh.d \\
\hline TANI & TDME & $\begin{array}{l}\text { L. L. } \\
\text { START }\end{array}$ & Lid & $\begin{array}{l}\text { PUTP } \\
\text { APS }\end{array}$ & TANX & STLER & EDD. & $\begin{array}{l}\text { PUFP } \\
\text { OFT }\end{array}$ & RBMARKS \\
\hline$\angle 06$ & $\cos 0$ & $22^{\prime} 3$ & 196 & 24.5 & 109 & $17^{\prime} 8$ & $19^{\prime} 7 \frac{1}{2}$ & 200 & Flehed \\
\hline $1 / 13$ & $2 / 0-6$ & $220 \frac{1}{2}$ & $19^{\prime} 2^{\prime \prime}$ & 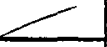 & 101 & 196 & 220 & 0545 & Flushed \\
\hline & & & & & & & & & \\
\hline & & & & & & & & & \\
\hline & & & & & & & & & \\
\hline & & & & & & & & $\cdot$ & \\
\hline
\end{tabular}

\begin{tabular}{|c|c|c|c|c|c|c|c|}
\hline TMI & & 0100 & 0700 & 0900 & 1500 & 1700 & 2300 \\
\hline 112 & & $21.9^{4}$ & $22 \cdot 4$ & $22^{\circ} 5^{\prime}$ & & 218 & $22^{\prime} a \frac{7}{2}$ \\
\hline 110 & & $19 \cdot 6$ & 19.4 & $19^{\circ} 4$ & & $20 \cdot 9$ & 206 \\
\hline 107 & & $19 \cdot .6$ & 19.6 & $19^{\prime} 6$ & 196 & 196 & 199 \\
\hline 108 & & $21 \cdot 7$ & 21,7 & $21^{\prime} 7$ & $2 \times 7$ & 217 & $21^{\prime 7}$ \\
\hline $10^{4}=$ & & 2J. 3 & 20.6 & $2 e^{\prime} 6$ & $20 \backslash 7$ & 207 & $21^{\prime} 7$ \\
\hline 1100 & $\ddot{*}$ & $22 \cdot 2$ & $22 \cdot 8$ & $23^{\prime} 8$ & 1228 & 22.8 & 22 \\
\hline 1103 & & $21 \cdot 10$ & $22 \cdot 0$ & $22^{\prime} .0$ & & 2204 & 21 \\
\hline 106 & 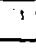 & $22 \cdot 3$ & $22 \cdot 3$ & $22^{\prime} 3$ & & $20 ! 3$ & 199 \\
\hline 1109 & . & $18 \cdot 5$ & $18 \cdot 0$ & $12^{\prime} 9$ & & $19^{\prime} 2$ & 195 \\
\hline & : & & & & & & \\
\hline$C_{\text {TOTLI }}$ & & $188^{\circ} 3^{\circ}$ & 188212 & $188^{\circ} 0$ & & $188^{\prime} 2 \frac{1}{2}$ & $188^{\circ} 0 \frac{1}{2}$ \\
\hline
\end{tabular}




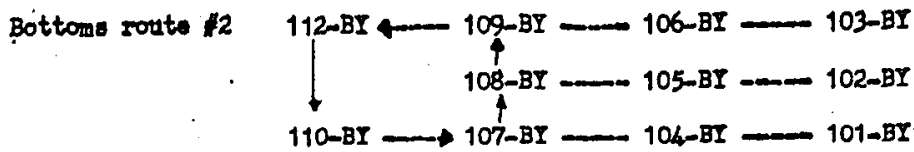

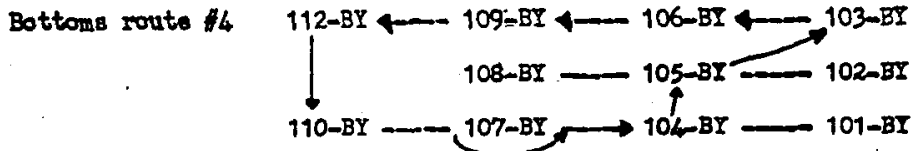

ITS-2 DNVENTORT AND PONPING

DATE $4-3-\not 1$

\begin{tabular}{|c|c|c|c|c|c|c|c|c|c|}
\hline 112-BI & TIMB & $\begin{array}{l}\text { L. L. } \\
\text { STARI }\end{array}$ & $\begin{array}{l}\text { L. L. } \\
\text { END }\end{array}$ & $\begin{array}{l}\text { POMP } \\
\text { AMPS }\end{array}$ & $110-B Y$ & $\begin{array}{l}\text { L. L. } \\
\text { STARE }\end{array}$ & $\begin{array}{l}\text { L. L. } \\
\text { END }\end{array}$ & $\begin{array}{l}\text { POMP } \\
\text { OFF }\end{array}$ & RDMARKS \\
\hline & 1005 & 22.9 & 216 & 23 & $t 0^{\prime}$ & $1 g^{\prime} 6$ & 21,5 & 1620 & 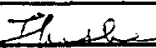 \\
\hline & & & & & & & & & \\
\hline & & & & & & & & & \\
\hline $110-8 I$ & TDME & $\begin{array}{l}\text { I. I. } \\
\text { STAREI }\end{array}$ & $\begin{array}{l}\text { L. L. } \\
\text { END. }\end{array}$ & $\begin{array}{l}\text { POMP } \\
\text { APS }\end{array}$ & TANK & $\begin{array}{l}\text { L. I. } \\
\text { START } \\
\end{array}$ & $\begin{array}{l}\text { L. L. } \\
\text { END }\end{array}$ & $\begin{array}{l}\text { PDMP } \\
\text { OFF }\end{array}$ & RUMARKS \\
\hline BATCH 1 & $2 \mathrm{sec}$ & $21: \frac{1}{2}$ & $19^{\prime} 6^{\prime \prime}$ & & $104-B Y$ & $20^{\prime} 5$ & $22^{\prime}$, & 0245 & Floshed \\
\hline BATCH 2 & & & & & 104-BY & & & & \\
\hline BATCR 3 & & & & & $107-\mathrm{BI}$ & & - & & . \\
\hline TANK & TDMB & $\begin{array}{l}\text { L. I. } \\
\text { STURE }\end{array}$ & $\begin{array}{l}\text { L. I. } \\
\text { BD }\end{array}$ & $\begin{array}{l}\text { PLVP } \\
\text { APS }\end{array}$ & TANX & $\begin{array}{l}\text { L. I. } \\
\text { STARI }\end{array}$ & Le L. & $\begin{array}{l}\text { Puitip } \\
\text { OFF }\end{array}$ & REMARES \\
\hline 105 & 0.550 & $22^{\prime} 8^{\prime \prime}$ & $20^{\prime} 1$ & 25 & 103 & $19^{\prime} 7^{\prime \prime}$ & 22 & $\angle 070$ & thushes \\
\hline 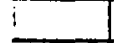 & & & & & & & & . & 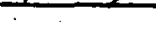 \\
\hline & & & & & & & & & \\
\hline & & & & & & & & & \\
\hline 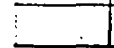 & & & & & & & &. & \\
\hline 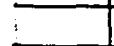 & & & & & & & & $\cdot$ & \\
\hline
\end{tabular}

\begin{tabular}{|c|c|c|c|c|c|c|c|}
\hline TMX & & 0100 & 0700 & 0900 & 1500 & 1700 & 2300 \\
\hline 112 & & $22^{\prime} 31^{\frac{1}{2}}$ & $22^{\circ} 4 \frac{11}{2}$ & $22^{\circ} 6$ & $21^{\prime} 8$ & 216 & $21^{7}$ \\
\hline 110 & & $19^{\prime} 11^{\frac{1}{2}}$ & $19^{\prime} 6^{\prime \prime}$ & $19^{\circ} 6$ & $20^{\prime} 8$ & 21.21 & 206 \\
\hline 107 & & $20^{\prime} 6^{\prime \prime}$ & $20^{\prime} 10^{1^{\prime \prime}}$ & $20^{\prime} 10$ & $20 \%$ & 2010 & 20.10 \\
\hline 108 & & $21^{\prime} 7^{\prime \prime}$ & $21^{\prime} z^{\prime \prime}$ & $21^{\prime} 6:$ & $21^{\prime} 6 \frac{1}{2}$ & 21 & 21 \\
\hline $10 \leqslant$ & & $20^{\prime} 6^{\prime \prime}$ & $20^{\prime} 6^{\prime \prime}$ & $20^{\circ} \sqrt{5}$ & $20^{\prime} 5$ & 205 & 21 \\
\hline 105 & $\because$ & $2^{\prime} 8^{\prime}$ & $22^{\prime} 3^{\prime \prime}$ & $21^{\prime} 8$ & $20^{\circ} 1$ & 201 & 20 \\
\hline 103 & - & $20^{\circ} 11^{\prime \prime}$ & $20^{\prime} 0^{\prime \prime}$ & $20^{\circ} 7$ & $22^{\prime} 0$ & 22 a $\frac{1}{2}$ & 22 \\
\hline$\angle 06$ & $\therefore$ & $20^{\prime} \cdot 8^{\prime \prime}$ & $22^{\circ} 0^{\prime \prime}$. & $22^{\prime} 0$ & $22^{\prime} 8$ & 220 & $22 c$ \\
\hline 109 & . & $19^{\prime} 5^{\prime}$ & $18^{-1} 10^{\prime \prime}$ & $18^{18}$ & $18^{\prime} 35$ & 182 & 1710 \\
\hline & ; & & & & & & 1 \\
\hline TOTAL & & $188^{\circ} \cdot 4^{\prime \prime}$ & $187^{\prime} 11^{\prime \prime}$ & $182^{\prime} 65$ & $182^{\prime} 6$ & $187^{10}$ & 1879 \\
\hline
\end{tabular}


Bottoms roate $2 \cdot 112-\mathrm{BI}+109-\mathrm{BY}=106-\mathrm{BY}-103-\mathrm{BI}$

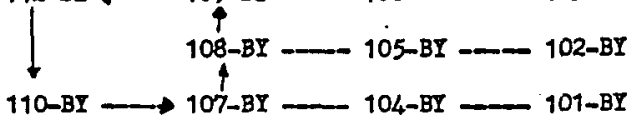

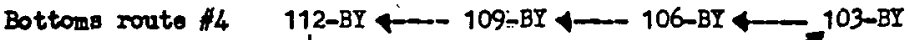

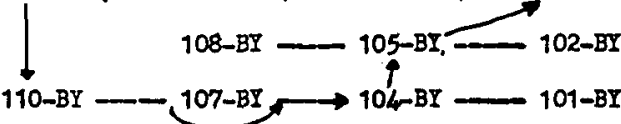

ITS-2 DNVDITORY AND PUMPING

DATE $4-5 \cdot 21$

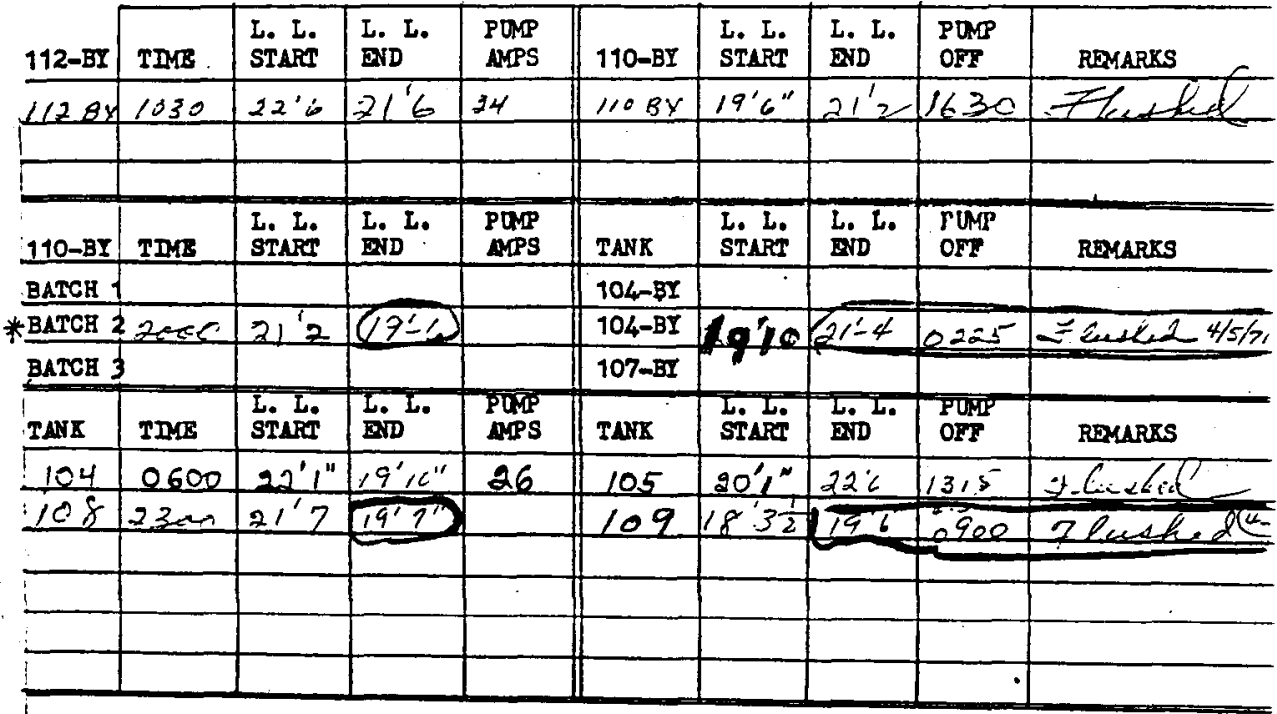

TANK

\begin{tabular}{l|c|c} 
& 0100 & 0700 \\
\hline 112 & $90^{\prime}$ &
\end{tabular}

\section{0}

$1 / 221^{\prime} 10^{\frac{1}{2}} 22^{\prime} 3 \frac{1}{1} 2^{\prime \prime} \quad 22^{\prime} 5^{\prime \prime}$ $110 \quad 19^{\prime} \quad 11^{\prime \prime} 19^{\prime} 6$ $107 \quad 20^{\prime} \quad 10^{\prime \prime} 20^{\circ} 10$

6 "19.6"

$7 " 21^{\prime 2} 2$

" $20^{\circ} 10^{\prime \prime}$

10.321

2

$21^{\prime} 7 \frac{10}{2}$

$8 \frac{1}{2}^{\circ} 21^{\prime} 9$

$105.20^{\circ}$

1 " $20^{\prime} 5$

103.22

$\frac{1}{2}=21^{1} \frac{1}{2}^{4} \frac{21 \%}{22^{\circ} 0^{2}}$

$106^{\circ} 22^{\prime}$

$0^{*} \mid 22^{\prime} 0$

10917

\begin{tabular}{l|ll}
$1 \frac{1}{2}$ & $19^{\circ} 3$ \\
\hline
\end{tabular}

$3^{\prime \prime}$

$\frac{R_{\text {se }} 103.8 x}{189^{\prime} \cdot 8}$

$12^{\prime} 0^{\prime \prime}$

\begin{tabular}{|c|c|c|}
\hline 1500 & 1700 & 2300 \\
\hline $21^{\prime} 8^{\prime \prime}$ & $21^{\prime} 6$ & $21^{\prime} 8$ \\
\hline $20^{\prime} 8^{\prime \prime}$ & $21^{\prime} 2$ & 20
\end{tabular}

$\frac{20^{\prime} 8^{\prime \prime}}{20^{\prime 10} 10^{\prime \prime}}$

212 an 6

\begin{tabular}{|l|l|}
$21^{\prime} 7 \frac{20}{2}$ & 20 \\
\hline & 21
\end{tabular}

2018

$1038 \times 70^{\circ}, 9098$,

TOTNI

\begin{tabular}{ll|l}
$187^{\prime}$ & $8^{\prime \prime}$ & $189^{\prime}$
\end{tabular}

$19^{\prime} 10^{\prime \prime}$

19 . le

205

$22^{\prime} 6^{\prime \prime}$

$22^{\circ} \circ \frac{1}{2}$

$22^{\prime} b$

$22^{\prime} 0$

220

22

:-

220

$19^{\circ} 3$

Inst $7 x$

$103 B X \ldots$

$189^{\prime} \mathrm{n}^{\prime \prime}$

$190^{\prime} 03$

$\begin{array}{ll}22 & 0 \\ 18 & 8\end{array}$

220 


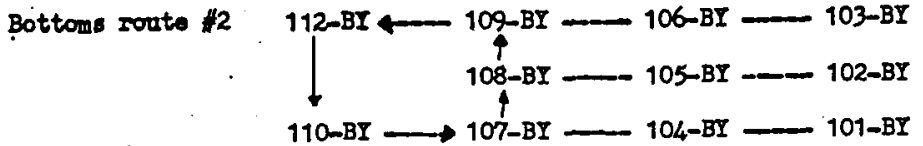

Bottoms route 4

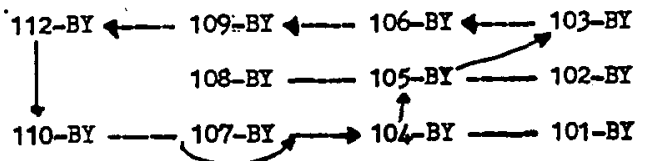

ITS-2 TNVDYTORI AND PUMPTNG

DATE $-4-5-71$

\begin{tabular}{|c|c|c|c|c|c|c|c|c|c|}
\hline 112-EI & TIMS & $\begin{array}{l}\text { L. L. } \\
\text { START }\end{array}$ & $\begin{array}{l}\text { L. L. } \\
\text { END }\end{array}$ & $\begin{array}{l}\text { POMP } \\
\text { AMPS }\end{array}$ & 110-BY & $\begin{array}{l}\text { L. L. } \\
\text { START }\end{array}$ & $\begin{array}{l}\text { L. L. } \\
\text { BND }\end{array}$ & $\begin{array}{l}\text { POAP } \\
\text { OFT } \\
\end{array}$ & RQMARKS \\
\hline $112 B y$ & 1050 & $22^{\prime} 6^{\prime \prime}$ & 216 & 24 & $\angle 10$ & 196 & 211 & 2030 & Flouked \\
\hline & & & & & & & & & 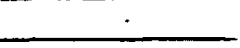 \\
\hline & & & & & & & & & \\
\hline $110-B Y$ & TDMS & $\begin{array}{l}\text { L. L. } \\
\text { START }\end{array}$ & $\begin{array}{l}\text { L. L. } \\
\text { BND }\end{array}$ & $\begin{array}{l}\text { PQMP } \\
\text { MAPS }\end{array}$ & TANK & $\begin{array}{l}\text { L. L. } \\
\text { STARI }\end{array}$ & $\begin{array}{l}\text { L. L. } \\
\text { BND }\end{array}$ & $\begin{array}{l}\text { POMP } \\
\text { OFF }\end{array}$ & RPMARS \\
\hline BAPGA & & & & & $10 \mathrm{E}=\mathrm{a}$ & & & & \\
\hline BATEA & & & & & 1048 & & & & \\
\hline BATCB 3 & 2306 & 211 & $19^{\prime}-11$ & & 107-BI & $209 \frac{1}{2}$ & $22^{\prime}-2$ & 0.345 & Flushed $4 / 6(x)$ \\
\hline TANX & IDAR & $\begin{array}{l}\text { L. Lut } \\
\text { START }\end{array}$ & Lo 10 & $\begin{array}{l}\text { PUTP } \\
\text { APS }\end{array}$ & TANS & $\begin{array}{l}\text { T. L: } \\
\text { STMRT }\end{array}$ & DND & $\begin{array}{l}\text { PUTP } \\
\text { OFF }\end{array}$ & RBMLRRS \\
\hline & & & & & & & & & \\
\hline & & & & & & & & & \\
\hline & & & & & & & & & \\
\hline & & & & & & & & & \\
\hline & & & & & & & & & \\
\hline & & & & & & & & & \\
\hline
\end{tabular}

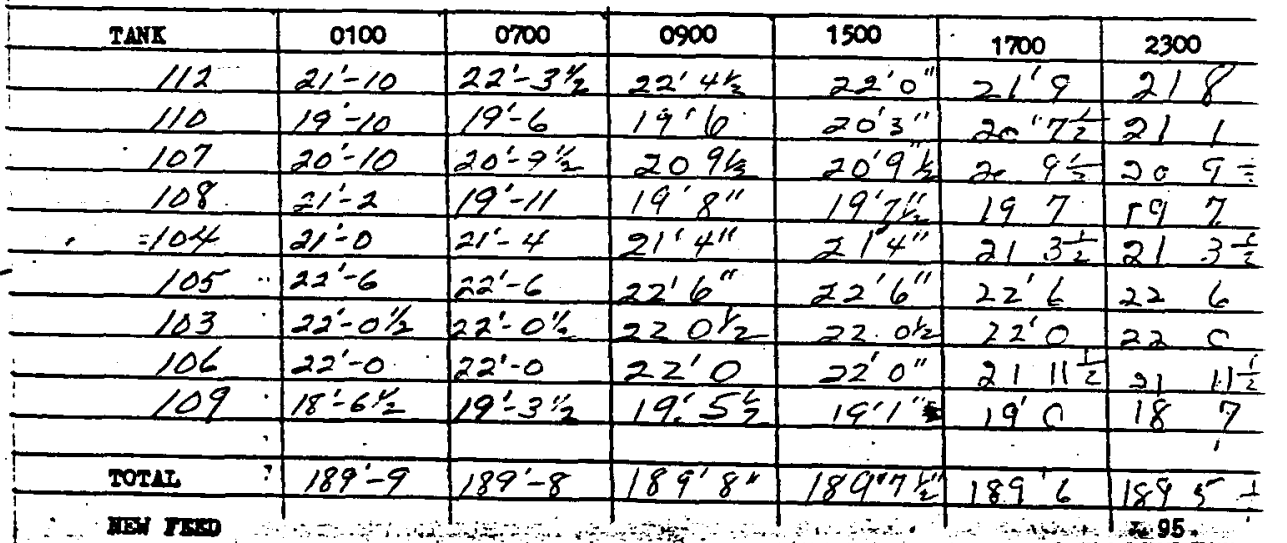




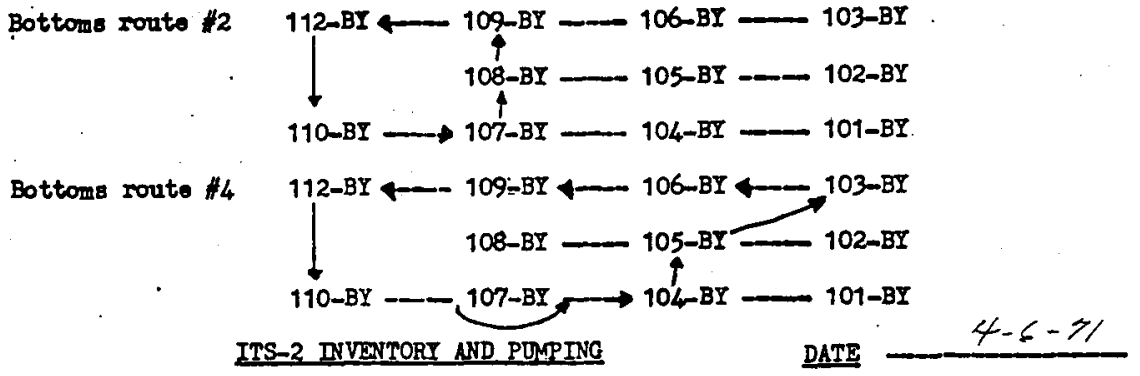

\begin{tabular}{|c|c|c|c|c|c|c|c|c|c|}
\hline 112-BI & $T A M$ & $\begin{array}{l}\text { I. I. } \\
\text { START }\end{array}$ & $\begin{array}{l}\text { I. L. } \\
\text { END }\end{array}$ & $\begin{array}{l}\text { POMP } \\
\text { AMPS }\end{array}$ & $110-B Y$ & $\begin{array}{l}\text { L. L. } \\
\text { START }\end{array}$ & L. L. & $\begin{array}{l}\text { POMP } \\
\text { OFP }\end{array}$ & REMARXS \\
\hline $1 / 2$ & 000 & 72.6 & $21 \%$ & 22. & 110 & $19 \%$ & $21 \% 1 / 2$ & 0421500 & choked \\
\hline & & & & & & & & & \\
\hline & & & & & & & & & \\
\hline $110-B Y$ & $\operatorname{Tng}$ & $\begin{array}{l}\text { L. L. } \\
\text { START }\end{array}$ & $\begin{array}{l}\text { L. L. L. } \\
\text { END }\end{array}$ & $\begin{array}{l}\text { POMP } \\
\text { AMPS }\end{array}$ & TANK & $\begin{array}{l}\text { L. L. } \\
\text { START }\end{array}$ & $\begin{array}{l}\text { L. L. } \\
\text { END }\end{array}$ & $\begin{array}{l}\text { PUMP } \\
\text { ORP }\end{array}$ & RPMARKS \\
\hline BATCH & 230 & $21 \frac{1}{2}$ & $19-11$ & & $10 / \overline{B Y}$ & $197 \frac{7}{2}$ & $x^{\prime}-2$ & 060 & Thenced $1 / 7 / 2$, \\
\hline BATCH 2 & & & & & $104-B I$ & & & & \\
\hline BATCH 3 & & & & & $107-8 I$ & & & & \\
\hline TAN & $\operatorname{Tng}$ & $\begin{array}{l}\text { L. L. } \\
\text { STLPI }\end{array}$ & LDD & $\begin{array}{l}\text { PUTP } \\
\text { APS }\end{array}$ & TARK & I. I. I. & $\begin{array}{l}\text { L. I. } \\
\text { END }\end{array}$ & $\begin{array}{l}\text { FuF } \\
\text { orf }\end{array}$ & REMURES \\
\hline$\angle \Delta Z$ & $06+0$ & $2=-2$ & $19^{\prime} 72$ & 15 & 109 & $17-11 \%$ & $19^{\prime} 4$ & 224.5 & ज"tudiod \\
\hline$j$ & & & & & & & & & \\
\hline & & . & & & & & & & \\
\hline & & & & & & & & & \\
\hline & & & & & & & & $\therefore$ & \\
\hline & & & & & & & & $\cdot$ & \\
\hline
\end{tabular}

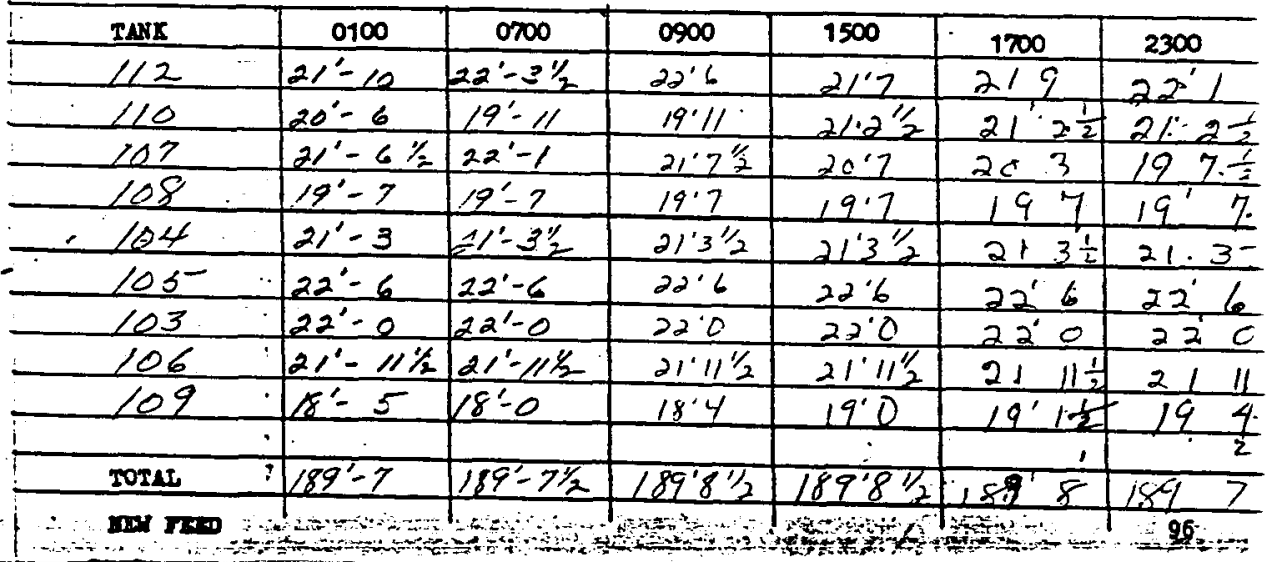




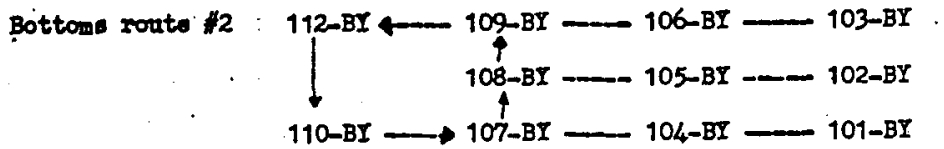

Bottoms route $: 4 \quad 112-\mathrm{BI}+109 \mathrm{BI} \rightarrow 106-\mathrm{BI}+10 \mathrm{BI}$

ITS-2 INVENTORY AND PUAPING

DATE $-4-7-y /$

\begin{tabular}{|c|c|c|c|c|c|c|c|c|c|}
\hline 112-BI & TIMS & $\begin{array}{l}\text { L. L. } \\
\text { START }\end{array}$ & $\begin{array}{l}\text { I. I. . } \\
\text { aND }\end{array}$ & $\begin{array}{l}\text { POMP } \\
\text { MMPS }\end{array}$ & $110-\mathrm{BY}$ & $\begin{array}{l}\text { L. L. } \\
\text { START }\end{array}$ & $\begin{array}{l}\text { L. I. } \\
\text { BND }\end{array}$ & $\begin{array}{l}\text { PURP } \\
\text { OFT }\end{array}$ & REMARKS \\
\hline$\angle 12$ & 0500 & $22 \div 6$ & 2116 & 23 & 110 & $19 \div 10$ & 2192 & $12: 00$ & LEs Let \\
\hline & & & & & & & & & \\
\hline & & & & & & & & & \\
\hline $110-B I$ & TDE. & $\begin{array}{l}\text { I. I. } \\
\text { START }\end{array}$ & $\begin{array}{l}\text { L. L. } \\
\text { END }\end{array}$ & $\begin{array}{l}\text { PONP } \\
\text { MAPS }\end{array}$ & TANK & $\begin{array}{l}\text { L. L. } \\
\text { START }\end{array}$ & $\begin{array}{l}\text { L. L. } \\
\text { END }\end{array}$ & $\begin{array}{l}\text { PUP } \\
\text { OEF }\end{array}$ & RDMARKS \\
\hline BATCH 1 & 1225 & 2/'Zs & $1910 x$ & 24 & $104-B I$ & $213 \frac{1}{2}$ & $2 z^{\prime} K^{\prime \prime}$ & 1730 & Flusteed \\
\hline BATCH 2 & & & & & $104-\mathrm{BI}$ & & & & \\
\hline BATCH 3 & & & & & 107-BI & & & & \\
\hline TANK & TIMB & $\begin{array}{l}\text { L. L. } \\
\text { START }\end{array}$ & $\begin{array}{l}\text { L. L. } \\
\text { EVD }\end{array}$ & $\begin{array}{l}\text { PUTP } \\
\text { MPS }\end{array}$ & TAKX & $\begin{array}{l}\text { L. I. } \\
\text { START }\end{array}$ & $\begin{array}{l}\text { L. } \\
\text { Exp }\end{array}$ & $\begin{array}{l}\text { FURP } \\
\text { OFF }\end{array}$ & RDMARKS \\
\hline 106 & 1630 & $2111 \frac{1}{2}$ & $69-6$ & 24 & 109 & $\angle E^{\prime}{ }^{\prime}$ & $19^{\prime}-1$ & 0230 & Sucle $2,4-2-26$ \\
\hline & & & & & & & & & \\
\hline & & & & & & & & & \\
\hline & & & & & & & & & \\
\hline & & & & & & & & & \\
\hline & & & & & & & & & \\
\hline
\end{tabular}

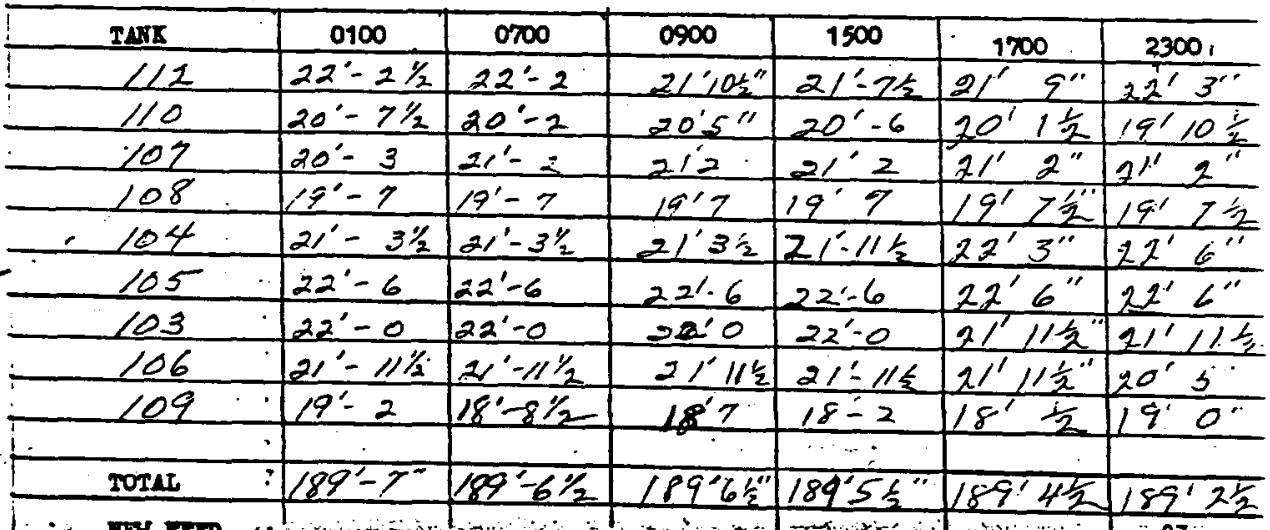



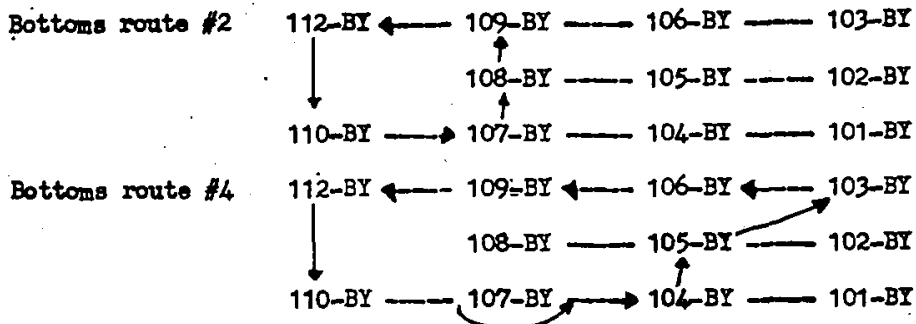

ITS=2 INVENTORY AND PIMPING

DATE $4-8-Z 1$

\begin{tabular}{|c|c|c|c|c|c|c|c|c|c|}
\hline $112-B I$ & TIMB & $\begin{array}{l}\text { L. L. } \\
\text { START }\end{array}$ & $\begin{array}{l}\text { L. L. } \\
\text { aND }\end{array}$ & $\begin{array}{l}\text { POMP } \\
\text { AMPS }\end{array}$ & 110-BI & $\begin{array}{l}\text { L. L. } \\
\text { START }\end{array}$ & $\begin{array}{l}\text { L. I. } \\
\text { DSA }\end{array}$ & $\begin{array}{l}\text { Parp } \\
\text { OrF }\end{array}$ & RIMARKS \\
\hline & $21 / 0$ & 226 & & $I$ & . & $20^{\prime} /{ }^{\prime \prime}$ & $21 \div 5$ & 0645 & 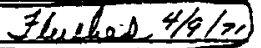 \\
\hline & & & & & & & & & \\
\hline $110-B I$ & TDR & $\begin{array}{l}\text { L. I. } \\
\text { STARS }\end{array}$ & $\begin{array}{l}\text { I. L. } \\
\text { END }\end{array}$ & $\begin{array}{l}\text { PQMP } \\
\text { APS }\end{array}$ & TANK & $\begin{array}{l}\text { L. L. } \\
\text { START }\end{array}$ & $\begin{array}{l}\text { L. I. } \\
\text { BND }\end{array}$ & $\begin{array}{l}\text { POMP } \\
\text { OF\% }\end{array}$ & RMMARKS \\
\hline BATCH 1 & & & & & 104-BY & & & & \\
\hline BATCH 2 & & & & & 104-BI & & & & \\
\hline BATCE 3 & 1715 & $2 I^{\prime \prime \prime}$ & 19195 & & $107-B I$ & $1 / 6^{\prime \prime}$ & $2010 \stackrel{1}{\sim}$ & 2220 & floshed \\
\hline TANK & TMM & $\begin{array}{l}\text { L. L. } \\
\text { STAPr }\end{array}$ & L. L. & $\begin{array}{l}\text { PUPP } \\
\text { MPS }\end{array}$ & $\max$ & $\begin{array}{l}\text { Lo Lo: } \\
\text { STARA }\end{array}$ & Lid ${ }^{2}$ & $\begin{array}{l}\text { Pupr } \\
\text { ort }\end{array}$ & REALARTS \\
\hline 103 & 0230 & $21-11 \%$ & $201 \frac{1}{2}$ & 26 & 106 & $19 \div 6$ & $211 / \frac{1}{2}$ & 1.515 & Flusked \\
\hline 107 & 2300 & $21-2$ & $19^{\prime} 6^{\prime r}$ & 15 & 108 & $19 \div 2$ & $213^{\prime \prime}$ & 1030 & Thenes \\
\hline $1 / 2$ & 0330 & $32^{\prime}-6$ & $2 l^{\prime} z^{\prime \prime}$ & 23. & 110 & $19 \div 10$ & $20 \% 1 \frac{1}{2}$ & 1000 & 2 ands \\
\hline 105 & 1300 & 2216 & $20^{\prime} 2^{\prime \prime}$ & 26 & 103 & $20^{\prime} \geq \%$ & $2 / 10$ & 1100 & flushed \\
\hline 106 & 2100 & $21^{\prime} 11 \frac{1}{2}$ & $20: 1 \%$ & 24 & 105 & $18^{\prime} 2^{\prime \prime}$ & & $0<50$ & Flush 0 \\
\hline
\end{tabular}

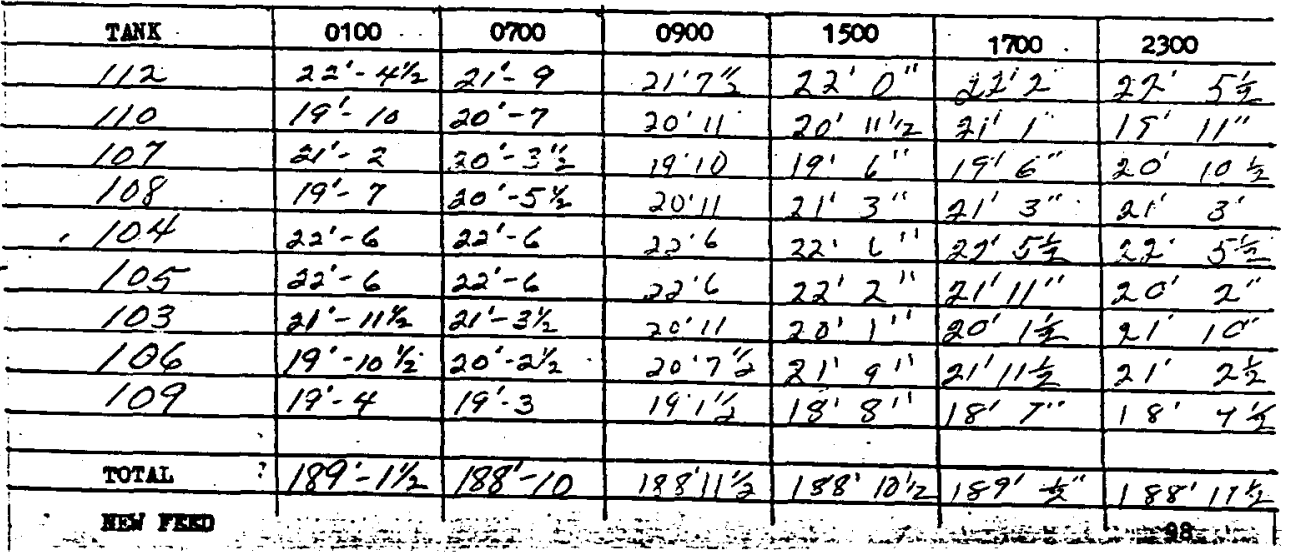




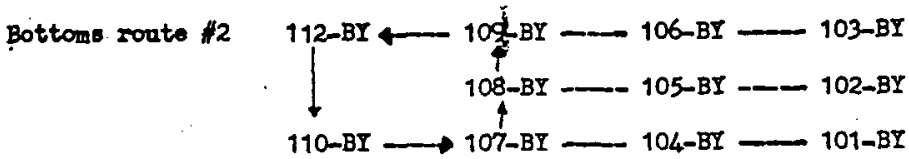

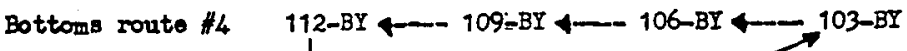

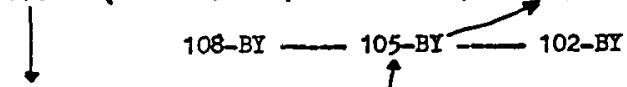

$110-\mathrm{BY}=107-\mathrm{BY} \longrightarrow 104-\mathrm{BY} \longrightarrow 101-\mathrm{BY}$

ITS-2 DNVDTORY AND PUMPING

DATE

\begin{tabular}{|c|c|c|c|c|c|c|c|c|c|}
\hline 112-BI & TIMB & $\begin{array}{l}\text { L. L. } \\
\text { START }\end{array}$ & $\begin{array}{l}\text { L. L. } \\
\text { END }\end{array}$ & $\begin{array}{l}\text { POMP } \\
\text { AMPS }\end{array}$ & 110-BI & $\begin{array}{l}\text { L. L: } \\
\text { START }\end{array}$ & $\begin{array}{l}\text { L. } L . \\
\text { BND }\end{array}$ & $\begin{array}{l}\text { PLMP } \\
\text { OFP }\end{array}$ & RDMARKS \\
\hline & & & & & & & & & \\
\hline & & & & & & & & & \\
\hline $110-B I$ & $\operatorname{TDMS}$ & $\begin{array}{l}\text { I. I. } \\
\text { STARE }\end{array}$ & $\begin{array}{l}\text { L. L. } \\
\text { DND }\end{array}$ & $\begin{array}{l}\text { POMP } \\
\text { AMPS }\end{array}$ & TANK & $\begin{array}{l}\text { L. L. } \\
\text { START }\end{array}$ & $\begin{array}{l}\text { L. L. } \\
\text { BND }\end{array}$ & $\begin{array}{l}\text { PUMP } \\
\text { OFP }\end{array}$ & RPMARKS \\
\hline BATCH 1 & squer & 2,5 & $19^{\prime} 10^{\prime \prime}$ & & $104-\mathrm{BI}$ & $20 \div \frac{7}{2}$ & $22^{\prime} 0^{\prime \prime}$ & 1615 & flushod \\
\hline BATCH 2 & & & & & $104-B I$ & & & & \\
\hline BATCH 3 & & & & & $107-\mathrm{BI}$ & & & & \\
\hline TANX & TDME & $\begin{array}{l}L_{0} L_{0} \\
\text { STARE }\end{array}$ & \begin{tabular}{ll|} 
L. L० \\
$\mathrm{END}$
\end{tabular} & $\begin{array}{l}\text { PUMP } \\
\text { AMPS }\end{array}$ & TANX & $\begin{array}{l}\text { L. L: } \\
\text { START }\end{array}$ & Bid & $\begin{array}{l}\text { PUNA } \\
\text { OFP }\end{array}$ & REYARKS \\
\hline$\angle 03$ & $0 / 55$ & $21-10 / 2$ & $20^{\prime}-0 \%$ & $2-$ & 106 & $20: 1 \%$ & $22^{\circ}-0$ & 0650 & Fluche \\
\hline 104 & 0200 & $22^{\prime}-5 / 2$ & $20 \div \frac{1}{2}$ & 26 & 105 & $20^{\prime}-1$ & $22^{\prime} 14$ & c 910 & the+ked \\
\hline 10.5 & cian & $22^{\prime}, \frac{1}{2}$ & $20^{\prime} \prime 3^{\prime \prime}$ & & 103 & $20^{\prime} \circ \frac{7}{2}$ & $27^{\prime} 0^{\prime}$ & 16,5 & Eloshed \\
\hline & & & & & & & & & \\
\hline & & & & & & & & & \\
\hline$i$ & & & & & & & & & $\cdot$ \\
\hline
\end{tabular}

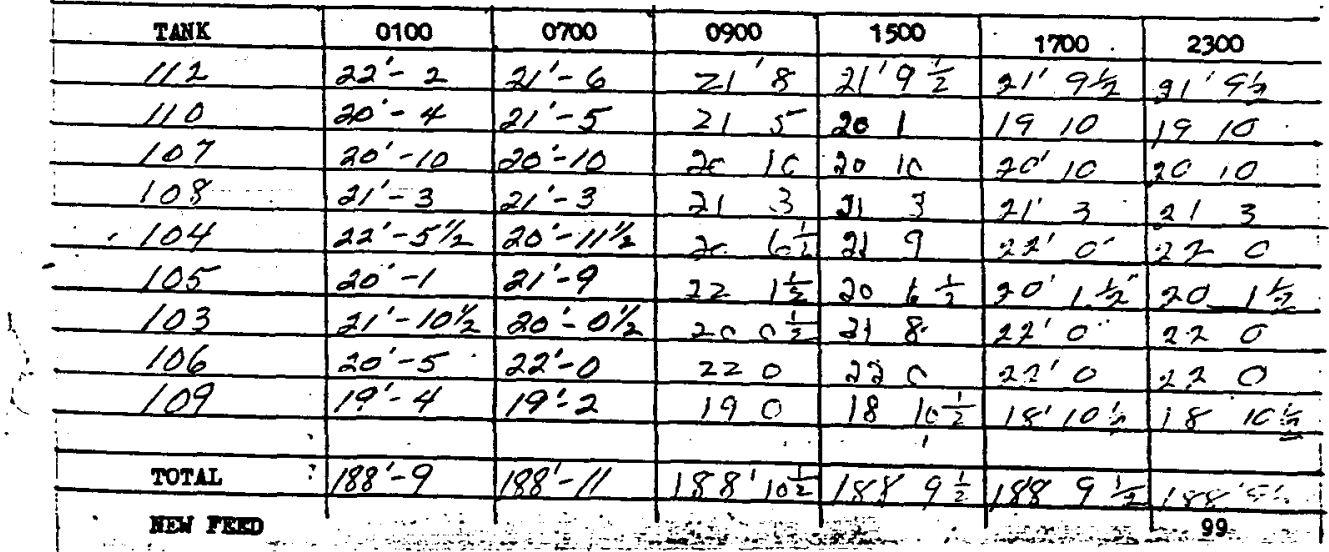


Bottons route /2

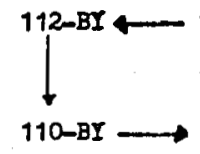

Bottoms route \#4

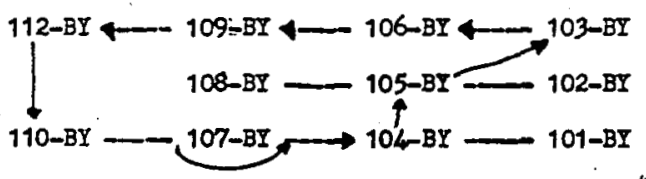
ITS-2 DIVENTORY AND POMPTNG

\begin{tabular}{|c|c|c|c|c|c|c|c|c|c|}
\hline 112-BI & IDMS & $\begin{array}{l}\text { L. L. } \\
\text { START }\end{array}$ & $\begin{array}{l}\text { L. L. } \\
\text { END }\end{array}$ & $\begin{array}{l}\text { POMP } \\
\text { MMPS }\end{array}$ & $110-B Y$ & $\begin{array}{l}\text { L. L. } \\
\text { START }\end{array}$ & $\begin{array}{l}\text { L. L. } \\
\text { BND }\end{array}$ & $\begin{array}{l}\text { POMP } \\
\text { OFP }\end{array}$ & REMARKS \\
\hline & & & & & & & & & \\
\hline & & & & & & & & & \\
\hline & & & & & & & & & \\
\hline $110-8 I$ & TAR & $\begin{array}{l}\text { L. Ins } \\
\text { STARS }\end{array}$ & E. L. & $\begin{array}{l}\text { POMP } \\
\text { AMPS }\end{array}$ & TANX & $\begin{array}{l}\text { L. L.: } \\
\text { START }\end{array}$ & L. $L$. & $\begin{array}{l}\text { POMP } \\
\text { OFP }\end{array}$ & REMARIKS \\
\hline BATCR 1 & & & & & 104 EI & & & & \\
\hline BATCH 2 & & & & & $104-B I$ & & & & \\
\hline BATCE 3 & & & & & $107-\mathrm{BI}$ & & & & \\
\hline TANE & $\operatorname{IDE}$ & $\begin{array}{ll}\text { L. Lo } \\
\text { STLPT }\end{array}$ & ${ }_{\text {B. }}^{\text {L. }}$ & $\begin{array}{l}\text { PUTP } \\
\text { DPPS }\end{array}$ & TLIS & STLE: & D. L. & $\begin{array}{l}\text { TUGF } \\
\text { OFT }\end{array}$ & REMARES \\
\hline 16.5 & c9en & $20^{\prime} 1 \frac{1}{2}$ & 19.5 & & 103 & 220 & 2115 & 1110 & Hecoshed \\
\hline 10.3 & $\Delta \varepsilon e 0$ & $22^{\prime} 0$ & 21《光 & & 106 & $22^{\prime} 0$ & $22^{\prime} 0$ & 210 & Zfendel' \\
\hline $\log 6$ & $c q<x$ & $22^{\circ} \mathrm{C}$ & $22^{\prime} \mathrm{c}$ & & 109 & $18^{\prime} 10^{\frac{1}{2}}$ & $196 \frac{1}{2}$ & 1110 & Ileseld \\
\hline Inot & 1300 & $198 / 0$ & 1925 & & 104 & $22^{\prime} \mathrm{O}$ & 2.214 & $14+5$ & Theothe \\
\hline 167 & 130 & $2 c^{\prime} 10$ & $20^{\prime} 2^{\circ}$ & & 108 & $21^{\prime} 3$ & $37^{\prime} c^{\prime \prime}$ & 160 & Flished \\
\hline & & & & & & & & & \\
\hline
\end{tabular}

\begin{tabular}{|c|c|c|c|c|c|c|}
\hline TAIK & 0100 & 0700 & 0900 & 1500 & $\therefore 1700$ & 2300 \\
\hline$\angle 12$ & $2119 \frac{1}{2}$ & 2195 & $21^{\prime} 2 \frac{1}{2}$ & $21^{\prime} 9 \frac{7}{2}$ & $2^{\prime} 9^{\prime}$ & $21^{\prime} \varepsilon \frac{1}{2}$ \\
\hline 110 & $14^{\prime} 10^{\prime \prime}$ & $19^{\prime} 10^{\circ}$ & $19^{\prime} 10^{\circ}$ & $196 \frac{1}{2}$ & $19^{\prime} 5 \frac{1}{2}$ & $\angle 5^{\prime} 5^{\prime} \frac{1}{2}$ \\
\hline 107 & $20^{\prime} \cdot 10^{\prime \prime}$ & $\pm 0^{\prime} 10^{\prime \prime}$ & $20^{\prime} 10$ & 2042 & $20^{\prime} 2^{\prime \prime}$ & $20^{\prime} 2^{\prime}$ \\
\hline 608 & $26^{\circ} 3$ & $21^{\prime} 3$ & $21^{\prime} 3$ & 21 & $22^{\prime} c$ & $22^{\prime} 0$ \\
\hline 104 & $22^{\prime} 0$ & $22^{\prime} 0$ & $22^{\prime} \mathrm{c}$ & 2214 & $22^{\prime} \quad 4$ & $22^{\prime} 4^{\prime}$ \\
\hline 105 & $20^{\circ} 115$ & $20^{\prime} 1 k_{s}$ & $20^{\prime}, \frac{1}{2}$ & 195 & $15^{\prime} \quad 5 \frac{1}{2}$ & $19^{\prime}$ \\
\hline$\angle 03$ & $22^{\prime} 0$ & $2 z$ & $22^{\prime} 0$ & $21 / 1 \frac{1}{2}$ & $21^{\prime} 1,2$ & $21^{\prime} 11 \frac{1}{2}$ \\
\hline 106 & 220 & 22 & 220 & $22^{\prime} 0$ & $22^{\prime} c$ & $22^{\prime} 0$ \\
\hline 107 & $\angle 810 \frac{1}{2}$ & 105 & $18^{\prime} 10^{7}=$ & $196 \frac{1}{2}$ & $19^{\prime} 6 \frac{1}{2}$ & $1 \dot{9}^{\prime} 6 \frac{1}{2}$ \\
\hline sorit & $188^{\prime} 95$ & $188^{\prime} 9 \frac{1}{2}$ & $188^{\prime} 8 \frac{1}{2}$ & 188 & $188^{\prime} 81$ & \\
\hline & & & & & & \\
\hline
\end{tabular}


Bottoms route $/ 2$

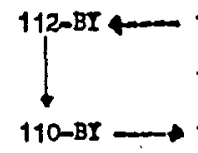

Bottoms route 4

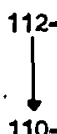

$109-12$<smiles>CC1(O)CCCCC1</smiles>

108-BY 4 107-BI 104-BI - 101-BI ITS-2 INVENTORT AND POMPING

108-8Y<smiles>[TeH2]</smiles>
104BY 101-BY

\begin{tabular}{|c|c|c|c|c|c|c|c|c|c|}
\hline 112-BY & $\operatorname{TAMS}$ & $\begin{array}{l}\text { I. I. } \\
\text { STARI }\end{array}$ & $\begin{array}{l}\text { L: L. } \\
\text { END }\end{array}$ & $\begin{array}{l}\text { POMP } \\
\text { AMPS } \\
\end{array}$ & $110-8 I$ & $\begin{array}{l}\text { L. L. } \\
\text { START }\end{array}$ & $\begin{array}{l}\text { L. L. } \\
\text { DND }\end{array}$ & $\begin{array}{l}\text { POAP } \\
\text { OFT }\end{array}$ & REMARKS \\
\hline & & & & & & & & & \\
\hline & & & & & & & & & \\
\hline & & & & & & & & & \\
\hline $110-B I$ & TDES & $\begin{array}{l}\text { I. L. } \\
\text { STARI }\end{array}$ & $\begin{array}{l}\text { L. I. } \\
\text { BND }\end{array}$ & $\begin{array}{l}\text { POMP } \\
\text { MMPS }\end{array}$ & TANK & $\begin{array}{l}\text { L. L. } \\
\text { START }\end{array}$ & $\begin{array}{l}\text { L. I. } \\
\text { END }\end{array}$ & $\begin{array}{l}\text { POMP } \\
\text { OFF }\end{array}$ & REMARKS \\
\hline \multicolumn{2}{|c|}{ BATCH $121+\infty$} & & & & 104-BI & & & & \\
\hline \multicolumn{2}{|c|}{ BATCH 2} & & & & 104-BI & & & & \\
\hline \multicolumn{2}{|l|}{ BATCH 3} & & & & 107-BI & & & & \\
\hline TANX & PDGE & $\begin{array}{l}\text { L. L. } \\
\text { START }\end{array}$ & Lid & $\begin{array}{l}\text { PASP } \\
\text { AMPS }\end{array}$ & TLAS & $\begin{array}{l}\text { L. L. } \\
\text { START }\end{array}$ & ${ }_{\text {EX }}^{1.0}$ & $\begin{array}{l}\text { PUTP } \\
\text { OST }\end{array}$ & ROPLARTS \\
\hline & & & & & & & & & \\
\hline & & & & & & & & & \\
\hline
\end{tabular}

.

TAMX

12

0100

(

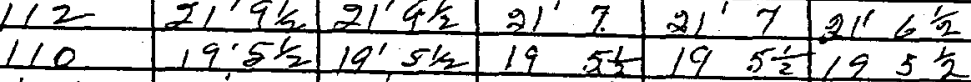

$107202 \quad 20 ;$ 20 $1 \frac{1}{2} 201 \frac{1}{2} 20^{\prime}+\frac{1}{2}$

108 . $22^{\prime} 0 \quad 22^{\prime} 0 \quad 2111 \frac{1}{2} 2111 \frac{1}{2} 22^{\prime} 0$

$r$ $+04$ $\partial 2^{\prime} 4$

\begin{tabular}{|c|c|c|c|c|}
\hline $\mathbf{0} 7 \mathbf{0}_{\gamma}$ & 0900 & 1500 & $\because 1700^{2}$ & 2300 \\
\hline $21^{\prime} 4 \sqrt{2}$ & $21^{\prime} 7$ & $21^{\prime} 7$ & $21^{\prime}<\frac{1}{2}$ & \\
\hline
\end{tabular}

105

$193 \frac{1}{2}$

$22^{\prime}$

$223 \div$

2

2

$9^{\prime} 5$

A

$103.2141 / 2195$

102

$2141 / 2$

$1+\frac{12}{2}$

21

2111

$121^{\prime} 4$

$19^{\prime} 5 \frac{1}{2}$

1.

109

20Tse

\begin{tabular}{|cc|cc}
22 & 0 & $92^{\prime}$ & 0 \\
14 & 65 & $14^{\circ}$ & $6 \%$
\end{tabular}

22

$6 \div$

$12^{\prime} 0$ 22'0

$19^{\prime} 6 !$

$19^{\prime} 6 \frac{1}{2}$

: $788 \frac{8}{2}$

$188^{19} / 218834$

$188^{\prime} 34 / 188^{\prime} 4 \frac{1}{2}$ 
PUMP OUT TANK

RECEIVING TANK

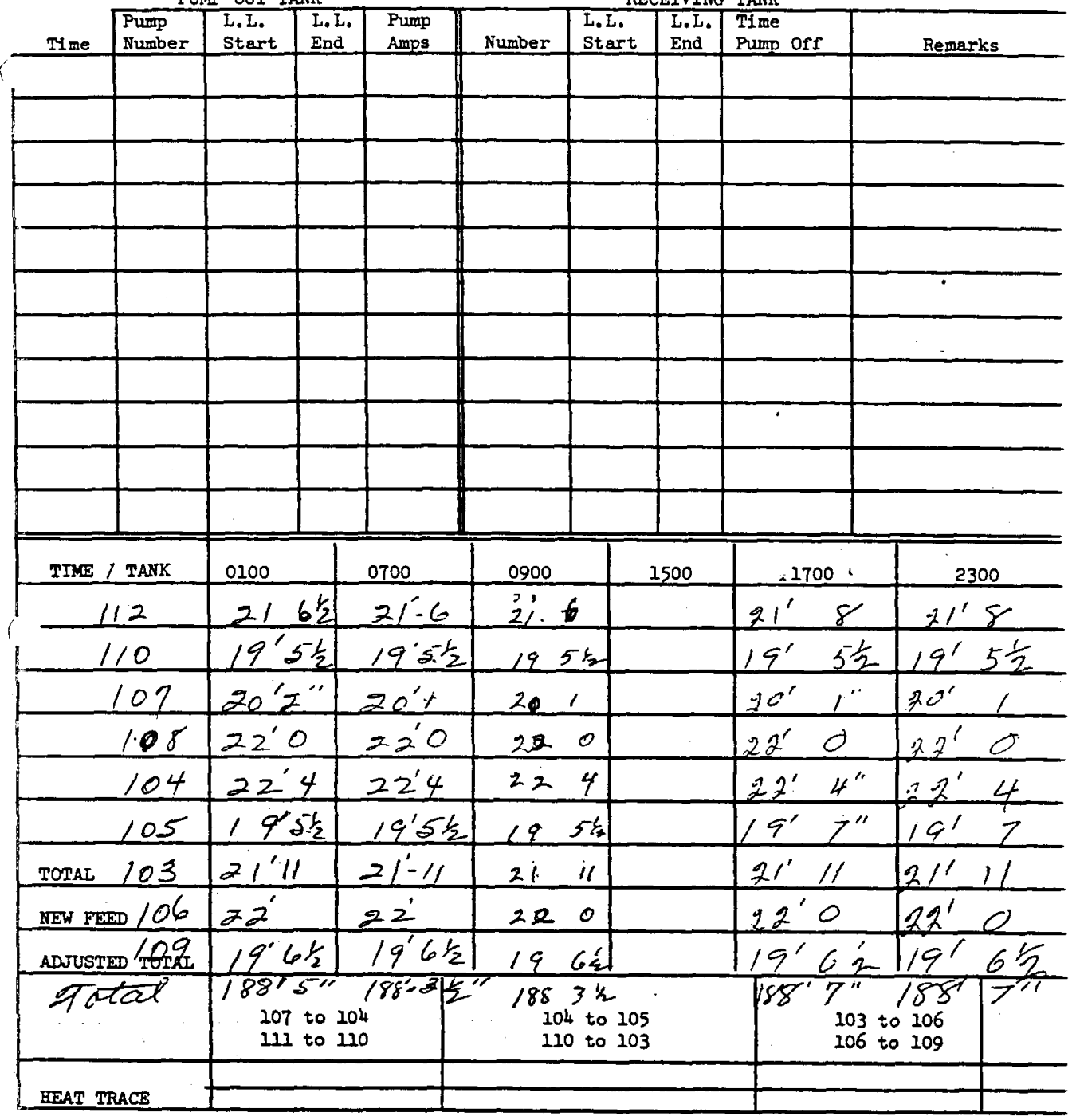

QI Walker:srb 9-29-70 
WHC-SD-WH-DP-209, ReV. 0

ITS-2 INVENTORY AND PUMPING

Date $4-13-71$

PUMP OUT TANK

RECEIVING TANK

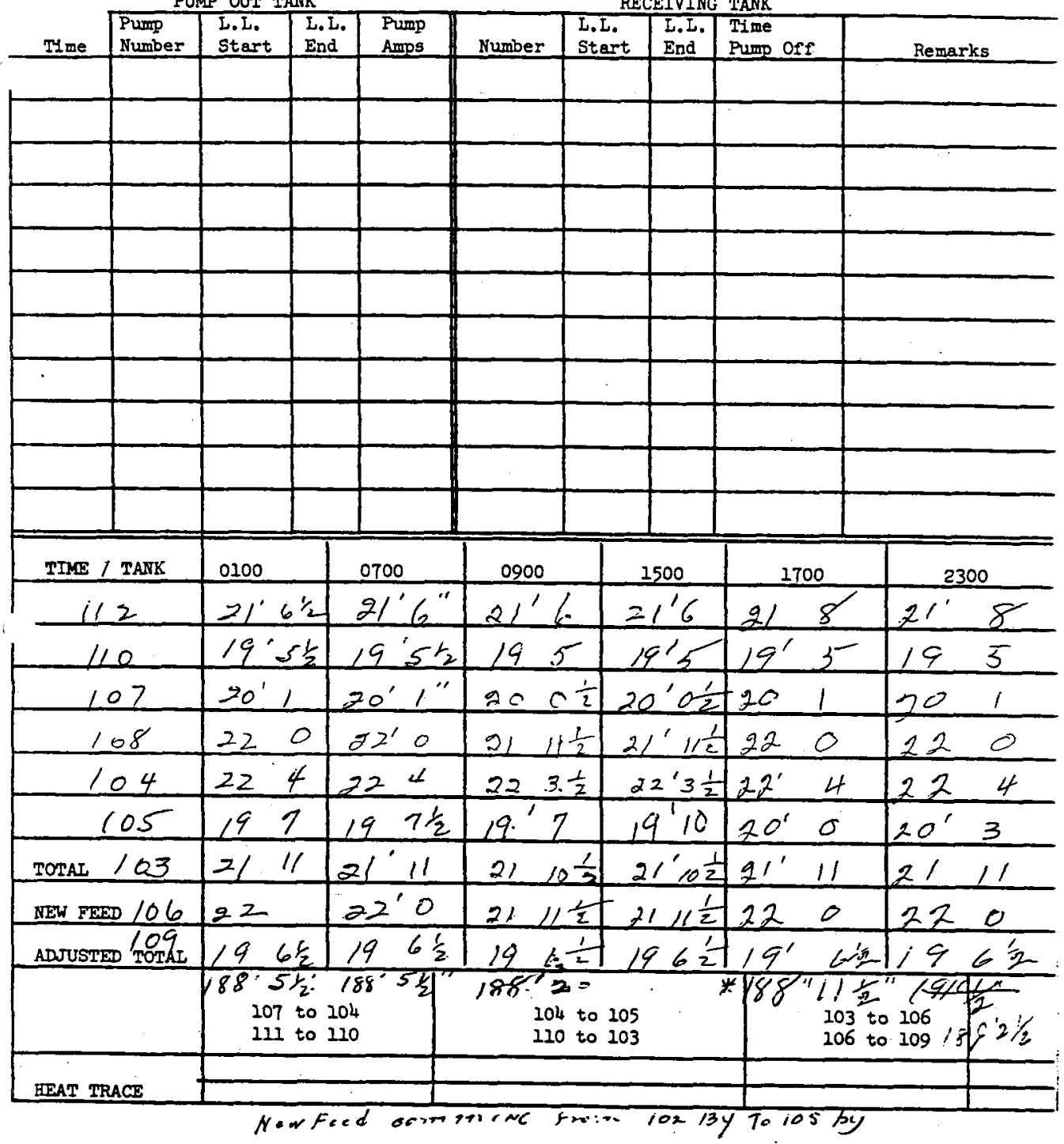

Qu Walker:srb 9-29-70

4. $1 / 2$

103 


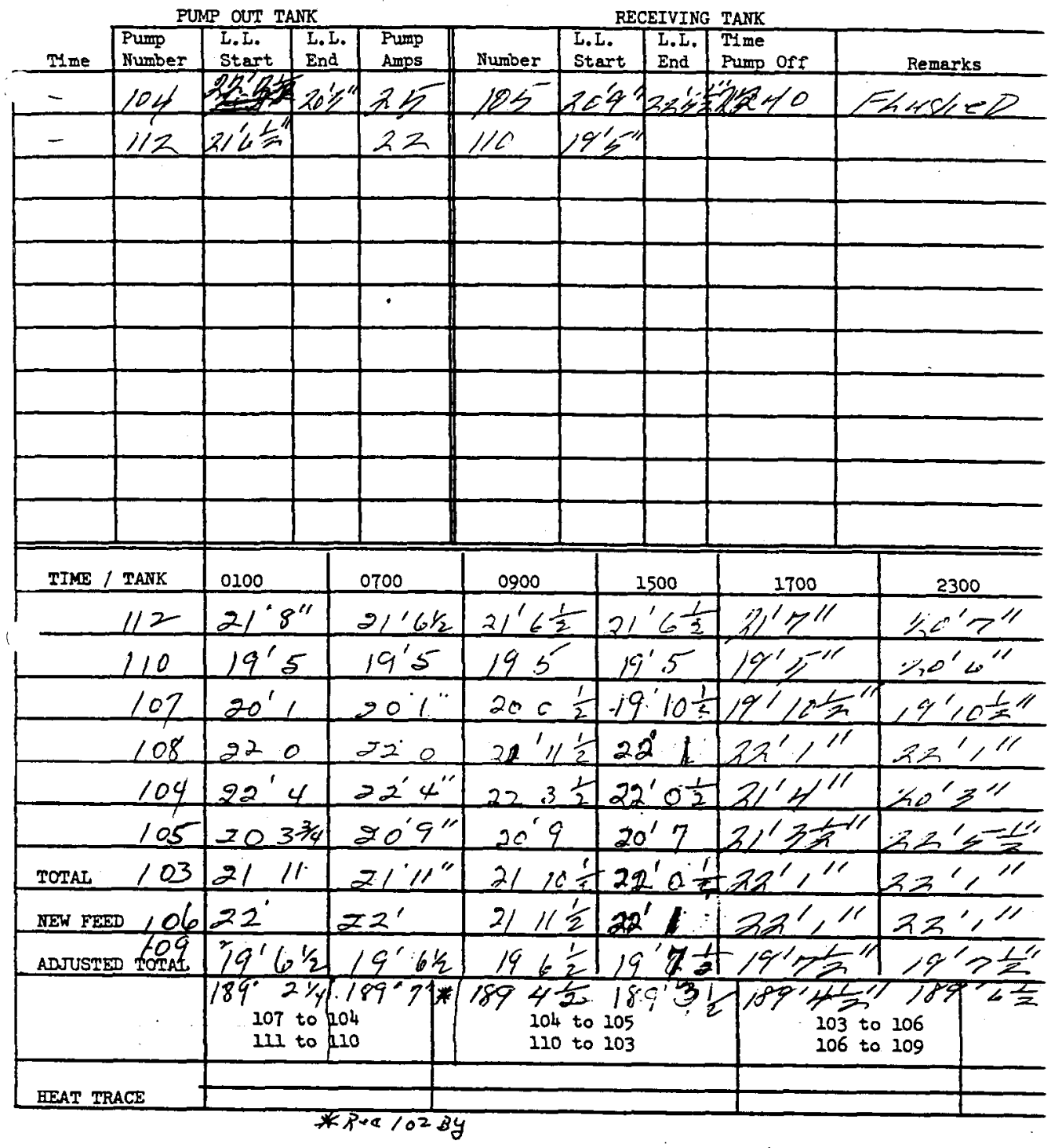

CM Walker:sIb 9-29-70 


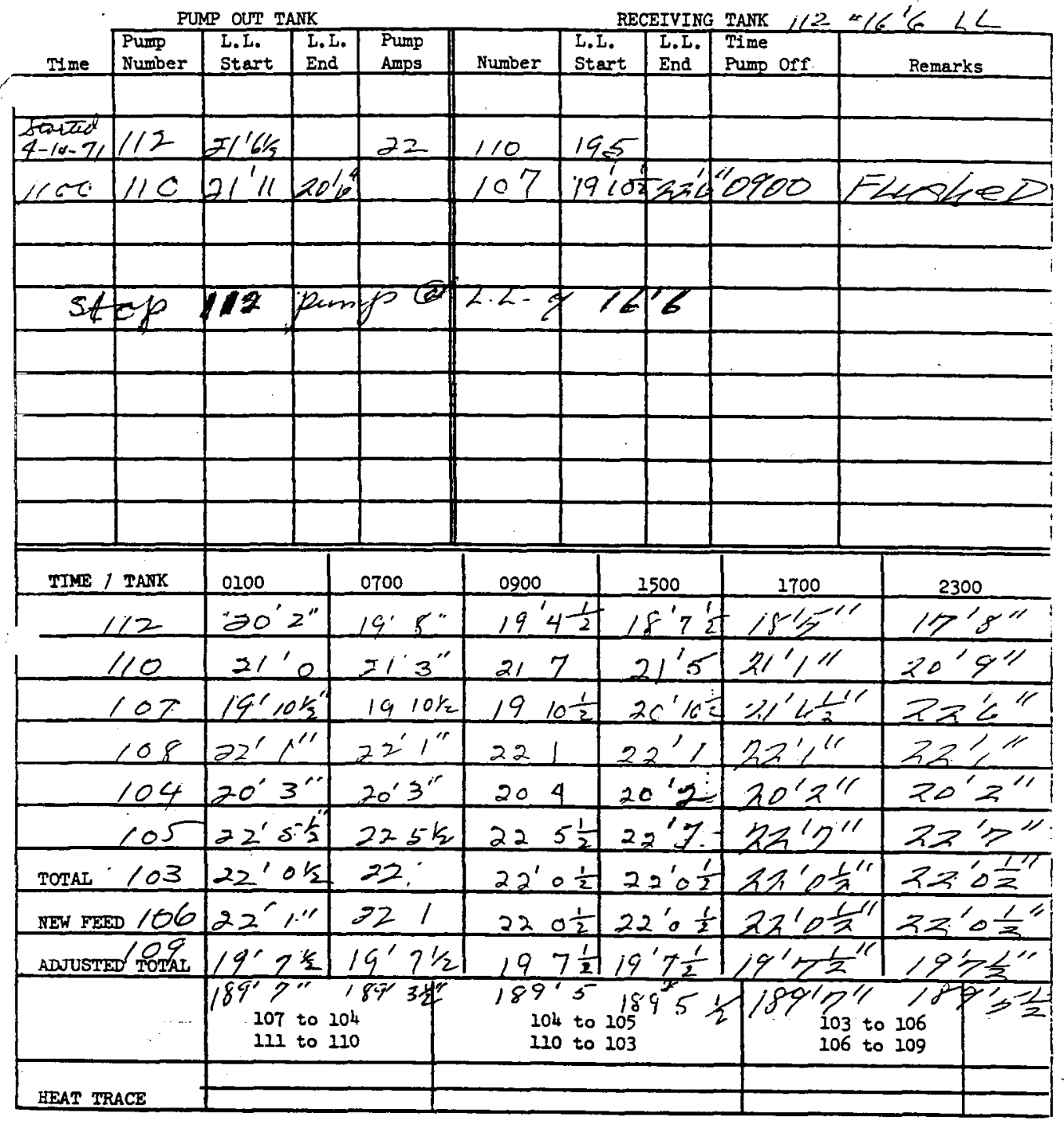

CA Walker:srb 9-29-70 
PUNP OUT TANK

RECETVING TAKK

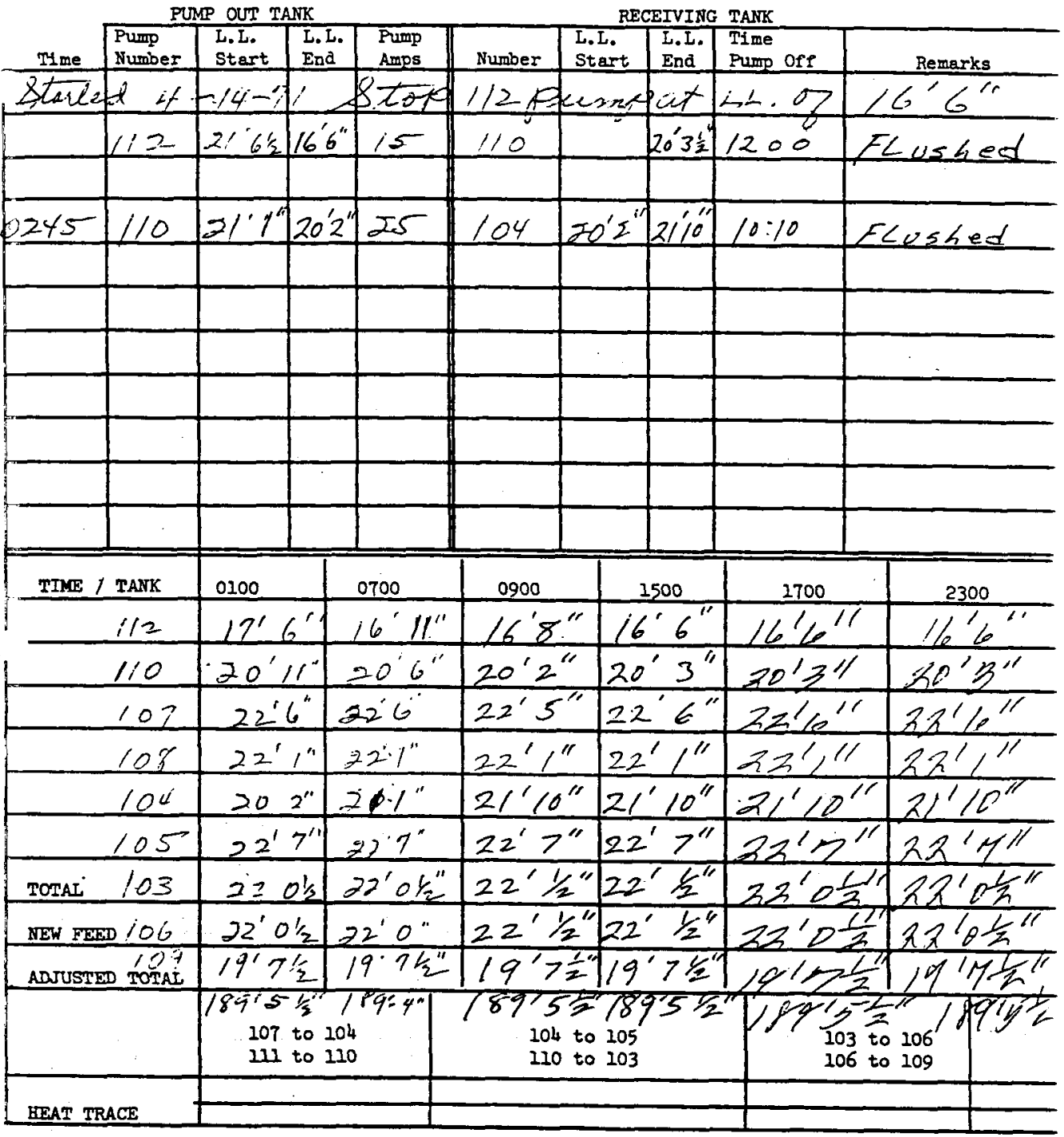

CM Walker:srb 9-29-70 
PUMP OUT TANK

RECEIVING TANK

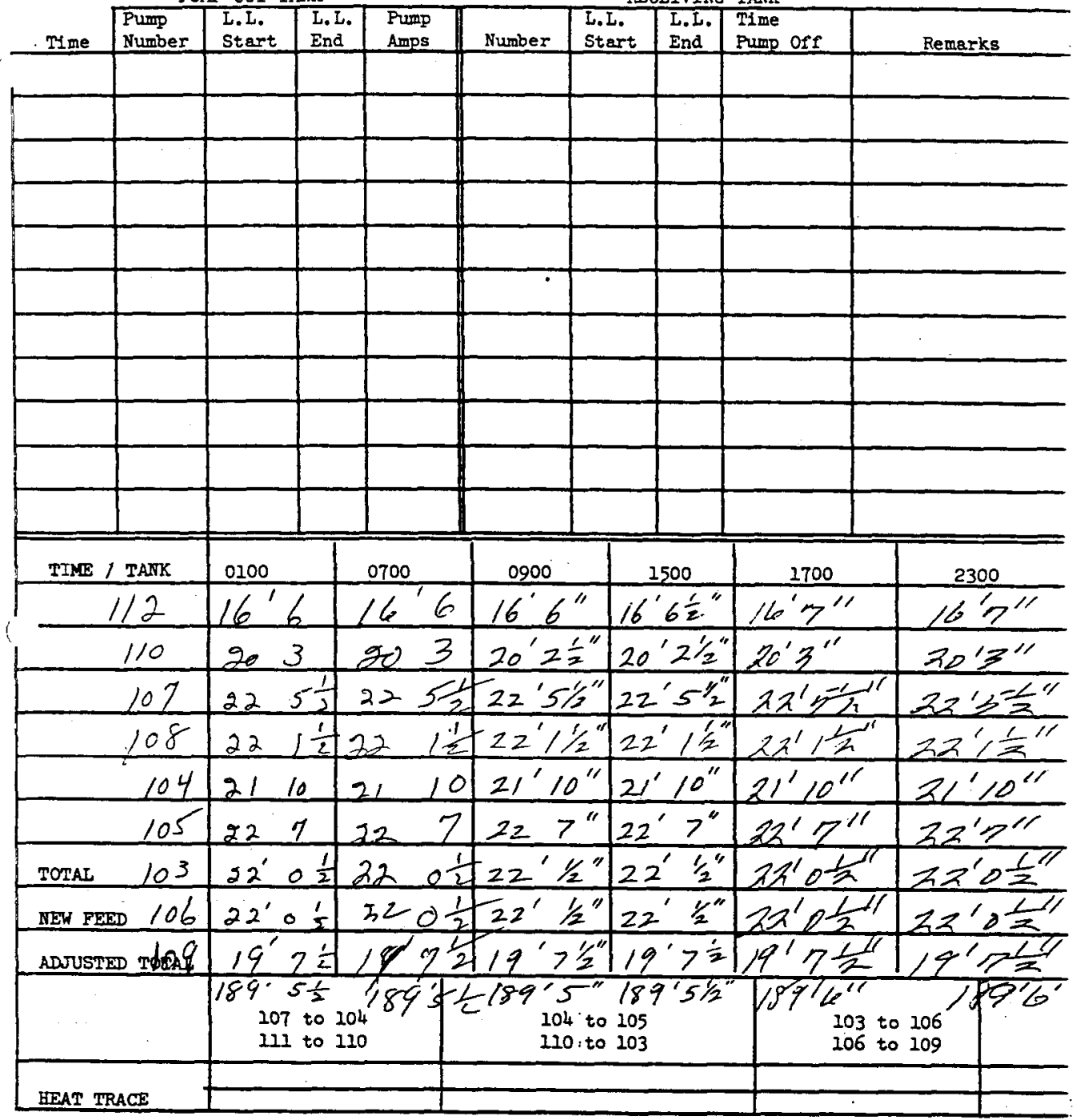


PUMP OUT TAMK

RECEIVING TANK

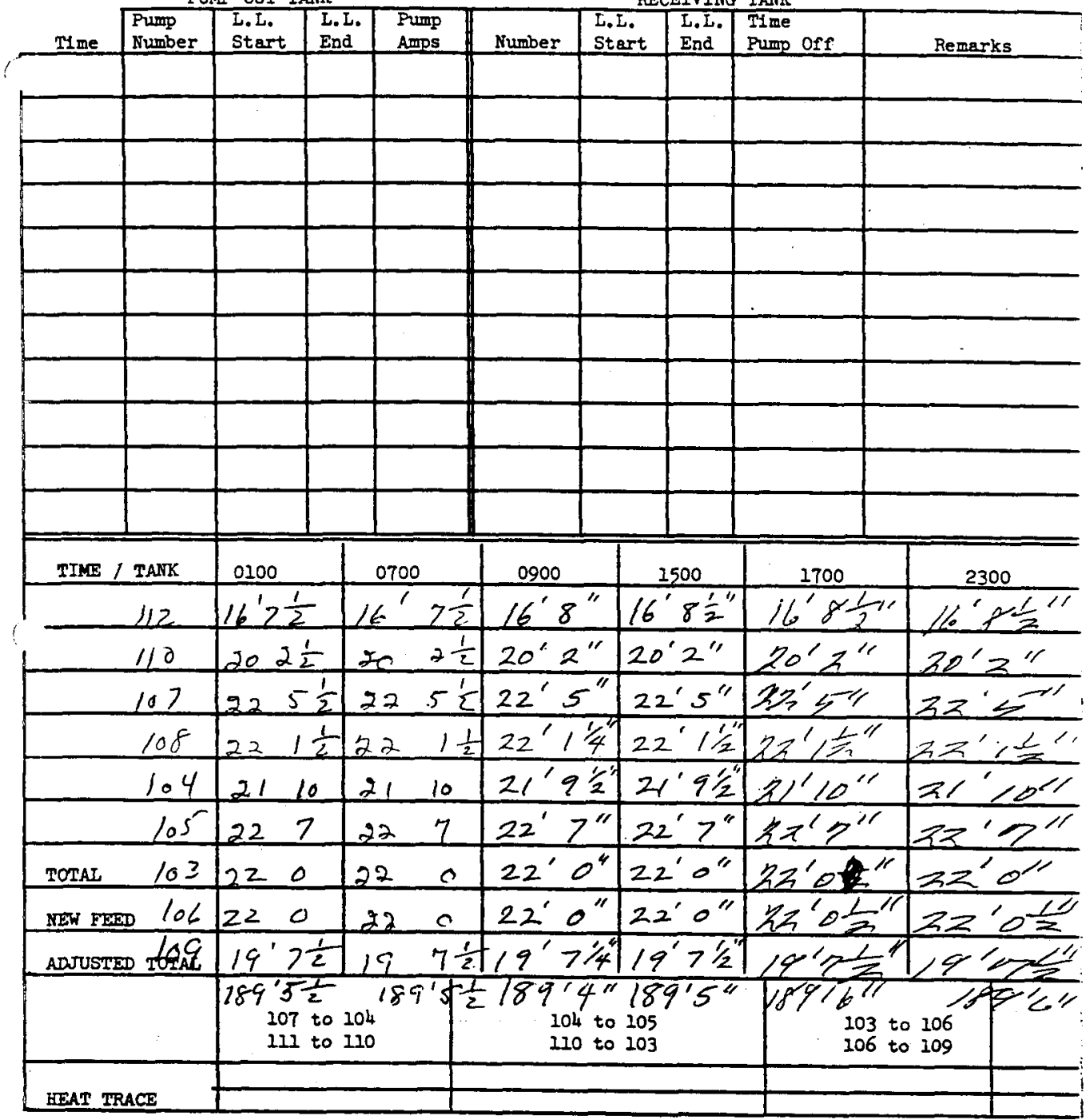

OM Walker:srb 9-29-70 
PUMP OUT TANK

RECEIVING TANK

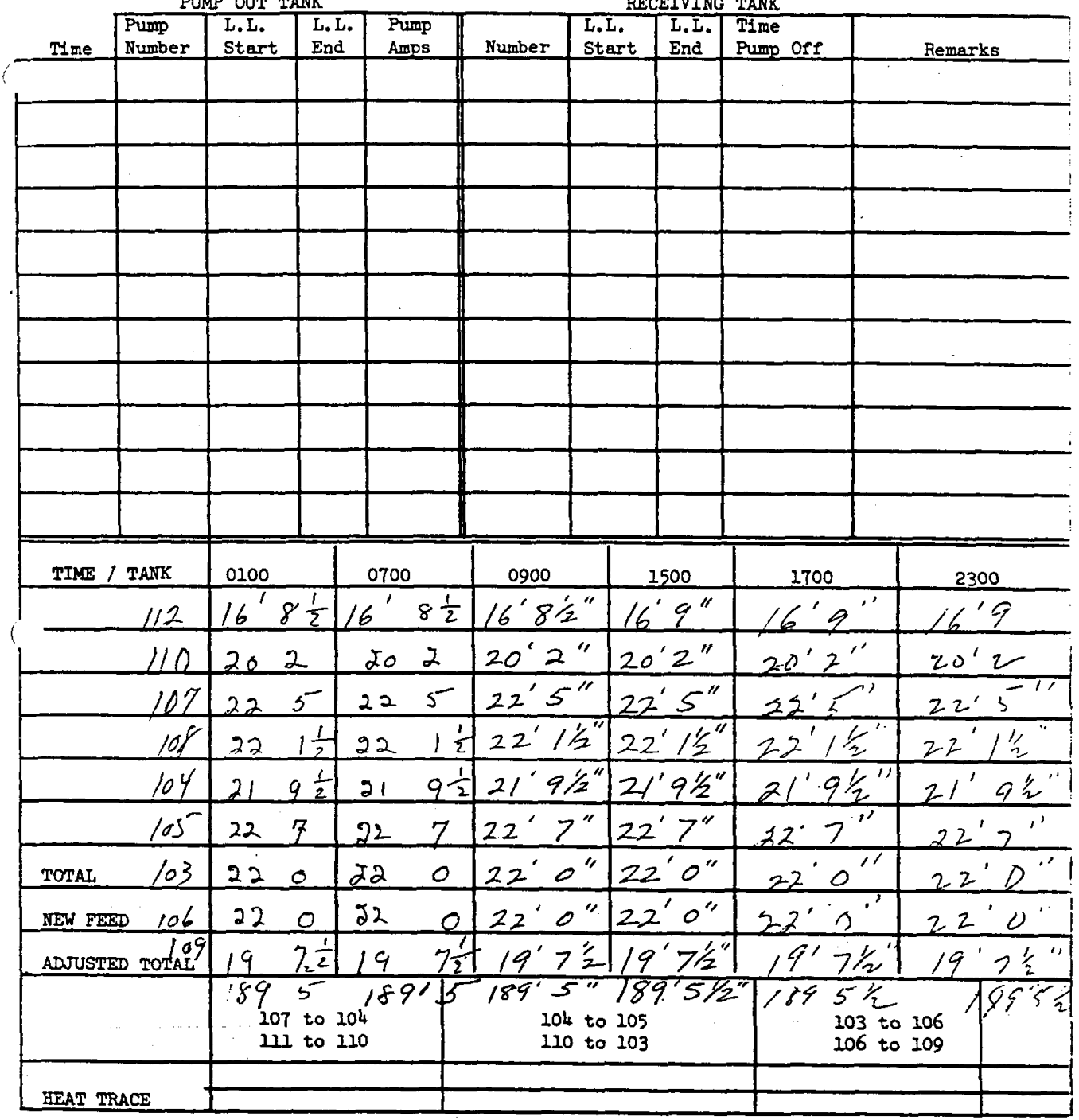

CM Walker:srb 9-29-70 
WHC-SD-LM-DP-209, ReV. D

ins

ITS-2 INVENTORY AND PUMPING

Date

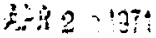

PUMP OUT TANK

RECEIVING TANK

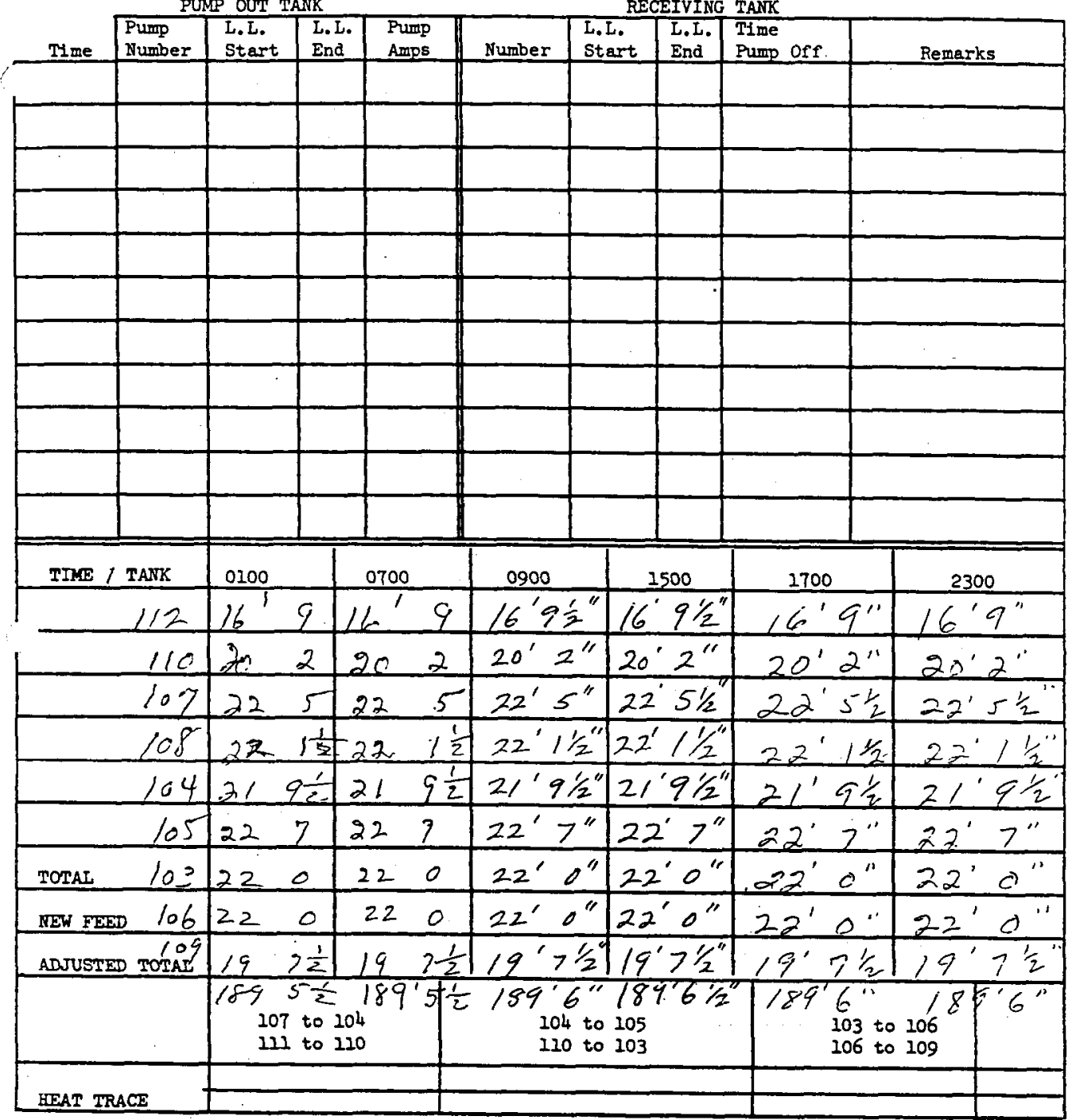

OM Wauker:srb 9-29-70 
PUMP OUT TANK

RECEIVING TANK

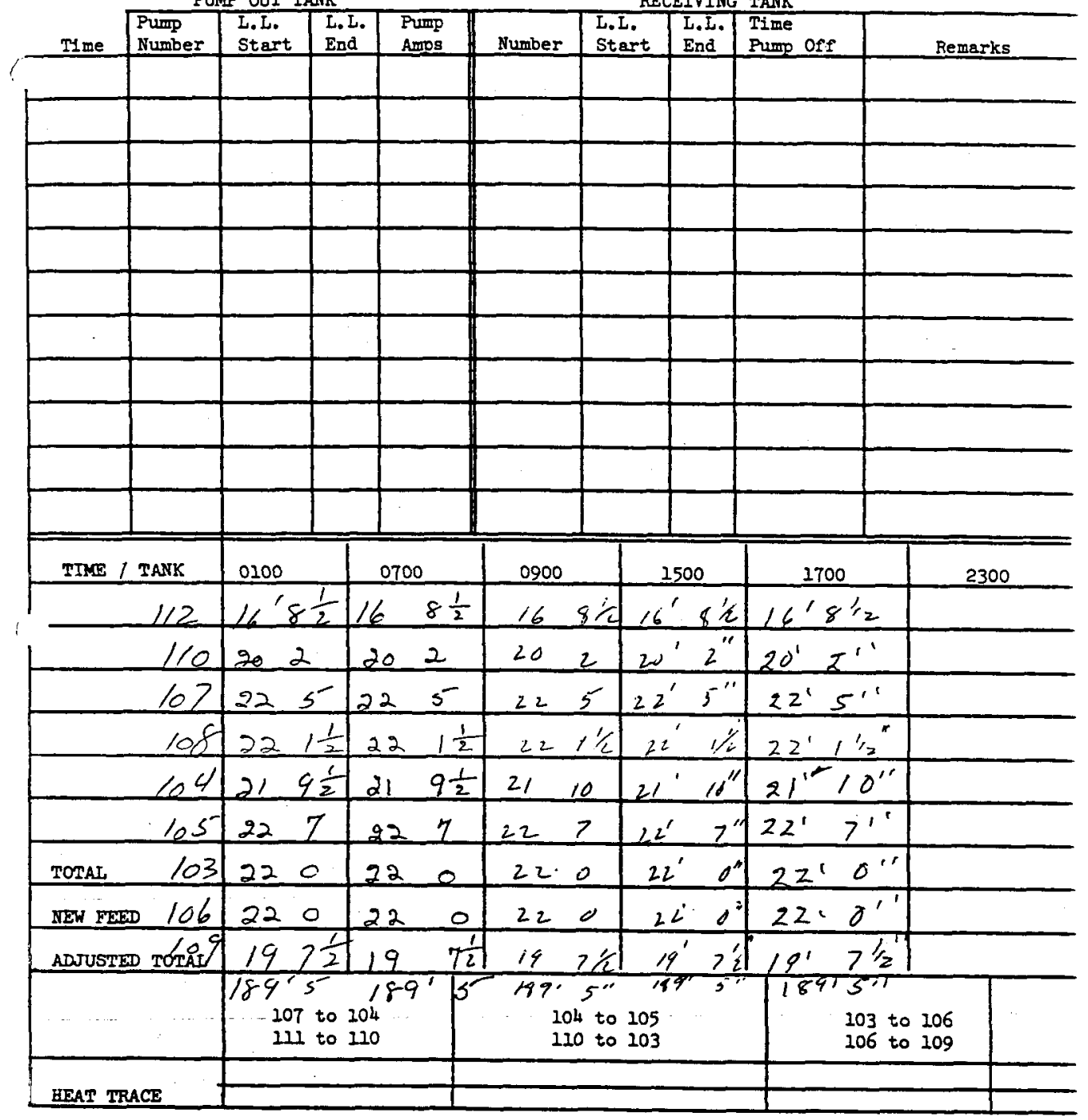

OM Wajker:s5b 9-29-70 


\section{ITS-2 INVENTORY AND PUMPING \\ Date APR 221971}

PUMP OUT TANK

RECEIVING TANK

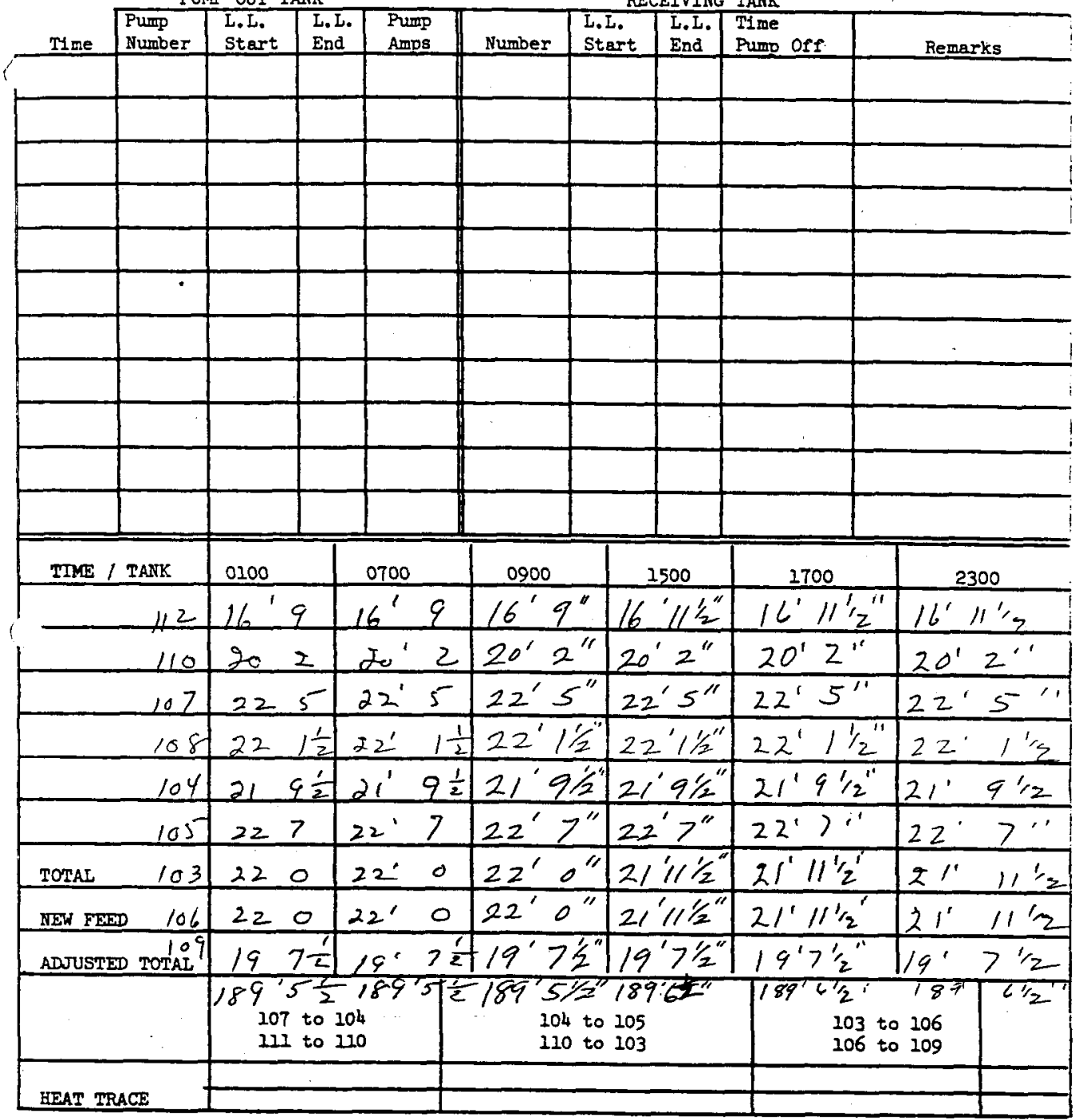

CM Walker:srb 9-29-70 
PUMP OUT TANK

RECEIVING TANK

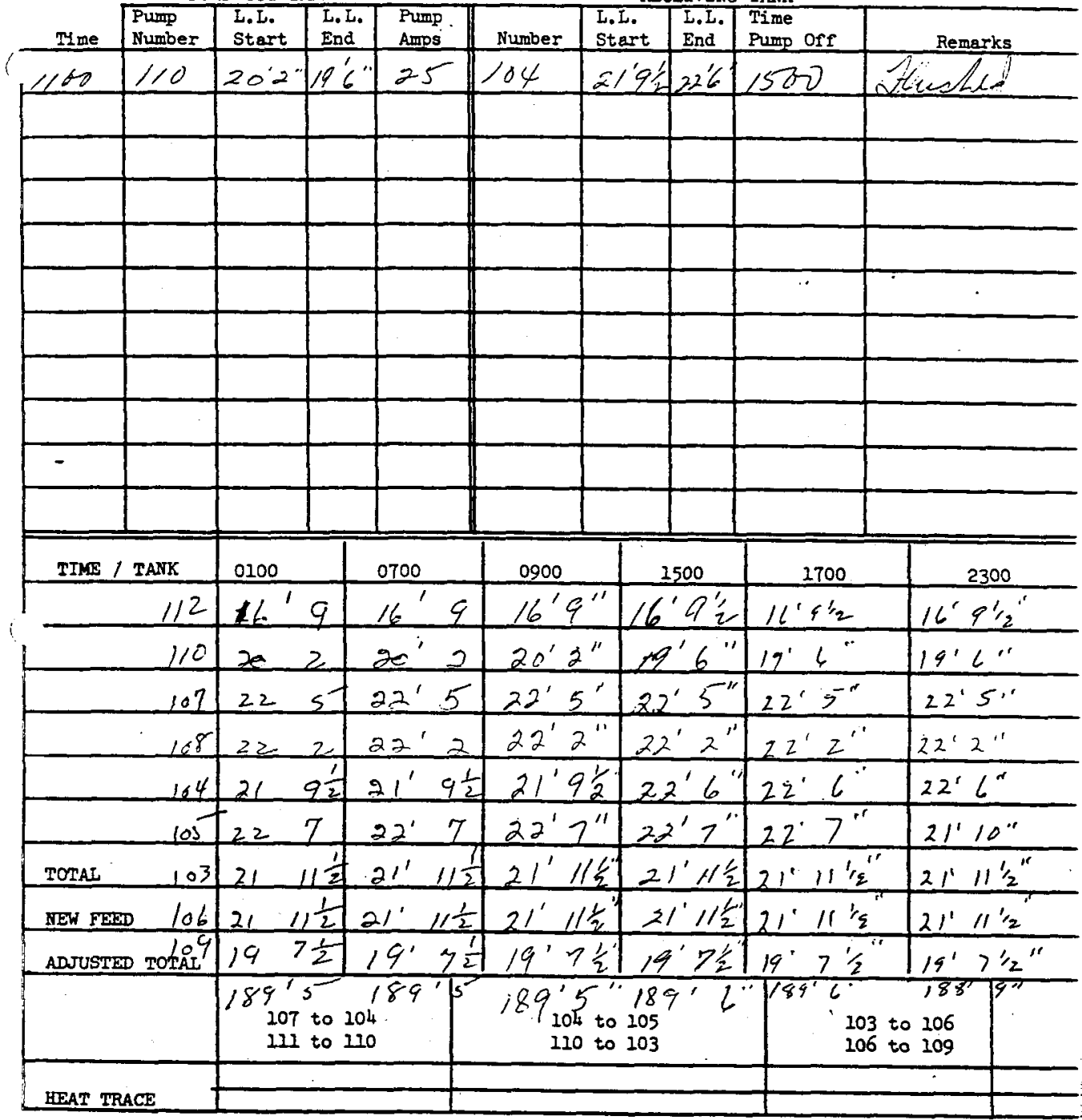

CM Walker:srb 9-29-70 
PUMP OUT TANK

RECEIVING TANK

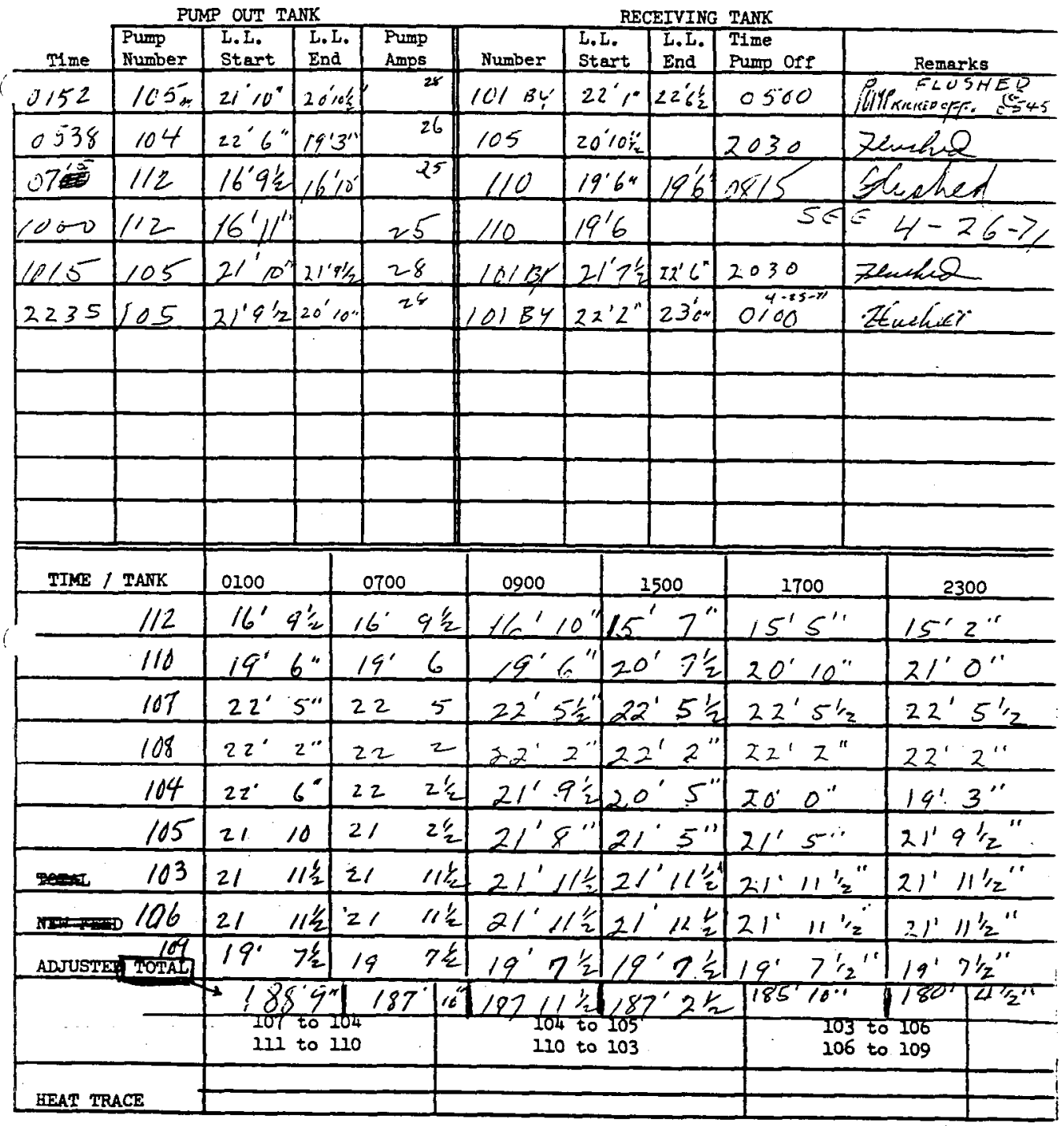

CM Walker:srb 9-29-70 
PUNP OUT TANK

RECEIVING TANK

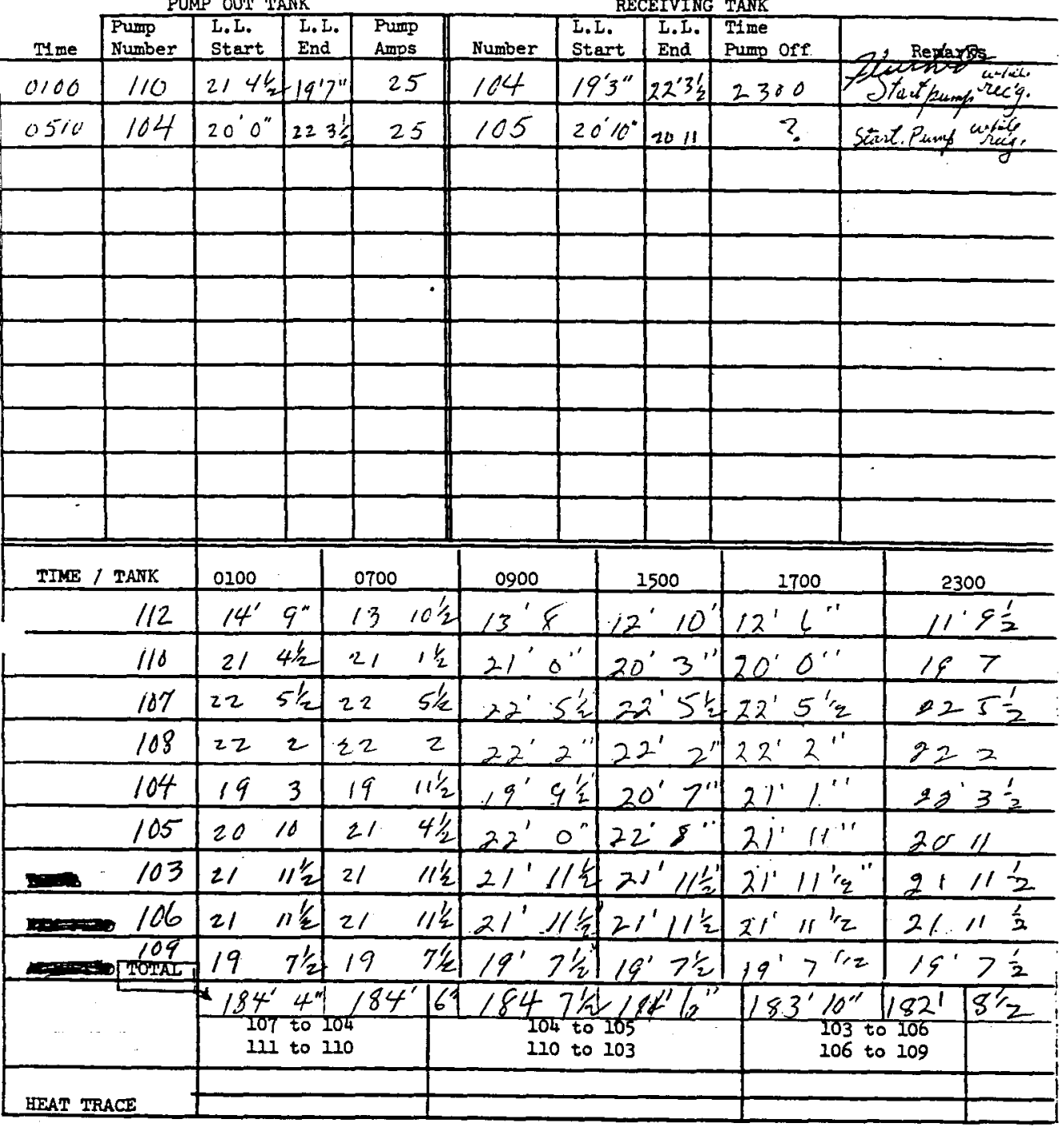

OM Wayker:srb 9-29-70 
PUMP OUT TANK

RECEIVING TANK

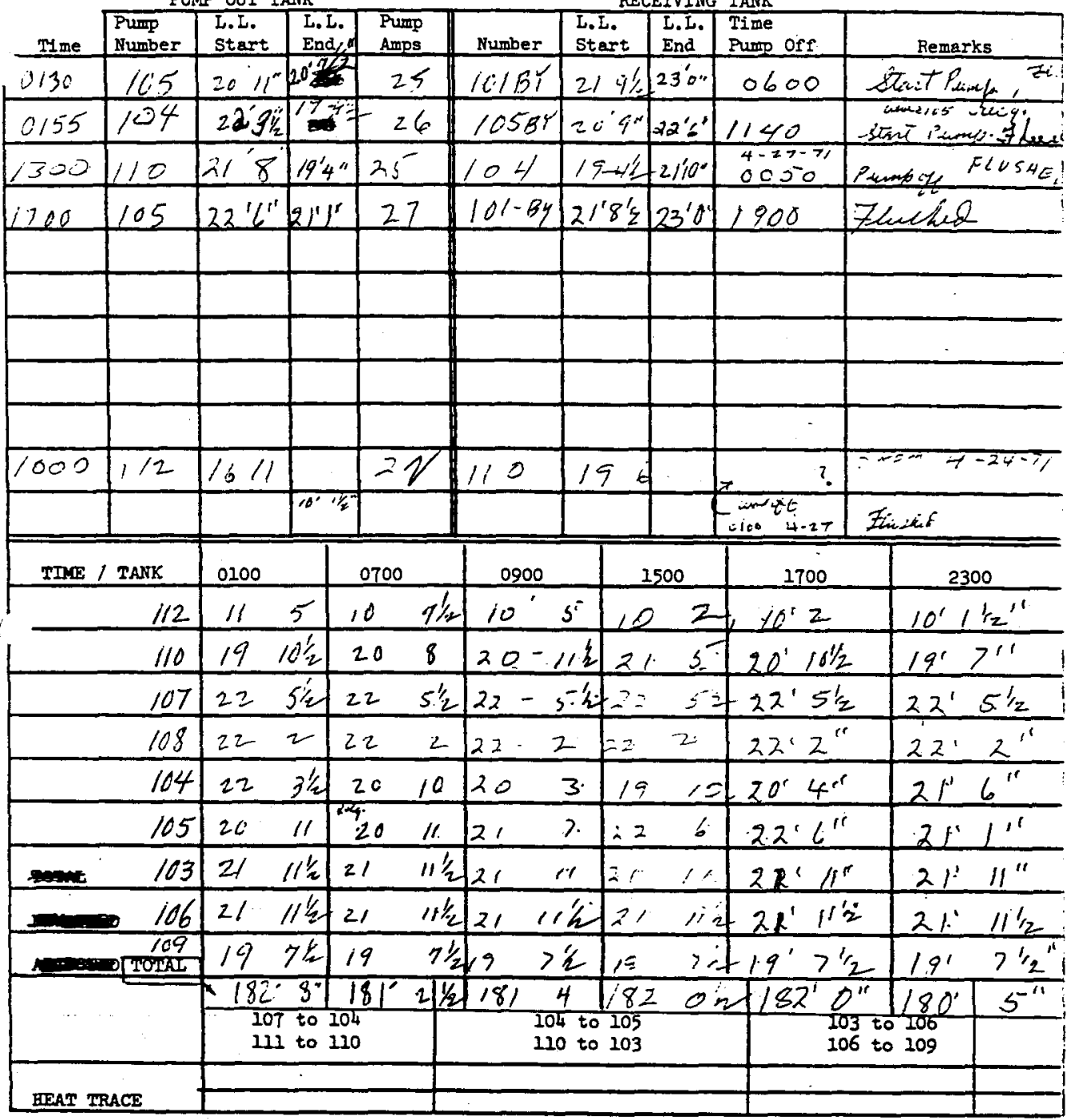


WHC-SD-WM-DP-209, ReV. O

ITS-2 INVENTORY AND PUMPING Date $4-27-71$

PUMP OUT TANK

RECEIVING TANK

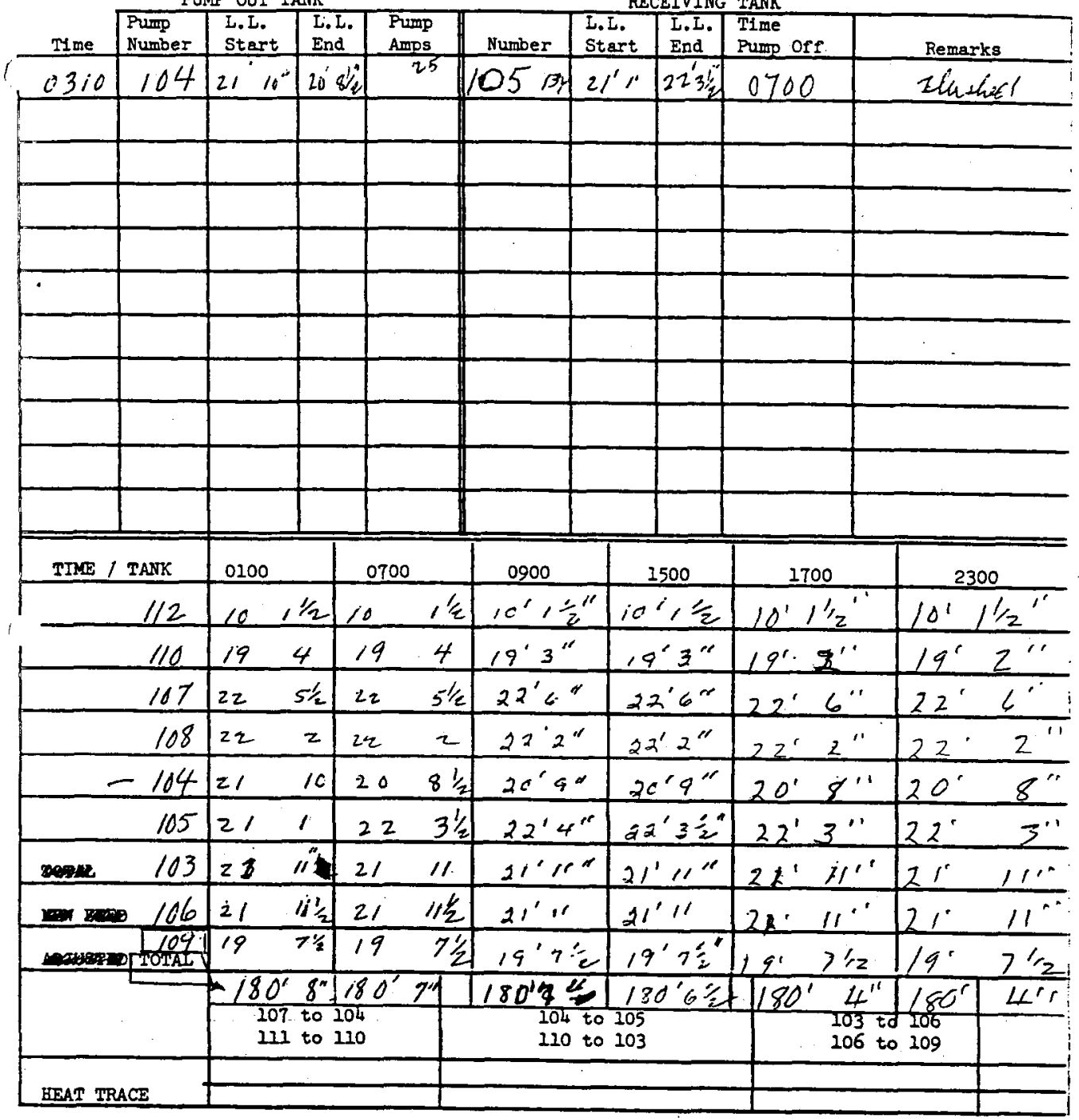

CM Walker:srb 9-29-70 
PUNP OUT TANK

RECEIVING TANK

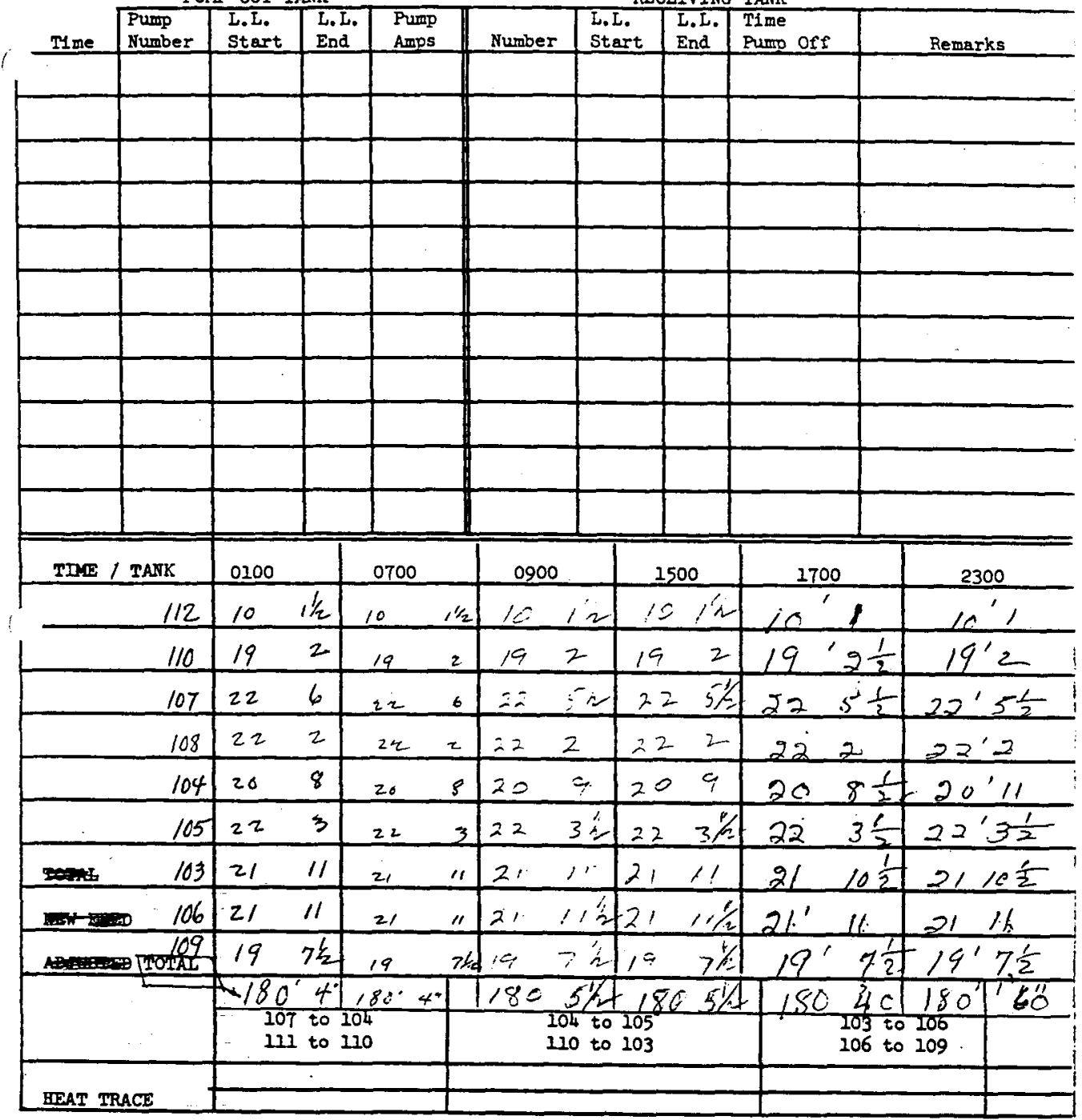


WHC-SD-LM-DP-209, ReV. 0

ITS-2 INVENTORY AND PUMPING Date $4.24 .7 \%$

PUMP OUT TANK

RECEIVING TANK $\$$ to vew Procedur

\begin{tabular}{|c|c|c|c|c|c|c|c|c|c|}
\hline Time & $\begin{array}{l}\text { Pump } \\
\text { Number }\end{array}$ & $\begin{array}{l}\text { L.L. } \\
\text { Start }\end{array}$ & $\begin{array}{l}\text { L. L. } \\
\text { End }\end{array}$ & $\begin{array}{l}\text { Prump } \\
\text { Amps }\end{array}$ & Number & \begin{tabular}{l}
\multicolumn{1}{c}{ LE } \\
L.I. \\
Stert
\end{tabular} & $\begin{array}{l}\text { LIVIN. } \\
\text { End }\end{array}$ & $\begin{array}{l}\text { TANK } \\
\text { Time } \\
\text { Pump off }\end{array}$ & Remarks \\
\hline$=0$ & 107 & $19>1$ & $18^{\prime} 6 /$ & 21 & $\therefore 2$ & $1=A$ & $10^{\prime} q^{\prime \prime}$ & 2855 & $\begin{array}{ll}12+\ln 12 & 12 \times \\
112 & \end{array}$ \\
\hline & & & & & & & & & Backflimed tum \\
\hline & & & & & & & & & \\
\hline
\end{tabular}

\begin{tabular}{|c|c|c|c|c|c|c|}
\hline TIME $/$ TAKK & 0100 & 0700 & 0900 & 1500 & 1700 & 2300 \\
\hline 112 & 10 & 10 & 12 & $\therefore 11 / 2$ & $10^{\circ} 1$ & $10^{\prime} 7 \frac{1}{2}$ \\
\hline 110 & 19 & 19 & 19 & 192 & $19, \frac{1}{2}$ & $\lg 1 \frac{1}{2}$ \\
\hline 167 & $2251 / 2$ & $S^{\prime} \varepsilon$ & $=-2$ & $22 \leq 4 /=$ & 225 & 225 \\
\hline 108 & $22=$ & 22 & $\therefore \approx$ & $=2 \quad 2$ & 222 & 222 \\
\hline 104 & 2011 & 20 & $2=$ & $20 \quad 11$ & 20 & $20 \quad 11$ \\
\hline 105 & $22 \quad 3 \frac{1}{2}$ & 22 & $3 \%$ & $223 \%$ & $223 \frac{1}{2}$ & $22 \quad 3 \frac{1}{2}$ \\
\hline 103 & $21 \quad 10 \frac{1}{2}$ & $10 k$ & 21 & 11.11 & $21 \quad 10 \frac{1}{2}$ & $10 \frac{2}{5}$ \\
\hline $10=x=0$ & $2 / \cdots 11$ & 21 & 21 & $21 \quad 11$ & 2111 & $21 / 1$ \\
\hline AD $=\frac{109}{10 T A L}$ & $19 \quad 7^{k}$ & $19 \quad 7 k$ & 191 & $15 \quad 7 / 2$ & $19^{\circ} \quad 6$ & $18^{\prime} 8 \frac{1}{2}$ \\
\hline & $\frac{180^{\prime}}{107} \frac{6^{\prime}}{111}$ & 104 & $\frac{17(4)}{104 \text { to }}$ & $\begin{array}{l}152-7 \\
105 \\
103\end{array}$ & 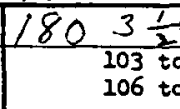 & \begin{tabular}{l|l}
189 & $0 \frac{1}{2}$ \\
106 & \\
109 &
\end{tabular} \\
\hline HEAT TRACE & & & & & & \\
\hline
\end{tabular}

C Wazker:srb 9-29-70 
WHC-SO-WM-DP-209, ReV. 0

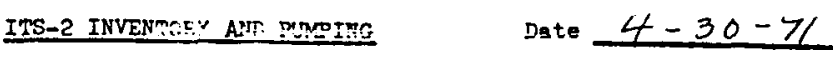

?UT.F OUT TANK

RECETVING TANK

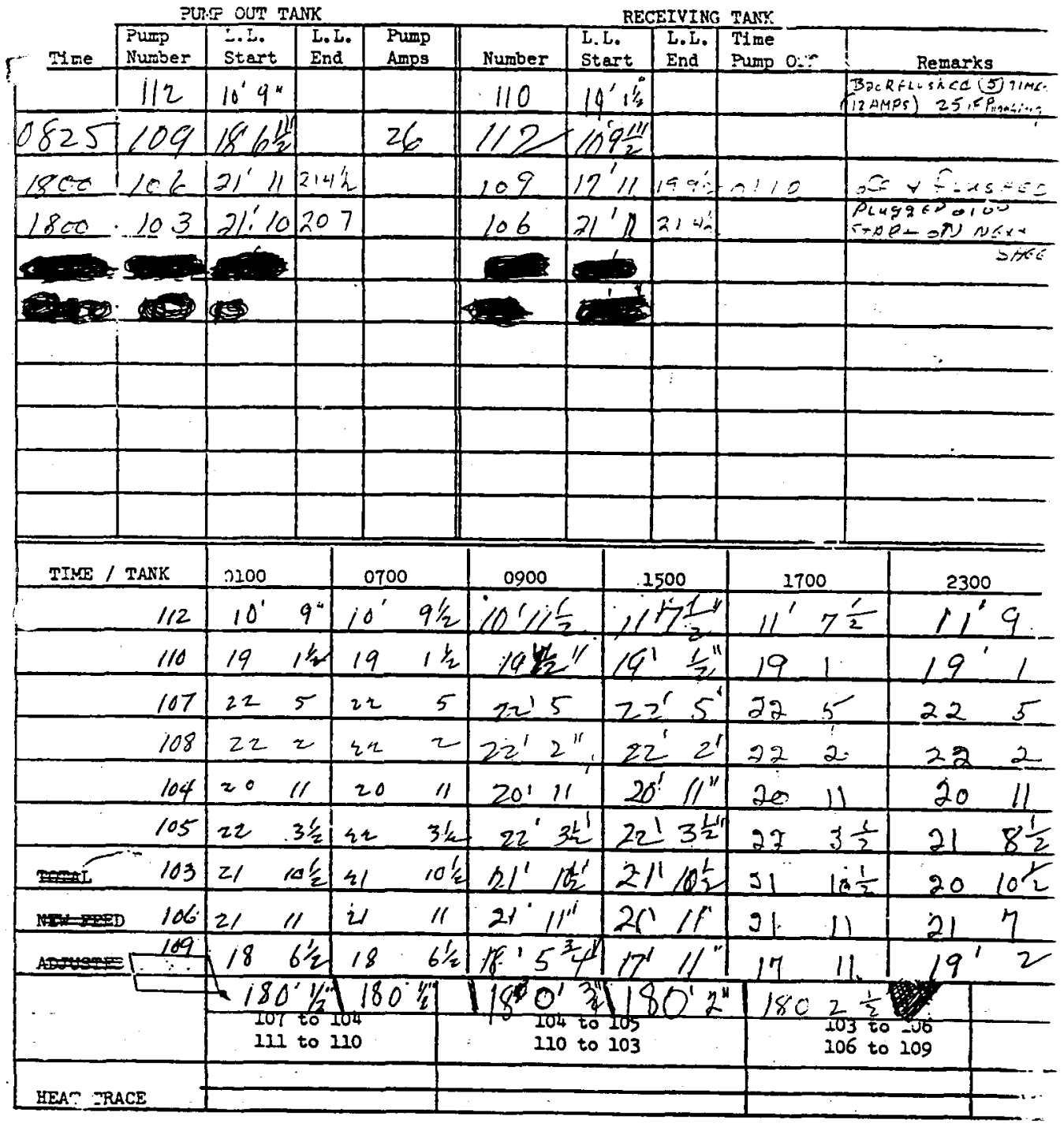

1. Wevirer:srt 9-29-70

120 
PURP OUT TANK

RECEIVING TANK

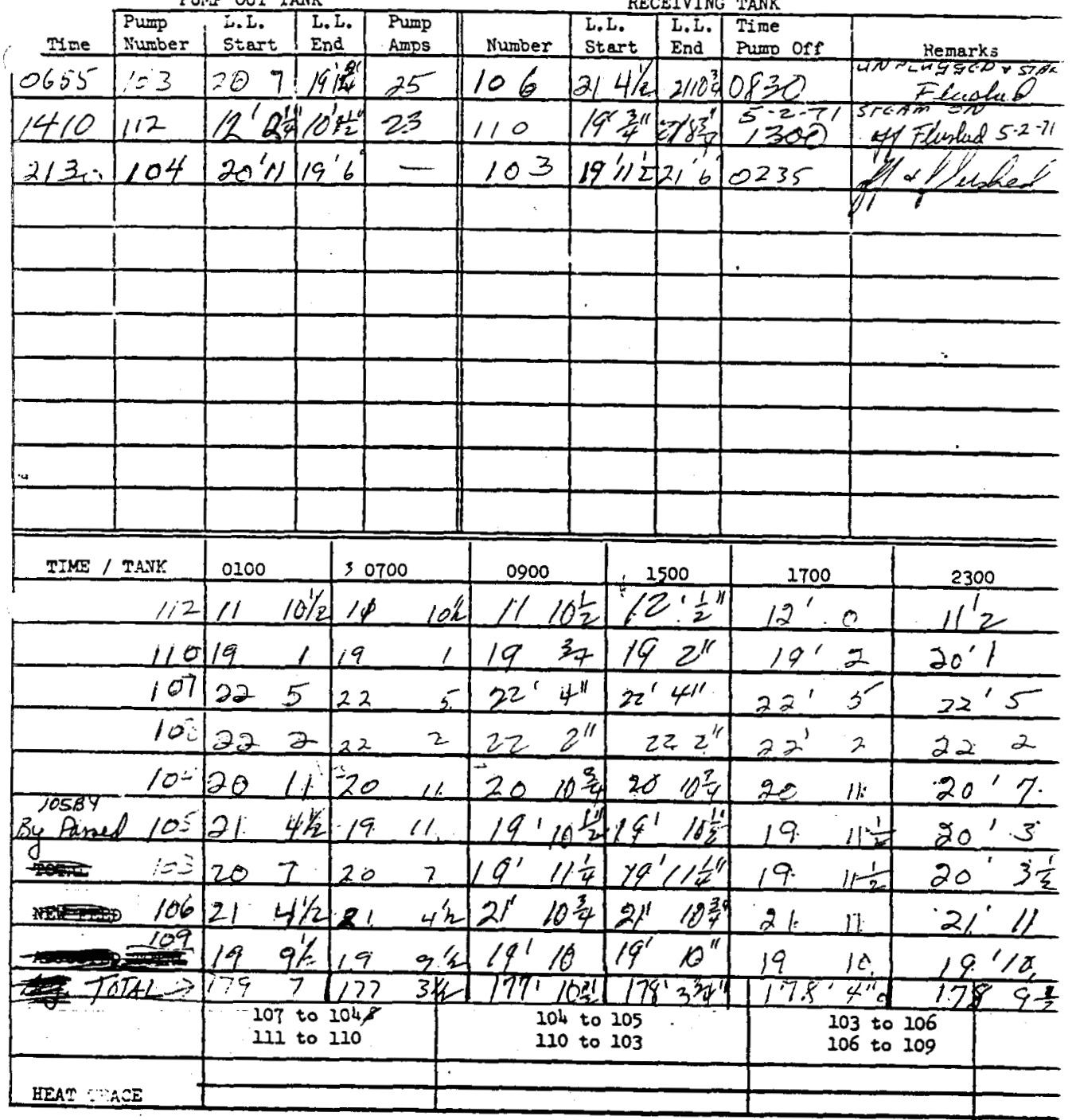

1... visior:srb 9-29-70

$-7.8$ 
PUIP OUT TANK

RECEIVING TANK

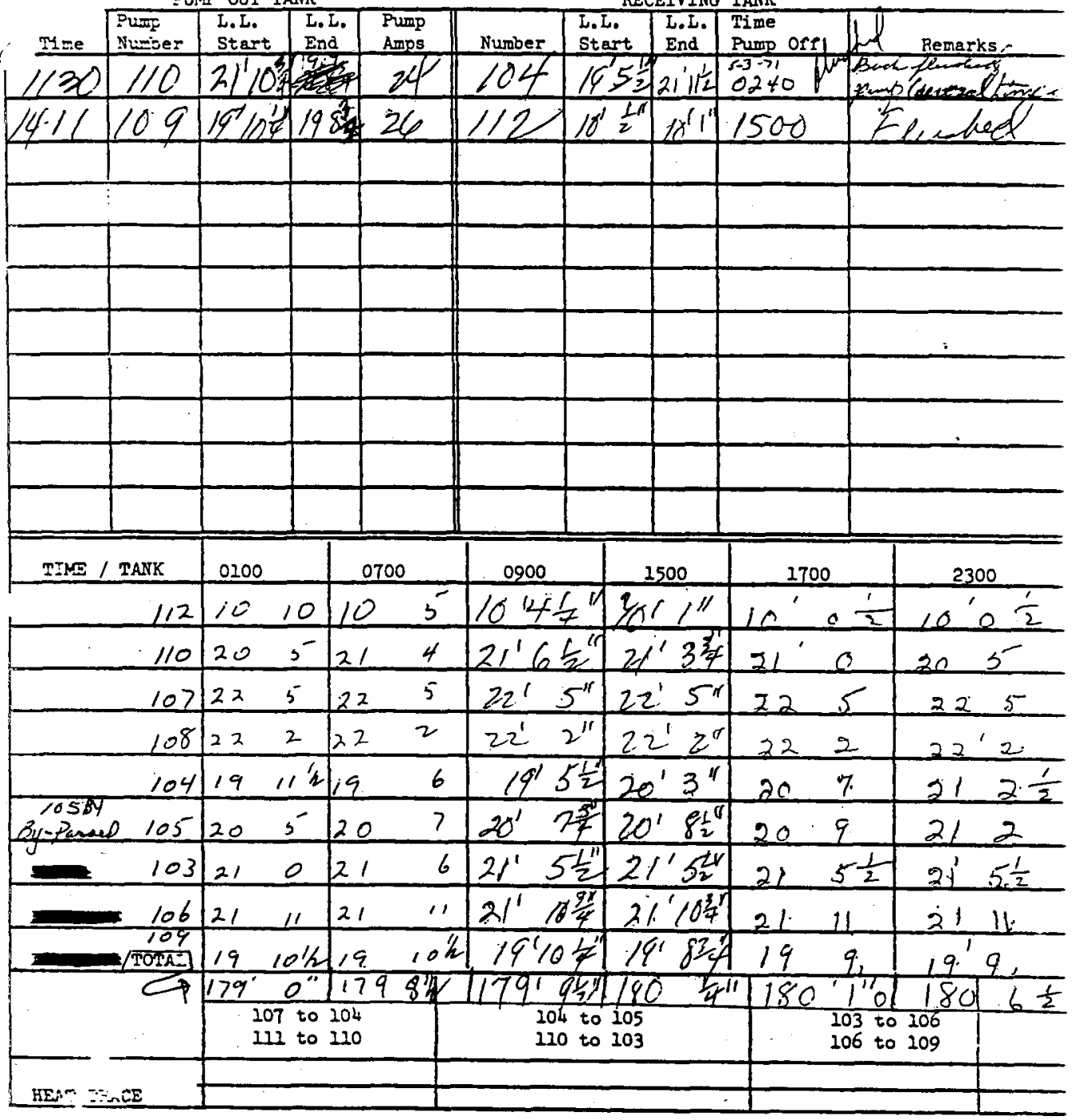

(W.. $5=b$ 2-29-70 
PUNP OUT TANK

RECEIVING TAITK

\begin{tabular}{|c|c|c|c|c|c|c|c|c|}
\hline Inse.e & $\begin{array}{l}\therefore= \\
\text {-jex }\end{array}$ & $\begin{array}{l}\text { L.I. } \\
\text { Start }\end{array}$ & $\begin{array}{l}\text { I. L. } \\
\text { End }\end{array}$ & $\begin{array}{l}\text { Fump } \\
\text { Amps }\end{array}$ & Number & $\begin{array}{l}\text { L.L. } \\
\text { Stert }\end{array}$ & $\begin{array}{l}\text { L.L. } \\
\text { End }\end{array}$ & $\begin{array}{l}\text { Time } \\
\text { Pump off }\end{array}$ \\
\hline 0110 & 109 & $19^{\prime} 9$ & $19.8 \frac{1}{2}$ & 27 & 112 & $100 \frac{1}{2}$ & $10^{\prime} 1$ & $0 / 40$ \\
\hline 1300 & 109 & $19^{\prime} 8^{\prime \prime}$ & $19^{\prime}{ }^{\prime}$ & 26 & 112 & $10^{\prime} / 2^{\prime \prime}$ & 100 & 1800 \\
\hline & & & & & & & & \\
\hline . & & & & & & & & \\
\hline & & & & & & & & \\
\hline & & & & & & & & \\
\hline & & & & & & & & \\
\hline & & & & & & & & \\
\hline & & & & & & & & \\
\hline & & & & & & & & \\
\hline & & & & & & & & \\
\hline
\end{tabular}

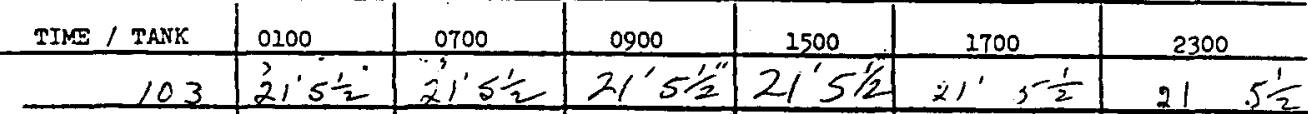

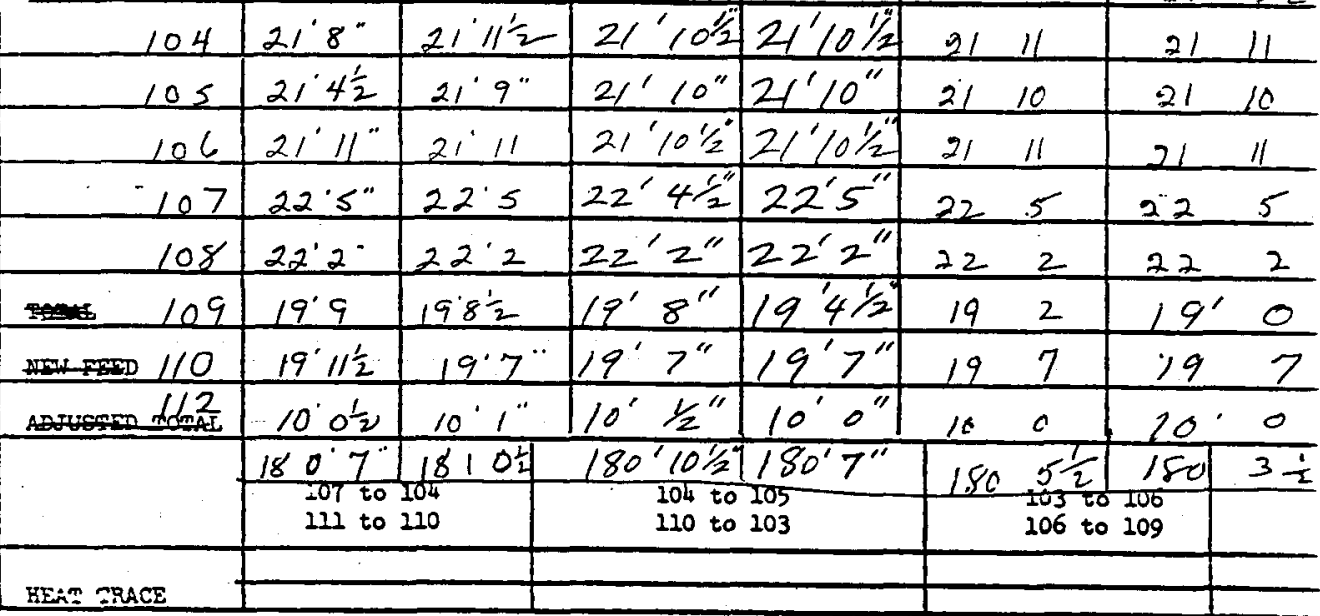

15944
-15950

(Walker:srb 9-29-70

$1599=$ 


\section{Whin \\ - oce 0L-6z-5 axs:-20xtry. ) \\ $8207 \%-0.0$}

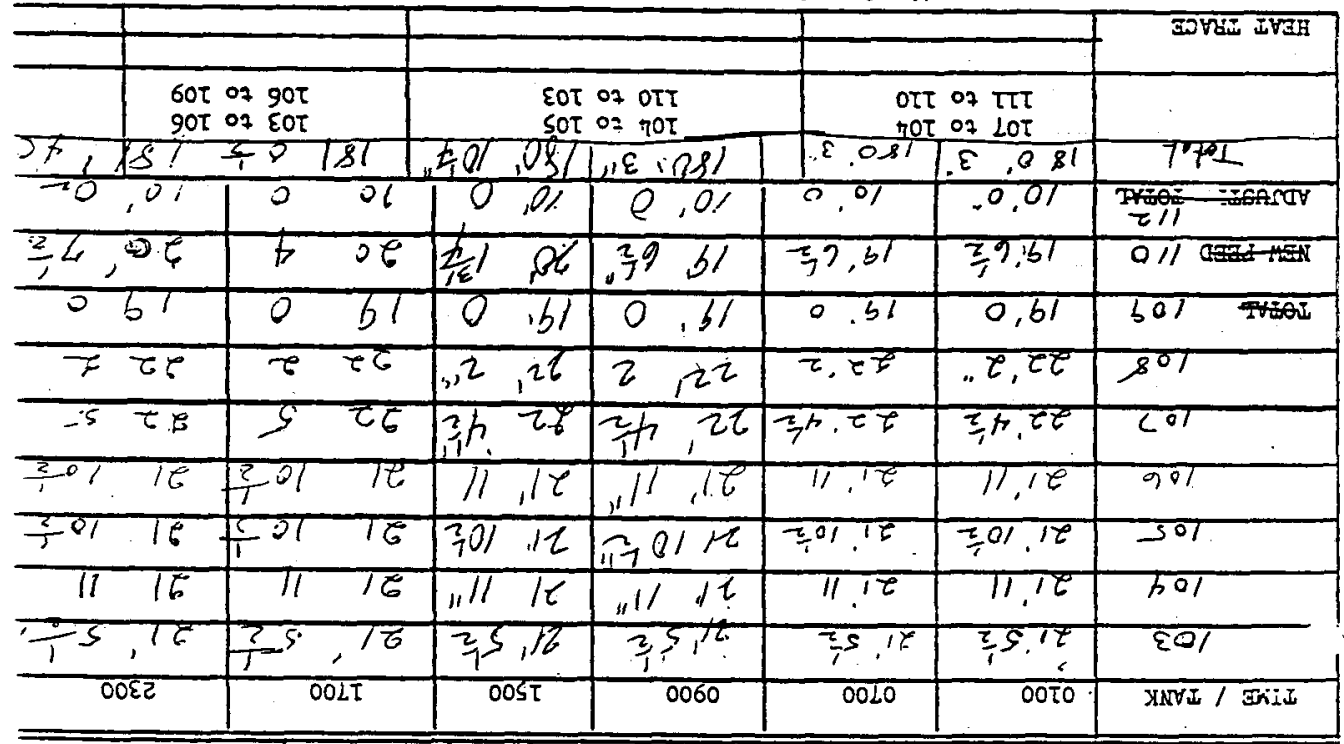

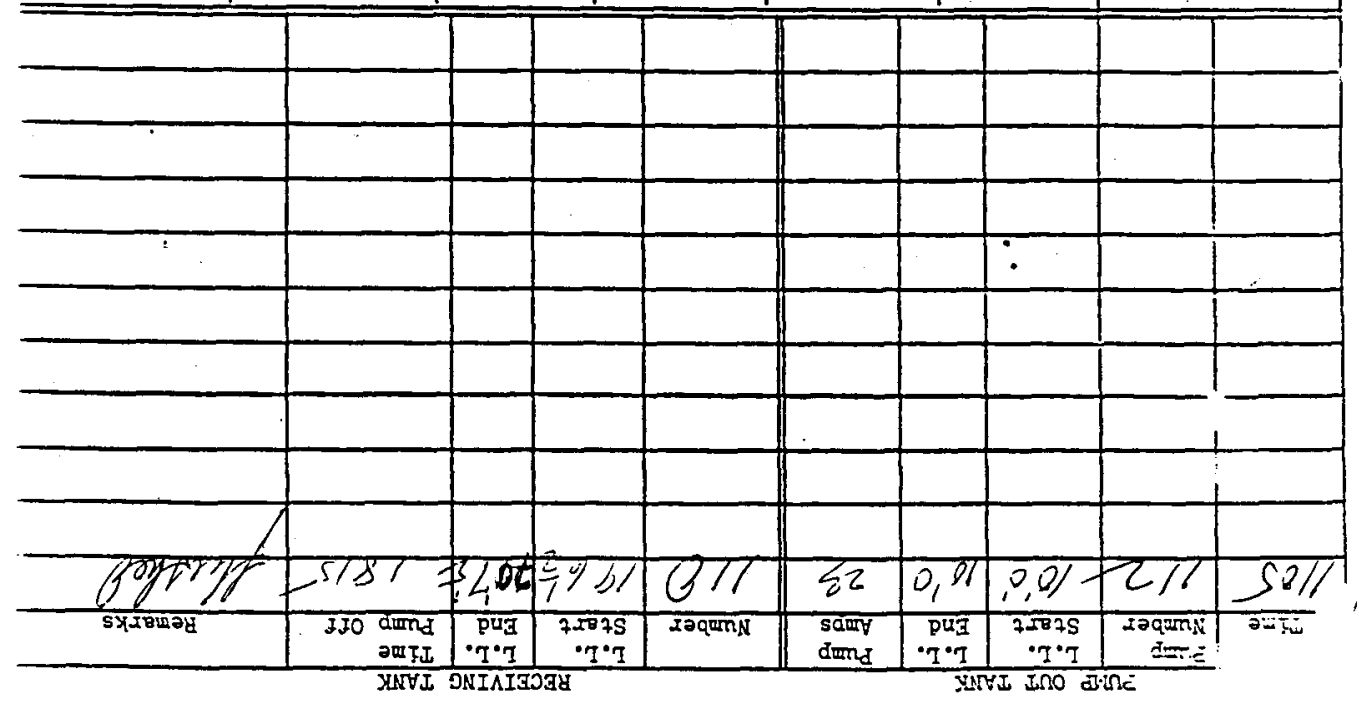

ll- 
WHC-SD-WA-DP-209. ReV. O

ITS-2 INVENTORY AND PUMPING Date $5-5-71$

PUNT OUT TANK

RECEIVING TANK

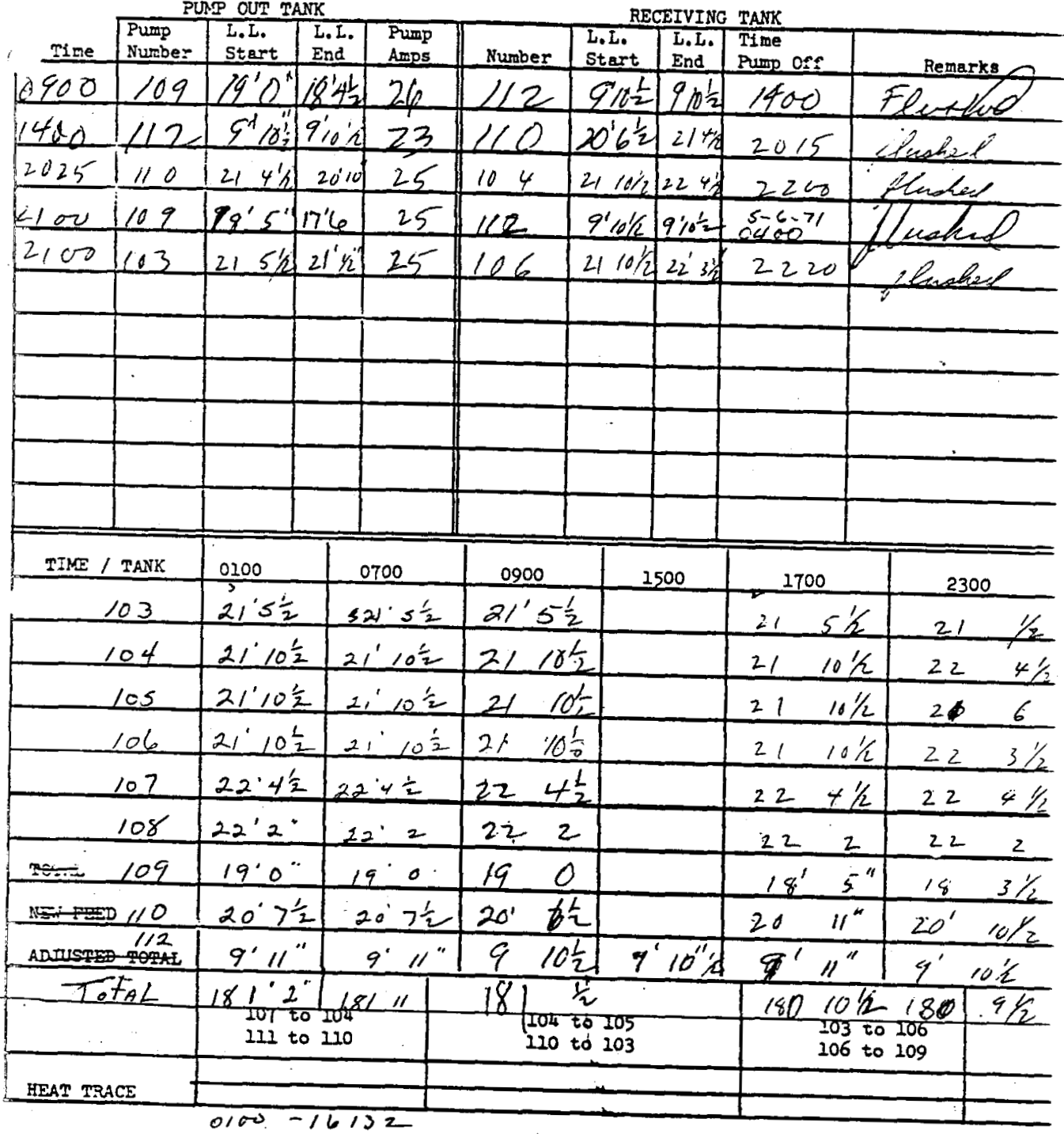

(Wake: arb 9-29-70 0700 -

125 
WL-SD-WM-DP-209, ReY. 0

ITS-2 INVENTORY AND PUMPING

Date $5-6-7 /$

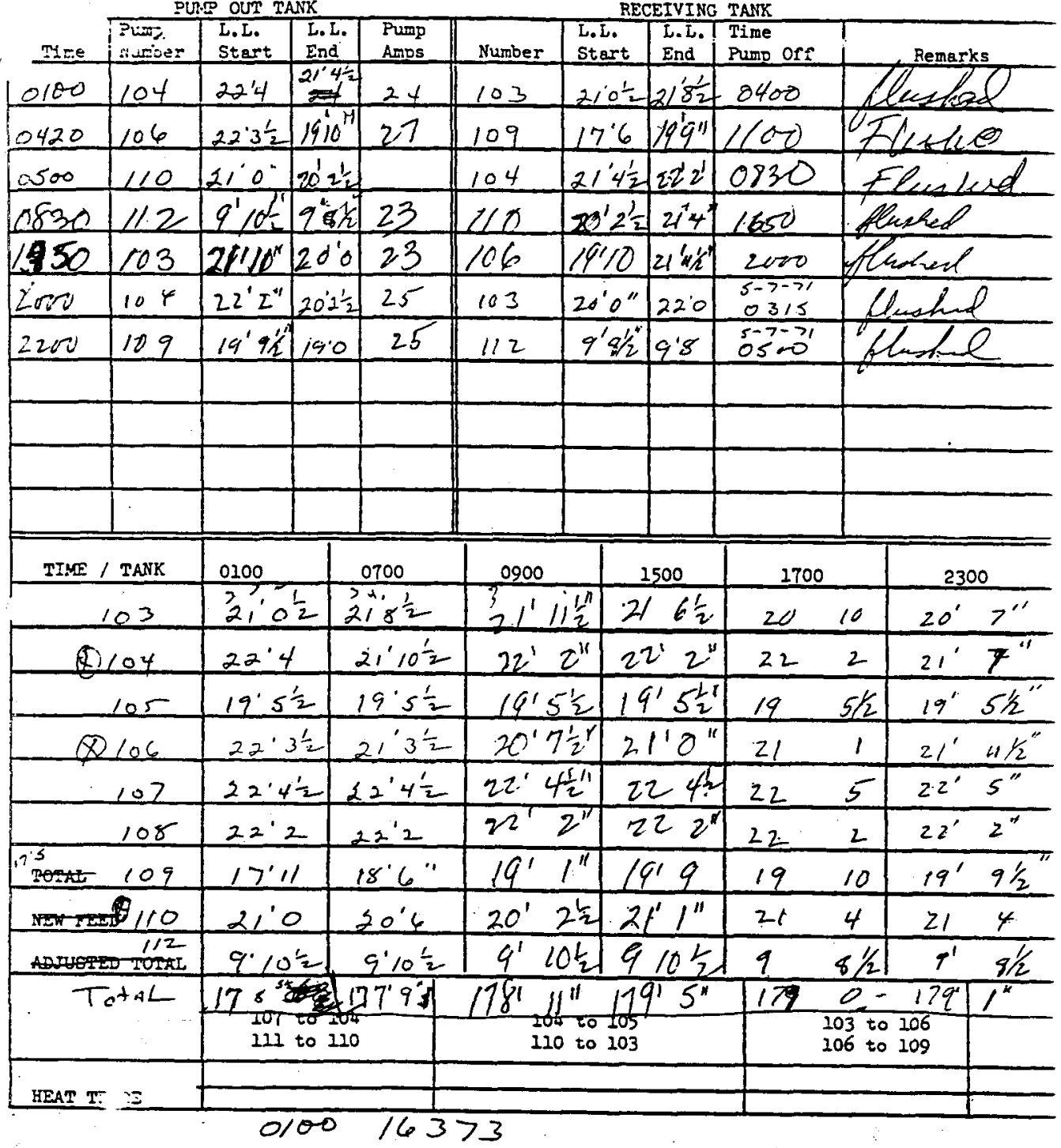

(. Walker:ist 9-29-70-yor 16555

126 
PUMP OUT TANK

RECEIVING TANK

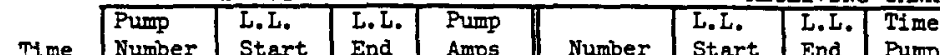

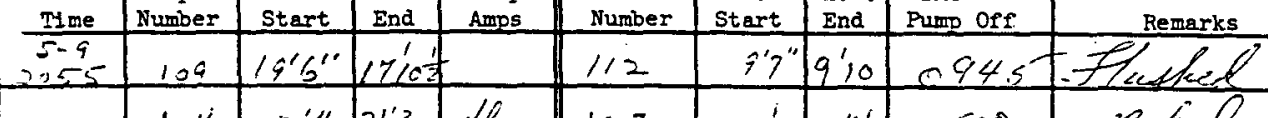

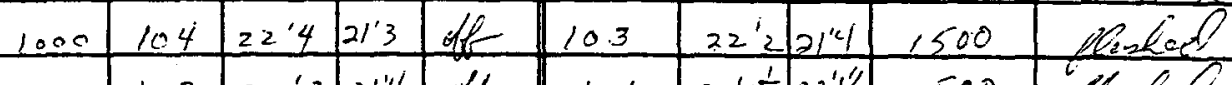

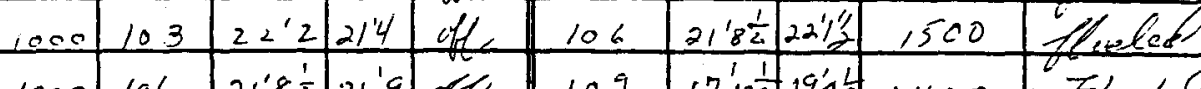

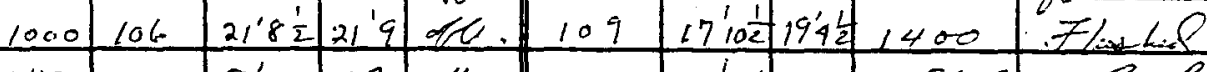

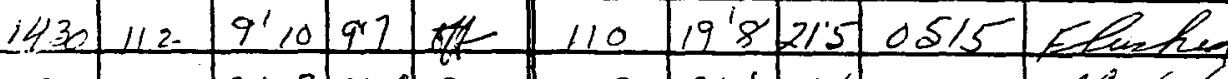

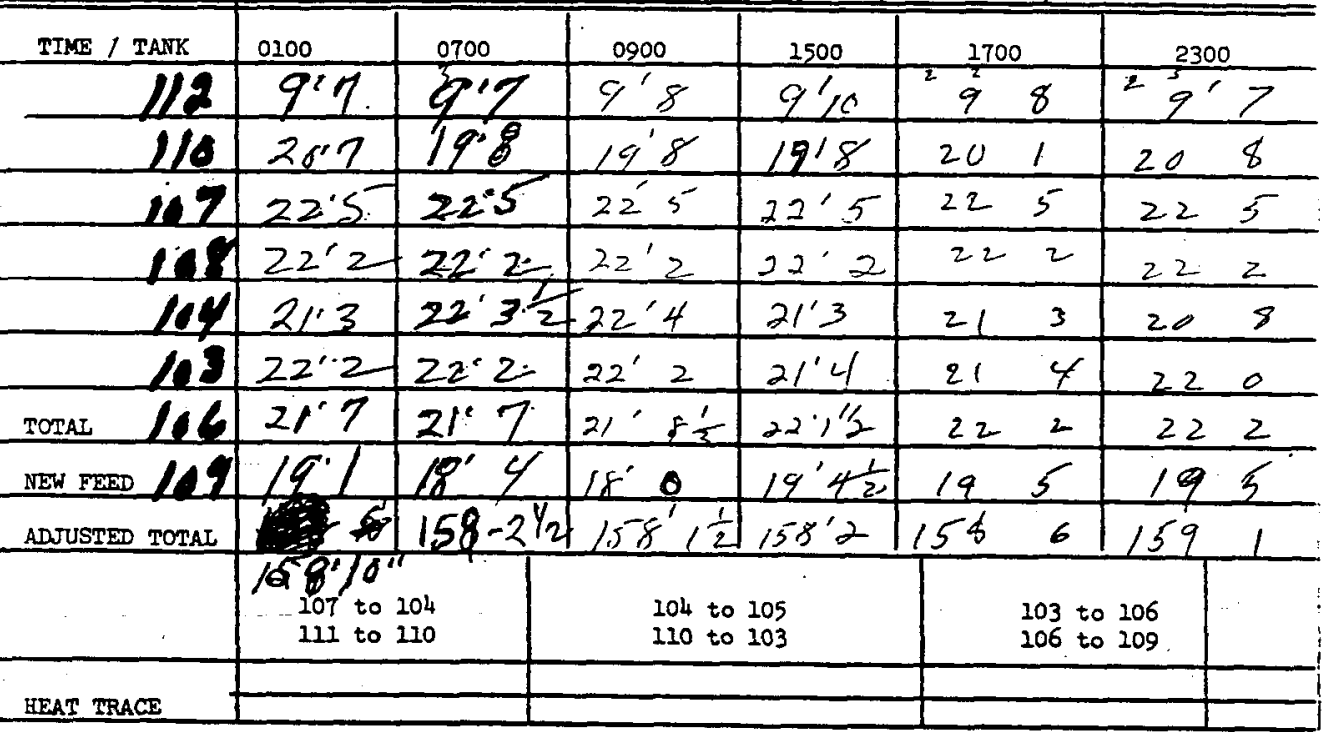

Walker:srb 9-29-70 
RECEIVING TANK

PUMP OUT TANK

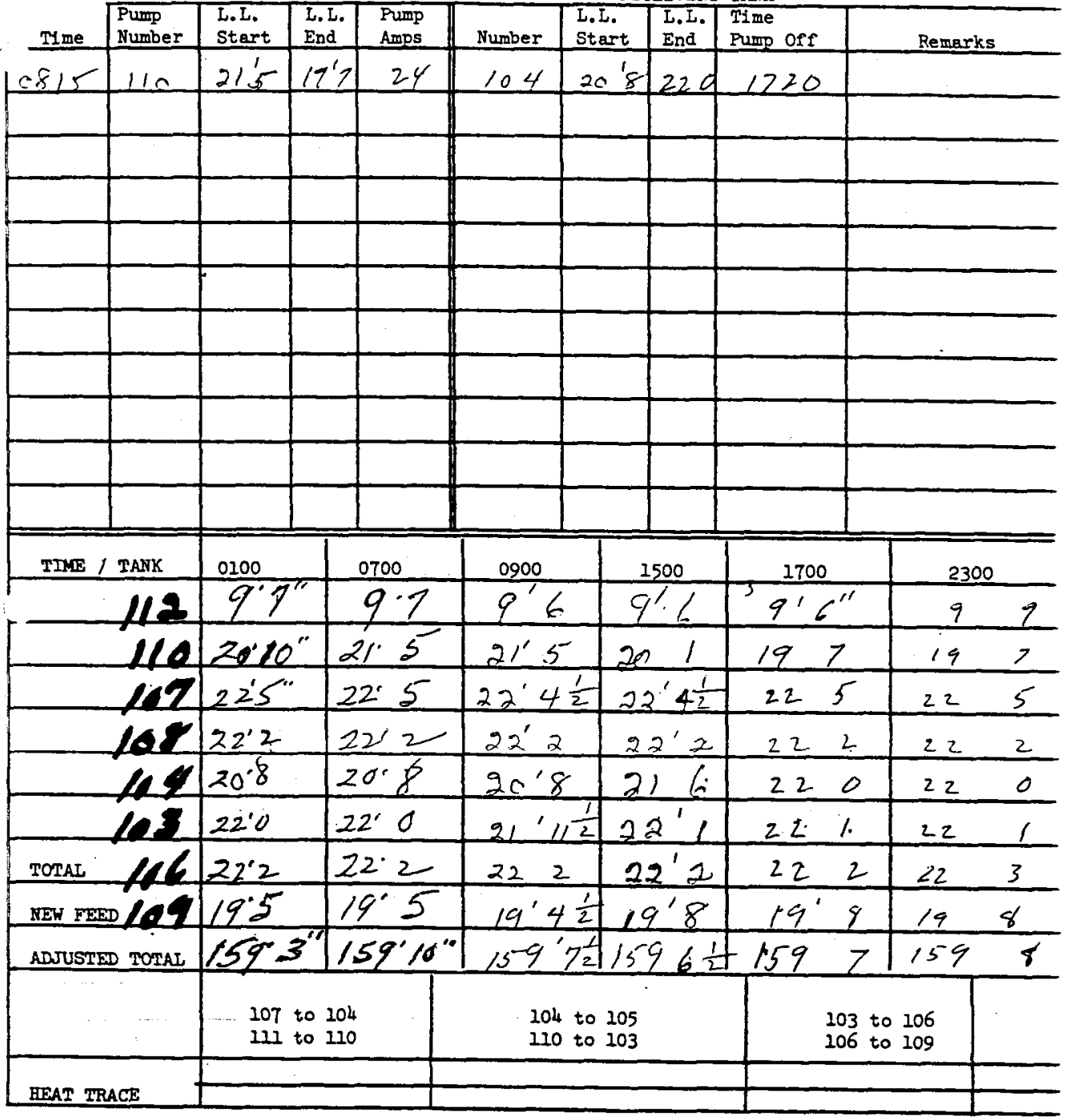

Walker:srb 9-29-70 
PUMP OUT TANK

RECEIVING TANK

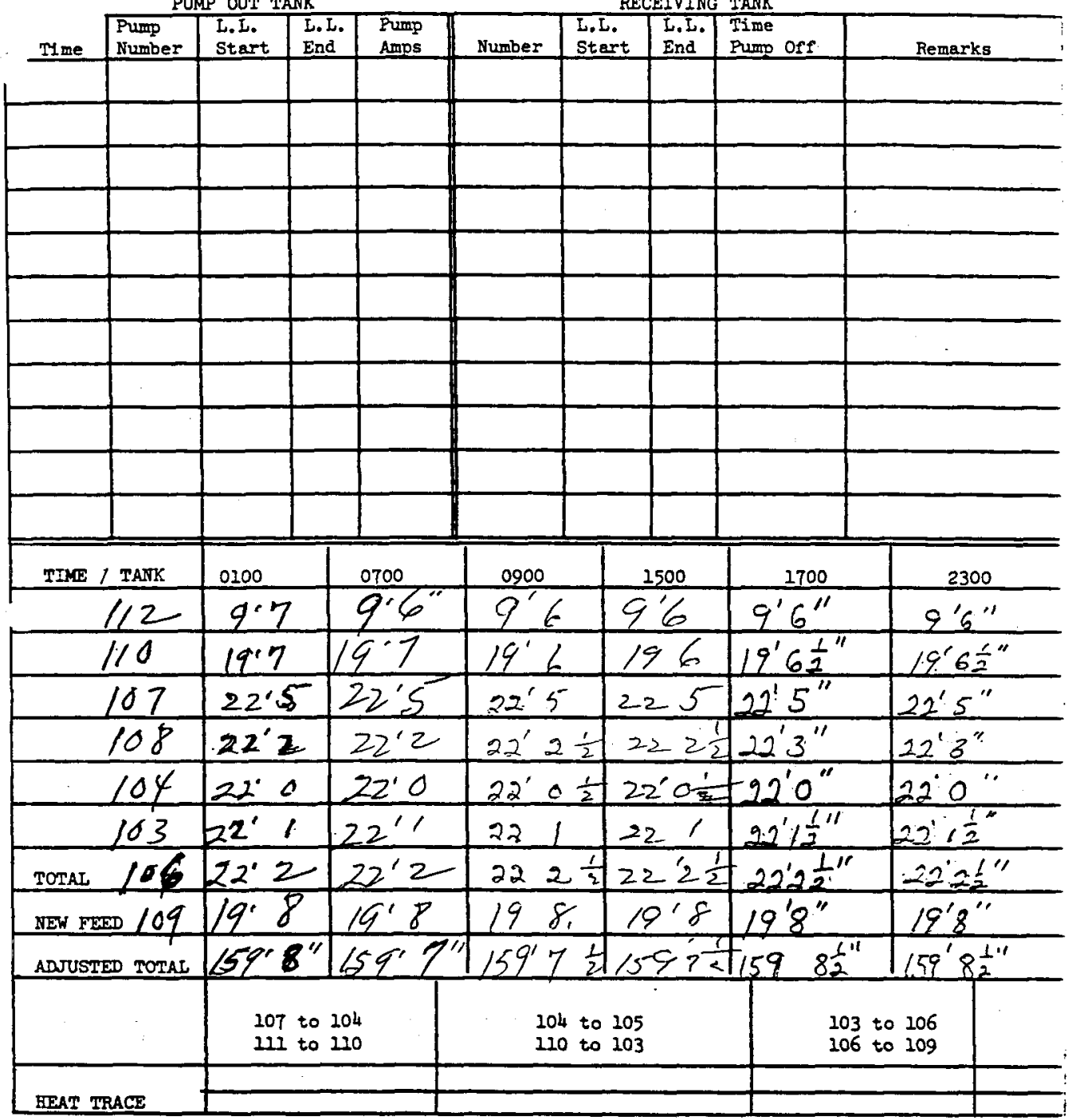

Waiker:srb 9-29-70 
PUMP OUT TANK

RECEIVING TANK

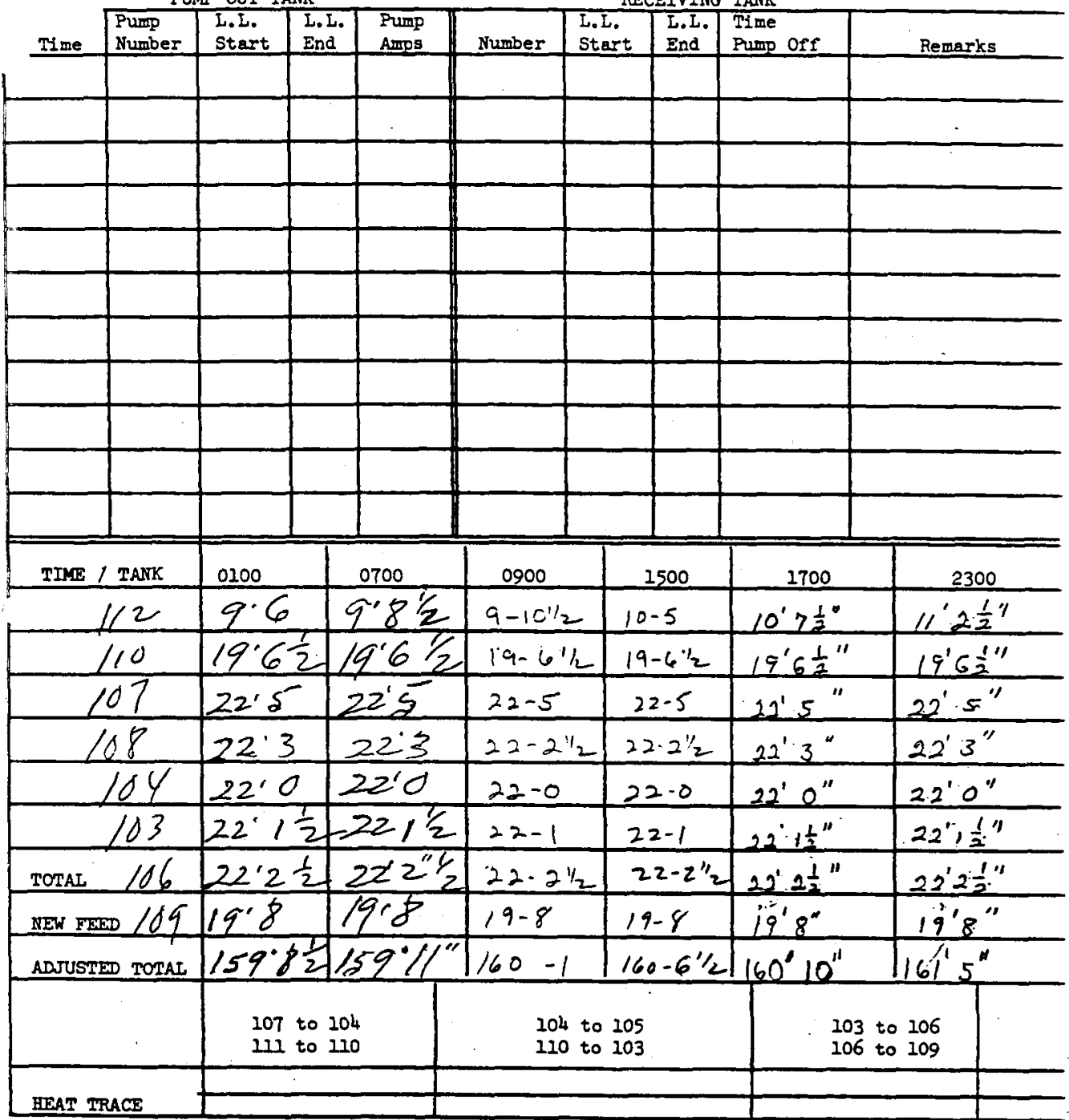

Walker:srb 9-29-70

an

ef $\quad=0 \div$ 
WHC-SD-LM-DP-209, ReV. O

ITS-2 INVENTORY AND PUMPING Date $s-19-71$

PUMP OUT TANK

RECEIVING TANK

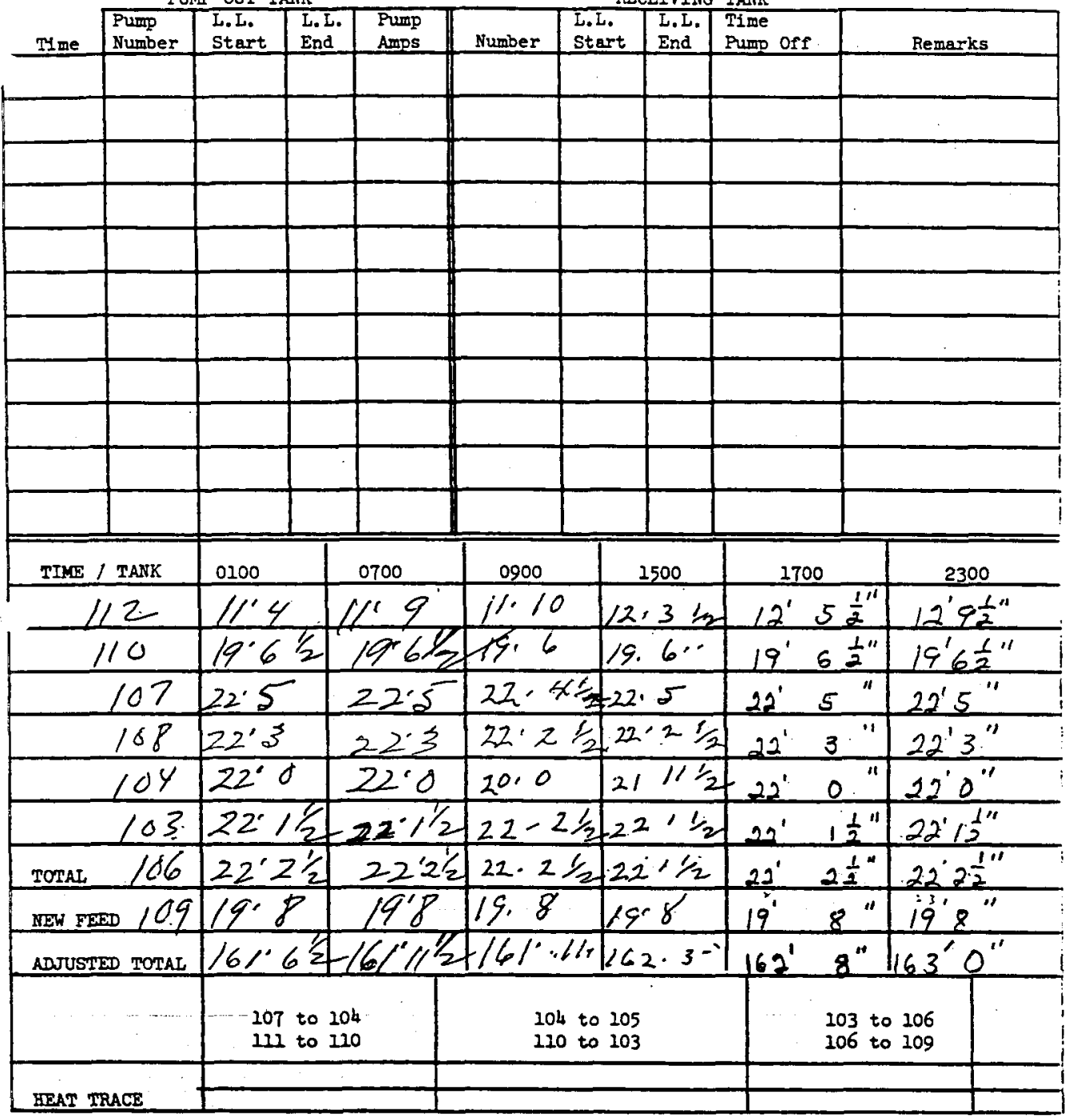

Walker:srb 9-29-70 


$$
\text { ITS-2 INVENTORY AND PUMPINC Date } 5-1.5
$$

PUNP OUT TANK

RECEIVING TANK

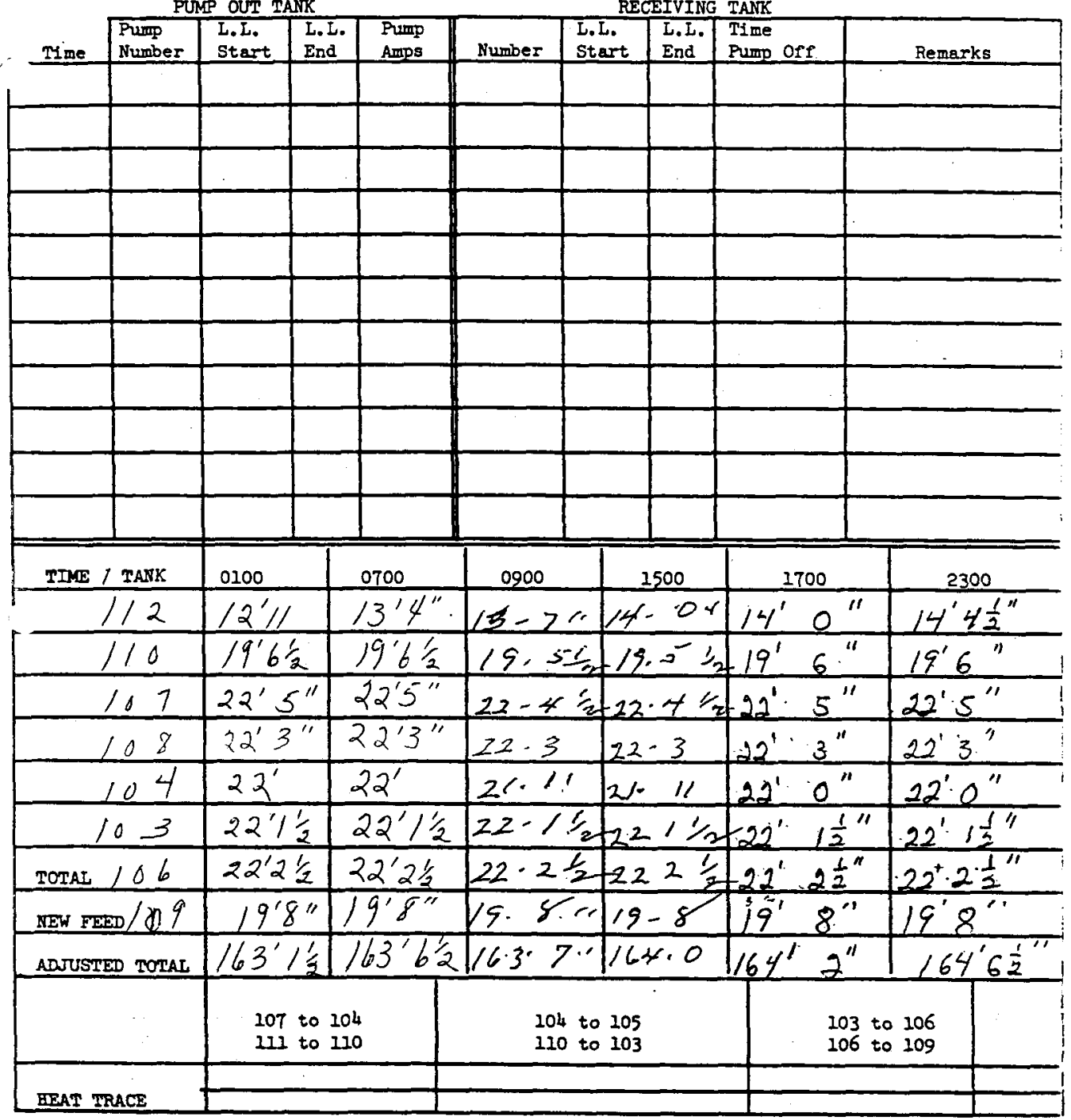

( Walker:srb 9-29-70 
PUMP OUT TANK

RECEIVING TANK

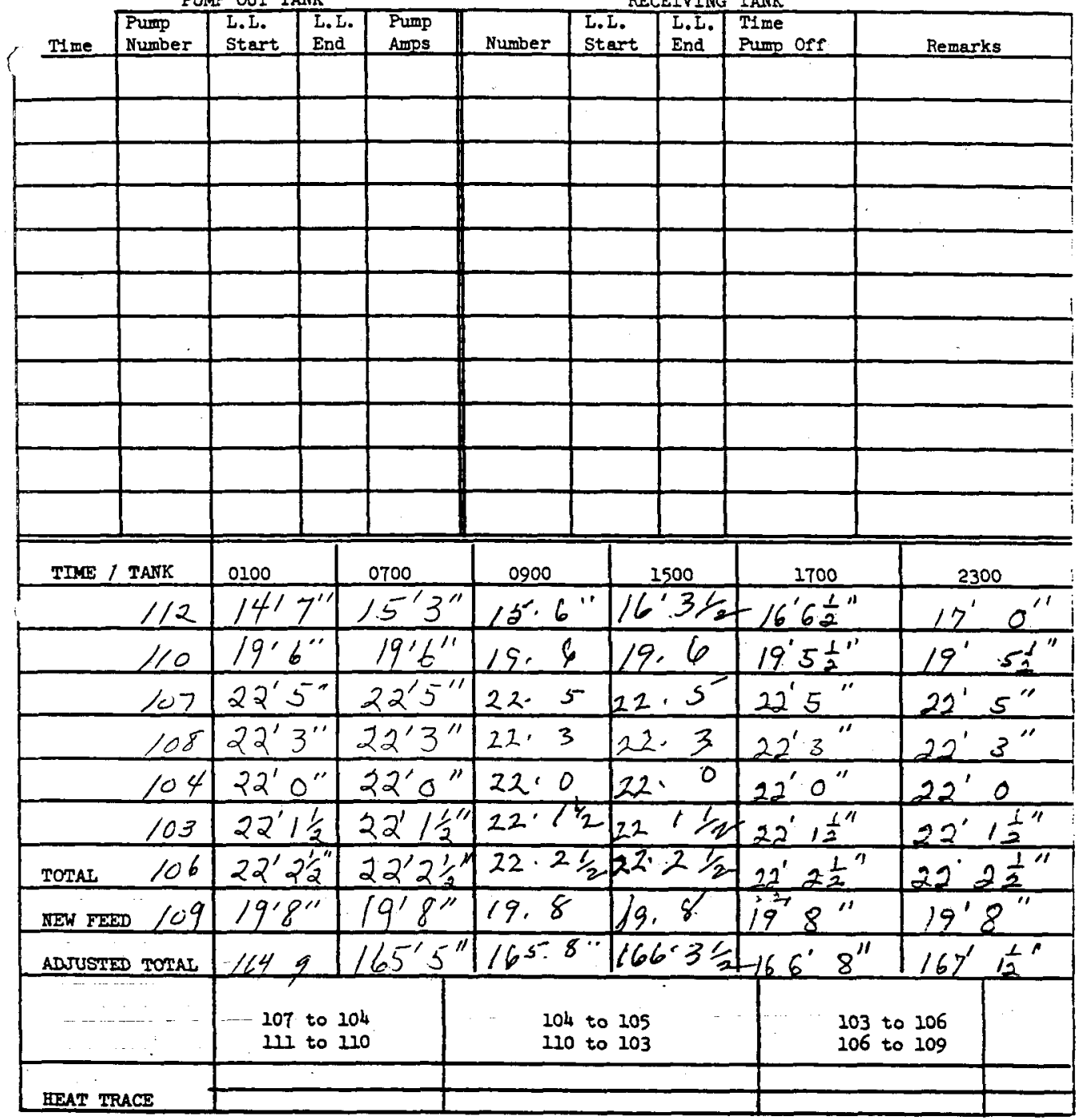

Walker :srb 9-29-70 
PUMP OUT TANK

RECEIVING TANK

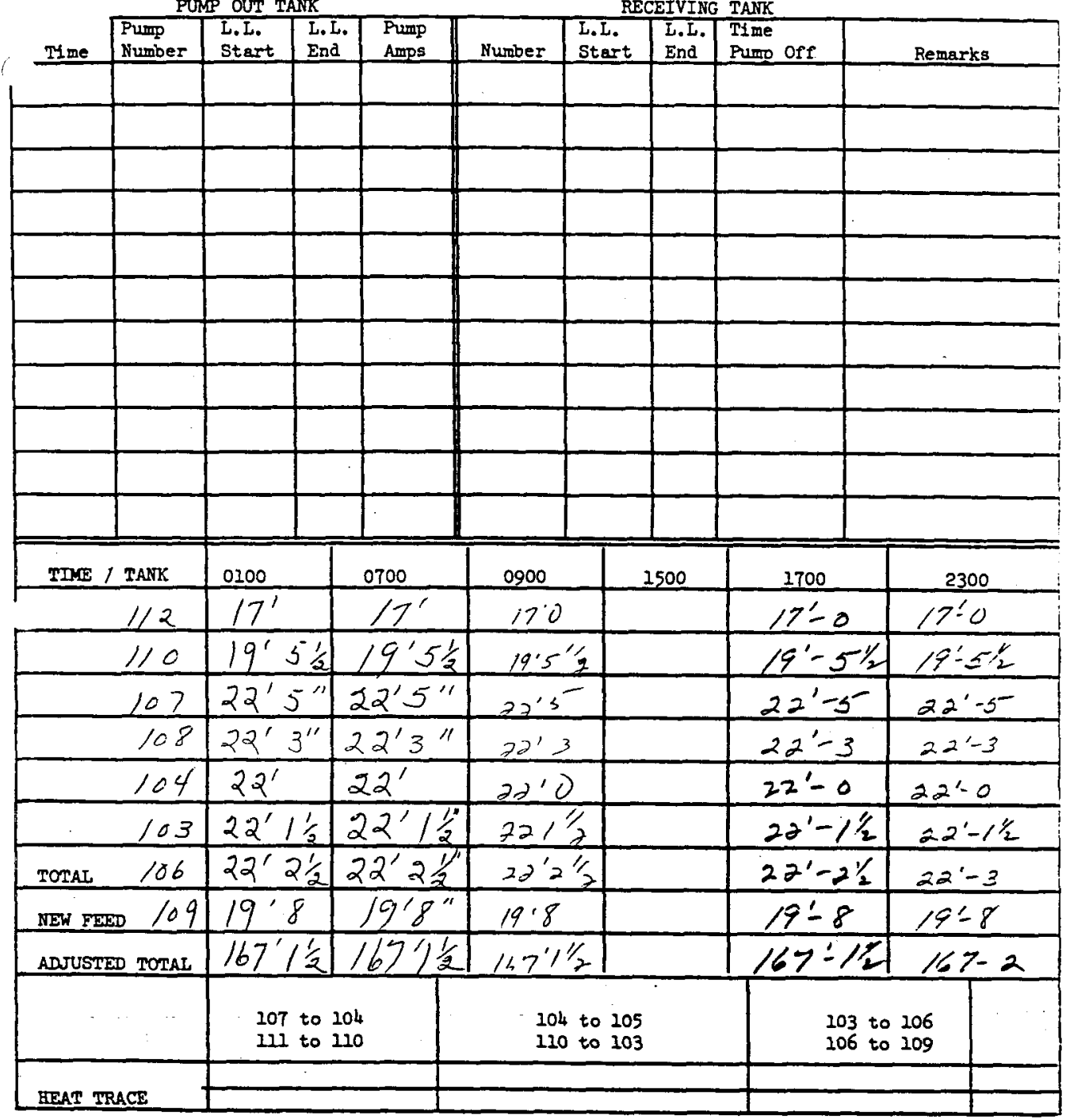

Walker:srb 9-29-70 
FUMP OUT TANK

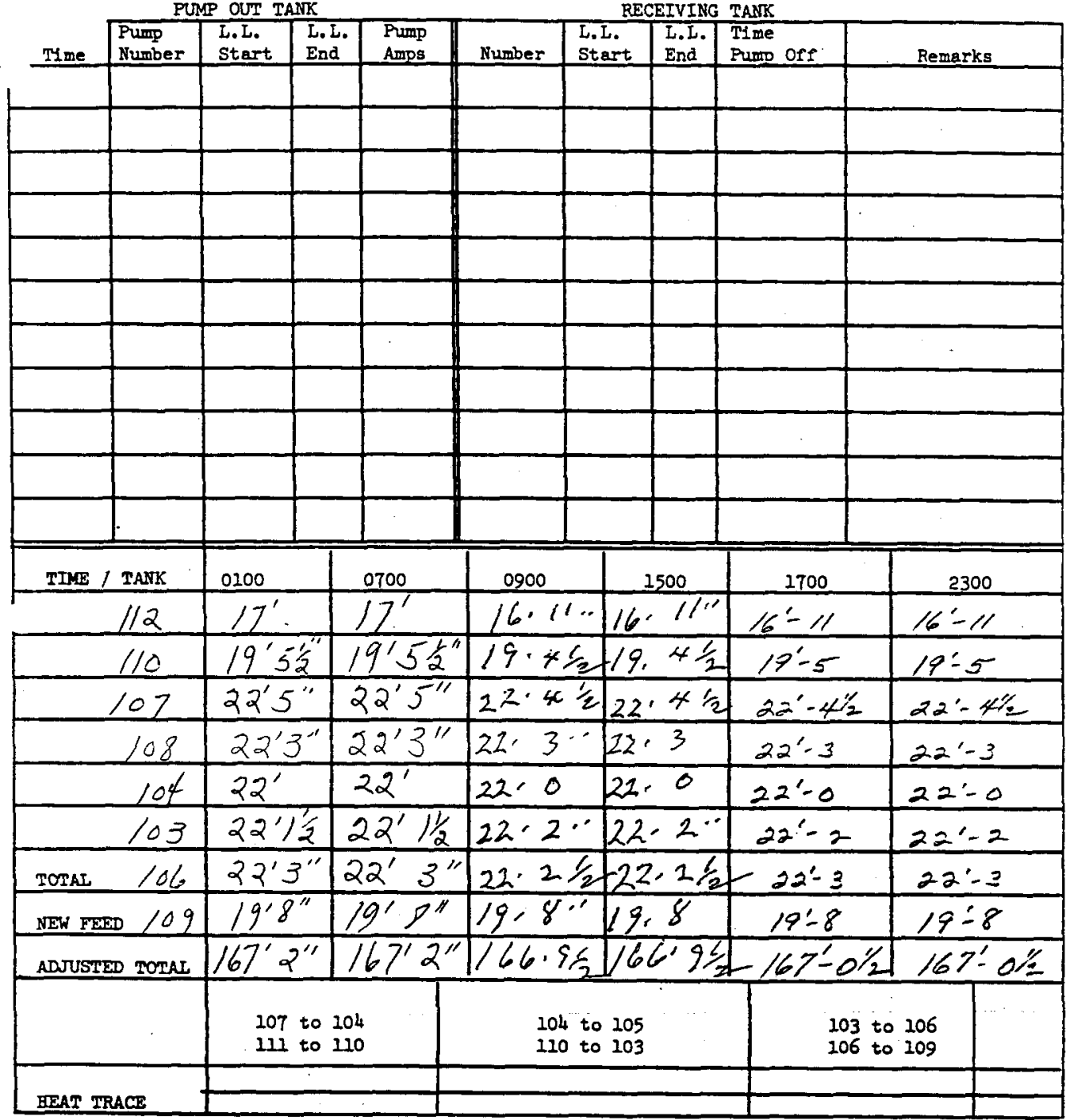


WHC-SD-LM-DP-209, REV. 0

ITS-2 INVENTORY AND PUMPING Date $5-19$

PUMP OUT TANK RECEIVING TANK

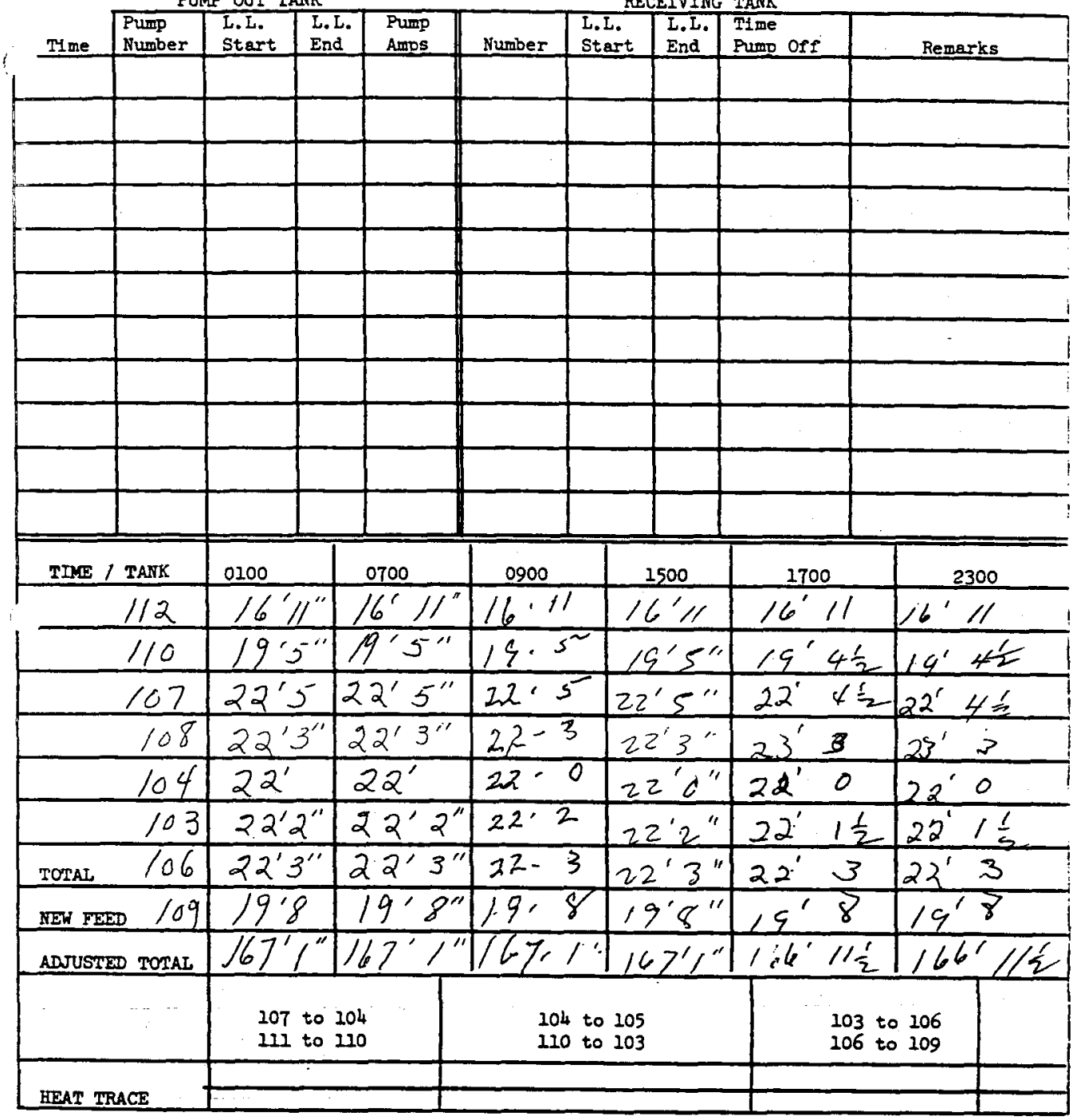

W"Walker:srb 9-29-70 
PUNP OUT TANK

RECEIVING TANK

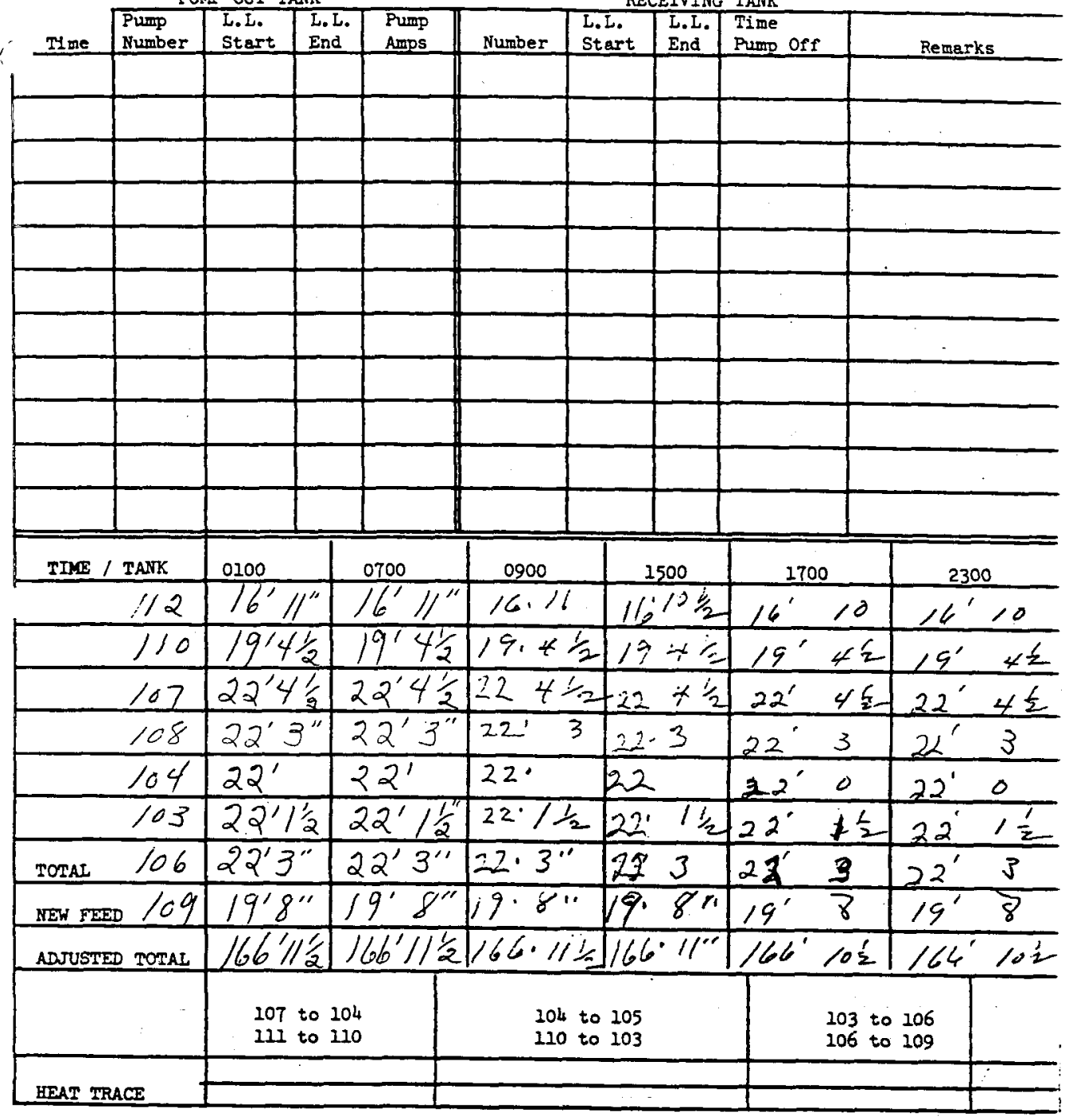

Walker:srb 9-29-70 
WHC-SO-WM-DP-209, ReV, 0

ITS-2 INVENTORY AND PUMPING Date $5-2 /$

PUMP OUT TANK RECEIVING TANK

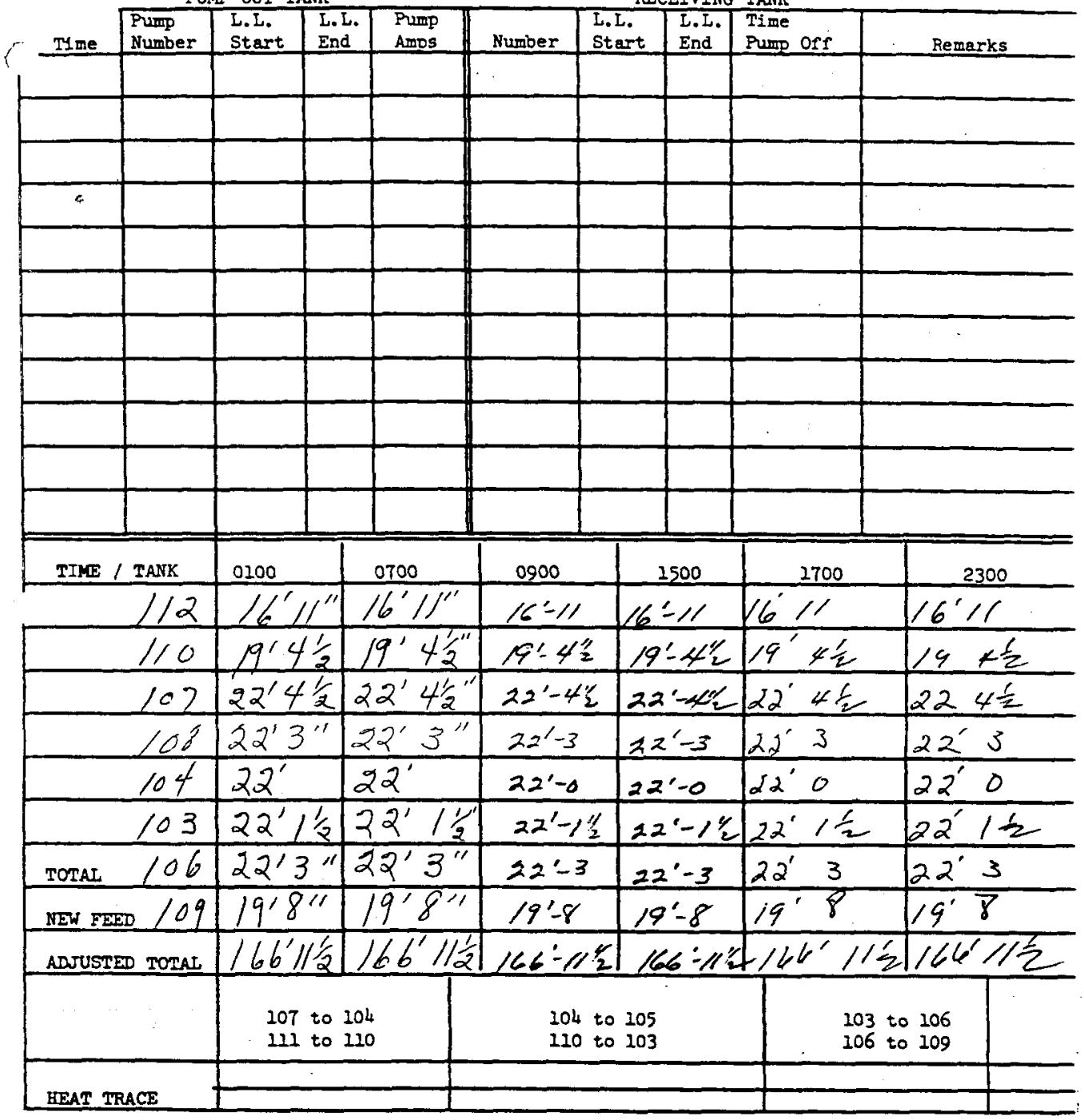

Walker:srb 9-29-70 
WHC-SD-WM-DP-209, Rev. 0

ITS-2 INVENTORY AID PUMPING

Dete $5-2 n-71$

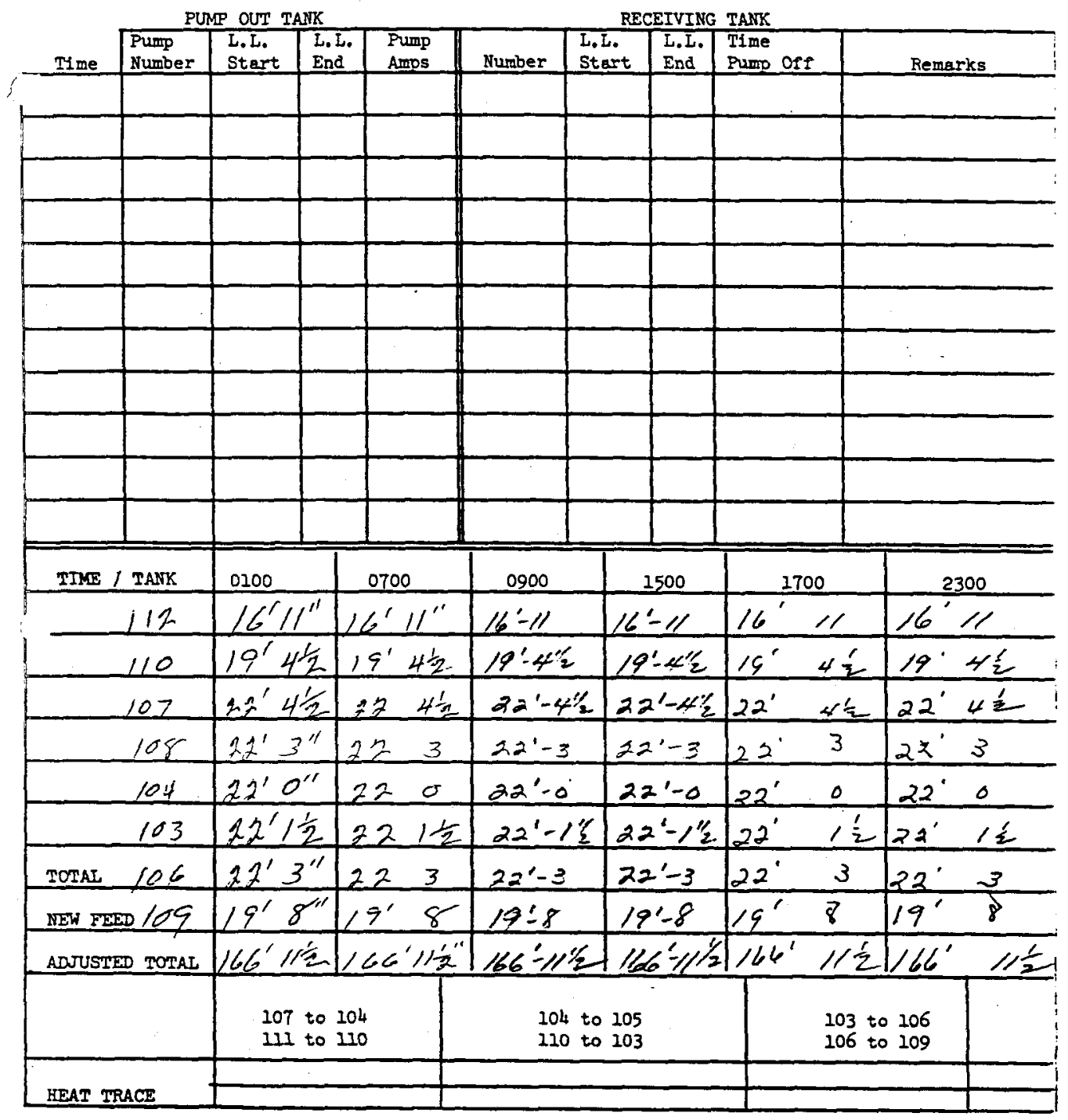

C Walker:srb 9-29-70 
ITS-2 INVENTORY AND PUMPING

Date $5-23-71$

PUNP OUT TANK RECEIVING TANK

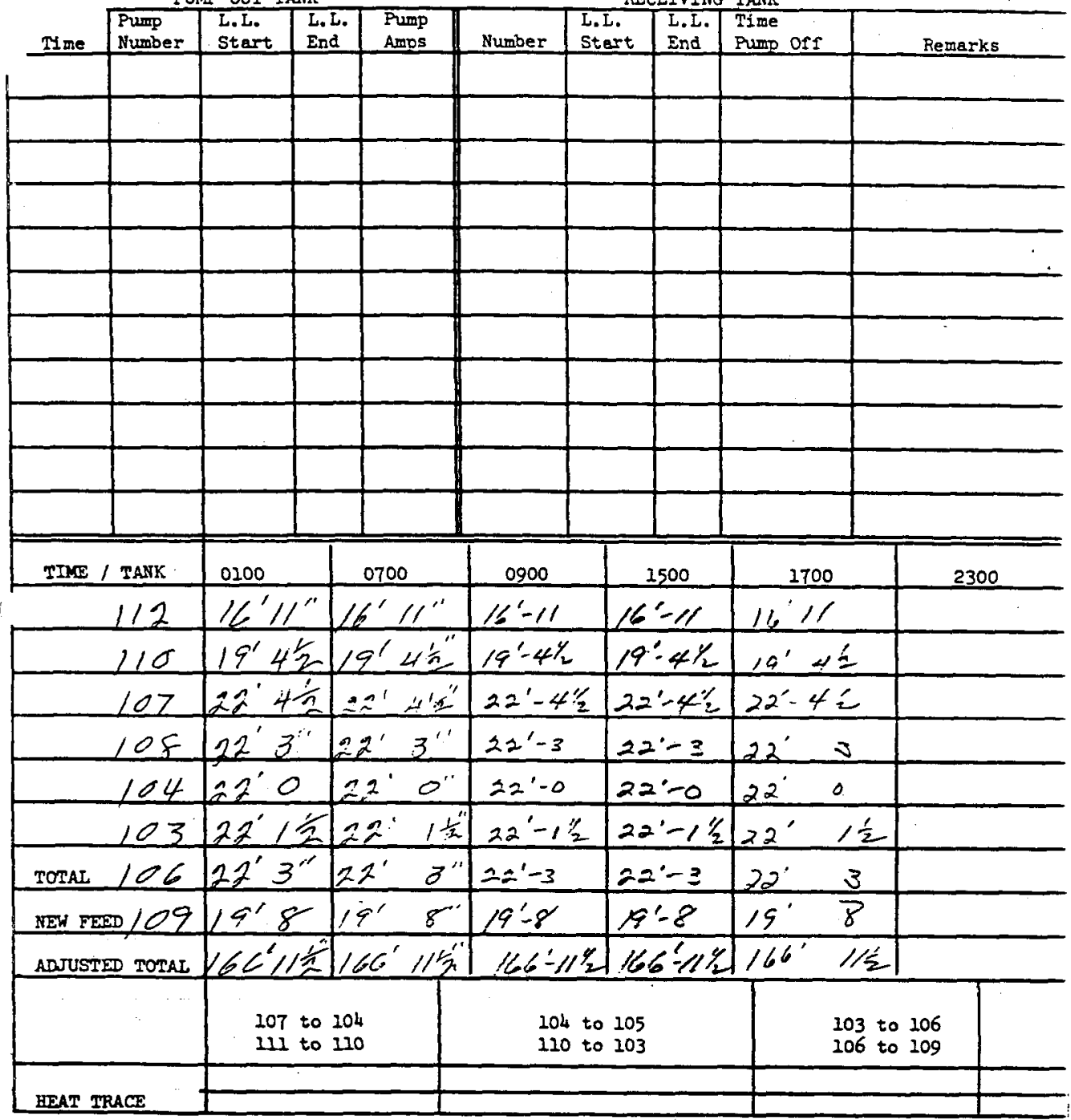

Walker:srb 9-29-70 
PUMP OUT TAMK

RECEIVING TANK

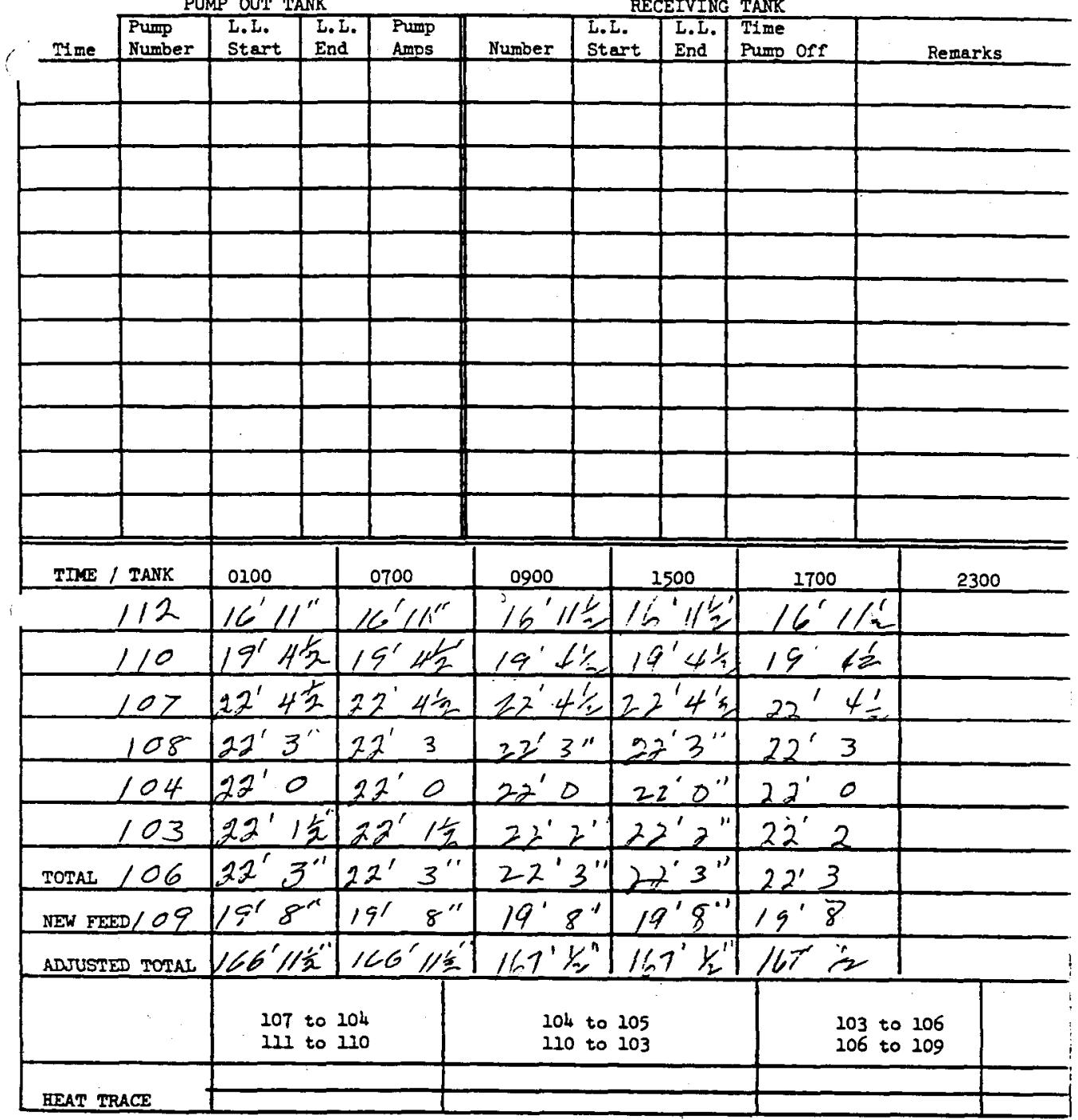

Walker:srb 9-29-70 
WHC-SO-LM-DP-209, ReV. D

ITS-2 INVENTORY AND FUMPING

Date MAY 251971

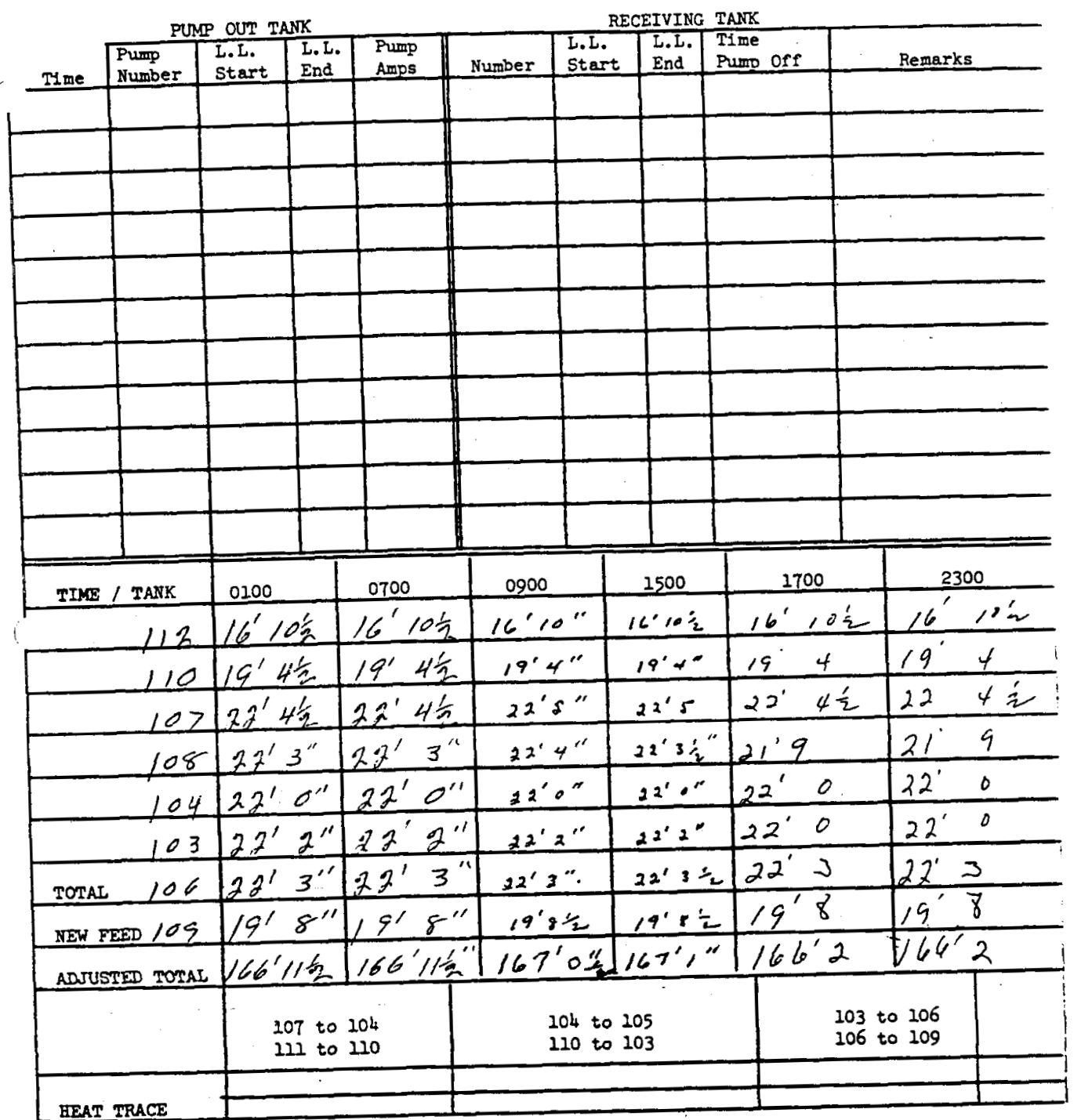

(. Walker:srb 9-29-70 
ITS-2 INVENTORY AND PUMPING

Date MAY 261971

FUMP OUT TANK

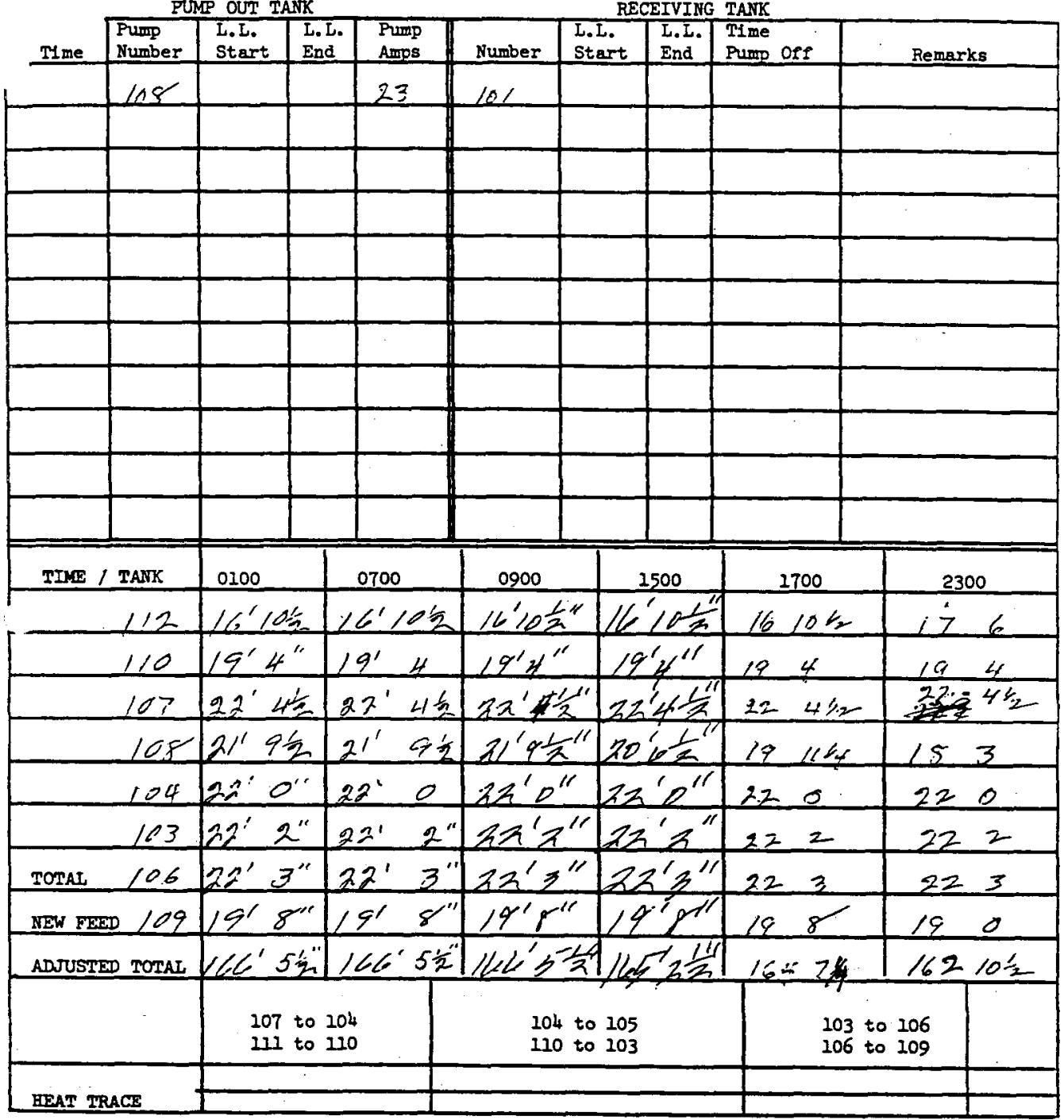

Wauker:srb 9-29-70 
PUMP OUT TANK

RECEIVING TANK

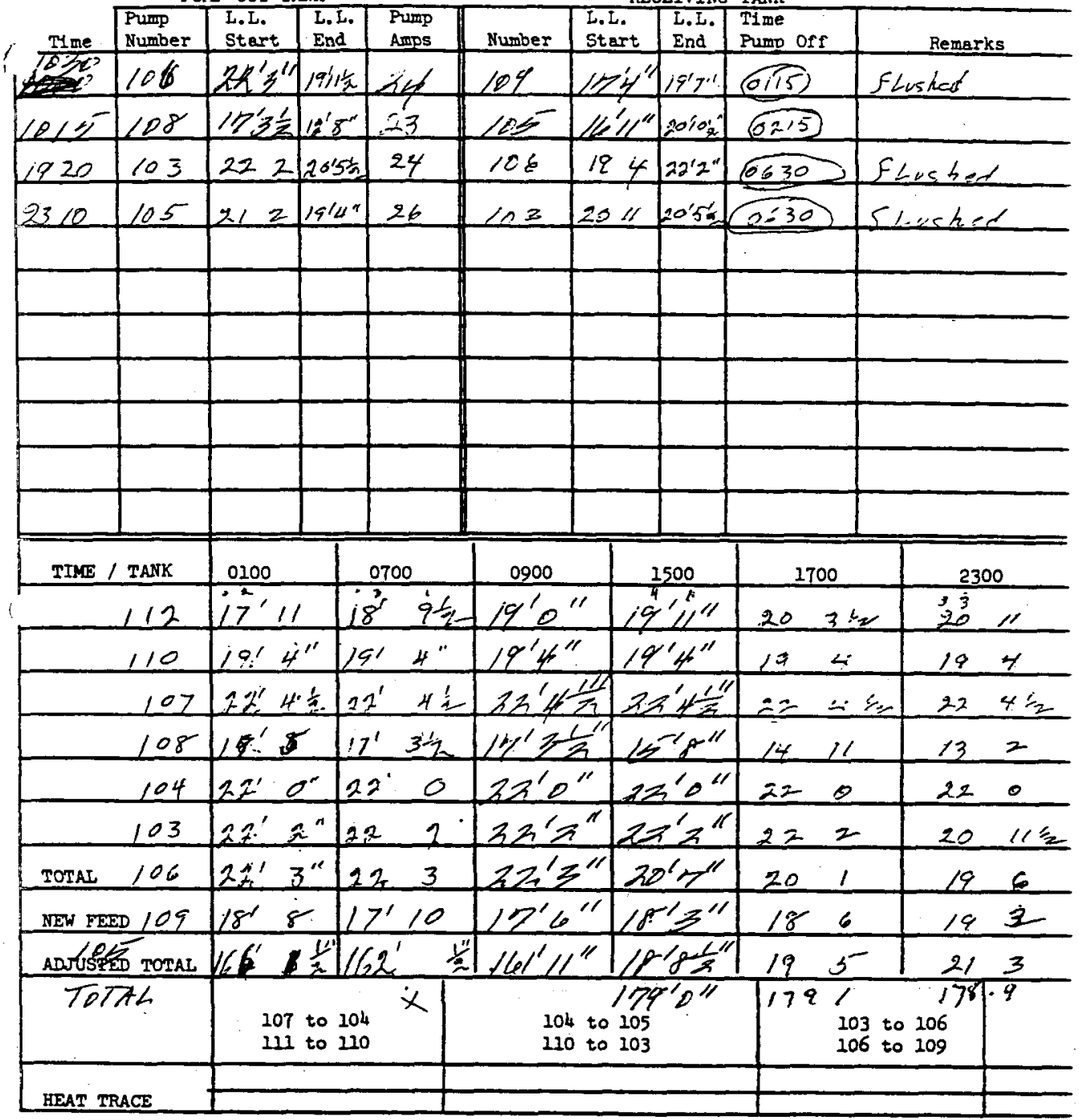

Walker:srb 9-29-70 
ITS-2 INVENTORY AND PUMPING Date MAY? 81971

PUNP OUT TAMK

RECEIVING TANK

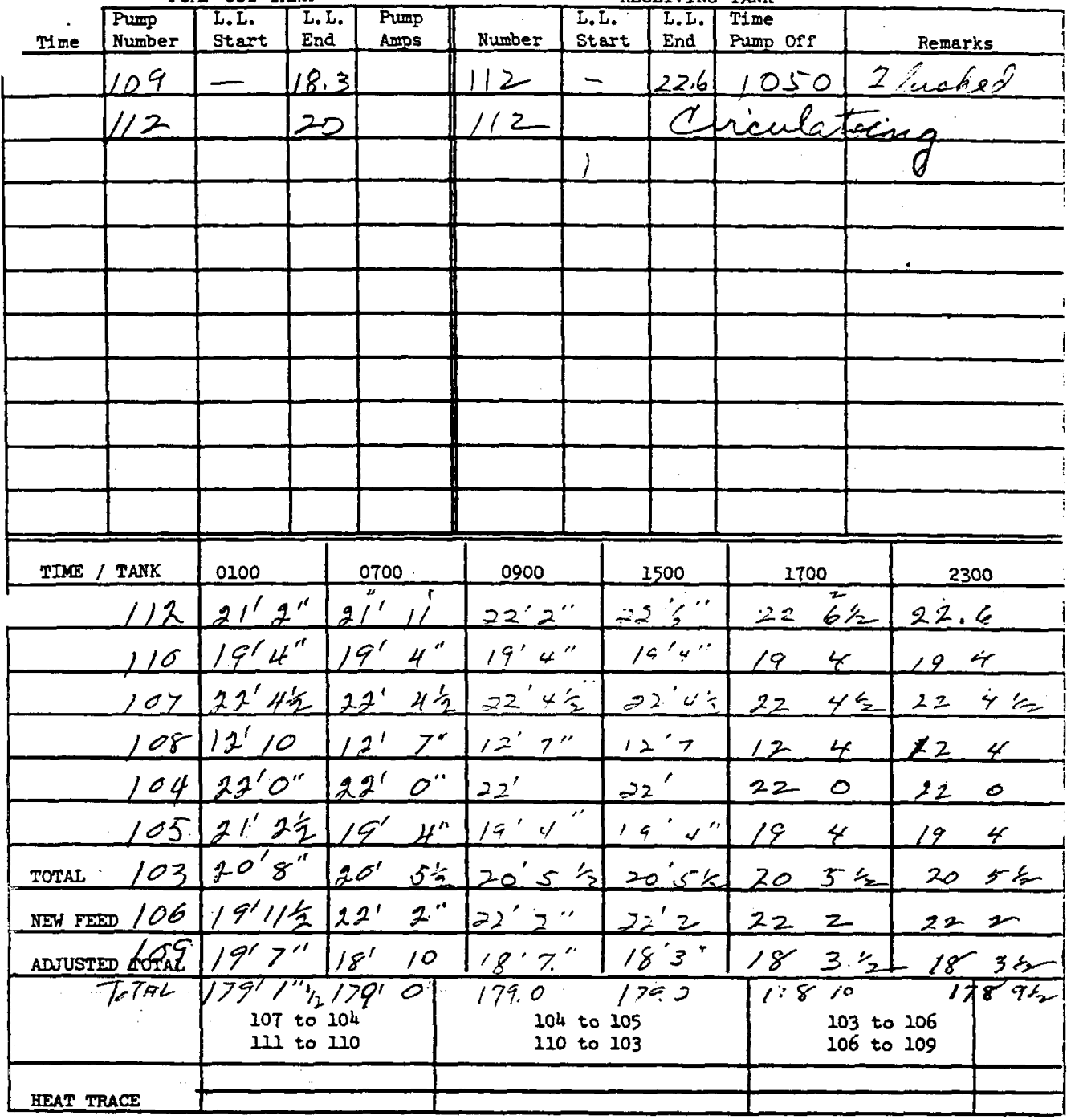

Lvater methe $000=20035$

Walker:srb 9-29-70 


\section{ITS-2 INVENTORY AND PUMPING Date $\overline{2}$ Z4-P1}

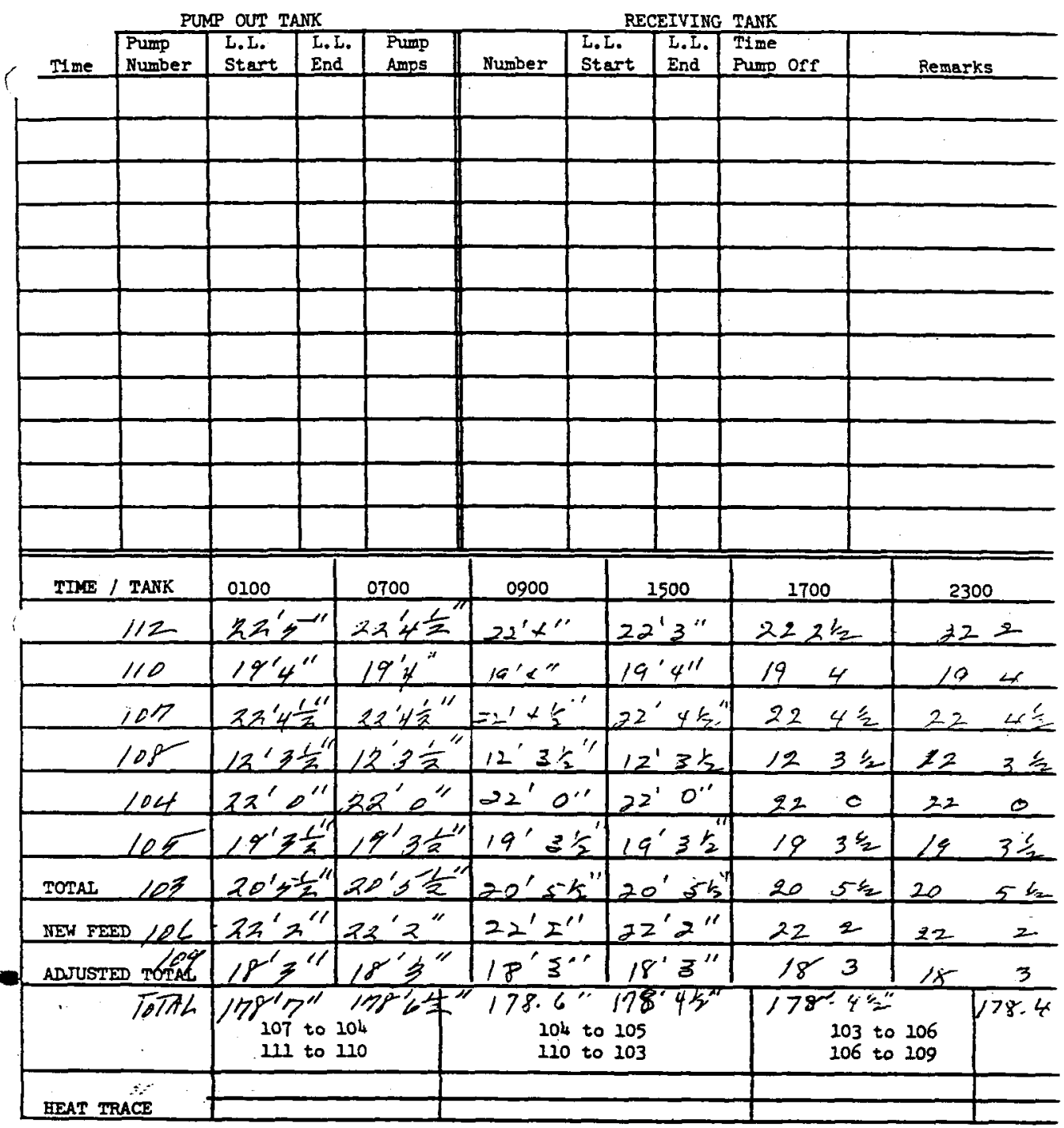

Walker:srb 9-29-70 
IIS-2 INVENTORY AND PUNPING Date $\angle^{-}-20-71$

PURP OUT TANK

RECEIVING TANK

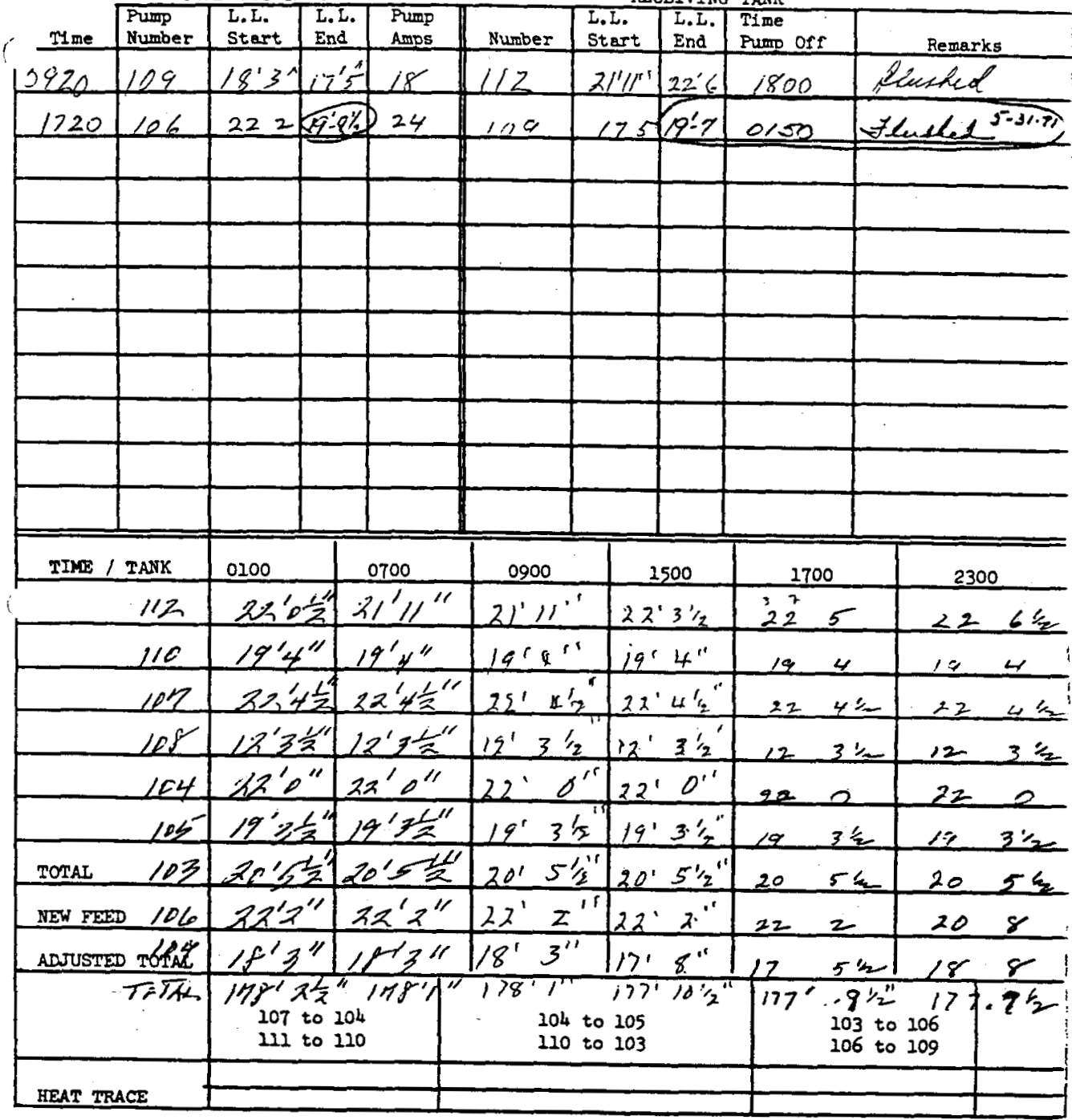

Helker :s5b 9-29-70 
ITS-2 INVENTORY AND PUMPING Dete $5-3 /-7 /$ PUMP OUT TANK RECEIVING TANK

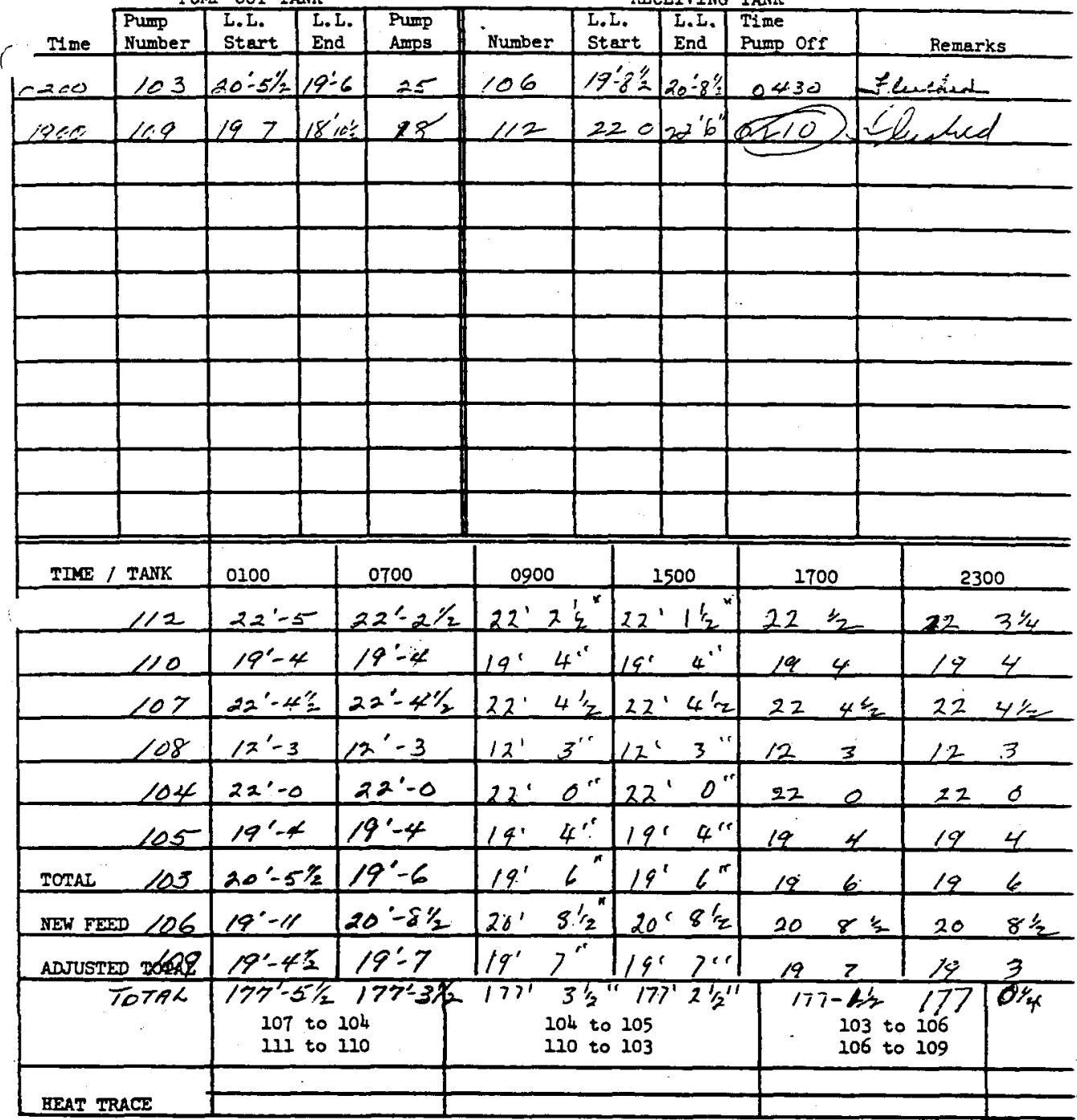

Walker:srb 9-29-70 
PUMP OUT TANK

RECEIVING TANK

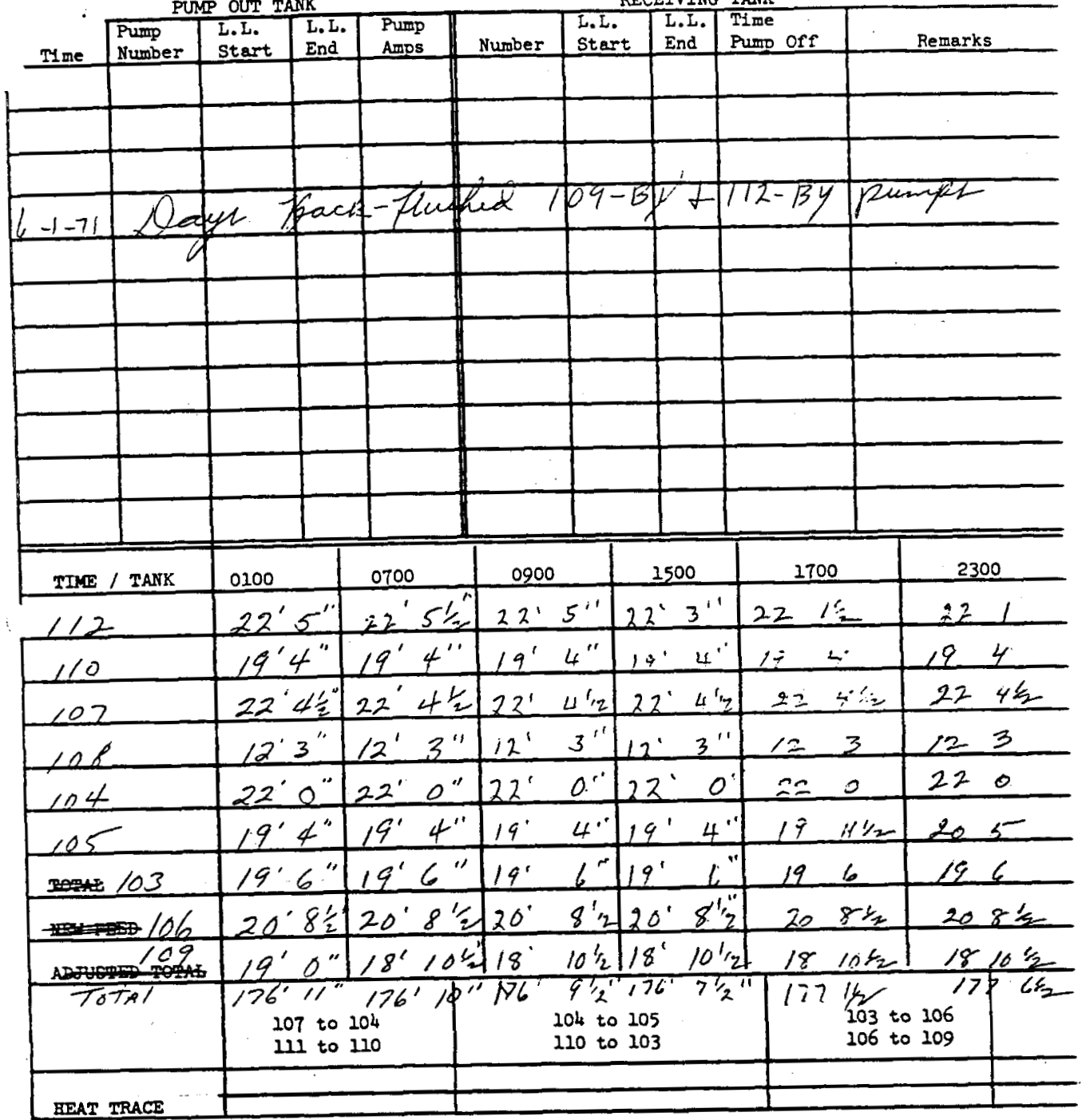

Halker:srb 9-29-70 
WHC-SD-LM-DP-209, ReV. 0

ITS-2 INVENTORY AMD PUMPING

Date $6 / 2 / 71$

PUMP OUT TANK

RECEIVING TANK

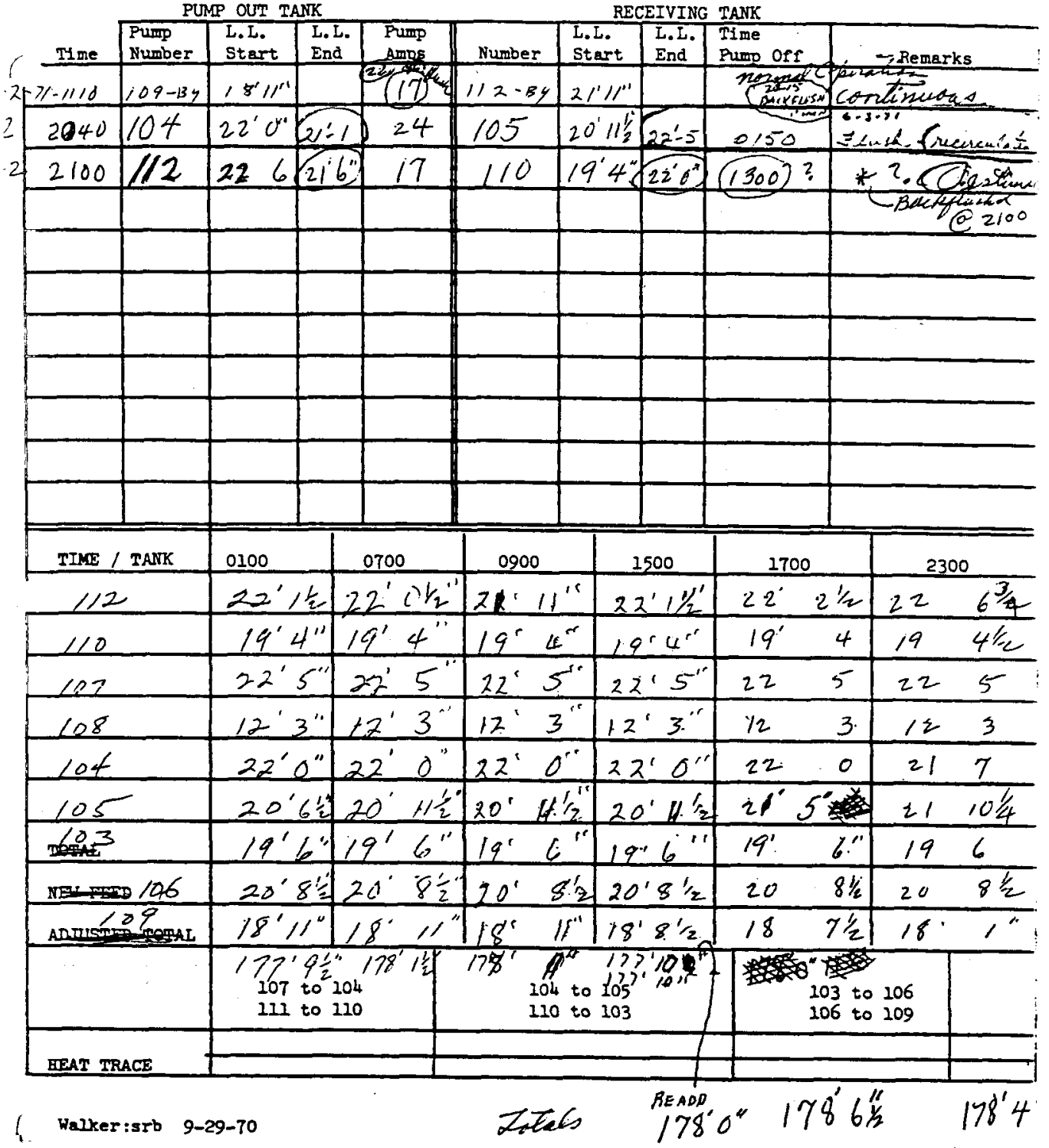

150 
ITS-2 INVENTORY AND PUMPING

Date $6-3-71$

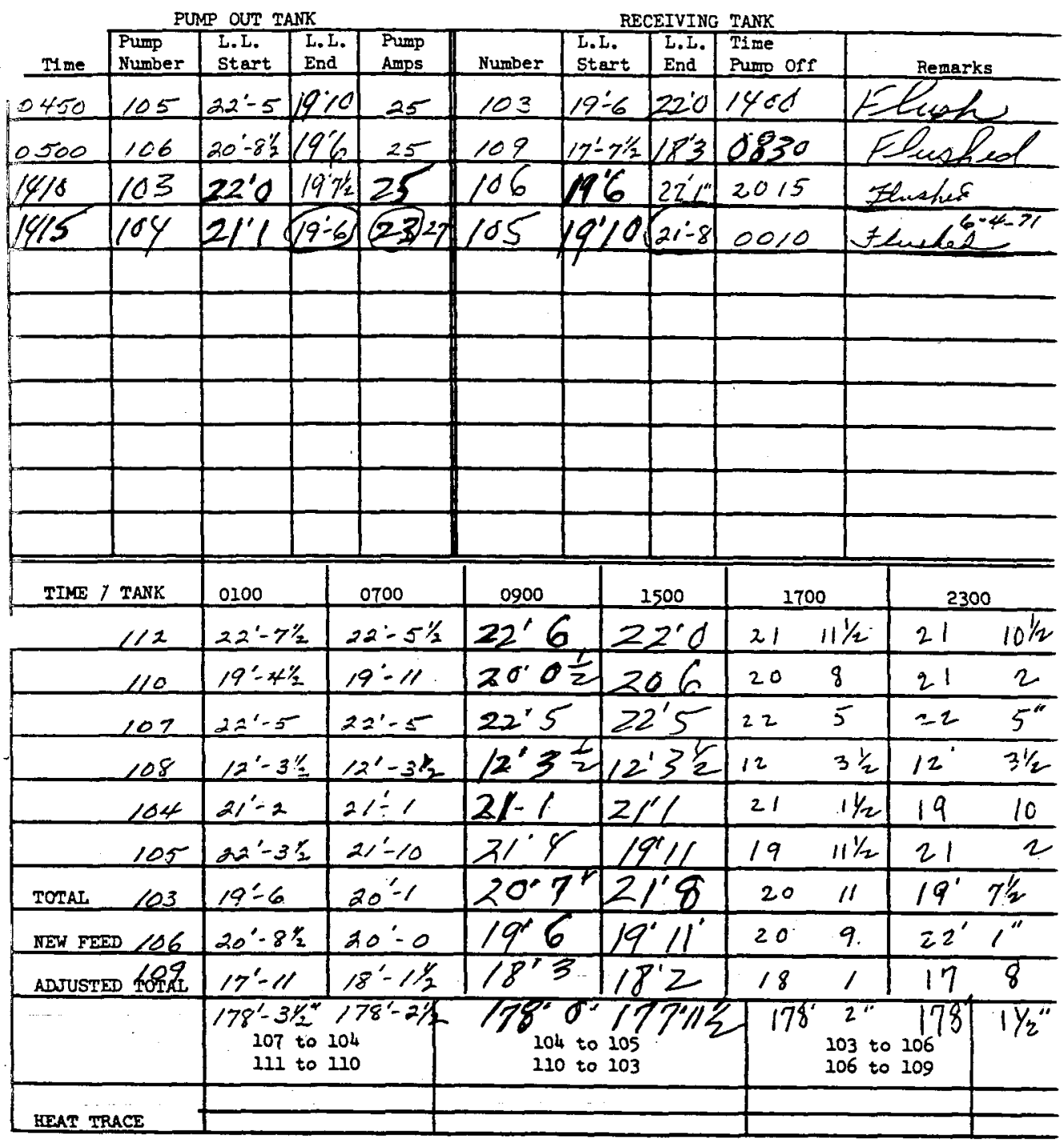

(. Walker:srb 9-29-70 
ITS-2 INVENTORY AND PUMPING

Date $6-4-71$

PUMP OUT TANK

RECEIVING TANK

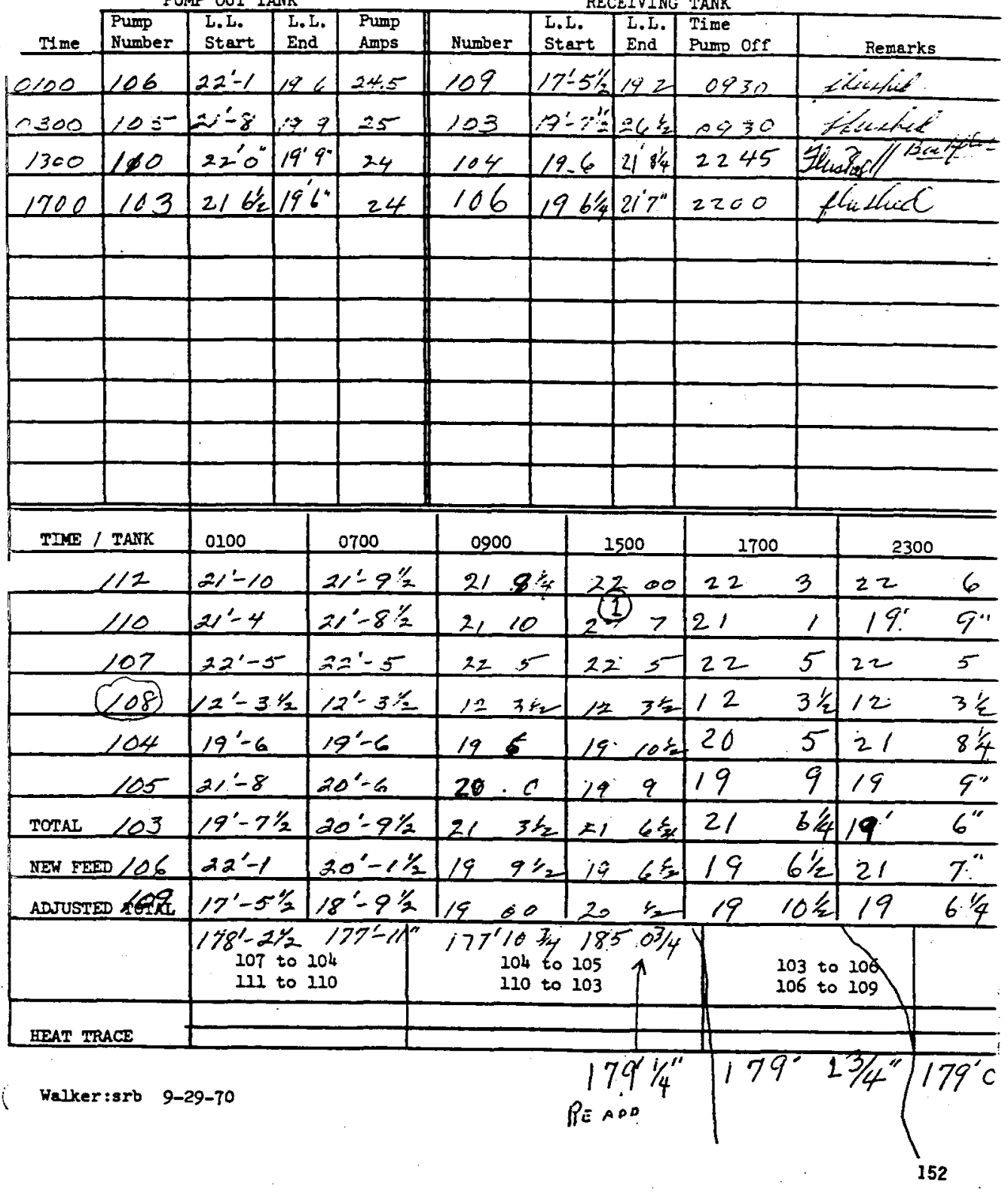


WHC-SD-MM-DP-209, REV. 0

ITS-2 INVENTORY ARD PUMPING Dete $(0-5-7)$

PUMP OUY TANK RECEIVING TANK

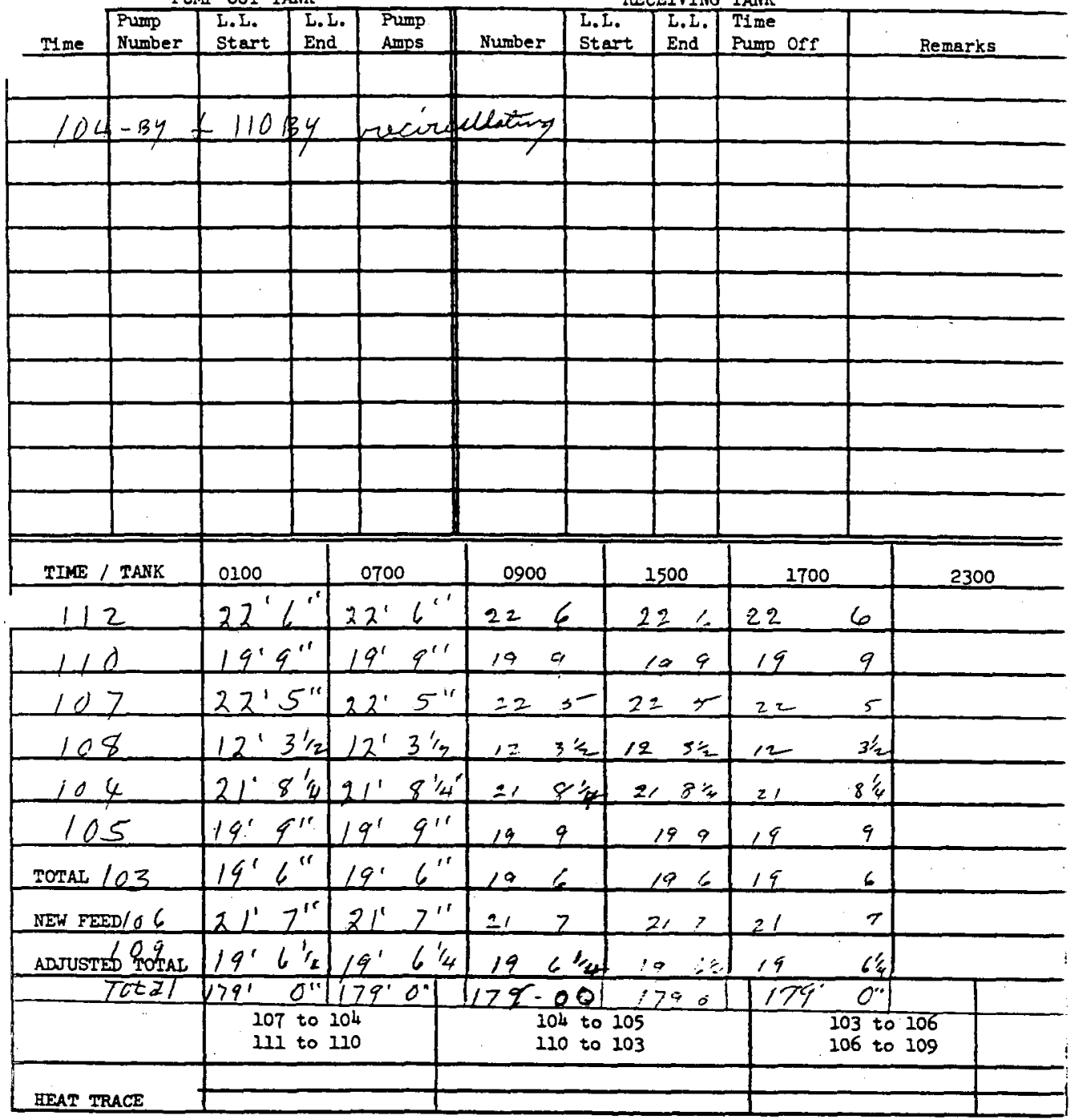

Waiker:srb 9-29-70 
ITS-2 INVENTORY AND PUMPING Date $6-6-21$

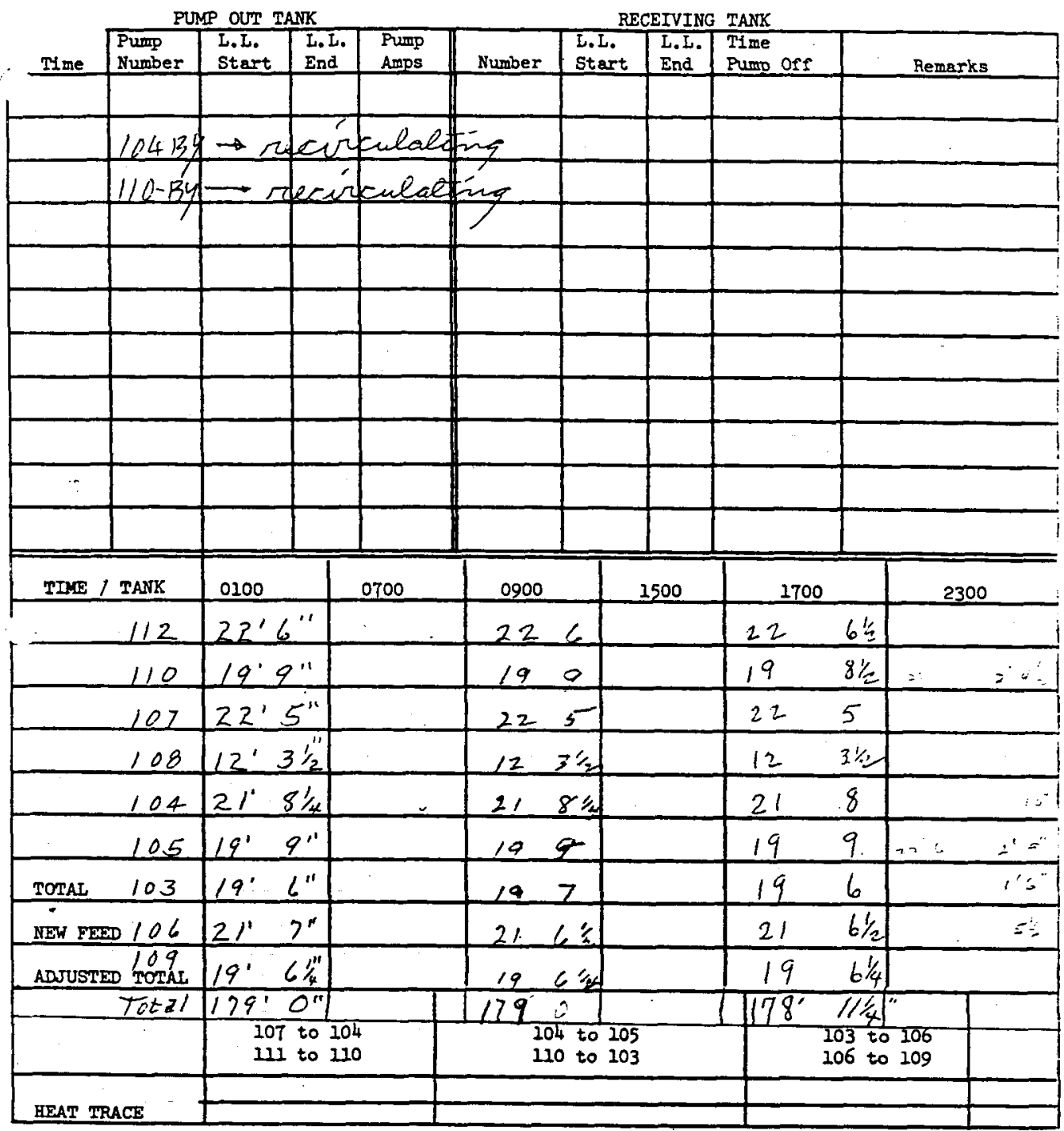

Wauker:srb 9-29-70 
ITS-2 INVENTORY AND PUNPING Date $6=7=21$

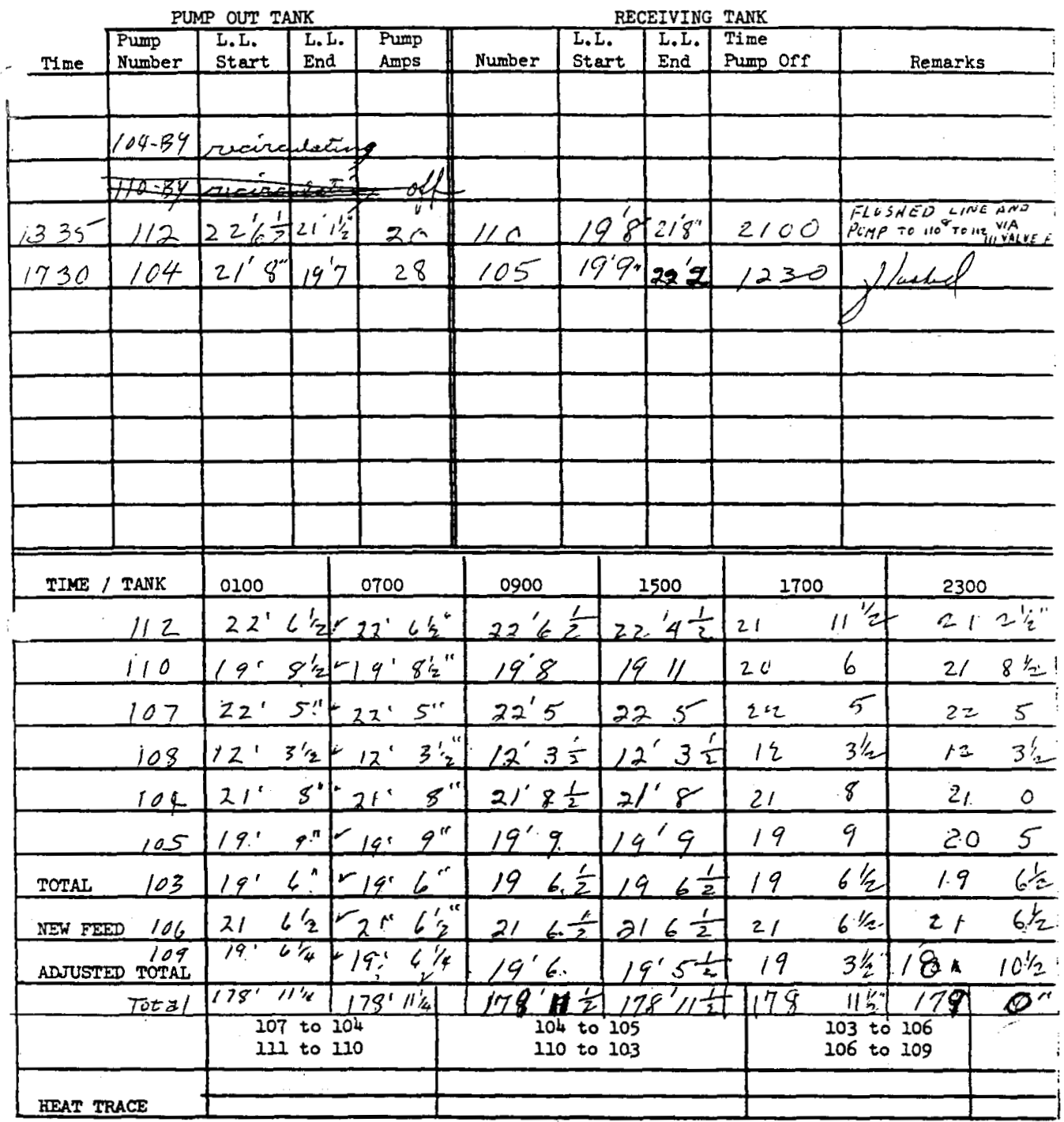

Walker:srb 9-29-70 
ITS-2 INVENTORY AND PUMPING $\quad$ Date $l-8.21$

PUMP OUT TANK

RECEIVING TAMTK

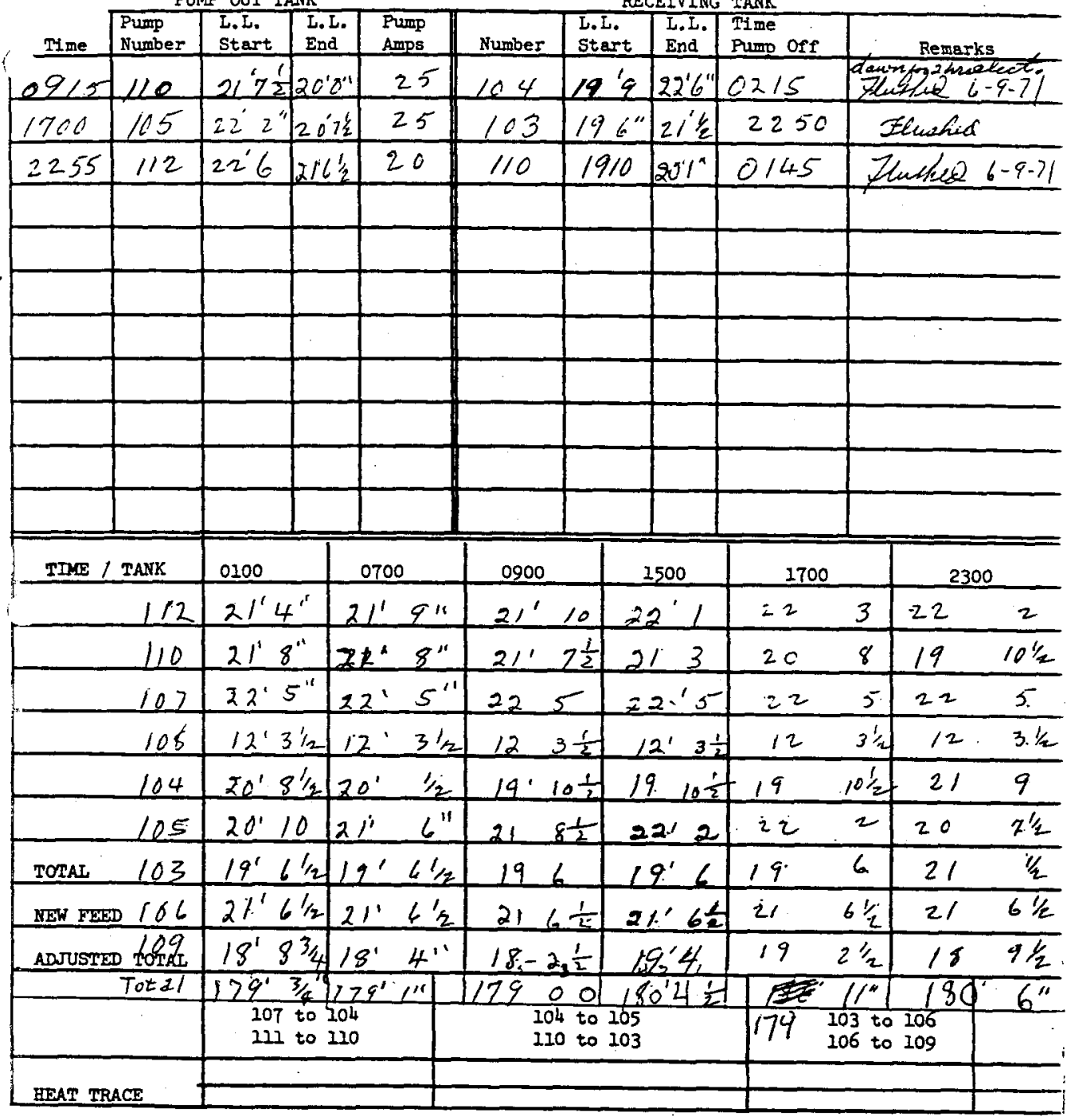

Walker:srb 9-29-70 
ITS-2 INVENTORY AND PUMPING

Date Ming 1971

PUMP OUT TANK

RECEIVING TANK

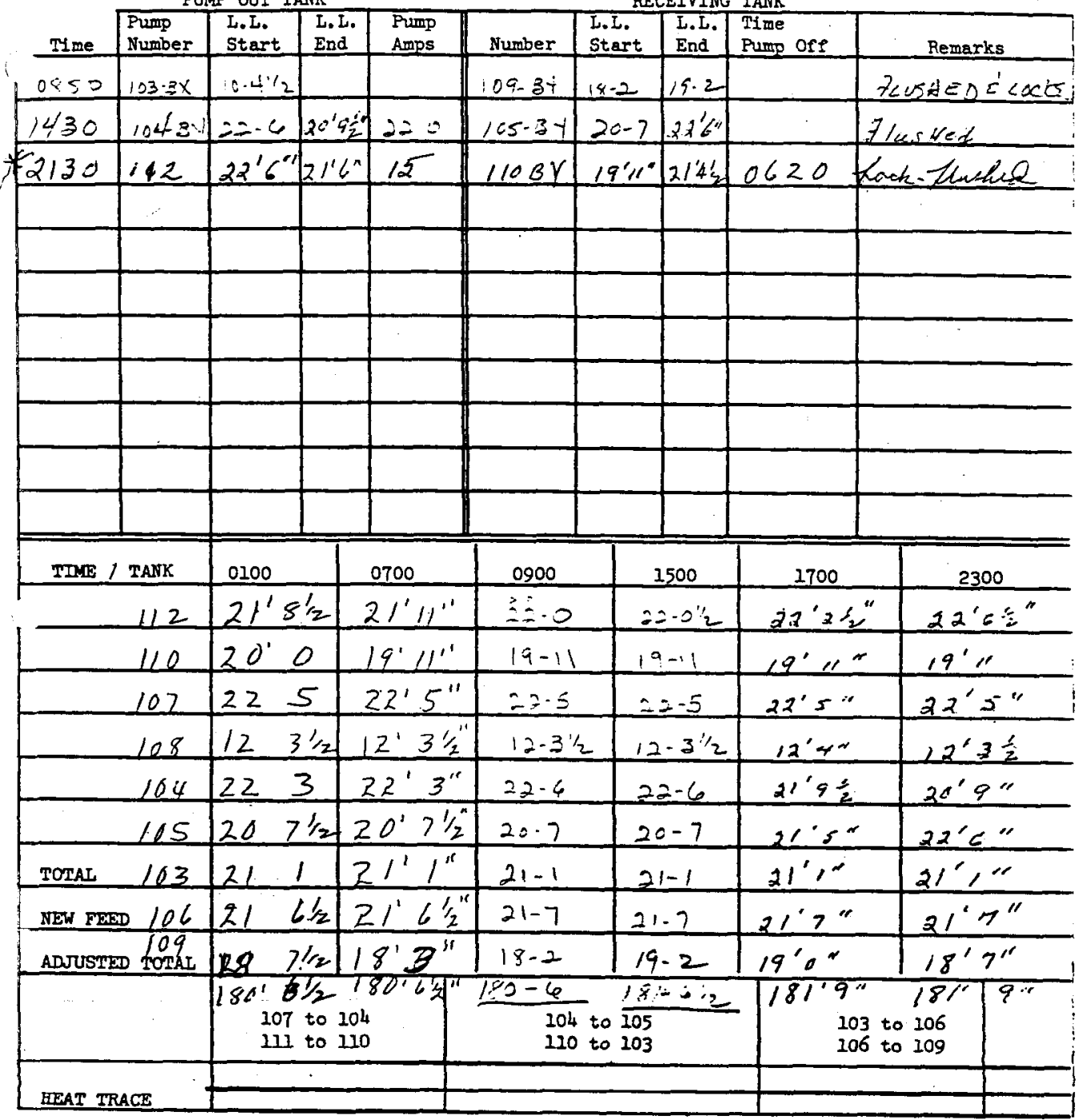

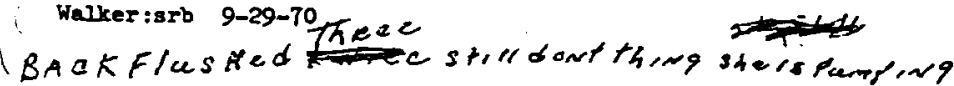

109 off + Flas $H C d 2330$ 
ITS-2 INVENTORY AND PUMPING

Date $6-10-71$

PUMP OUT TANK

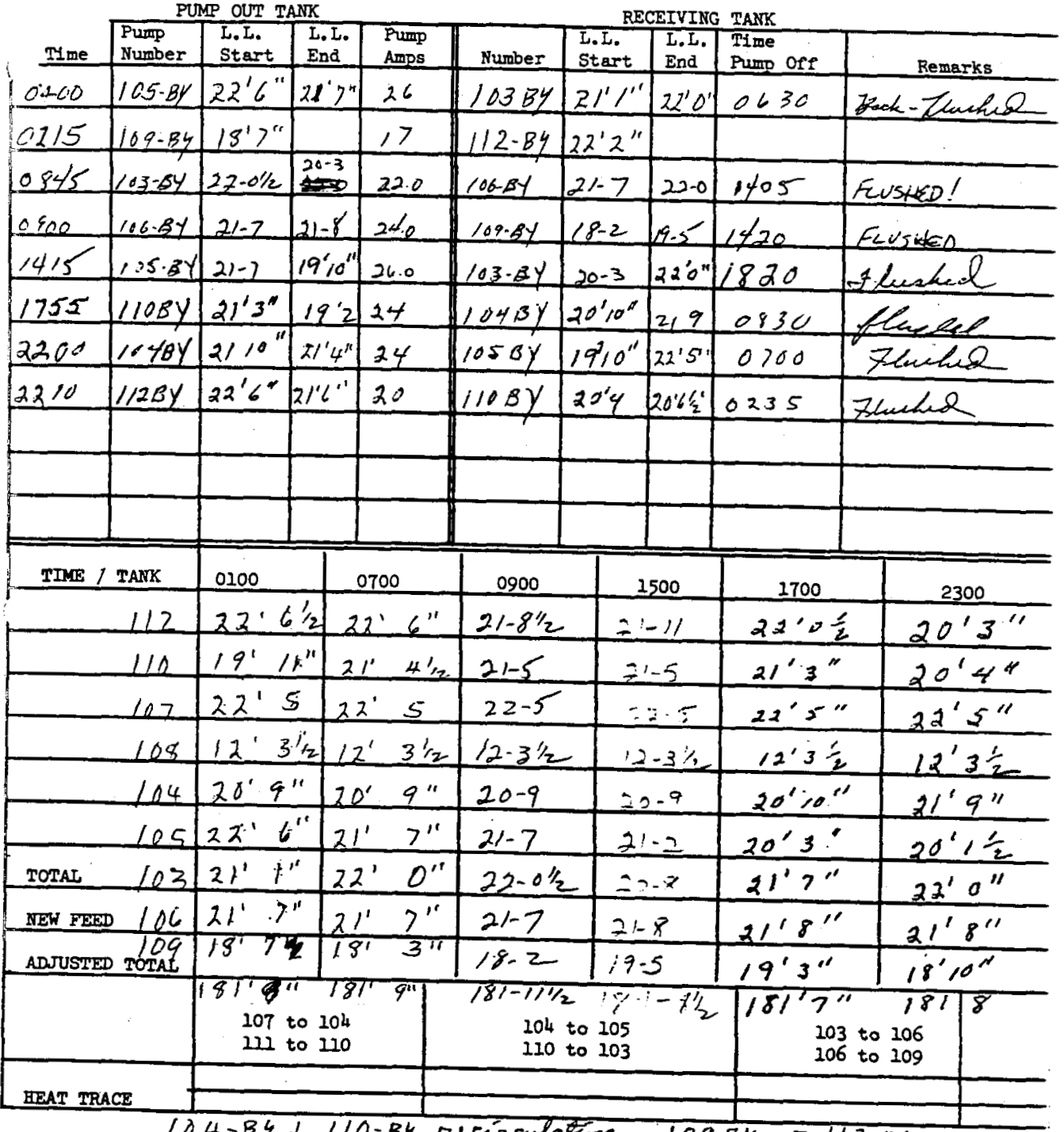

$104-8 y+110-8 y$ recículatey $10984=112-8 y$

Walker:srb 9-29-70 /12-BY Condendete samplen is 1410 6c @8 06x0

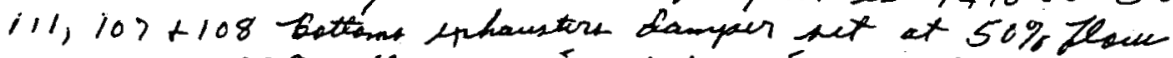
110 at $100 \%$ thow will take piotares sin 110-15y tank 
ITS-2 INVENTORY AND PUMPING Date TJN1+10274

PUMP OUT TANK

RECEIVING TANK

JUN 11197

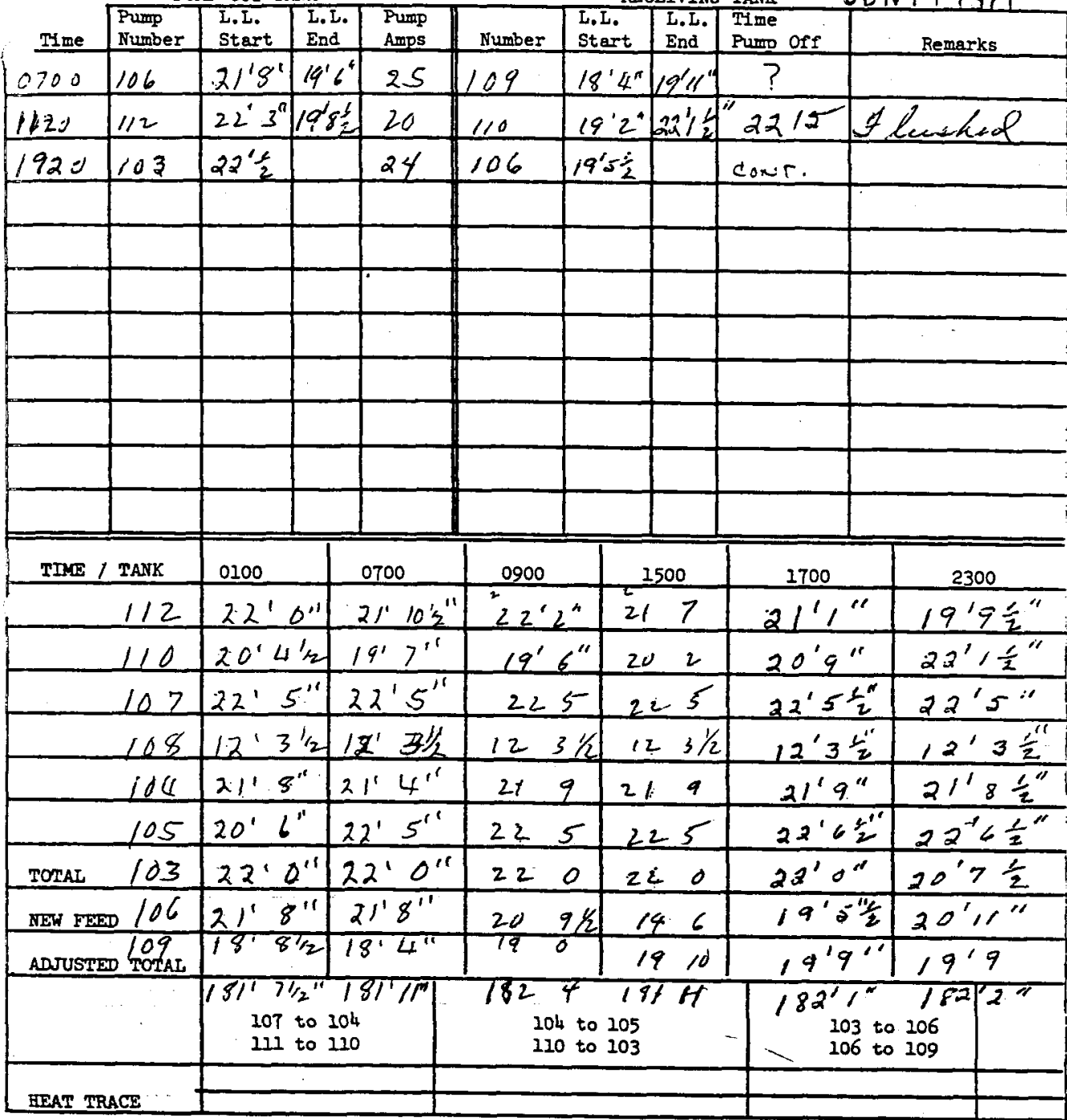

Walker :srb 9-29-70 
ITS-2 INVENTORY AND PUMPING Date $6-12-71$

PUMP OUT TANK

RECEIVING TANK

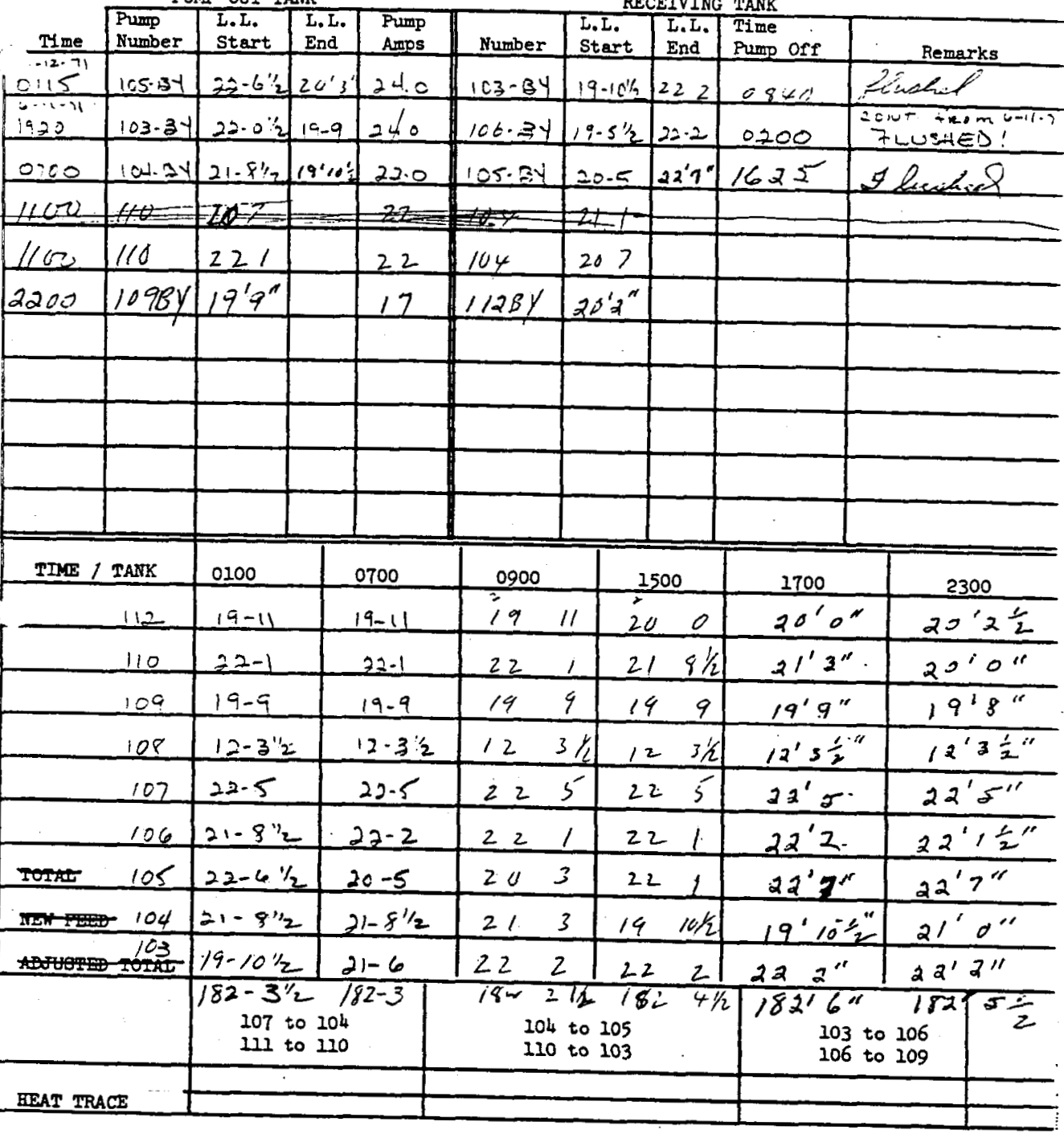

Walker:srb 9-29-70 0700-KMU CLECKED 11-BY PUmp Pr.

$$
\frac{300 \mathrm{w} / \mathrm{s}}{100 \mathrm{~m} / \mathrm{c}}
$$




$$
\text { ITS-2 INVENTORY AND PUMPING Date } 6.13 .7
$$

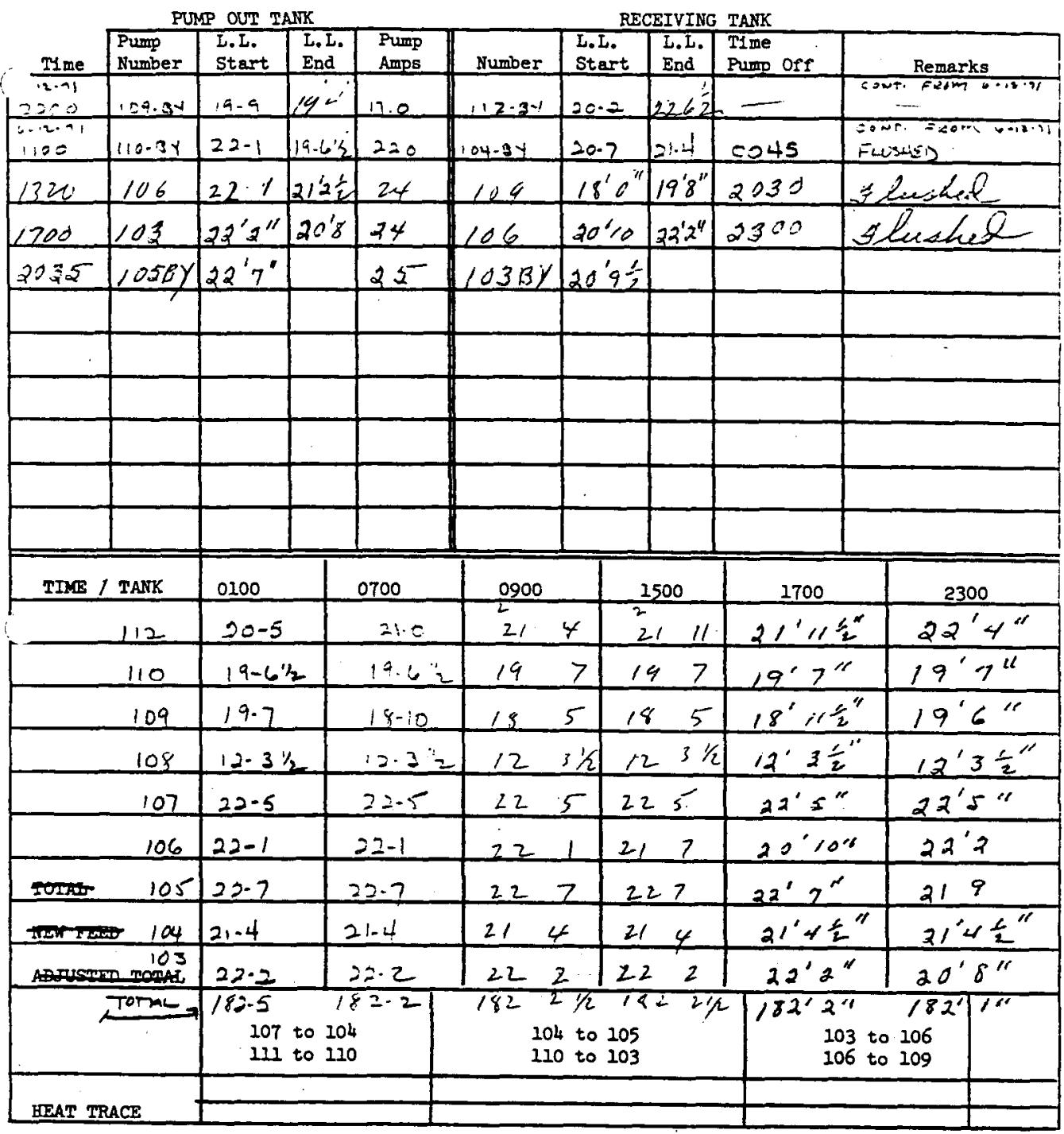

(Wayker:srb 9-29-70 $\left[\begin{array}{r}0045-118-84 \text { is Recrecine. } \\ 104-84 " 14\end{array}\right.$ 
ITS-2 INVENTORY AND PUMPING Date $6-14-71$ PUNP OUT TANK RECEIVING TANK

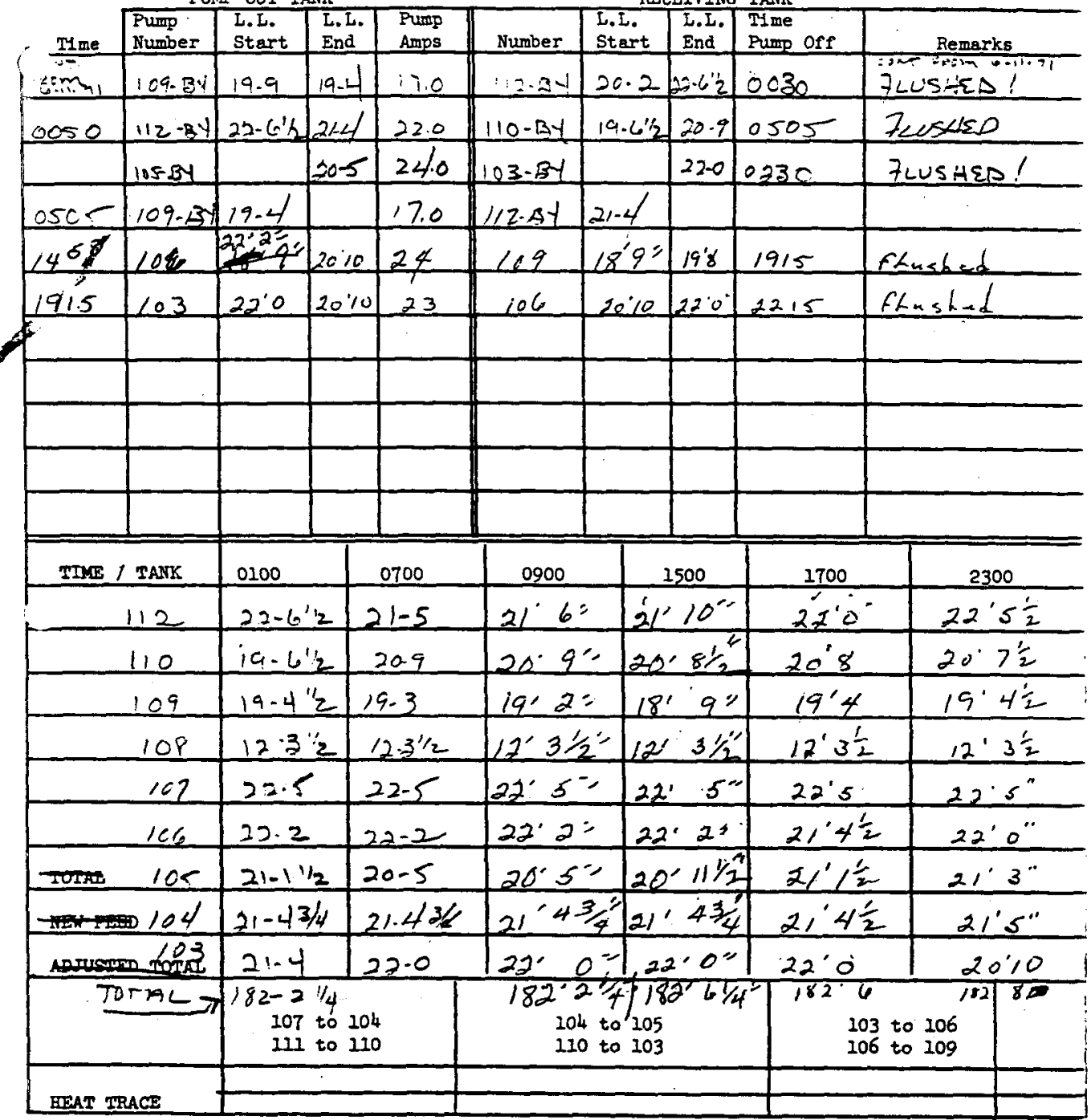

(Walker:srb 9-29-70 
PUMP OUT TANK

RECEIVING TANK

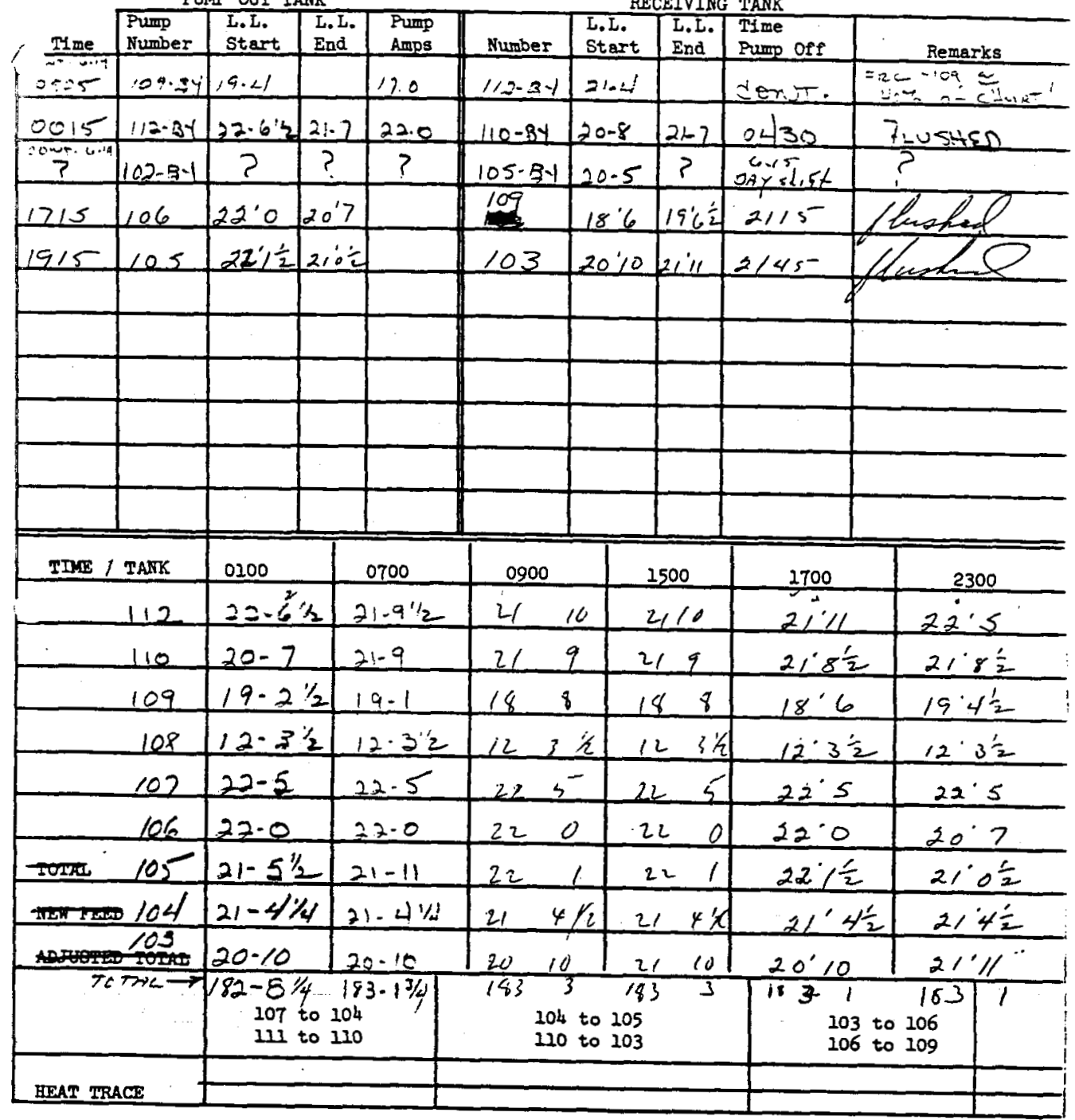

(Walker:srb 9-29-70 
ITS-2 INVENTORY AND PUMPING

Date $6-16-71$

PUMP OUT TANK

RECEIVING TANK

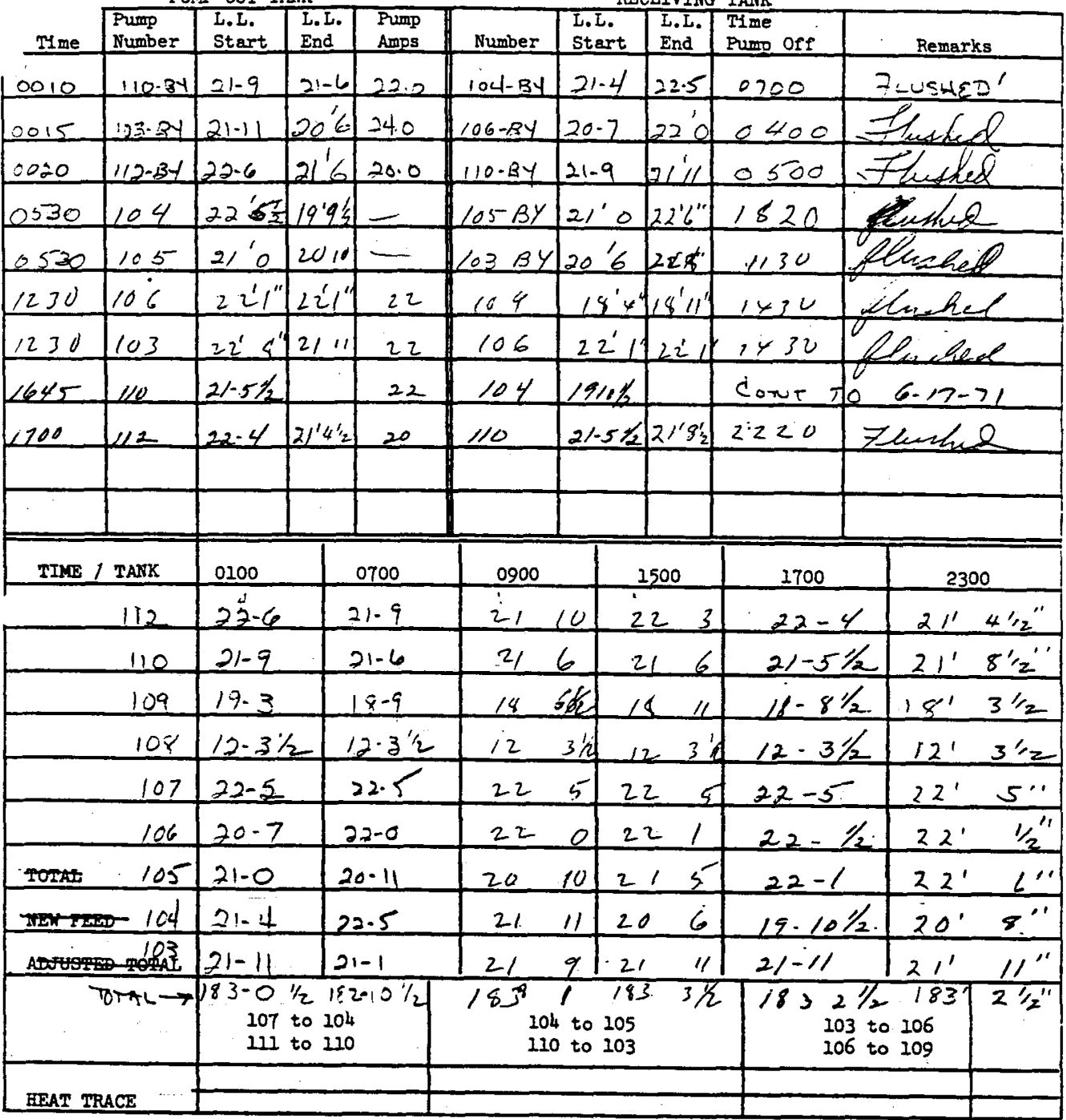

Walker:srb 9-29-70 
ITS-2 INVENTORY AND PUMPING

Dete $6.17-71$

PUMP OUT TANK

RECEIVING TANK

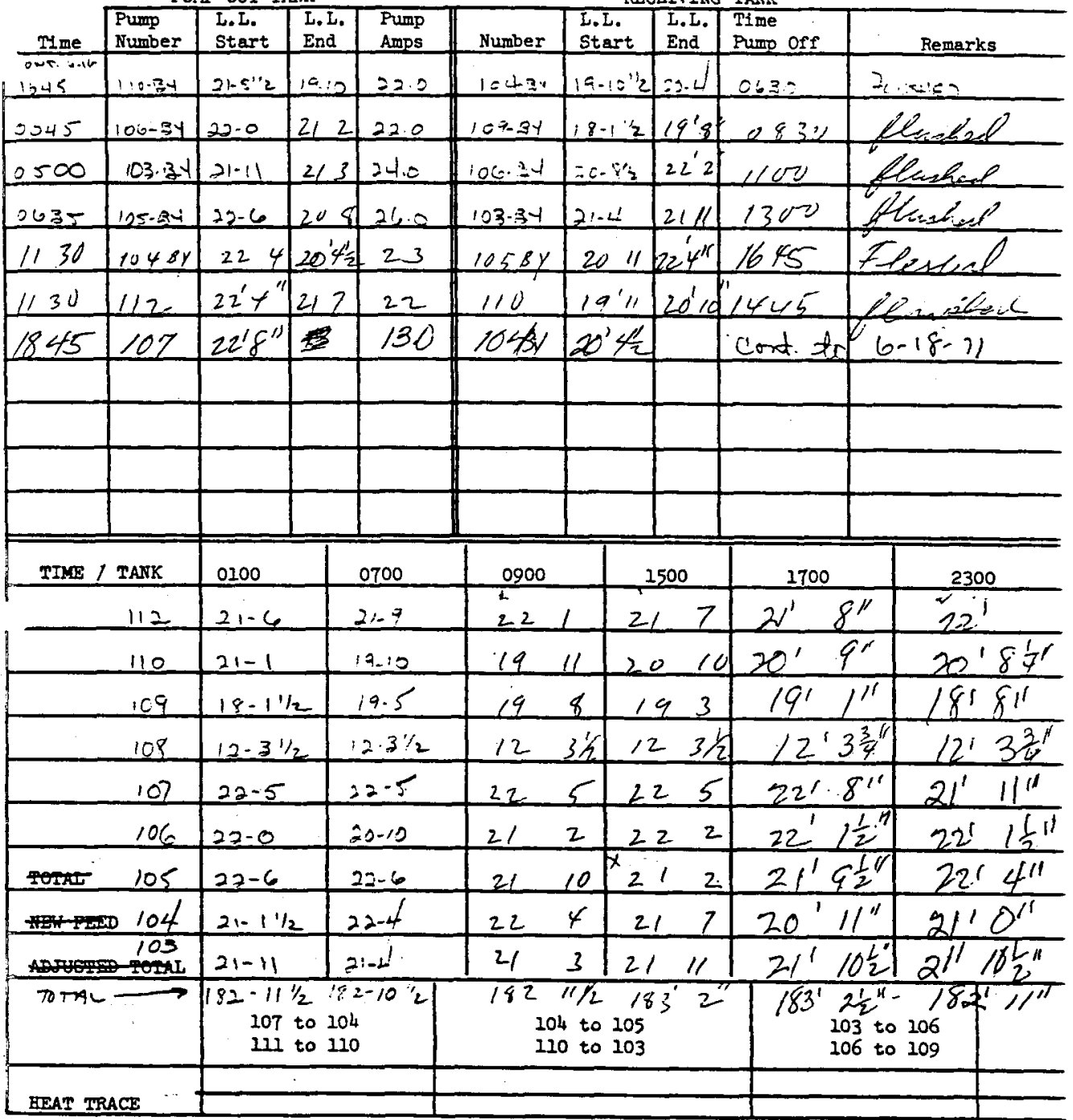

Walker: $85 b \quad 9-29-70$ 
ITS-2 INVENTORY AND PUMPING

Date $(e-18-2)$

PUMP OUT TAMK

RECEIVING TANK

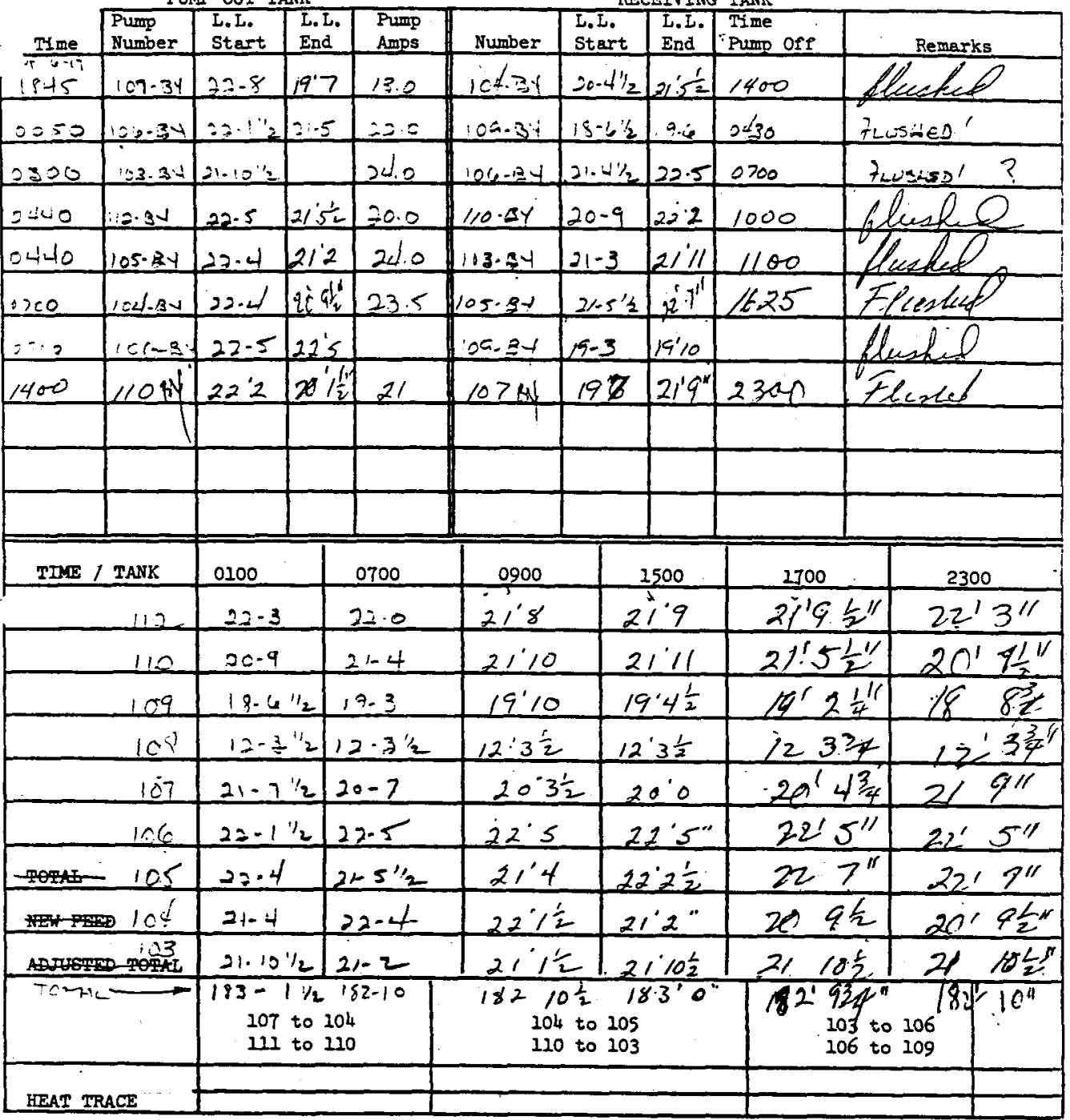

WaIker:srb 9-29-70 
ITS-2 INVENTORY AND PUMPING Date $6-197$

PUMP OUT TANK

RECEIVING TANK

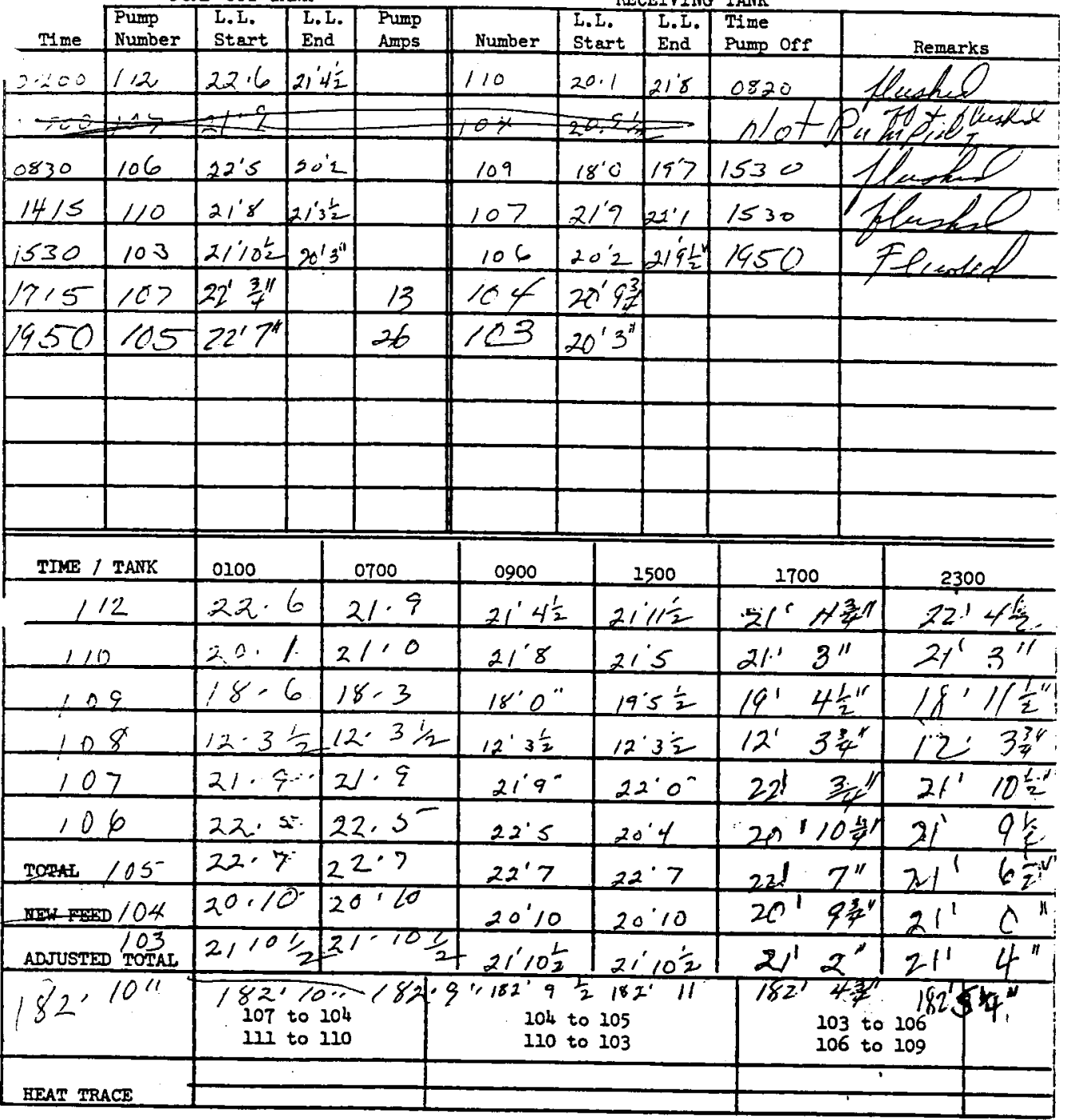

Walker:srb 9-29-70 
ITS-2 INVENTORY AND PUMPING

Date $6-207$

PUMP OUT TANK

RECEIVING TANK

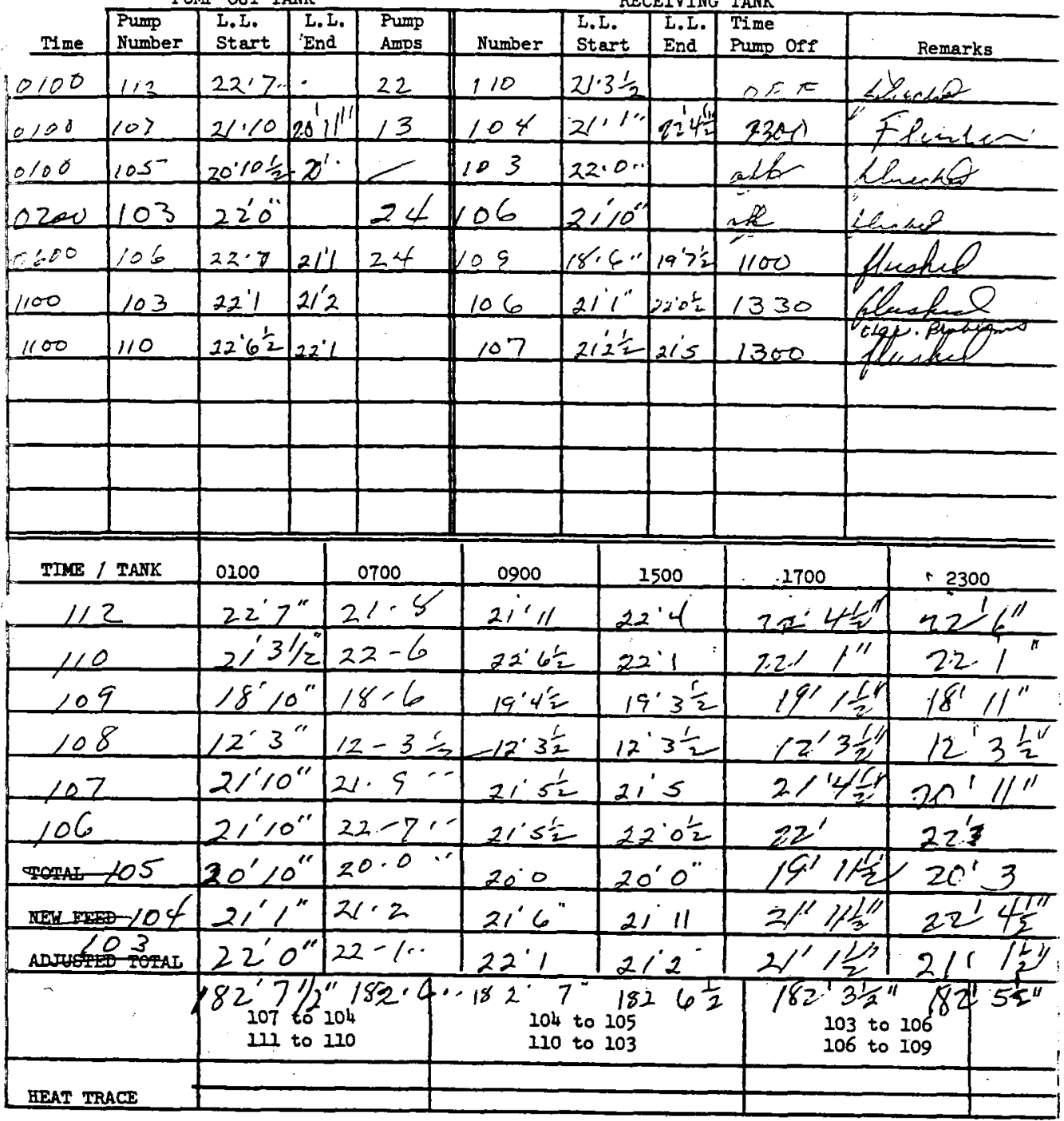

Wulker:srb 9-29-70 
ITS-2 INVENTORY AND PUMPING Date $6.2,7 /$

PUMP OUT TANK

RECEIVING TANK

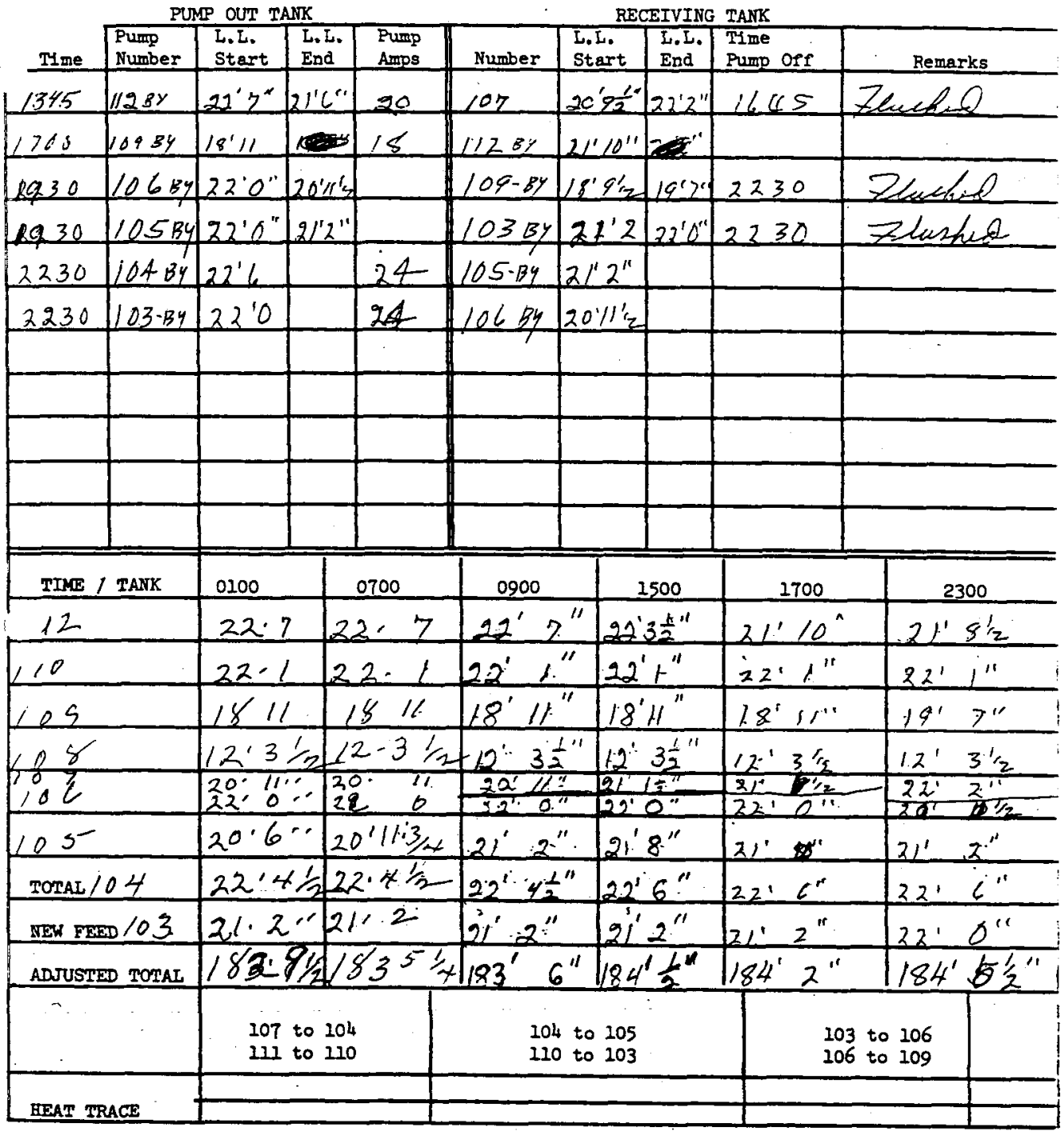

Waiker :srb 9-29-70 
ITS-2 INVANTORY AND PUNPING Date $\frac{6-22-7}{7}$

PUMP OUT TANK

RECEIVING TANK

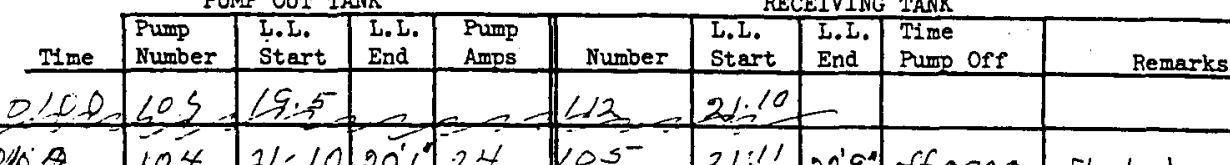

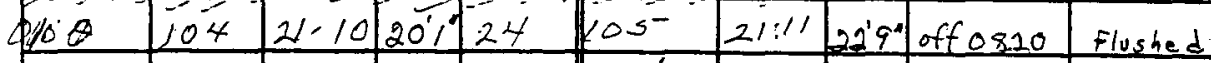

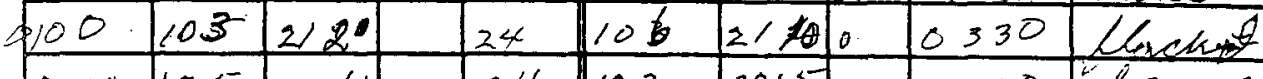

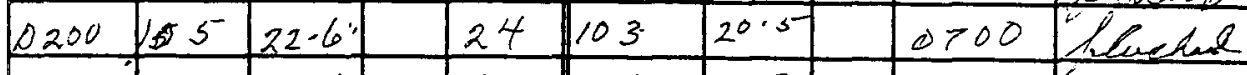

\begin{tabular}{l|l|l|l|l|l|l}
0200 & 107 & 22.1 & & 13 & 104 & 21.3
\end{tabular}

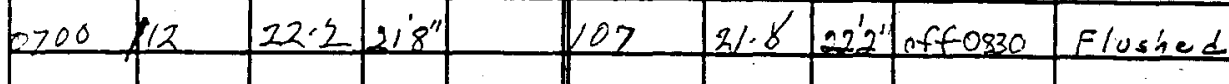

\begin{tabular}{l|l|l|l|l||}
\hline & & & & \\
\hline & & & & \\
\hline & & & & \\
\hline
\end{tabular}

\begin{tabular}{|c|c|c|c|c|c|c|}
\hline TIME / TANK & 0100 & 0700 & 0900 & 1500 & 1700 & 2300 \\
\hline$\angle 1 Z$ & $2110^{\prime \prime}$ & $22-2$ & & $21^{\prime} 8^{\prime \prime}$ & $21^{\prime}$ & $C^{\prime \prime}$ \\
\hline 110 & $22^{\prime} 1^{\prime \prime}$ & $2 z-1$ & $22^{\prime} 1$ & $22^{\circ} 1^{\prime \prime}$ & $22^{\prime}-\frac{1}{2}$ & 22 \\
\hline 109 & $1935^{\circ}$ & 19.6 & $19^{\prime} 5$ & $19^{\prime} 5^{\prime \prime}$ & $19^{\prime} 3^{\prime \prime}$ & $10 \frac{1}{2}$ \\
\hline 108 & $12.31 / 2^{\prime \prime}$ & $12-3$ & & $1.2^{\prime} \quad 3 \frac{1}{2}$ & $3 k$ & 12 \\
\hline 102 & $22^{\prime} 1^{\prime \prime}$ & 21 & $\alpha$ & $91^{\prime} 5^{\prime \prime}$ & $21^{\prime}$ & $2 e^{\prime} 4 \frac{1}{1}^{\prime}$ \\
\hline 106 & $21^{\prime} 10^{\circ}$ & $22 \cdot 0$ & $21^{\prime}$ & $2.2^{\prime} 0 \frac{1}{2}$ & $22^{\prime}$ & $22 \frac{1}{4}$ \\
\hline $\operatorname{sos} 2105$ & $21^{\prime \prime} 1^{\circ}$ & $22-$ & $22^{\prime} 9$ & $22^{\prime} 8 \frac{1}{2}^{\prime \prime}$ & $22^{\prime}$ & $2.2 \cdot 8$ \\
\hline $\operatorname{Iax} 104$ & $21^{\prime} 10^{\prime \prime}$ & $20^{\circ} \mathrm{S}$ & $20^{\circ} 1$ & $20^{\circ} 9^{\frac{1}{2}}$ & $21^{\prime}$ & $21^{\prime} 9^{\prime \prime}$ \\
\hline A 103 & $21^{\prime} 2^{\prime \prime}$ & $21-8$ & 2111 & $21^{\prime} 11^{\prime \prime}$ & $21^{\prime} \quad 10 \frac{1}{2}$ & $2110 \frac{1}{2}$ \\
\hline- & $\begin{array}{r}84 \cdot 5 \% \\
107 \text { to } \\
111 \text { to }\end{array}$ & $\begin{array}{l}18 \% \\
104 \\
110\end{array}$ & $\begin{array}{l}10 \\
11\end{array}$ & $\begin{array}{l}105 \\
103\end{array}$ & $\begin{array}{r}183^{1} \quad 17^{3} \\
103 \text { to } \\
106 \text { to }\end{array}$ & $\begin{array}{l}183 \\
106 \\
109\end{array}$ \\
\hline HEAT TRACE & & & & & & \\
\hline
\end{tabular}

Walker:srb 9-29-70 
ITS-2 INVENTORY AND FUMPING Date $6-2-3-7$

PUMP OUT TANK

RECEIVING TANK

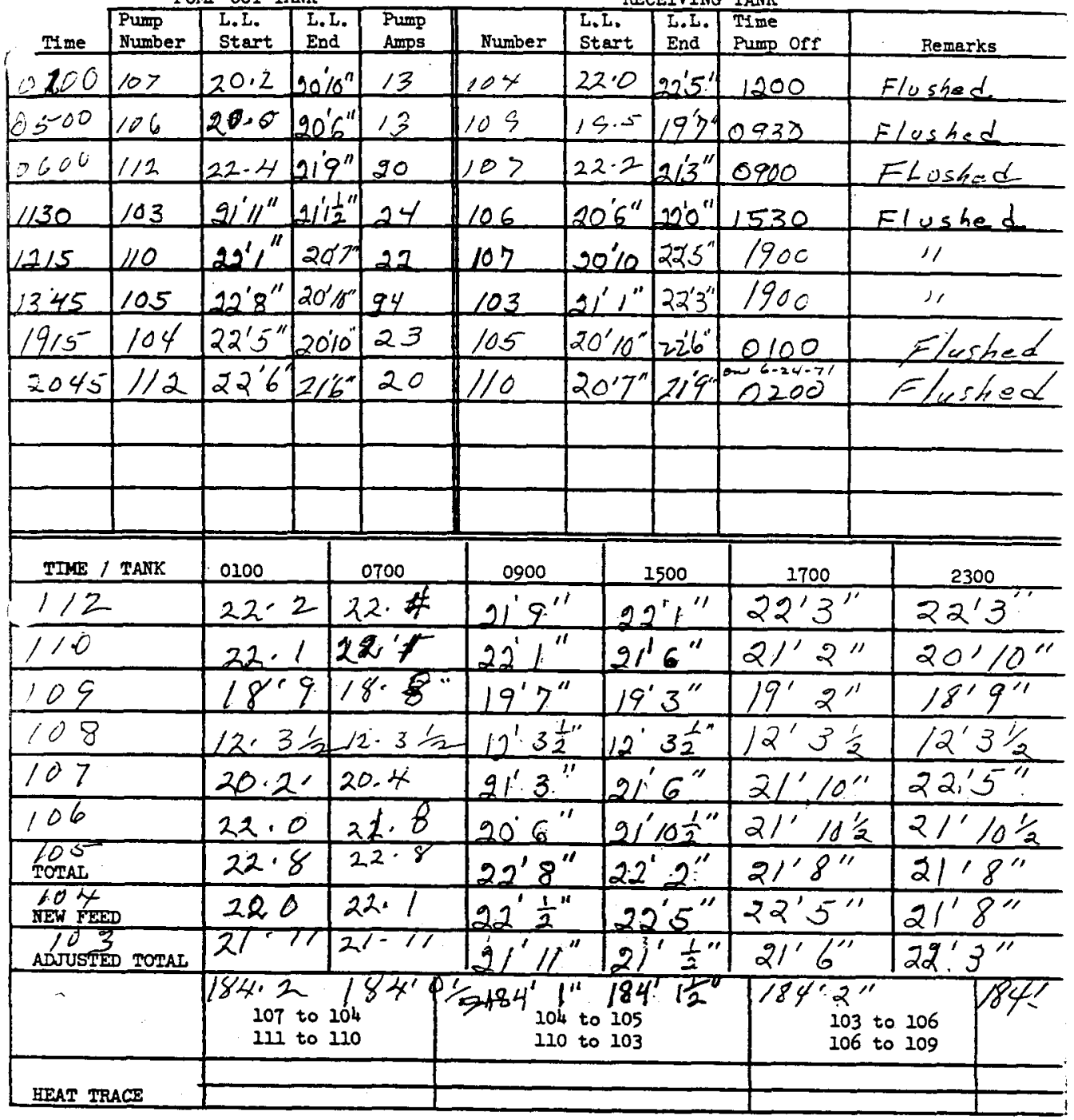

Walker:srb 9-29-70 


$$
\text { IIS-2 INVENTORY AND PUMPING Dete } 6-24-7 /
$$

PUMP OUT TANK

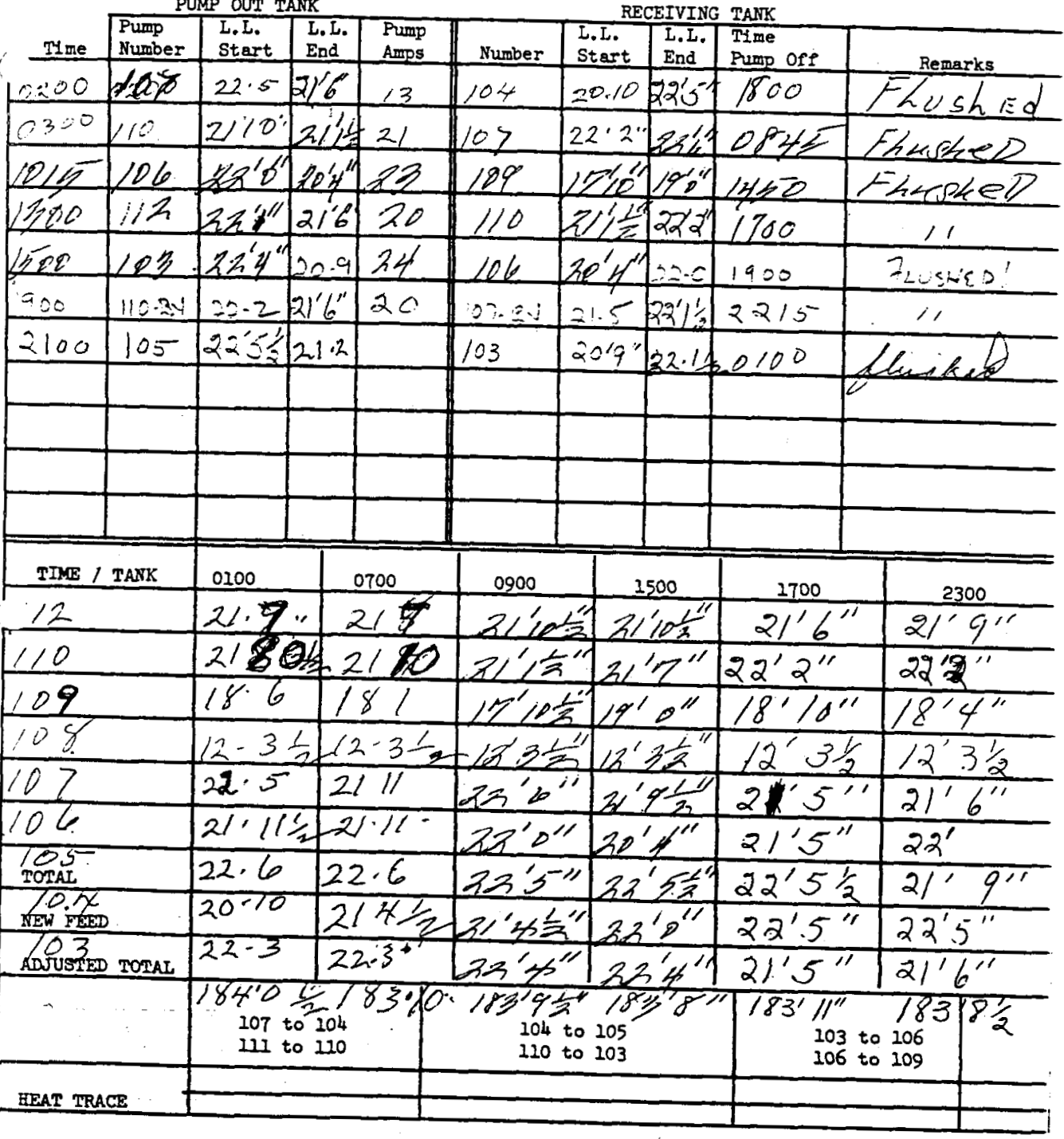

Walker : 8 rb 9-29-70 
WHC-SD-LM-DP-209, ReV. 0

ITS-2 INVENTORY AND PUMPING

Date $U .25^{-}-7$

PUMP OUT TANK

RECEIVING TANK

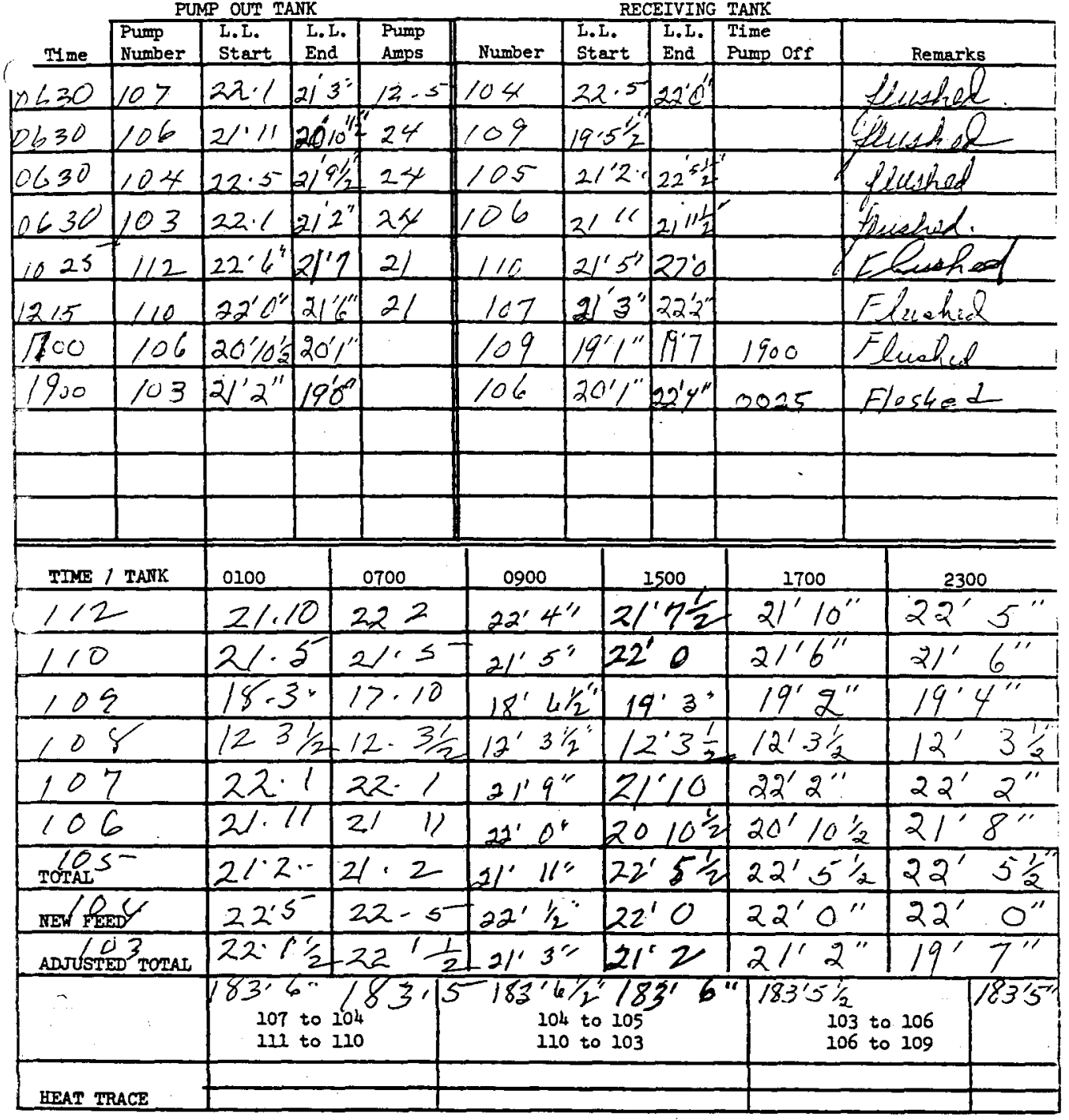

(Walker:srb 9-29-70

173 
WHC-SD-WM-DP-209, ReV. 0

ITS-2 INVENTORY AND PUMPING

Date $6.26 \cdot 21$

PUMP OUT TANK

RECEIVING TANK

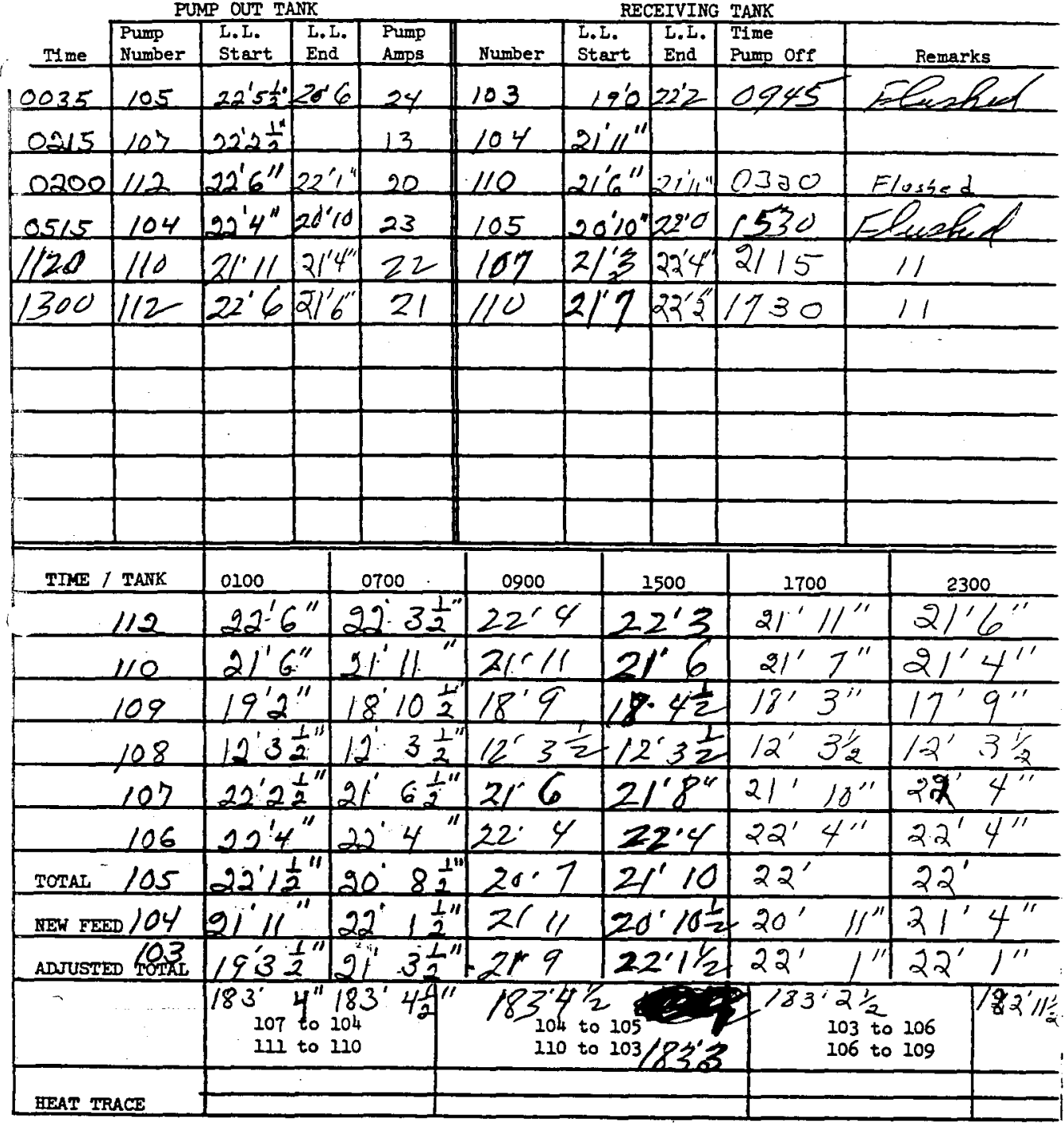

Walker:srb 9-29-70

174 
PUMP OUT TANK

RECEIVING TANK

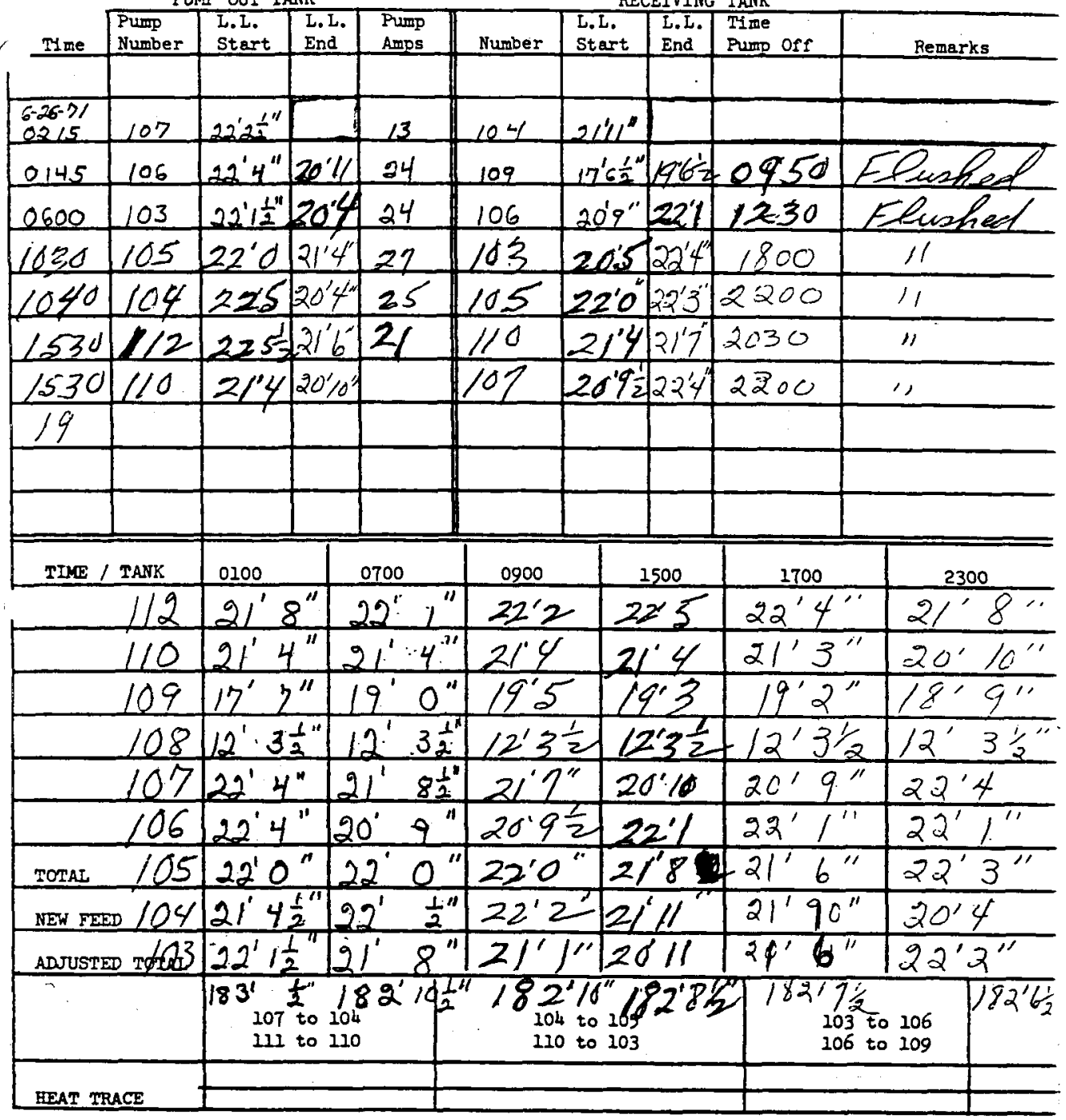

Walker:srb 9-29-70 
PUNP OUT TANK

RECEIVING TANK

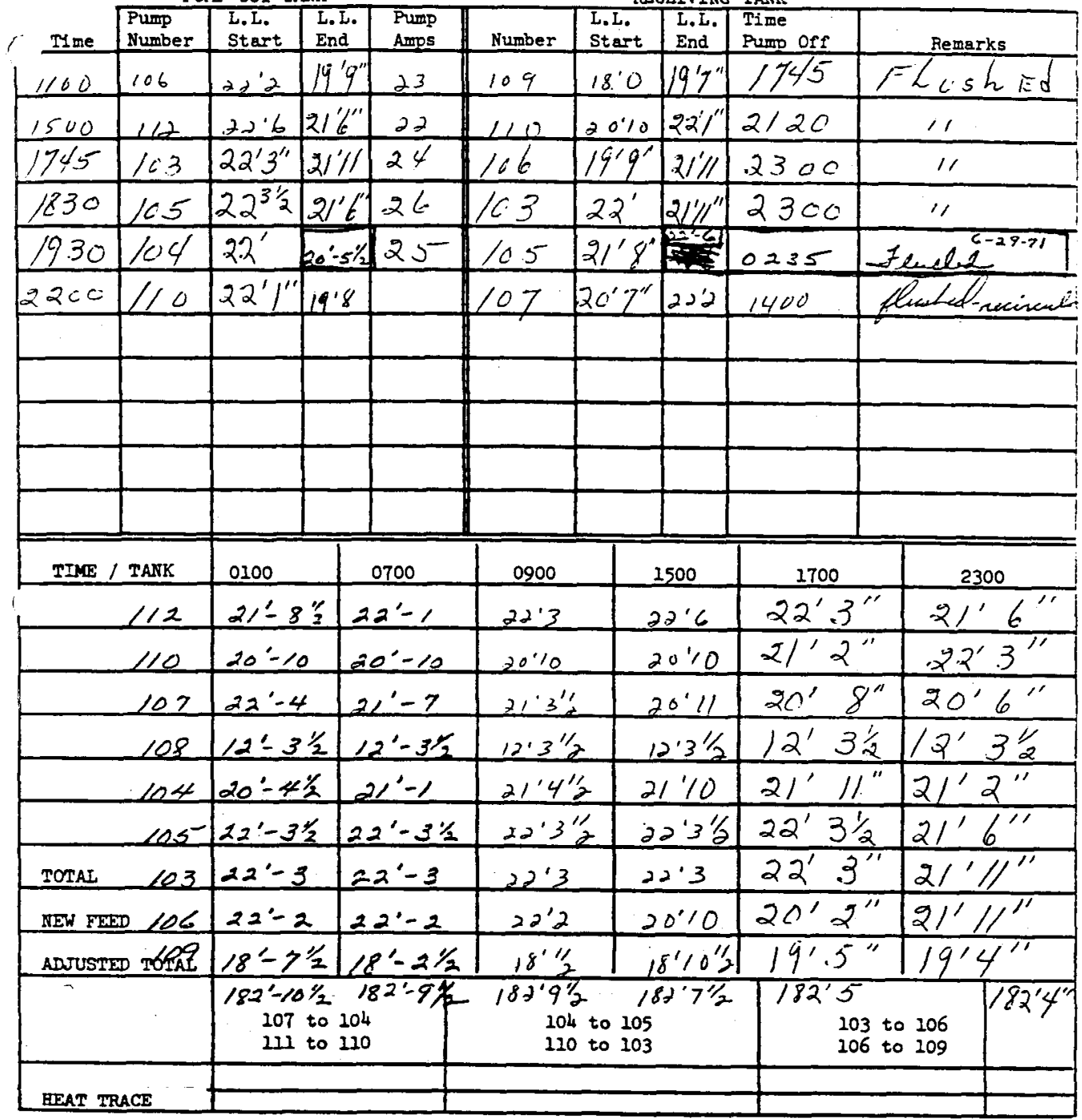

Walker:srb 9-29-70 
ITS-2 INVENTORY AND PUMPING

Date $(2-29-71$

PUNP OUT TANK

RECEIVING TANK

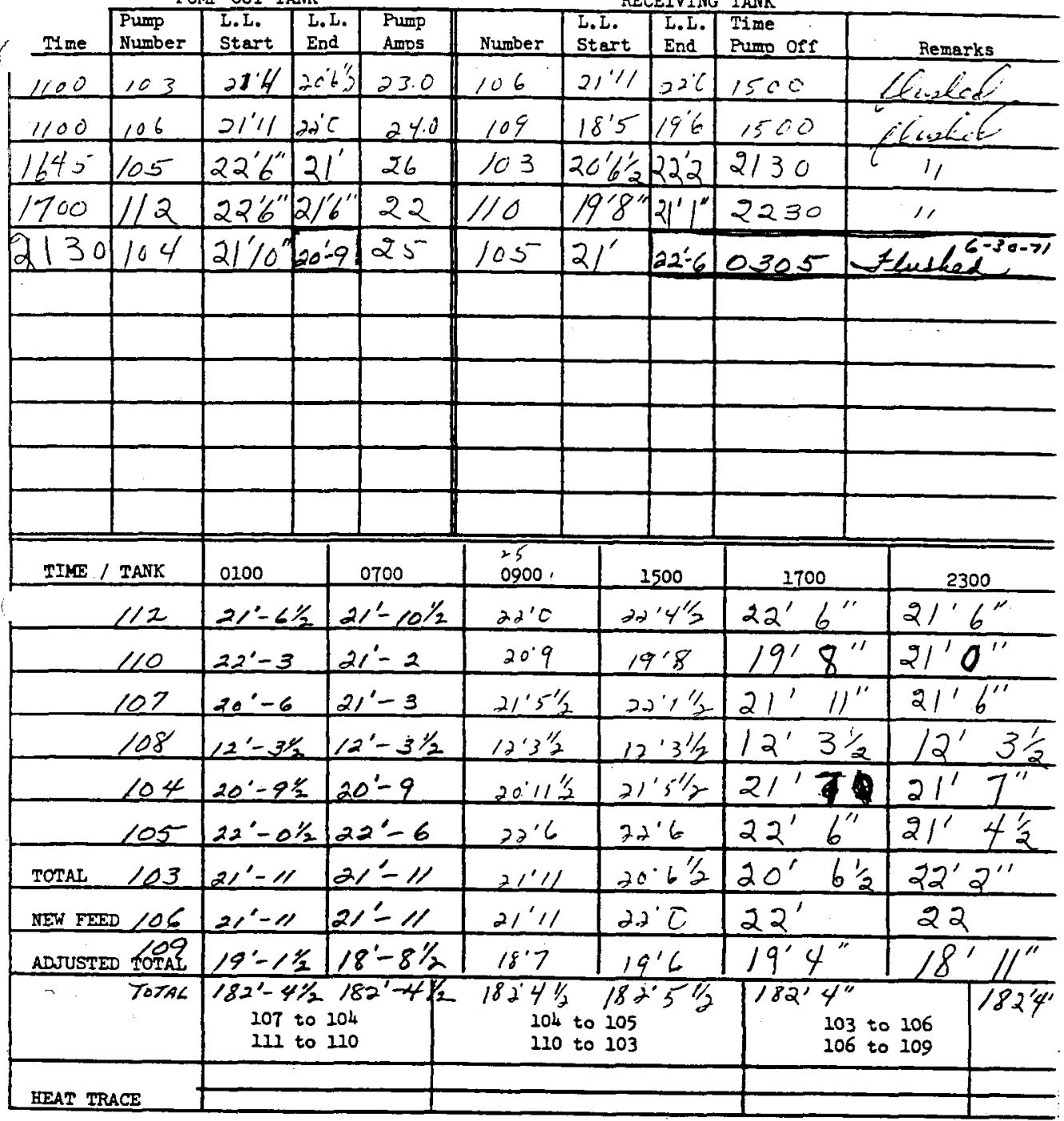

Walker:srb 9-29-70 
WHC-5D-LM-DP-209, Rev. O

ITS-2 INVENTORY AND PUMPING Date $6-30-\% /$

PUMP OUT TANK

RECEIVING TANK

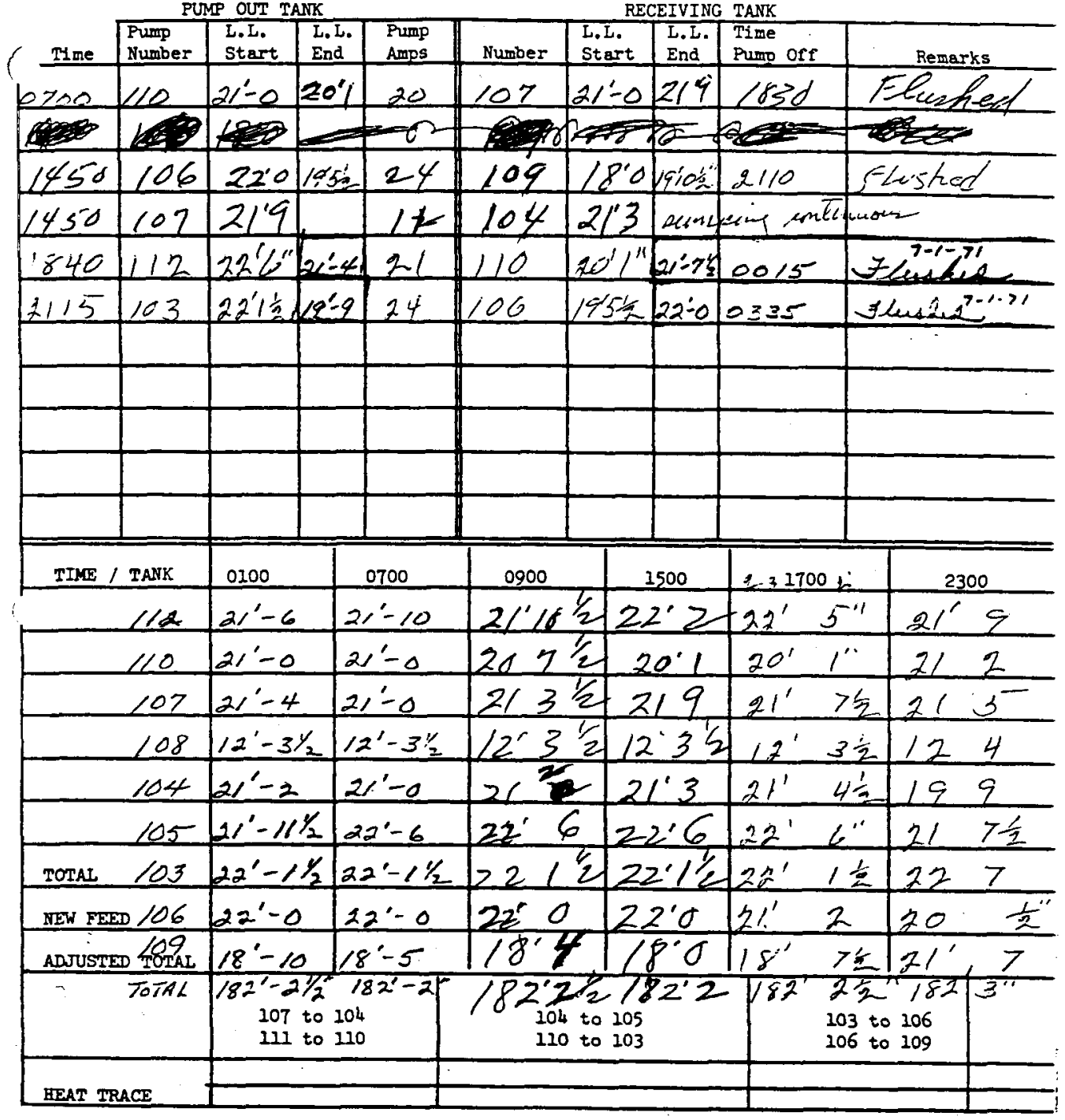

L'Walker:srb 9-29-70

178 
WHC-SD-WM-OP-2OQ, Rev. 0

ITS-2 INVENTORY AND PUMPING

Date $7-1-71$

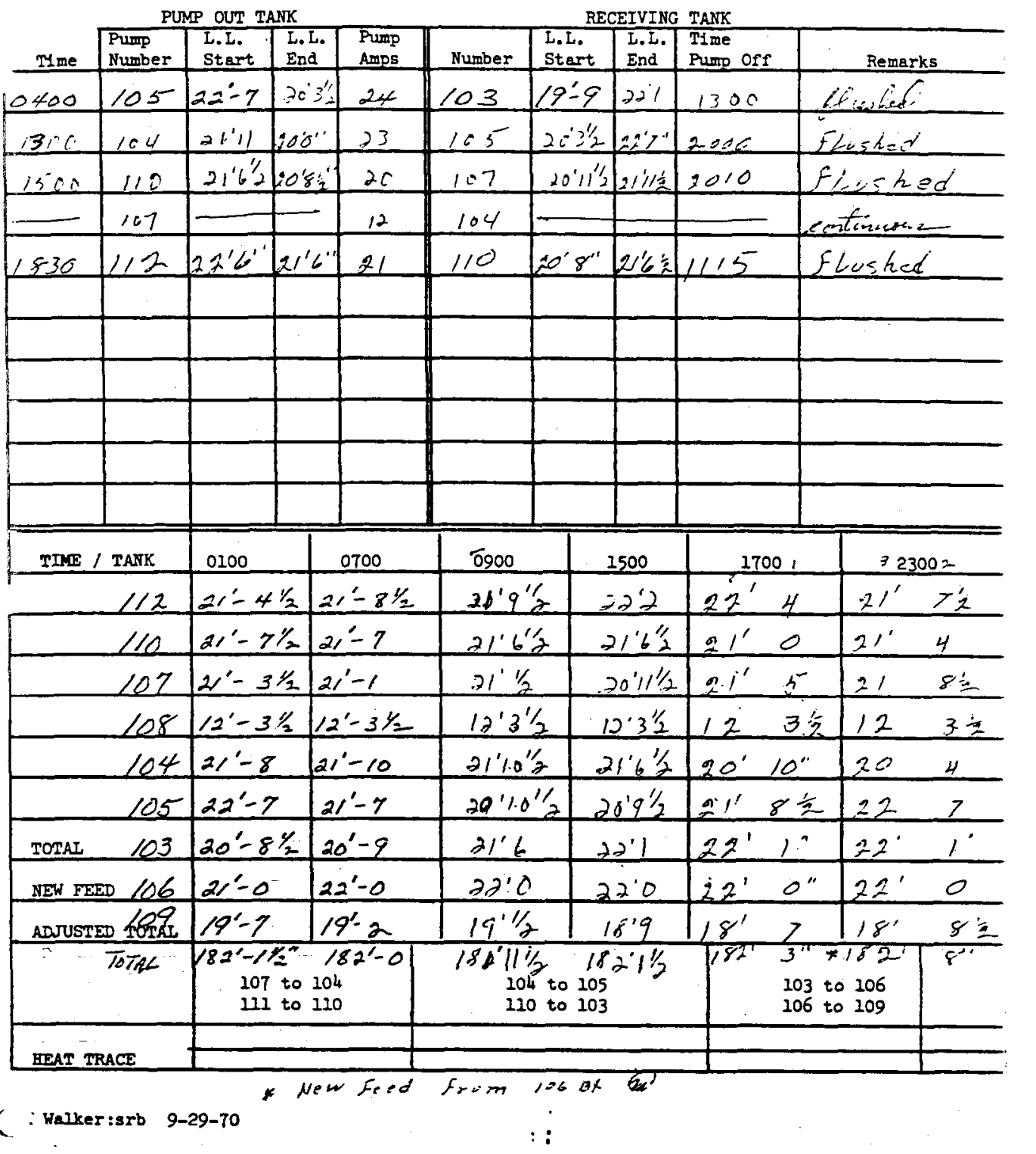


PUMP OUT TANK

RECEIVING TANK

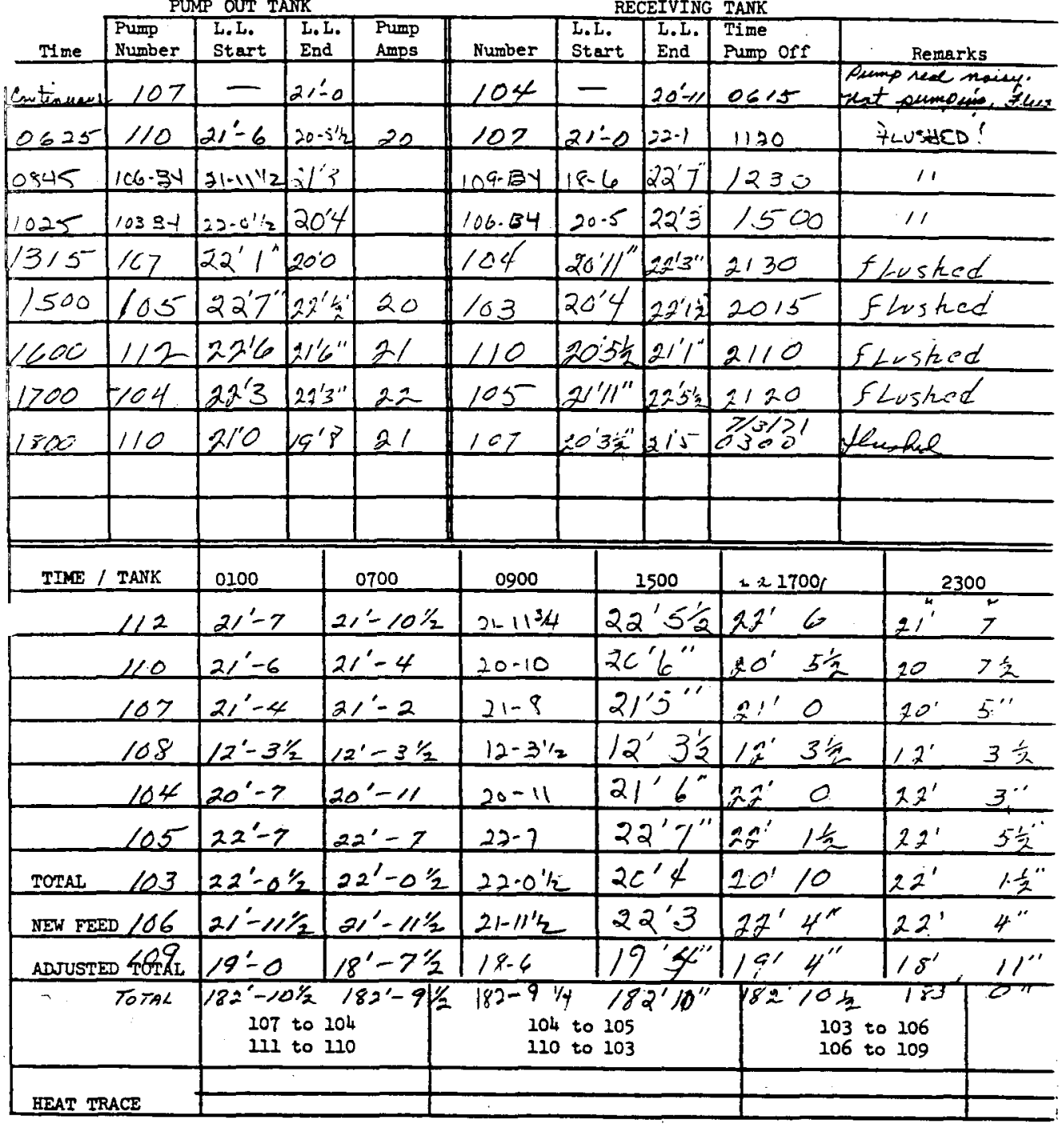

Walker:srb 9-29-70 
ITS-2 INVENTORY AND PUMPING

Date $263 / 21$

PUMP OUT TANK

RECEIVING TANK

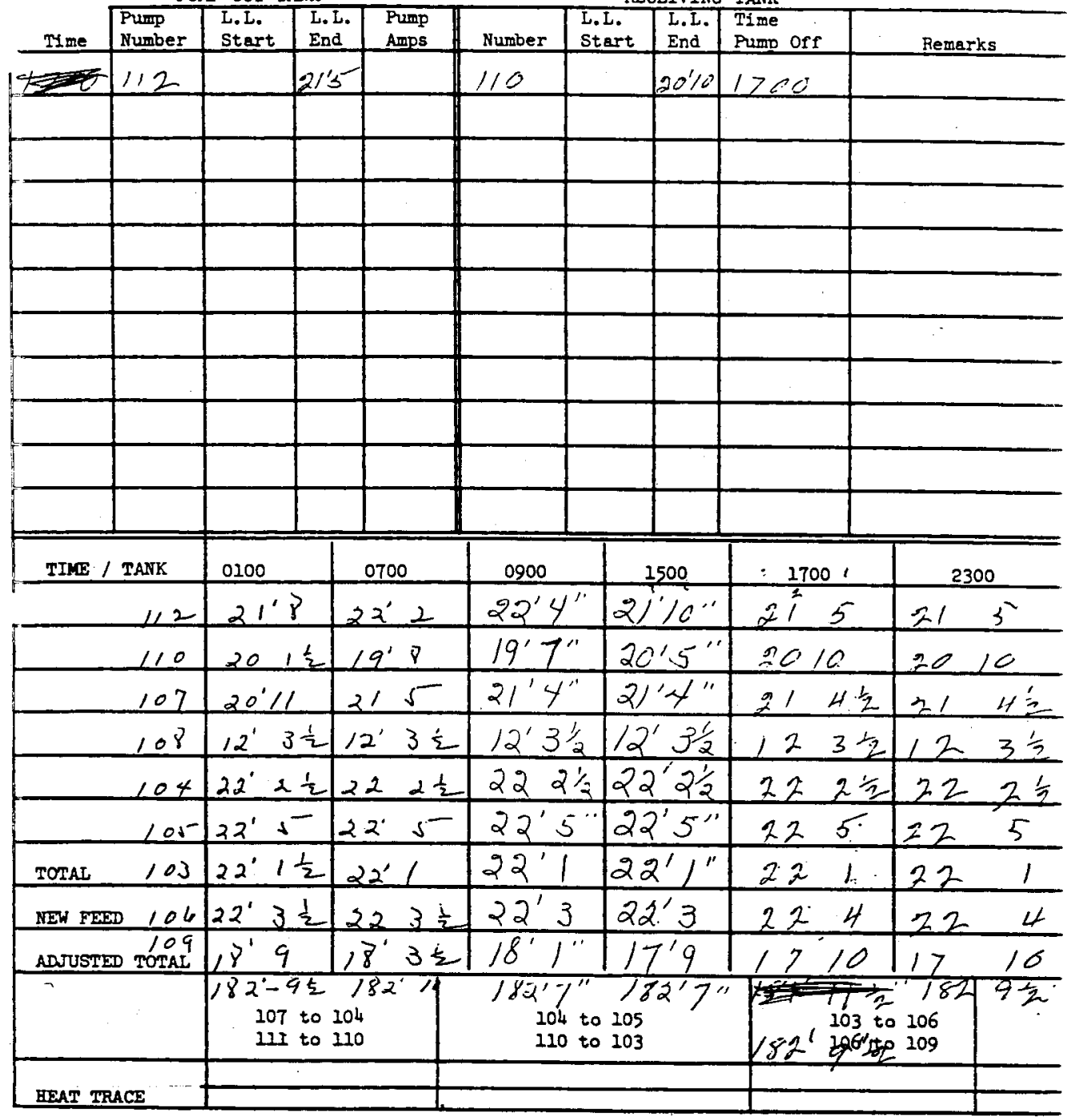

$\{$ I Walker:srb 9-29-70 


\section{ITS-2 INVENTORY AND PUMPING Date $2 / 4 / 21$}

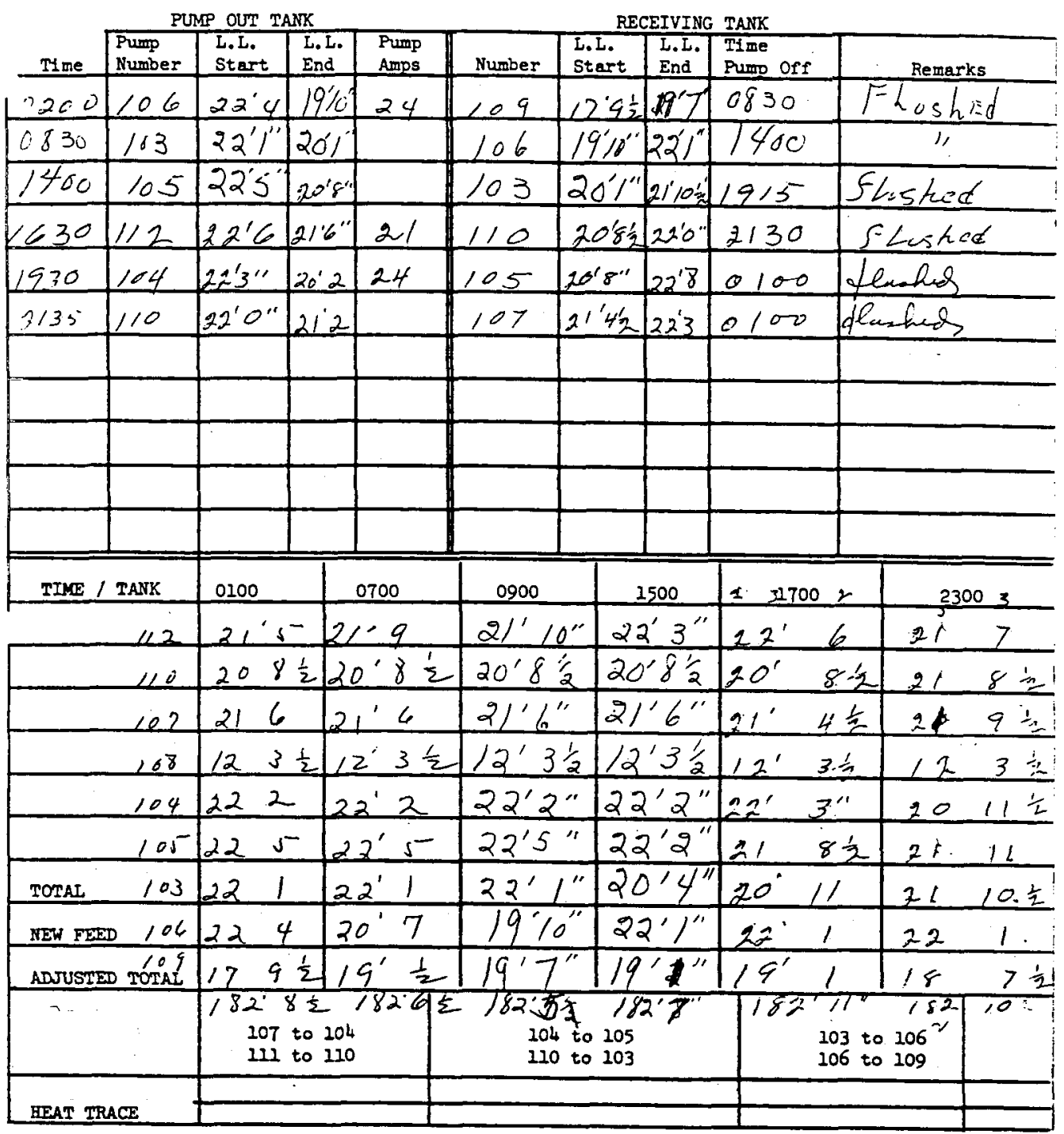

i Walker:srb 9-29-70 
ITS-2 INVEXTORY AND PUMPING

Dete $2 / 5-17$

PUMP OUT TANK

RECEIVING TANK

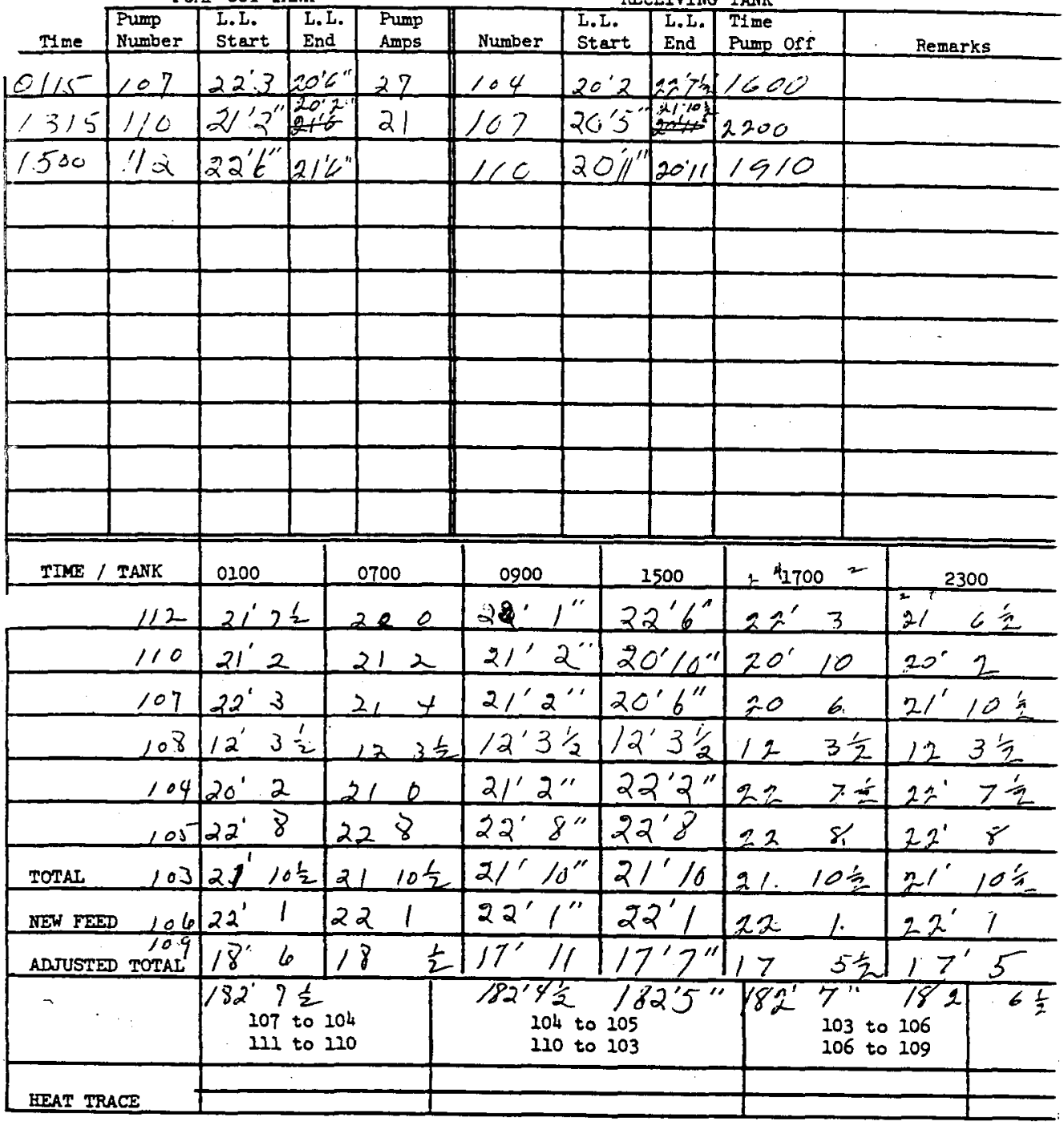

\} Walker:srb 9-29-70 
PUMP OUT TANK

RECEIVING TANK

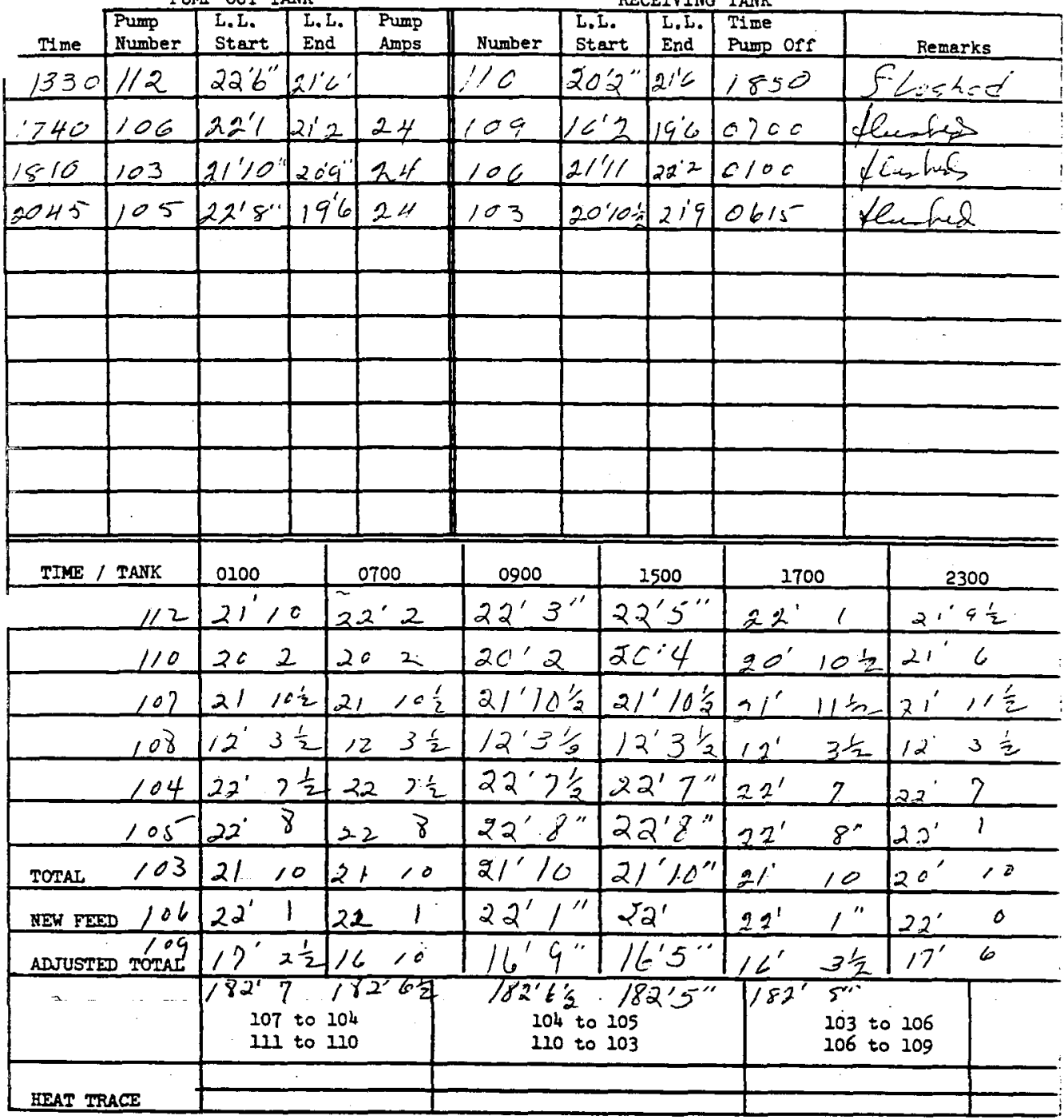

I Walker:srb 9-29-70 
ITS-2 INVENTORY AND PUMPING

Date $2 / 2 / 21$

PUNP OUT TANK

RECEIVING TANK

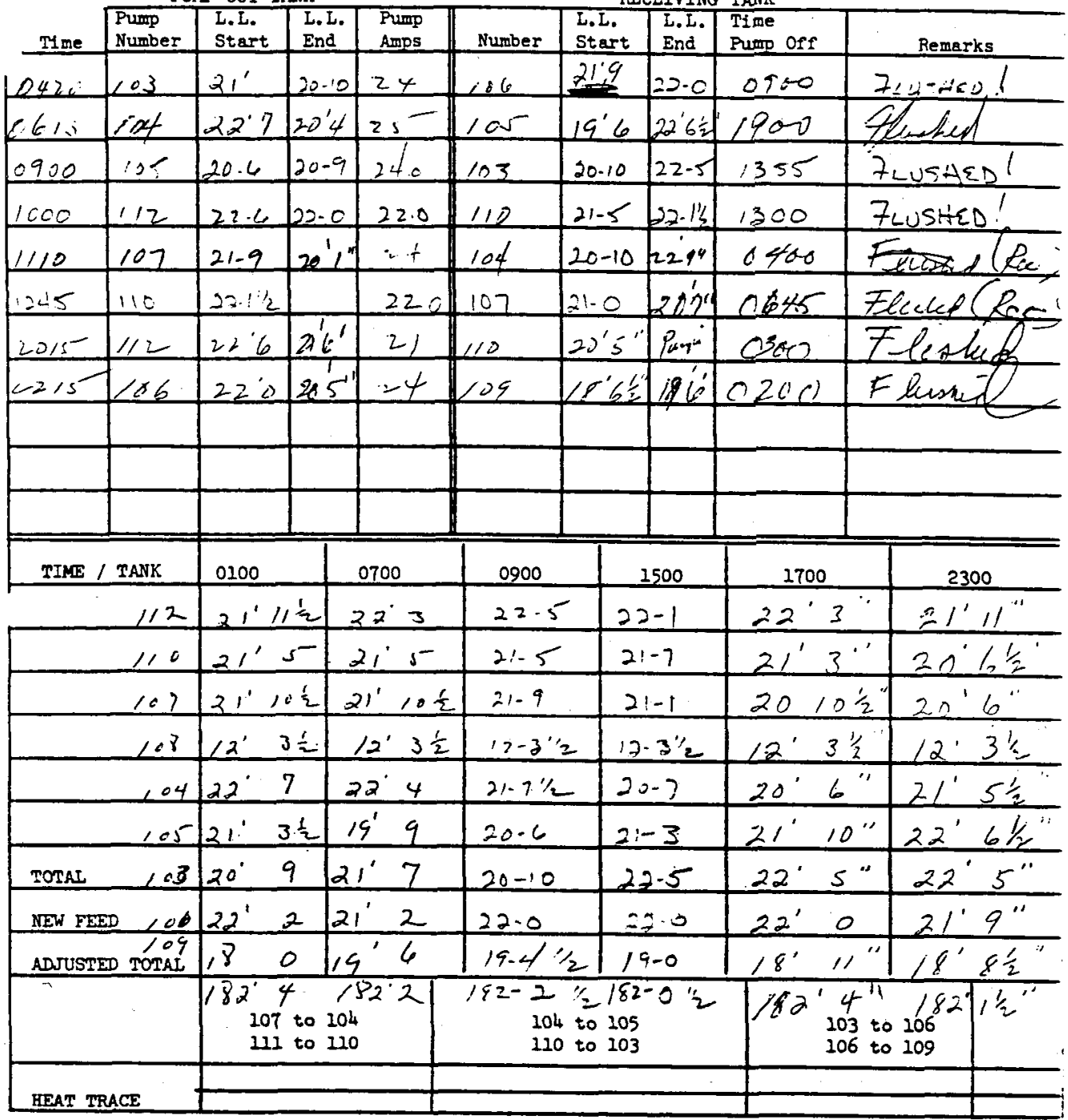

Walker:srb 9-29-70 
ITS-2 INVENTORY AND PUMPING

Date $7-8-7$

PUMP OUT TANK

RECEIVING TANK

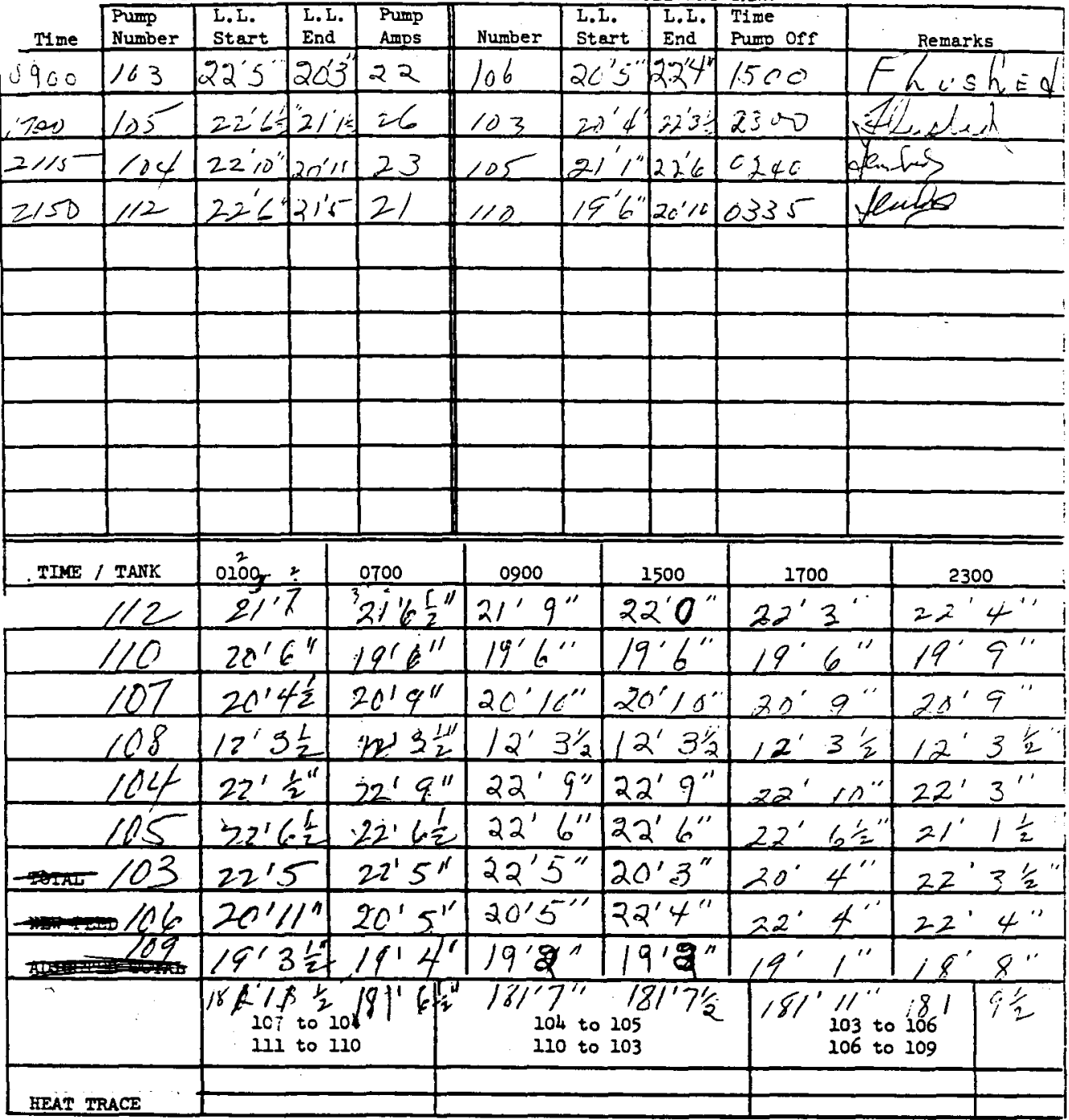

Walker:srb 9-29-70 
ITS-2 INVENTORY AND PUMPING

Date $219 / 21$

PUMP OUT TANK

RECEIVING TANK

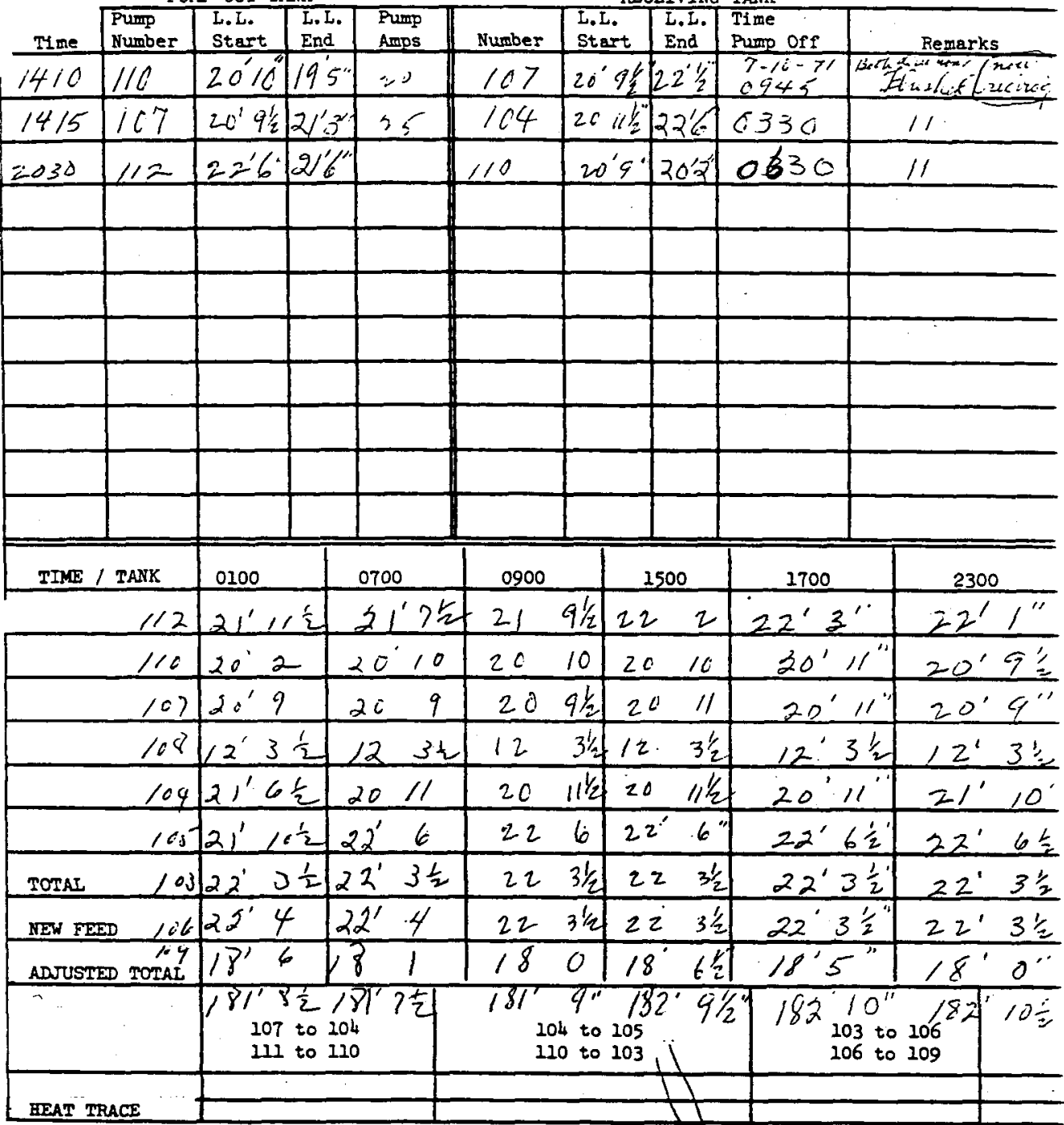

Walker:srb 9-29-70 
ITS-2 INVENTORY AND PUMPING

Date $7-\mid c-71$

PUMP OUT TANK

RECEIVING TANK

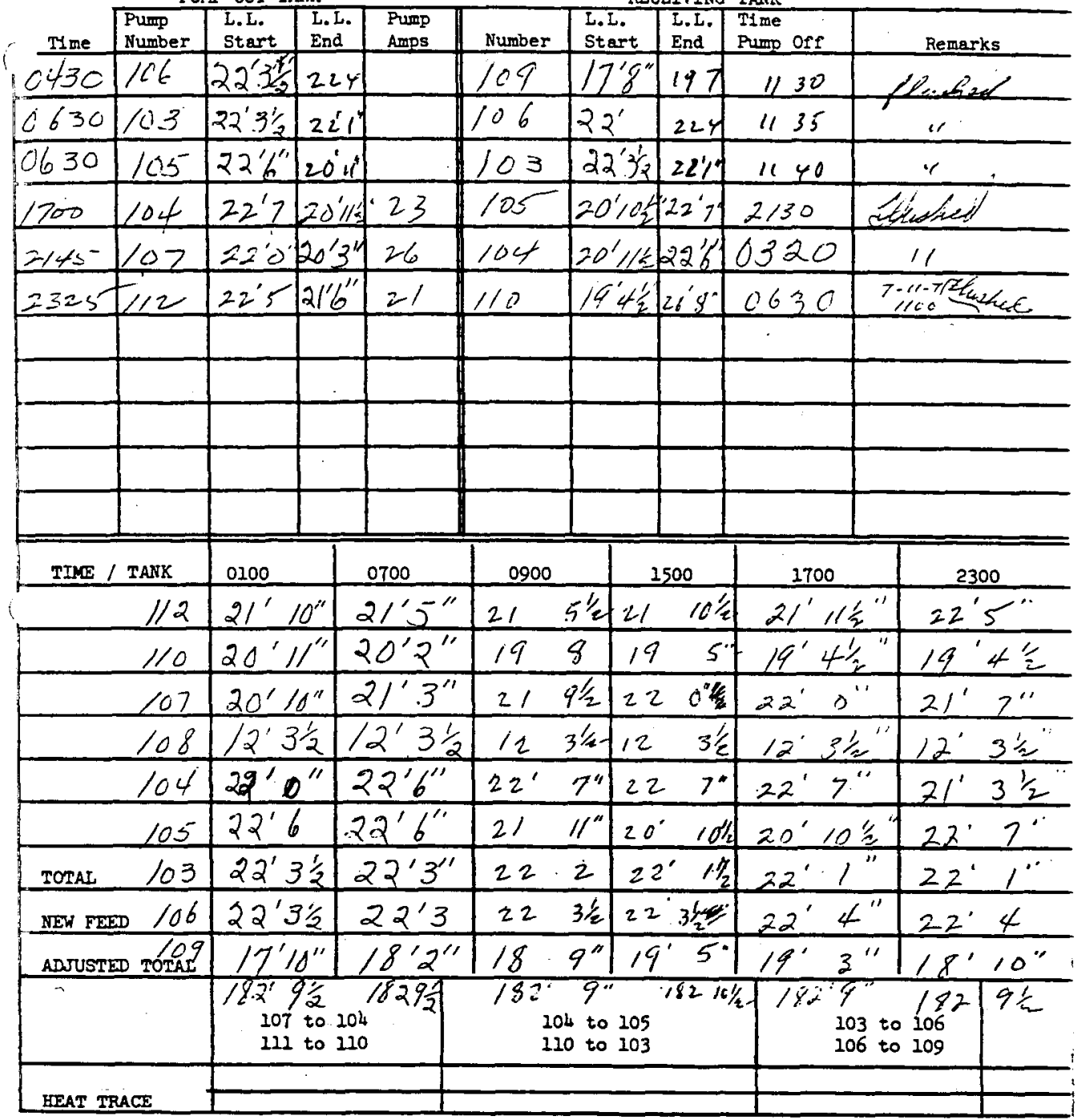

Welker :srb 9-29-70 
ITS-2 INVENTORY AND PUMPING $\quad$ Date $7-1 /-71$

PUMP OUT TANK RECEIVING TANK

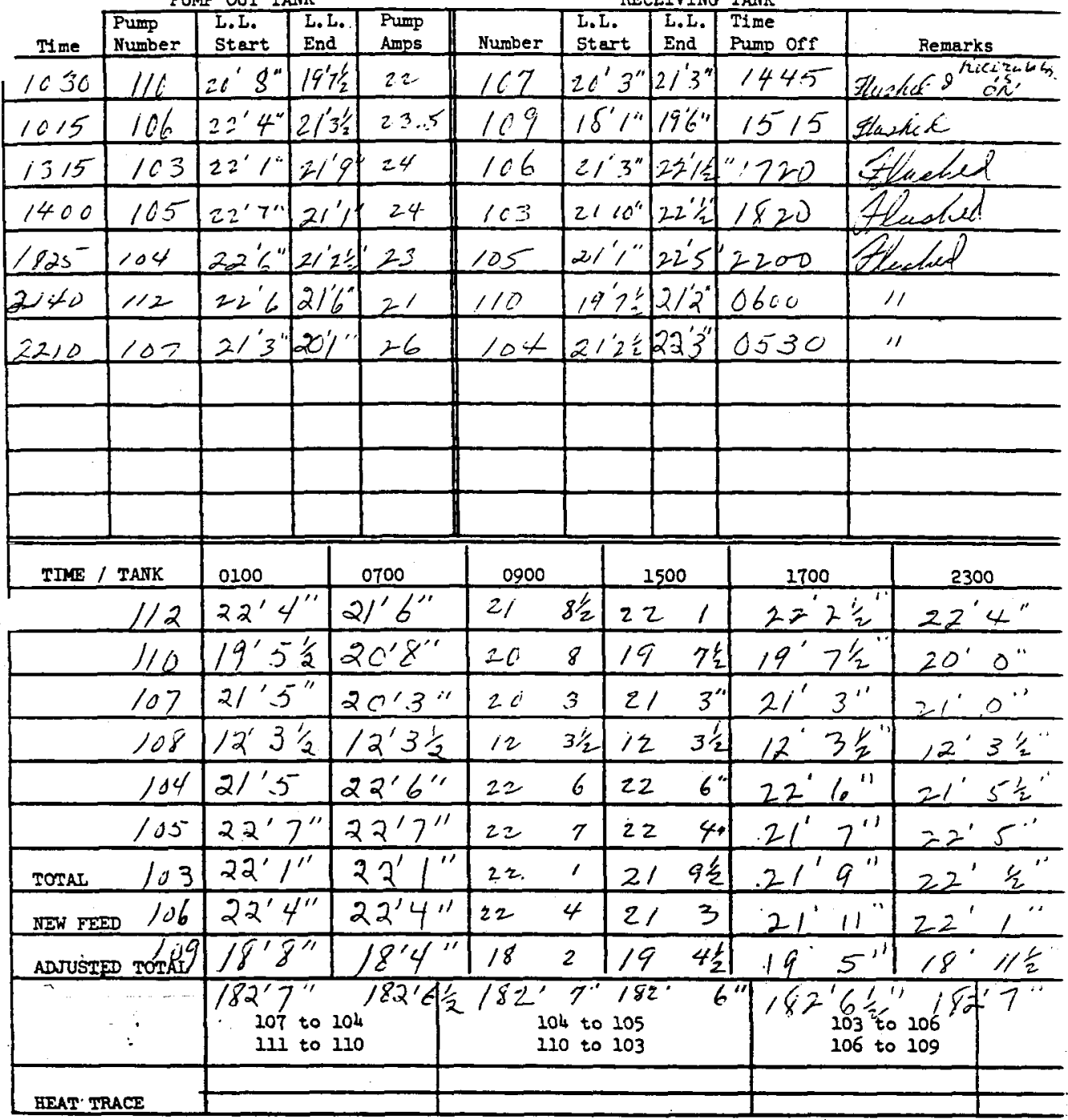

Walker:sirb 9-29-70 
WHC-SD-WM-DP-209, ReV. O

ITS-2 INVENTORY AND FUMPING Date $7-12-71$

PUMP OUT TANK

RECEIVING TANK

\begin{tabular}{|c|c|c|c|c|c|c|c|c|c|}
\hline Time & \begin{tabular}{|l|} 
Pump \\
Number \\
\end{tabular} & $\begin{array}{l}\text { L.I. } \\
\text { Start }\end{array}$ & $\begin{array}{l}\text { L.L. } \\
\text { End }\end{array}$ & $\begin{array}{l}\text { Pump } \\
\text { Amps }\end{array}$ & Number & $\begin{array}{l}\text { L.I. } \\
\text { Start }\end{array}$ & $\begin{array}{l}\text { L.I. } \\
\text { End } \\
\end{array}$ & $\begin{array}{l}\text { Time } \\
\text { Pump off }\end{array}$ & Remarks \\
\hline 6945 & 110 & 212 & $19^{\prime} 9$ & 21 & 162 & 20.2 & $2 i^{\prime \prime}$ & 1520 & Alestion \\
\hline 1760 & $10 \%$ & $22^{\prime} 1^{\prime}$ & $19^{\circ} 9$ & 24 & 109 & $17^{\prime} 9^{\prime \prime}$ & 196 & $2.24_{5}$ & SUlesheil \\
\hline $2 / 5-5$ & 112 & 226 & $2 / 6$ & 20 & 110 & $\angle C^{\prime} 8=$ & $21^{\prime \prime}$ & $040^{7} 0^{-12}$ & 1, \\
\hline 2360 & 103 & $22^{\prime} k^{\circ}$ & $20^{\prime} 6^{\prime \prime}$ & $2 L$ & $10 \%$ & $19 ' 9$ & 224 & $0.50 \frac{7.13}{0}$ & $1 /$ \\
\hline & & & & & & & & & \\
\hline & & & & & & & & & \\
\hline & & & & & & & & & \\
\hline & & & & & & & & & \\
\hline & & & & & & & & & \\
\hline & & & & & & & & & \\
\hline & & & & & & & & & \\
\hline
\end{tabular}

TIME / TANK

\begin{tabular}{l|l}
0100 & 0700 \\
\hline
\end{tabular}

$1 / 222^{\prime} 2^{\prime \prime} 21^{\prime} 7^{\prime \prime}$

0900

218

1500

1700

2300

\begin{tabular}{|c|c|c|c|c|c|}
\hline $1 / 0$ & $20^{\prime} 2^{\prime \prime}$ & $21^{\prime} 2^{\prime \prime}$ & & 2 & $19^{\prime}$ \\
\hline
\end{tabular}
107

$20^{\prime} 1^{\prime \prime} 202$

\begin{tabular}{l|l|l|ll}
108 & $12^{\prime} 3 \frac{1}{2}$ & $12^{\prime} 3 \frac{1}{2}$ & 12 & $3 / 2$ \\
\hline
\end{tabular}

218

$22^{\prime} x^{\prime \prime \prime} / 1$

$12 \quad 3 / 2$

$19^{\prime} 8 \%$

$22^{\prime \prime} 4^{\prime \prime}$

\begin{tabular}{ll|l|ll}
104 & $21^{\prime} 8^{\prime \prime}$ & $22^{\prime} .3$ & 22 &
\end{tabular}

223

\begin{tabular}{l|l|l|}
\hline $1^{\prime \prime}$ & $27^{\prime \prime} 7^{\prime \prime}$ \\
\hline
\end{tabular}

$105^{-} 22^{\prime} 5^{\prime \prime} 22^{\prime} 5^{\prime \prime} 225$

$2<5$

Torar $/ c 322^{\prime} \frac{1}{2} 2^{\prime \prime} 2^{\prime} y^{\prime \prime}$

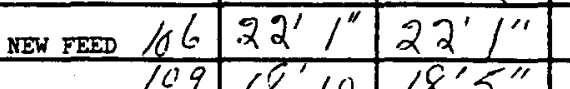

andustad total $1 / 8^{\prime} 1018^{\prime} 5^{\prime \prime}$

$\checkmark \quad \begin{gathered}182^{\prime} 5^{\prime \prime} / 82^{\prime} 4 \\ 107 \text { to } 104 \\ 111 \text { to } 110\end{gathered}$

HEAT TRACE

22

\begin{tabular}{ll|l|}
221 & 221 \\
184 & $17 !$
\end{tabular}

1

$22+22$

$12^{\prime} 3 \frac{1 / 2}{2} 12^{\prime} 3 \frac{1}{2}$

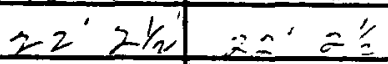

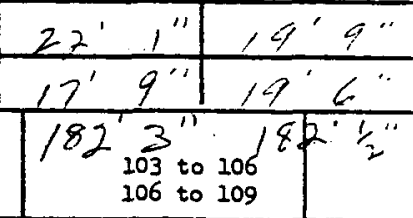

104 to 105

110 to 103

$82 \mathrm{C}$ 22 
WHC-SD-WM-DP-209, ReV. 0

ITS-2 INVENTORY AND PUMPING

Date $7-13-7 !$

PUMP OUT TANK

RECEIVING TANK

\begin{tabular}{|c|c|c|c|c|c|c|c|c|c|}
\hline Time & umber & $\begin{array}{l}\text { L. L. } \\
\text { Start }\end{array}$ & $\begin{array}{l}\text { L. L. } \\
\text { End }\end{array}$ & ump & Number & $\begin{array}{l}\text { L.L. } \\
\text { Start } \\
\end{array}$ & $\begin{array}{l}\text { L.L. } \\
\text { End }\end{array}$ & $\begin{array}{l}\text { Time } \\
\text { Pump off }\end{array}$ & $\mathrm{rks}$ \\
\hline cifuc & $105^{-}$ & $224^{\prime}$ & $205^{\prime \prime}$ & 24 & 103 & $20^{\prime} 8^{\prime \prime}$ & $22^{\prime} 2^{\prime \prime}$ & 1000 & Eluekt \\
\hline$C \& 6$ & 104 & $=22 / 2$ & $19^{\prime} 11$ & 24 & 165 & $204 \%$ & $22^{\prime} 4^{\prime \prime}$ & 1520 & NOW \\
\hline incth & 107 & $21^{\prime} 21 \%$ & $20^{\prime} s^{\prime} / 2$ & 26 & 104 & $20^{\circ} 0^{\circ}$ & $22^{\prime} 4^{\prime \prime}$ & $110^{7-14}$ & \\
\hline 755 & 106 & $27^{\prime} 31$ & $3 x^{\prime \prime}$ & 23 & 109 & $18^{\prime} 3^{\prime \prime}$ & 195 & 2150 & zlliestod \\
\hline$=155$ & 103 & $22^{\prime} 2^{\prime}$ & $20^{\prime} 9^{\prime \prime}$ & 23 & $10 \%$ & $20^{\prime} 8$ & $22^{\prime} 2^{\prime \prime}$ & $020^{7} 0^{14}$ & 11 \\
\hline & $\angle / 0$ & $21^{\prime}, 1$ & $20^{\circ} 0^{\prime \prime}$ & $=1$ & 197 & $2,5^{\prime}$ & $20^{\circ}, 0^{1} / z$ & 0945 & 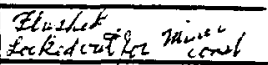 \\
\hline 2115 & $1 / 2$ & $\equiv z^{\prime} 6^{\prime \prime}$ & $216^{\prime \prime}$ & 20 & 110 & 2111 & $21^{\prime}$ & 0.530 & $11 \div$ \\
\hline
\end{tabular}

\begin{tabular}{ll|l|l|l|l|l|l|l|l|l}
\hline & & & & & & & & & \\
\hline & & & & & & & & & \\
\hline & & & & & & & & & \\
\hline
\end{tabular}

\begin{tabular}{|c|c|c|c|c|c|c|c|}
\hline TIME / TANK & 0100 & 0700 & \multicolumn{2}{|c|}{0900} & 2500 & 2700 & 2300 \\
\hline $1 / 2$ & $22^{\prime} 2^{\prime \prime}$ & $90^{\prime \prime} 6^{\prime \prime}$ & 21 & $81 / 5$ & $2=1 "$ & $22^{\prime} 2 \frac{1}{2}{ }^{\prime \prime}$ & 225 \\
\hline 110 & $20^{\prime} 1^{\prime \prime}$ & $21^{\prime} 1^{\prime \prime}$ & 21 & $1 / 2$ & $1 / 2$ & $\approx 1^{\prime},{ }^{\prime \prime}$ & $21^{\prime} 1 \frac{1}{2}^{\prime \prime}$ \\
\hline 102 & $21^{\prime} 7^{\prime \prime}$ & $21^{\prime} 7^{\prime \prime}$ & 21 & $7 \frac{2}{2}$ & $7 \leq$ & $21^{\prime} 71^{\prime \prime}$ & $20^{\prime} 6^{\prime \prime}$ \\
\hline 108 & $12^{\prime} 3 / 2$ & $12^{\prime} .3 \frac{1}{2}$ & 12 & $3 \frac{1}{2}$ & $3 \frac{1}{2}$ & $31 / 2$ & $12^{\prime} 3 \frac{1}{2}$ \\
\hline 104 & $22^{\prime} 2 / 2$ & $22^{\prime} 2 \frac{1}{2}$ & 22 & $2 \frac{1}{2}$ & 20 & $=0^{\circ} 0^{\prime \prime}$ & $211^{\prime} 4 \frac{1}{2}$ \\
\hline 105 & $22^{\prime} 4 \frac{1}{2}$ & $21^{\prime} 1^{\prime \prime}$ & 20 & $4 / 2$ & $1 / 2$ & $22^{\prime} 4^{\prime \prime}$ & $224^{\prime \prime}$ \\
\hline TOTAL 103 & $22^{\prime} 0^{\prime \prime}$ & $21^{\prime}$ & 21 & $9 \frac{1}{2}$ & $22^{\prime}$ & $22^{\prime} 2^{\prime}$ & $21^{\prime} 9^{\prime \prime}$ \\
\hline NEW FEED 106 & $19^{\prime} / 0^{\prime \prime}$ & $22^{\prime} 4^{\prime \prime}$ & 22 & $3 \frac{1}{2}$ & $22 \quad 3 \%$ & $22^{\prime} 3 \frac{1}{2}$ & $21^{\prime}, " 1$ \\
\hline ADUUSTED TOTA & $195^{\prime \prime}$ & $19^{\prime}$ & $18^{\circ}$ & $10^{\prime \prime}$ & $18 \quad 54$ & $184^{\prime \prime}$ & $195+1 / 2$ \\
\hline$\because \quad \ldots$ & $\begin{array}{l}181^{\prime} / 1 / 2 \\
107 \text { to } \\
111 \text { to }\end{array}$ & $\begin{array}{l}182^{\prime} \phi \\
110\end{array}$ & & $\begin{array}{l}28 \\
104 \text { to } \\
110 \text { to }\end{array}$ & $\begin{array}{l}1 S 2^{\prime} 4^{\prime \prime} \\
105 \\
103\end{array}$ & $\begin{array}{r}/ 8=f^{\prime \prime} \\
103 \text { to } \\
106 \text { to }\end{array}$ & $\begin{array}{l}1873 \\
106 \\
109\end{array}$ \\
\hline HEAT TRAC & & & & & & & \\
\hline
\end{tabular}

1 Walker:srb 9-29-70 
PUNP OUT TANK

RECEIVING TANK

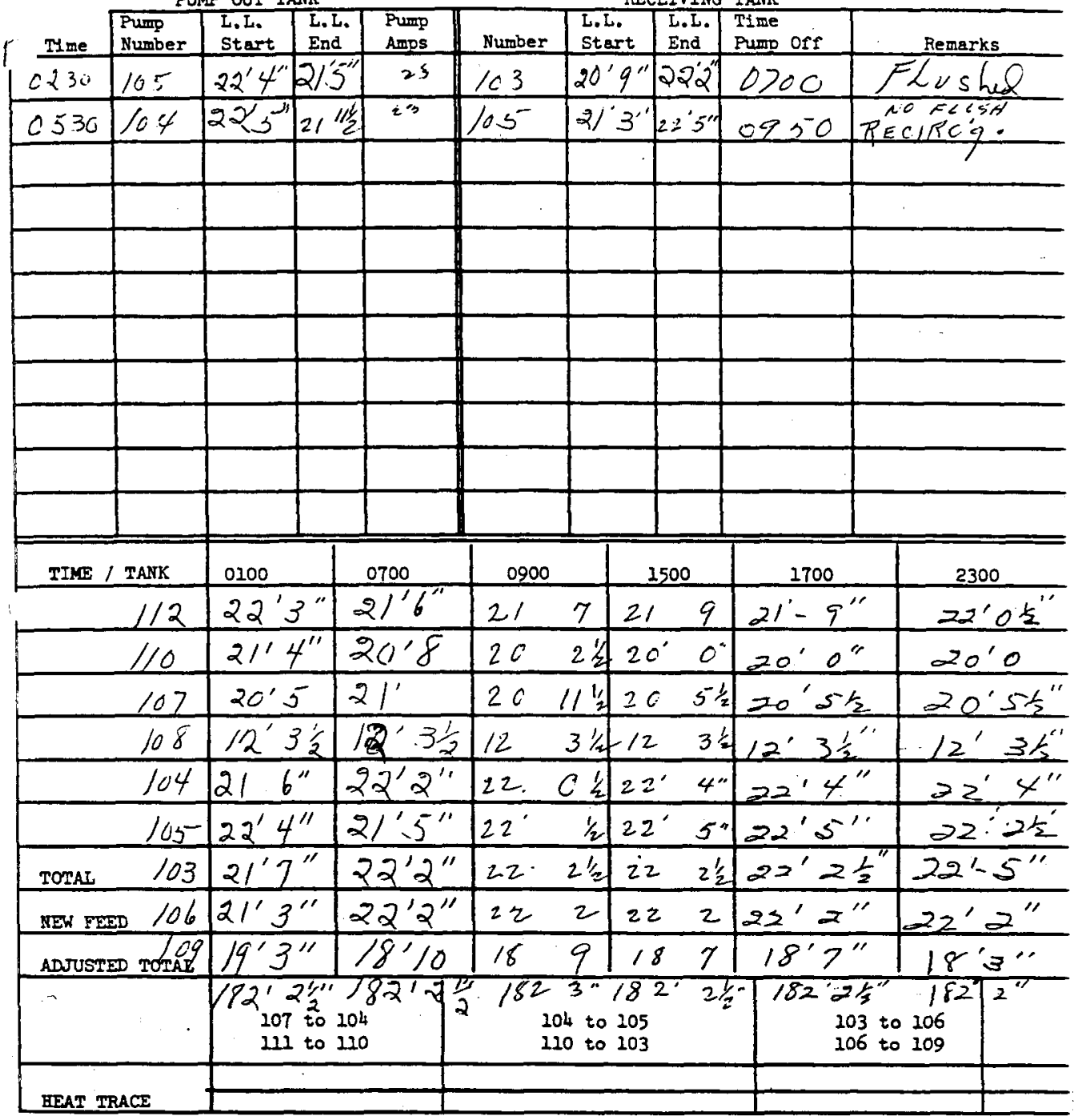

Walker:srb 9-29-70 
PUMP OUT TANK

RECEIVING TANK

\begin{tabular}{|c|c|c|c|c|c|c|c|c|c|}
\hline Tyme & $\begin{array}{l}\text { Pump } \\
\text { Number }\end{array}$ & $\begin{array}{l}\text { L.L. } \\
\text { Start } \\
\end{array}$ & $\begin{array}{l}\text { L. L. } \\
\text { End }\end{array}$ & $\begin{array}{l}\text { Pump } \\
\text { Amps }\end{array}$ & Number & $\begin{array}{l}\text { L.L. } \\
\text { Start }\end{array}$ & $\begin{array}{l}\text { L.L. } \\
\text { End }\end{array}$ & $\begin{array}{l}\text { Time } \\
\text { Pump off }\end{array}$ & Remarks \\
\hline 0600 & 106 & $22^{\prime} 2^{\prime \prime}$ & $1901 / 2$ & 24 & 109 & $17^{9} 9^{\prime \prime}$ & $19^{\prime} 6^{\prime}$ & 1146 & weshot \\
\hline 0700 & 112 & $226^{\prime \prime}$ & 216 & 20 & 110 & 2018 & $217^{\prime \prime}$ & $13=5$ & thentex \\
\hline 1510 & $1<5$ & $22 \quad 4 \%$ & $21 \%$ & $2 \%$ & 103 & $2, " 1 \prime^{\prime \prime}$ & $22^{\prime \prime} 2^{\prime}$ & 0200 & Fluobel \\
\hline 1425 & 103 & $222 \frac{1}{2}$ & II & $2 \varepsilon$ & 106 & $19^{\circ} 10^{\prime}=$ & $22^{\prime} 4$ & 2050 & 7luskel \\
\hline $15 \div$ & 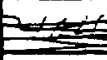 & & & & & & & & $\tan \theta$ \\
\hline
\end{tabular}

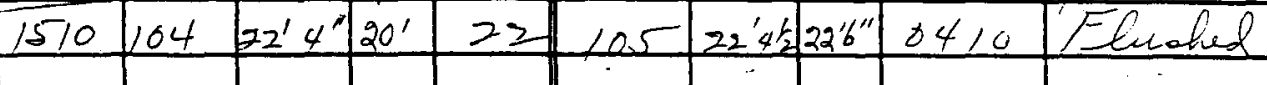

TIME / TANK

\begin{tabular}{l|l|l|ll|l}
\hline 12 & $22^{\prime} 2^{\prime} / 2$ & $22^{\prime} 6^{\prime \prime}$ & 22 & 2 & 21500 \\
\hline
\end{tabular}

$11020^{\prime} 0^{\prime \prime} 20^{\prime} 0^{\prime \prime}$

26 $75217 \%$

$10720^{\prime} 5 \frac{1}{2} 20^{\prime} 5 \frac{1}{2}$

$20 \quad 5 \frac{1}{2} 20 \quad 5 \frac{1}{2} 20^{\prime} 5 s^{\prime \prime}$

\begin{tabular}{l|l|l|}
108 & $12^{\prime} 3 \frac{1}{2}$ & $12^{\prime} 3 \frac{1}{2}$
\end{tabular}

$10422^{\prime} 4^{\prime \prime} 22^{\prime} 4^{\prime \prime}$

$12 \quad 3 / 21$

$10522^{\prime} 2 \frac{1}{2} \quad 22^{\prime} 2 \frac{1}{2}$

22 if $=2$ if $22^{\prime} 10 \%$

3/2

\begin{tabular}{l|l}
\hline $0^{\prime}$ & $20^{\prime} 5 \kappa^{\prime \prime}$ \\
\hline $2^{\prime} 3 k^{\prime \prime}$ & $12^{\prime} 3 \kappa^{\prime \prime}$
\end{tabular}

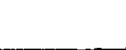

TOTAL 103

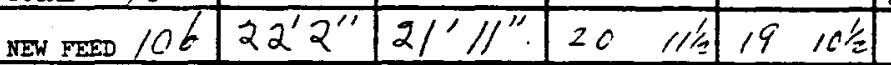
ADUSTED TORA 9 $18^{\prime \prime} 1^{\prime \prime}$ $182^{\prime} 2^{\prime \prime} 182^{\prime} /$ Ch" $^{\prime \prime}$ 10 to 104

111 to 110 \begin{tabular}{l|l}
$22^{\prime} 5^{\prime \prime}$ & 22 \\
\hline $21^{\prime} /^{\prime \prime}$ & 20 \\
\hline
\end{tabular}

$2 \quad 4 / 2$ 22 4/2 $22^{\prime}$ $21^{\prime} 10^{\prime \prime}$

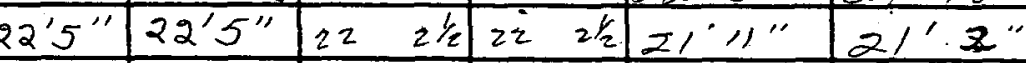


ITS-2 INVENTORY AND PUMPING

Date $7-16-71$

PUMP OUT TANK

RECEIVING TANK

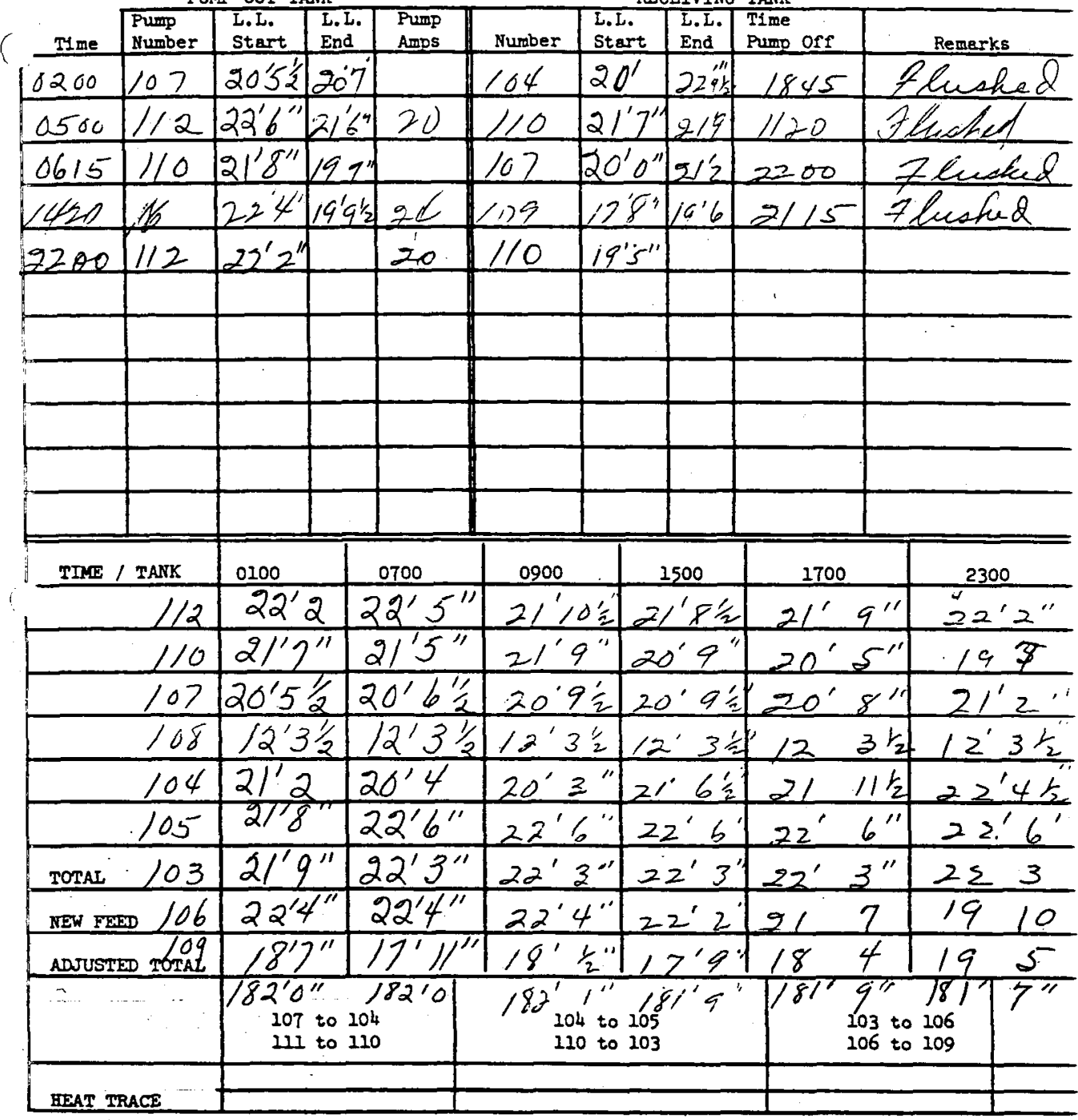

I i Walker:srb $9-29-70$ 
PUMP OUT TANK

RECEIVING TANK

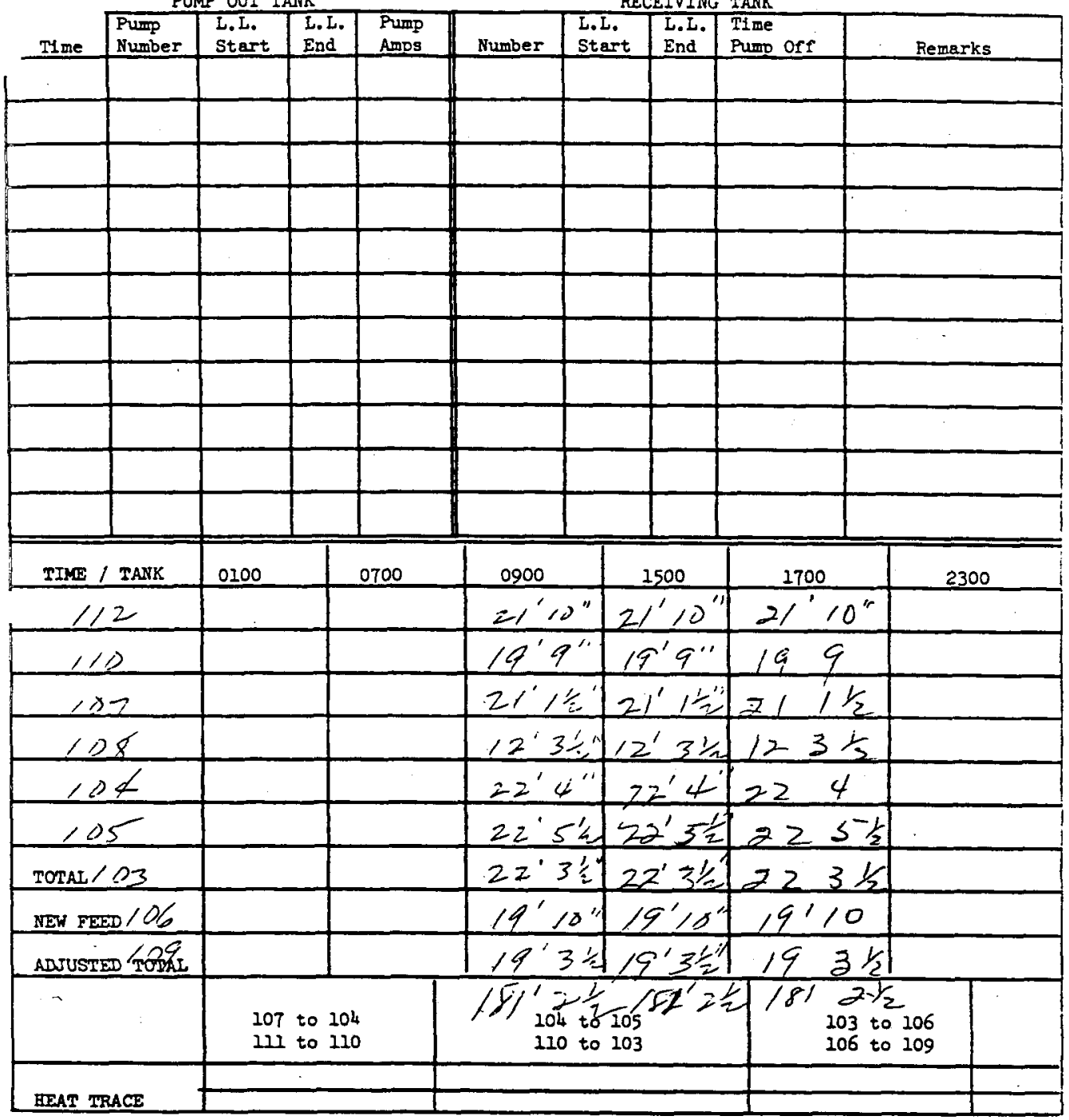

L walker:srb 9-29-70 
WHC-SD-WM-DP-209, ReV. 0

ITS-2 INVENTORY AND PUNPING

Date $Z-18-7$

PUMP OUT TANK

RECEIVING TANK

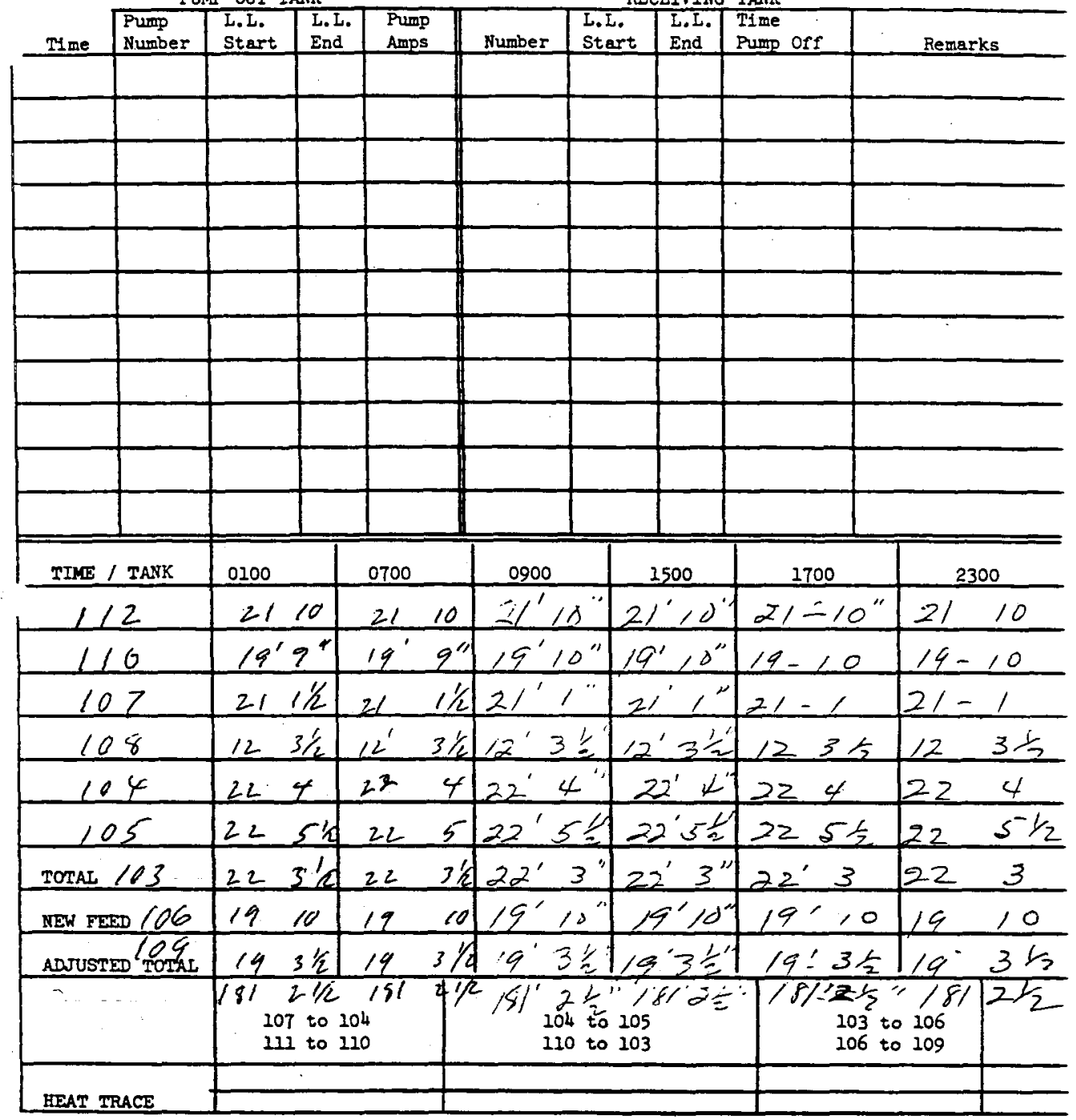

(..t walker:srb 9-29-70 
ITS-2 INVENTORY AND PUNPING

Date $7-1$ - 71

PUNP OUT TANK

RECEIVING TANK

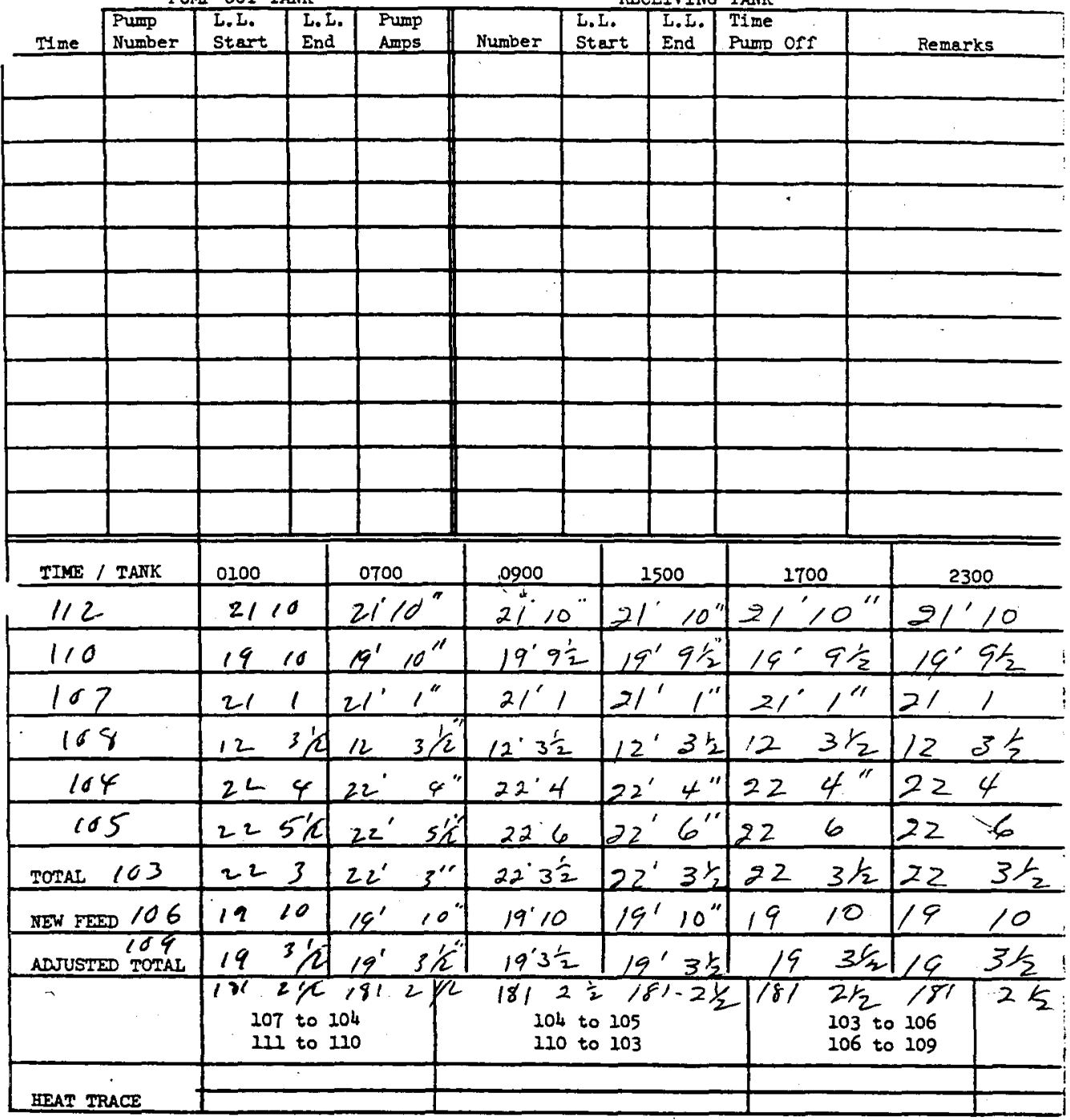

... Walker:srb 9-29-70 
IMS-2 IMVENTORY AND PUMPING

Dete $7-20-71$

PUMP OUT TANK RECEIVING TANK

\begin{tabular}{|c|c|c|c|c|c|c|c|c|c|}
\hline THme & \begin{tabular}{|l|} 
Pump \\
Number
\end{tabular} & $\begin{array}{l}\text { I. L. } \\
\text { Start } \\
\end{array}$ & $\begin{array}{l}\text { L. L. } \\
\text { End }\end{array}$ & $\begin{array}{l}\text { Pump } \\
\text { Amps }\end{array}$ & Number & $\begin{array}{l}\text { L. L. } \\
\text { Start }\end{array}$ & $\begin{array}{l}\text { L.L. } \\
\text { End }\end{array}$ & $\begin{array}{l}\text { Time } \\
\text { Pump orf }\end{array}$ & Remarks \\
\hline & 110 & & & & & & & & \\
\hline & 107 & & $>$ & Cur & uli & $\operatorname{sen} 4$ & & & \\
\hline & 104 & & ) & & & t & & & \\
\hline & & & & & & & & & \\
\hline & & & & & & & & & \\
\hline & & & & & & & & & \\
\hline & & & & & & & & & - \\
\hline & & & & & & & & & \\
\hline & & & & & & & & & \\
\hline & & & & & & & & & \\
\hline & & & & & & & & & \\
\hline
\end{tabular}

TIME / TANK

\begin{tabular}{l|l}
0100 & 0700 \\
\hline
\end{tabular}

\begin{tabular}{l|l}
0900 & 1500 \\
\hline
\end{tabular}

1700

2300

\begin{tabular}{l|ll}
112 & 21 & 10
\end{tabular}

\begin{tabular}{l|l}
110 & $19 \cdot 10$ \\
\hline
\end{tabular}

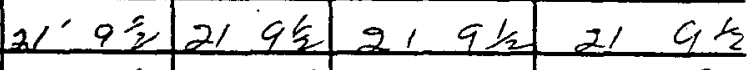

\begin{tabular}{l|ll|ll|ll}
$1910 \frac{6}{2}$ & $1910 \%$ & 199 & 199
\end{tabular}

\begin{tabular}{l|ll}
107 & 21 & 1 \\
\hline 109 & 12 & $3 / 4$
\end{tabular}

$108 \cdot 12 \quad 3 / 2$

\begin{tabular}{l|l}
104 & 22.4 \\
\hline
\end{tabular}

\begin{tabular}{l|ll}
105 & 22 & 5
\end{tabular}

\begin{tabular}{l|ll} 
TOTAL 103 & $22 \quad 3$ \\
\hline
\end{tabular}

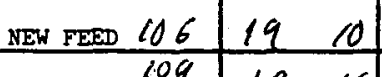

ADUSTED TORL $19 \quad 4$

$1912 \frac{1}{2}$

107 to 104

211 to 210

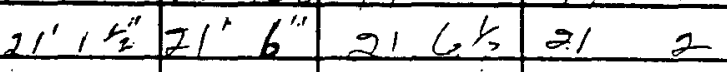

$12.4 " 123 \frac{1}{2} 123 / 5123 \frac{1}{2}$

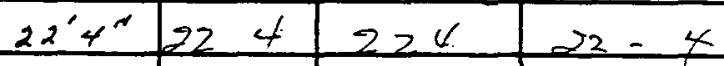

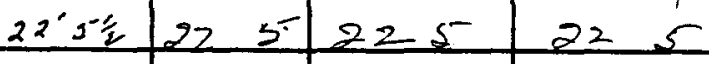

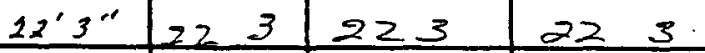

\begin{tabular}{l|l|l|l|l}
$19^{\prime} 10$ & 19.10 & 1910 & 1910 \\
\hline
\end{tabular}

$1913 \% 1 / 9$ 3/ $1931 / 193 / 2$

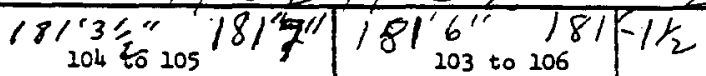

110 to 103

106 to 109

HEAT TRACE

1

Walker:srb 9-29-70 
ITS-2 INVENTORY AND PUMPING

Date $7-21-71$

PUMP OUT TANK

RECEIVING TANK

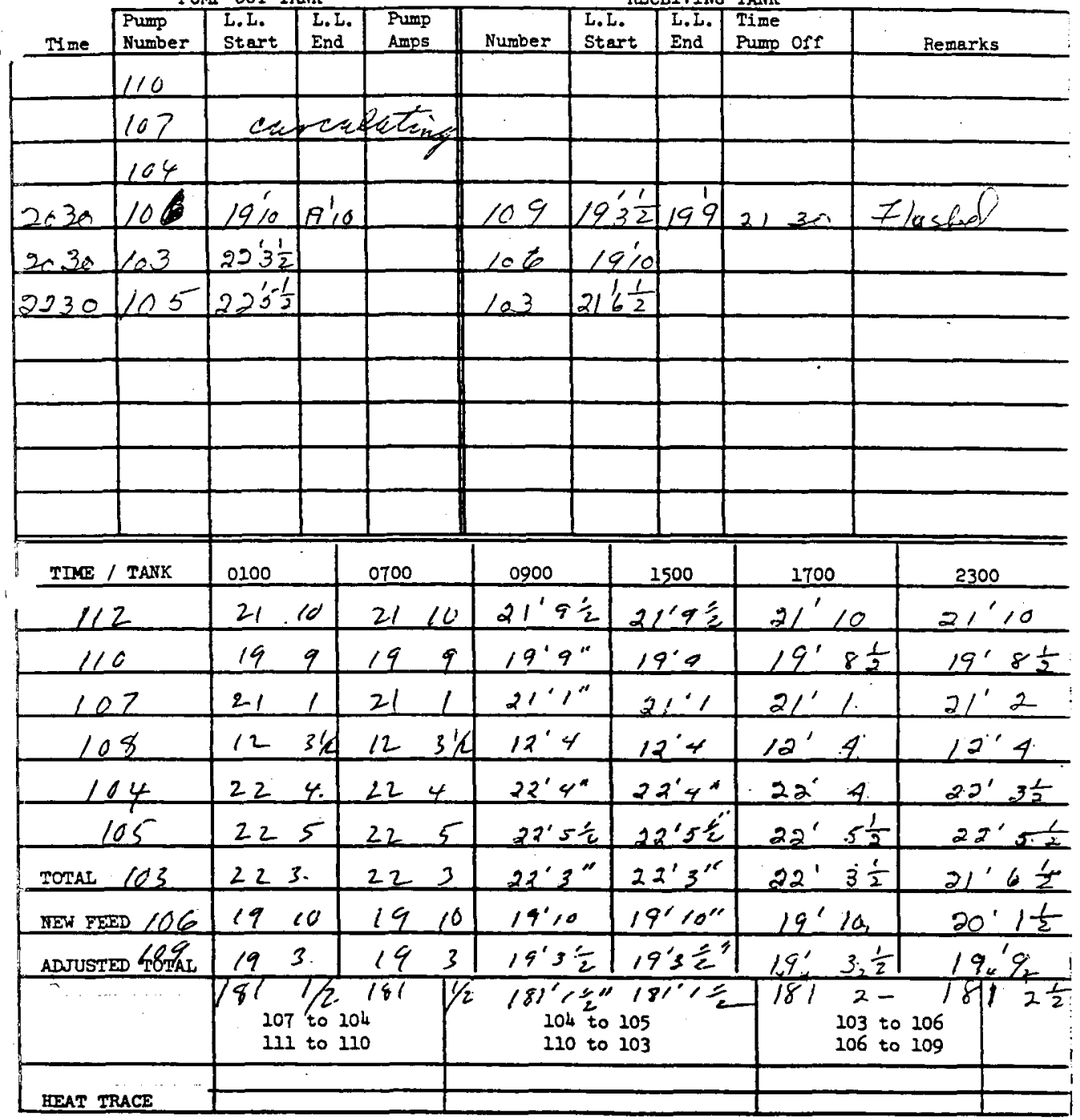

Cu Walker:srb 9-29-70 
WHC-SD-LN-DP-209, ReV. O

ITS-2 INVENTORY AND PUNPING

Date $7-22-71$

PUNP OUT TANK

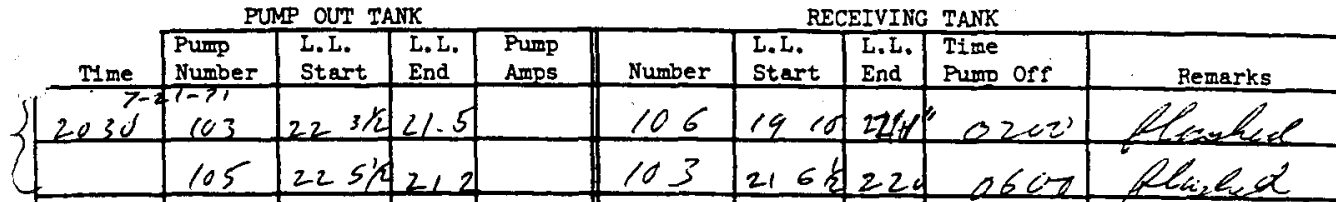

\begin{tabular}{l|l|l|l|}
\hline 530 & $10 \%$ & 224 & $21 / 4$ \\
\hline
\end{tabular}

\begin{tabular}{|l|l|l|l|}
\hline 1600 & 107 & 217 & 2012 \\
\hline
\end{tabular}

110 \& 104 Recurenlat

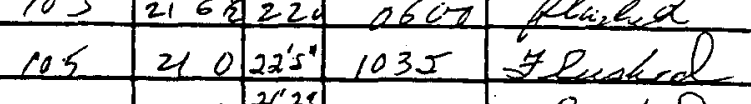

\begin{tabular}{|l|l|l|l|l|}
\hline & & & & \\
\hline 110 o & 104 & Recurenlat \\
\hline & & & & \\
\hline & & & & \\
\hline & & & & \\
\hline & & & & \\
\hline
\end{tabular}

TIME / TANK

0100

0700 104 $224 t^{2}=$

1220

ylushod

\begin{tabular}{|c|c|c|c|c|c|c|}
\hline IINE / TANK & 0100 & 0700 & 0900 & 2500 & 1700 & 2300 \\
\hline 112 & $21 \quad 10$ & 2110 & $21^{\prime} 9 \leq$ & $21^{\prime} 10^{\prime \prime}$ & $21^{\prime} 10$ & $21^{\prime} 10$ \\
\hline 110 & 199 & 199 & $19^{i} 9^{\prime \prime}$ & $19^{\prime} 9$ & $9^{\prime} q$ & $19^{\prime} 9$ \\
\hline 107 & 212 & 210 & 20.9 & $20^{\prime} 2^{\prime}$ & $20^{\prime} \mathrm{I}$ & $20^{\prime} 2$ \\
\hline 108 & $12 \quad 3 / 2$ & $12 \quad 3^{\prime} \Omega$ & $12^{\prime} 4$ & $12^{\prime} 4$ & $12^{\prime} 4$ & $12+4$ \\
\hline 104 & 224 & 216 & $2 \cdot 2^{\prime \prime}$ & 212 & $21^{\prime} 2$ & $21 \cdot 2$ \\
\hline 105 & 217 & 212 & $21 \cdot 11$ & $22^{\prime} 5^{\prime \prime}$ & $27^{\prime} 5$ & $22 ! 5$ \\
\hline TOTAL 103 & $21 \quad 6$ & 220 & $22^{\prime} 0 \frac{1}{2}$ & $22^{\prime} \circ \frac{1}{2}$ & $22^{\prime} 0 \frac{1}{2}$ & $22^{\prime} \cdot \frac{1}{2}$ \\
\hline NEW FEED 106 & 212 & 221 & $22^{\prime \prime \prime}$ & $22^{\prime} ! " 1$ & 291 & $22^{\prime} 1$ \\
\hline ADJUSTED toTh & $19 \quad 9 / 2$ & $199 / 2$ & $199 \frac{1}{2}$ & $199 \frac{2}{2}$ & $19^{\circ} 9 \frac{1}{2}$ & $19^{\prime} 9 \frac{1}{2}$ \\
\hline & $\begin{array}{ll}|8| & 5 \\
107 & \text { to } \\
111 & \text { to }\end{array}$ & $1041^{181^{\prime}}$ & $\begin{array}{r}1817 \frac{1}{2} \\
104 \text { to } \\
110 \text { to }\end{array}$ & $\begin{array}{l}181^{\prime} 7 " 1 \\
105 \\
103\end{array}$ & $\begin{array}{l}1817 \\
103 \text { to } \\
106 \text { to }\end{array}$ & \begin{tabular}{l|l}
181 & $7-$ \\
106 &
\end{tabular} \\
\hline & & & & & & \\
\hline
\end{tabular}

Lus Walker:srb 9-29-70 
ITS-2 INVENTORY AND PUMPING

Date $>-23-71$

PUMP OUT TANK

RECEIVING TANK

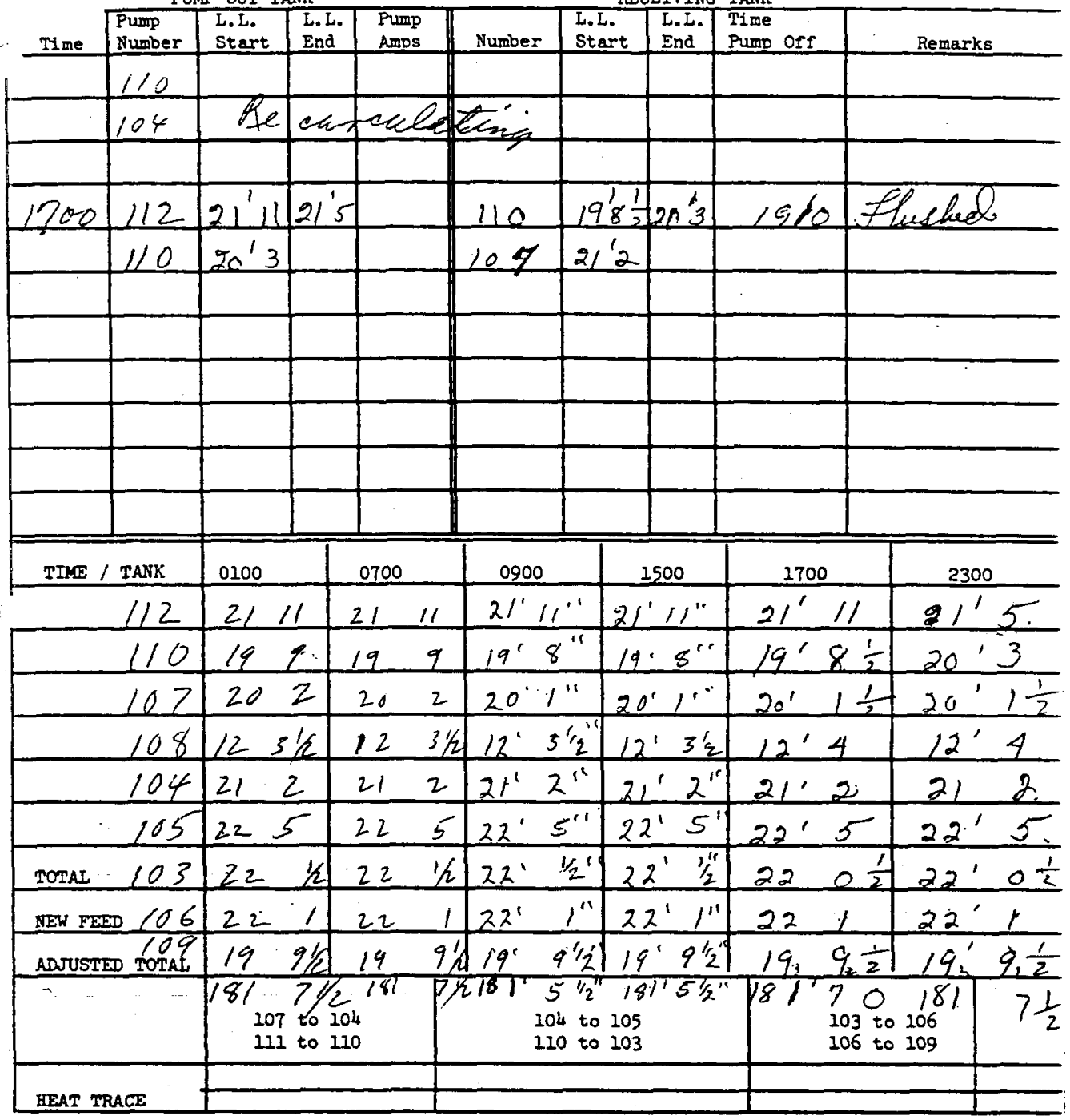

Walker:srb 9-29-70 
PUMP OUT TANK

RECEIVING TANK

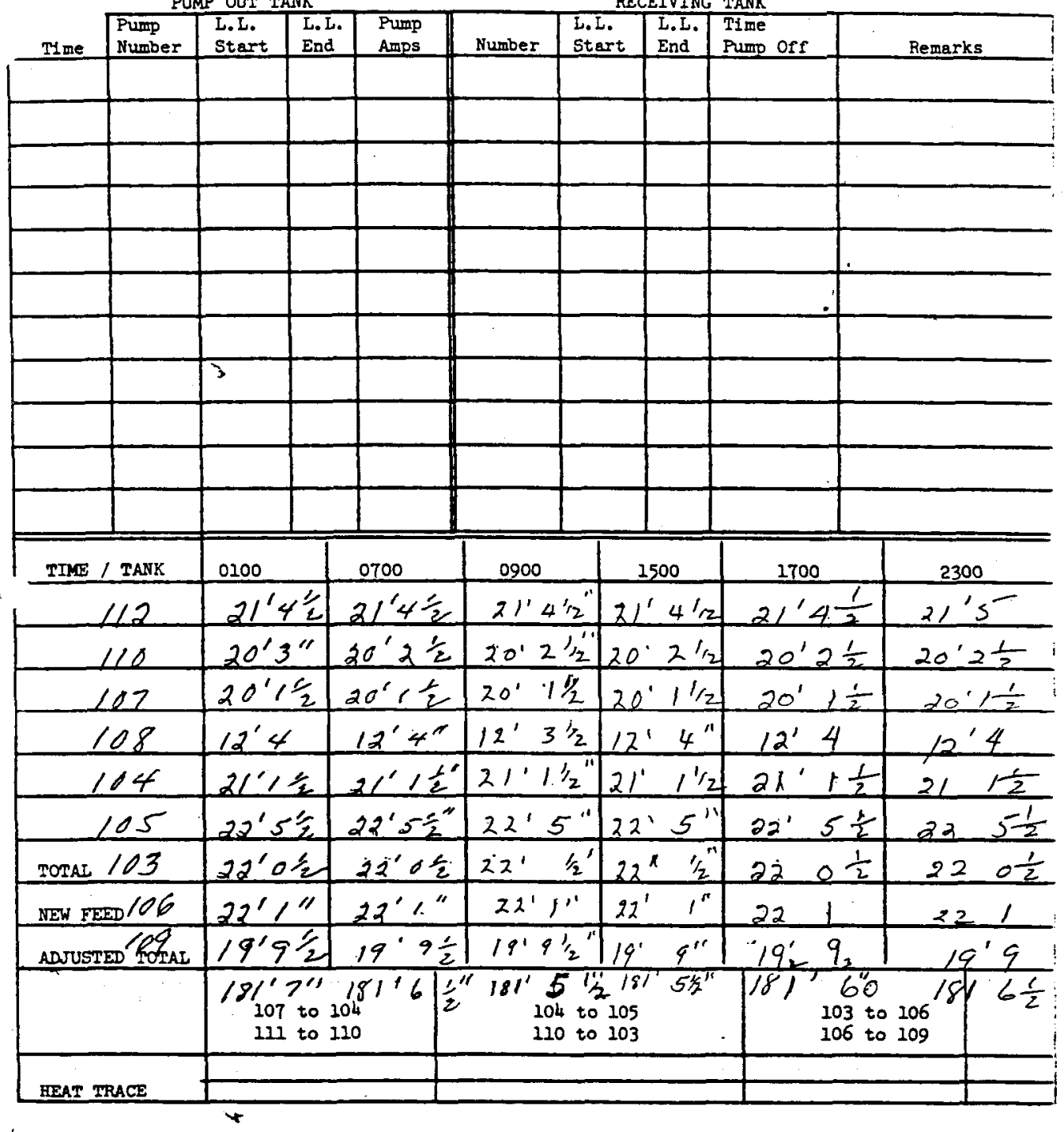

walker:srb 9-29-70 
ITS-2 INVENTORY AND PUNPING

Date $7-25-71$

PUMP OUT TANK

RECEIVING TANK

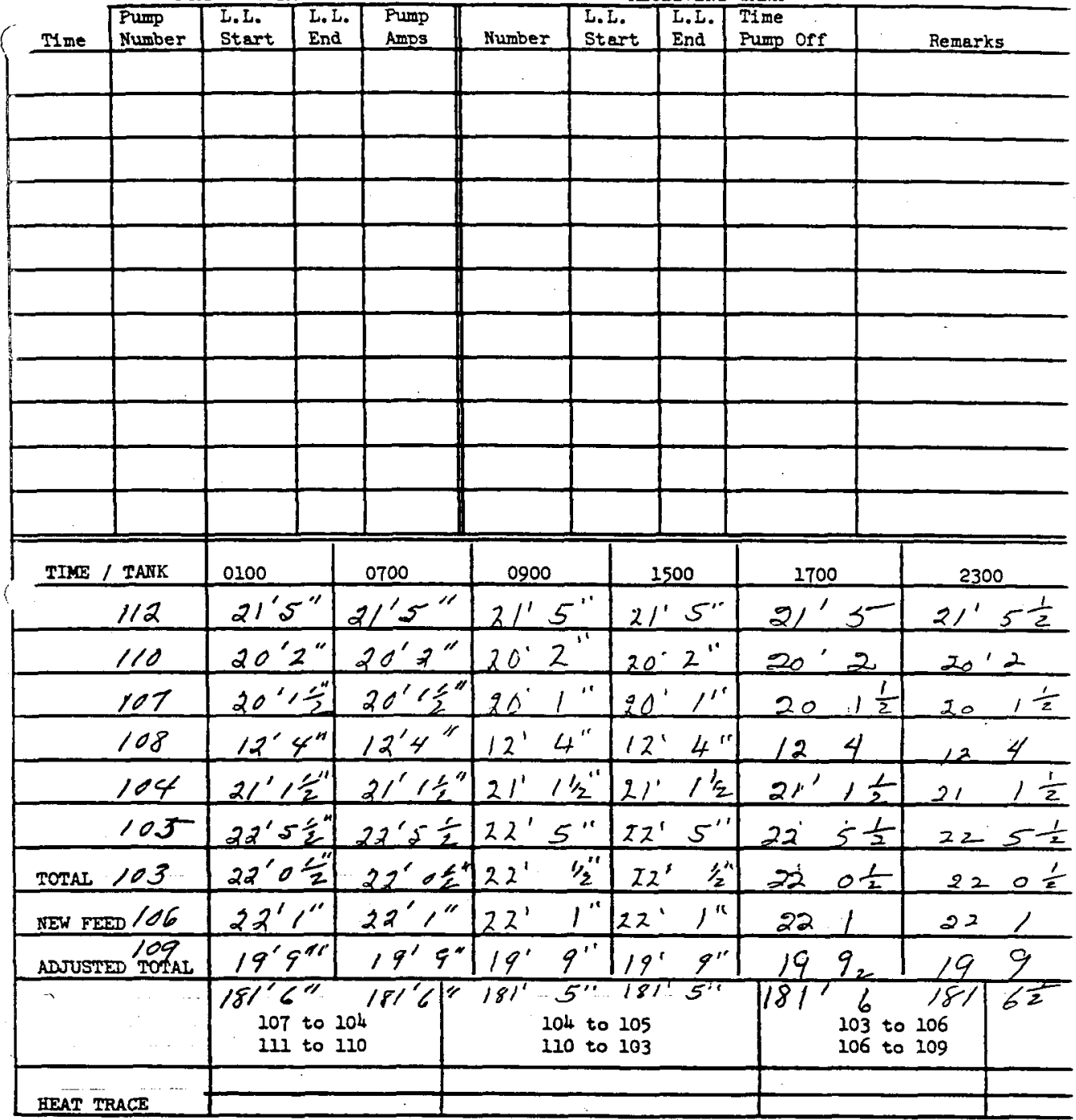

wa Walker : s5b 9-29-70 
HHC-SD-LM-DP-209, ReV. 0

ITS-2 INVENTORY AND PUMPING

Date $7-26-7 /$

PUMP OUT TANK

RECEIVING TANK

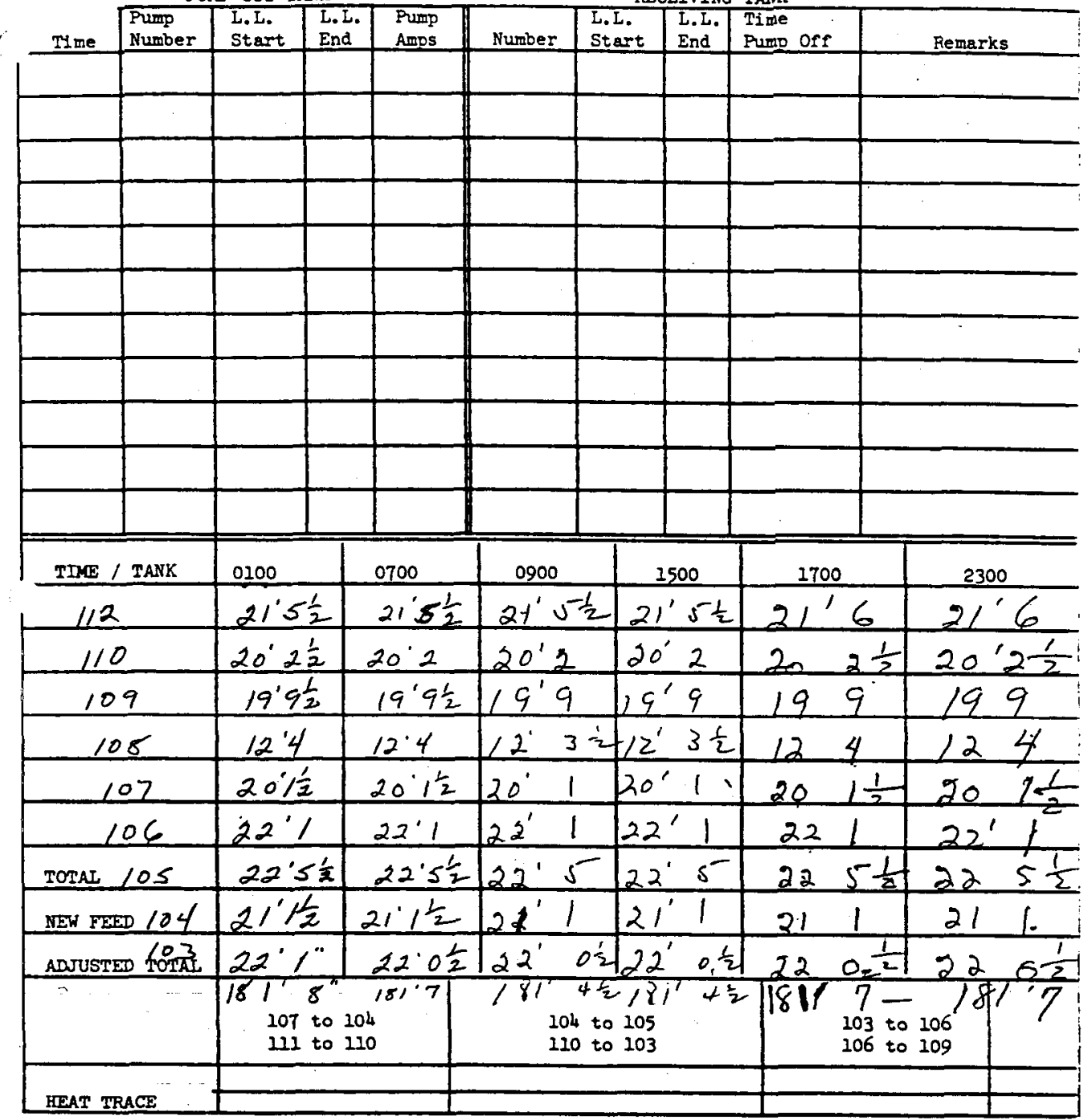

L. Wauker:strb 9-29-70

204 
ITS-2 INVENTORY AND PUMPING

Date $7-27-71$

PUMP OUT TANK

RECEIVING TANK

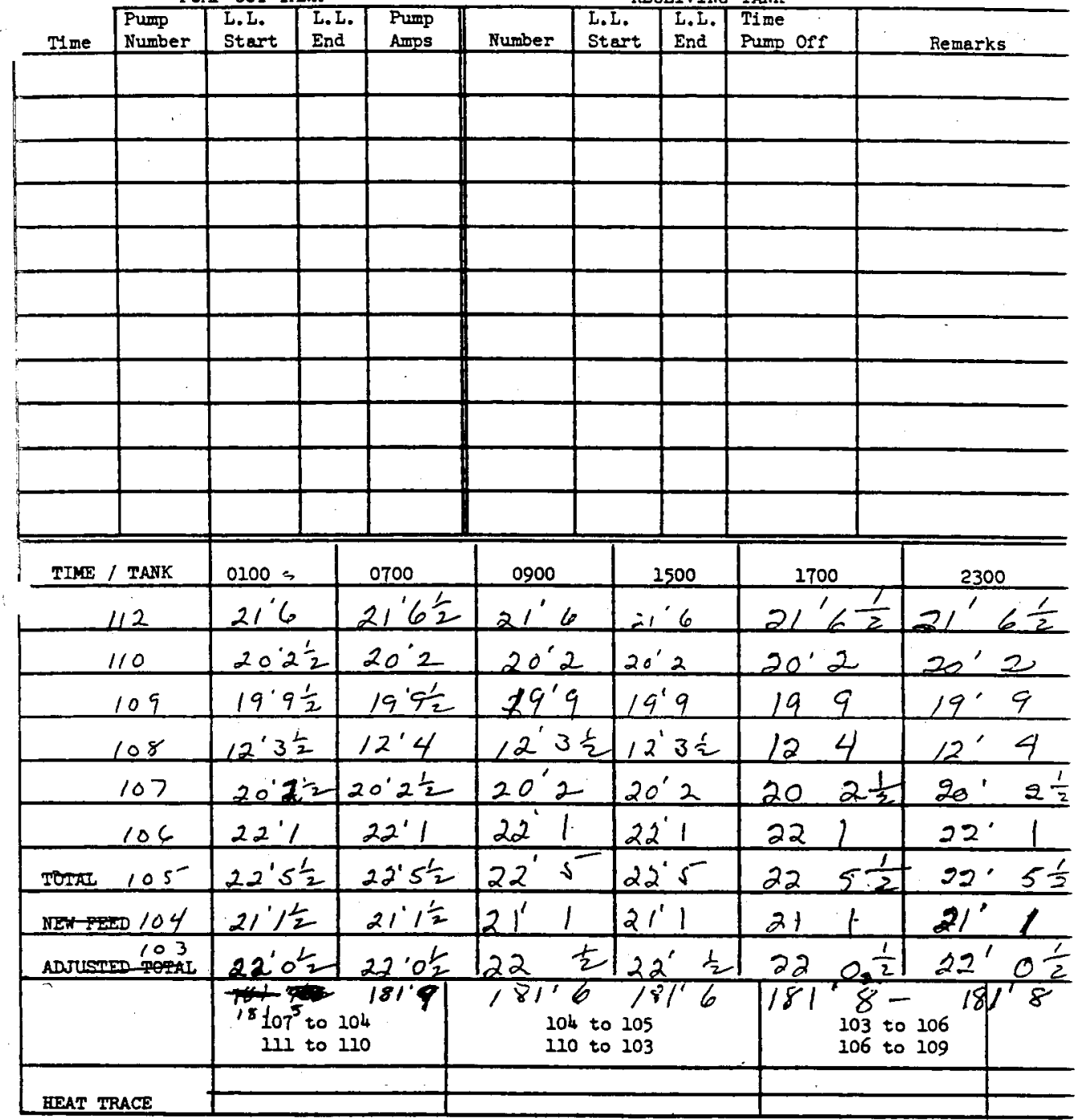

Walker:srb 9-29-70 
ITS-2 INVENTORY AND PUNPING

Date $7-28-71$

PUMP OUT TANK

RECEIVING TANK

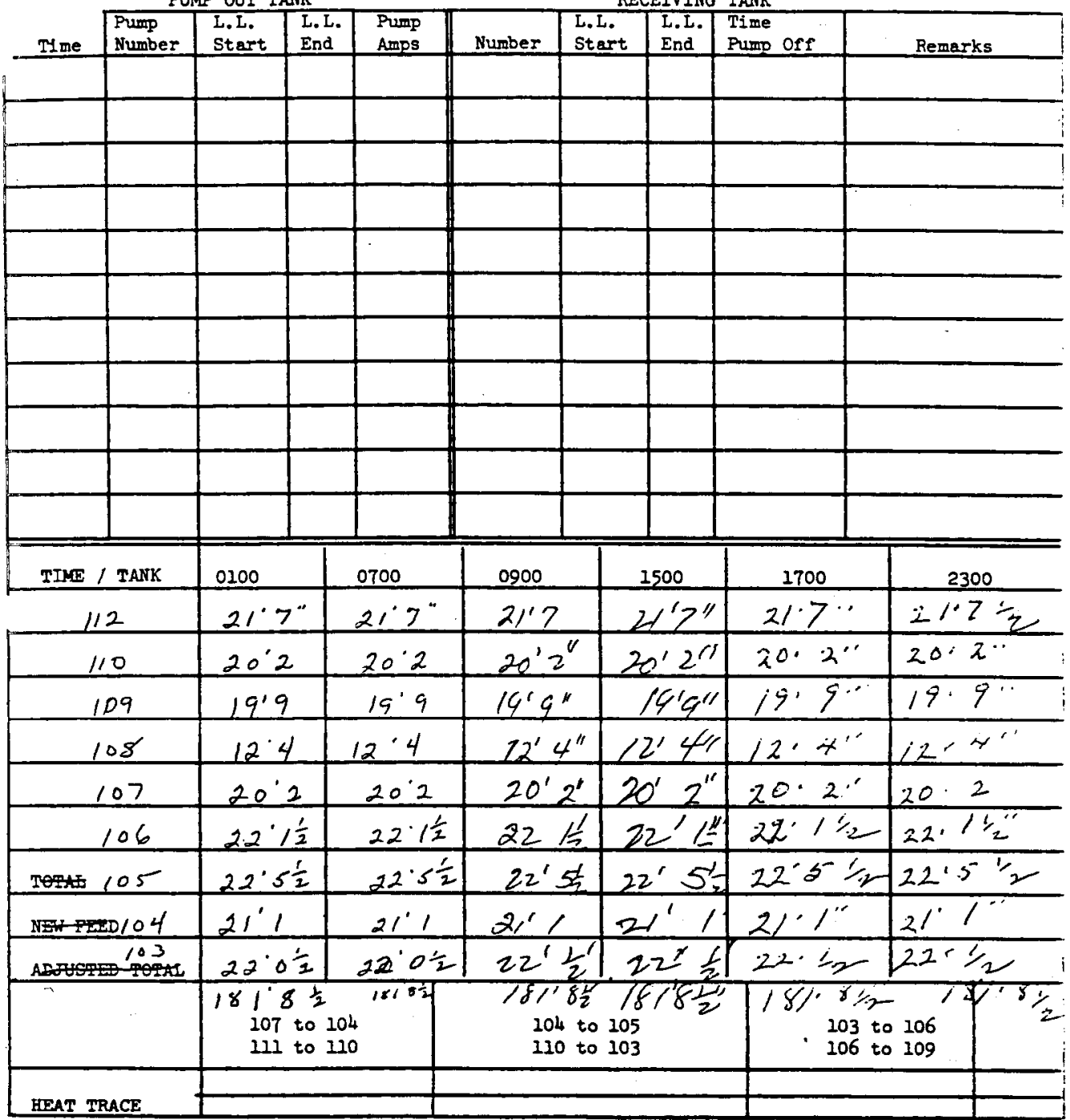

Walker:srb 9-29-70 
ITS-2 INVENTORY AND PUMPING Date 7-29-71

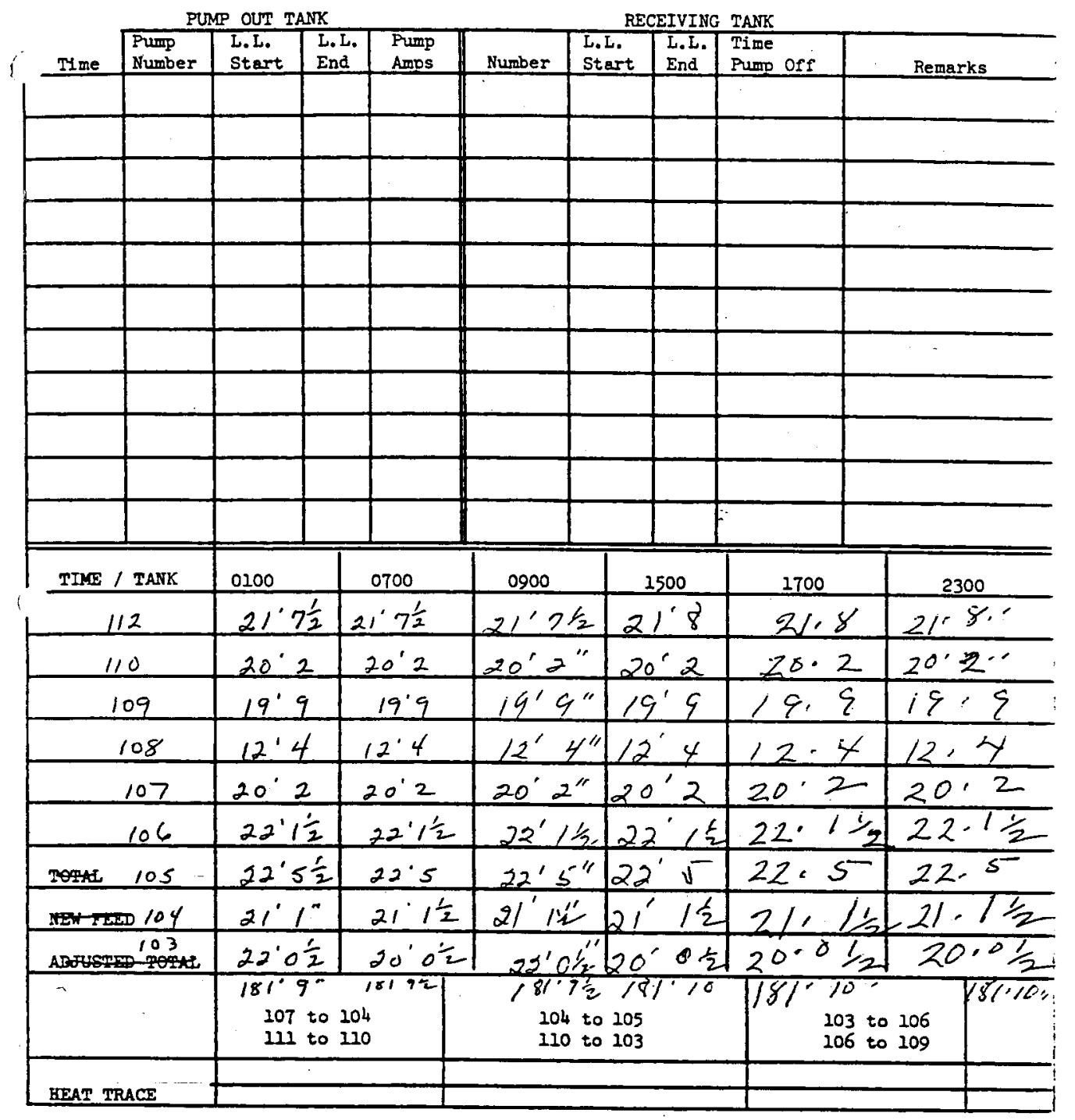

Walker:srb 9-29-70 
PUMP OUT TANK

RECEIVING TANK

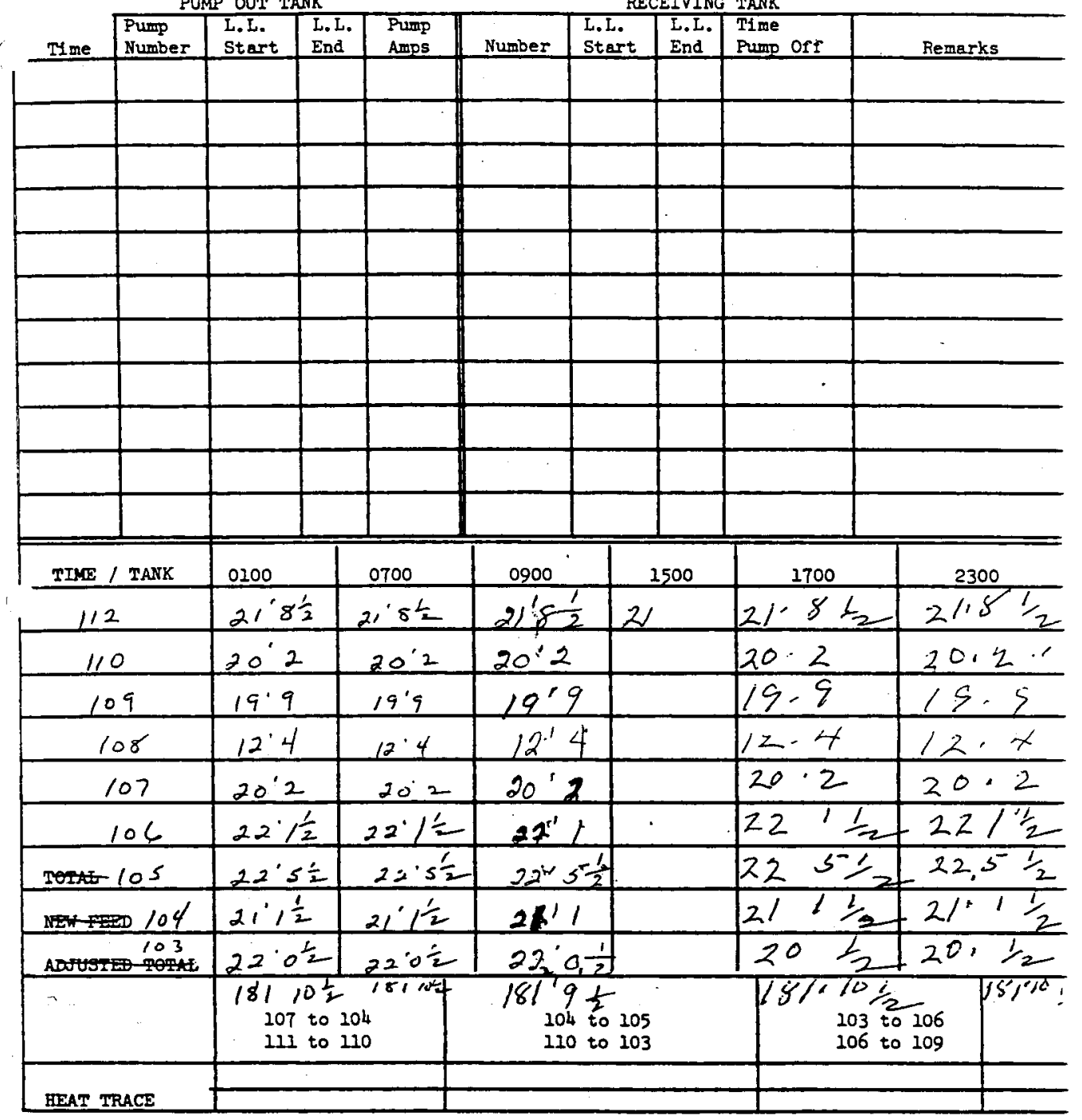

Valker:srb 9-29-70 
WHC-50-WHA-DP-209, ReV, O

ITS-2 INVENTORY AND PUMPING

Date $7-3 /-71$

PUMP OUT TANK

RECEIVING TANK

\begin{tabular}{|c|c|c|c|c|c|c|}
\hline $\begin{array}{l}\text { Pump } \\
\text { Number }\end{array}$ & \begin{tabular}{l|l} 
L.L. & L. L. \\
Start & End \\
\end{tabular} & $\begin{array}{l}\text { Pump } \\
\text { Amps }\end{array}$ & Number & \begin{tabular}{l|l|} 
L. & L.L.L. \\
art & End \\
\end{tabular} & $\begin{array}{l}\text { Time } \\
\text { Pump orf }\end{array}$ & Remarks \\
\hline & & & & & & \\
\hline & & & & & & \\
\hline & & & & & & \\
\hline & & & & & & \\
\hline & & & & & & \\
\hline & & & & & & \\
\hline & & & & & & \\
\hline & & & & & & \\
\hline & & & & & & \\
\hline & & & & & & \\
\hline & & & & & & \\
\hline TIME / TANK & 0100 & 0700 & 0900 & 1500 & 1700 & 2300 \\
\hline $1 / 2$ & $2 / 8 \frac{1}{2}$ & 2185 & $21^{\prime} 8 \frac{1}{2}$ & $21^{\prime} 8 \frac{1}{2}$ & $21.8 \frac{1}{2}$ & $21.8 \%$ \\
\hline 110 & $202^{\prime \prime}$ & 202 & 202 & $20 \cdot 2$ & $20 \cdot 20$ & 20.2 \\
\hline 109 & $19^{\prime} .97$ & $14^{\prime} 9^{\prime \prime}$ & 199 & $19^{\prime 9}$ & 19.3 & $14-q$ \\
\hline 108 & $12^{\prime} y^{\prime \prime}$ & $1214^{8}$ & 124 & $10^{\prime} 4$ & $12-4$ & 12.4 \\
\hline 107 & $20 \cdot 2^{\prime \prime}$ & 212 & $201 \div$ & $20^{\circ} 1 \frac{1}{2}$ & $201 / 2$ & $201 / 2$ \\
\hline 106 & $22^{\prime} 1 \frac{1}{2}$ & $221 \% \frac{1}{2}$ & $22, \frac{1}{2}$ & $22, \frac{1}{2}$ & $221 \frac{1}{2}$ & $221 \frac{1}{2}$ \\
\hline$=105$ & $225 \frac{1}{2}$ & $225 \leq 1$ & $225 \frac{1}{2}$ & $22^{\prime} 5 \frac{1}{2}$ & $22 \cdot 5=\frac{1}{2}$ & $22.5 \frac{1}{2}$ \\
\hline 104 & $2 \% 1 \frac{1}{2}$ & $41 \%$ & $21 \times \frac{1}{2}$ & $211 \frac{1}{2}$ & $2 \sigma^{\prime} / \frac{1}{2}$ & $-2 / 1 / 2$ \\
\hline $\begin{array}{r}103 \\
\end{array}$ & $20 \quad L$ & $28^{\prime} \geq$ & $220 \frac{1}{2}$ & $920 \frac{1}{2}$ & $22-0 \frac{1}{2}$ & $220 \%$ \\
\hline-1 & $\begin{array}{l}19110 \frac{1}{2} \\
107 \text { to } 10 \\
111 \text { to } 1\end{array}$ & $\begin{array}{l}.04^{10110 k} \\
.10\end{array}$ & $\begin{array}{l}18110 \\
104 \text { to } \\
110 \text { to }\end{array}$ & $\begin{array}{l}181^{\prime} \% \\
105 \\
103\end{array}$ & $\begin{array}{l}\left.18\right|^{1} 10 \\
103 \text { to } \\
106 \text { to }\end{array}$ & \begin{tabular}{l|l} 
& \\
106 & \\
109 &
\end{tabular} \\
\hline & & & & & & \\
\hline
\end{tabular}

(') Wauker:8rb 9-29-70 
WHC-SD-LM-DP-209, Rev. 0

ITS-2 INVENTORY AND PUMPING

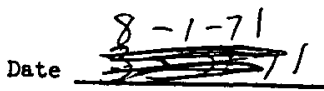

PUMP OUT TANK

RECEIVING TANK

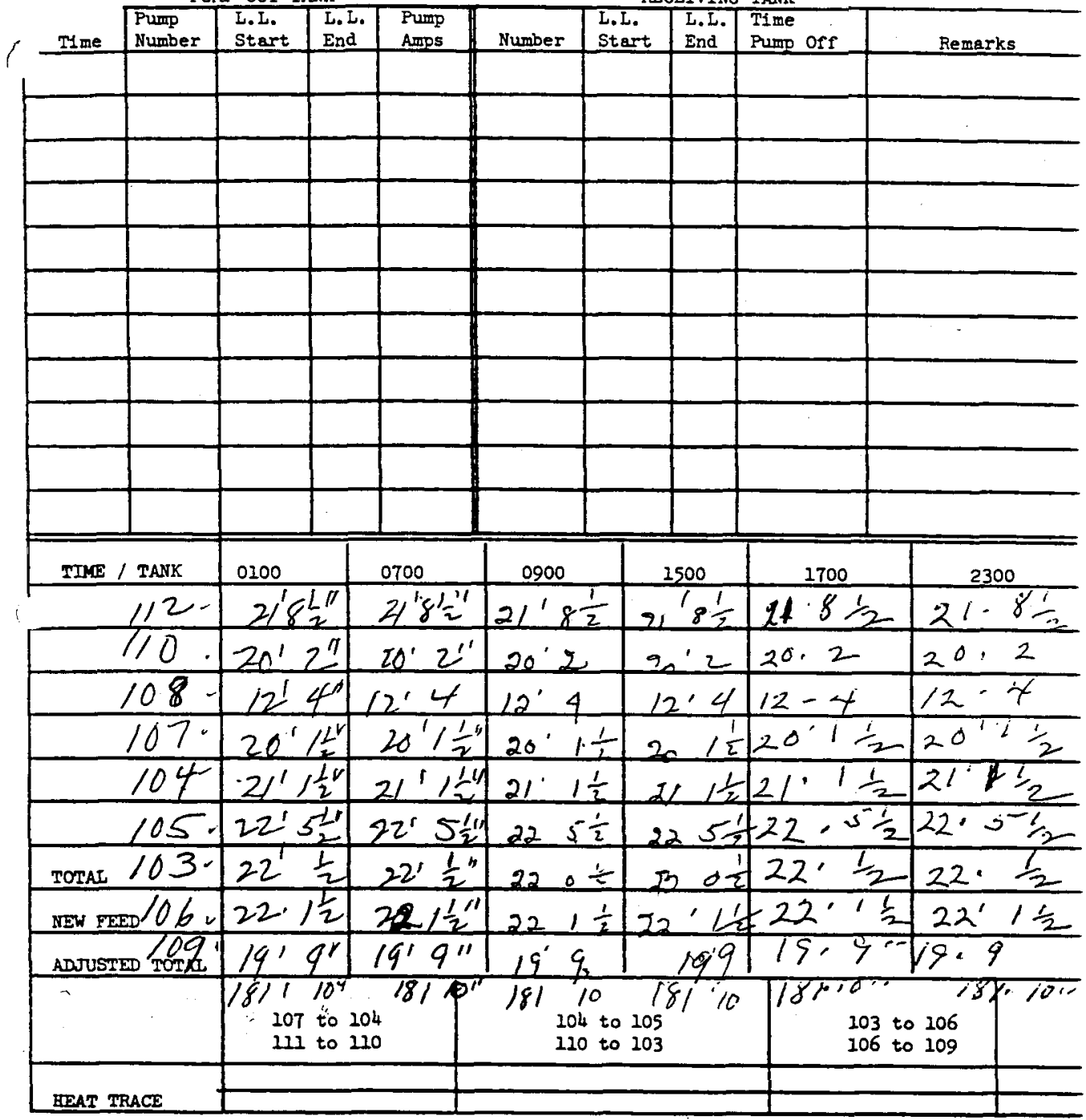

Walker:srb 9-29-70 503 
WHC-SD-WM-OP-209, ReV. 0

ITS-2 INVENTORY AND PUMPING

Date $8-2-71$

PUMP OUT TANK

RECEIVING TANK

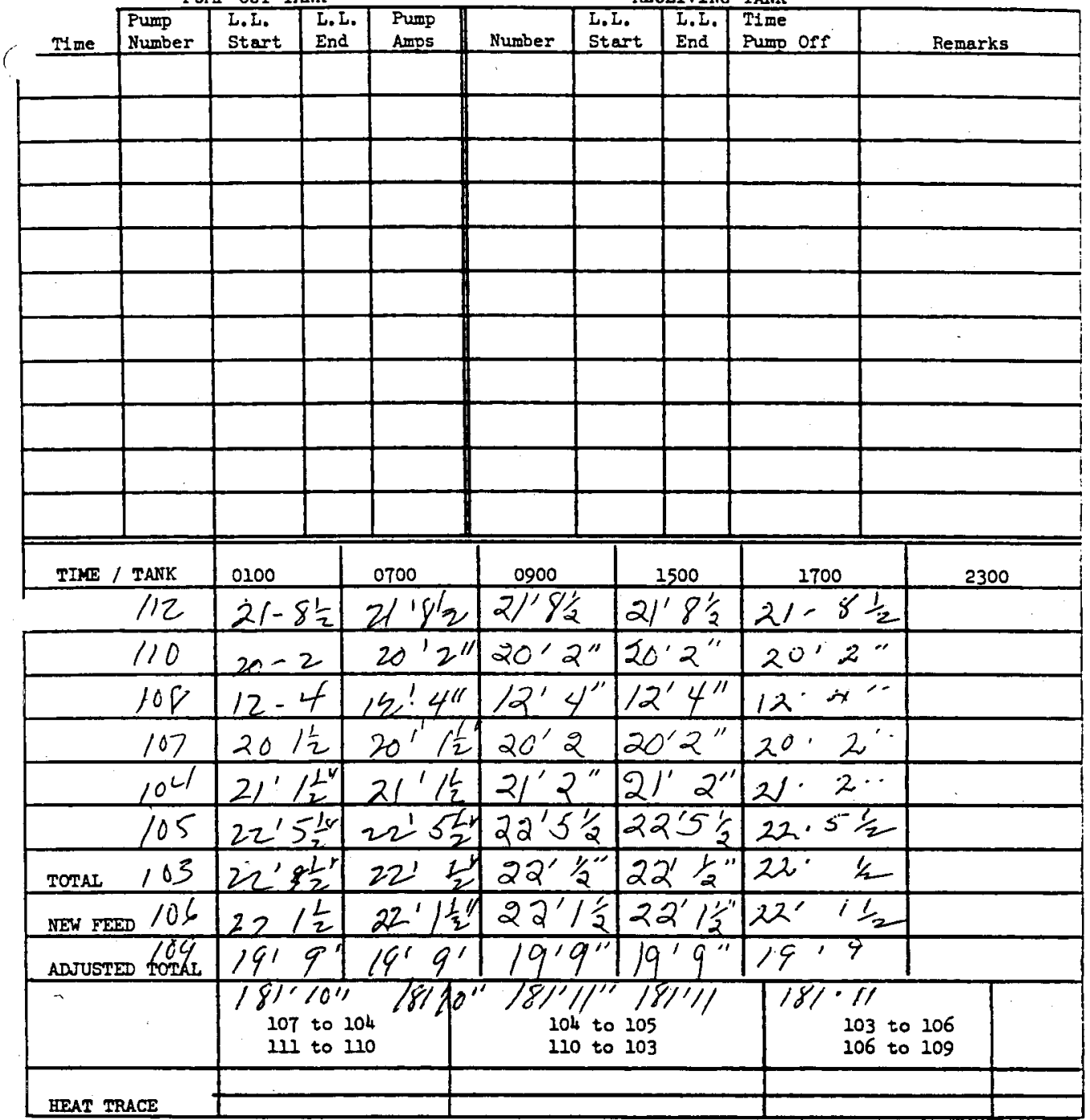

(. Walker:srb 9-29-70 
ITS-2 INVENTORY AND PUMPING Date $8-3.71$ PUNP OUT TANK RECEIVING TANK

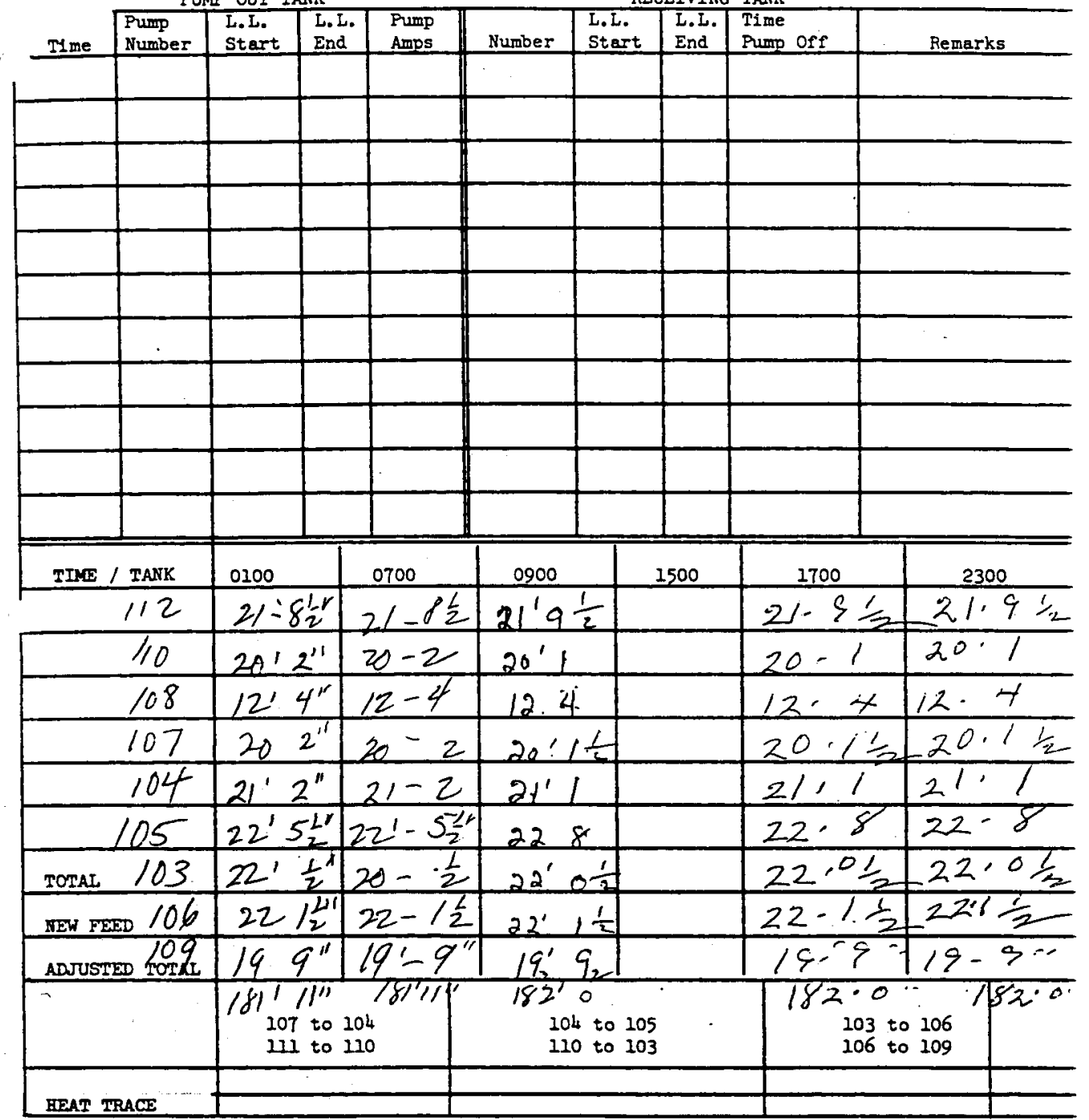

Walker:srb 9-29-70 
WHC-SD-WM-DP-209, ReV. O

ITS-2 INVENTORY AND PUMPING Date 8-471

PUNP OUT TANK RECEIVING TANK

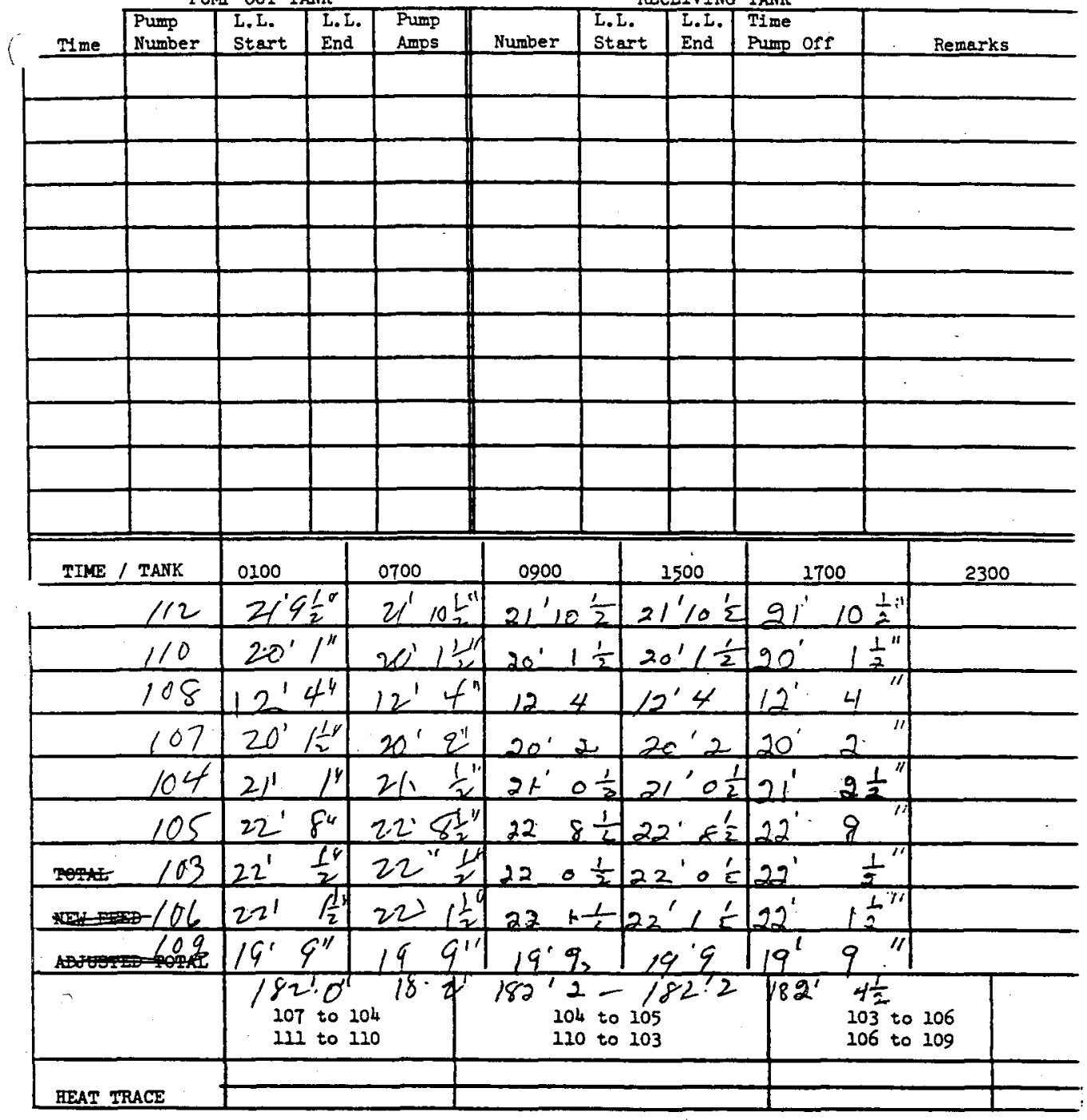

Sil wauker:srb 9-29-70 
WHC-SD-WM-DP-209, ReV. O

ITS-2 INVENTORY AND PUMPING Date $8-5-71$

PUMP OUT TANK

RECEIVING TANK

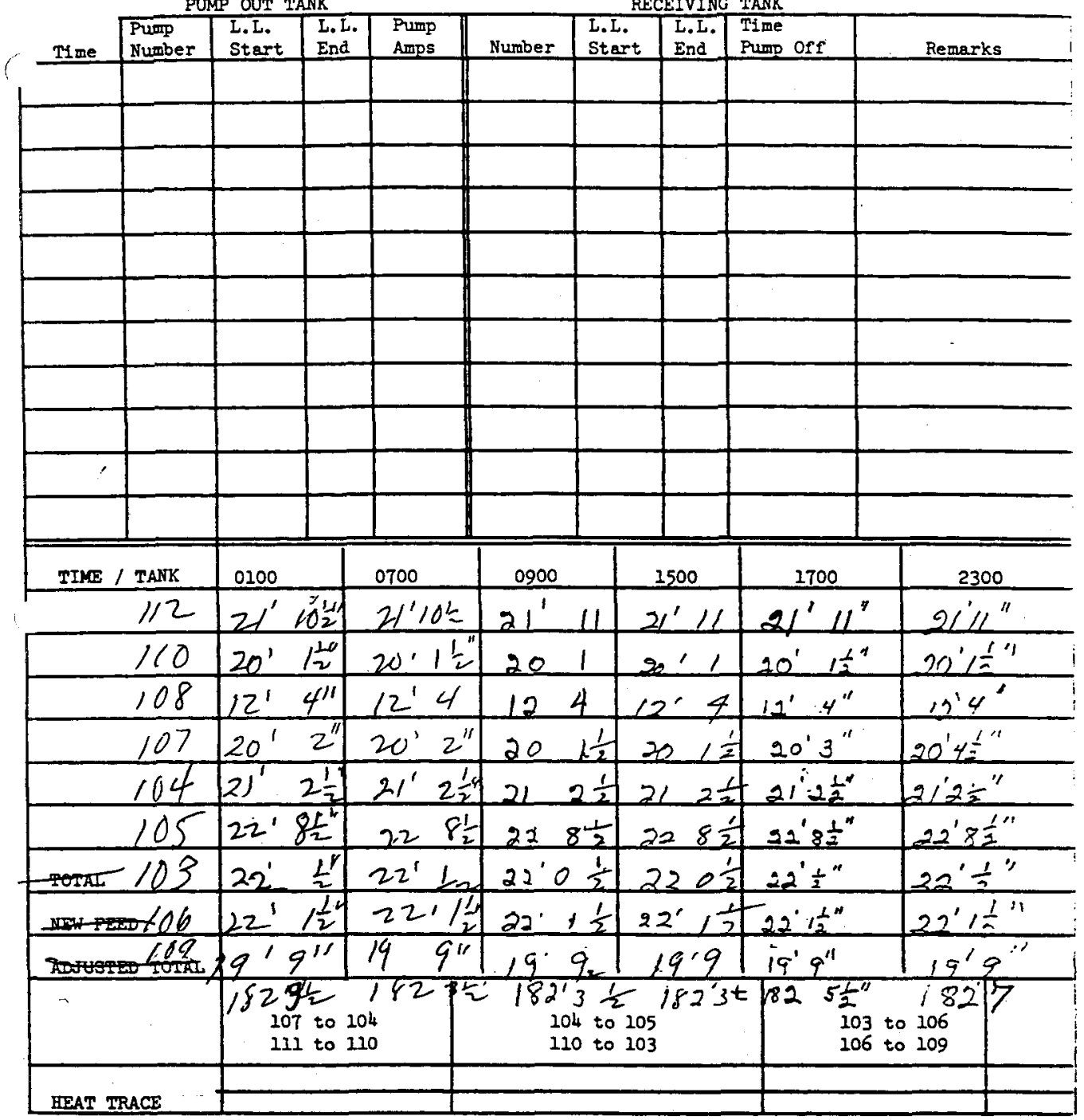

(Walker:sro 9-29-70 
PUNP OUT TANK

RECEIVING TANK

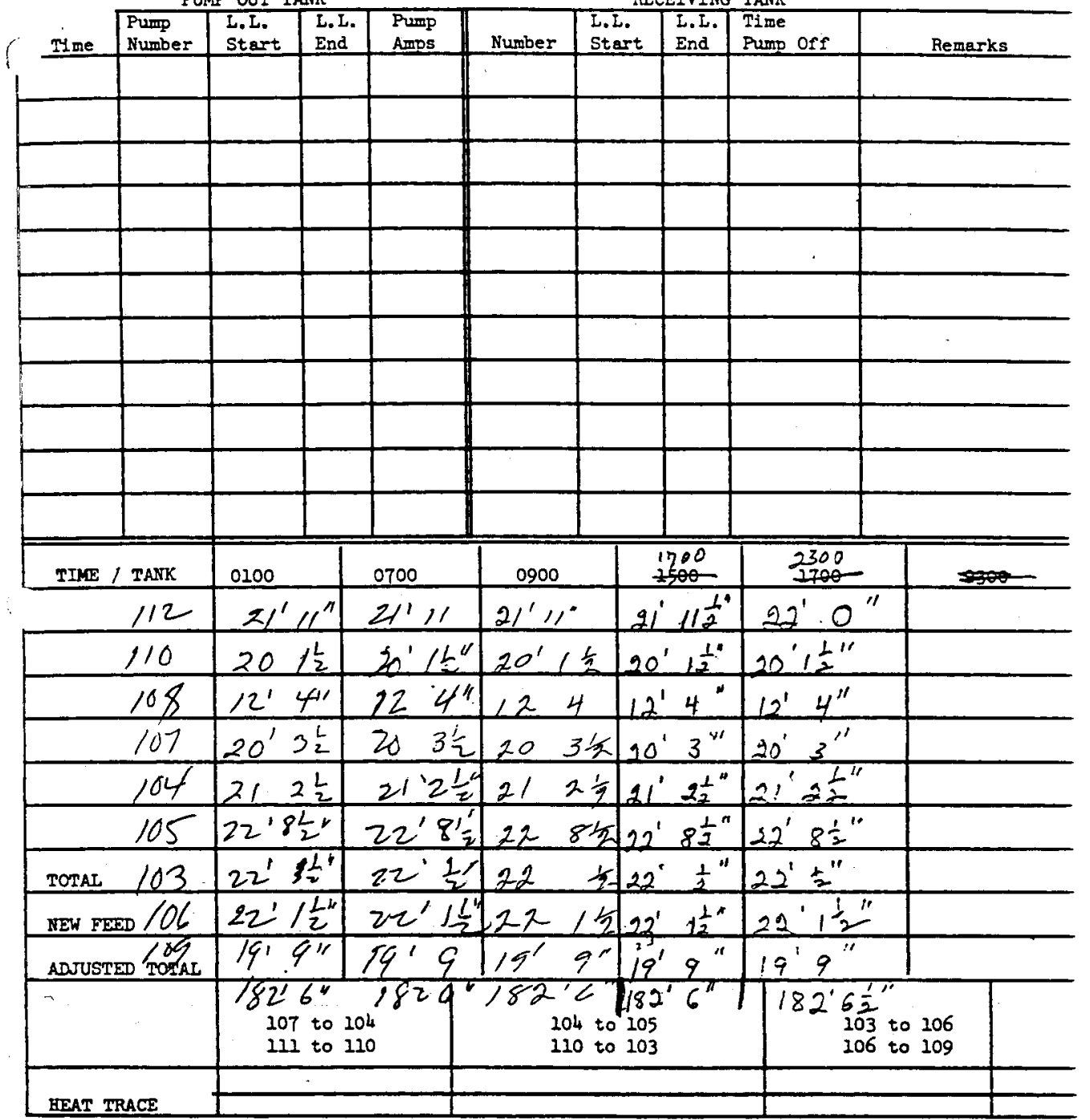

1 Walker:srb 9-29-70 
PUMP OUT TANK

RECEIVING TANK

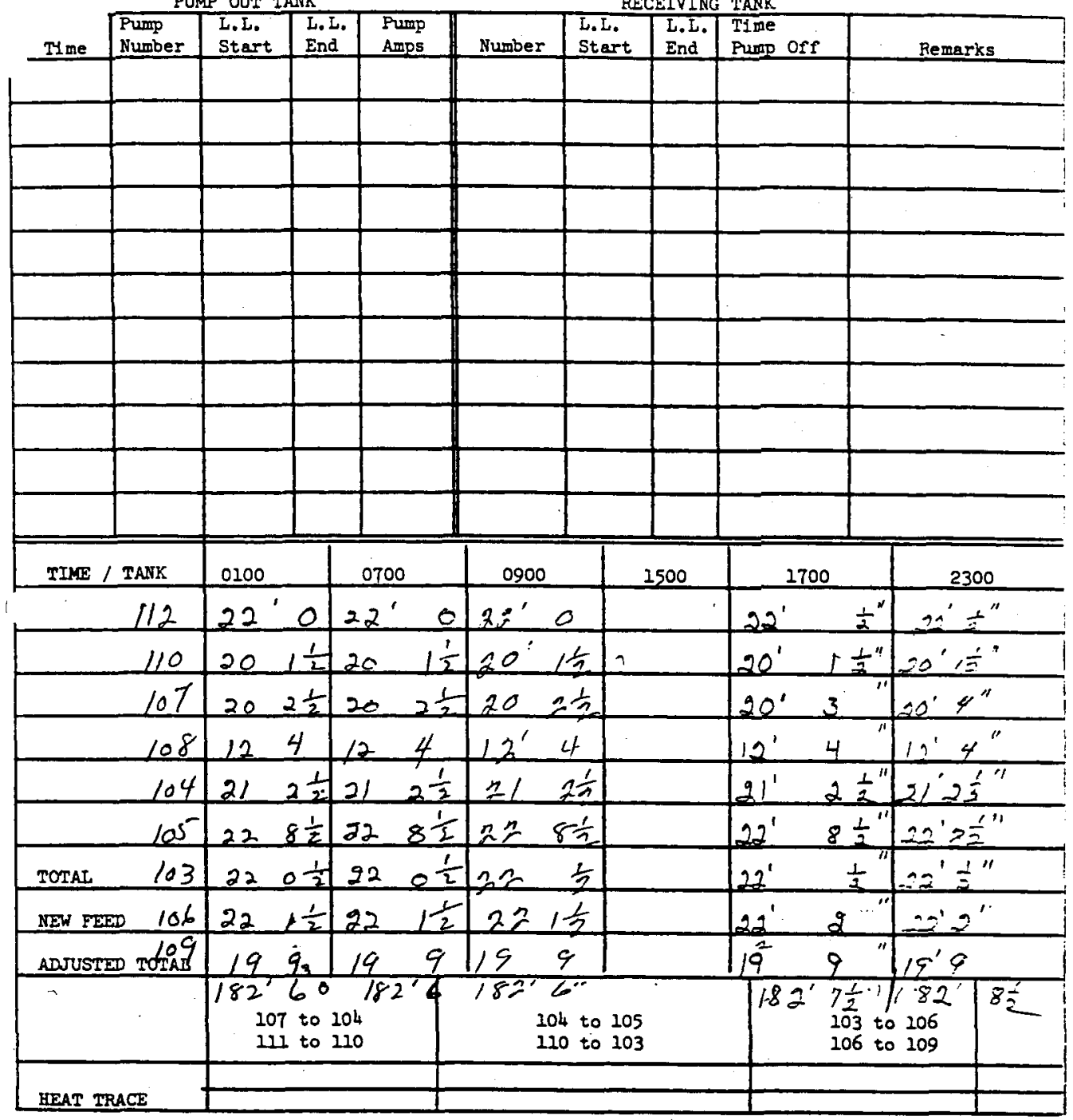

Walker:srb 9-29-70 
ITS-2 INVENTORY AND PUMPING Date AUG 819\%:

PUMP OUT TANK

RECEIVING TANK

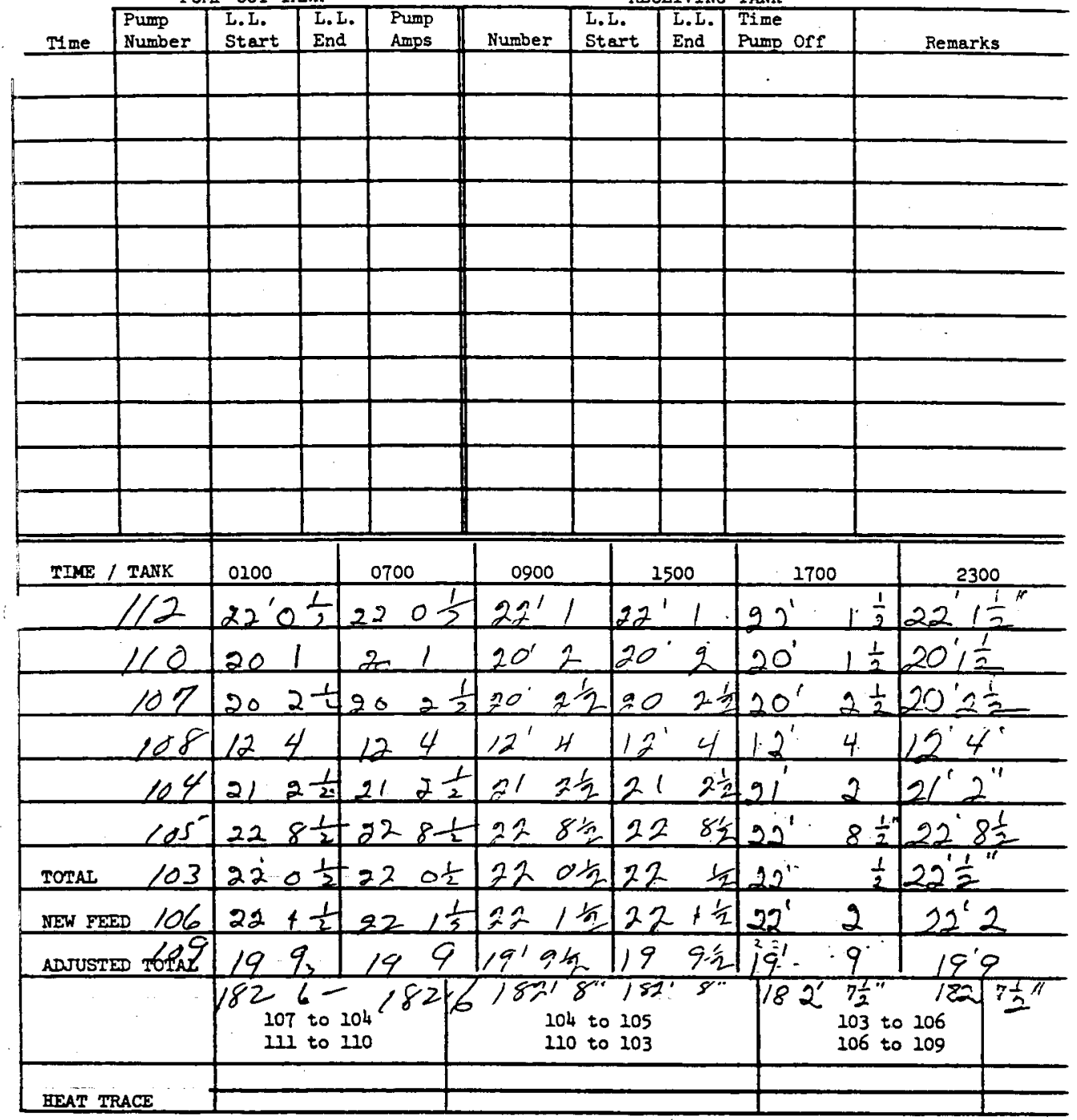

WaIker:srb 9-29-70 
WHC-SD-WM-OP-209, ReV. 0

ITS-2 INVENTORY AND PUMPING Date $\mathrm{AU6} \times 9197:$

PUNP OUT TANK RECEIVING TANK

\begin{tabular}{|c|c|c|c|c|c|c|}
\hline \multirow[b]{2}{*}{ Tyme } & & \\
\hline & \begin{tabular}{l|l} 
L.L. & L.I \\
Start & End \\
\end{tabular} & $\begin{array}{l}\text { Pump } \\
\text { Amps }\end{array}$ & Number & $\begin{array}{l}\text { L.L. } \\
\text { End }\end{array}$ & \begin{tabular}{|l} 
Time \\
Pump off \\
\end{tabular} & Remarks \\
\hline & & & & & & \\
\hline & & & & & & \\
\hline & & & & & & \\
\hline & & & & & & \\
\hline & & & & & & \\
\hline & & & & & & . \\
\hline & & & & & & \\
\hline & & & & & & . \\
\hline . & & & & & & \\
\hline & & & & & & \\
\hline TIME / TANK & 0100 & 0700 & 0900 & 1500 & 2700 & 2300 \\
\hline 112 & $22^{\circ} 1 \frac{1}{2}$ & $22^{\prime} 1 \frac{1}{2}$ & $2211 / 2$ & $22 \quad 1 \frac{1}{2}$ & $22^{\prime}-1$ & $22^{\prime}-1 / 2$ \\
\hline 110 & $20 \quad 1 \frac{1}{2}$ & $20 \quad 1 \frac{1}{2}$ & $201 / 41$ & $20 \quad 1 / 2$ & $20 \div 1$ & $20^{\prime}-1$ \\
\hline 109 & $20 \cdot 2 \frac{1}{2}$ & $20 \quad 2 \frac{1}{2}$ & $20 \quad 2 \%$ & $20 \quad 2 \%$ & $20 \div-1 / 2$ & $20^{\circ}-1 / 2$ \\
\hline 108 & $12^{\circ} 4$ & 124 & 124 & 124 & $12^{\prime}-4$ & $12 \cdot 4$ \\
\hline 104 & $21.2 \frac{1}{2}$ & $212 \frac{1}{2}$ & 21212 & $21 \quad 2 \frac{1}{2}$ & $21^{\prime}-2$ & $2 \prime^{\prime}-2$ \\
\hline $105^{-}$ & $228 \frac{1}{2}$ & $228 \frac{1}{2}$ & $2281 / 2$ & $22 \quad 8 \%$ & $22-8 \%$ & $22^{\prime}-8 / 2$ \\
\hline TOTAL 103 & $22 \quad 0 \frac{1}{2}$ & $220 \frac{1}{2}$ & $220 \%$ & $220 \frac{1}{2}$ & $22^{\prime}-01 / 2$ & $22 \% 0 \%$ \\
\hline NEW FEED 106 & 222 & 222 & $22=$ & 222 & $22^{\prime}-2$ & $22^{\prime}-2$ \\
\hline ADUUSTED TOTAL? & 19.9 & 199 & 199 & 199 & $19^{\prime}-9$ & $19: 9$ \\
\hline & $\begin{array}{l}182,8 \\
107 \text { to } \\
111 \text { to }\end{array}$ & \begin{tabular}{l|l}
182 & 8 \\
104 &
\end{tabular} & $\begin{array}{l}1828 \\
104 \text { to } \\
110 \text { to }\end{array}$ & $\begin{array}{l}1828 \\
105 \\
103\end{array}$ & $\begin{array}{r}182:-5 / 2 \\
103 \\
106\end{array}$ & $\begin{array}{l}182^{\prime}-K^{\prime \prime} \\
106 \\
109\end{array}$ \\
\hline HEAT TRACE & & & & & & \\
\hline
\end{tabular}

- il Walker:srb 9-29-70 
PUMP OUT TANK

RECEIVING TANK

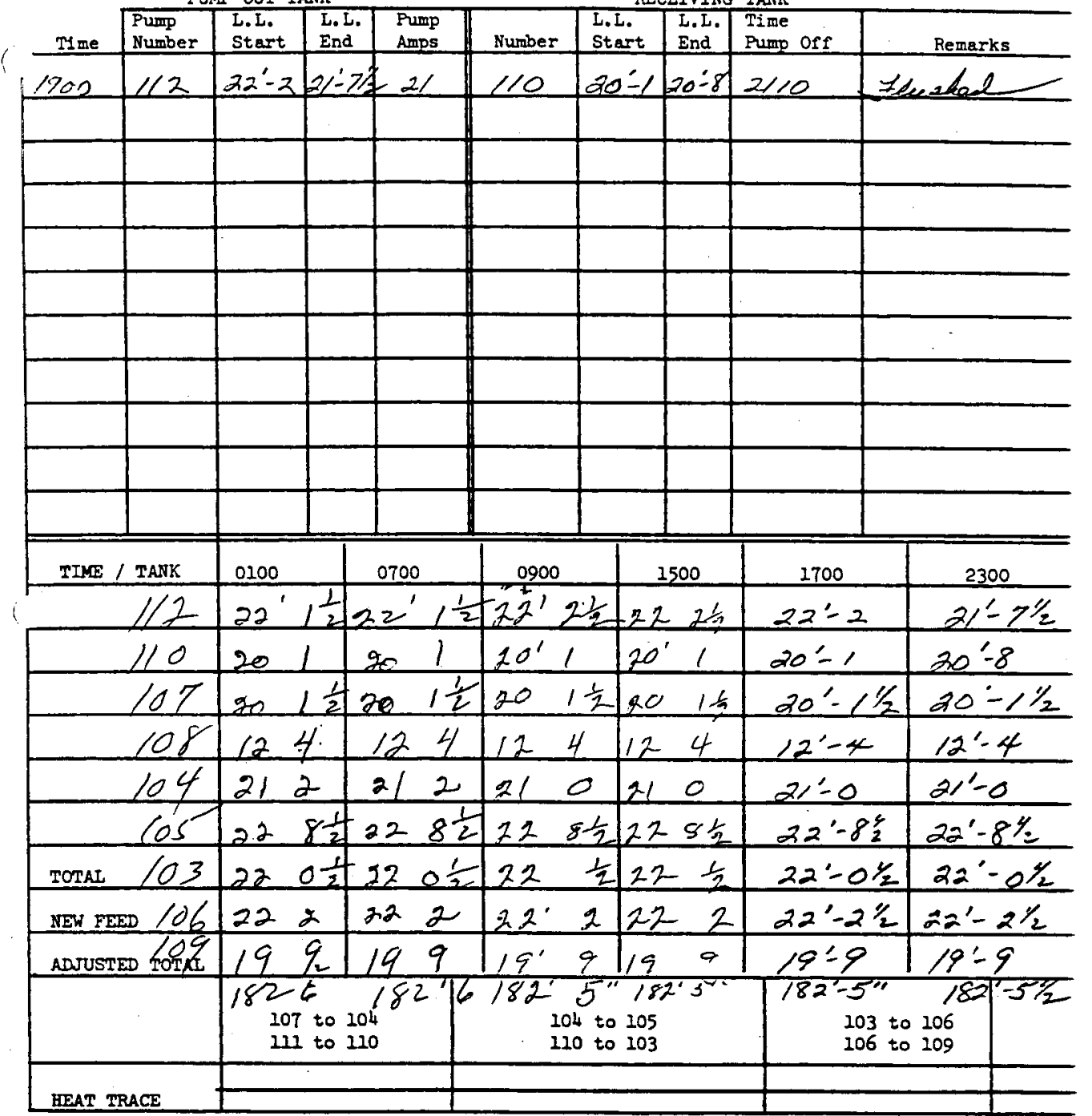

(Walker:srb 9-29-70 
ITS-2 INVENTORY AND PUMPING

Date AUG it

PUMP OUT TANK

RECEIVING TANK

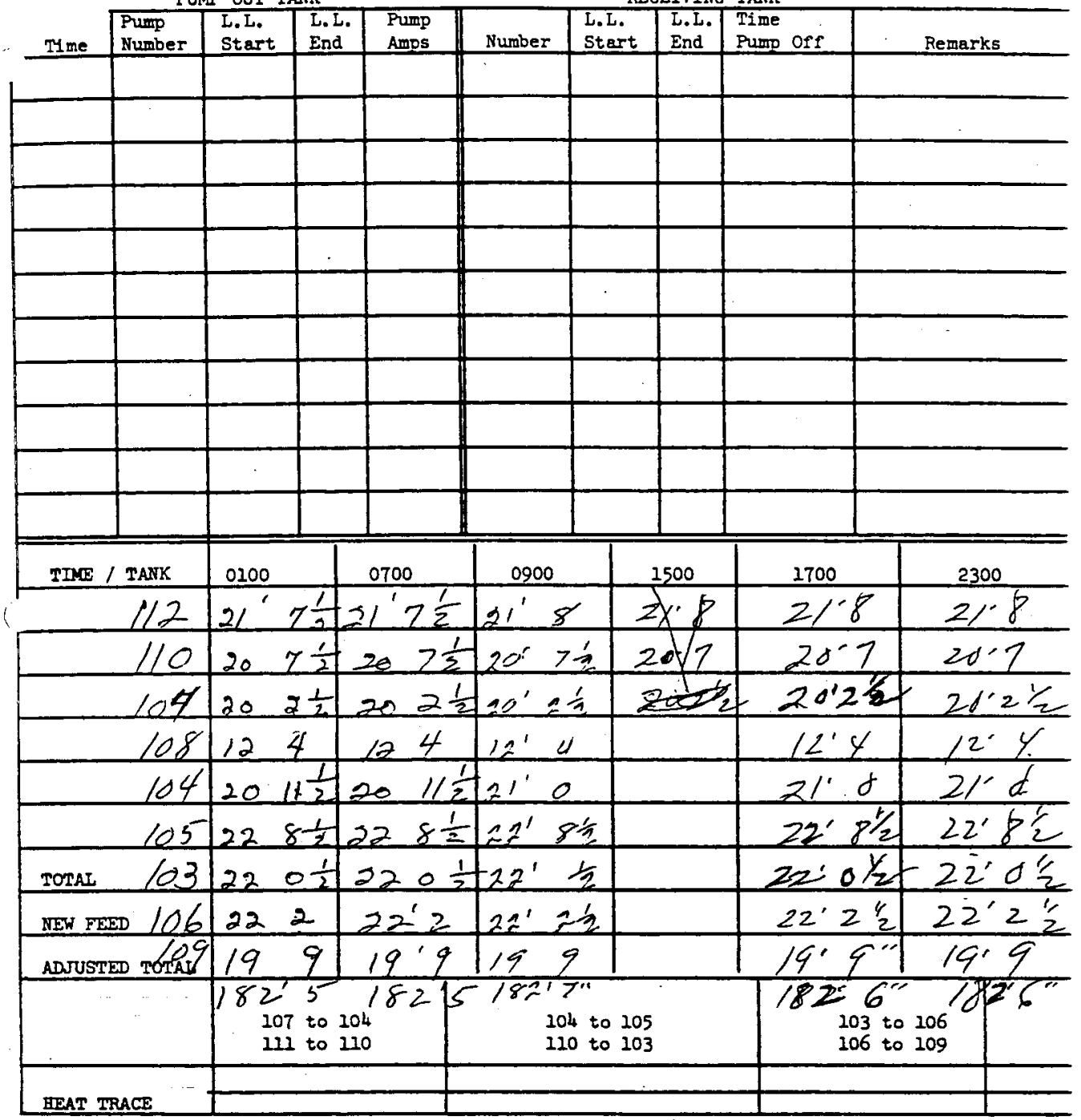

(Walker:srb 9-29-70 
PUMP OUT TANK

\begin{tabular}{l|l|l|l|l|l|l|l|l|l|}
\cline { 2 - 7 } & Tume & $\begin{array}{l}\text { Pump } \\
\text { Number }\end{array}$ & $\begin{array}{l}\text { L.L. } \\
\text { Start }\end{array}$ & $\begin{array}{l}\text { L. L. } \\
\text { End }\end{array}$ & $\begin{array}{l}\text { Pump } \\
\text { Amps }\end{array}$ & Number & $\begin{array}{l}\text { L.L. } \\
\text { Start }\end{array}$ & $\begin{array}{l}\text { L.L. } \\
\text { End }\end{array}$ & $\begin{array}{l}\text { Time } \\
\text { Pump orf }\end{array}$ \\
\hline
\end{tabular}

RECEIVING TANK

\begin{tabular}{|l|l|l|l|l|l|l}
\hline & & & & & & \\
\hline
\end{tabular}

$+5$

TIME / TANK \begin{tabular}{l|ll|llllllll}
110 & 20 & 7 & 20 & 7 & 10 & 8 & 20 & 8 & $20^{\prime} 8$ \\
\hline
\end{tabular}

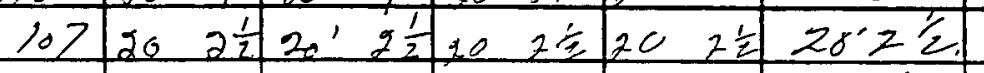

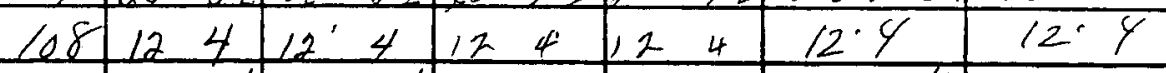
1042011 年20 11章2011.

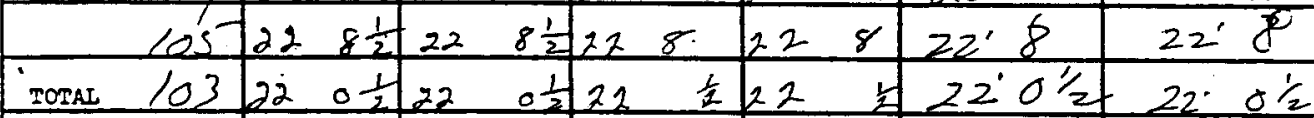

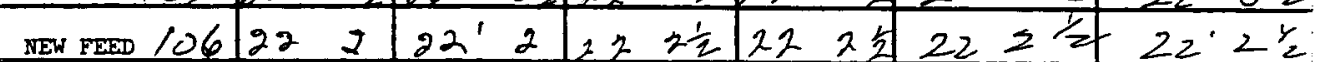
\begin{tabular}{|l|ll|ll|ll|ll|l|l}
\hline ADUUSTED TOTÁ & 19 & 9 & 19 & 9 & 19 & 9 & 19 & 9 & $19^{\prime} 9^{\prime \prime}$ & $19^{\prime} 9$ \\
\hline
\end{tabular}

\begin{tabular}{|c|c|c|c|}
\hline . & $\begin{array}{l}182^{\prime} 5182^{\circ} \\
107 \text { to } 104 \\
111 \text { to } 110\end{array}$ & $\begin{array}{c}5182 c c 1826 \\
104 \text { to } 105 \\
110 \text { to } 103\end{array}$ & $\begin{array}{c}182^{2} 6 \\
103 \text { to } 106 \\
106 \text { to } 109\end{array}$ \\
\hline TRACE & & & \\
\hline
\end{tabular}

i Walker:srb 9-29-70 
WHC-SO-WM-DP-209, REV. 0

\section{ITS-2 INVENTORY AND PUMPING Dete All6 31971}

PUMP OUT TANK RECEIVING TANK

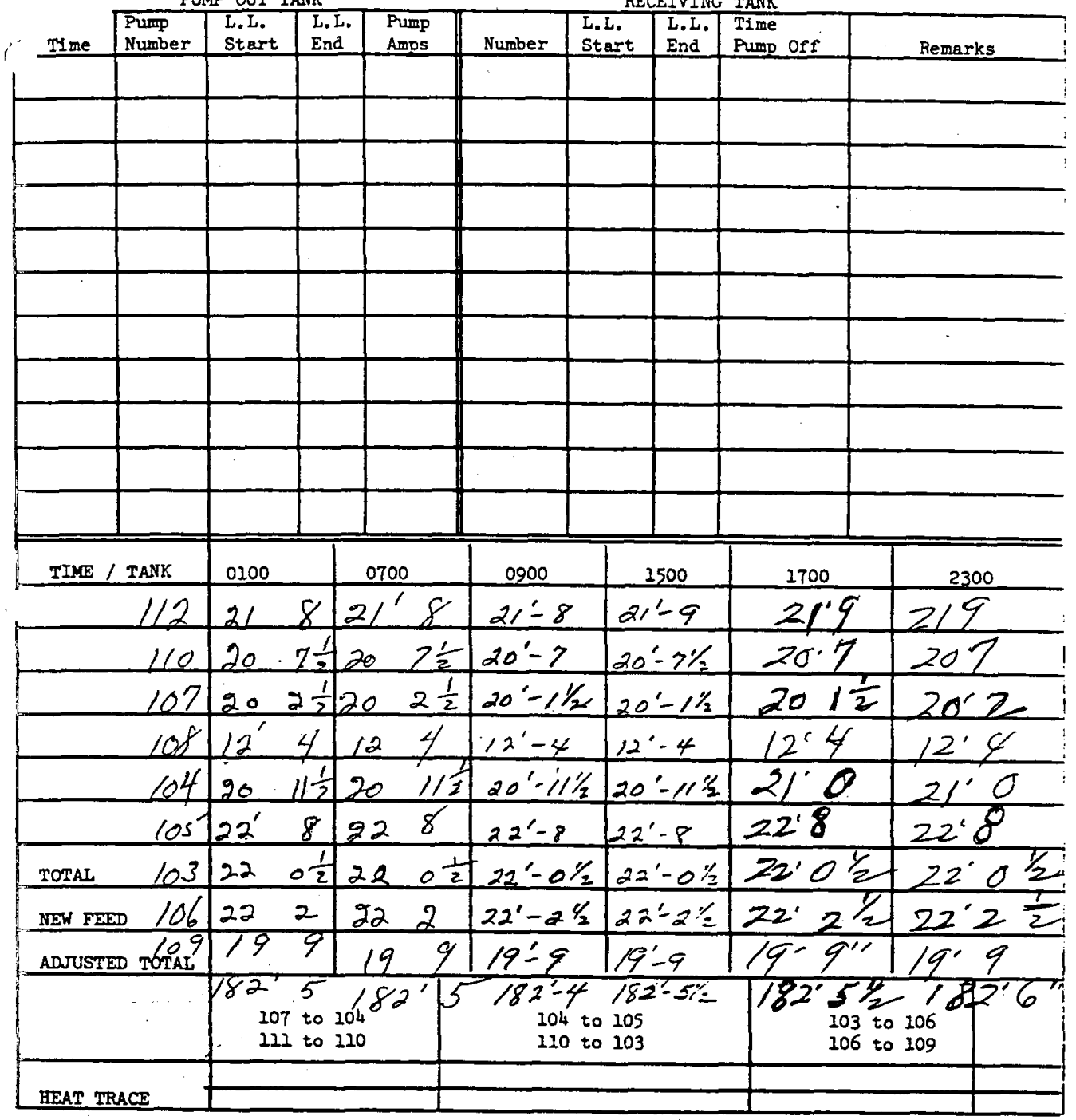

I Wauker:srb 9-29-70 
ITS-2 INVENTORY AND PUMPING

Date $8-14-7$

PUMP OUT TANK

RECEIVING TANK

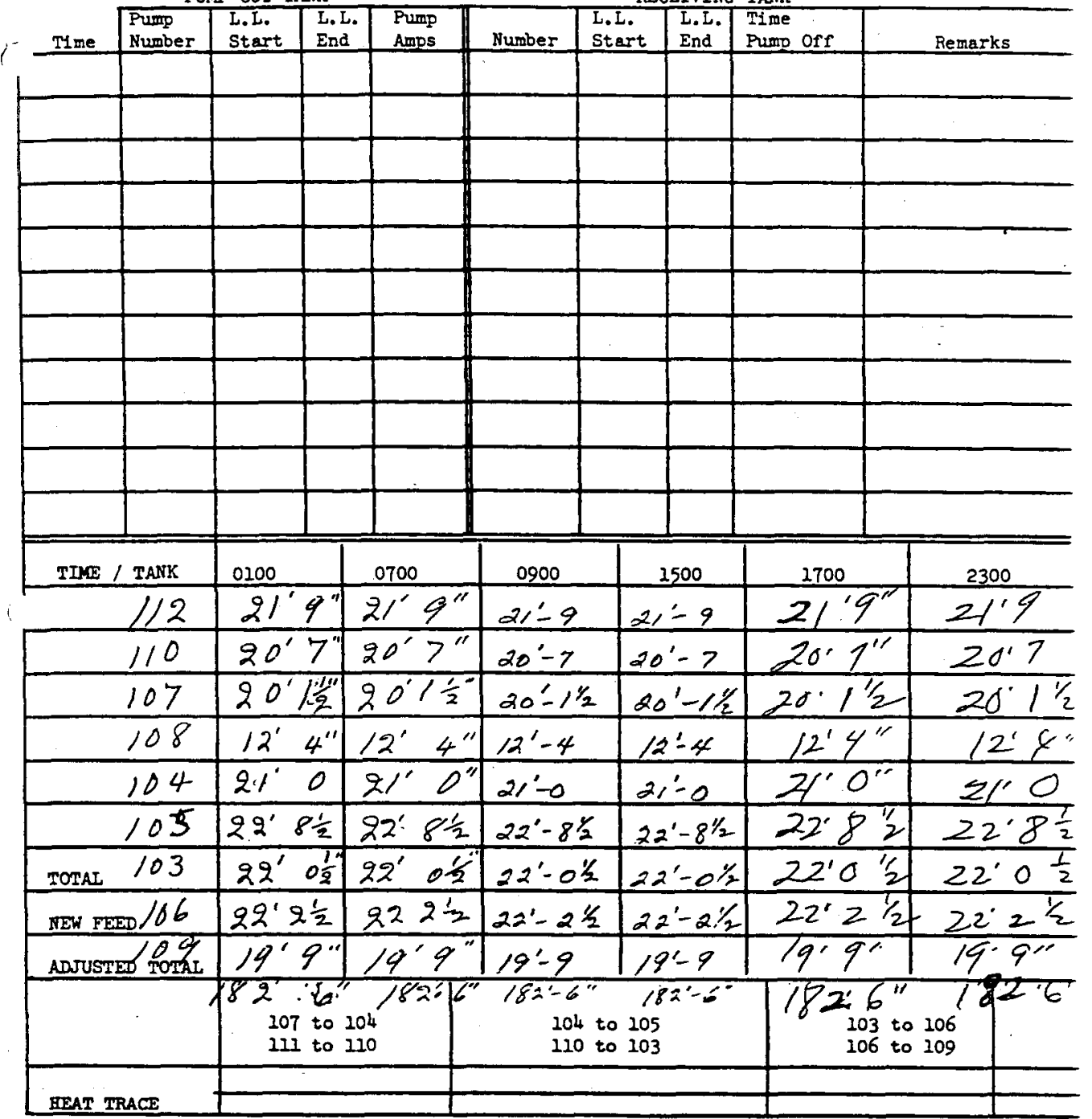

I Walker:srb 9-29-70 
IIS-2 INVENTORY AND PUMPING Date 8-15-7/

PUMP OUT TANK RECEIVING TANK

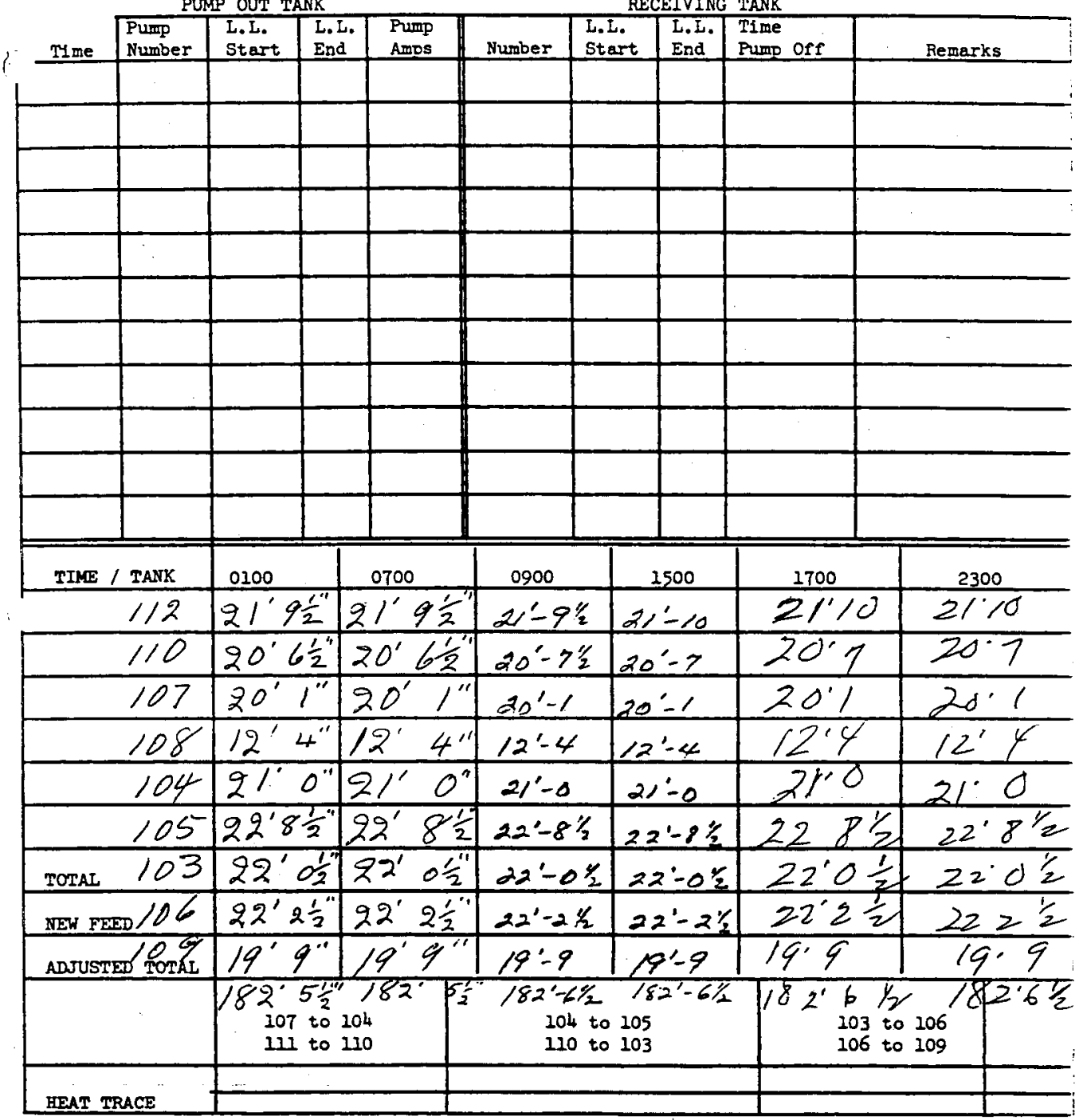

( I WaJker:srb 9-29-70 
WHC-SD-LMA-DP-209, REV. 0

ITS-2 INVENTORY AND PUMPING

Dete $3-16-71$

PUMP OUT TANK

RECEIVING TANK

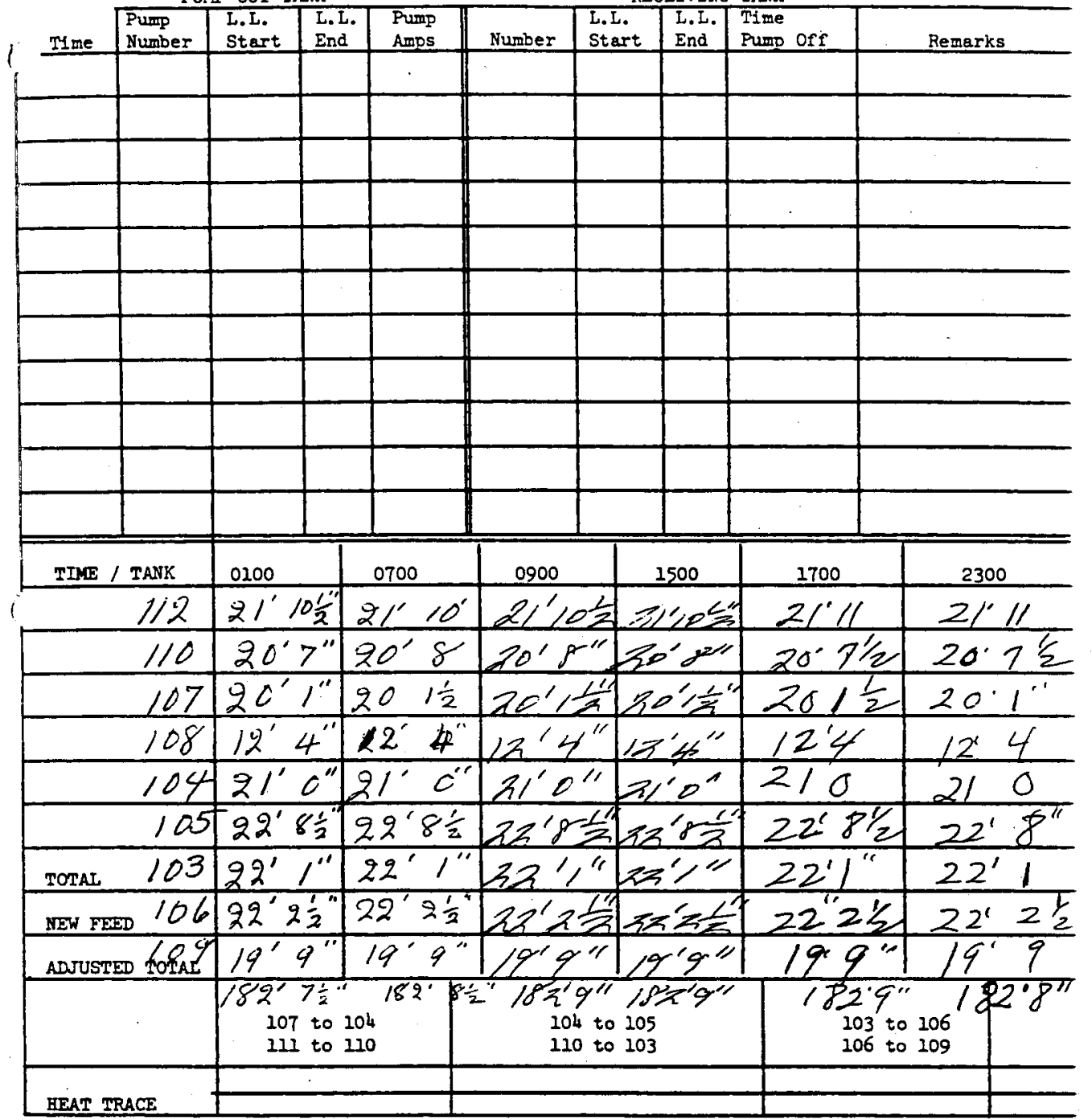

(.Walker:srb 9-29-70 ilo Pumep Locped ocex + FLUSHED $0>00$ 
ITS-2 INVENTORY AND PUMPING Date $17>7$ PUMP OUT TANK RECEIVING TANK

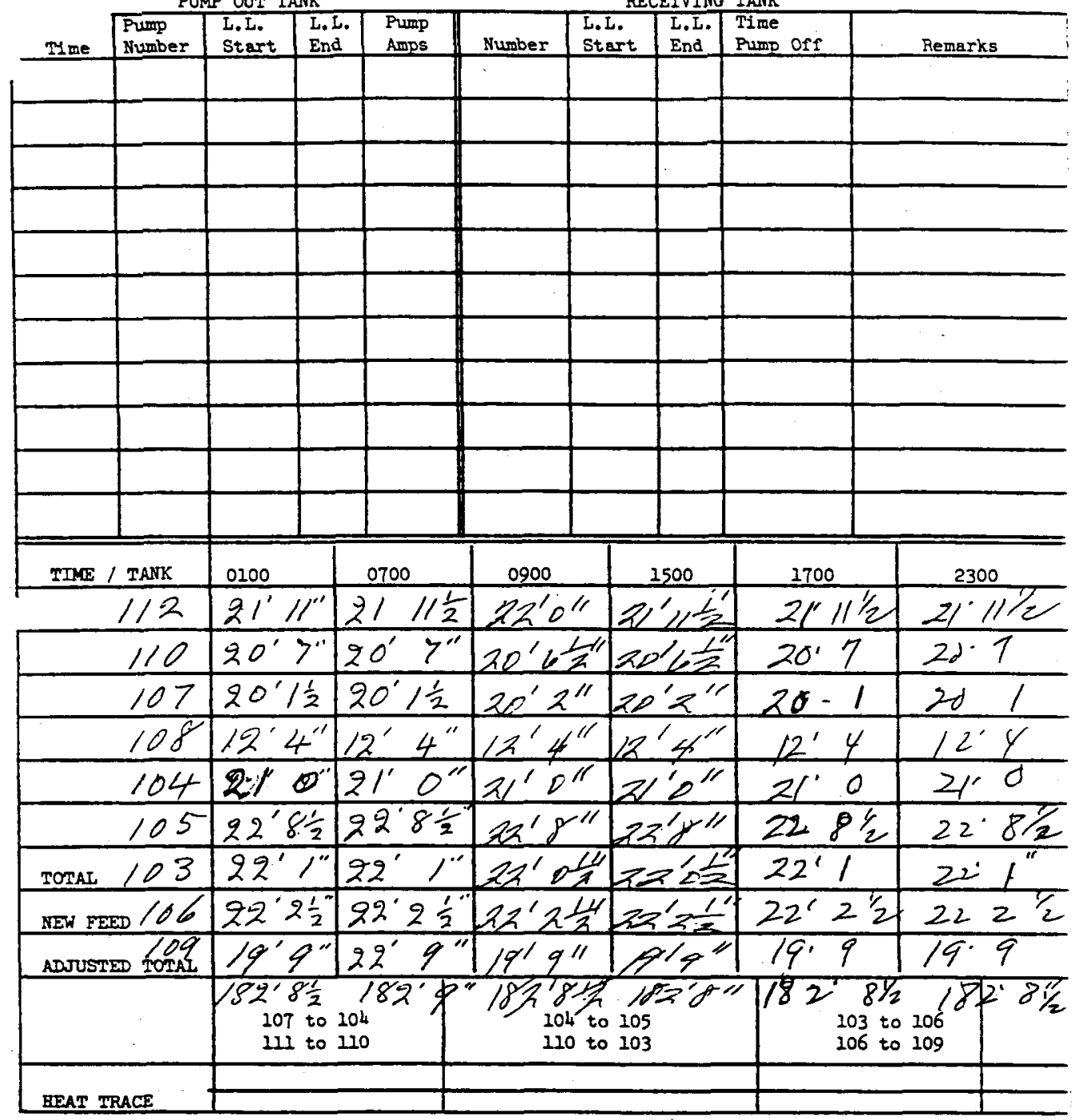

: Walker:sro 9-29-70 $104-64$ TOMP OFF, FLUSHED

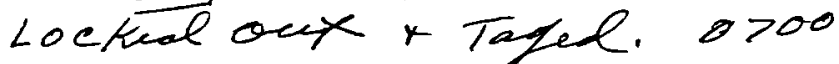
11D-BS ST, 
ITS-2 INVENTORY AND PUMPING Date $8-18-71$

PUMP OUT TANK

RECEIVING TANK

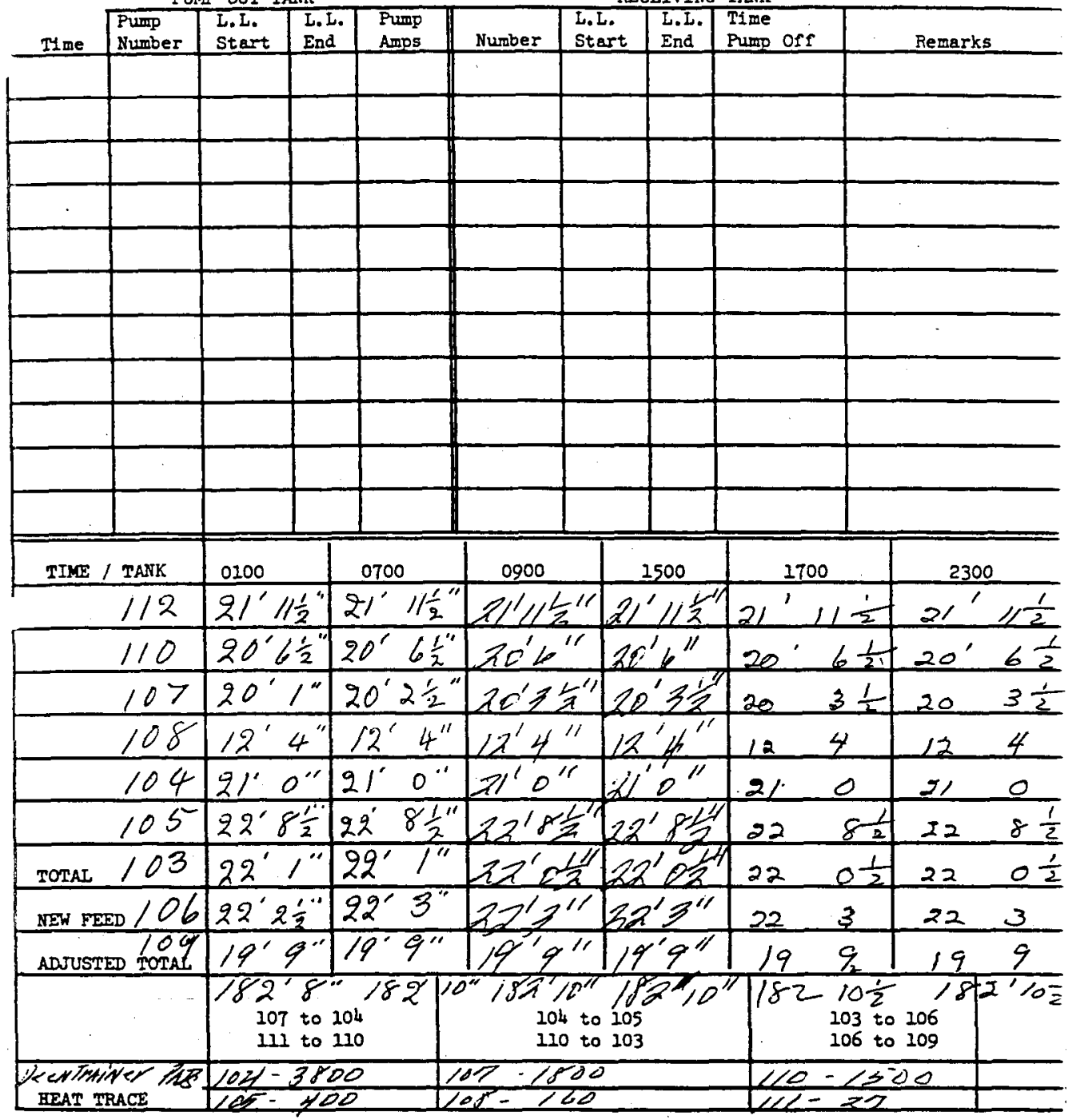

WaIker:srb 9-29-70 


\section{IIS-2 INVENTORY AND PUMPING Date $P-19-21$}

PUMP OUT TANK

RECEIVING TANK

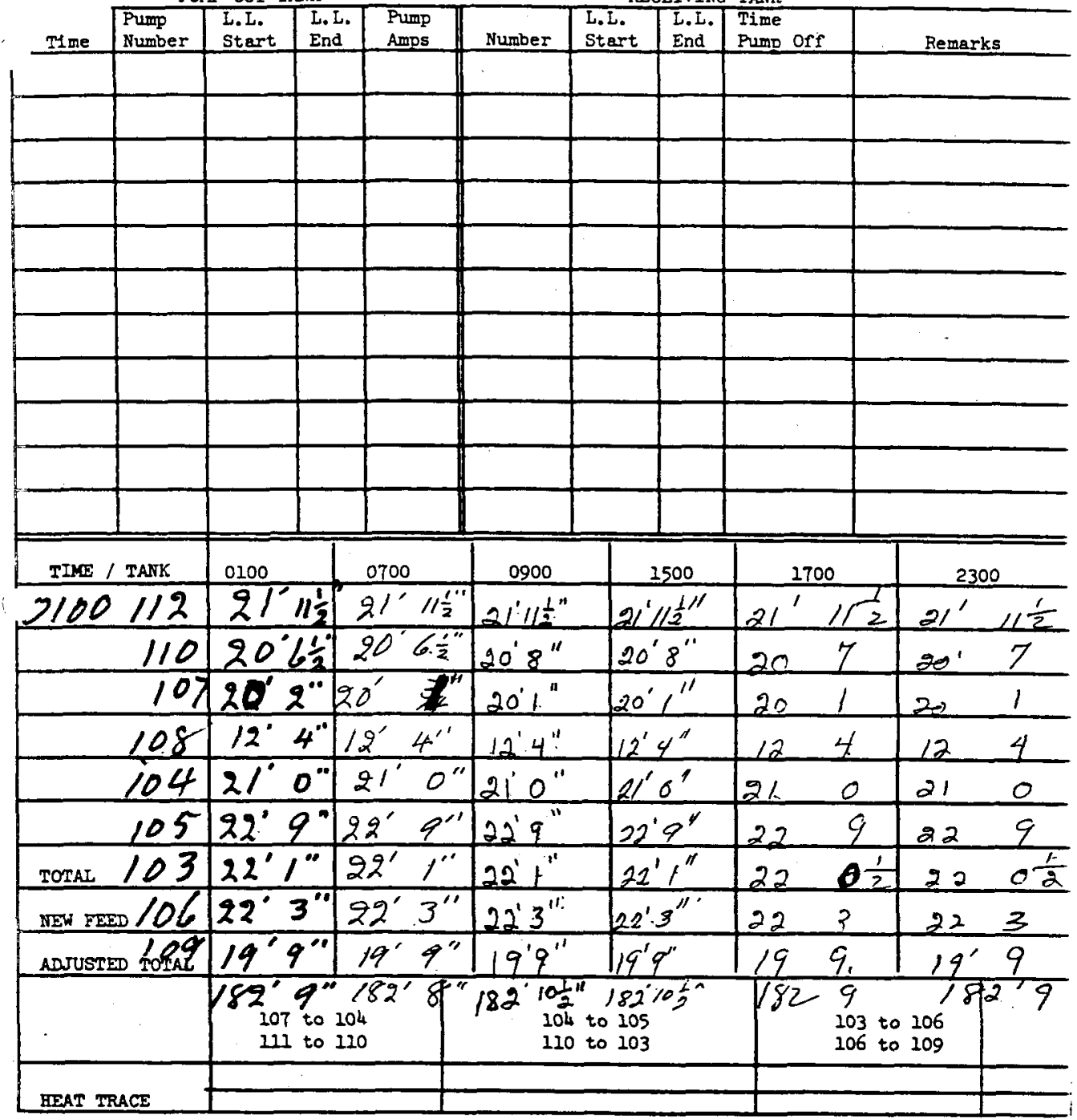

i Walker:srb 9-29-70

$4:-$ 
WHC-SD-WH-DP-209, ReV. 0

ITS-2 INVENTORY AND PUMPING Date $8-20-71$

PUMP OUT TANK

RECEIVING TANK

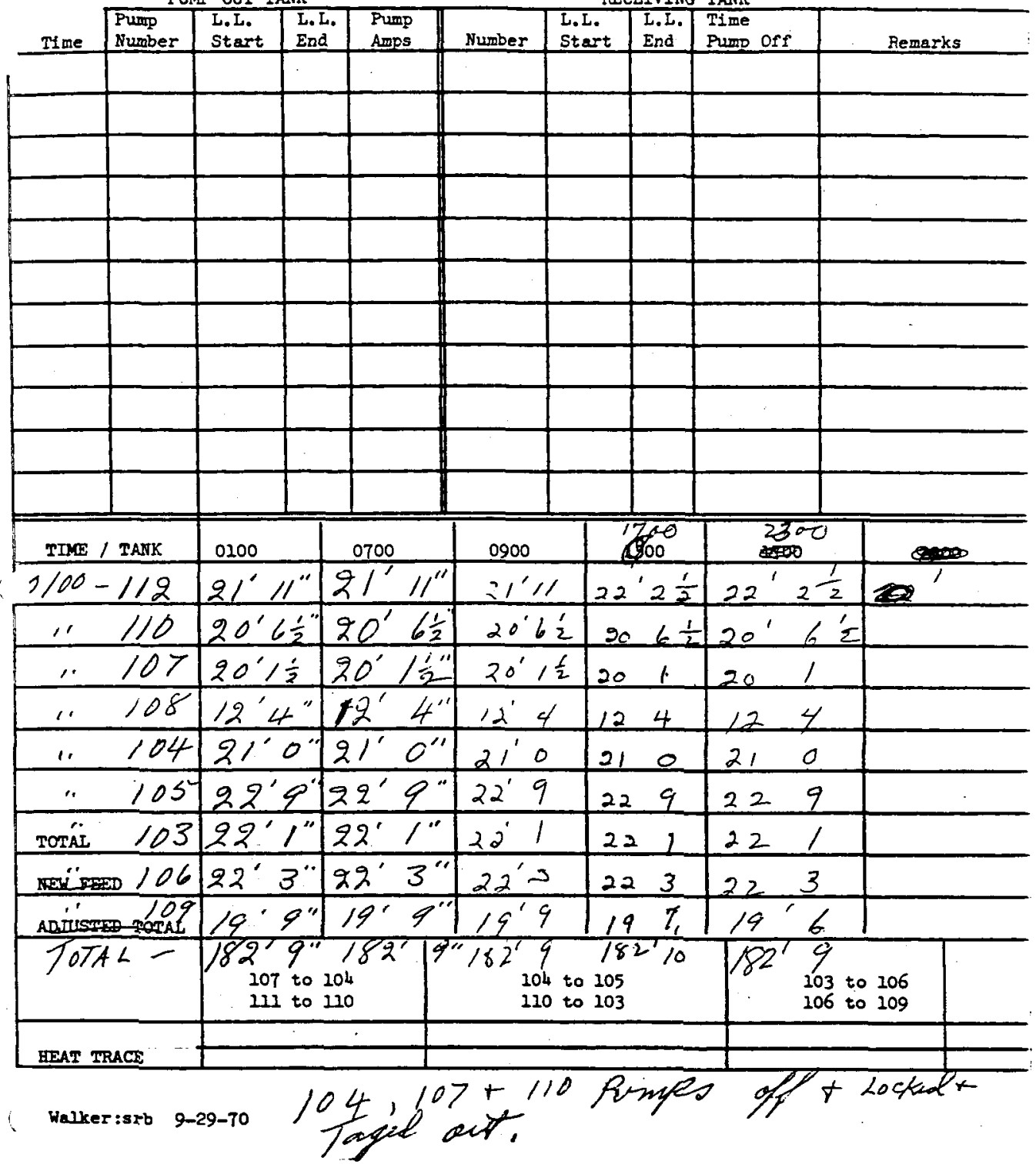


WHC-SD-WM-DP-209, Rev. 0

ITS-2 INVENTORY AND PUNPING

Date $e^{2}-21-01$

PUNP OUT TANK

RECEIVING TANK

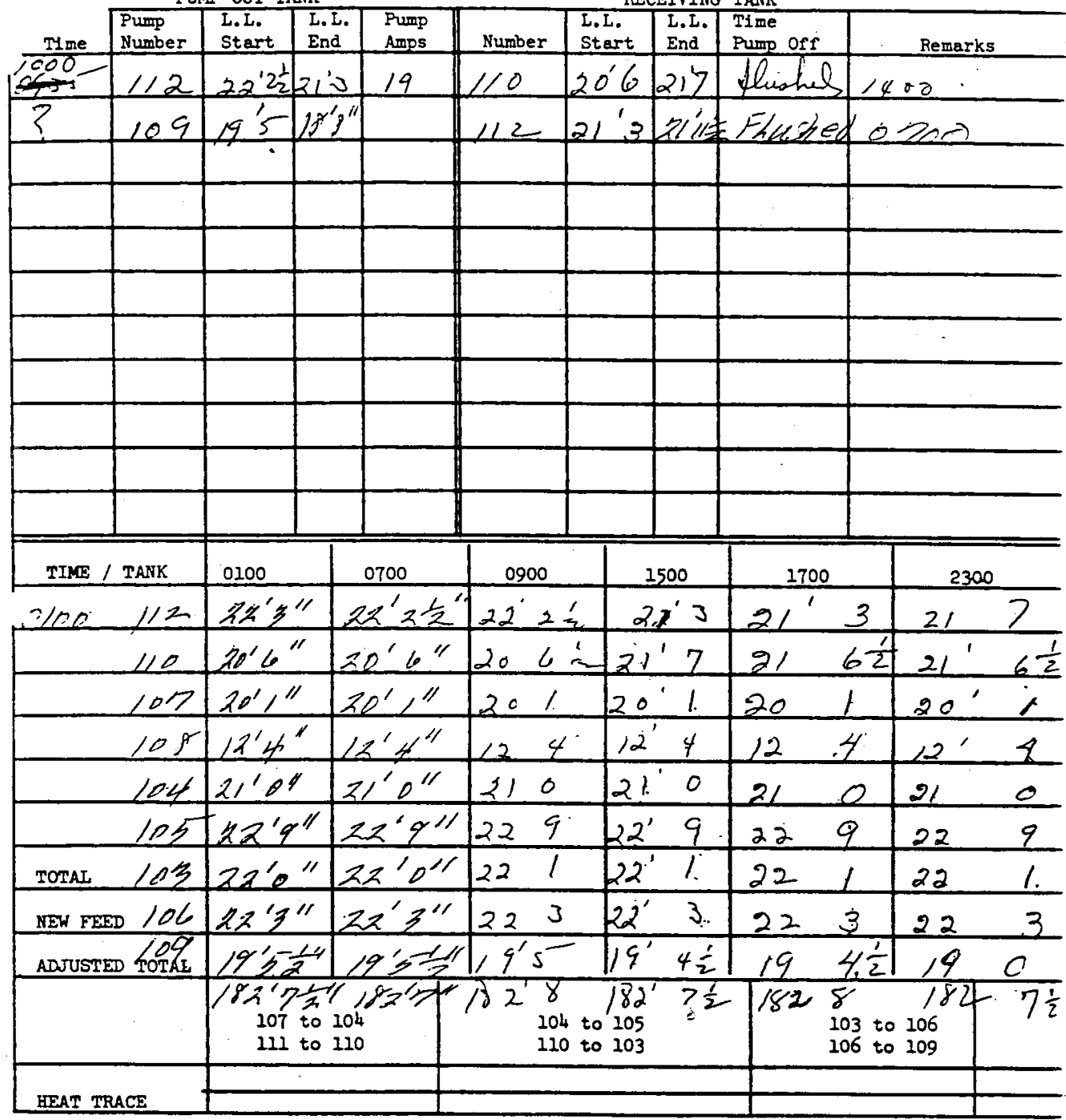

Walker:srb 9-29-70 
PUMP OUT TANK

RECEIVING TANK

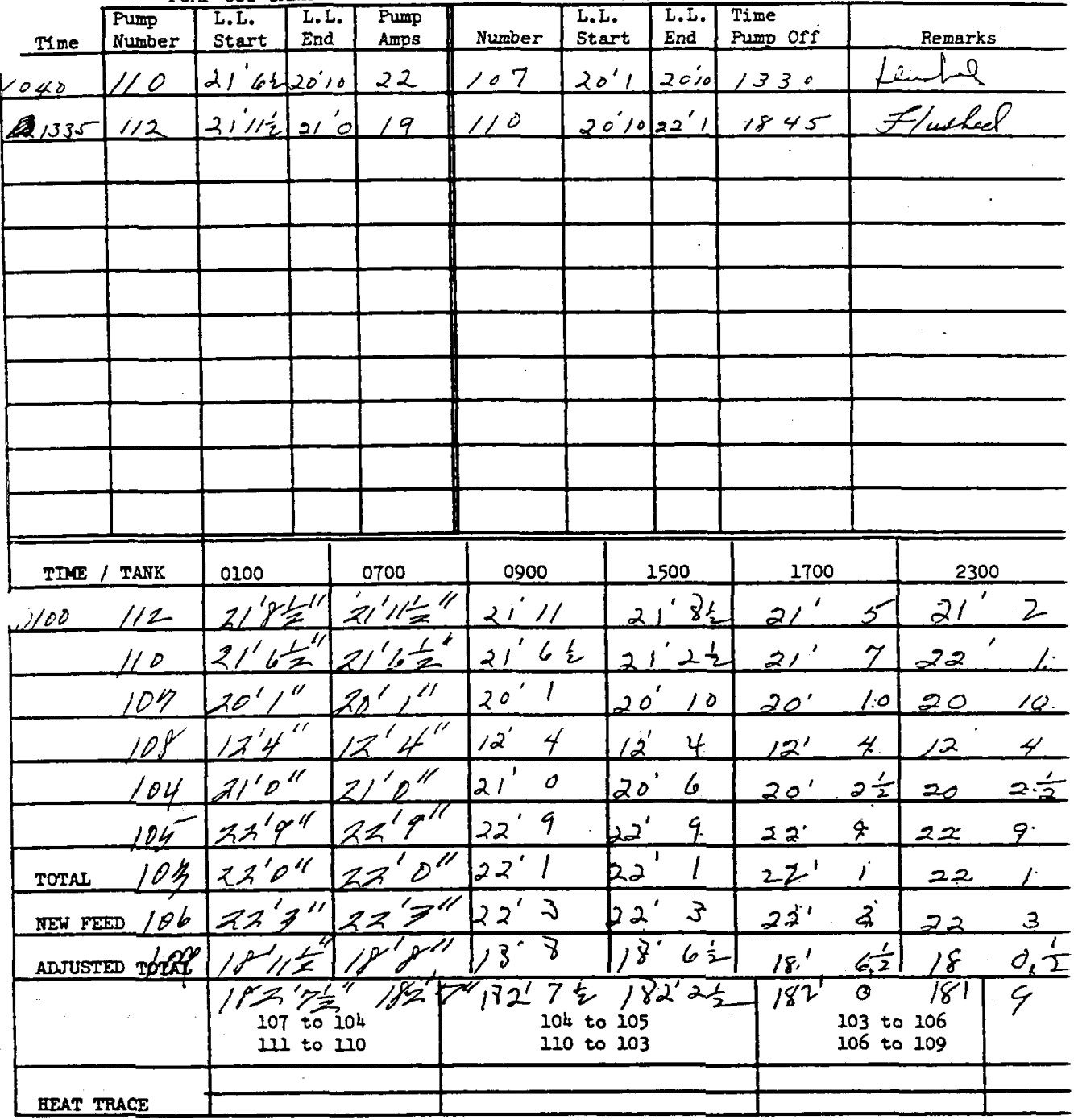

Waiker:srb 9-29-70 
PUNP OUT TANK

RECEIVING TANK

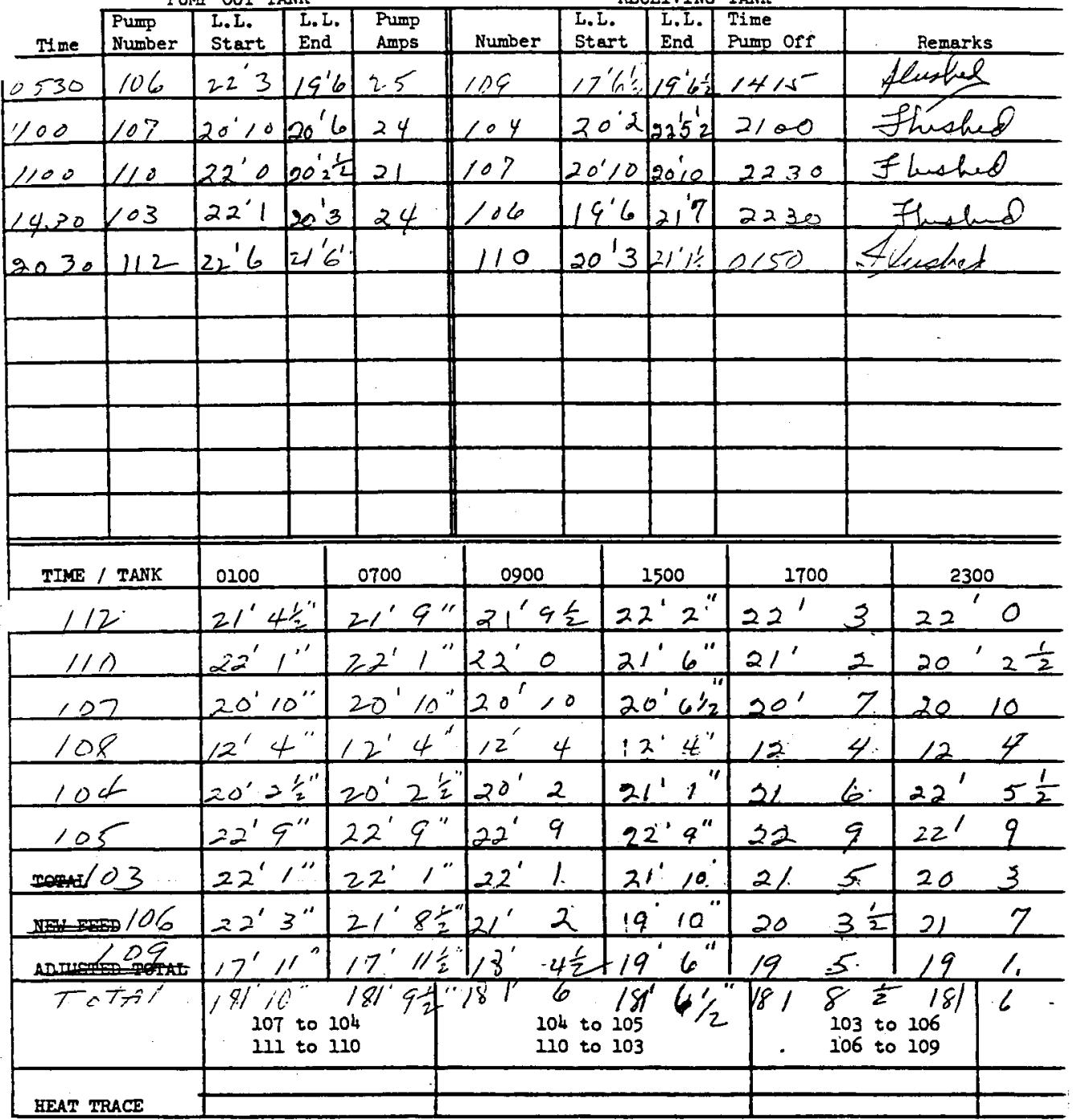

Walker:srb 9-29-70 
WHC-50-WM-DP-209, ReV. 0

ITS-2 INVENTORY AND PUMPING

Date $8 / 24 / 71$

PUMP OUT TANK

RECEIVING TANK

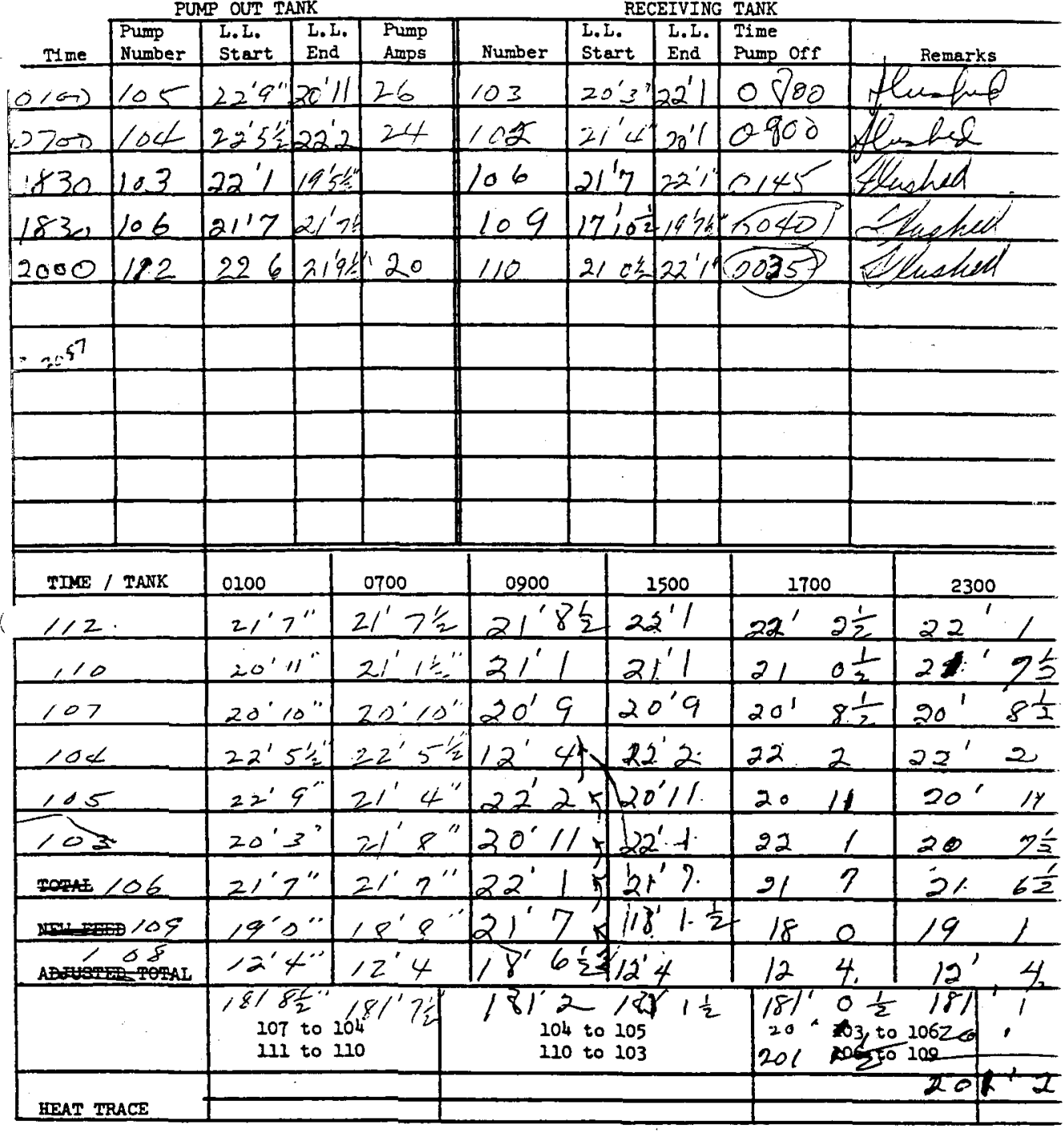

( Walker:srb 9-29-70

233 
ITS-2 INVENTORY AND PUMPING. . Dete . $8 / 25 / 21$

PUMP OUT TANK

RECEIVING TANK

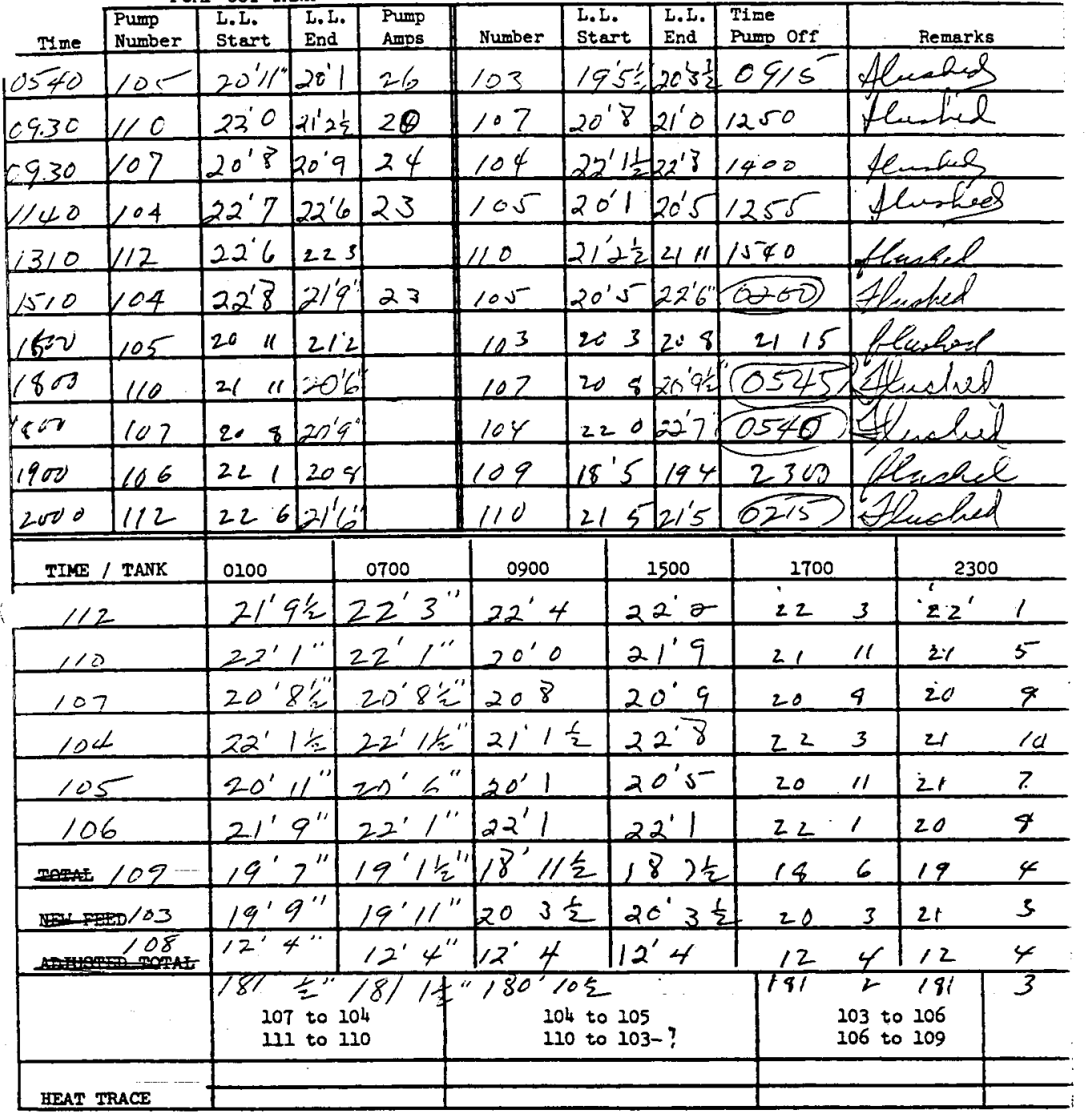

Walker:srb 9-29-70 
ITS-2 INVENTORY AND PUMPING

Date $8 / 26 / 71$

PUMP OUT TANK

RECEIVING TANK

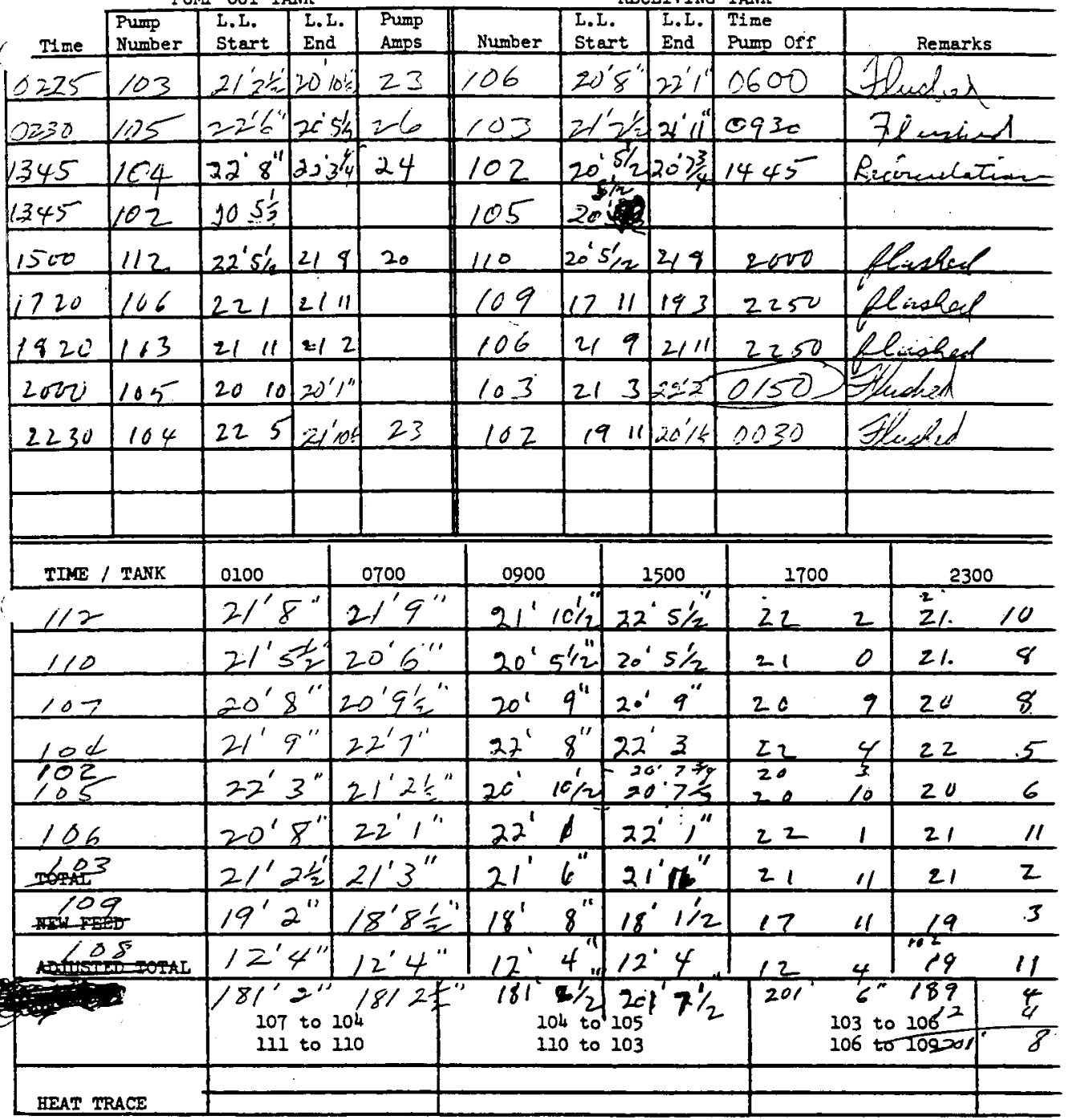

( I Walker:srb 9-29-70 


\section{ITS-2 INVENTORY AND PUMPING \\ Date $8 / 27 / 71$}

PUMP OUT TANK

RECEIVING TANK

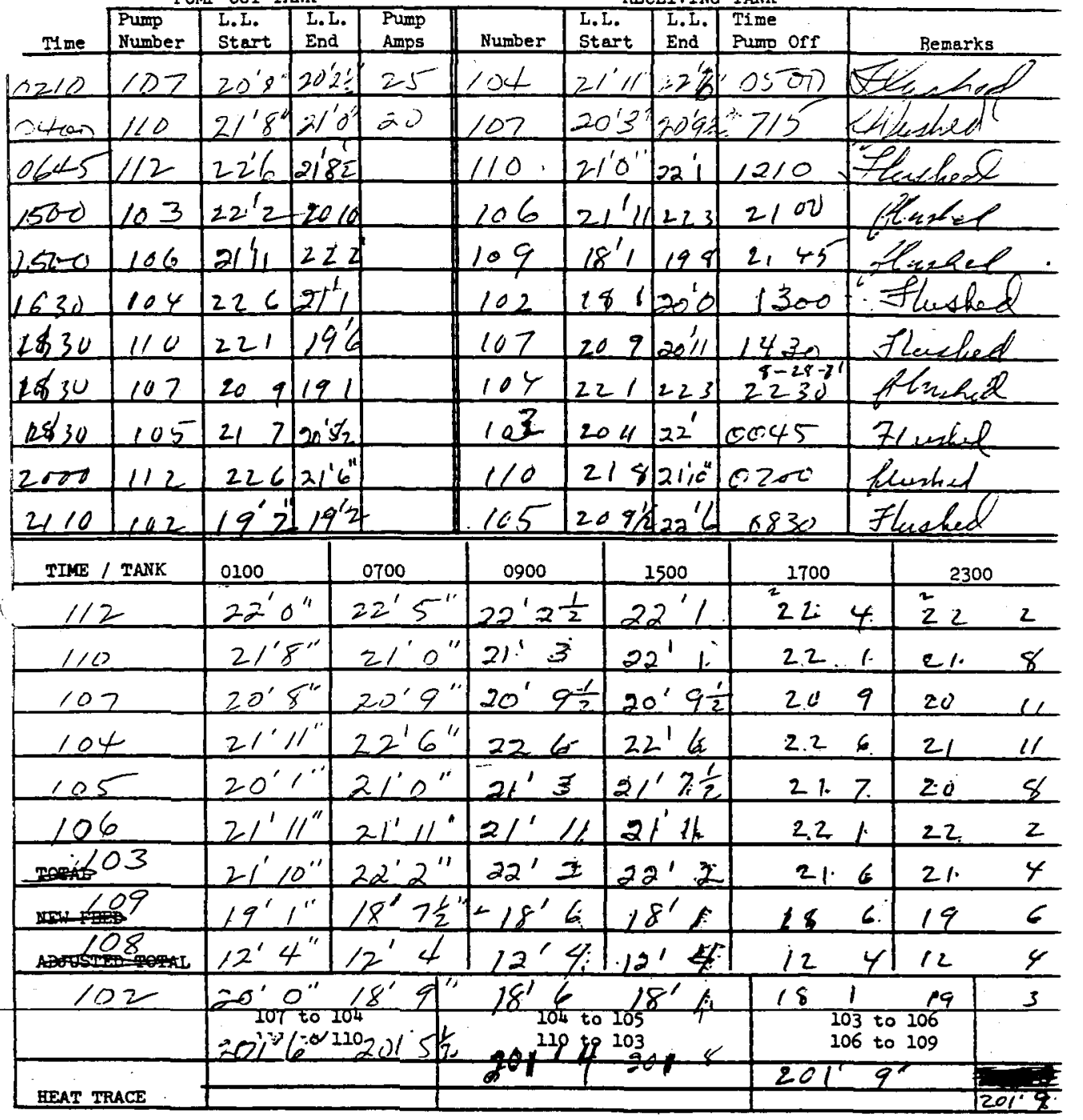

('Wauker:srb 9-29-70' 
ITS-2 INVENTORY AND PUMPING

Date $8-28 \cdot 71$

PUMP OUT TANK

RECEIVING TANK

\begin{tabular}{|l|l|l|l|l|l|l|l|}
\hline Pump & L.L. & L.I. & Pump & & L.L. & L.L. Time \\
\hline
\end{tabular}

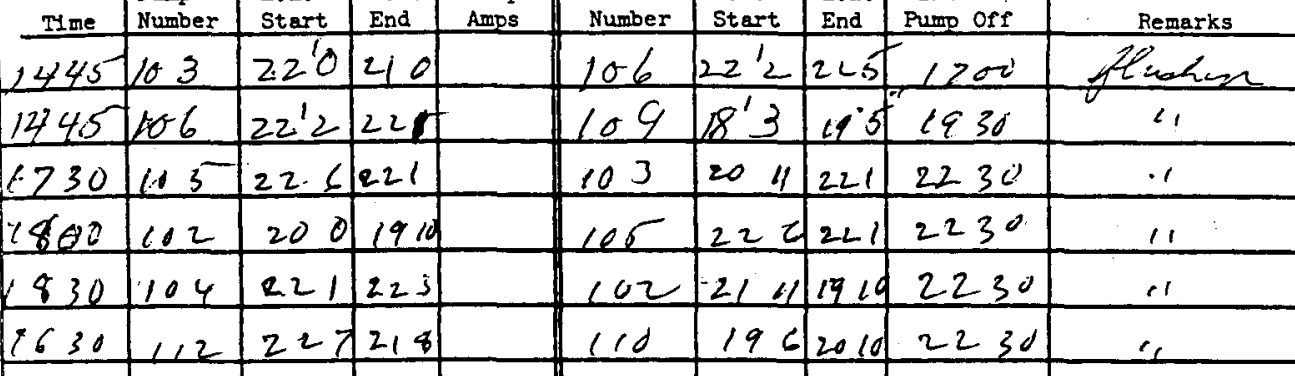

\begin{tabular}{|l|l|l|l|l|}
\hline & & & & \\
\hline & & & & \\
\hline & & & & \\
\hline & & & & \\
\hline & & & & \\
\hline
\end{tabular}

TIME / TANK

112

110

107

104

10.2

105

$\operatorname{TOTAL} / 03$

NEW FEED $/ C 6$

\begin{tabular}{l|ll|lll|lll} 
ADUSTENCOFAL & $19^{\prime} 5^{\prime} / 2$ & $18^{\prime}$ & $10^{\prime \prime}$ & $18^{\prime}$ & 8 & $18^{\prime} 3$. \\
\hline
\end{tabular} 108

\section{\begin{tabular}{l|l}
0100 & 0700 \\
\hline
\end{tabular}}

$1^{\circ} 71^{\prime} t^{\prime} 9^{\prime} \quad 11$

$21^{\circ} 7 \frac{1}{2}$

$\left.2110^{\prime} / 2\right) 20912$

$-1,=\frac{22^{\prime} 4}{19^{\prime}}$

$20^{\prime}, 0^{\prime \prime} 20^{\prime} \prime \prime \prime$

$21^{\circ} 10^{\prime \prime} 21^{\circ} 6$

$19^{\circ}$

$20^{\prime}-1 / 22^{\prime} 0^{\prime \prime}$

21

\begin{tabular}{|l|l|}
$20.5 / 2$ & $22^{\circ} 0$ \\
$22^{\circ} 0^{\prime \prime}$ & $22^{\circ}$
\end{tabular}

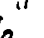

21

$0 \frac{1}{2}$

$\frac{1500}{22^{\prime} 4}$

2700

22

19

$2 c$

\begin{tabular}{l|l}
4 & $2 ! 4$ \\
\hline
\end{tabular}

$22^{\prime} e^{\prime \prime} 2 \lambda^{\prime \prime} 0$

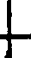

$22^{\prime} 622^{\prime} 6$

24

$22^{\prime} 0$

0

$22 \cdot 0$

20

\begin{tabular}{|l|l|ll|}
$22^{\prime}$ & $+22^{\prime} 2$ & 20 & 4 \\
\hline
\end{tabular}

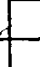

HEAT TRACE

$$
\begin{aligned}
& 107 \text { to } 104 \\
& 111 \text { to } 110
\end{aligned}
$$$$
104 \text { tol्es }
$$$$
110 \div 203
$$

$201^{\prime} 8^{\prime \prime} 2017$ 20 $1^{\prime} 6 \frac{2}{2} 20$

\begin{tabular}{|l|l|}
\hline 103 to & 106 \\
106 to & 109 \\
\hline
\end{tabular}

. 
WHC-SO-WM-OP-209, ReV. 0

ITS-2 INVENTORY AND PUMPING

Date $8-29-7$

PUMP OUT TANK

RECEIVING TANK

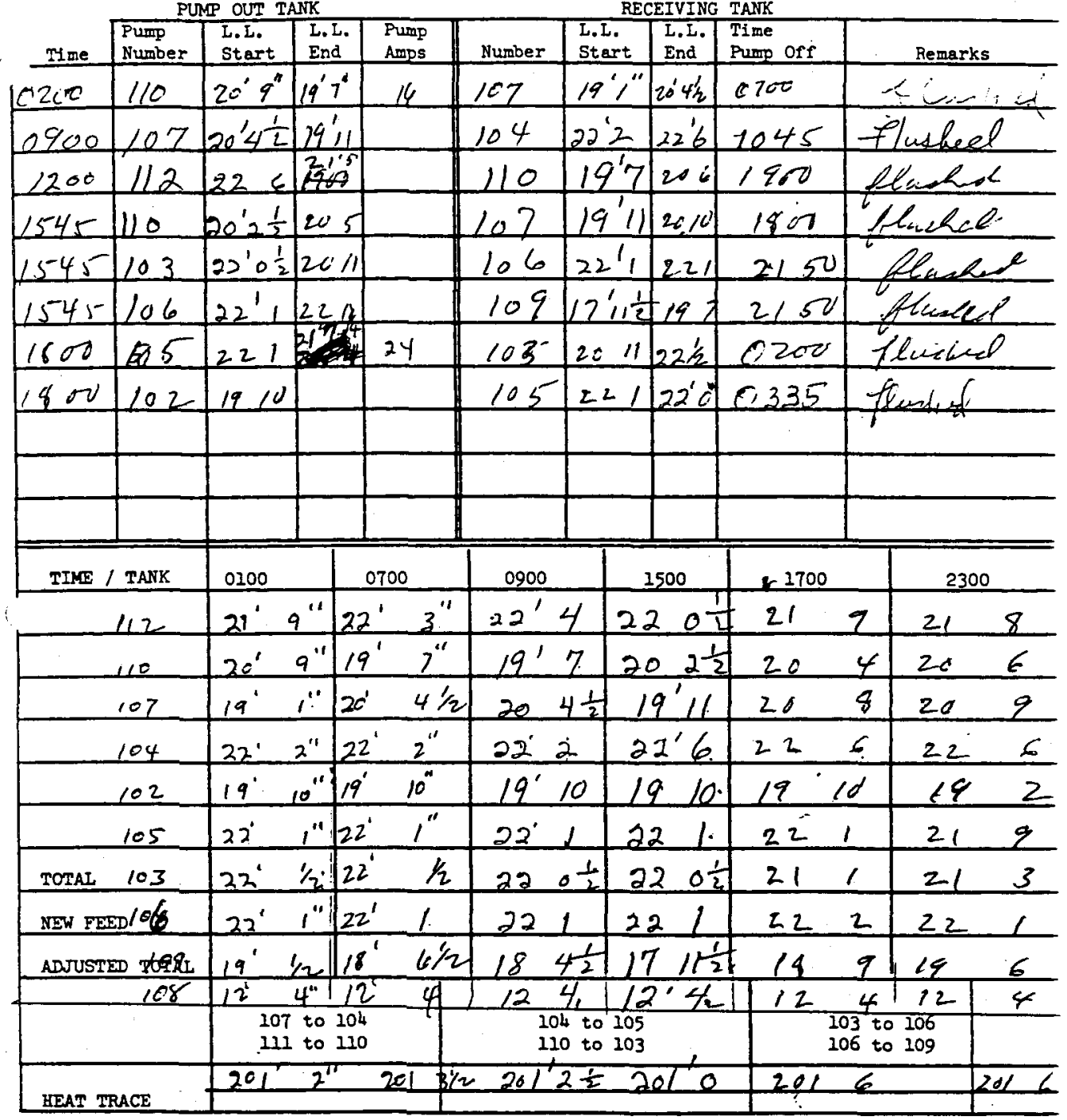

(. Wayker:srb 9-29-70

238 
WHC-SO-WM-DP-209, ReV. 0

ITS-2 INVENTORY AND PUMPING

Date $8.30 .7 \%$

PUMP OUT TANK

RECEIVING TANK

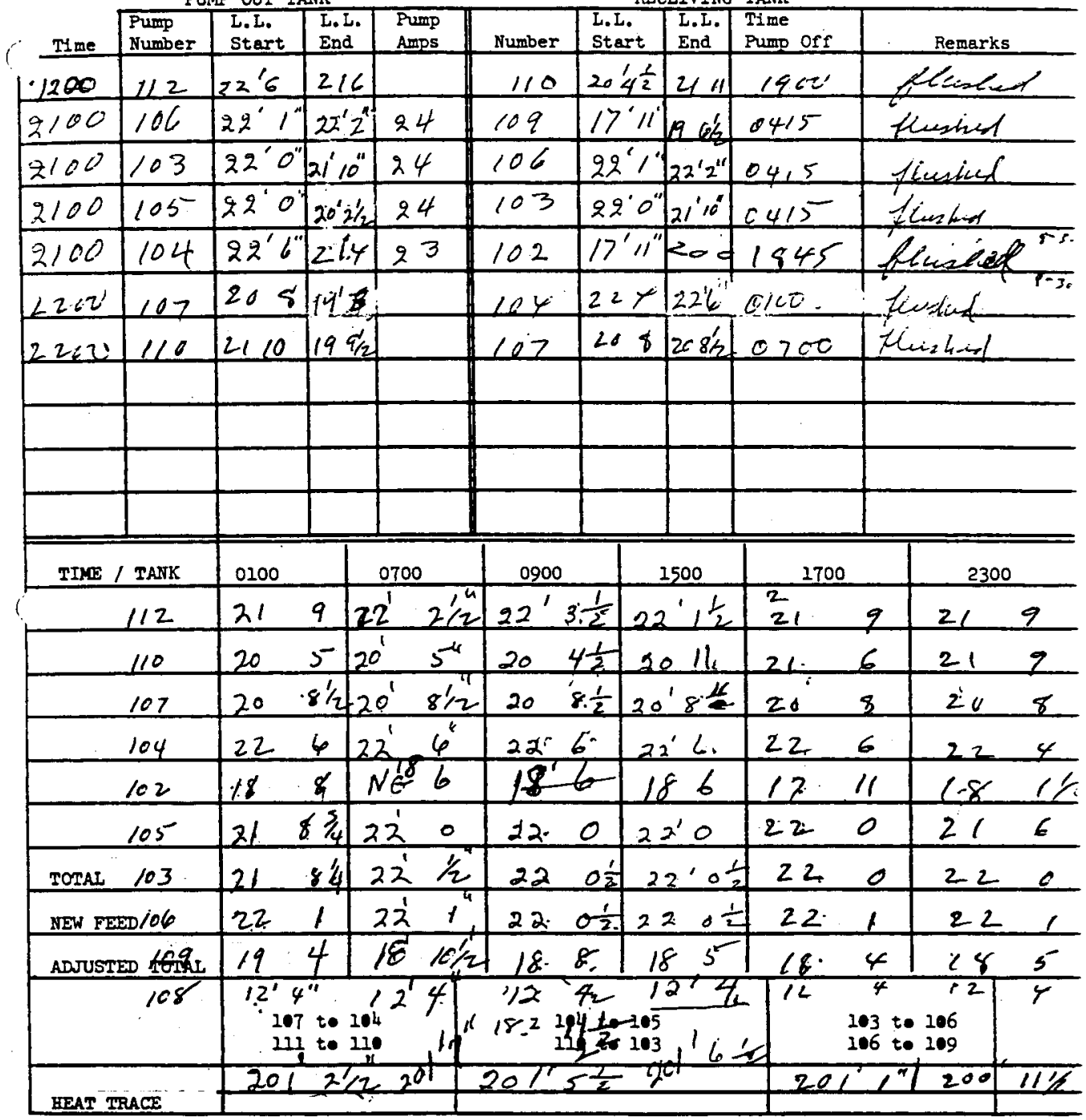

('Wayker:srb 9-29-70

239 
PUMP OUT TANK

RECEIVING TANK

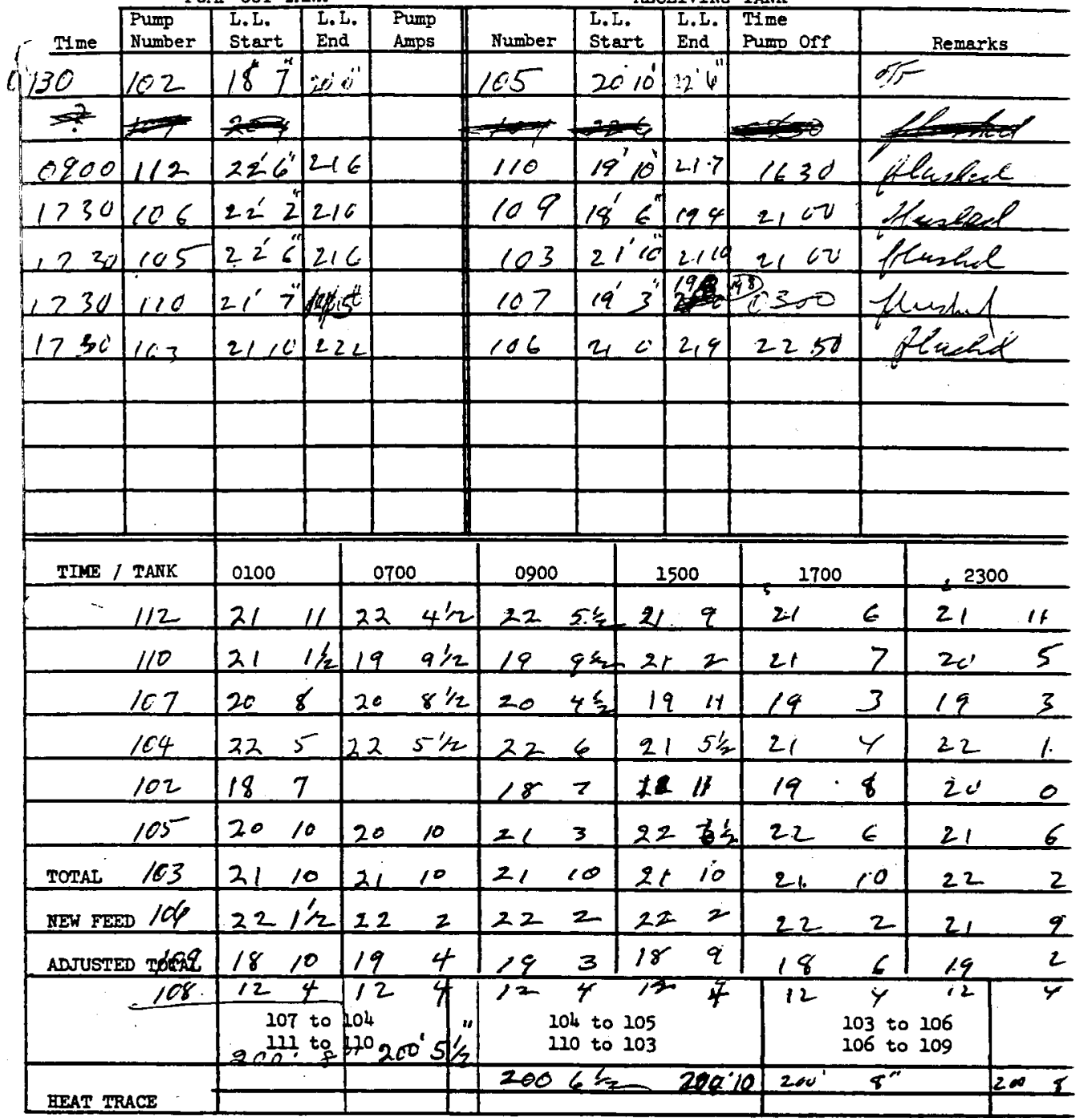

i. Nouker:srb 9-29-70 
PUMP OUT TANK

RECEIVING TANK

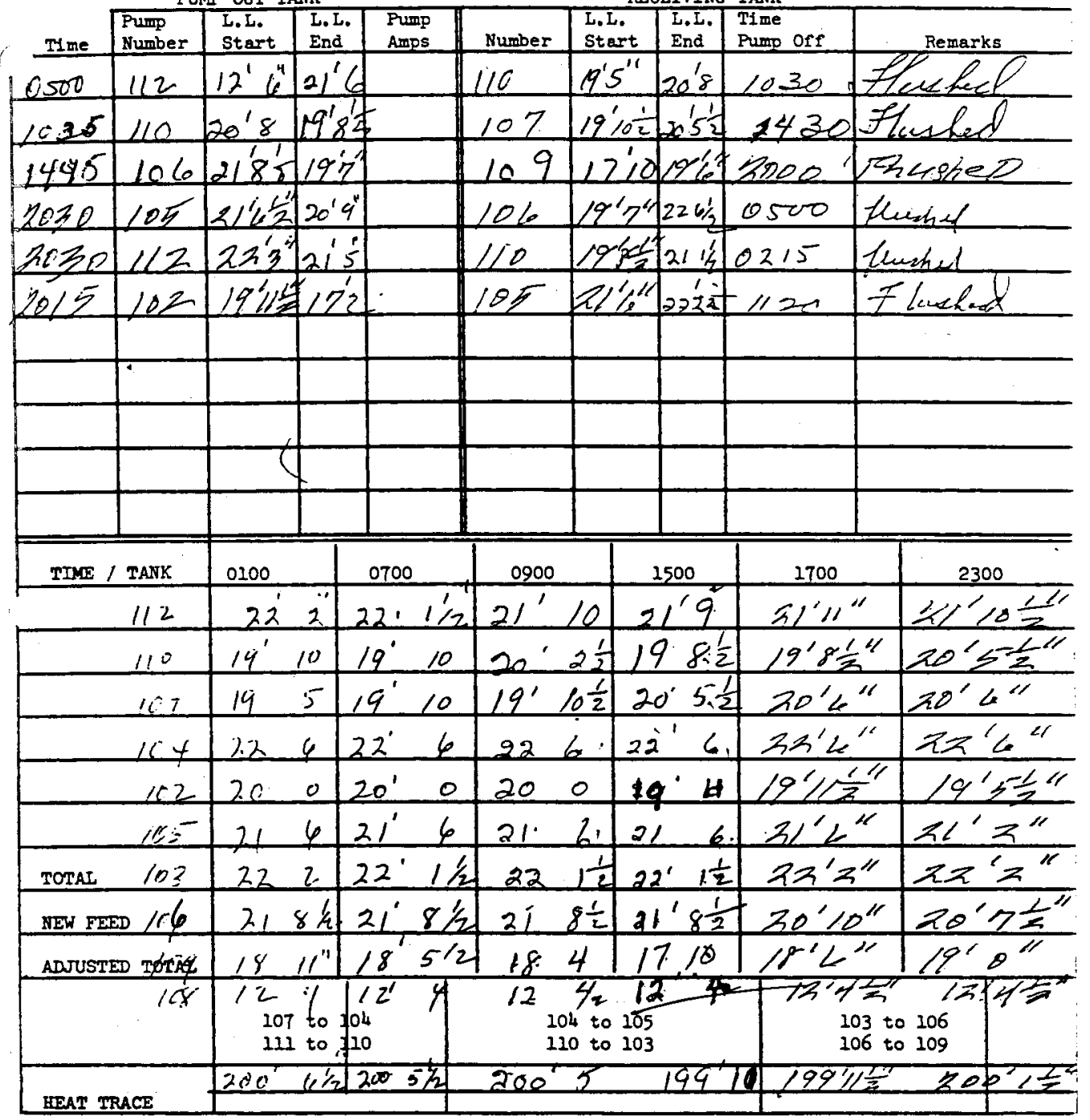

Walker :srb 9-29-70 
WHC-SD-WM-DP-209, ReV. O

ITS-2 INVENTORY AND PUMPING

Date $9-2-71$

PUMP OUT TANK

RECEIVING TANK

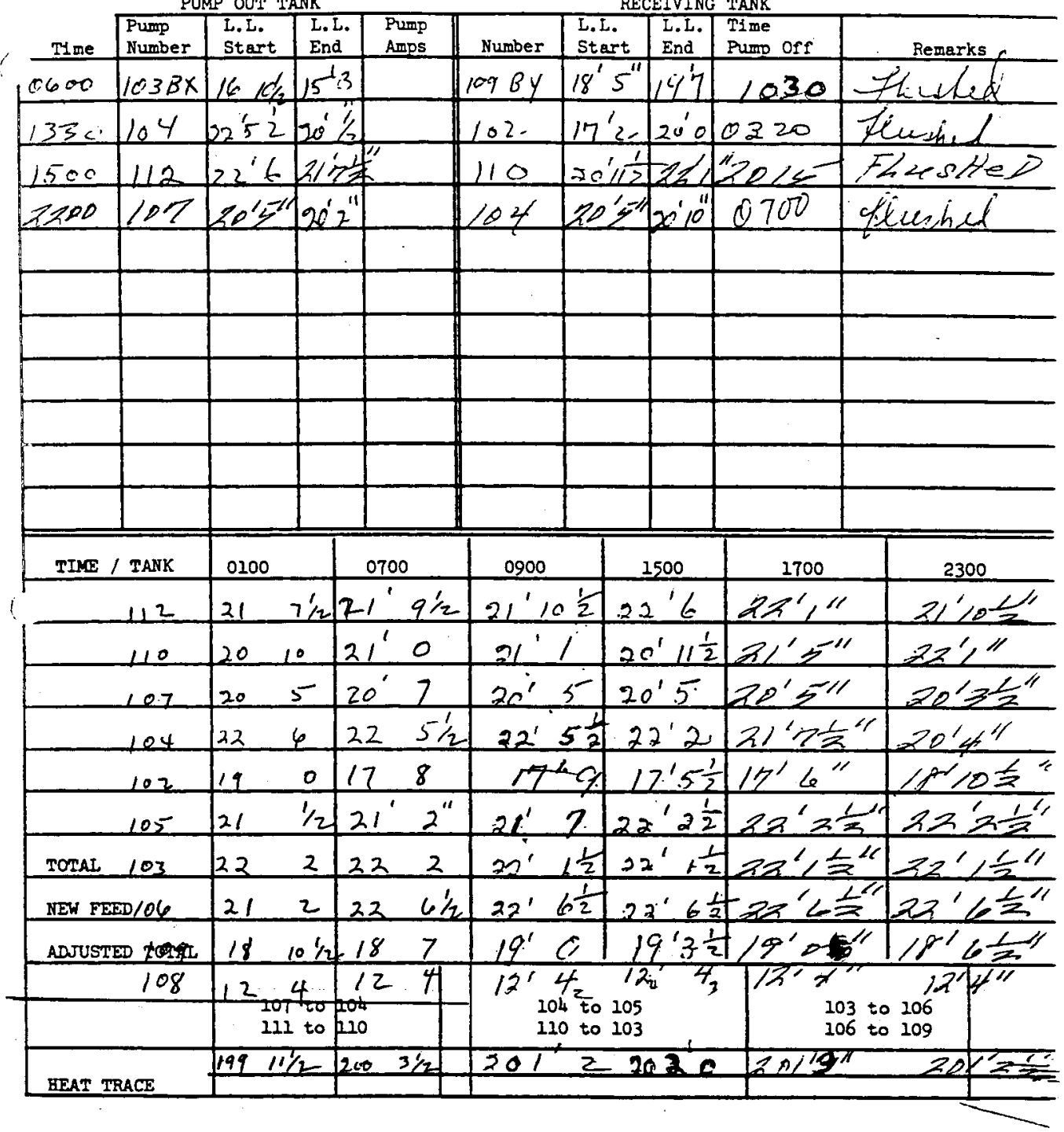

i Wayker:srb 9-29-70

242 
PUMP OUT TANK

RECEIVING TANK

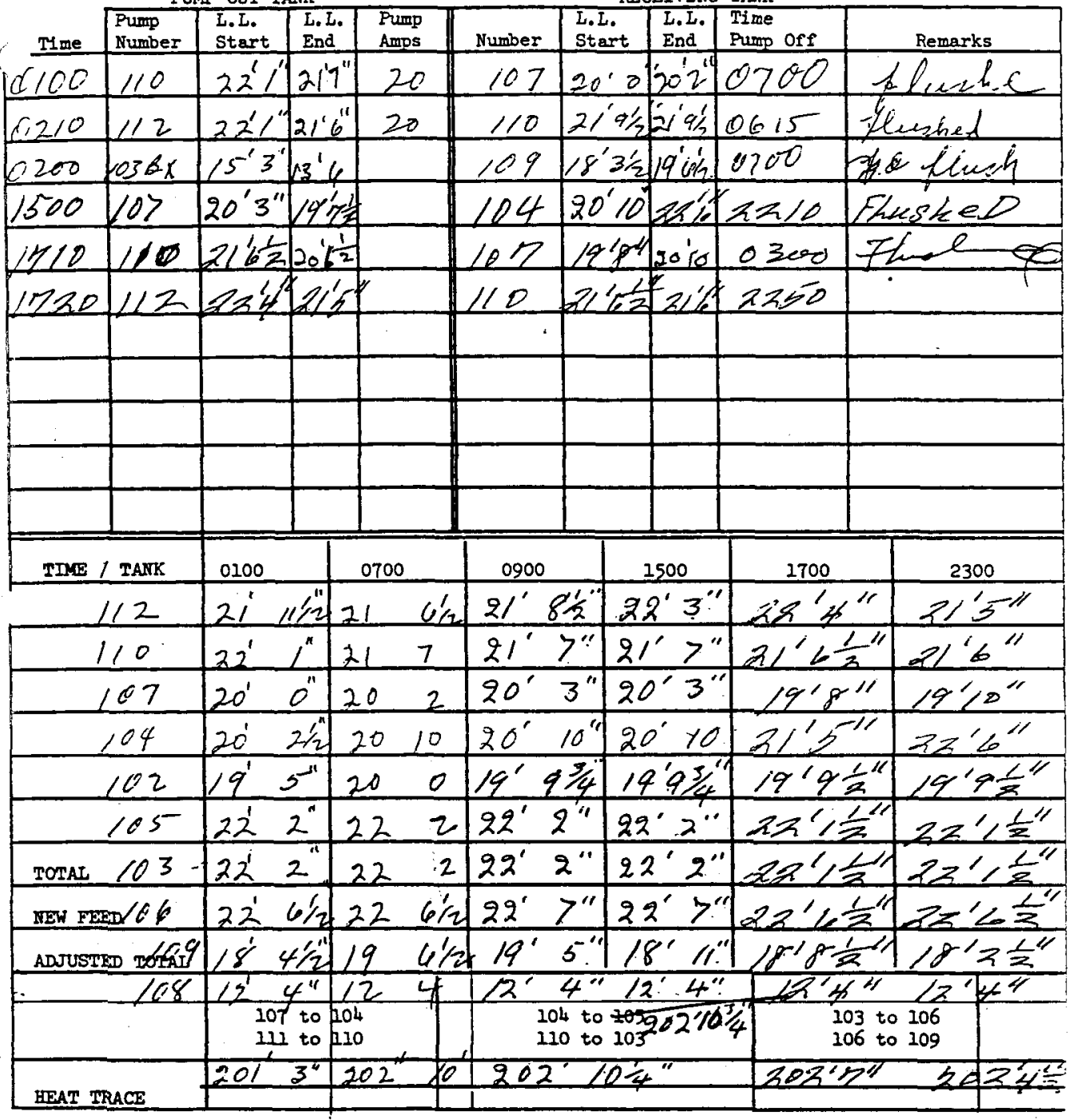


PUNP OUT TANK

RECEIVING TANK

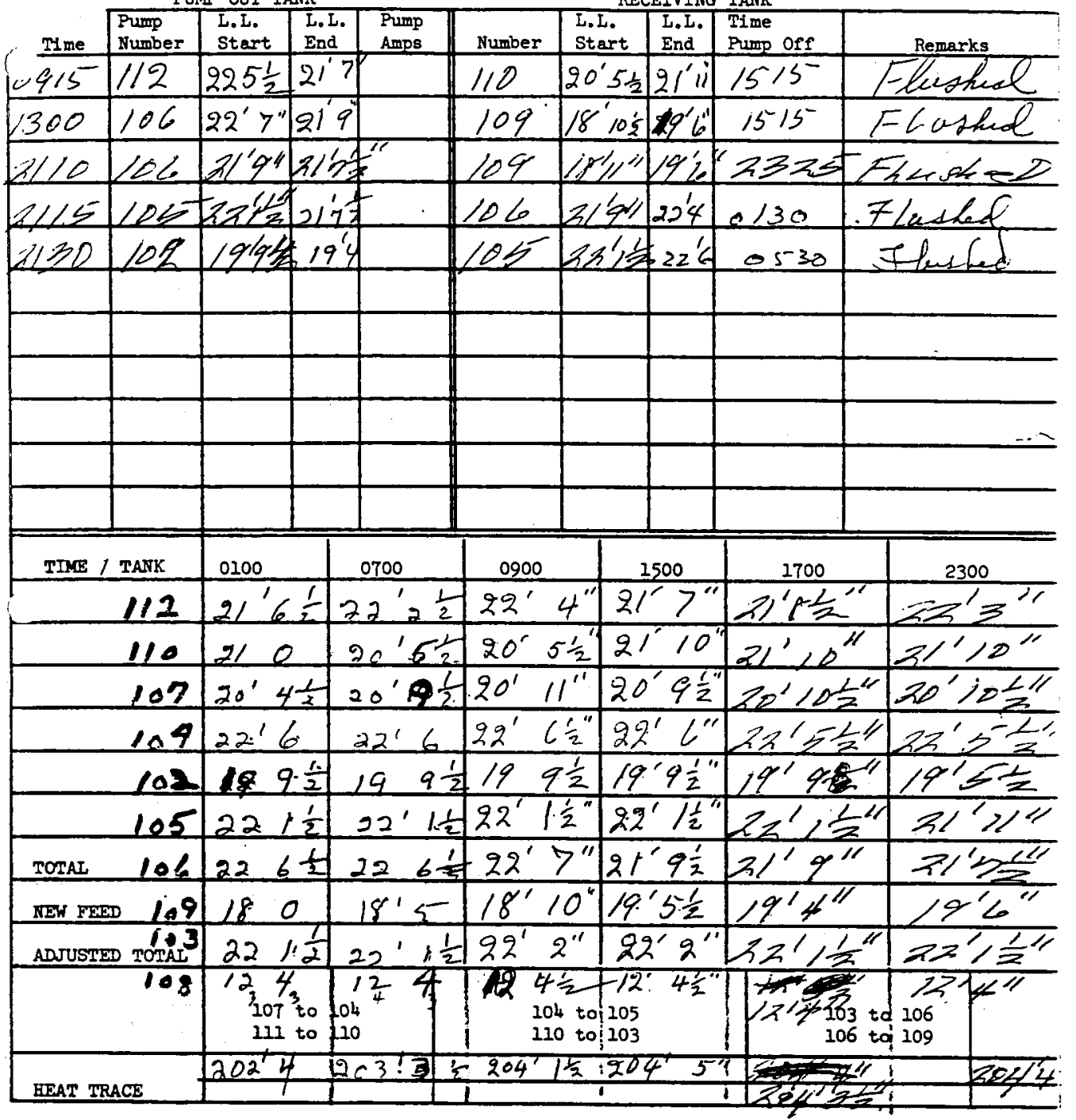

Walker:srb 9-29-70 
WHC-SD-WM-DP-209, Rev. 0

ITS-2 INVENTORY AND PUMPING

Date

SEP 5 197,

PUMP OUT TANK

RECEIVING TANK

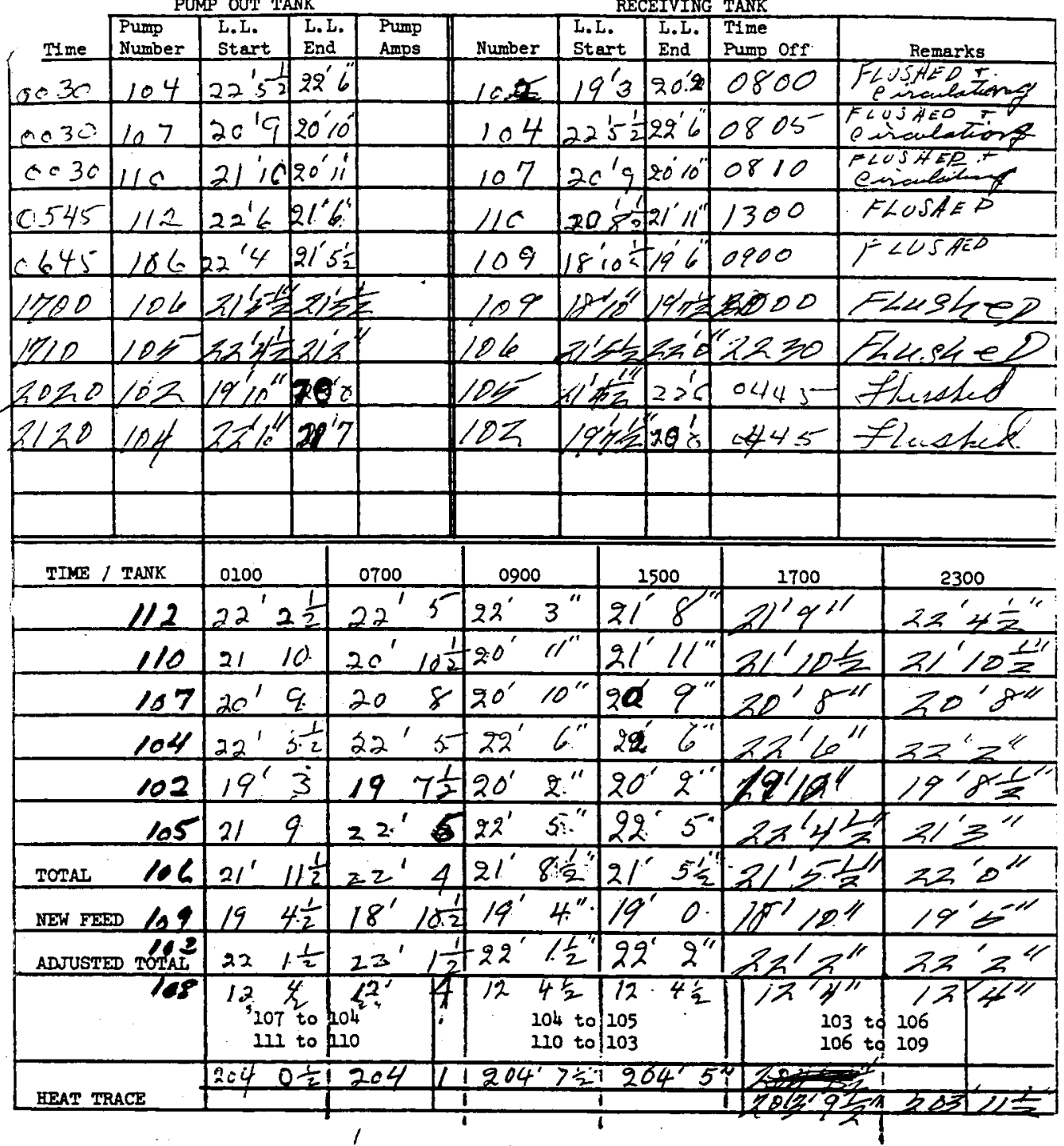

i. Wauker:srb 9-29-70

245 
PUMP OUT TANK

RECEIVING TANK

\begin{tabular}{|c|c|c|c|c|c|c|c|}
\hline $\begin{array}{l}\text { Pump } \\
\text { Number } \\
\end{array}$ & $\begin{array}{l}\text { L.L. } \\
\text { Start } \\
\end{array}$ & $\begin{array}{l}\text { Pump } \\
\text { Amps }\end{array}$ & Number & \multicolumn{2}{|c|}{\begin{tabular}{l|l|} 
L.L. & L.L. \\
Start & End \\
\end{tabular}} & $\begin{array}{l}\text { Time } \\
\text { Pump off }\end{array}$ & Remarks \\
\hline 0.30 & $20^{\prime} 8 / 19^{\prime}$ & & 104 & \multicolumn{2}{|c|}{$x^{\prime} 9 \frac{1}{2} 22^{2}+4$} & $0 \times 40$ & $\begin{array}{c}107 \times 104 \\
\text { mes. }\end{array}$ \\
\hline 0445 & $22.6211^{\prime}$ & & 160 & $2110 \frac{1}{2}$ & 2210 & 1212 & $F<\cup S A \&=0$ \\
\hline 0445 & $2110 \frac{1}{4}-199$ & & 107 & 1994 & 26,95 & 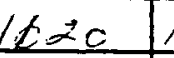 & $1 / 0-R \cdot e c$ \\
\hline 0.645 & $22^{\prime} 020^{\circ}$ & & 109 & \multicolumn{2}{|c|}{$18^{\prime} 919^{\prime \prime}$} & 1010 & "Ylusked. \\
\hline $12 / 5$ & $22^{\prime} 6^{\prime \prime} 211$ & & 106 & $20^{\prime} 8^{\prime \prime}$ & $22^{\prime} 2$ & 1730 & cleste \\
\hline- & & & & $=1$ & & & toen \\
\hline $19 \angle 5$ & $20^{\circ} 0 / 19^{\prime}$ & & 105 & \multicolumn{2}{|c|}{$21 \% 22 ' 6$} & 0305 & thuaked \\
\hline 2230 & $2110 \frac{1}{2} 211$ & & 102 & \multicolumn{2}{|c|}{$19^{\prime} 1020^{\prime} 1$} & 2330 & \\
\hline 2230 & $2 i^{\prime}>\frac{1}{2} x^{\prime \prime}$ & & 104 & \multicolumn{2}{|c|}{$2110 \quad 22^{\prime} 4 \frac{1}{4}$} & 0600 & thusbel \\
\hline 2230 & $2110 \frac{1}{2}+208$ & & 107 & \multicolumn{2}{|c|}{$21^{\prime}>\frac{1}{4} 208 \frac{1}{2}$} & 0640 & Atated \\
\hline 106 & $22^{\prime} 220^{\circ} 4$ & $20^{\circ} 44$ & 109 & $18^{\prime} 6$ & $1+834$ & 02750 & ELUSHED \\
\hline TIME / TANK & 0100 & 0700 & 0900 & & .500 & 1700 & 2330 \\
\hline 112 & $22^{\prime} 4$ & $2.2^{\prime} \cdot 3$ & $\approx 2=$ & & $y^{\prime} q^{\prime \prime}$ & $219 \frac{1}{2}$ & $223 \frac{1}{2}$ \\
\hline 110 & $21 \quad 10 \frac{1}{2}$ & $21.9 \frac{1}{2}$ & $71^{\prime} 4^{\circ}$ & 21 & $1-1 \mid$ & 2,11 & 218 \\
\hline 117 & $20^{\prime} 8$ & $1910 \frac{1}{2}$ & $19^{\prime}: "$ & 20 & -9 & $20 \cdot 8 \frac{1}{2}$ & $20^{\circ} 8$ \\
\hline 104 & $21 \quad 9 \frac{4}{2}$ & 2111 & $22^{\circ} 3^{\circ}$ & 22 & $-\psi^{\prime \prime}$ & $22^{\prime} 3 \frac{1}{2}$ & 2110. \\
\hline 102 & $19^{\prime} 16$ & 20.0 & $20^{\prime} 0$ & 20 & -0 & $20^{\circ} 0$ & $20 \%$ \\
\hline 105 & $21^{\prime 7} 7$ & 226 & $2 \geq 6$ & 21 & -6 & $21^{\prime} 4$ & $21 \cdot 9 \frac{7}{2}$ \\
\hline TOTAL 106 & $22^{\prime} 0$ & $22^{\prime} \mathrm{c}$ & $21^{\prime} 3^{\prime \prime}$ & 21 & $\left(-7 \frac{1}{5}\right.$ & $21^{\circ} 10 \frac{1}{2}$ & $22: 2$ \\
\hline NEW FEEDD 119 & $19^{\prime} 3$ & $18^{\prime} \%$ & $14^{\prime} z$ & & 'E' & $19^{\circ} 1$ & $18^{\circ} 6$ \\
\hline ADUUSTED TOTAE & 22,4 & $22.1 \frac{1}{3}$ & $22^{\circ} 1 \%$ & & $11 \frac{1}{2}$ & $221 \frac{1}{2}$ & $221 \frac{1}{2}$ \\
\hline 128 & $\begin{array}{l}12^{\prime} 4 / 1 \\
107 \text { to } 1 \\
111 \text { to } 1\end{array}$ & $\frac{12}{10} 4$ & $\begin{array}{l}1244 \prime \\
=104 \text { tc } \\
110 \text { to }\end{array}$ & $\begin{array}{l}12 \\
\text { tof } 105 \\
\text { to } 103\end{array}$ & $4 y^{\prime \prime}$ & $\begin{array}{r}12 \cdot 4 \frac{2}{2} \\
103 \\
106\end{array}$ & $\mid \begin{array}{l}12 \\
\text { to } 106 \\
\text { to } 109\end{array}$ \\
\hline HEAT TRACE & 3039 हा & $2 x^{3}$ & $20 E^{\prime} 5 \varepsilon^{\prime \prime}$ & $\frac{\pi 203}{1}$ & & 2037 & $2: 20 \sqrt{3} 6 \frac{1}{2}$ \\
\hline
\end{tabular}

1 Wadker:srb 9-29-70 
PUMP OUT TANK

RECEIVING TANK

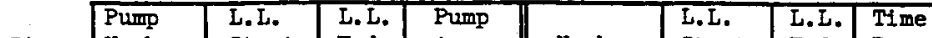

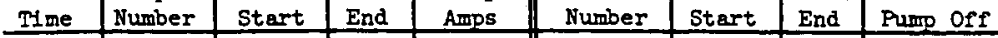

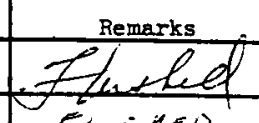

$10030 / 104 \quad 220 \frac{1}{22} 21$

$10219^{\prime} 920^{\circ} \cdot 420$

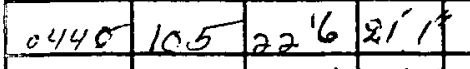

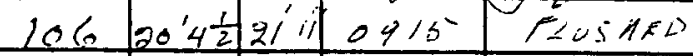

$10440112 \quad 22^{\circ} 6216$

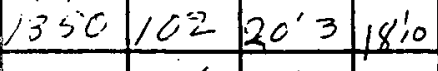

\begin{tabular}{|l|l|l|l|}
1615 & 106 & 2111 & 211 \\
\hline
\end{tabular}

\begin{tabular}{|l|l|l|l|}
1830 & 105 & $22 \%$ & $2110 \frac{1}{2}$ \\
\hline
\end{tabular}

\begin{tabular}{|l|l|l|l|}
2100 & 104 & $224 \frac{1}{2}$ & 22.15 \\
\hline
\end{tabular}

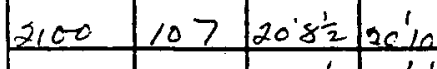

\begin{tabular}{l|l|l|l|}
2100 & 110 & $219 \frac{1}{2}$ & 19 \\
\hline
\end{tabular}

TIME / TANK

\begin{tabular}{l|l|l|l|l} 
& 0100 & 0700 & 0900 & 1500 \\
\hline 12 & $22^{\prime} 4$ & $22^{\prime} 4$ & $92^{\prime} 0^{\prime \prime}$ & $21^{\prime} 8$
\end{tabular}

$110 \quad 21 \quad 4 \frac{1}{2} 20 \quad 8 \frac{1}{2}$

$21^{\prime \prime} 3^{\prime \prime}$

$\ln 20 \quad 8 \frac{1}{2}$ 20 $8 \frac{1}{2}$

110 20'9 $21^{\prime} \% 1135$

105

$21^{\prime} 1^{\prime \prime} 22^{\prime} 7$

thentich

109

$18^{\circ} 11+19^{\prime} \mathrm{C}$

0030

Hench

.

[

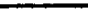

$1,4 \quad 22 \quad 0 \frac{1}{2} 22^{1} 4 \frac{1}{2}$

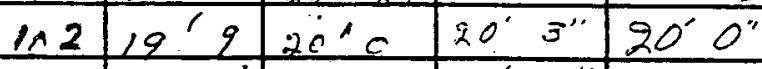

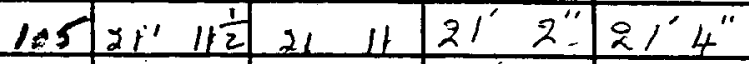

$107208 \frac{1}{2} 20 \%$ 0630

1830

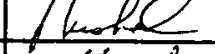


PUMP OUT TANK

RECEIVING TANK

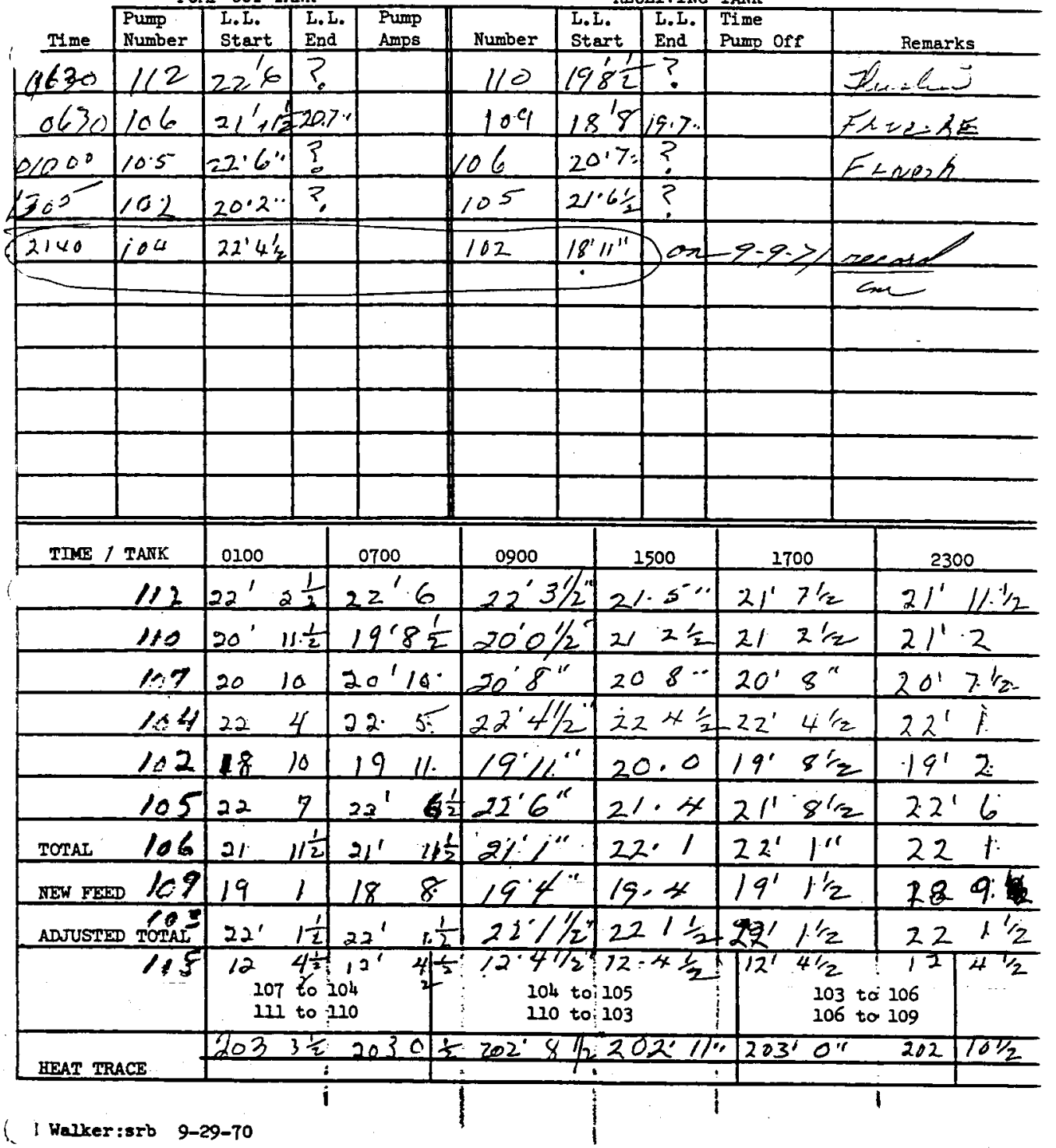


WHC-SO-WM-DP-209, REV. 0

ITS-2 INVENTORY AND PUMPING

Date $\operatorname{sep} g-7$

PUMP OUT TANK

RECEIVING TANK

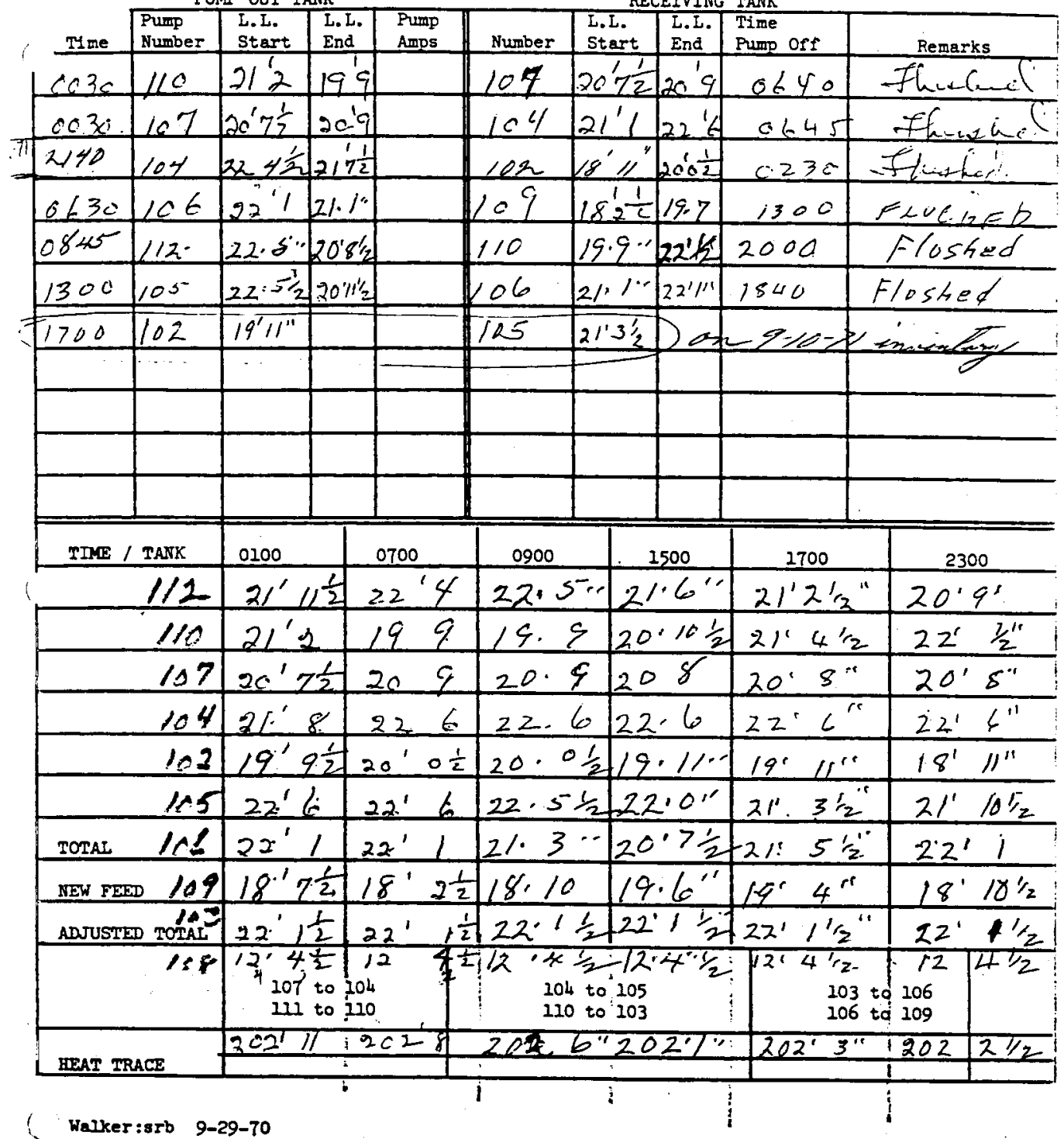

249 
PUMP OUT TANK

RECEIVING TANK

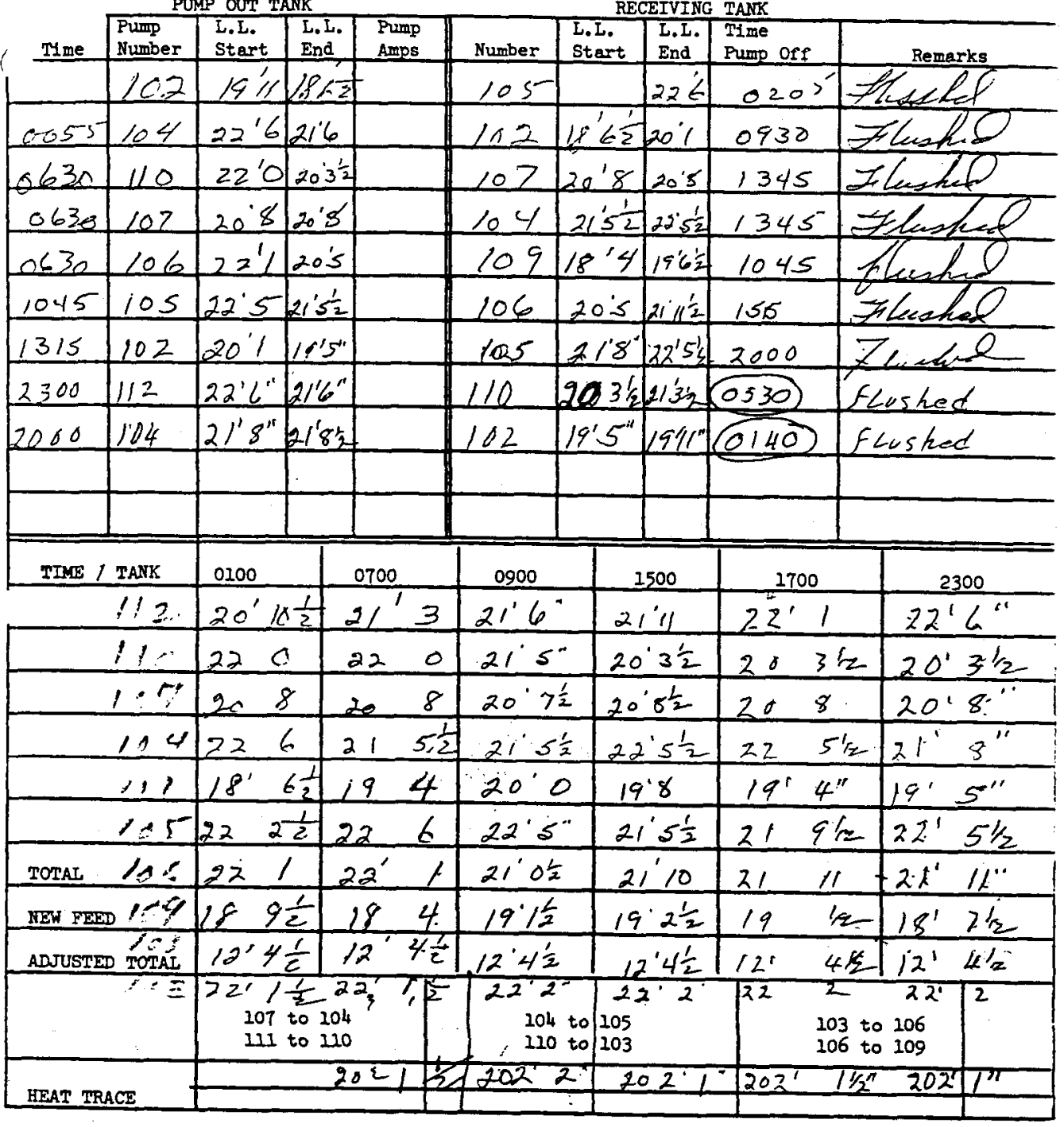

$M$ Walker:srb 9-29-70 
PUMP OUT TANK

RECEIVING TANK

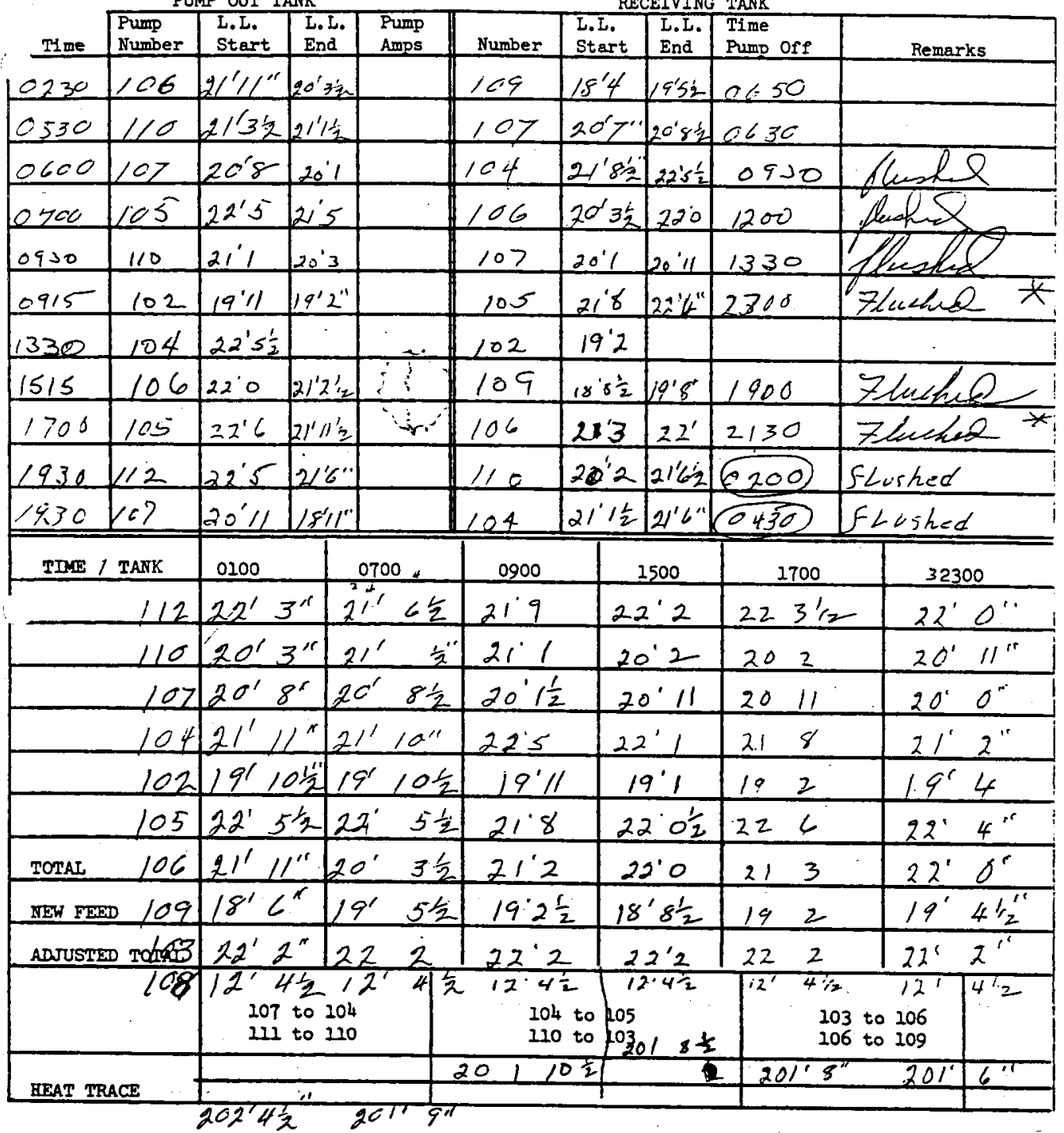

Walker:srb 9-29-70 
WHC-SD-HM-DP-209, ReV. D

ITS-2 INVENTORY AND PUMPING

Date CFE!; 1071

PUMP OUT TANK

RECEIVING TANK

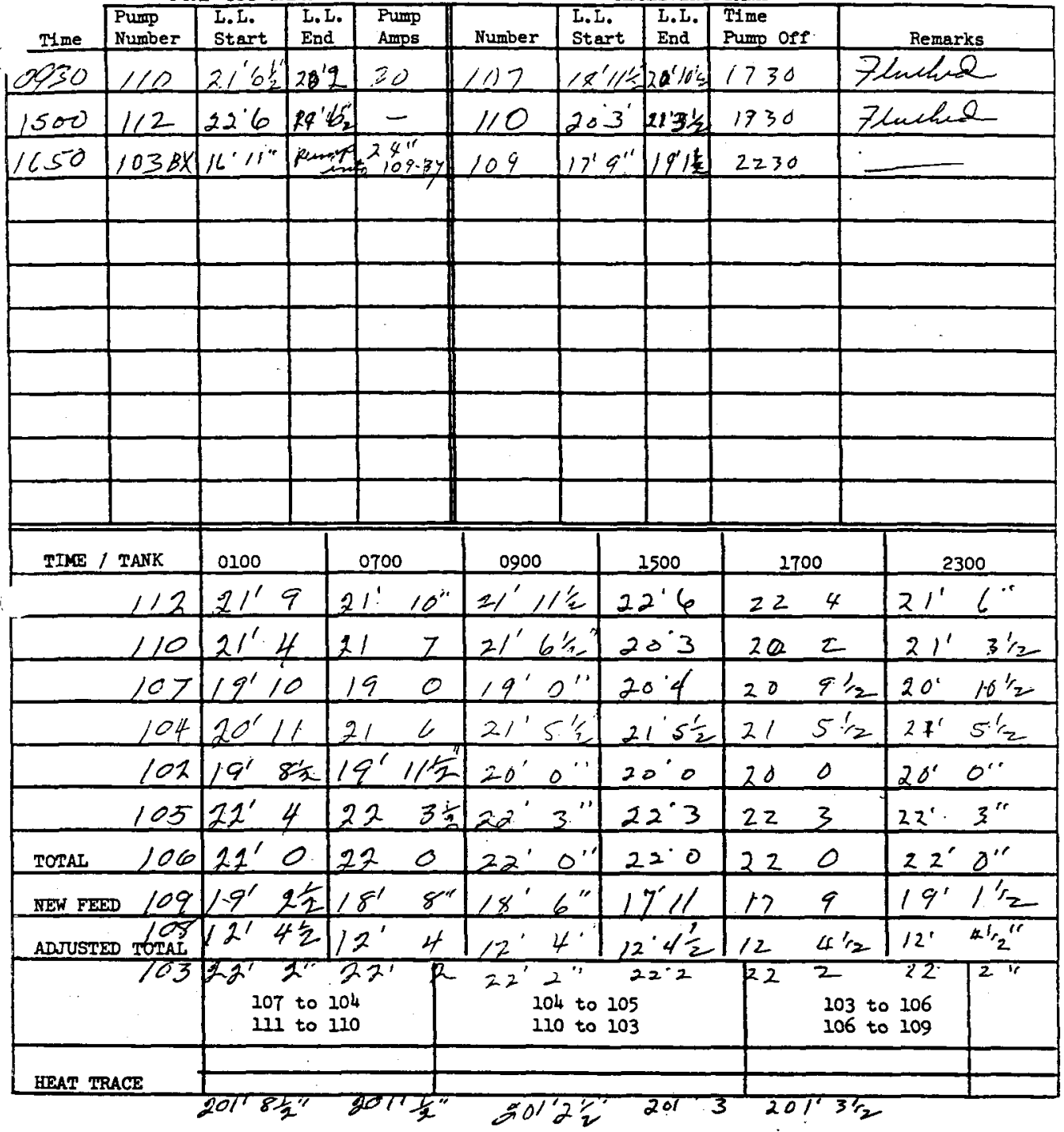

Walker:srb 9-29-70 
WHC-SO-LW-DP-209, Rev. 0

ITS-2 INVENTORY AND PUMPING

Date SEP 131971

PUMP OUT TANK

RECEIVING TANK

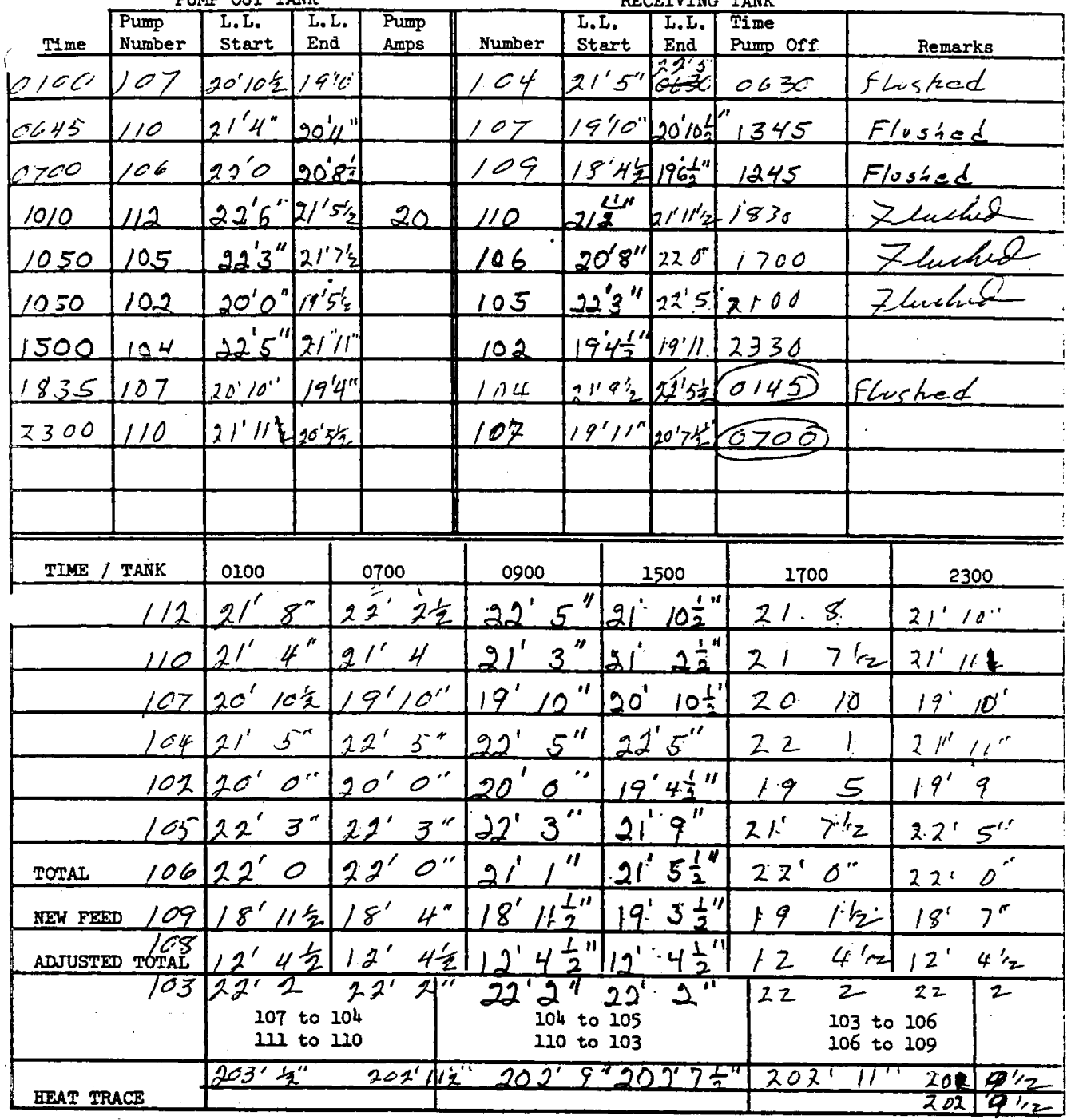

1. Wolker:srb 9-29-70

$$
=\frac{5 \frac{5}{8} 5}{7 \frac{1}{2}}
$$

253 


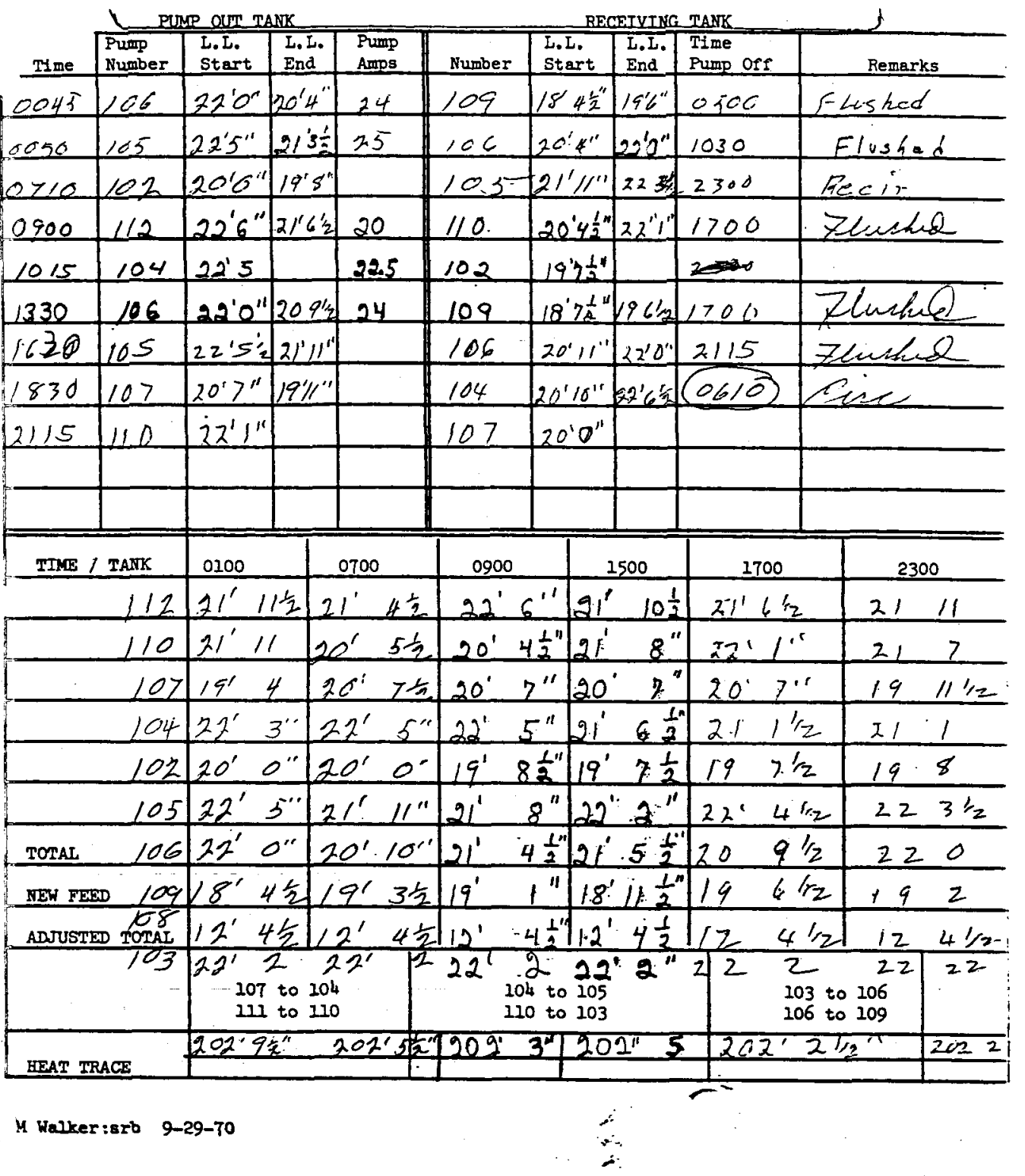


WHC-SD- BM-OP-209, REV. O

ITS-2 INVENTORY AND PUMPING

Date

SEP : 1971

PUMP OUT TANK

RECEIVING TANK

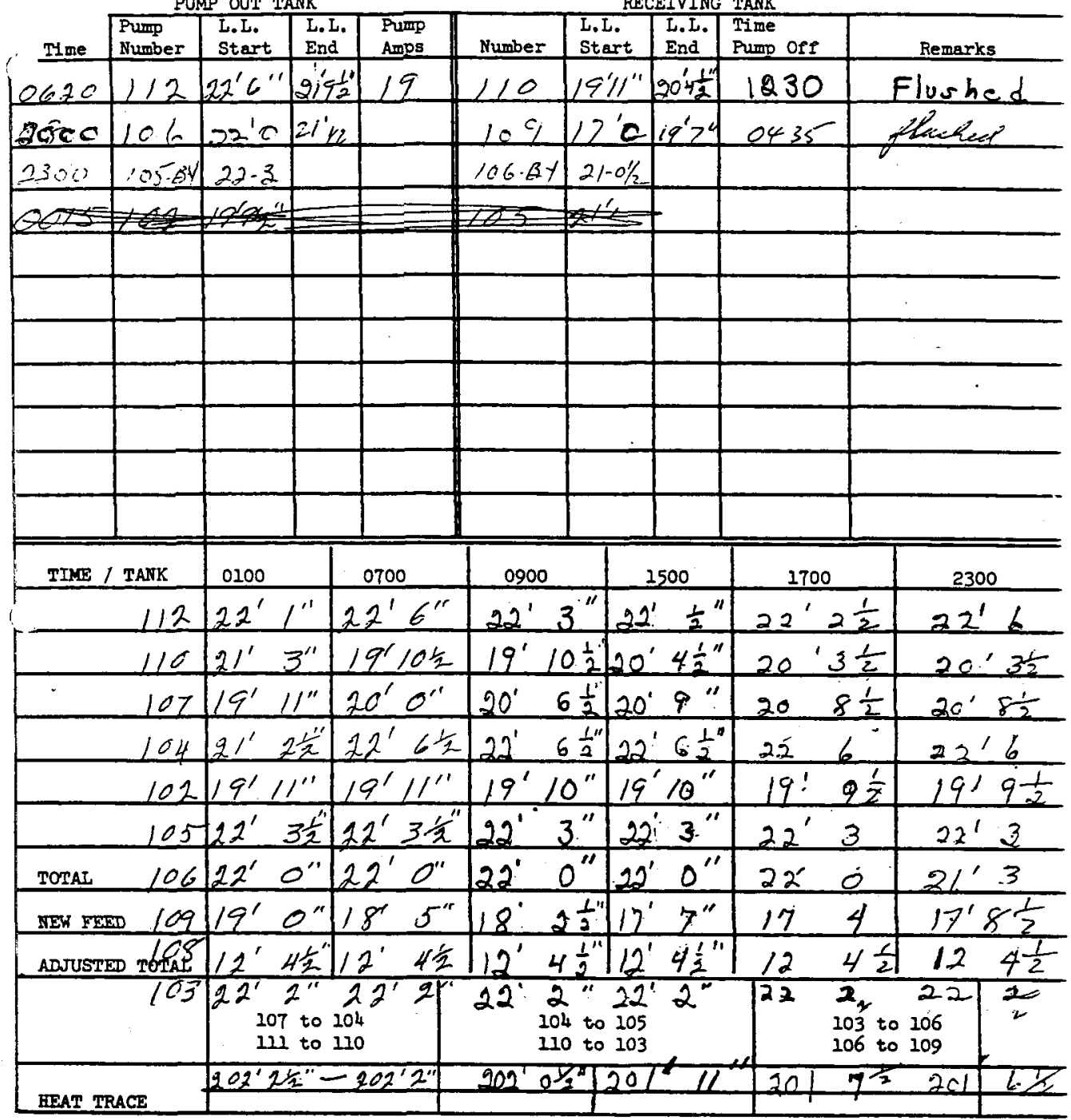

( Walker:srb 9-29-70

255 
WHC-SD-WM-DP-209, ReV. O

ITS-2 INVENTORY AND PUMPING

Date

PUMP OUT TANK

RECEIVING TANK

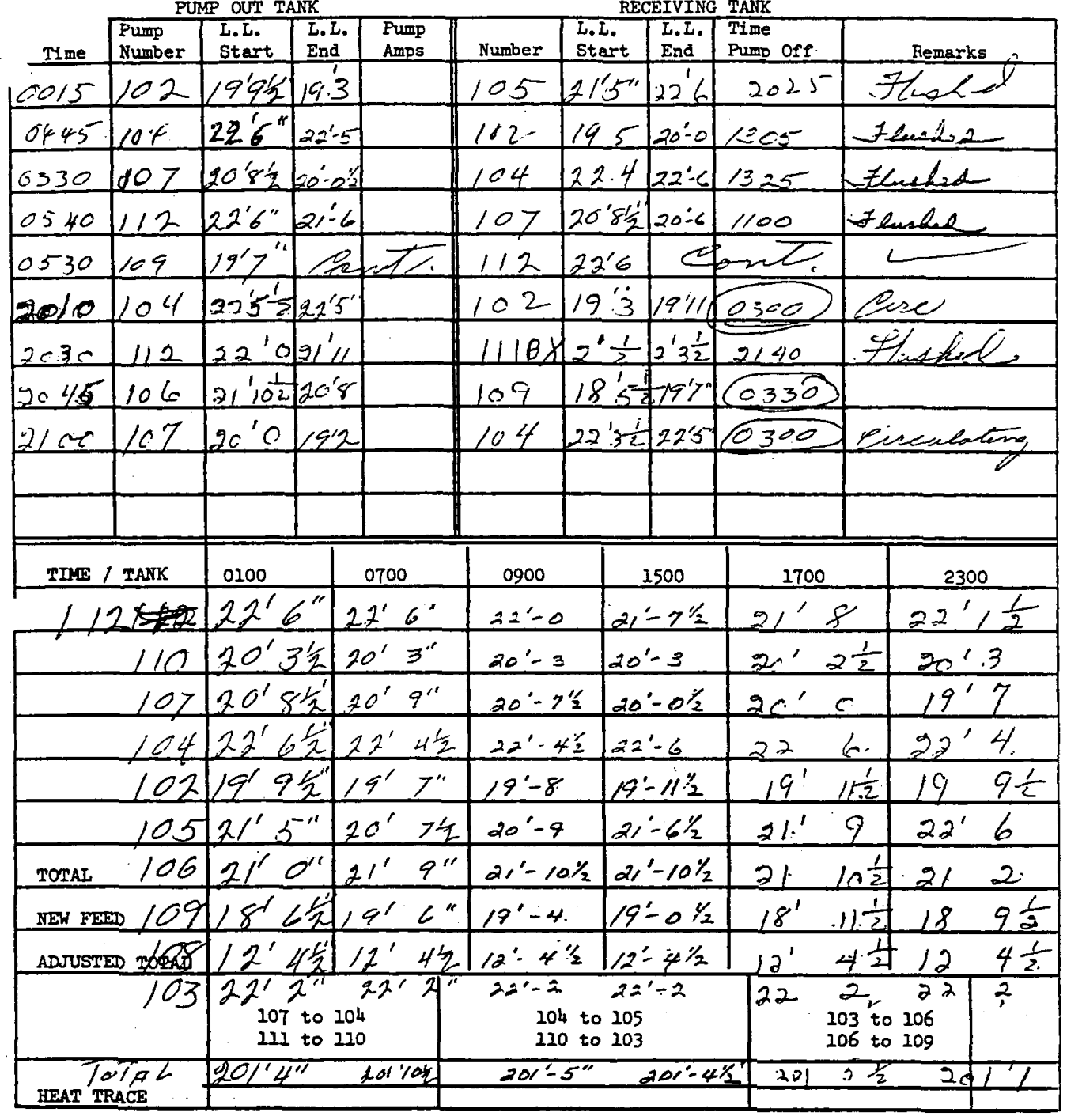

M Walker:srb 9-29-70

256 
Dete

SEP+ $+9+94$

PUMP OUT TANK

RECEIVING TANK

\begin{tabular}{|c|c|c|c|c|c|c|c|c|c|}
\hline Thme & $\begin{array}{l}\text { Pump } \\
\text { Number }\end{array}$ & $\begin{array}{l}\text { L.L. } \\
\text { Start }\end{array}$ & $\begin{array}{l}\text { L.L. } \\
\text { End }\end{array}$ & $\begin{array}{l}\text { Pump } \\
\text { Amps }\end{array}$ & Number & $\begin{array}{l}\text { L.L. } \\
\text { Start }\end{array}$ & $\begin{array}{l}\text { L. I. } \\
\text { End }\end{array}$ & $\begin{array}{l}\text { Time } \\
\text { Pump off }\end{array}$ & Remarks \\
\hline 10005 & 105 & $22^{\prime} 56$ & $\begin{array}{l}21^{\prime} 3 \frac{5}{5} \\
34\end{array}$ & 24 & 106 & $20^{\prime} 7$ & $2 / 11^{\prime \prime}$ & 0645 & \\
\hline 0005 & 102 & $20^{\prime} 0$ & $195 \frac{1}{2}$ & & 105 & $22^{\prime} 5 \frac{1}{2}$ & $2 / 8^{\prime}=$ & 1030 & Evarlesp \\
\hline 0130 & 112 & $22^{\prime} 6 \frac{1}{2}$ & $16^{\prime} 6^{\circ}$ & 20 & 107 & $19^{\prime} 2^{\prime \prime}$ & & 1215 & Elepteen \\
\hline
\end{tabular}

-

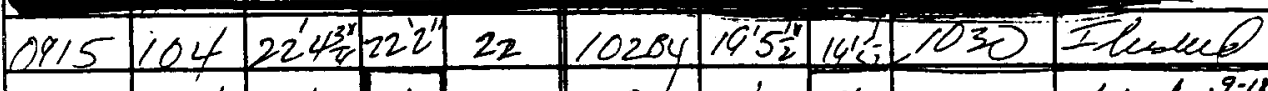
$1: 45 \quad 106211121-4$

$1925-105-21860^{\circ}-x$

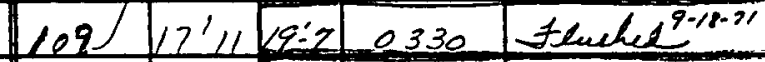
\begin{tabular}{l|l|l|l|}
200 & 104 & $22^{\prime} 2$ & $2 y^{2}$
\end{tabular} $2.30112 \quad 22^{\prime} 6216^{\prime \prime}$

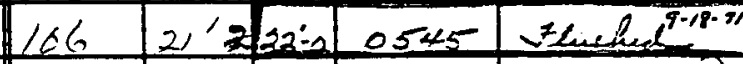
2ive 107 20'9 $18^{\prime} 9^{\prime \prime}$ $10219^{\prime} \cdot \frac{1}{2}\left(9^{\prime} 951515\right.$ Tleuch 1955 16.2 $19^{\prime} 5^{\prime}+19^{\prime} 9^{\prime \prime}$ $10720^{\prime} 7 \frac{1}{2} 80^{\prime \prime} 0900$ Flewed 9 .

\begin{tabular}{|c|c|c|c|c|c|c|}
\hline TANK & 0100 & 0700 & & 50 & 1700 & 2300 \\
\hline 112 & $22^{\prime} 5^{\prime \prime}$ & $22^{\prime} 3^{\prime \prime}$ & $2^{\prime} \pi^{\frac{1}{2}}{ }^{\prime}$ & $219 \frac{1}{2}$ & $21^{\prime} 11 \frac{1}{2}$ & $22^{\prime} 4$ \\
\hline 110 & $20^{\prime} 3^{\prime \prime}$ & $70^{\prime} 3$ & $20^{\prime} 3^{\prime \prime}$ & $20^{1} 5^{11}$ & $20^{\prime} 3$ & $x_{0}^{\prime}$ \\
\hline 107 & $\angle 9^{\prime} \quad z^{\prime}=$ & $191 \quad 7 \frac{1}{2}$ & $20^{\prime}$ & $20^{\prime} 8^{\prime \prime}$ & $7 \cdot \frac{1}{2}$ & $20 \cdot 8 \frac{1}{2}$ \\
\hline 104 & $22^{\prime} \quad 4^{\prime \prime}$ & $22^{\prime} \quad 5$ & $22^{\prime} 44^{3}$ & $22^{\prime} 2^{\prime \prime}$ & 22 & 92 \\
\hline 102 & $20^{\prime} 0^{\prime \prime}$ & $19^{\prime} 10 \frac{1}{2}$ & $\left.14^{\prime} 5 \frac{1}{2}\right)^{\prime \prime}$ & $19^{\prime} \overline{52}$ & $19^{\prime} 5 \frac{1}{2}$ & $5 \frac{1}{2}$ \\
\hline 105 & $225 \frac{1}{2}$ & $21^{\prime} 3 \frac{1}{2}$ & $2^{\prime \prime} 6^{\prime \prime}$ & $218 \frac{2}{2}$ & $21^{\prime} \quad 8 \cdot \frac{1}{2}$ & $4 \frac{1}{2}$ \\
\hline 106 & $20^{\prime} 7^{\prime \prime}$ & $2 \prime^{\prime \prime} / 1{ }^{\prime \prime}$ & $21^{\prime \prime} 10^{\prime \prime}$ & $2 \% 10^{\prime \prime}$ & $21 \quad 15$ & $1 \frac{1}{2}$ \\
\hline $\mathrm{KEH}$ FERD & $19^{\prime} / \frac{1}{2}$ & $19^{\prime} 2^{\prime \prime}$ & $\left.18^{\prime} 10^{3}\right)^{\prime \prime}$ & $18^{\prime} 4^{\prime \prime}$ & $18^{\prime}$ & $1 \frac{1}{2}$ \\
\hline NUSTED T6TAP & $12^{14}$ & $12^{\prime} 4 \frac{1}{2}$ & $1214 \frac{1}{2}$ & $124 \frac{1}{2}$ & $4 \frac{1}{2}$ & $12^{\prime}$ \\
\hline & $\begin{array}{r}22^{\prime} 2^{11} \\
107 \text { to } \\
\text { III to to }\end{array}$ & & $\begin{aligned} 22^{\prime} 2^{\prime \prime} \\
104 t \\
110 \\
110\end{aligned}$ & & $\begin{array}{cc}272 & 22 \\
103 \\
106\end{array}$ & $\begin{array}{l}27 \\
106 \\
109\end{array}$ \\
\hline $\begin{array}{l}\text { To TAPL } \\
\text { AT TRAB }\end{array}$ & $200^{\prime}$ & $20011^{11}$ & $\tan 10$ & 209 & 2008 & 2019 \\
\hline
\end{tabular}

4 Walker:srb 9-29-70 


\section{ITS-2 INVENTORY AND PUMPING Date $9-18^{2}-7 /$}

PUMP OUT TANK

RECEIVING TANK

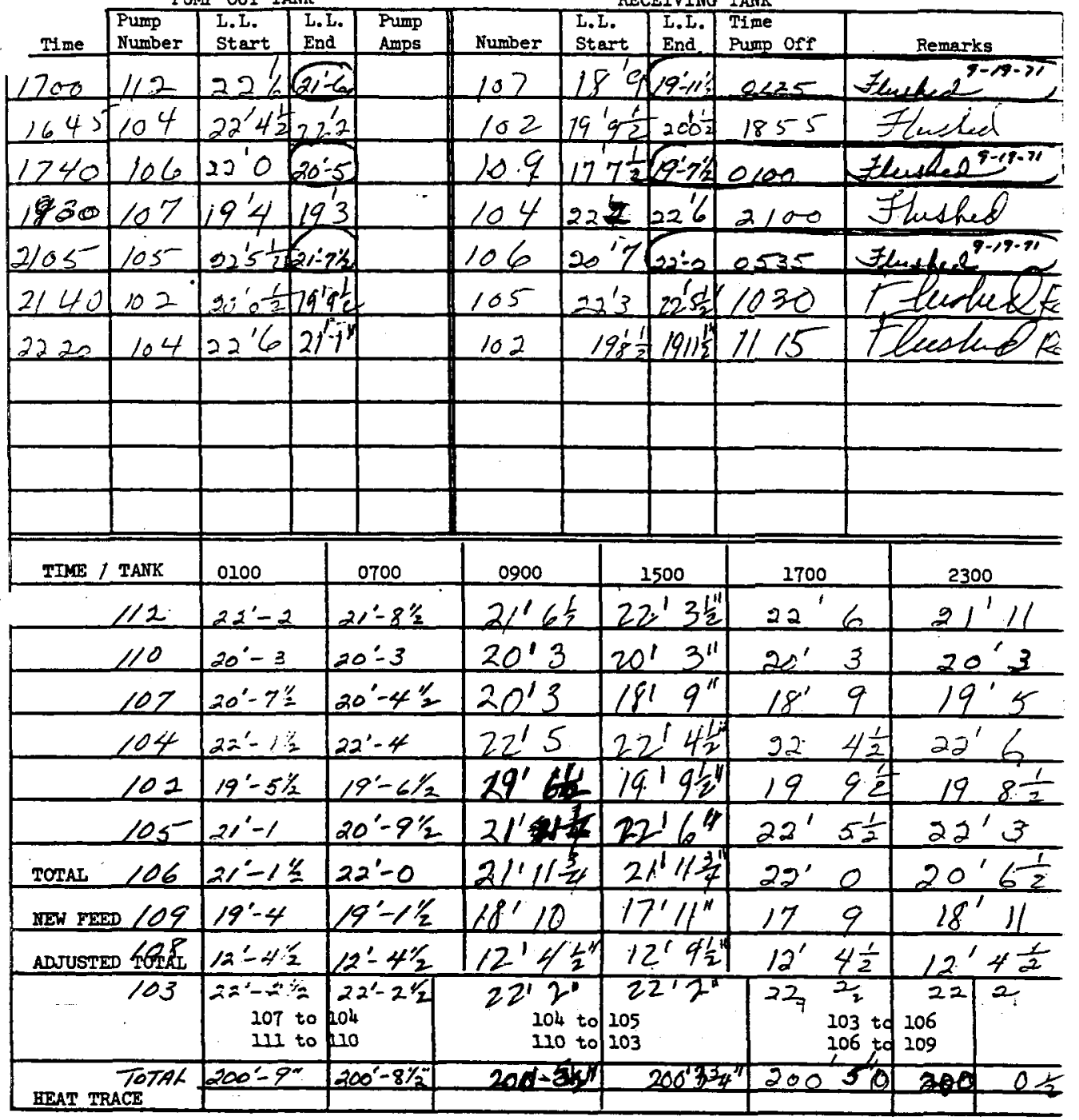

(I Wauker:srb 9-29-70 
ITS-2 INVENTORY AND PUMPING

Date $9-14-7 /$

PUMP OUT TANK

RECEIVING TANK

\begin{tabular}{l|l|l|l|l||l|l|l|l|l|}
\cline { 2 - 4 } & $\begin{array}{l}\text { Pump } \\
\text { Number }\end{array}$ & $\begin{array}{l}\text { L.L. } \\
\text { Start }\end{array}$ & $\begin{array}{l}\text { L. L. } \\
\text { End }\end{array}$ & $\begin{array}{l}\text { Pump } \\
\text { Amps }\end{array}$ & Number & $\begin{array}{l}\text { L.L. } \\
\text { Start }\end{array}$ & $\begin{array}{l}\text { L. L. } \\
\text { End }\end{array}$ & $\begin{array}{l}\text { Time } \\
\text { Pump off }\end{array}$ & Remarks \\
\hline
\end{tabular}

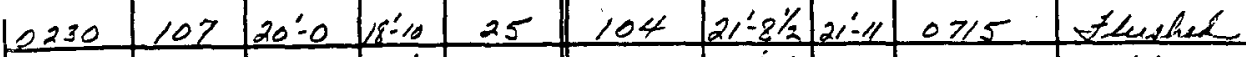

\begin{tabular}{|l|l|l|l|}
\hline 300 & 112 & $22^{\prime} 6^{\prime \prime}$ & 216 \\
\hline
\end{tabular}

$1630107 / 19^{\prime} 7189$

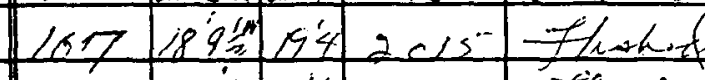

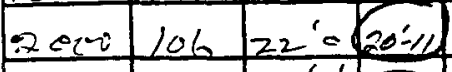

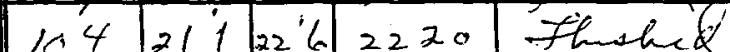

$12.30 / 0.5225 \frac{1}{20}-5$

$10 \% 18^{\prime} \circ 69^{\prime} 7220$ 7kedes

$12.10+252252$

$106 \quad 212,22: 00530$

$32.462^{9-20}$

\begin{tabular}{l|l|l|l|l|}
\hline & & & & \\
\hline & & & & \\
\hline & & & & \\
\hline & & & & \\
\hline & & & & \\
\hline
\end{tabular}

TIME $/$ TANK

\begin{tabular}{l|l|l|l|}
0200 & 0700 & 0
\end{tabular}

\section{The 1}

\begin{tabular}{l|l|l|l|ll|l|l|l}
112 & $21-6 \%$ & $21^{\prime}-11$ & $22^{\prime} 1$ & 22 & $2 \frac{1}{2}$ & $21^{\prime} 11 \frac{1}{2}$ & $21^{\prime} 72$
\end{tabular}

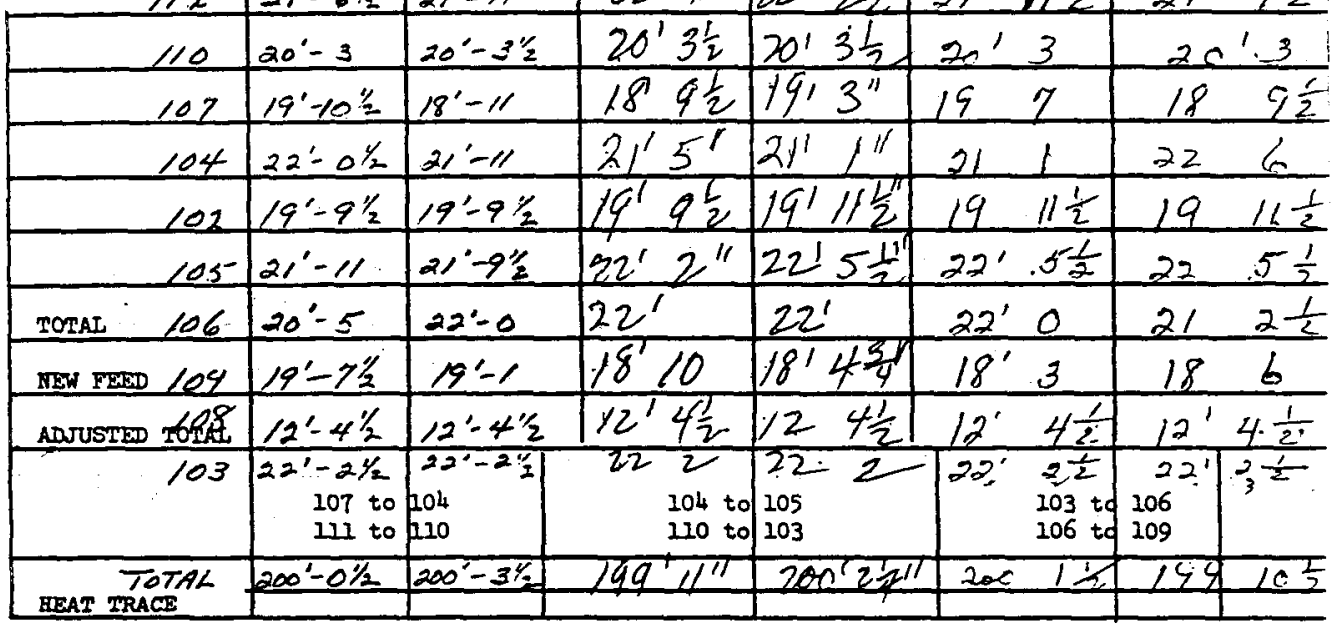

1 Walker:srb 9-29-70 
ITS-2 INVENTORY AND PUMPING Date 9-20-7/

PUMP OUT TANK RECEIVING TANK

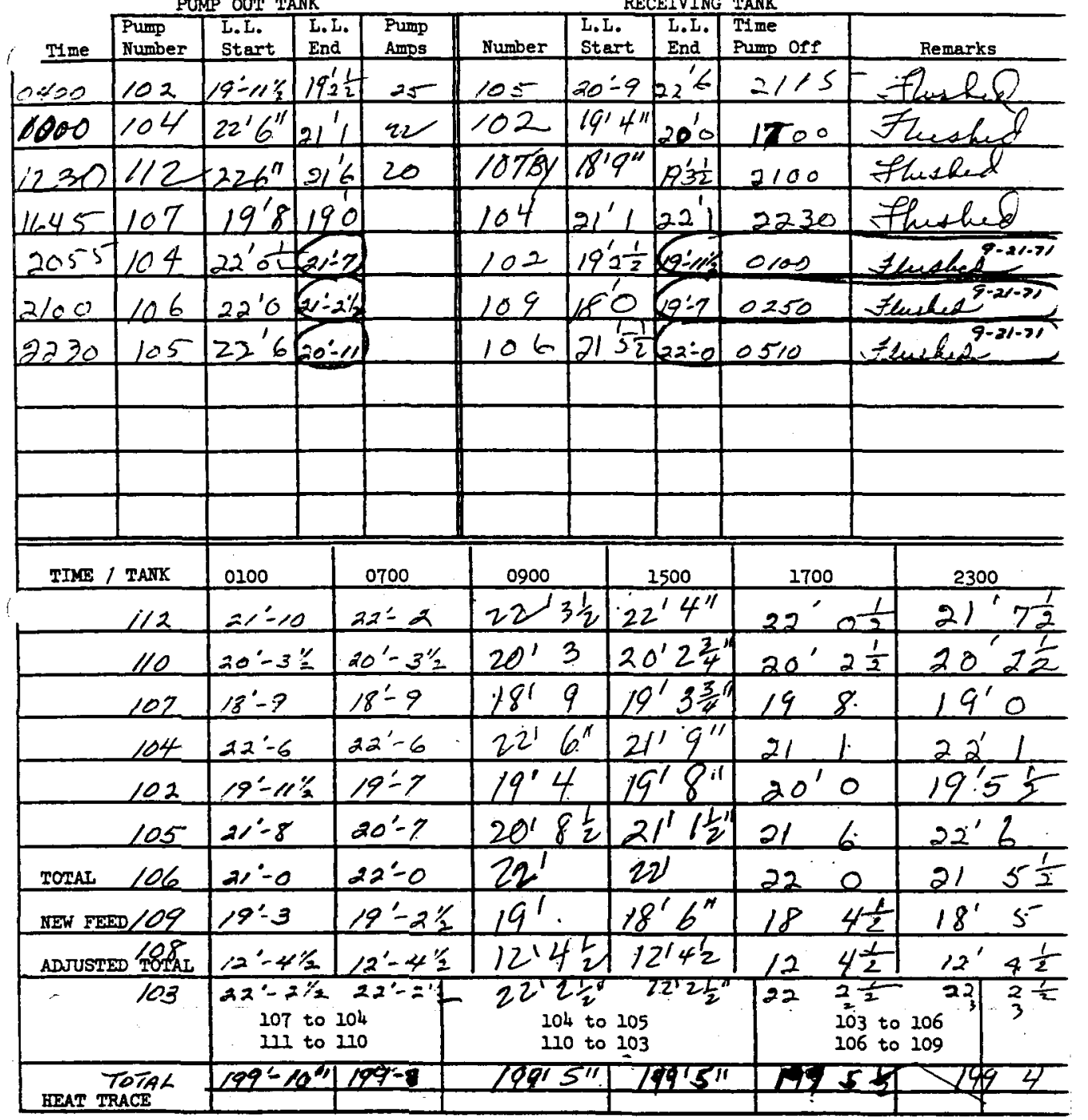

Walker:srb 9-29-70 
ITS-2 INVENTORY AND PUMPING Date $4-2 /-7 /$

PUMP OUT TANK RECEIVING TANK

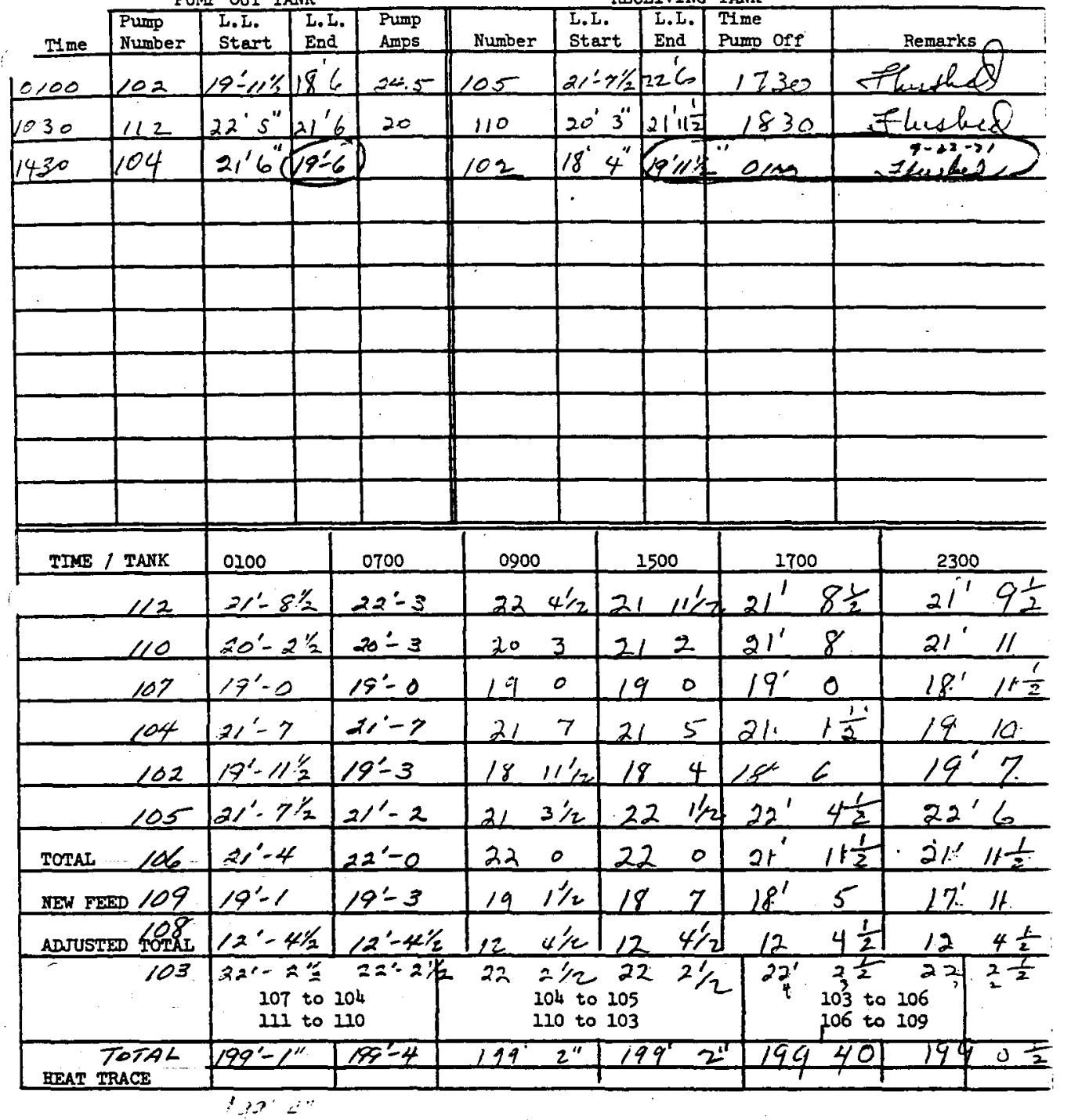

Walker:srb 9-29-70 
WHC-SD-WM-OP-209, REV. 0

ITS-2 INVENTORY AND PUMPING

Date $8-2 z-y$

PUMP OUT TANK

RECEIVING TANK

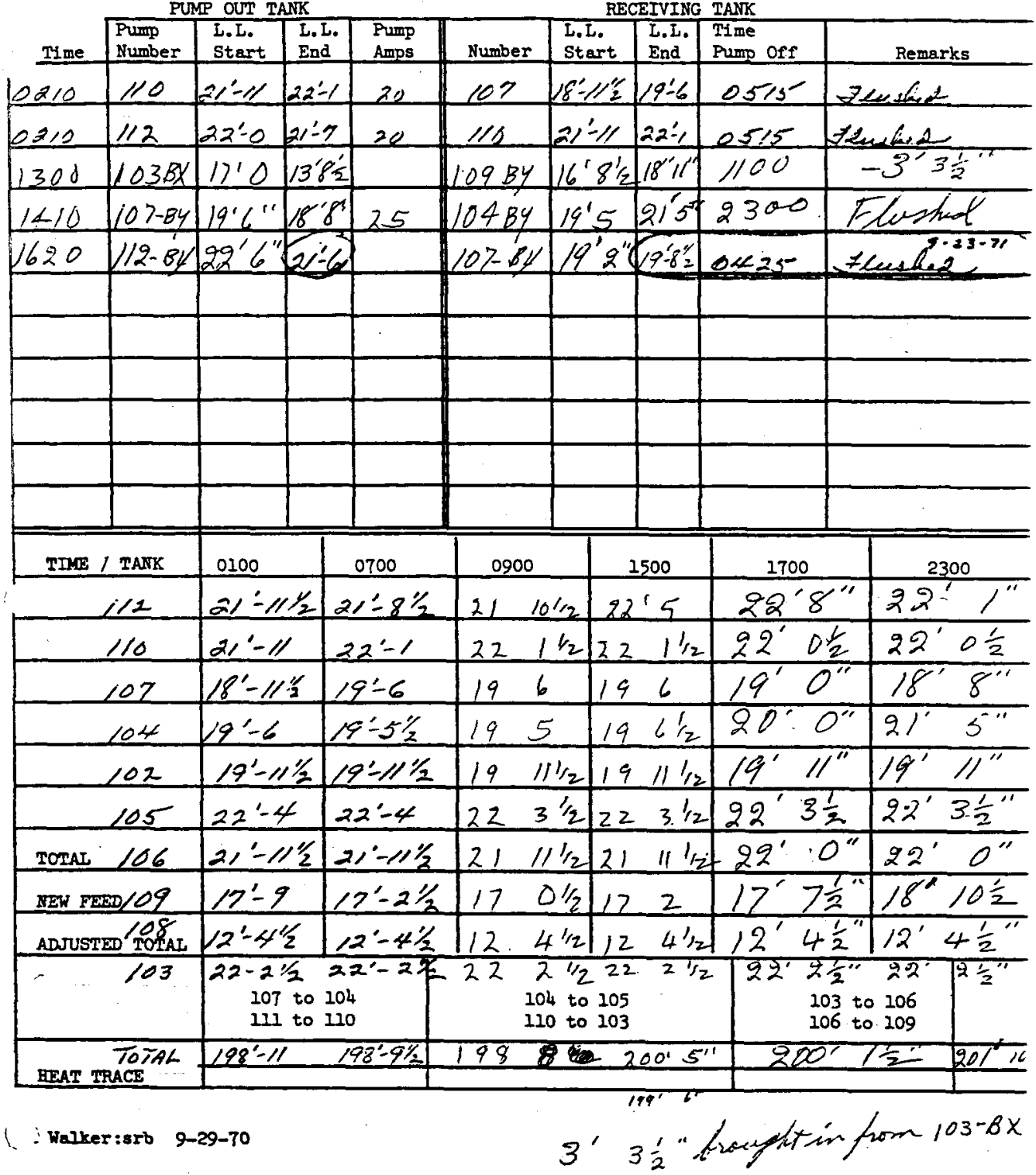

262 
PUMP OUT TANK

RECEIVING TANK

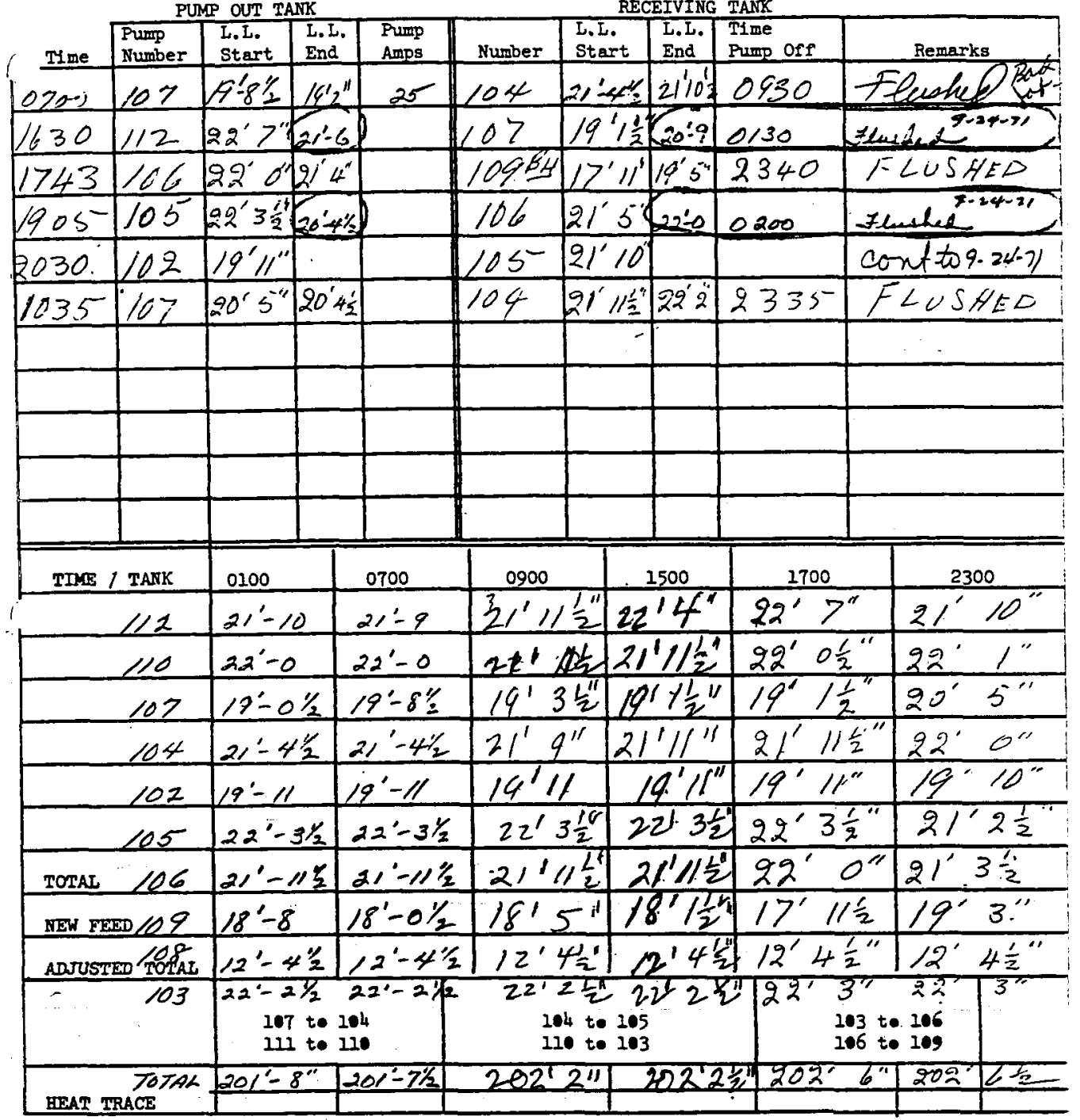


ITS-2 INVENTORY AND PUMPING Date $9-24-7 /$

PUMP OUT TANK

RECEIVING TANK

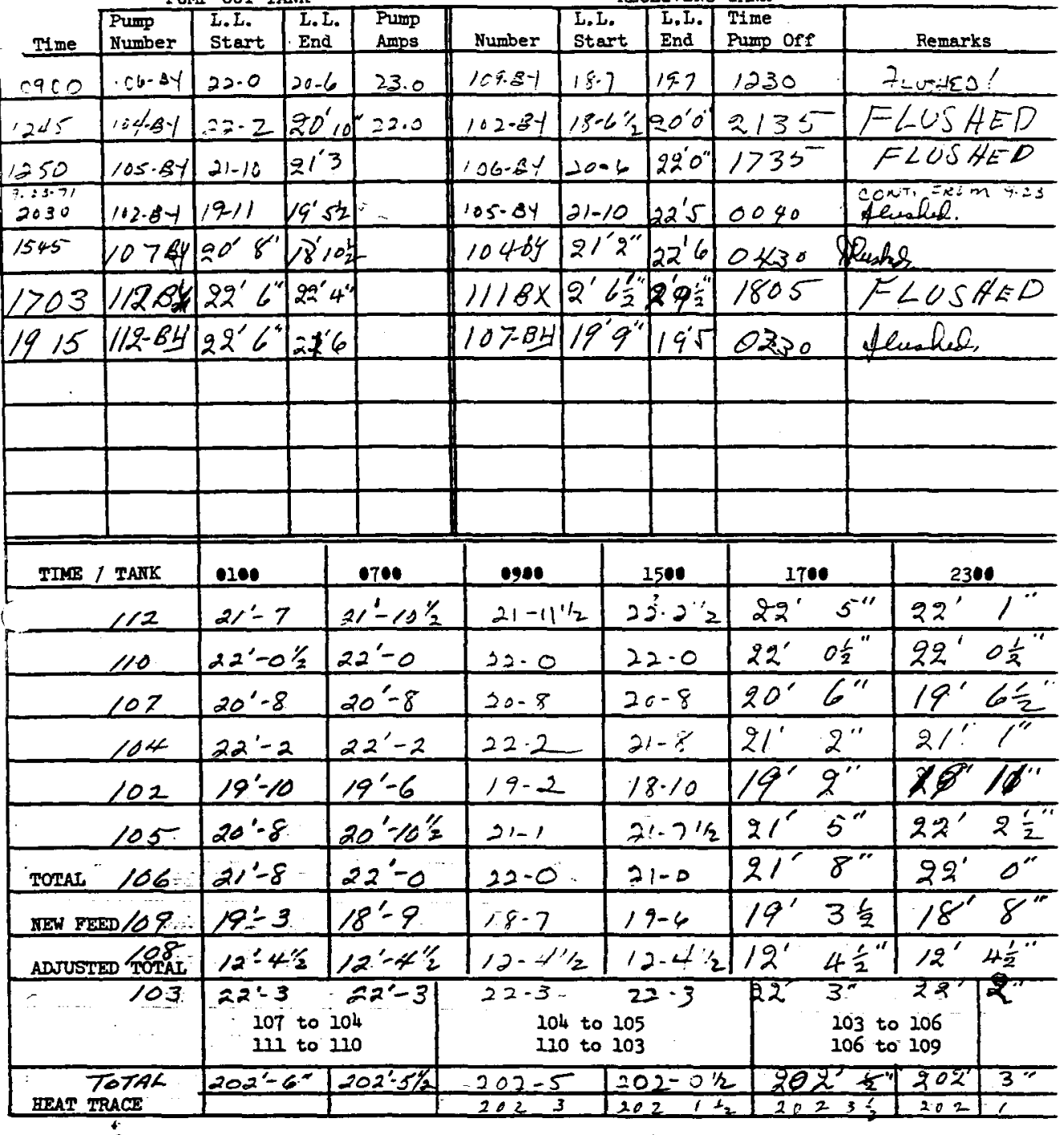

Walker:srb 9-29-70 
PUMP OUT TANK

RECEIVING TANK

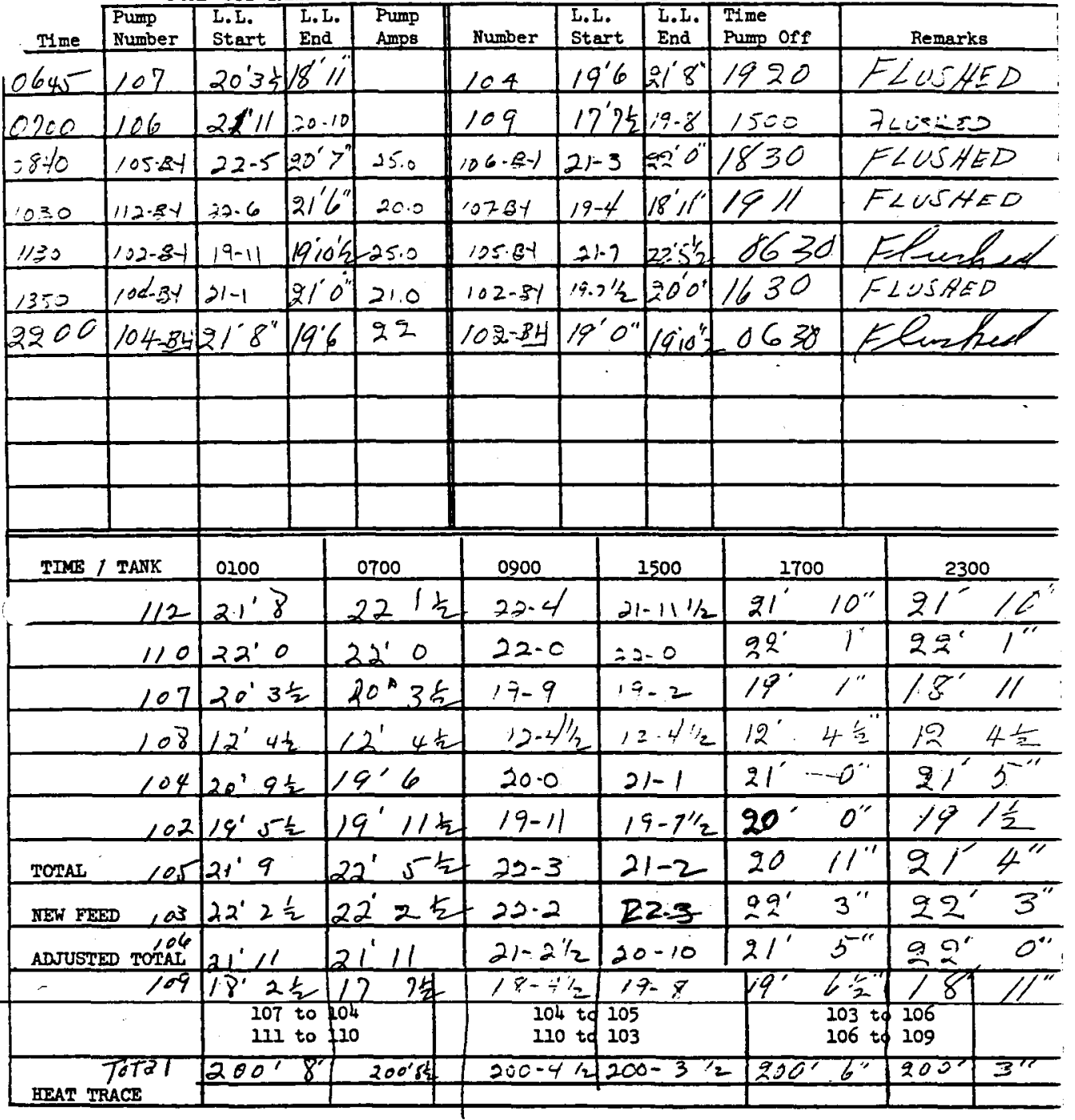

Wajker:srb 9-29-70 
WHC-SD-WM-DP-209, REV. 0

ITS-2 INVENTORY AND PUMPING

Date $4-27-71$

PUMP OUT TANK

RECEIVING TANK

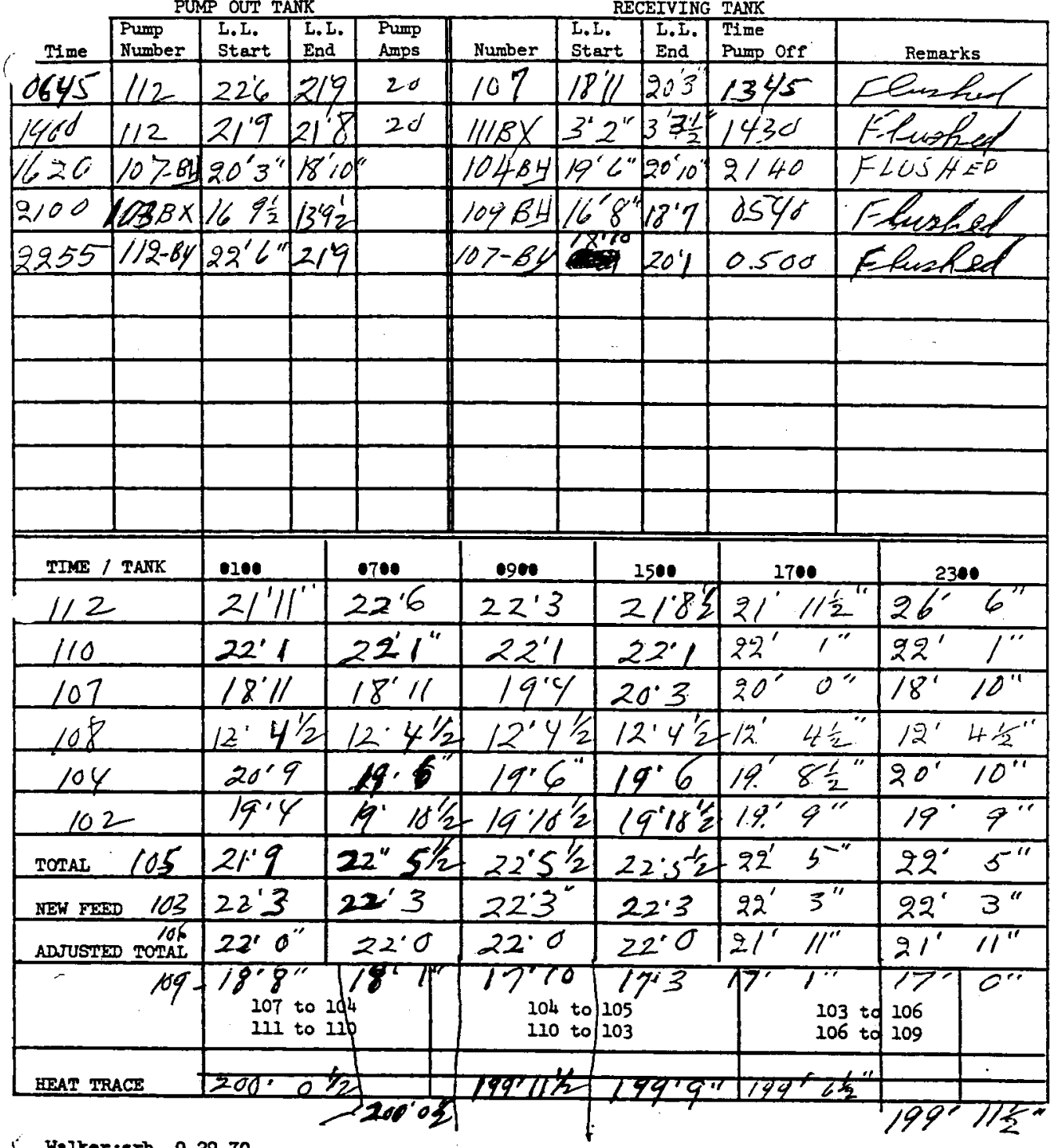

267 
PUMP OUT TANK

RECEIVING TANK

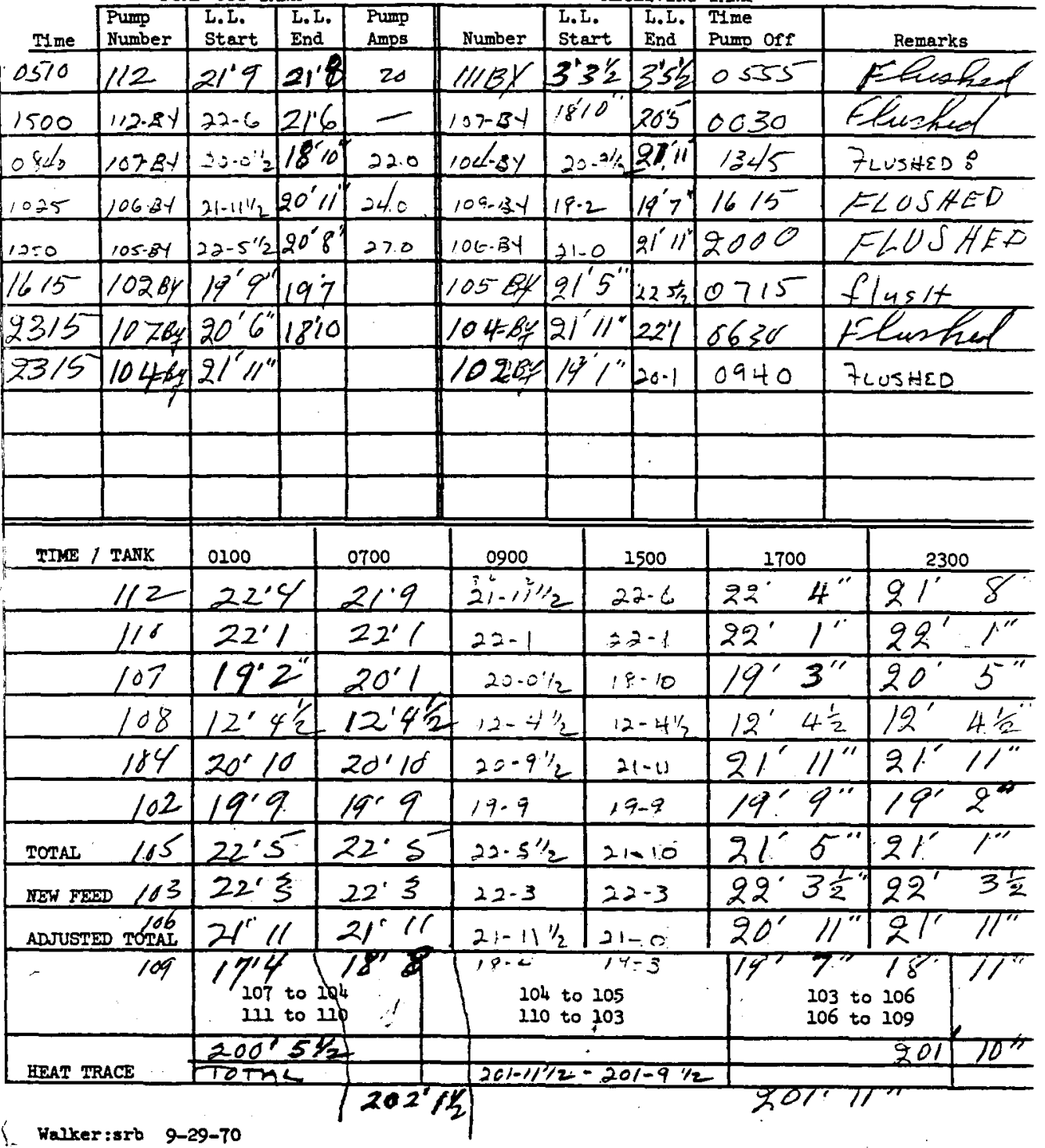


PUMP OUT TANK

RECEIVING TANK

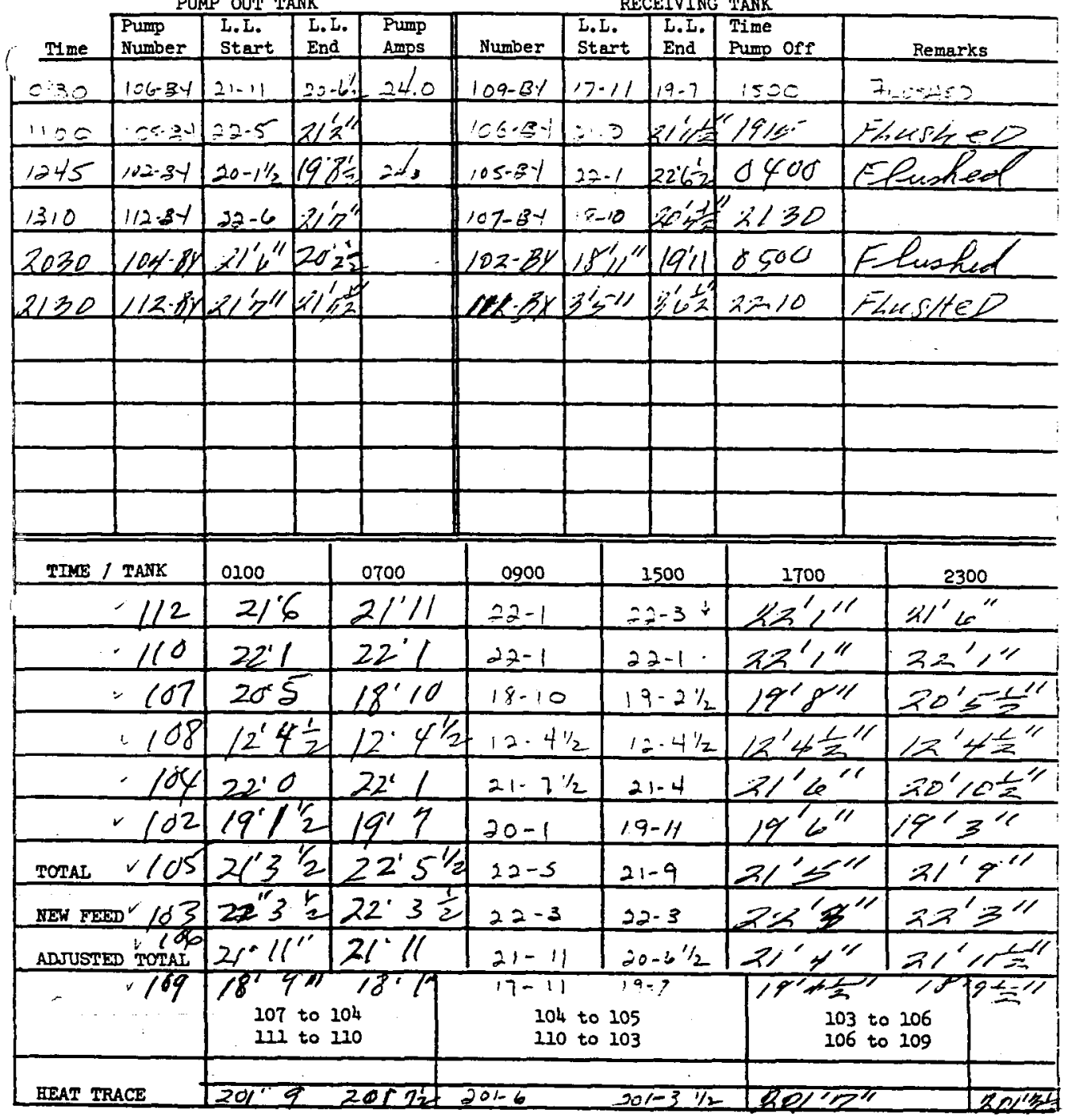

I. Walker:srb 9-29-70 
ITS-2 INVENTORY AND PUMPING

Date $9-36-71$

PUMP OUT TANK

RECEIVING TANK

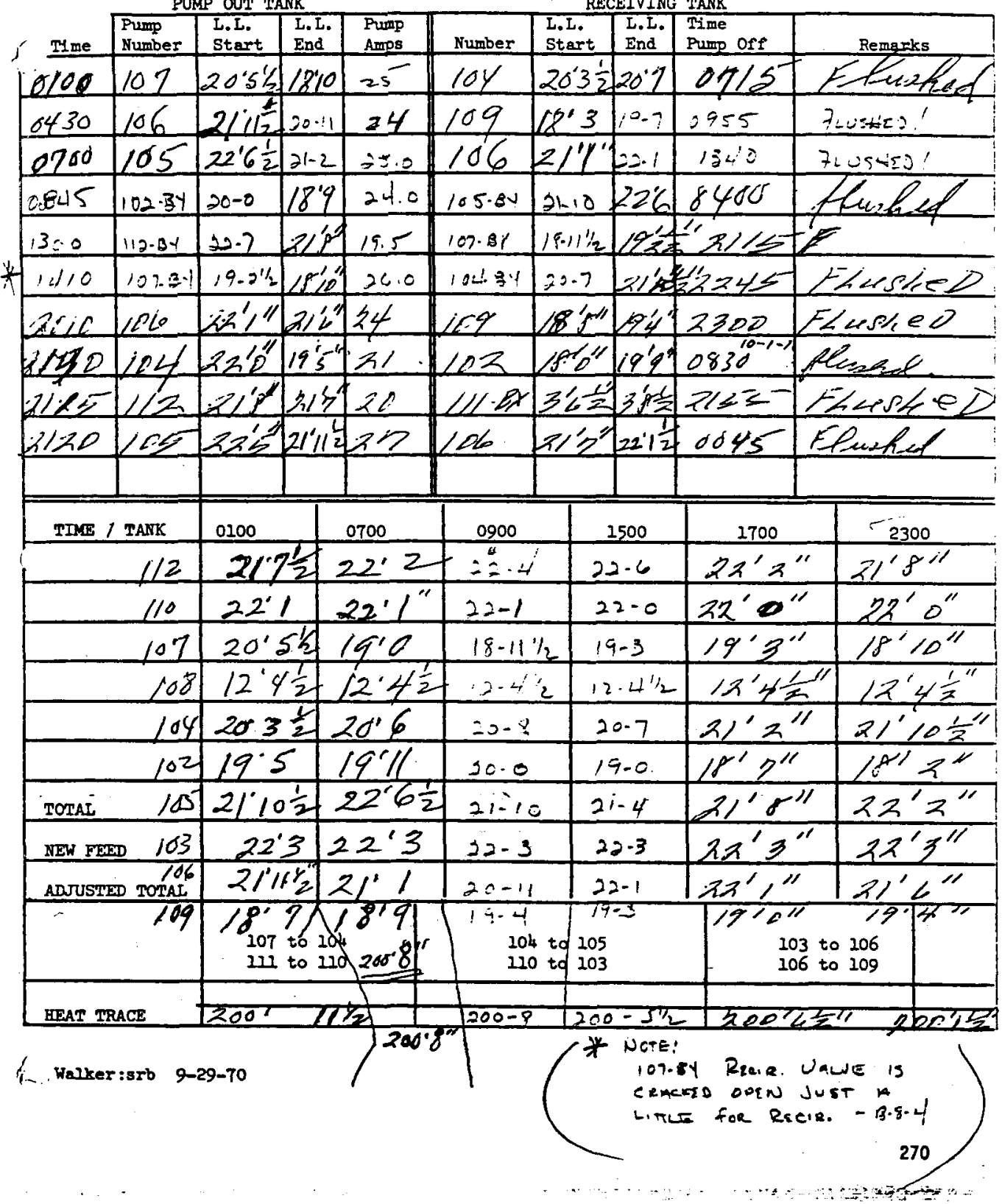


PUNP OUT TANK

RECEIVING TANK

\begin{tabular}{|c|c|c|c|c|c|c|c|c|c|}
\hline Time & $\begin{array}{l}\text { Pump } \\
\text { Number }\end{array}$ & $\begin{array}{l}\text { L.L. } \\
\text { Start }\end{array}$ & $\begin{array}{l}\text { L.L. } \\
\text { End }\end{array}$ & $\begin{array}{l}\text { Pump } \\
\text { Amps }\end{array}$ & Number & $\begin{array}{l}\text { L.L. } \\
\text { Start }\end{array}$ & $\begin{array}{l}\text { L.I. } \\
\text { End }\end{array}$ & $\begin{array}{l}\text { Time } \\
\text { Pump off }\end{array}$ & Remariks \\
\hline 0715 & 112 & 225 & 217 & & 107 & $18 \%$ & 1918 & 1500 & \\
\hline 0715 & 106 & 2719 & $20 \%$ & & 109 & 186 & $19 ' 9$ & 1230 & \\
\hline 1240 & 105 & $2 i 5$ & $\left.2 e^{\prime}\right|^{\prime}$ & & 186 & $20^{\prime} \prime^{\prime \prime}$ & 运程 & 1900 & FHUSHEL \\
\hline 1250 & 107 & $19^{\prime} 11^{\prime \prime}$ & 189 & & $\cos$ & $11^{\prime} 4^{\prime \prime}$ & 213侳 & -1906 & FhasheD \\
\hline 1430 & 102 & 1911 & & & 105 & 411 & & CENT. T & $10-2.71$ \\
\hline KSOD & $i 1 z$ & $2 x^{\prime} 4$ 年 & & 20 & 102 & $15^{\prime \prime} q^{\prime \prime}$ & & Cont. Th & $70-2-71$ \\
\hline & & & & & & & & & \\
\hline & & & & & & & & & \\
\hline & & & & & & & & & \\
\hline & & & & & & & & & \\
\hline
\end{tabular}

TIME / TANK

\begin{tabular}{l|l|l|ll|c|c}
112 & 0200 & 0700 & 0900 & 1500 & 1700 \\
\hline 19 & $22^{\prime} 4 \frac{1}{2}$ & 22 & 4 & 217 & $2^{\prime} 9^{\prime}{ }^{\prime \prime}$
\end{tabular}

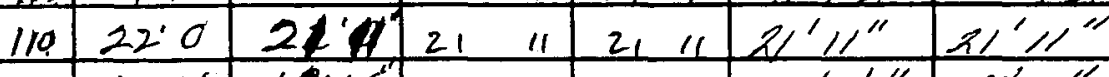

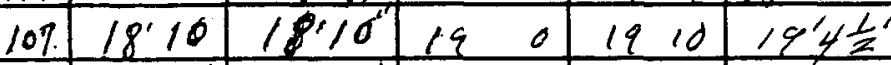

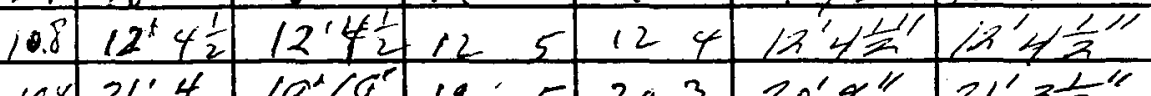

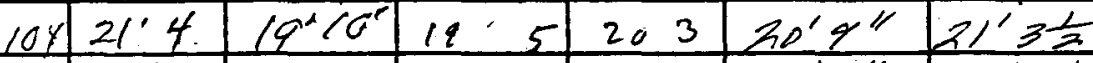

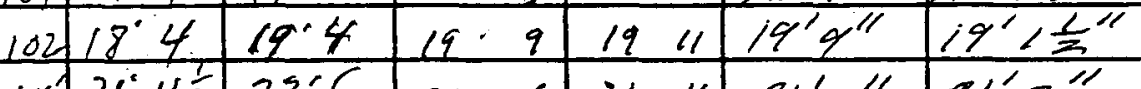

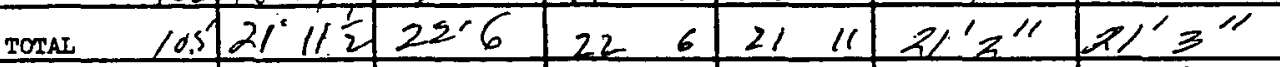

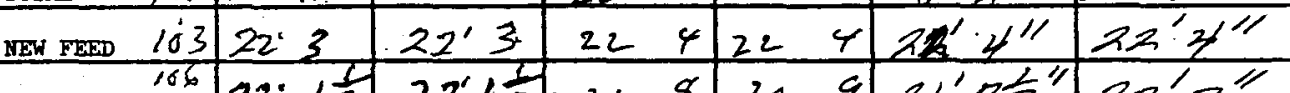

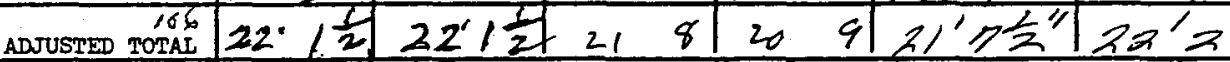

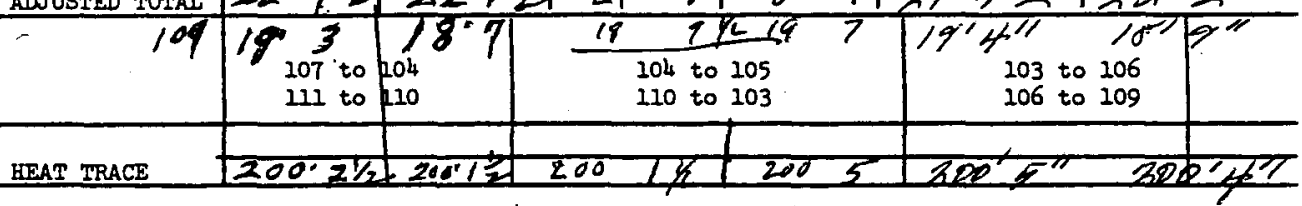

Walker:srb 9-29-70 
WHC-SD-WM-DP-209, ReV. O

ITS-2 INVENTORY AND PUMPING

Date

$10-2.71$

PUMP OUI TANK

RECEIVING TANK

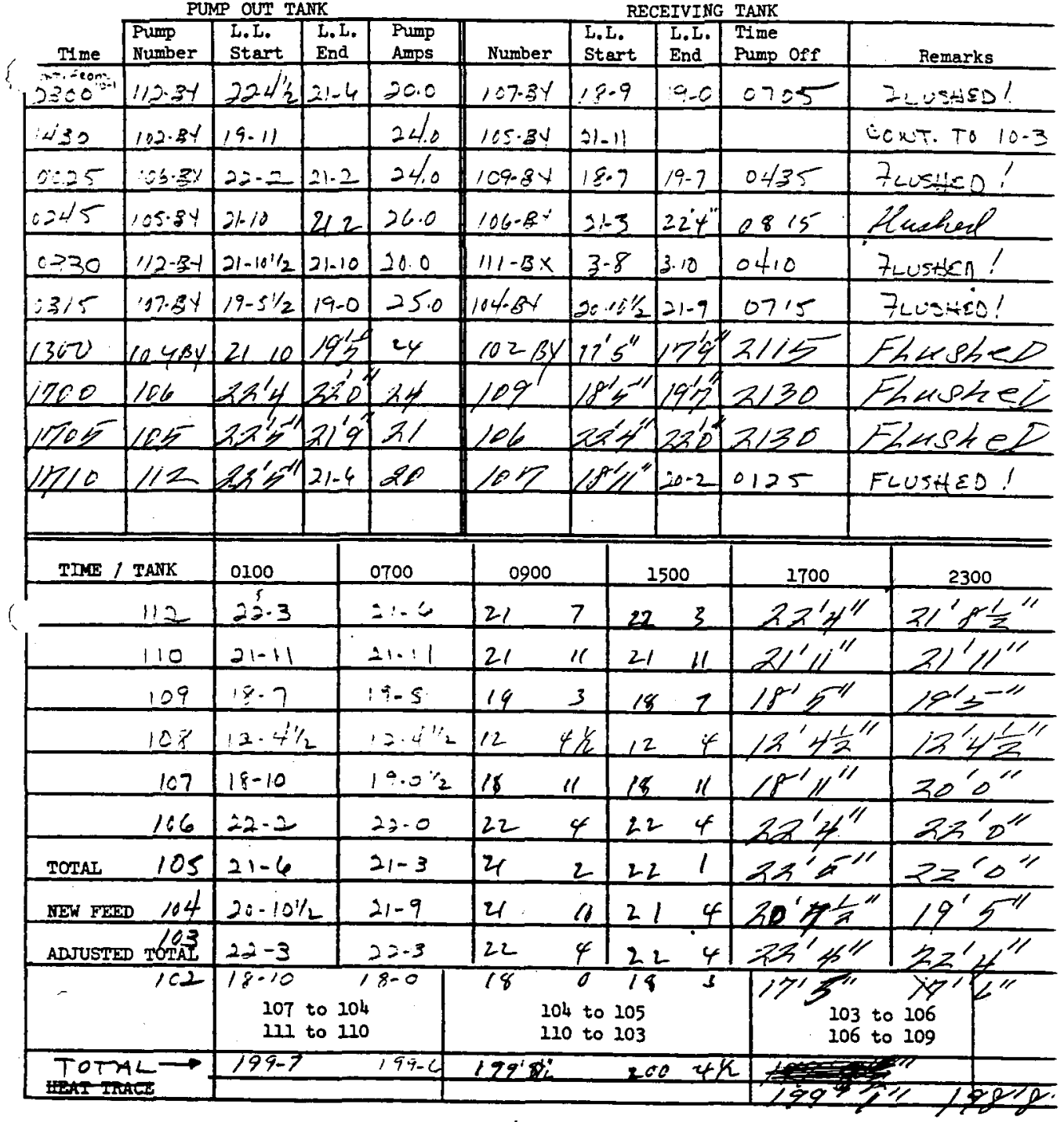

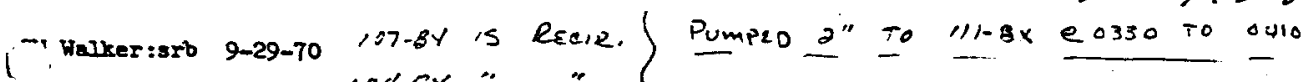
$1046 \mathrm{EY}$ $00700 \quad 5.12 \cdot 8$

272

$1.9 \sigma^{\prime} 8$ 
WHC-SD-WM-DP-209, Rev. 0

ITS-2 INVENTORY AND PUMPING

Date

$10-3.71$

PUMP OUT TANK

RECEIVING TANK

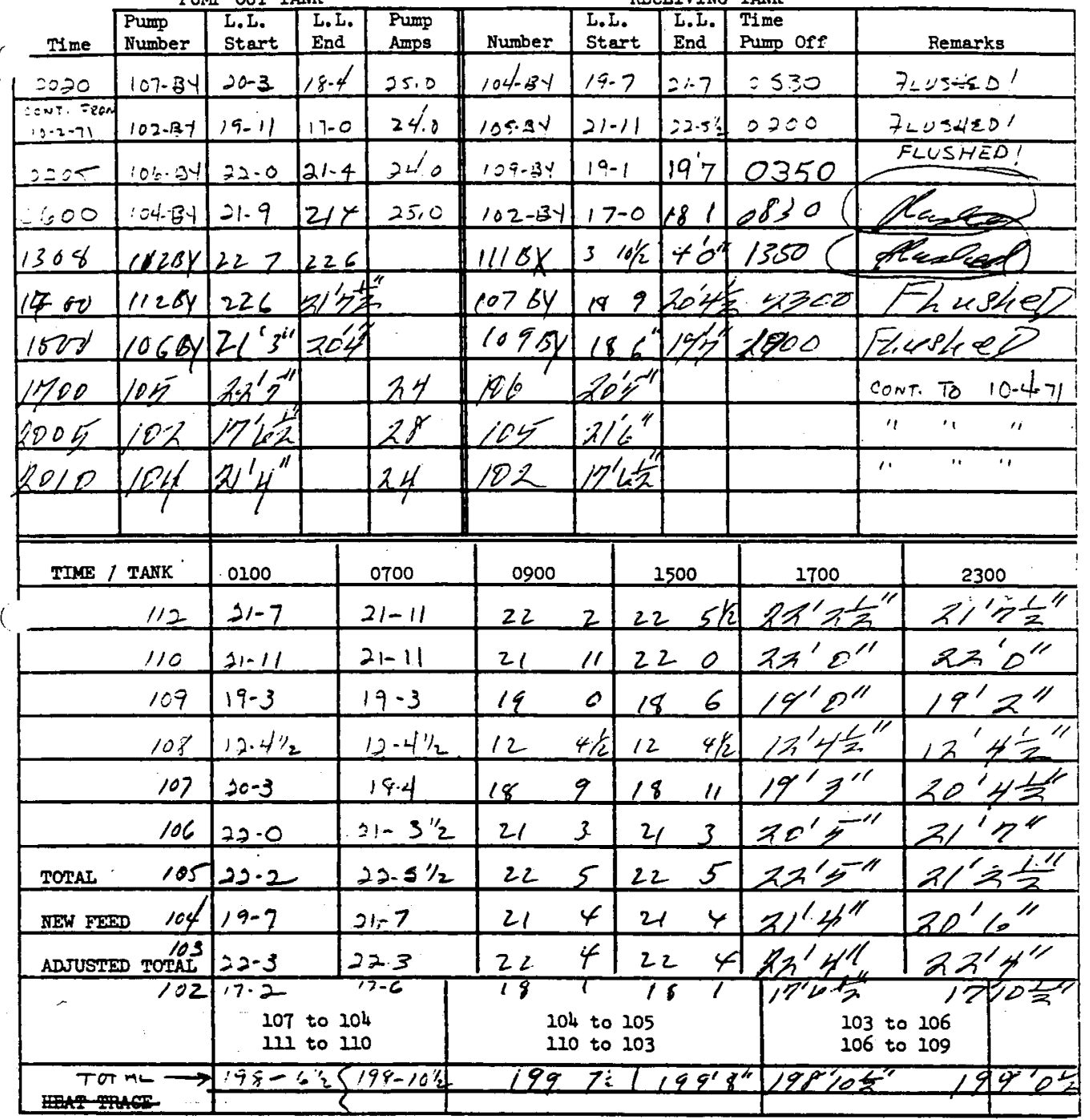

- Walker:srb 9-29-70

107.84 is RE=IR. 00780 
WHC-SO-WM-DP-209, Rev. O

ITS-2 INVENTORY AND PUMPING Date $10-4671$

PUMP OUT TANK

RECEIVING TANK

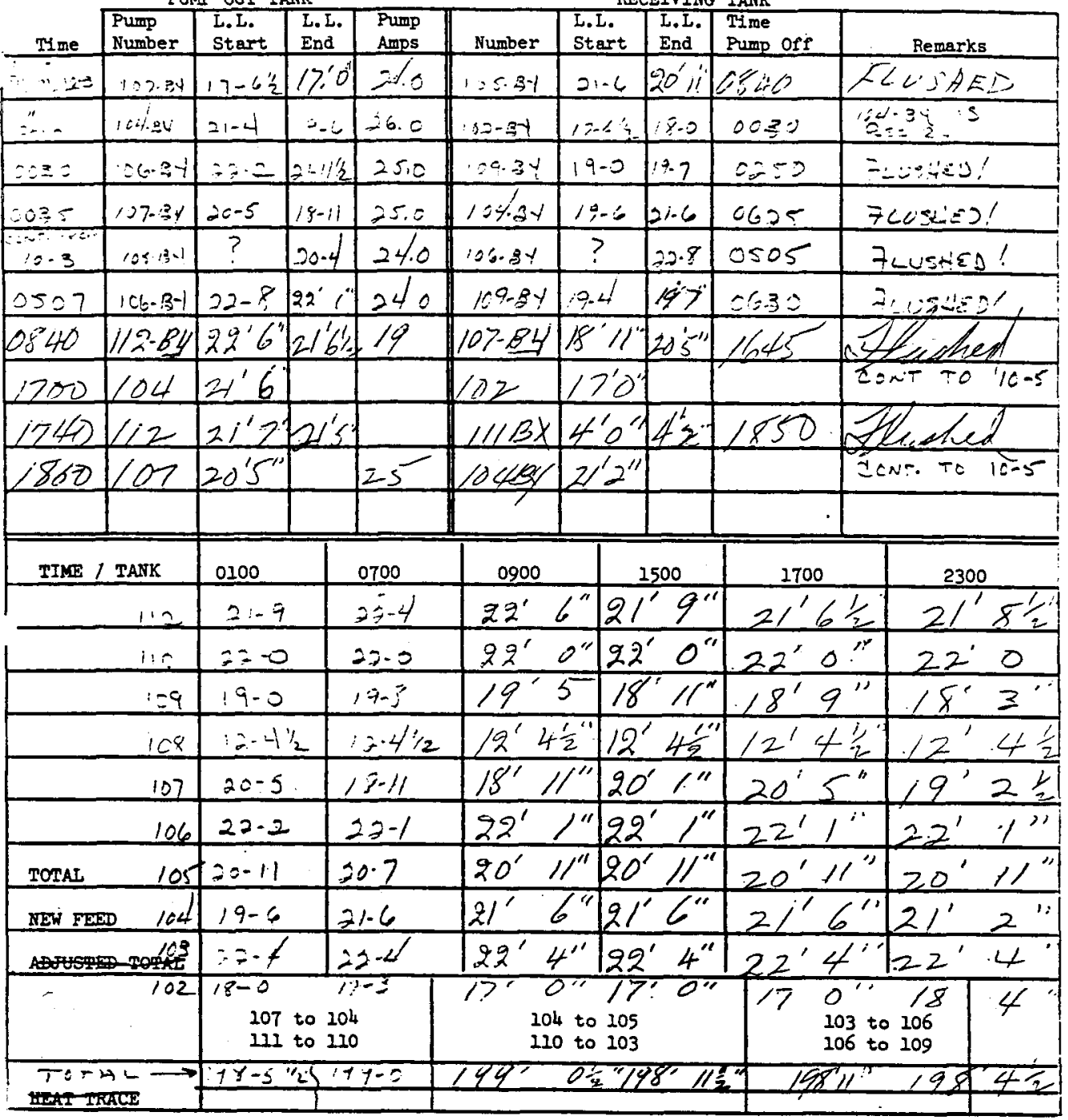

, W Walker:srb 9-29-70 
WHC-SD-WM-DP-2OQ, REV. O

ITS-2 INVEYTORY AND PUMPING Date $|t \cdot 5-1|$

PUMP OUT TANK

RECEIVING TANK

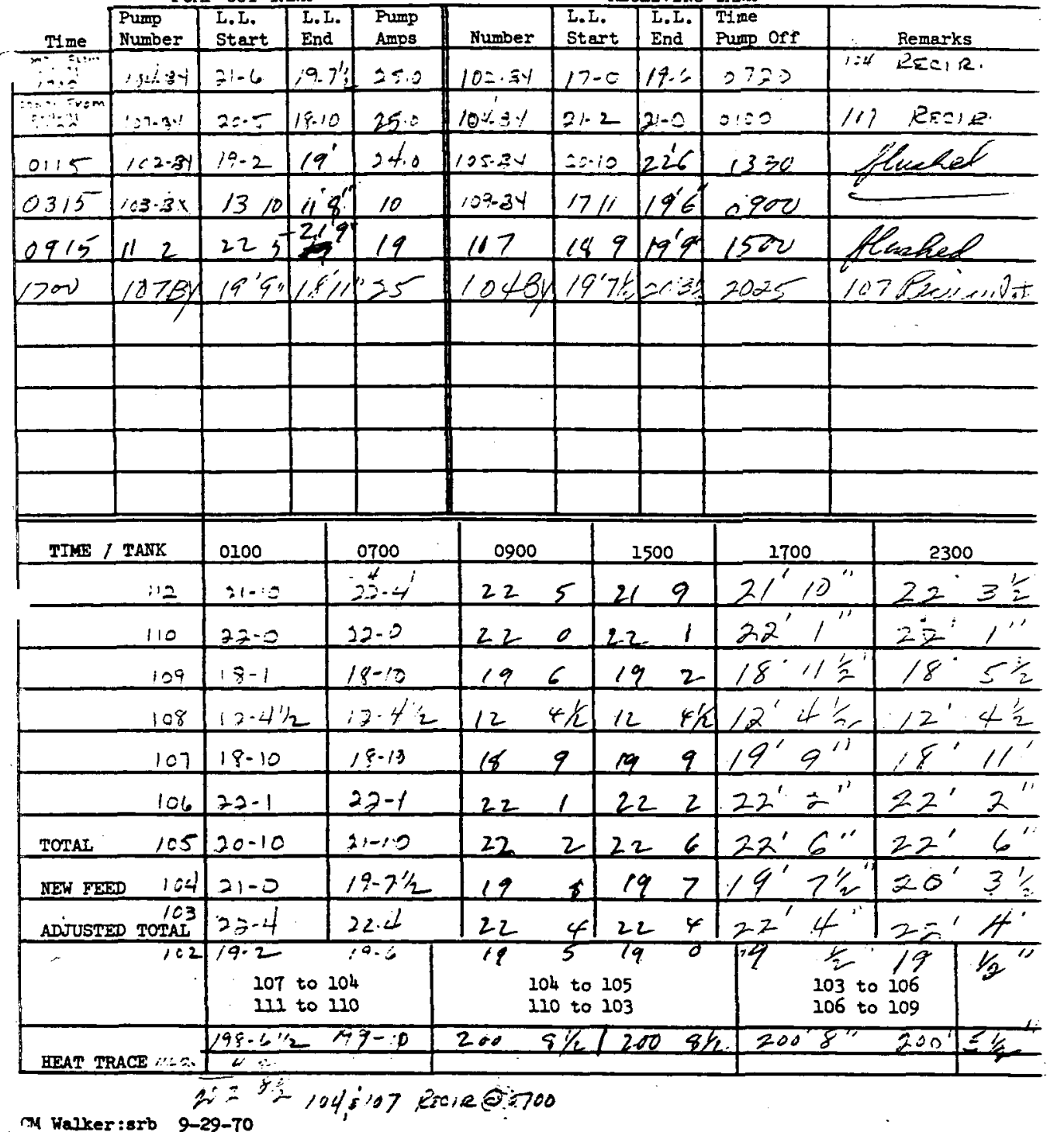


WHC-SD-LAM-DP-209, ReV. 0

ITS-2 INVENTORY AND PUMPING

Date $10-6.71$

PUMP OUT TANK

RECEIVING TANK

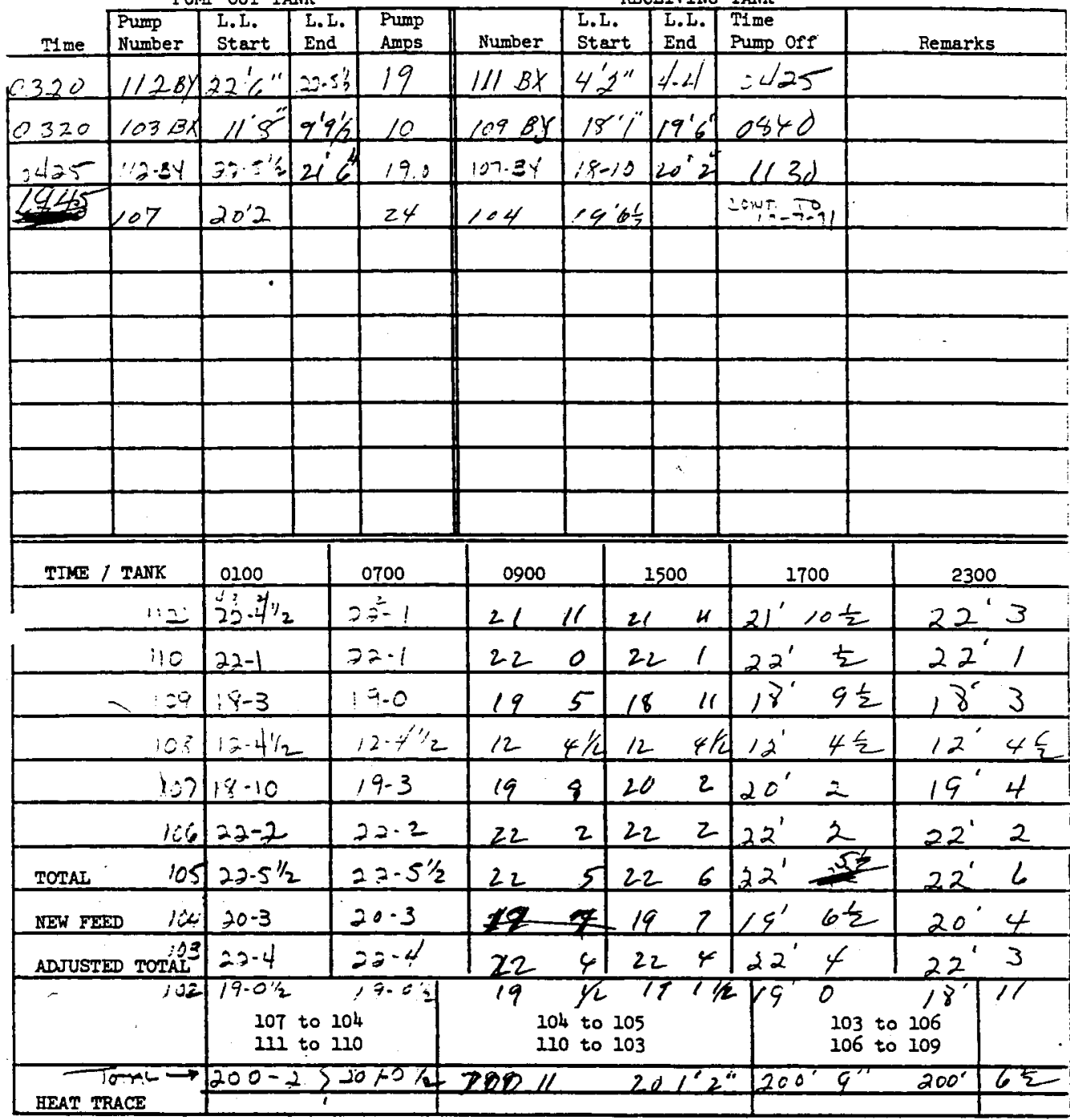

M Walker:srb 9-29-70 
PUMP OUT TANK

RECEIVING TANK

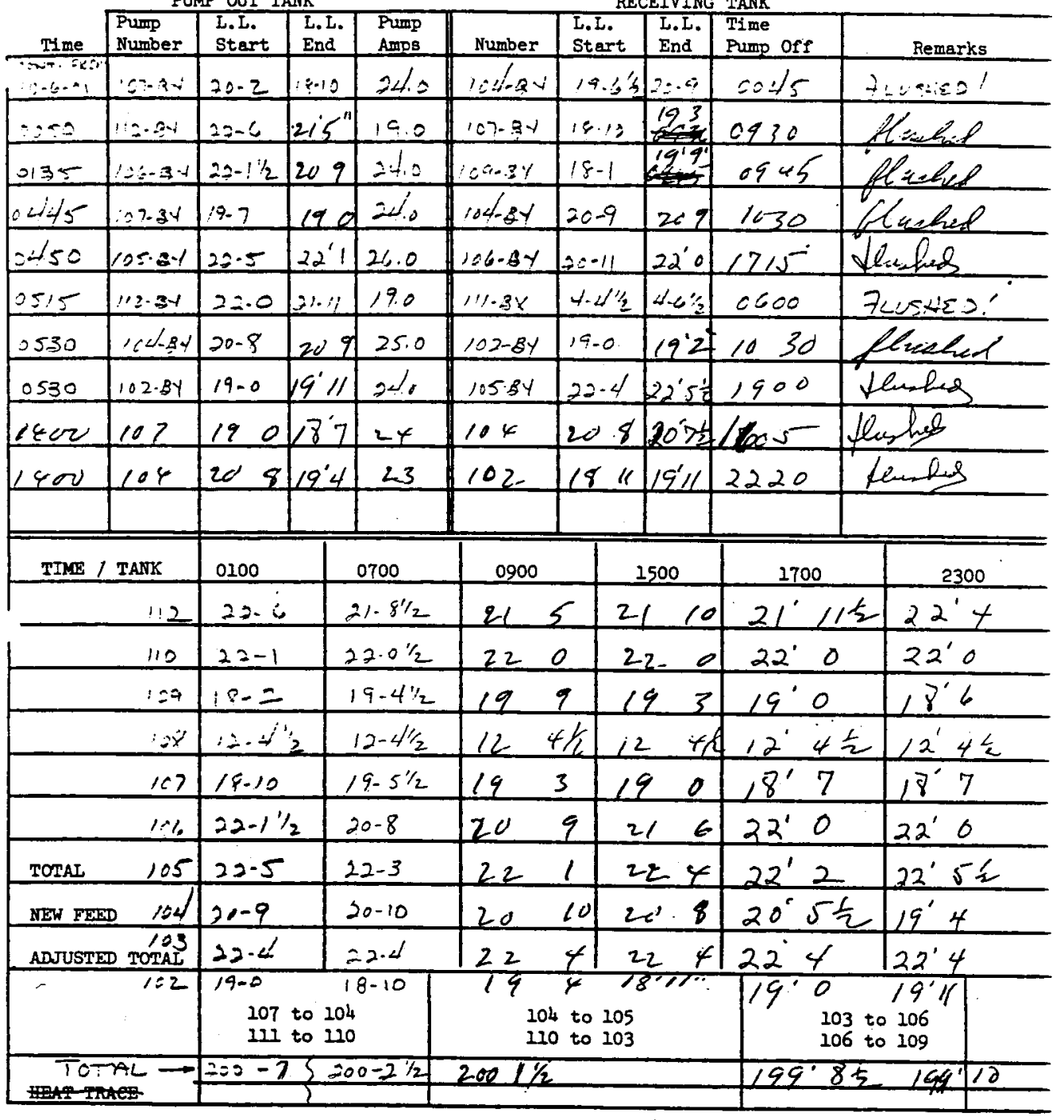

Pumpes 2" $1 / 2.8 y-111-8 x$

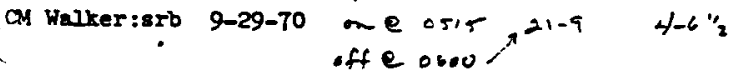


Date $-16-5-71$

PUMP OUT TANK

RECEIVING TANK

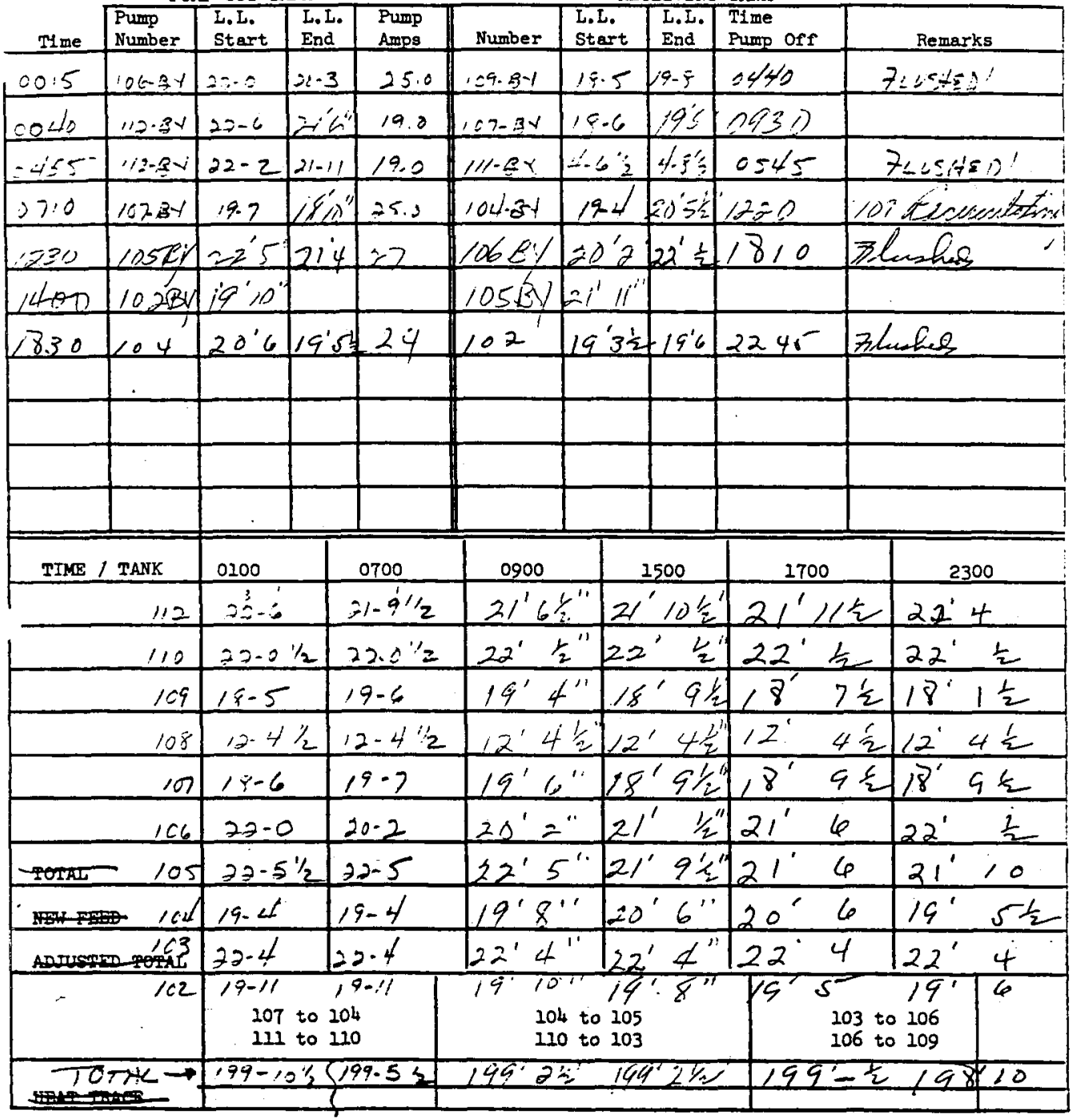

$\mathrm{m}$ Walker:srb 9-29-70 
WHC-SD-WM-DP-209/ReV. O

ITS-2 INVENTORY AND PUMPING

Date $109-71$

PUMP OUT TANK

RECEIVING TANK

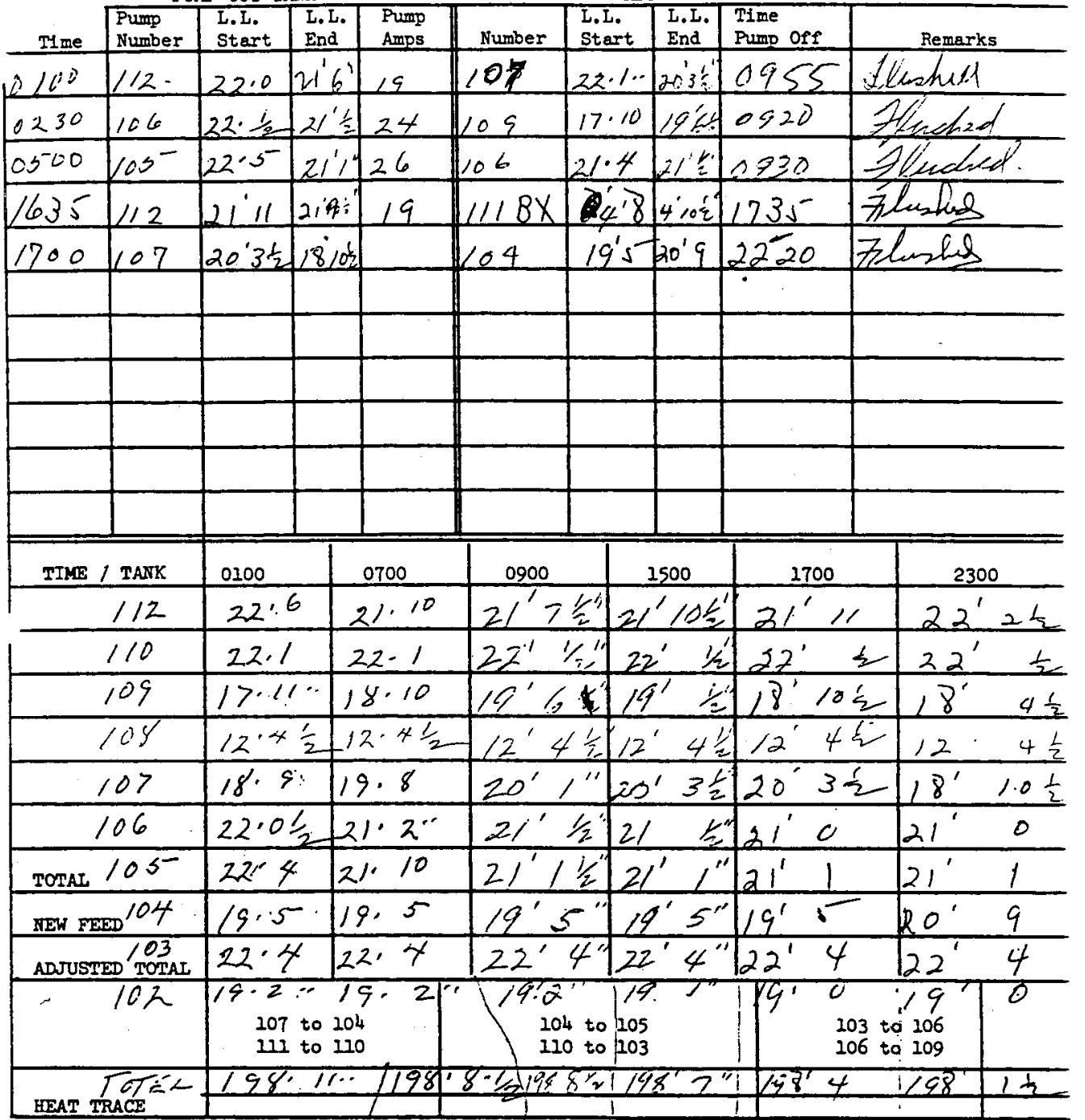

M Walker:srb 9-29-70 
WHC-SD-WM-DP-209, Rev, 0

ITS-2 INVENTORY AND PUMPING

Date $1210>1$ PUMP OUT TANK

RECEIVING TANK

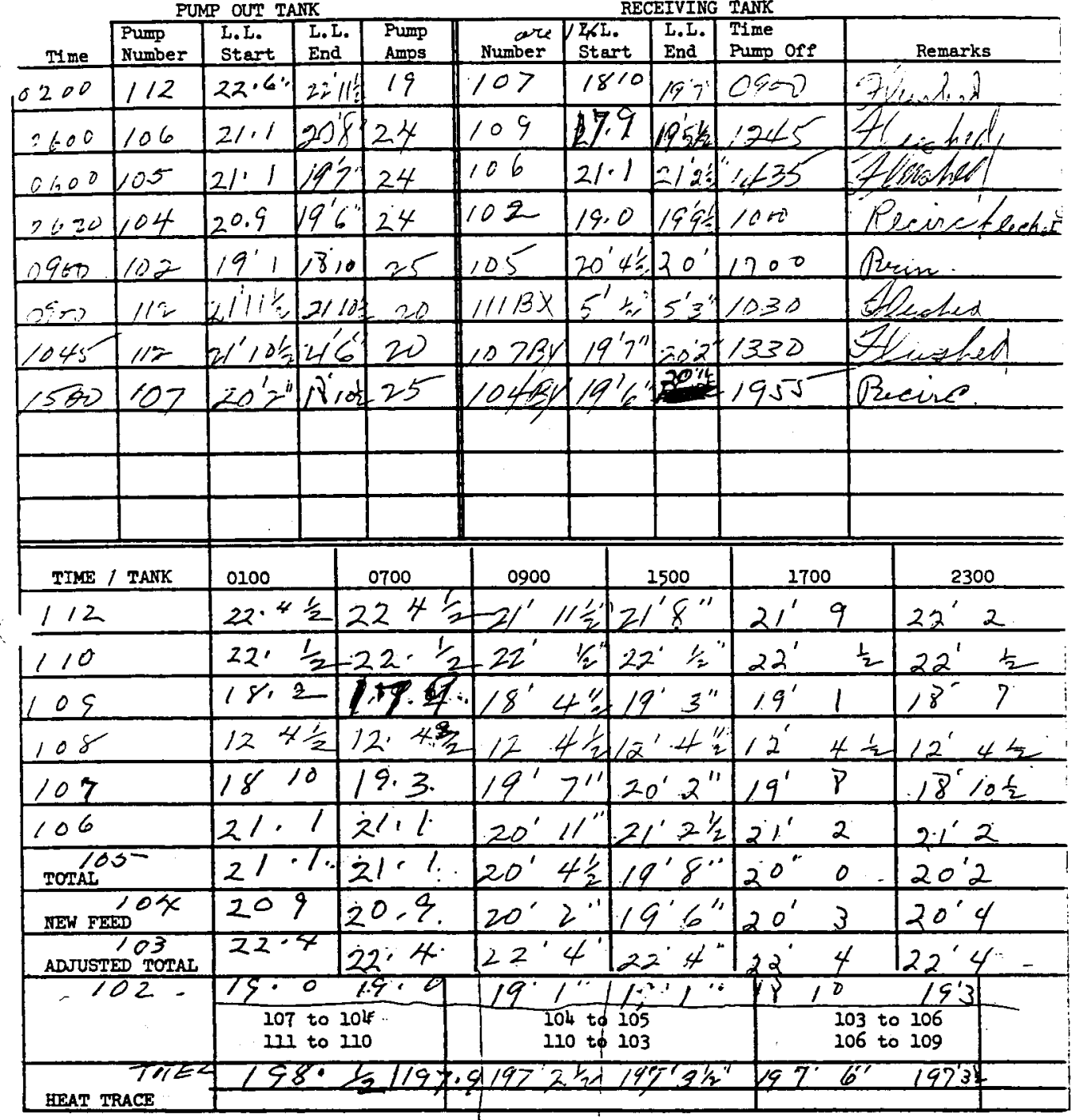

Wauker:srb 9-29-70

280 


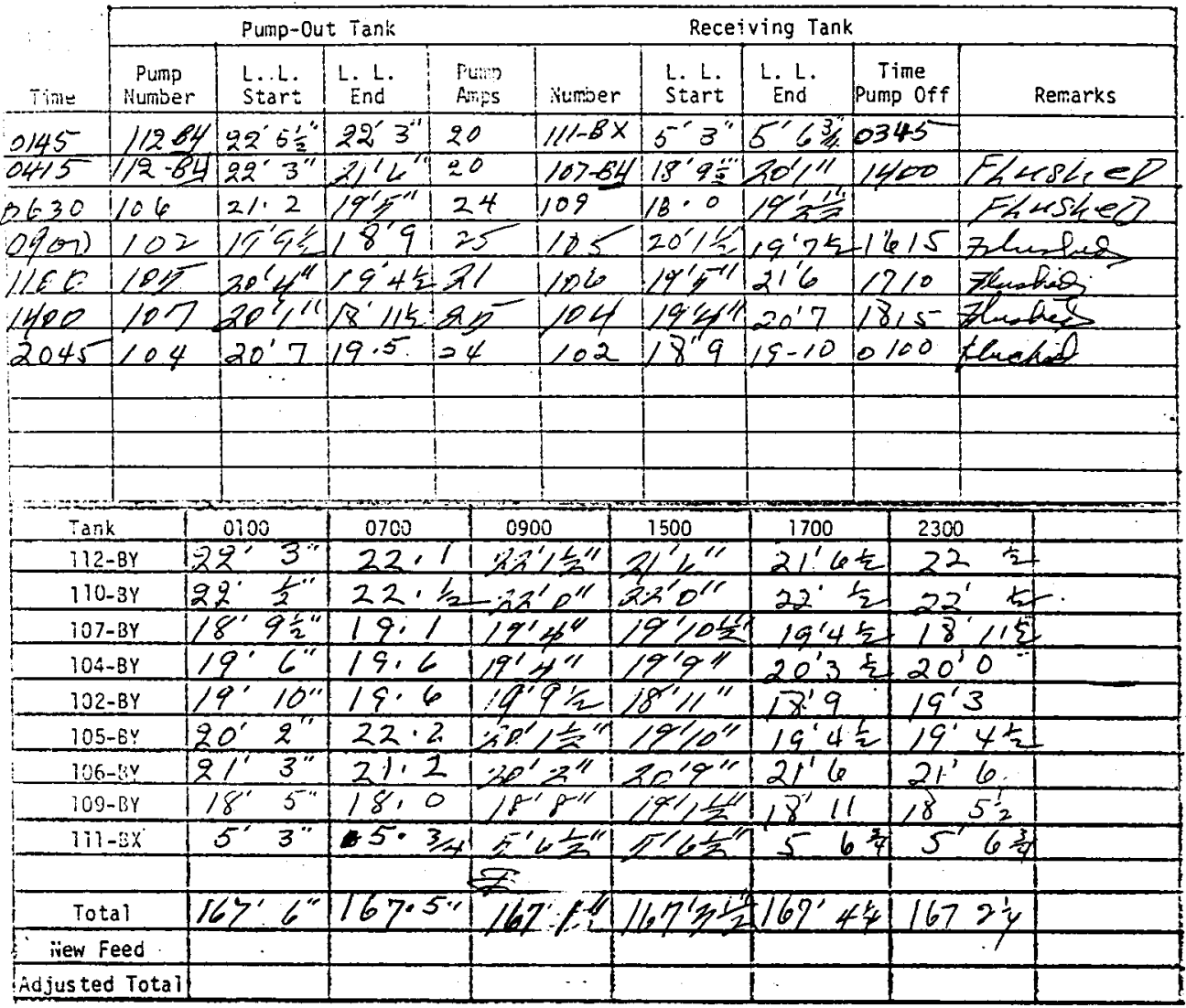

INACTIVE TANKS

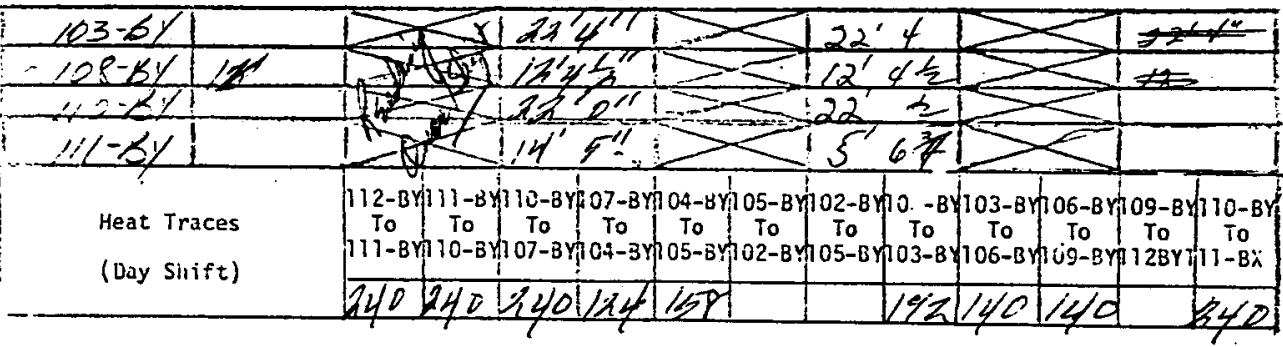




\section{ump-Out Tank Receiving Tank}

\begin{tabular}{|c|c|c|c|c|c|c|c|c|c|}
\hline \multirow[b]{2}{*}{ inte } & \multicolumn{4}{|c|}{ Pump-Out Tank } & \multicolumn{4}{|c|}{ Receiving Tank } & \multirow[b]{2}{*}{ Remarks } \\
\hline & $\begin{array}{c}\text { Pump } \\
\text { Number }\end{array}$ & $\begin{array}{l}\text { L..L. } \\
\text { Start }\end{array}$ & L. L. & $\begin{array}{l}\text { Plilo? } \\
\text { Alips }\end{array}$ & irumber & $\begin{array}{l}\text { L. L. } \\
\text { Start }\end{array}$ & L. L. & $\begin{array}{l}\text { Time } \\
\text { Pump off }\end{array}$ & \\
\hline 33 & 172 & $225^{2}$ & $21 / 2 \frac{2 x}{3}$ & 19 & 402 & 1811 & Best & $1 / 500$ & FhasheD \\
\hline
\end{tabular}

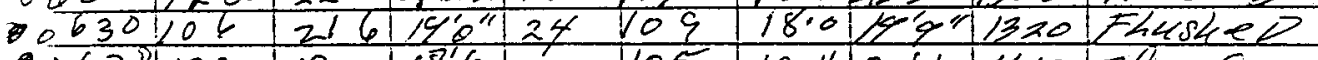

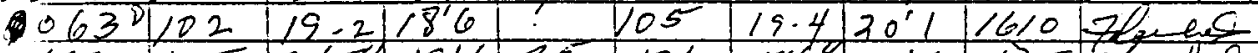

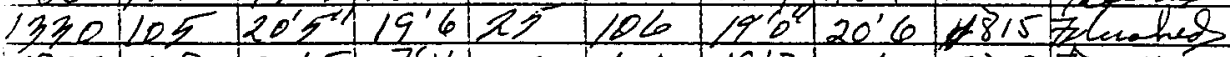

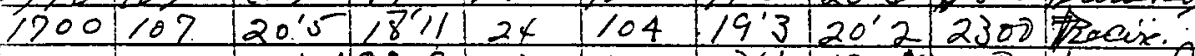

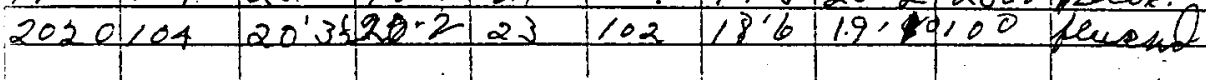

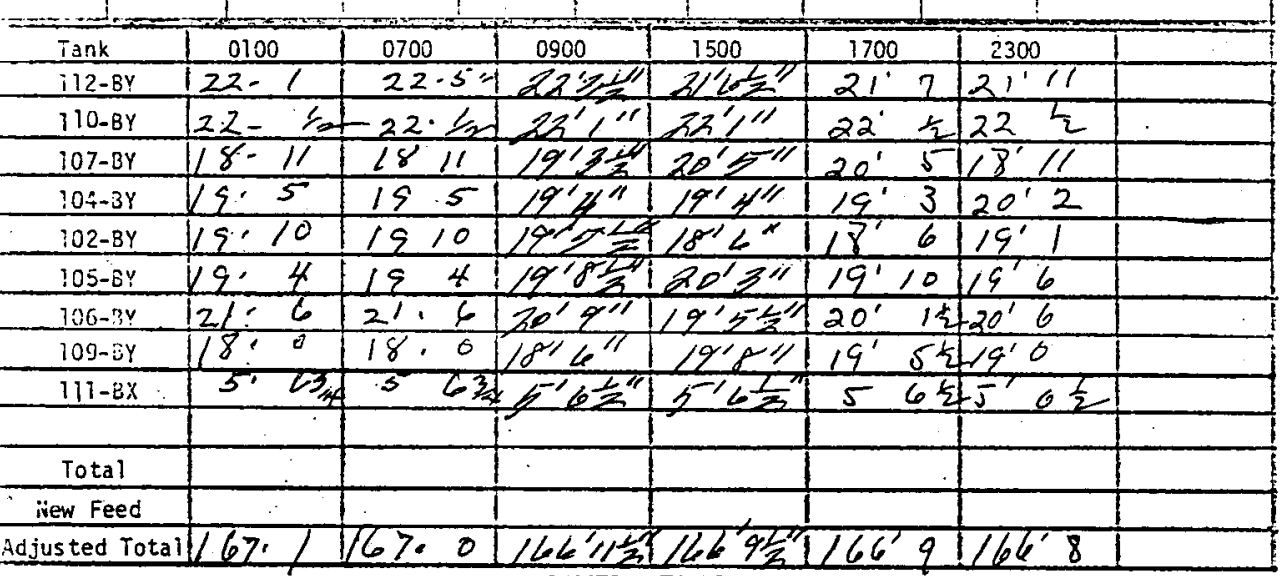

$\frac{103}{108}-\frac{172^{\prime \prime}}{1 / 2^{\prime} 4^{\prime \prime}}$

Heat Traces

(Day Shift)

\section{INACTIVE TANKS \\ INACTIVE TANKS}

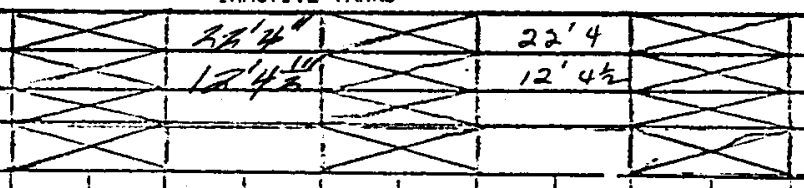

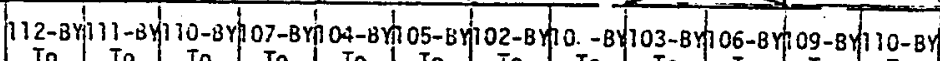

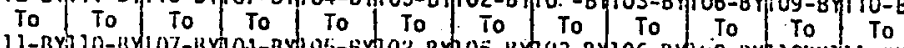

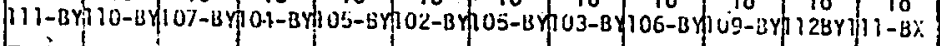

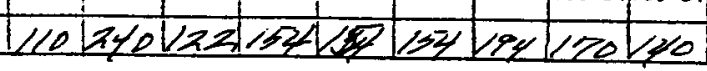
10 
IIS-2 INVENTORY ANO PUMPING

Date $10-13-71$

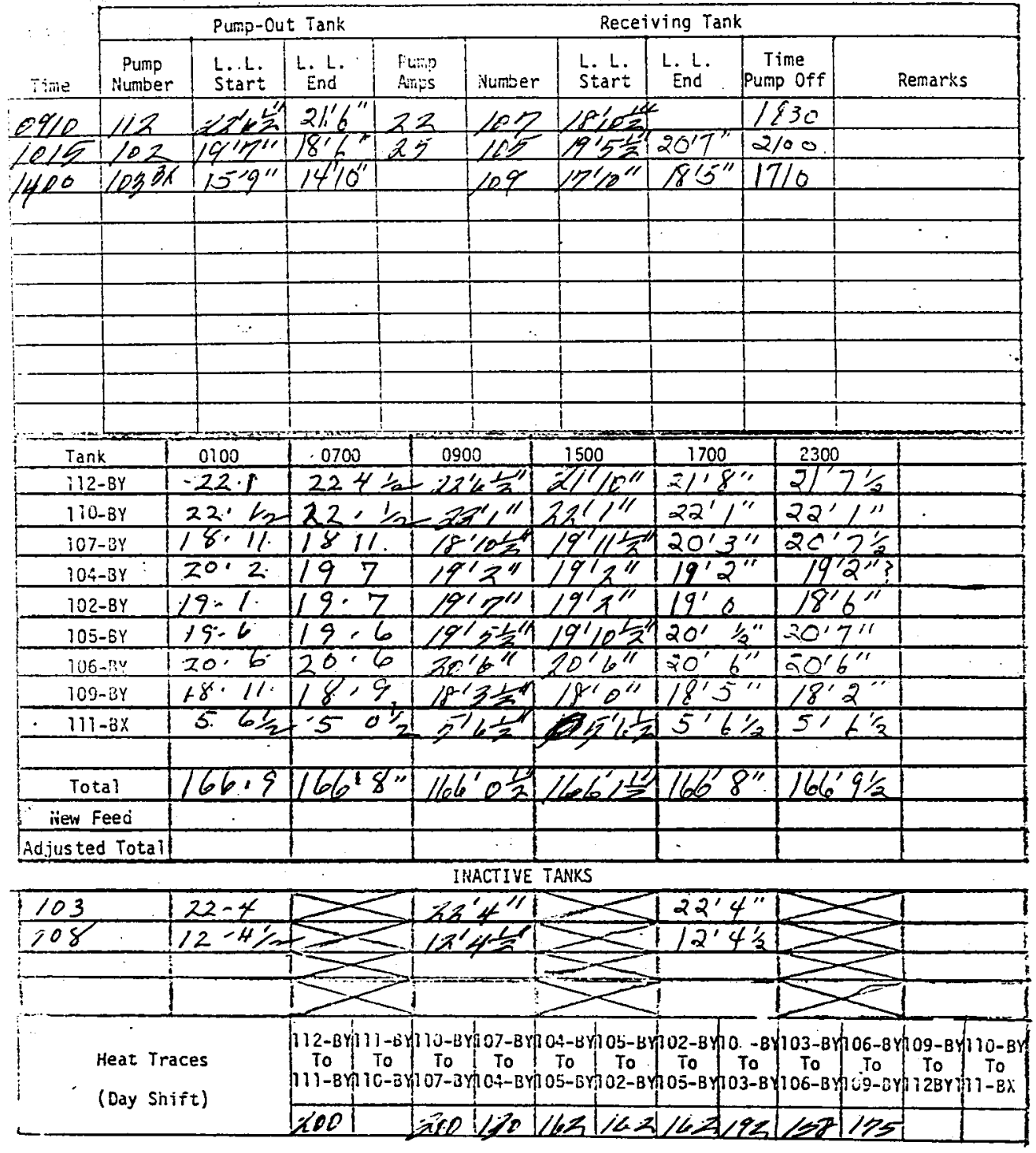


七82

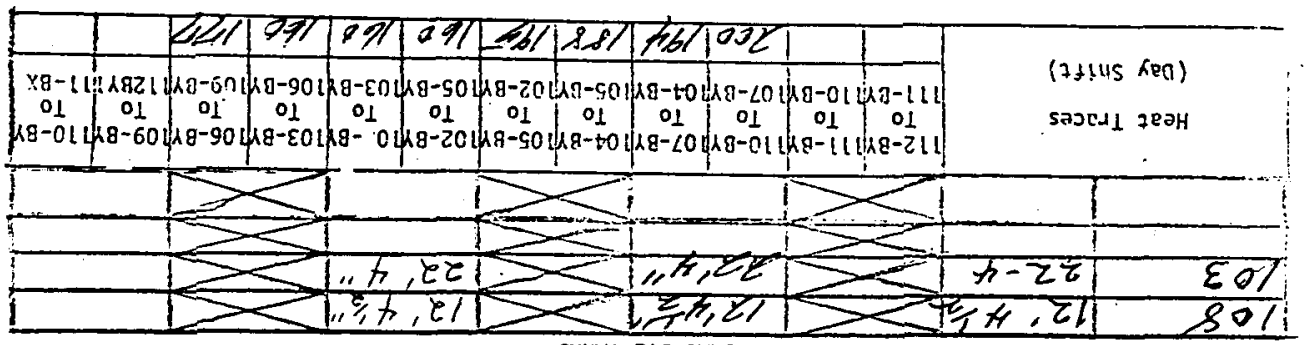

SXNVI 3 \ILJYNI

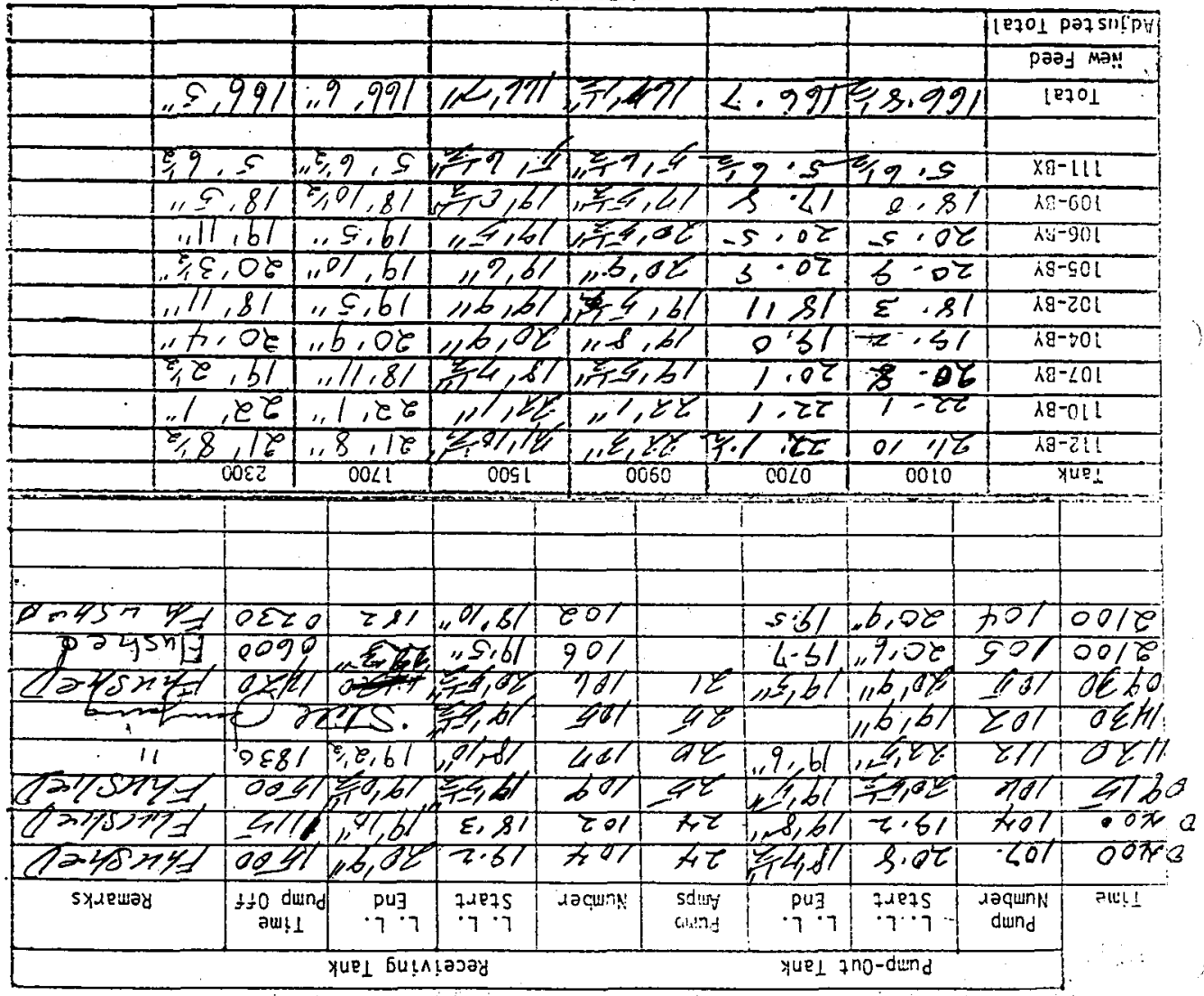


ITS-2 INVENTORY AIUD PUAPING

Date $10 \cdot 13^{2}>5$

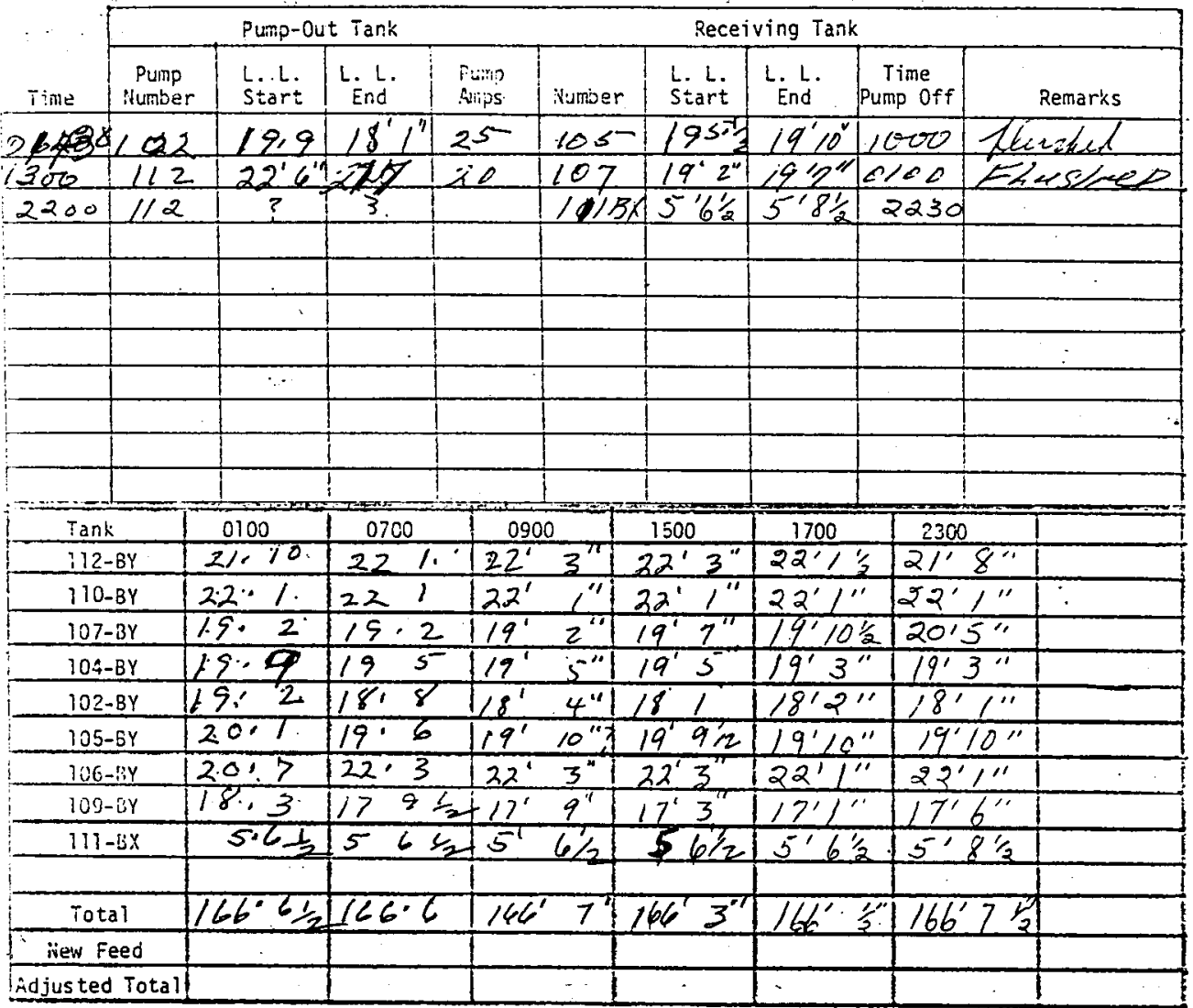

INACTIVE TANKS

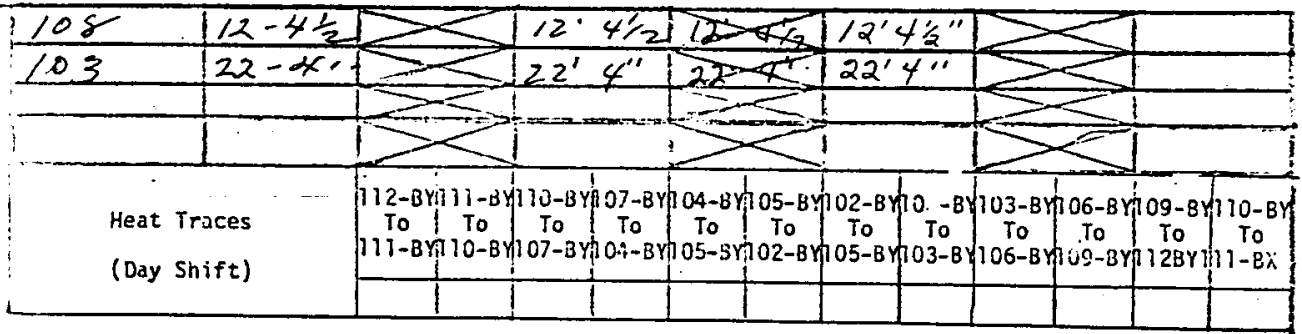




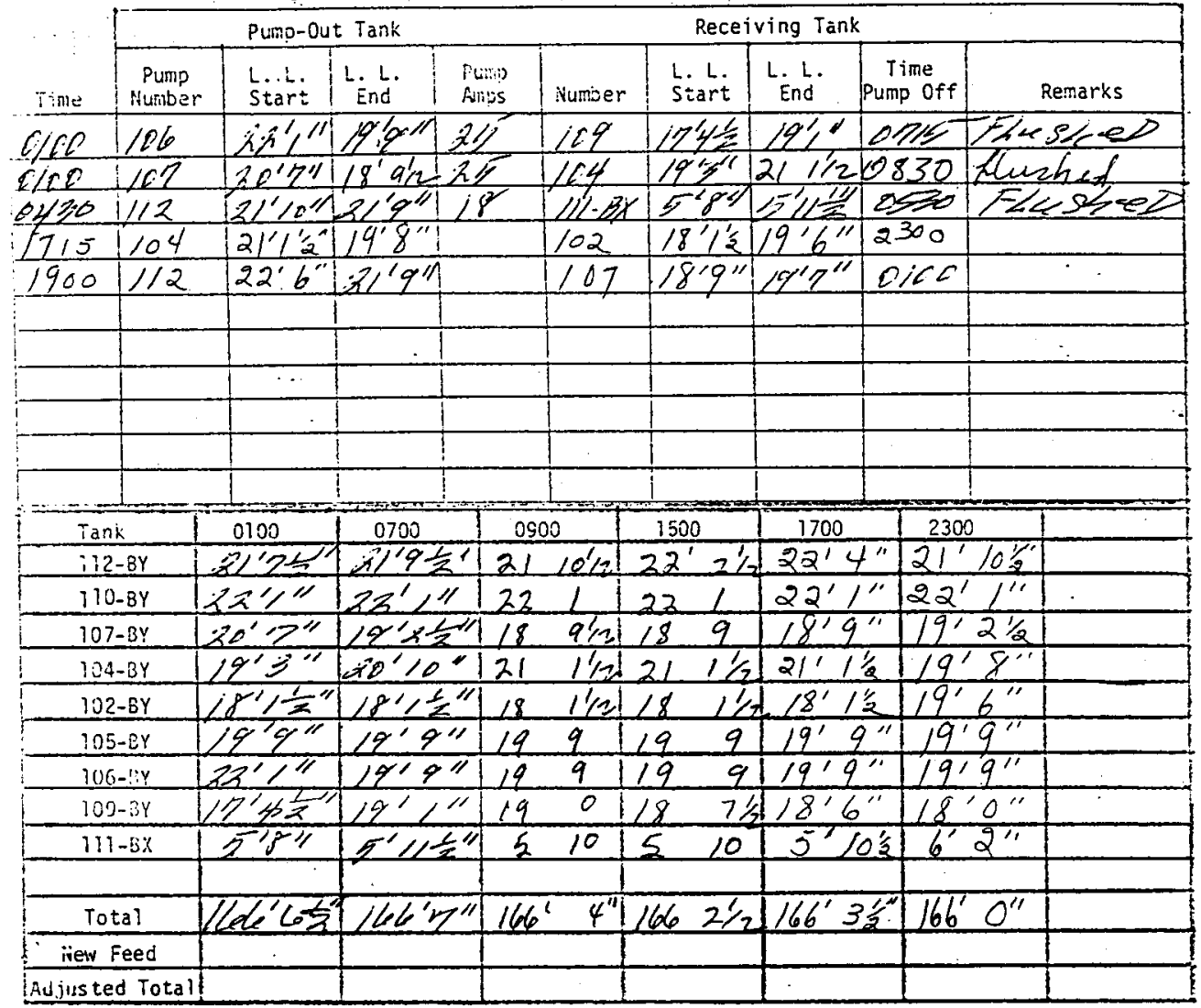

\section{INACTIVE TANKS}

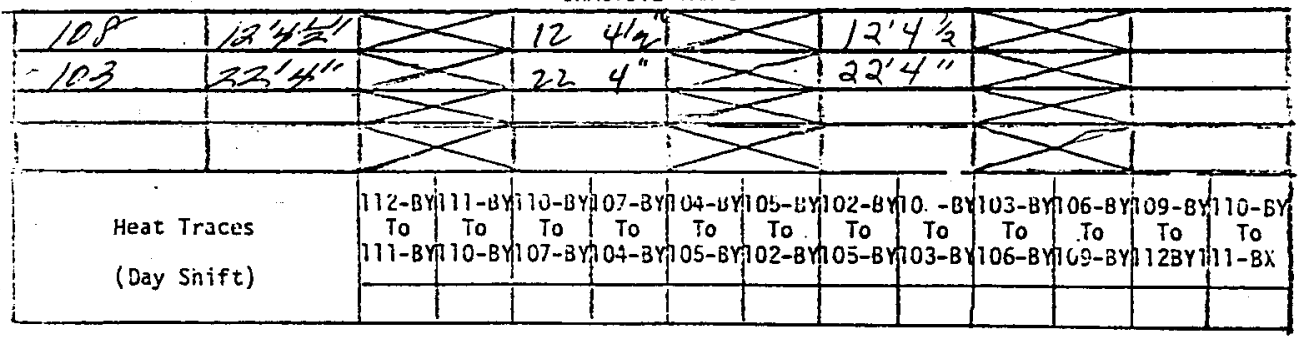


IIS-2 INVENTORY ANU PURPING

Date $10-1 P-P$

\section{:-i. : ITS IN}

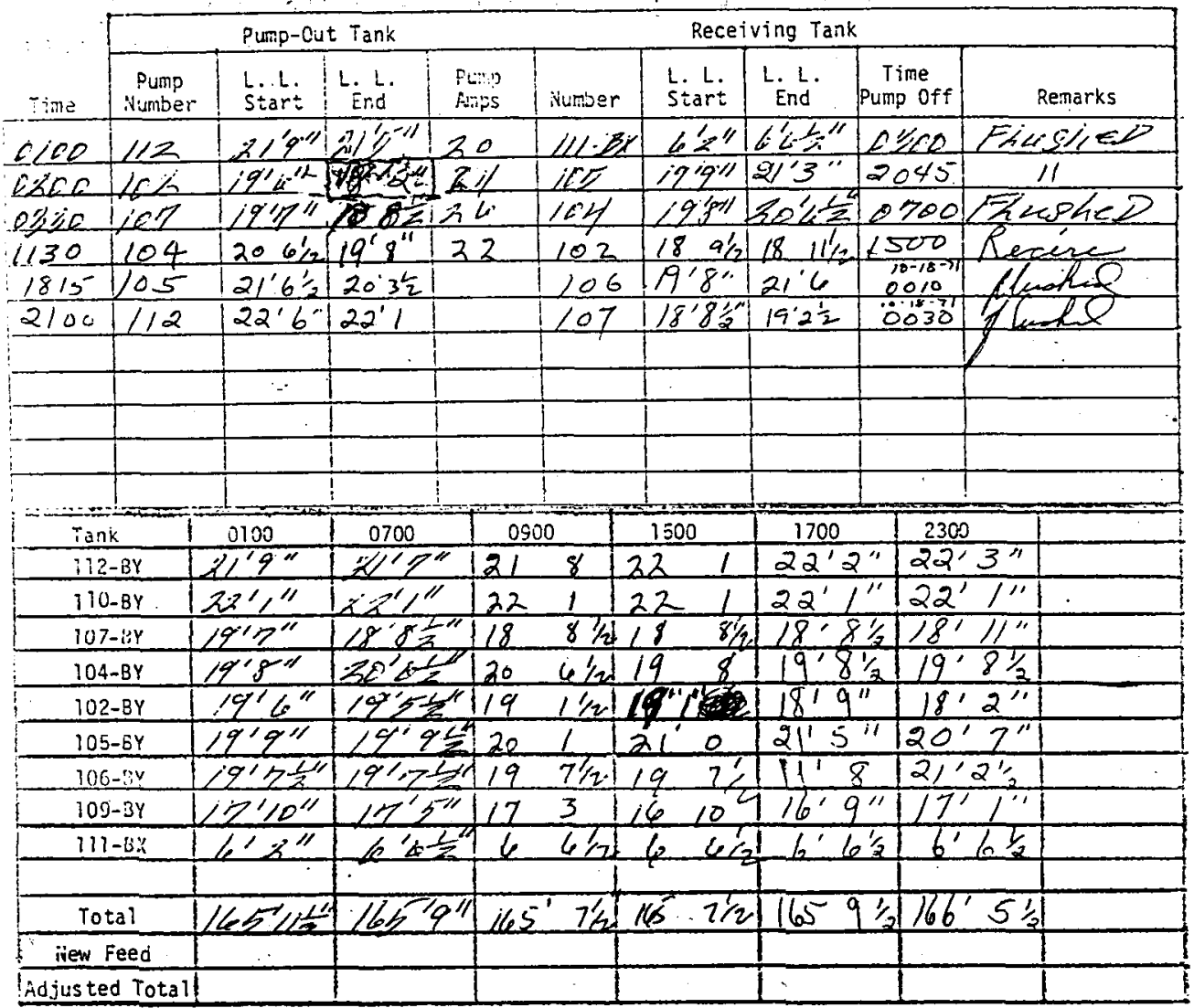

\section{INACTIVE TANKS}

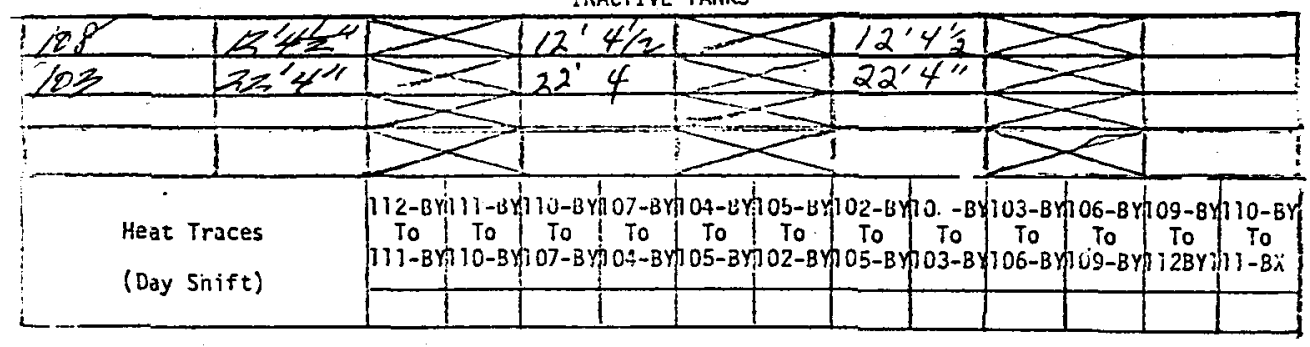

Heat Traces

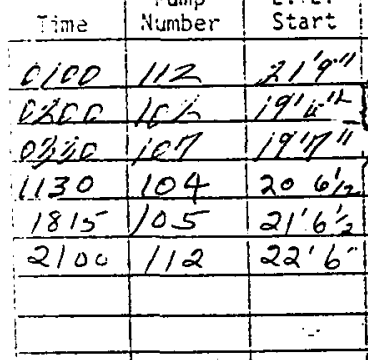


ITS-2 IAIVENTORY ANO PUNPING

Date $10-18-71$

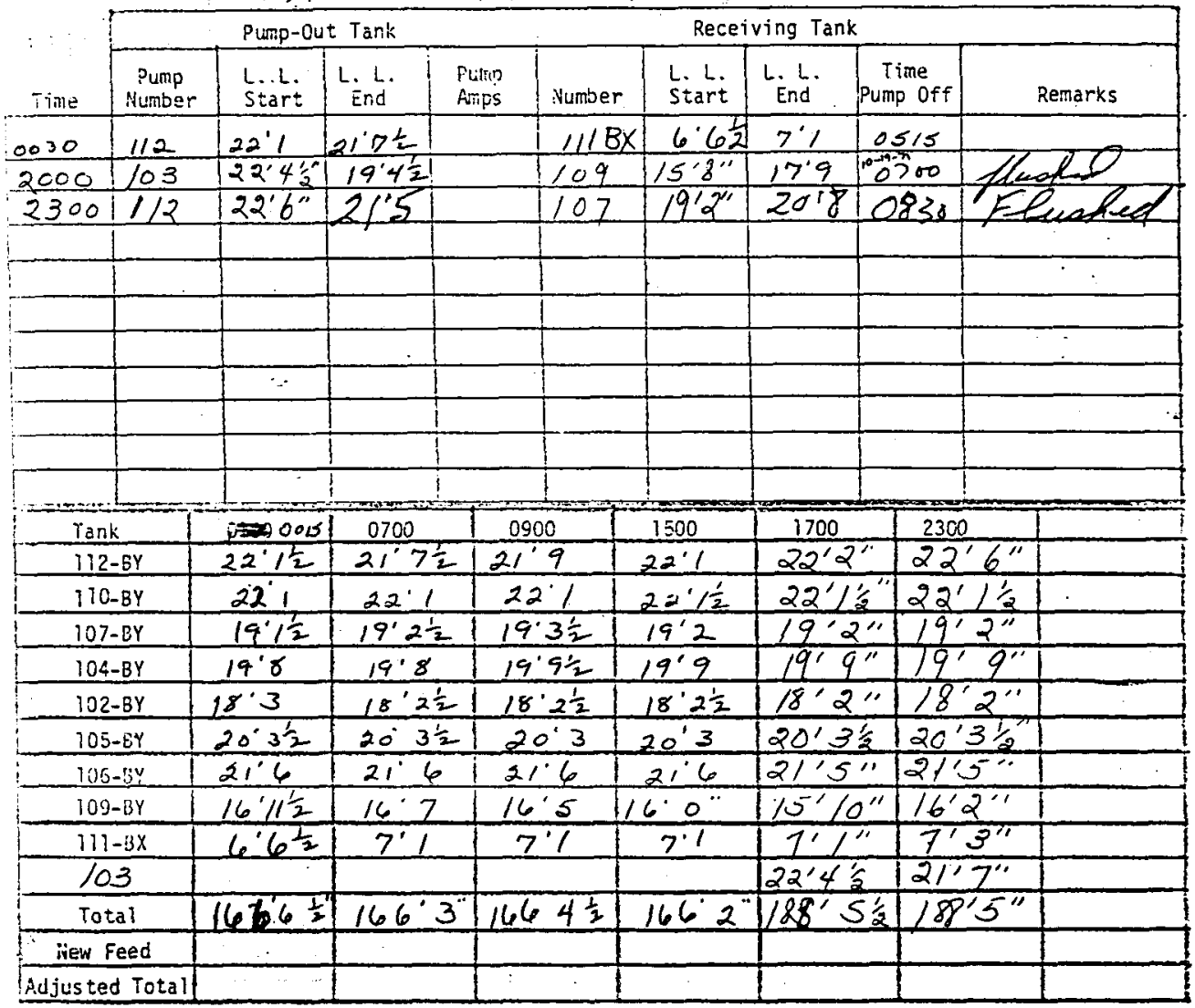

INACTIVE TANKS

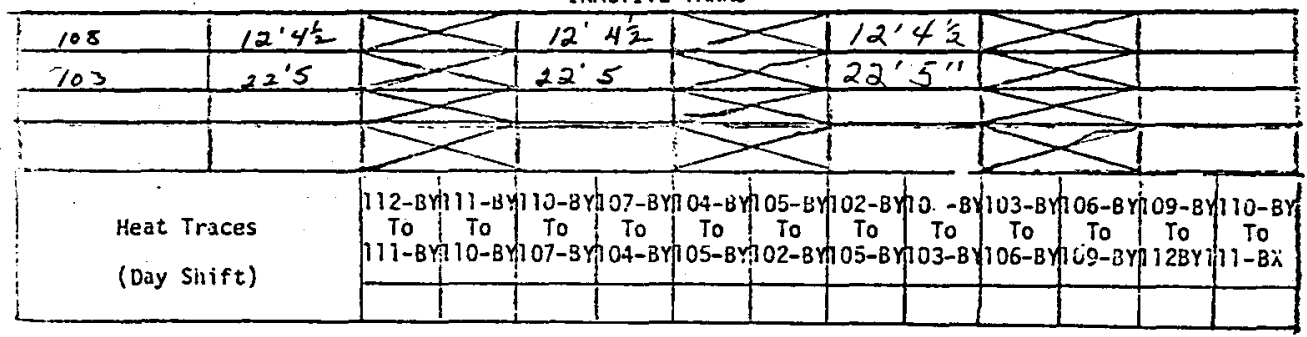




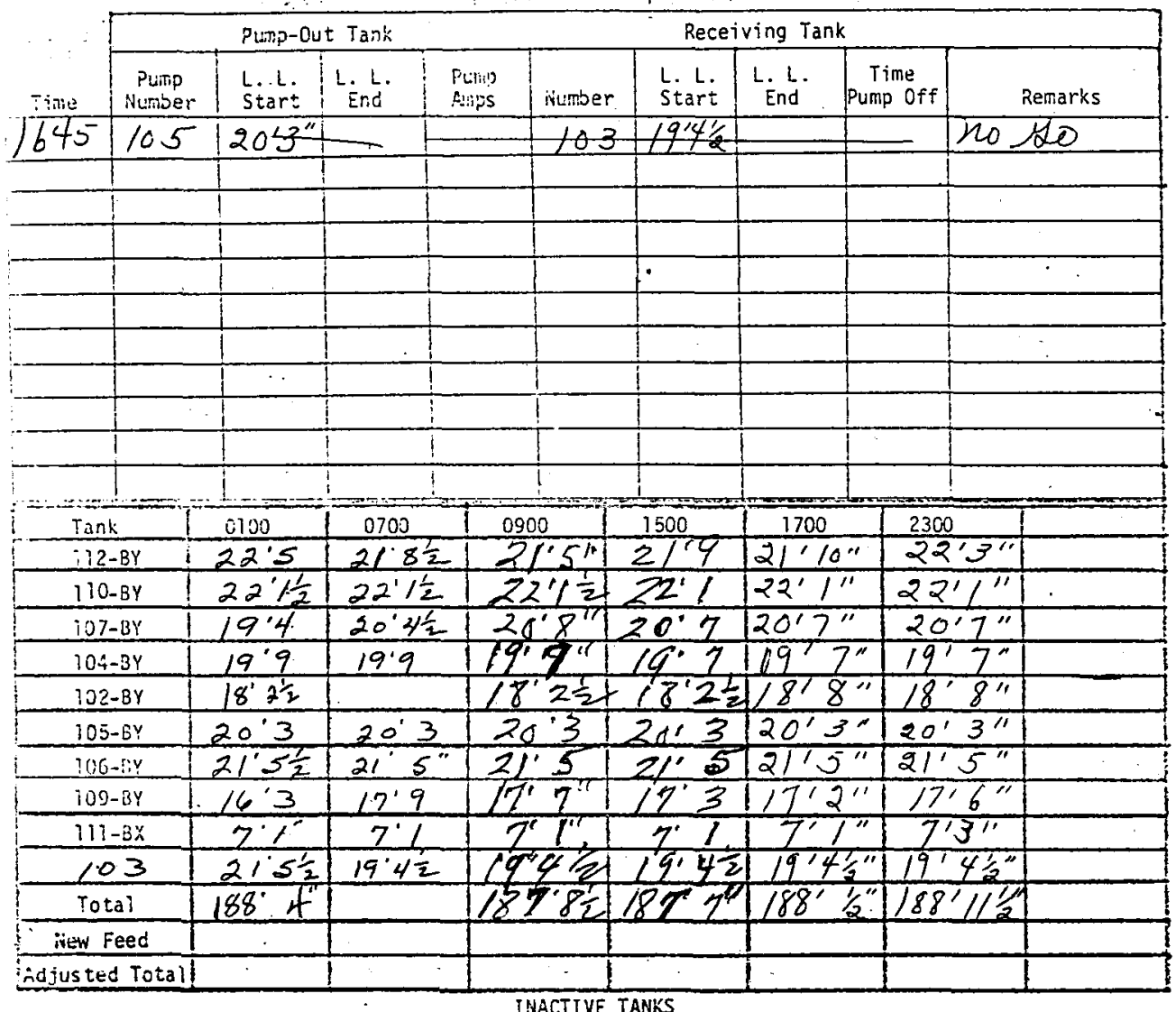

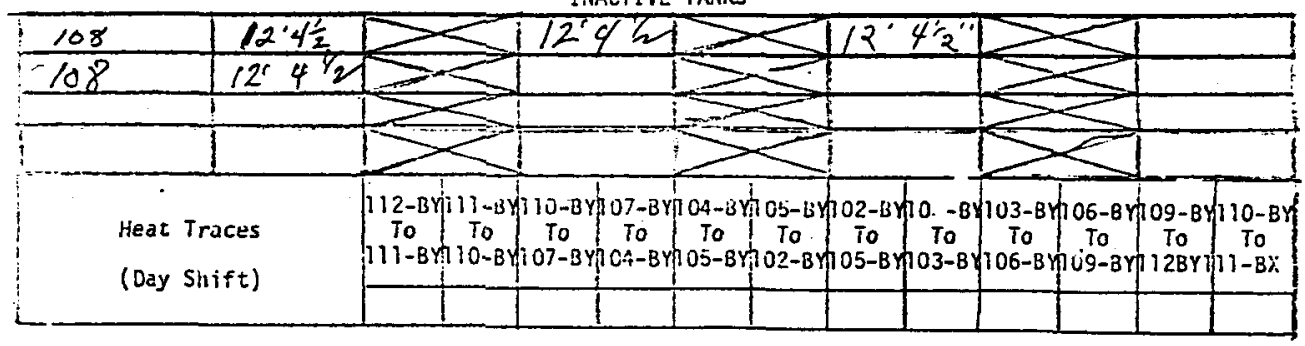




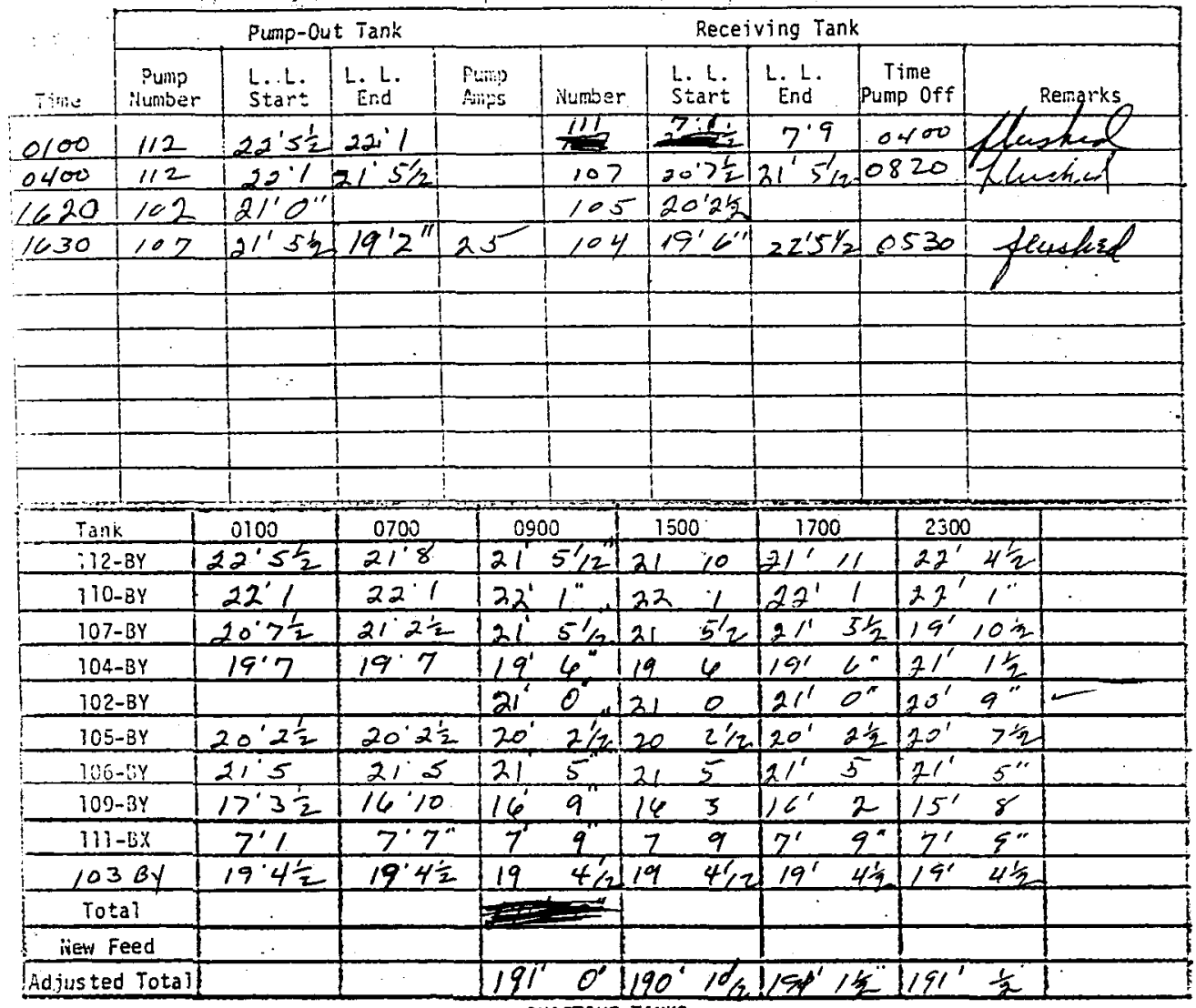

\section{INACTIVE TANKS}

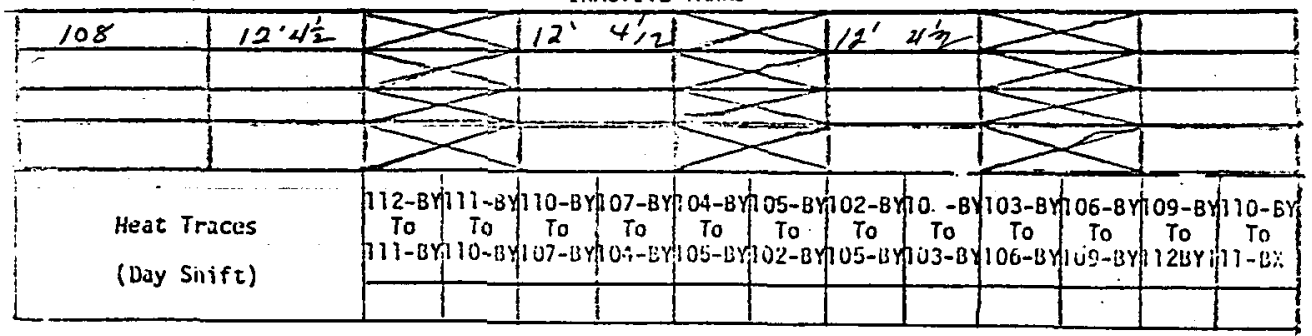




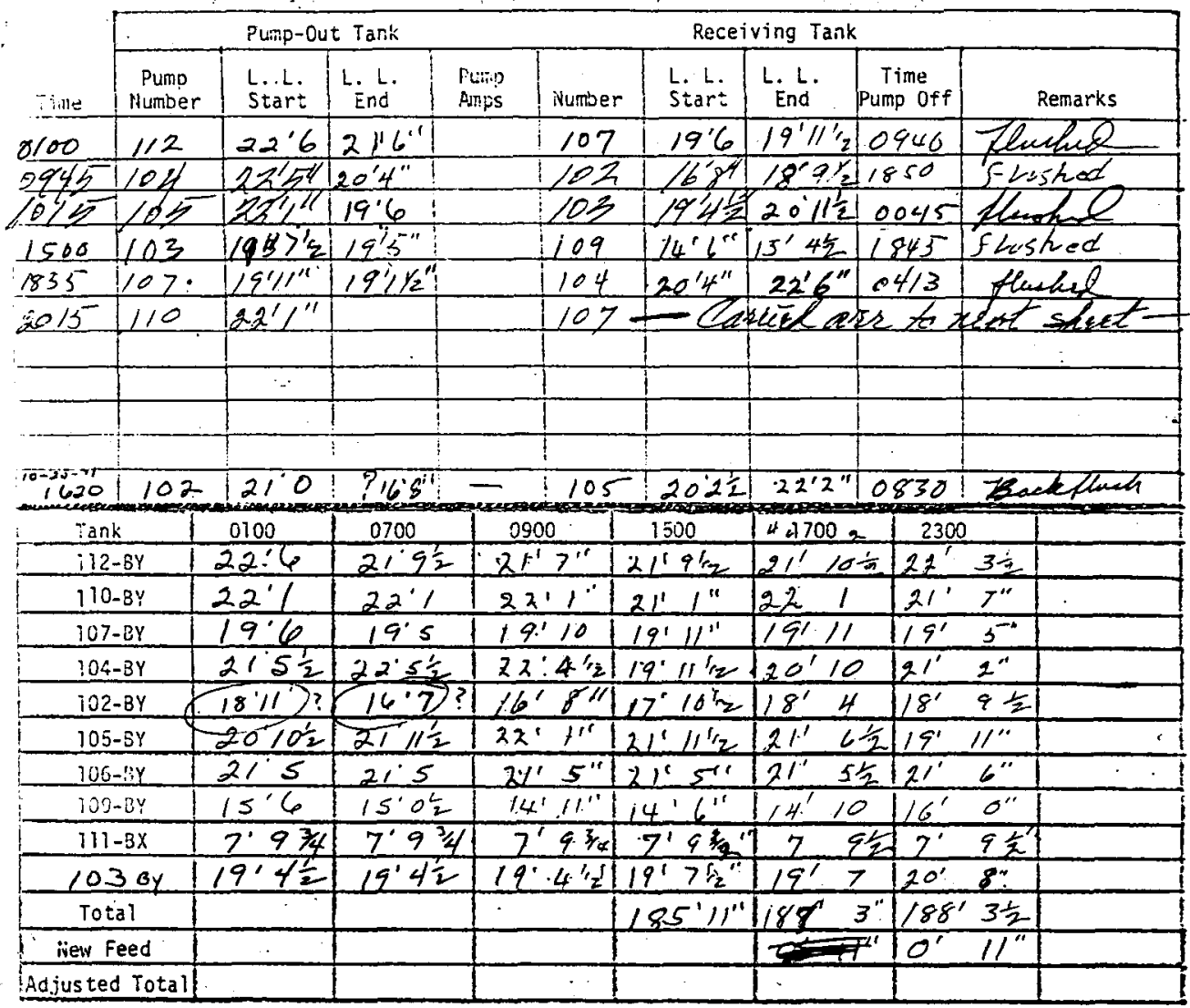

INACTIVE TANKS

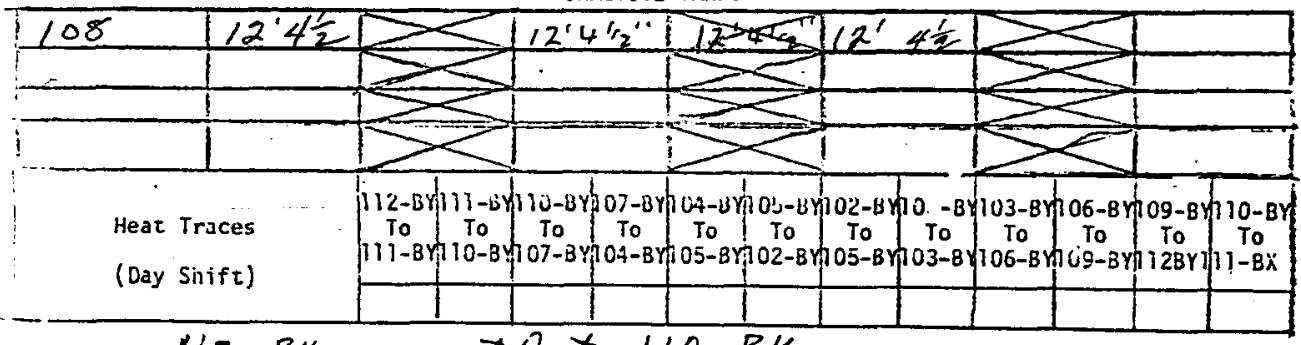
$112-B y$ renouted $t 110-B y$ 


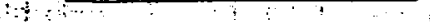

Pump-Out Tank Receiving Tank

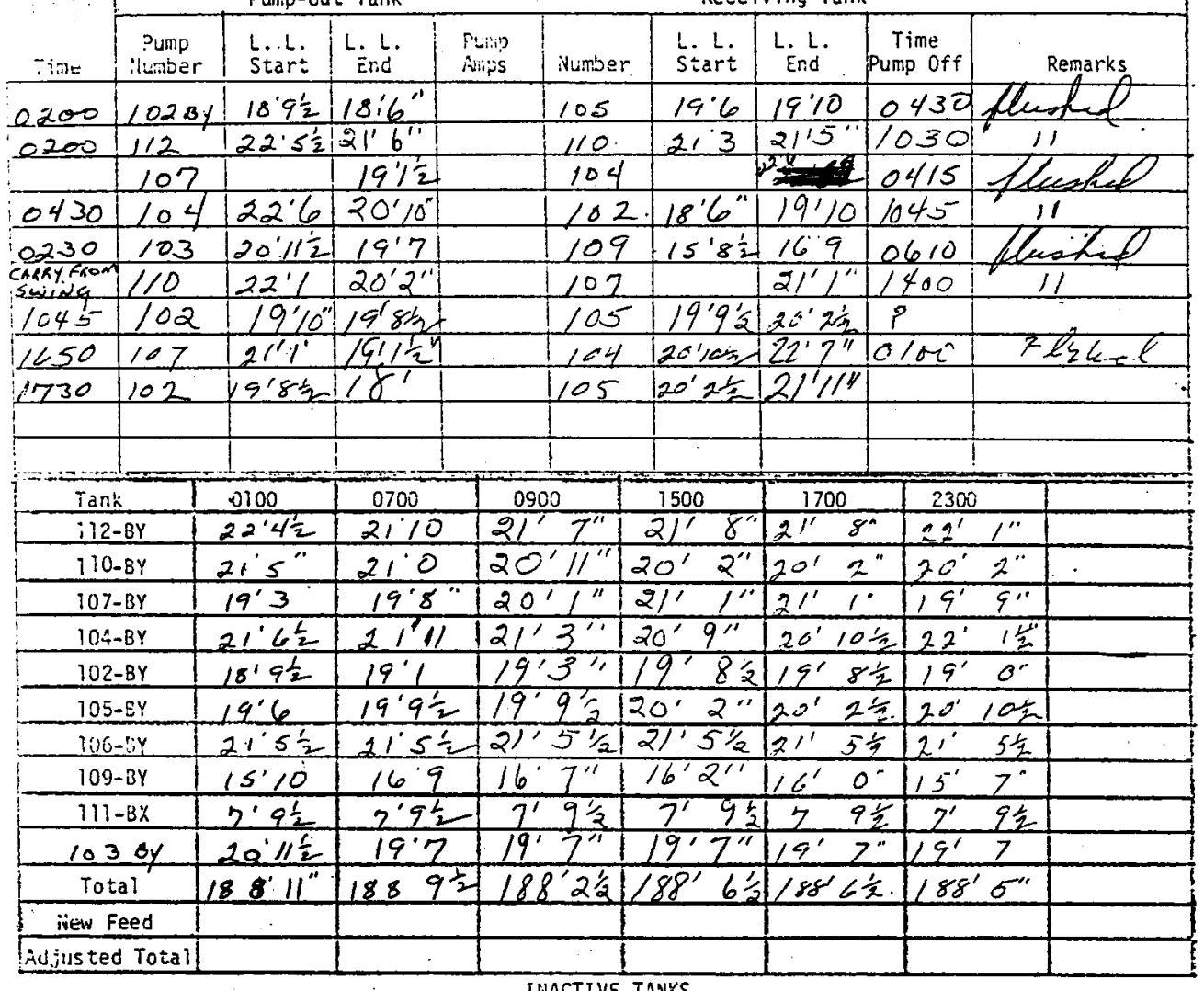

INACTIVE TANKS

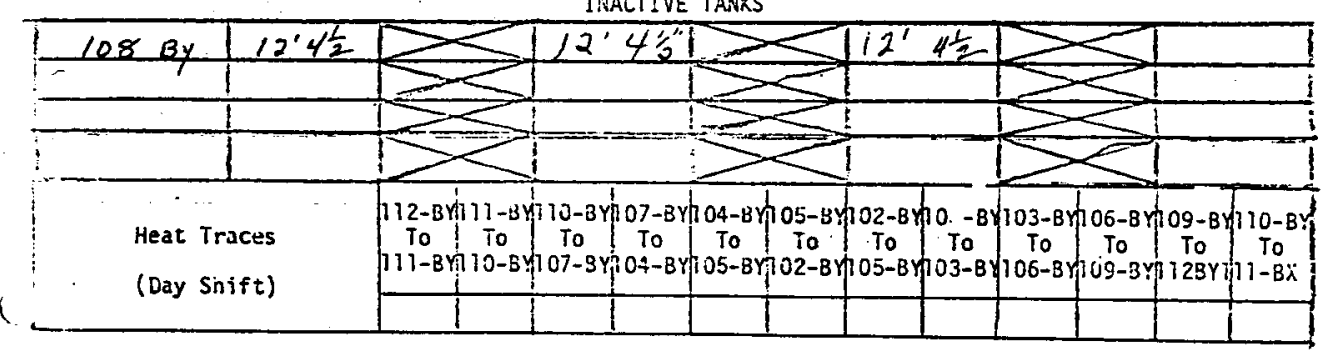

(Day Sinift) 


\section{$\therefore=$}

Receiving Tank

Pump-Out Tank

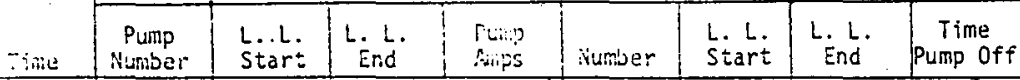

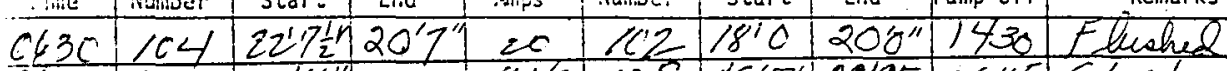

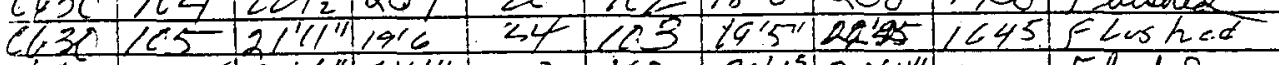

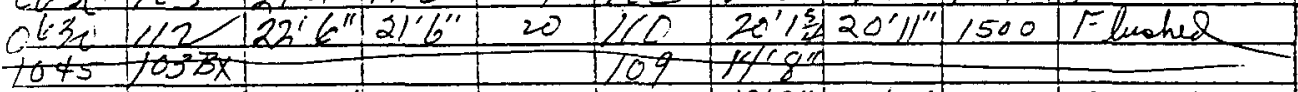

$1100 \quad 110 \quad 21^{\prime} 0^{\prime \prime} \mid 15^{\prime} 5^{\prime \prime}$

$16451102120^{\circ} 0^{\prime 1} / 8^{\prime} \mathrm{C}$

$1>15 \mid 103 \quad 22^{\prime} 2 / 19 \%$

$115 \mid 107120^{\prime} 719^{\prime} 0$

$107 \cdot 19^{\prime} 2^{\prime \prime} 120^{\prime} 2^{\prime \prime} 2100 \mid$ Flustied.

1105

\begin{tabular}{|c|c|c|c|}
\hline 156 & $21^{\prime} 7$ & 0645 & \\
\hline 1510 & $17^{\prime} 10$ & 0102 & \\
\hline $20^{\prime}-6$ & $22 \div 6$ & 0700 & $030 \times 2 c$ \\
\hline
\end{tabular}

\begin{tabular}{|c|c|c|c|c|c|c|c|}
\hline Tunk & 0100 & .0700 & 0900 & 1500 & 1700 & 2300 & \\
\hline $112-5 y$ & $122^{\prime}, 6^{\prime \prime}$ & $22^{\prime} 6^{\prime \prime}$ & $22^{\prime} 4^{\prime \prime}$ & $21^{\prime} 6^{\prime \prime}$ & $21^{\prime} 8$ & $2 \pi$ & \\
\hline $110-B Y$ & $80^{\prime} 13$ & $x^{\prime} 1^{\frac{3}{4}}$ & $20^{\prime} 6^{\prime \prime}$ & $20^{\prime} / 1^{\prime \prime}$ & $20^{\prime} 8$ & 19 & . \\
\hline $107-3 Y$ & $191<2^{11}$ & $16^{\prime} \times \frac{11}{2}$ & $19^{\prime} 2^{\prime \prime}$ & $19^{\prime} 9^{\prime \prime}$ & $20^{\prime} \quad 1$ & 2011 & \\
\hline$: 04-8 Y$ & $22172^{\prime \prime}$ & $\left.22^{\prime} 7^{\prime}\right)^{\prime \prime \prime}$ & $22^{\prime} 4^{\prime \prime}$ & $150^{\prime} 7^{\prime \prime}$ & $20 \quad 7 / 2$ & 217 & \\
\hline $102-8 y$ & $18^{1} 8^{11}$ & $\pm 8^{\prime} \mathrm{C}$ & $18^{\prime} 3^{\prime \prime}$ & $120^{\prime} 0^{\prime \prime}$ & $20^{\prime}$ & 19 & \\
\hline 105-BY & $2113^{\prime \prime}$ & $21^{\prime} 11^{\prime \prime}$ & $21^{\prime}$ & $20^{\prime} 1^{\prime \prime}$ & $15^{\prime} 6$ & 20 & \\
\hline $106-10$ & $121^{\prime} 5 y^{4}$ & $24^{\prime} 5 \frac{1}{2} r$ & $21.5 \%$ & $21,5 \frac{1}{2}$ & $5 \frac{1}{2}$ & 21 & \\
\hline $109-3 Y$ & $15^{\prime} 4^{\prime \prime}$ & $1511^{11}$ & $14^{\prime} 1.0^{\prime \prime}$ & $15^{\prime} 6^{\prime \prime}$ & $15^{1} 10$ & 17 & \\
\hline $311-8 x$ & $7^{\prime 9} 92^{\prime \prime}$ & $2^{\prime} \quad q^{\prime}$ & $7^{\prime} 91 / 2$ & $7^{\prime} 9 \frac{1}{2}$ & 792 & $9 \frac{1}{2}$ & \\
\hline $103 B G$ & 191511 & $14^{\prime} .5^{\prime \prime}$ & $19^{\prime} 11^{\prime \prime} 2$ & $21^{\prime} 7^{\prime \prime}$ & $21^{\circ} 2^{\prime \prime}$ & 20 & \\
\hline Total ${ }^{\sigma}$ & $187^{\prime} \cdot 10^{3}$ & $18.7^{1} 8^{11}$ & $188^{\prime} 12^{\prime \prime}$ & $188^{\prime} 2^{\prime \prime}$ & $189^{\prime} 8 \frac{1}{2}$ & 1898 & \\
\hline irew $f$ & & & & & & & \\
\hline$s t$ & & & & & & & \\
\hline
\end{tabular}

Adjus ted Total

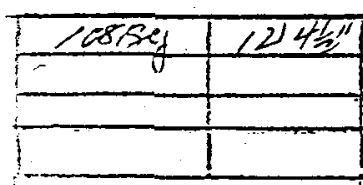

\section{INACTIVE TANKS}

Heat Traces

(Day Shift)

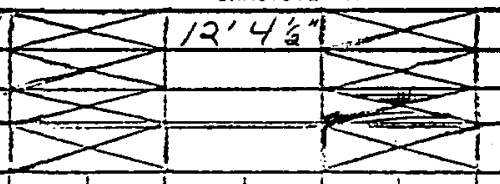

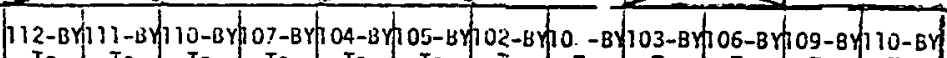

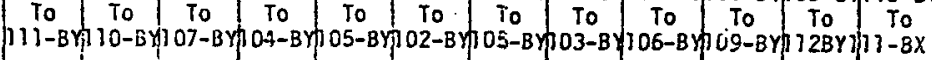


ITS-2 INVENTORY AND PUNPING

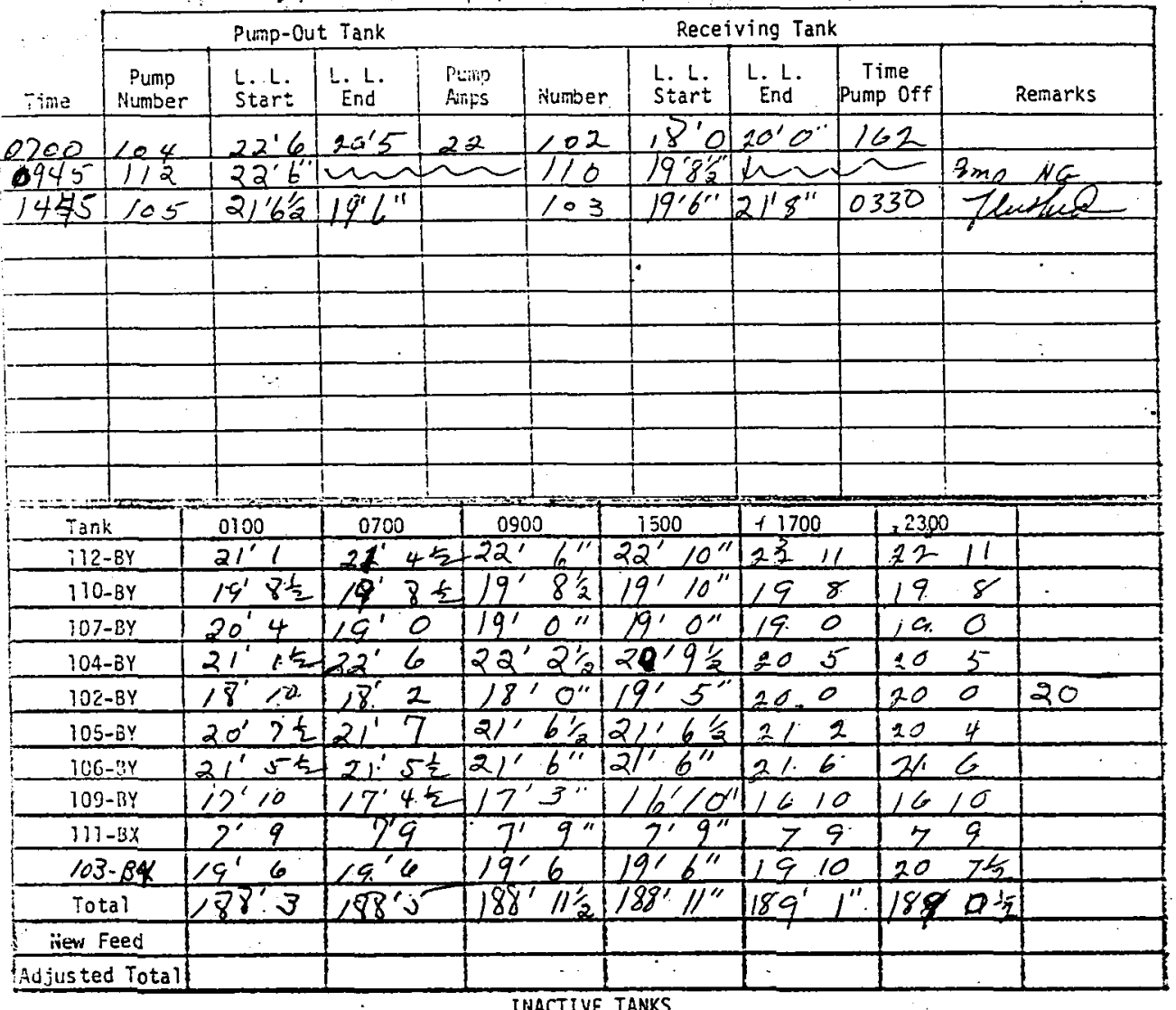

Date $10 / 24 / 21$ 


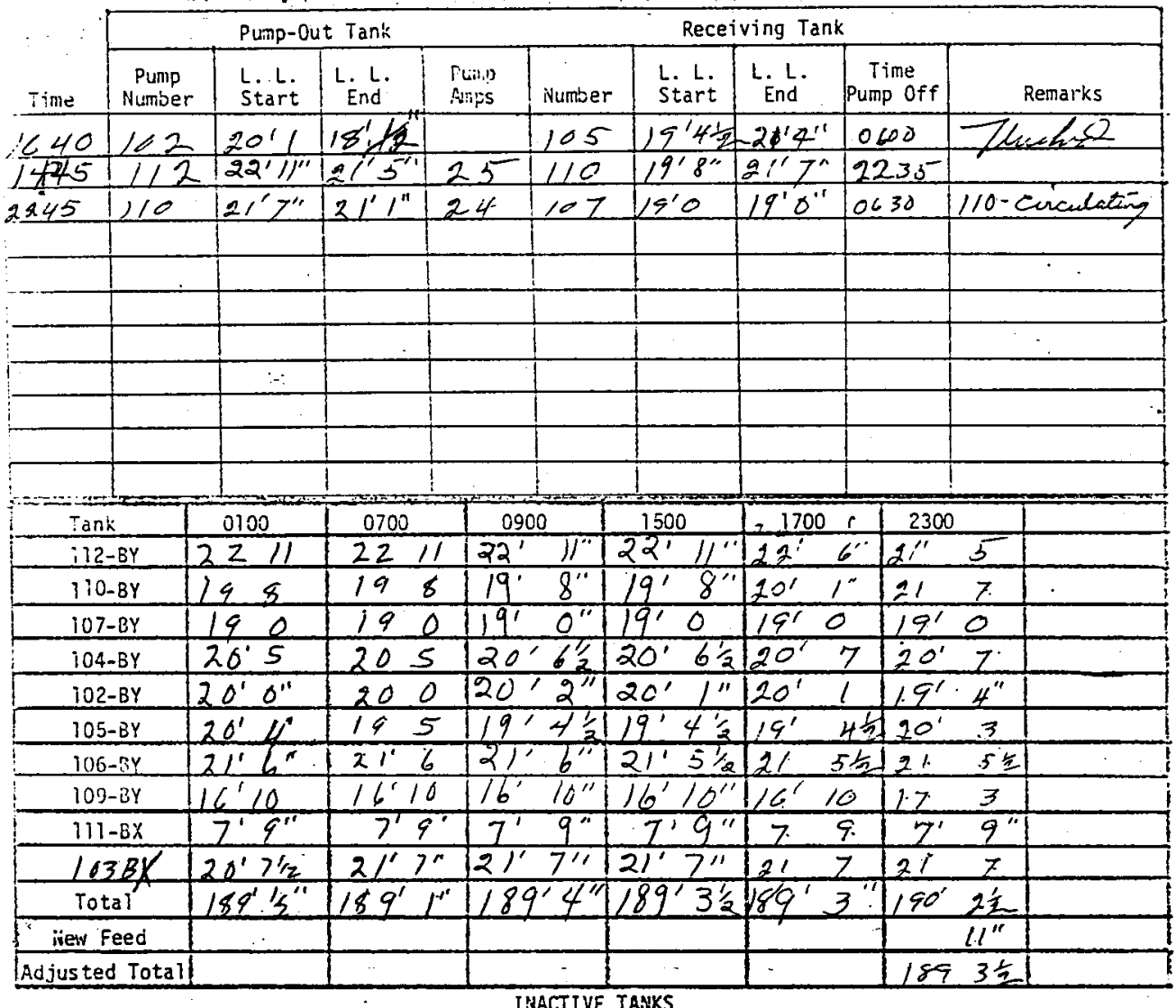

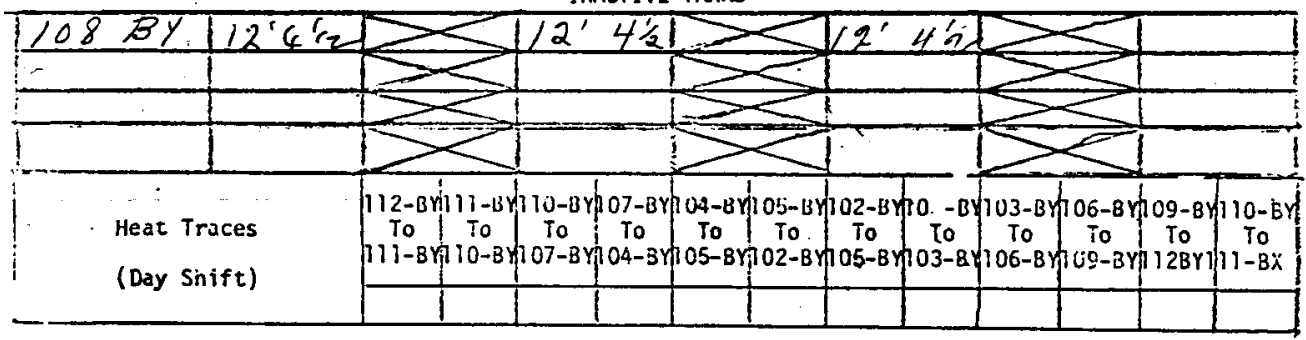




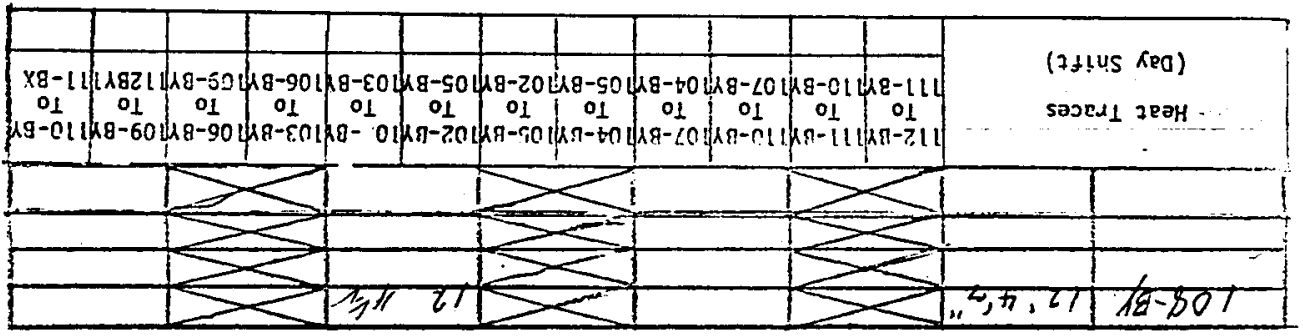

\section{SXNV1 3AILONI}

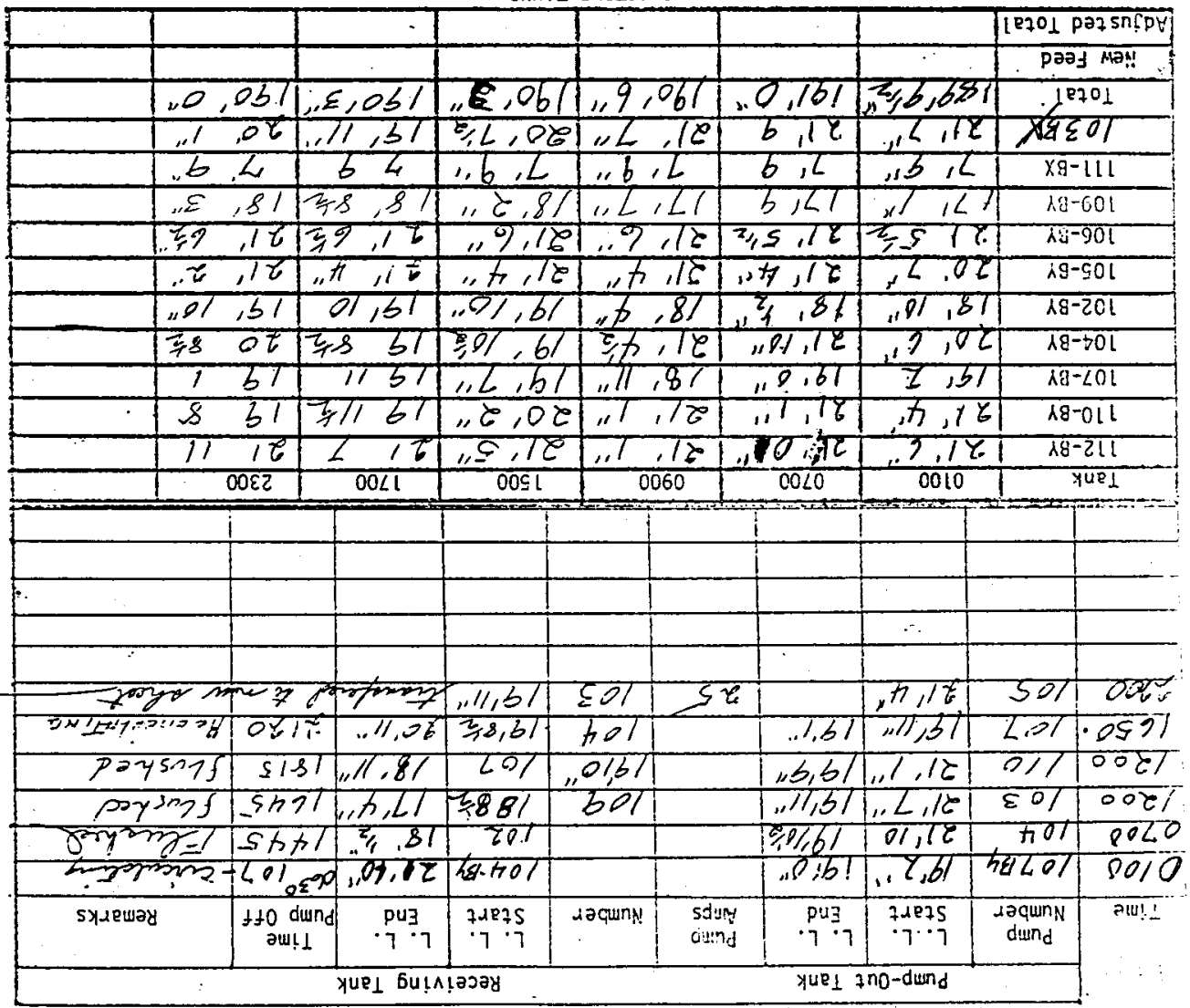




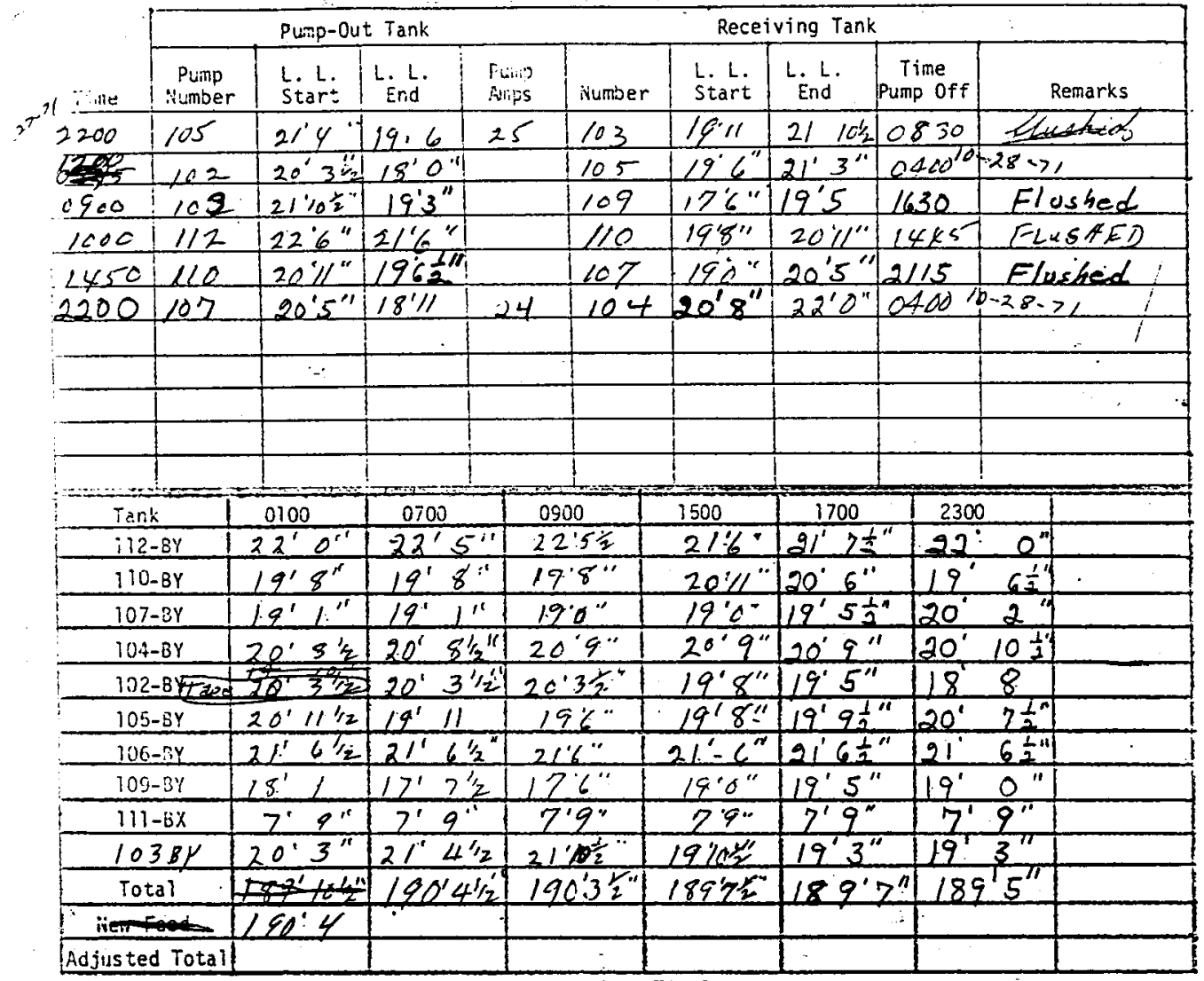

IRACT I VE TANKS

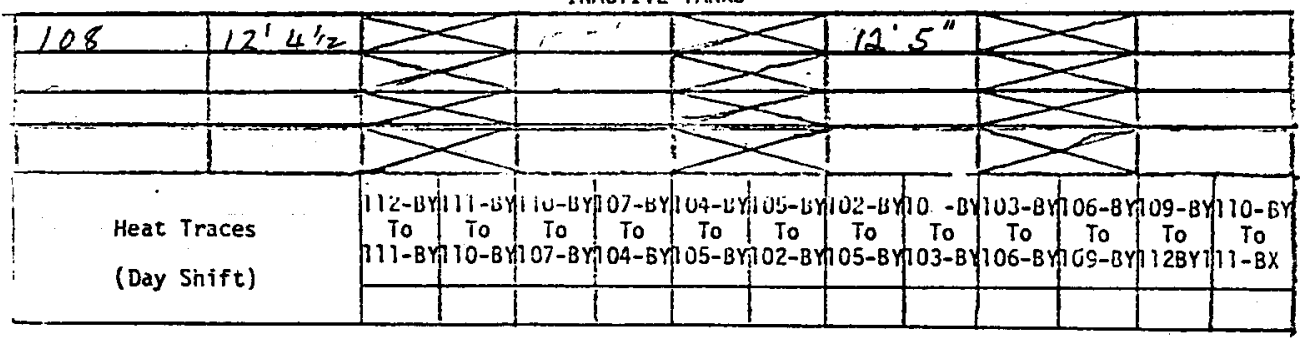




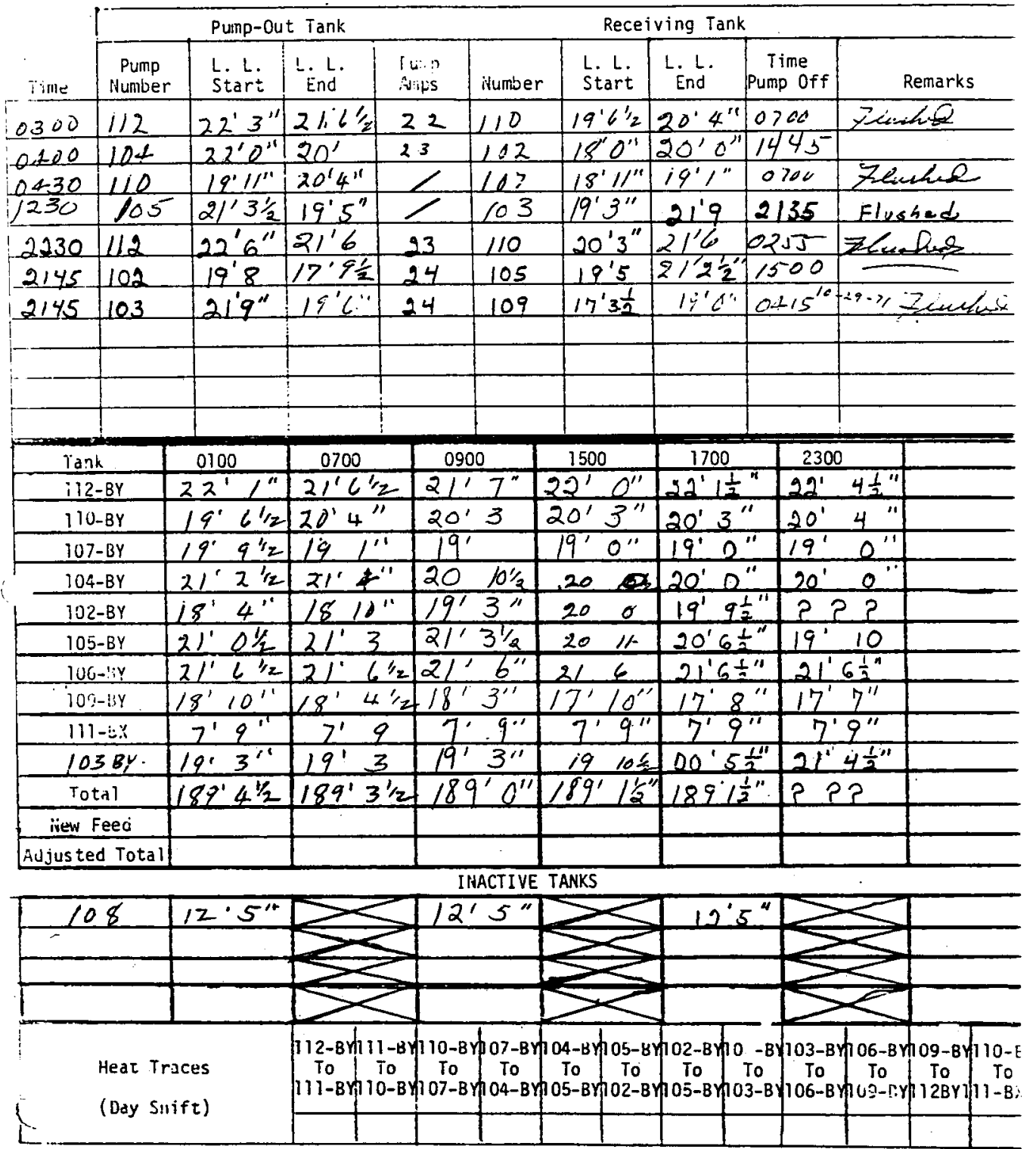




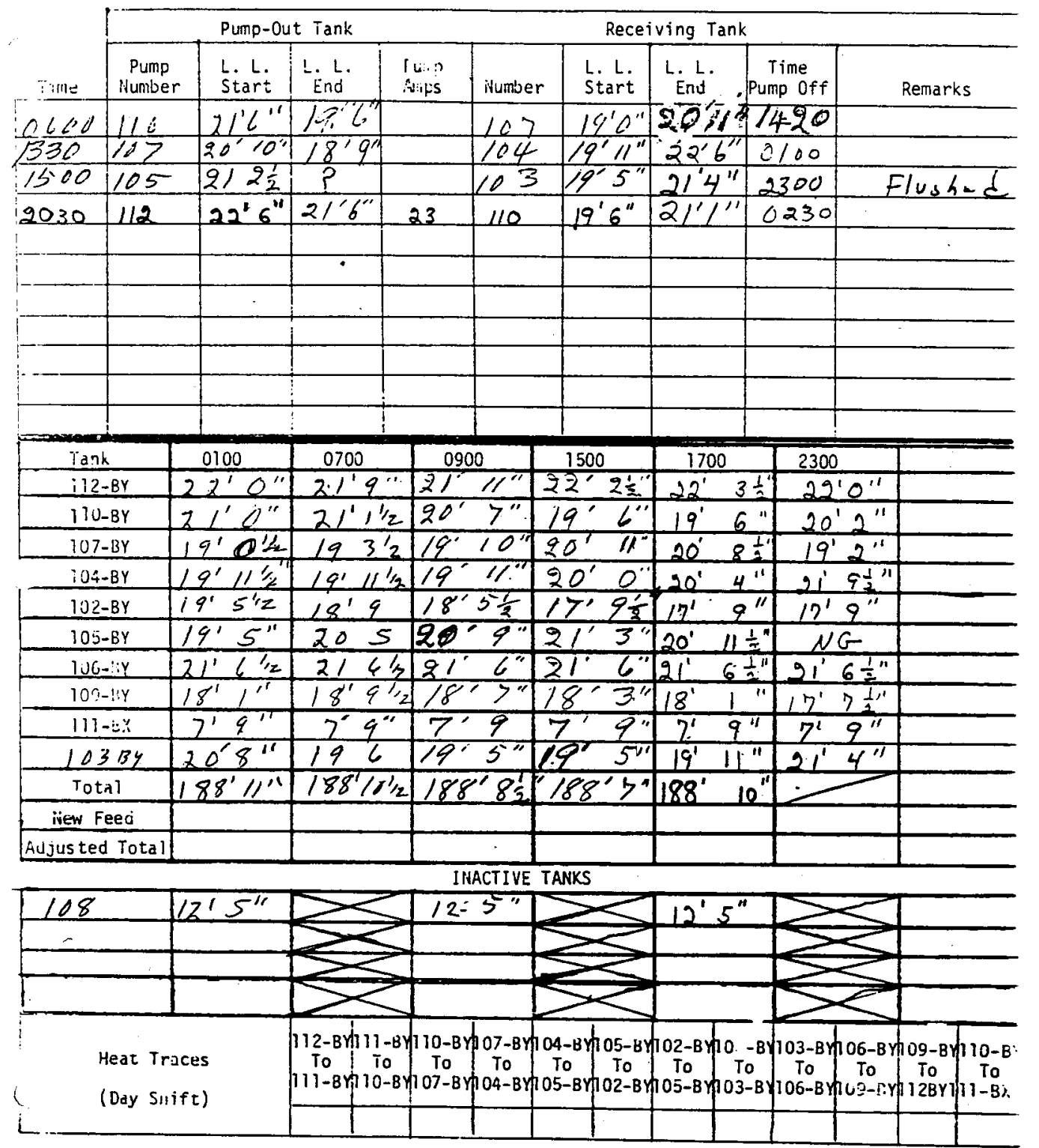




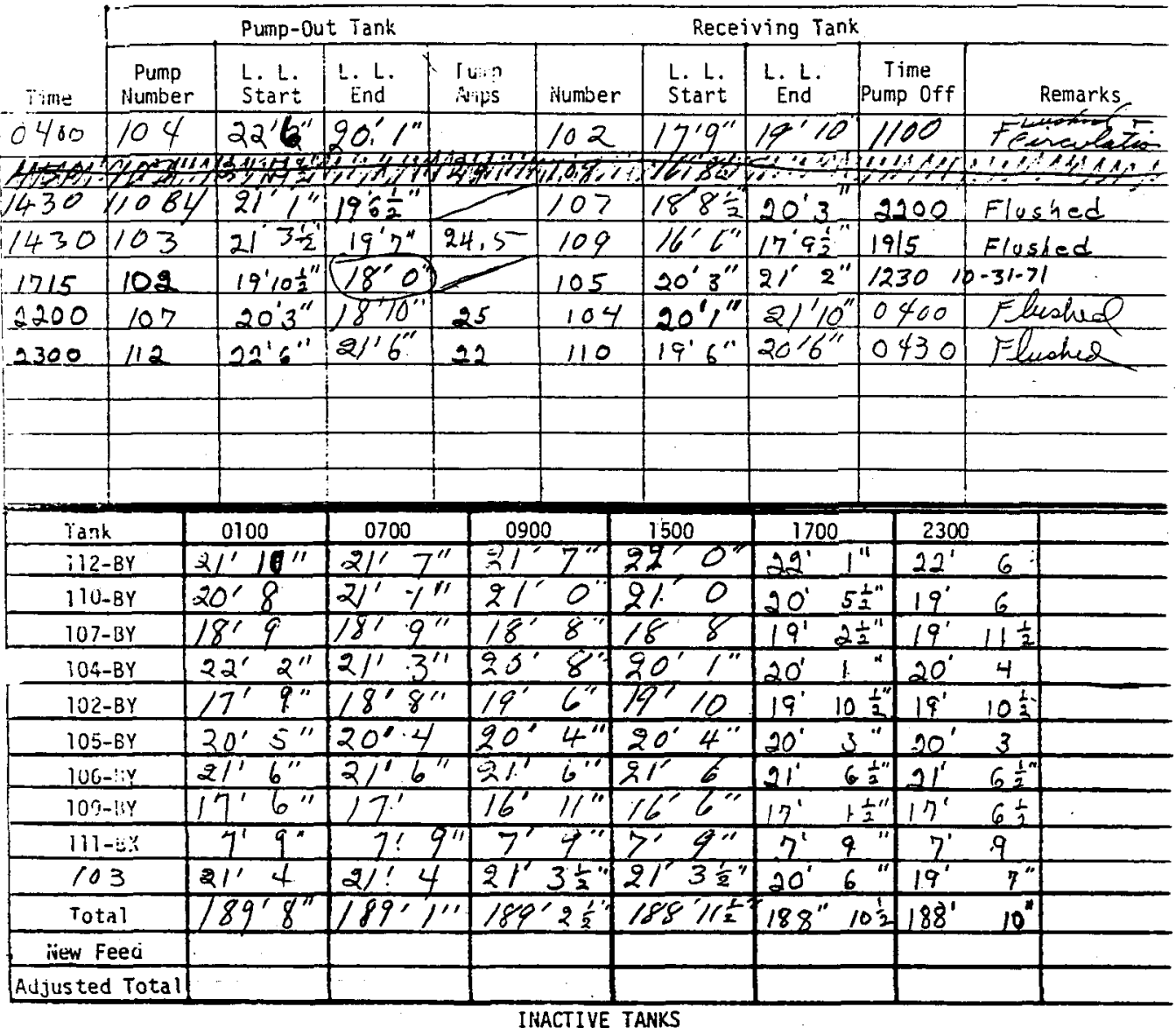

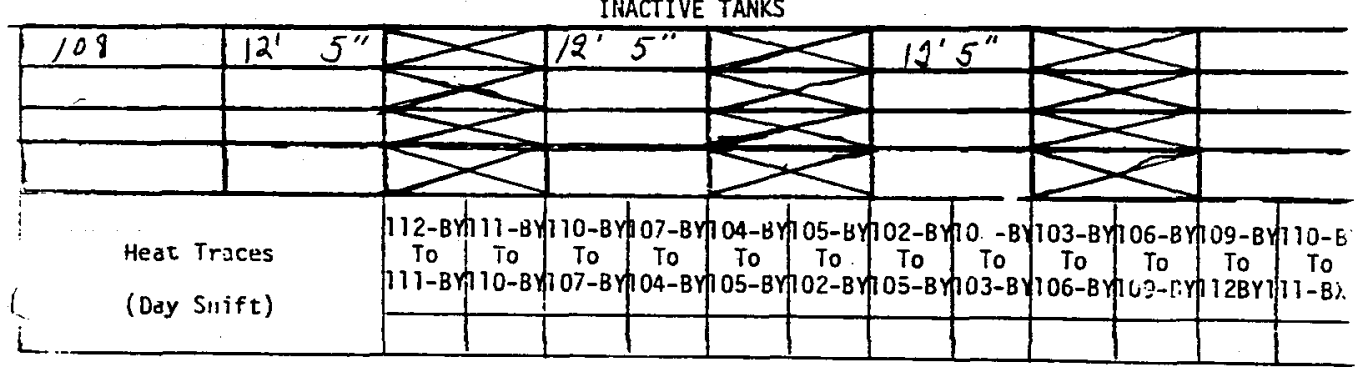




\section{ITS-2 INUENTORY ANU PUMPING \\ Date $|0-3|-7 \mid$}

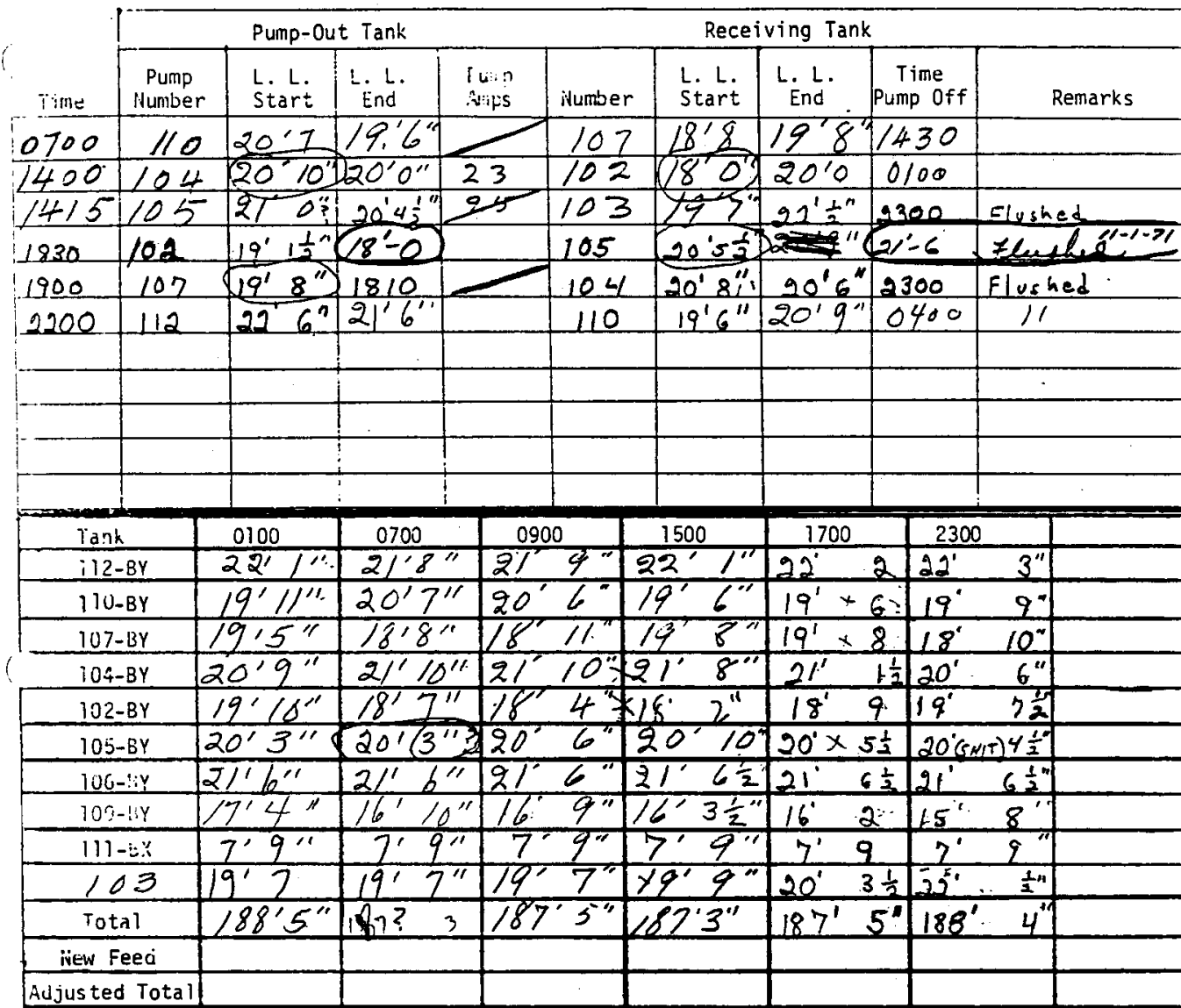

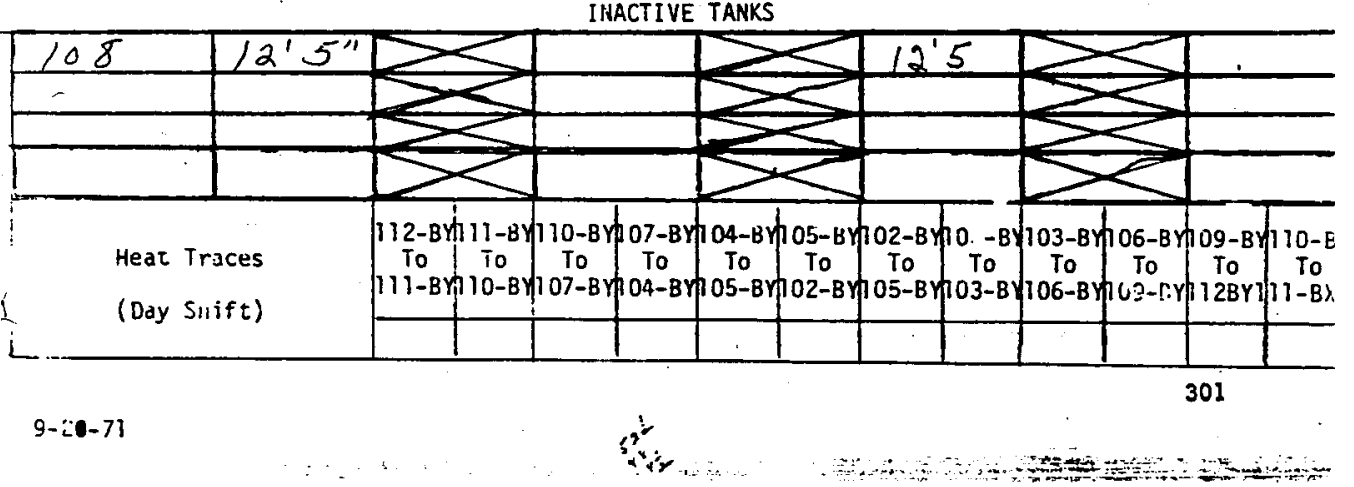




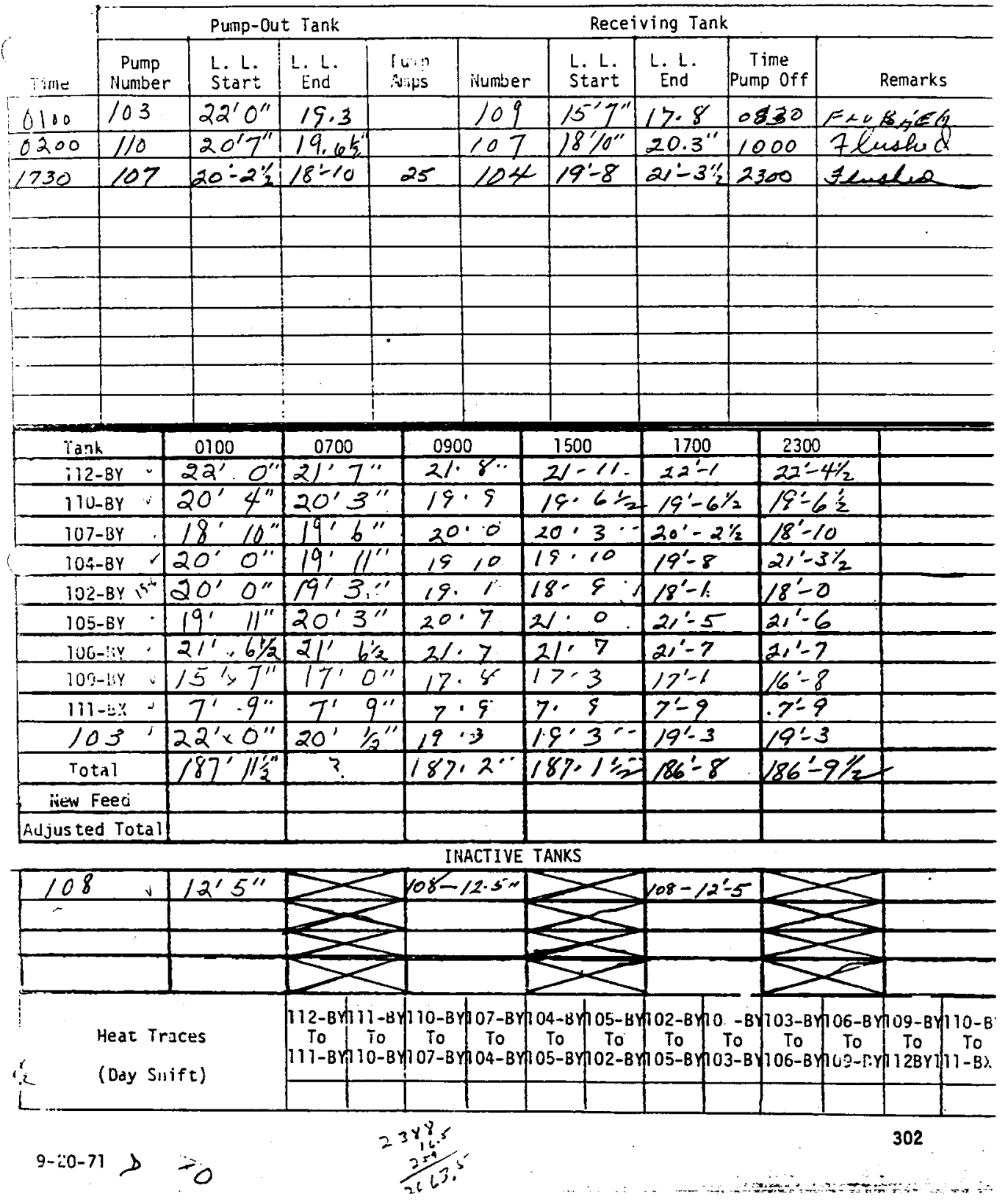




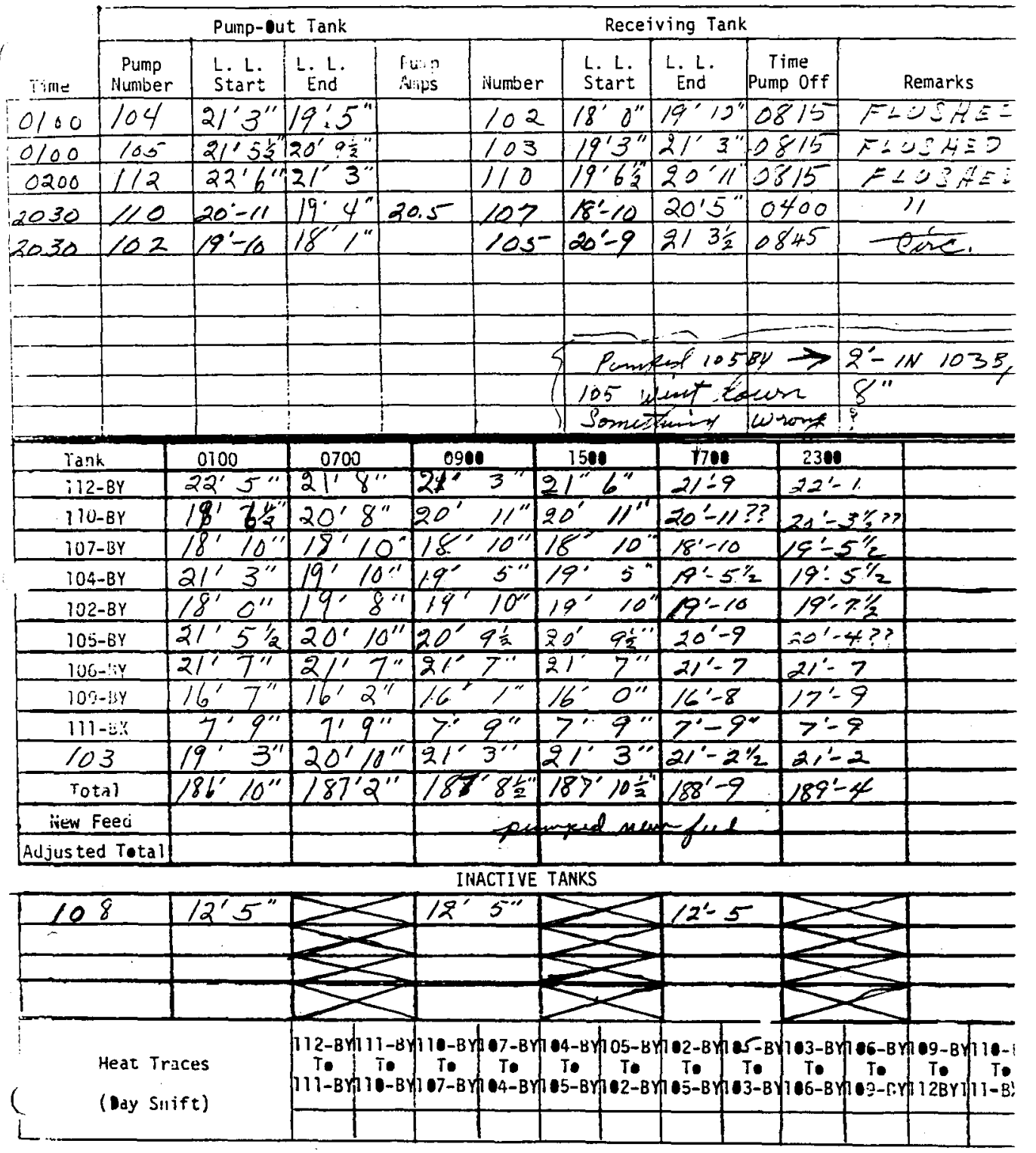




\begin{tabular}{|c|c|c|c|c|c|c|c|c|c|}
\hline \multirow[b]{2}{*}{ Tne } & \multicolumn{4}{|c|}{ Pump-Out Tank } & \multicolumn{4}{|c|}{ Receiving Tank } & \multirow[b]{2}{*}{ Remarks } \\
\hline & $\begin{array}{l}\text { Pump } \\
\text { Number }\end{array}$ & $\begin{array}{l}\text { L. L. } \\
\text { Start }\end{array}$ & E. L. & $\begin{array}{l}\text { fwin } \\
\text { Alitis }\end{array}$ & Number & $\begin{array}{l}\text { L. L. } \\
\text { Start }\end{array}$ & $\begin{array}{l}\text { L. L. } \\
\text { End }\end{array}$ & $\begin{array}{c}\text { Time } \\
\text { Pump off }\end{array}$ & \\
\hline 0630 & 112 & $22^{\prime} 6^{\prime \prime}$ & $9 l^{\prime} 6^{\prime \prime}$ & & 110 & $19^{\prime} 4^{\prime \prime}$ & $20^{\circ} 5$ & 1210 & \\
\hline 1645 & 107 & $20^{\prime} 4^{\prime \prime}$ & $14: 2$ & 24 & 104 & $19^{\prime} 4^{\prime \prime}$ & $20^{\prime} 9^{\prime \prime}$ & 2230 & Ilenced \\
\hline & & & & & & & & & \\
\hline & & & & & & & & & \\
\hline & & & & & & & & & - \\
\hline & & & & & & & & & \\
\hline & & & & & & & & & \\
\hline & & & & & & & & & \\
\hline & & & & & & & & & \\
\hline & & & & & & & & & \\
\hline & & & & & & & & & \\
\hline
\end{tabular}

\begin{tabular}{|c|c|c|c|c|c|c|c|}
\hline Tank & 0100 & 0700 & 0900 & 1500 & 1700 & 2300 & \\
\hline i12-BY & $2274^{\prime \prime}$ & $22^{\prime} 5^{\prime \prime}$ & $220^{\prime \prime}$ & 21.81 & $21^{\prime} 8 \frac{1}{2}$ & $22^{\prime}$ & \\
\hline $110-B Y$ & $19^{\prime} 9^{\prime \prime}$ & $19 ' 5 ?$ & $19^{\prime} 10^{\prime \prime}$ & $20^{\prime} 5^{\prime \prime}$ & $28^{\prime} 6^{\prime \prime}$ & $20^{\prime}$ & \\
\hline $107-B Y$ & $1911^{\prime \prime}$ & $20^{\prime} 5^{\prime \prime}$ & $20^{\circ} 5 \frac{1}{2}$ & $20^{\prime} \quad 5 \frac{7}{2}$ & $20^{\prime} 4^{3} 3^{\prime \prime}$ & $19^{\prime} 2^{\prime \prime}$ & \\
\hline iOA-BY & $19^{\prime} 12$ & $14^{\prime} 4^{\prime \prime}$ & $19^{\prime} 4^{\prime \prime}$ & $19^{\prime} 4^{\prime \prime}$ & $19^{\circ} 4^{\prime \prime}$ & $20^{\prime} 9^{\prime \prime}$ & \\
\hline $10 Z-B Y$ & $9^{\prime} 3^{\prime \prime}$ & $188^{\prime} 4^{\prime \prime}$ & $18^{\prime} /^{\prime \prime}$ & $18^{\prime} 1^{\prime \prime}$ & $18^{11} 11$ & 1811 & \\
\hline $105-B Y$ & $20^{\prime} 72$ & $21^{\prime} 2 \div 2$ & $21^{\prime} 3 \frac{1}{2}$ & $21^{\prime} 9 \frac{1}{2}$ & $21^{\prime} 2^{\prime \prime}$ & $21^{\prime} 2^{\prime \prime}$ & \\
\hline $106-: y$ & $21 / 71 / 2$ & $2117 \%$ & $2 l^{\prime} Z^{\prime \prime}$ & $21 \quad 2$ & $21^{\prime} 7 \frac{1}{2}$ & $2117 \frac{1}{2}$ & \\
\hline $10 r_{i-1 i \gamma}$ & $12^{\prime} 8^{\prime}$ & $17^{\prime} 3^{\prime \prime}$ & $17^{\prime} 2$ & $16^{\prime} 9 \frac{1}{2}$ & $16^{\prime} 811$ & $16^{\prime} 3^{\prime \prime}$ & \\
\hline $111-\div: 2$ & $1^{\prime} 9^{\prime \prime}$ & $7^{\prime} 9^{\prime \prime}$ & $7^{\prime} 9^{\prime \prime}$ & $7^{\prime} 9^{\prime \prime}$ & 71 & $7^{\prime} 9^{\prime \prime}$ & \\
\hline 103 & $21^{\prime} 2^{\prime \prime}$ & $2 / 2^{\prime} 2^{\prime \prime}$ & $212{ }^{\prime \prime}$ & $2 t^{\prime} 2^{\prime \prime}$ & $21^{\prime} 2^{\prime \prime}$ & $212^{\prime}$ & \\
\hline Total & $189^{\prime} 2 \frac{1}{2}$ & $188^{\prime} 10^{\prime \prime}$ & $188^{\prime} 7 \frac{1}{2}^{\prime \prime}$ & $188^{7} 5 \frac{1}{2}$ & $18814 \frac{3}{4}$ & $188^{\circ} 5 \frac{1}{2}^{\prime \prime}$ & \\
\hline ivew Feed & & & & & & & \\
\hline jus ted To & & & & & & & \\
\hline
\end{tabular}

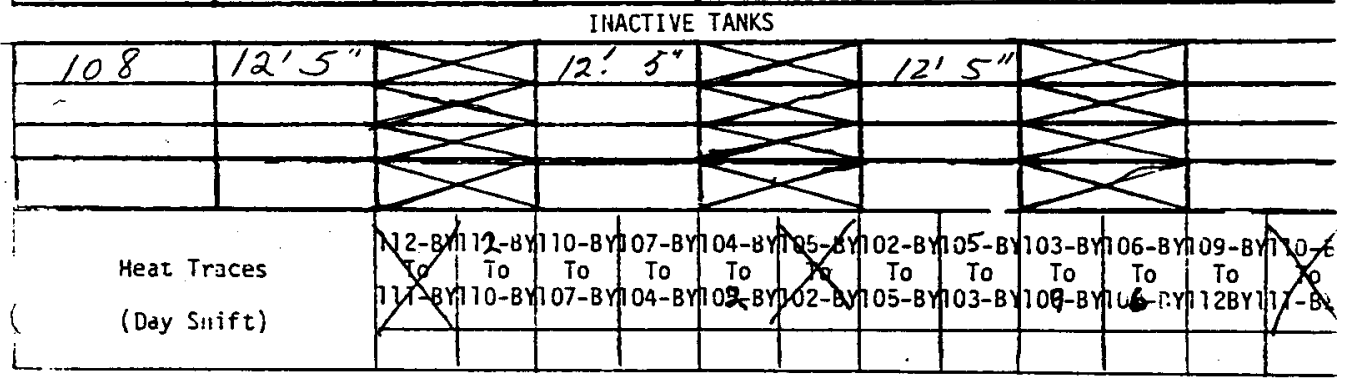


WHC-SD-WM-OP-200, ReV. 0

ITS-2 INVENTORY AND PUMPING

cate $|1-4-7|$

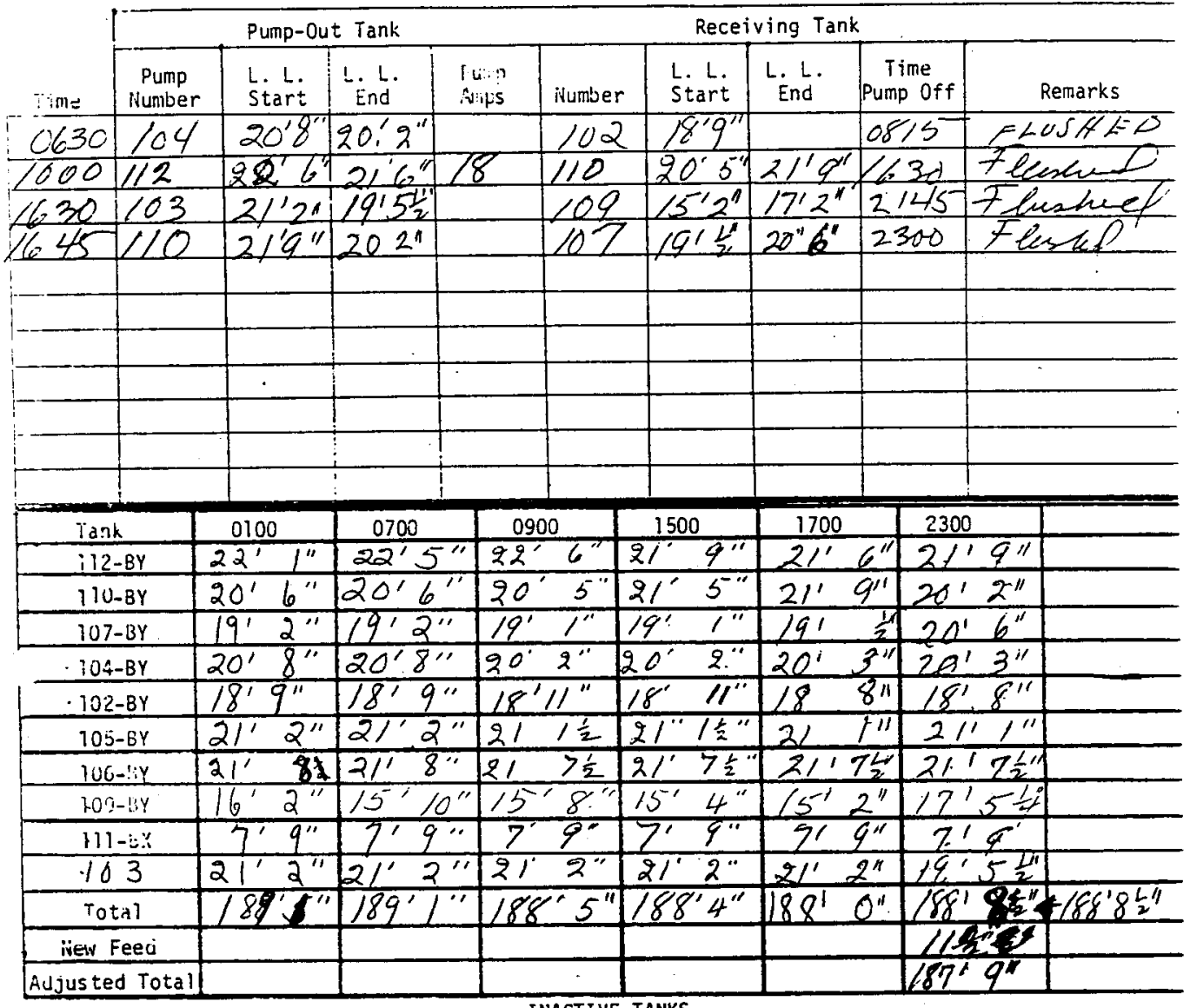

INACTIVE TANKS

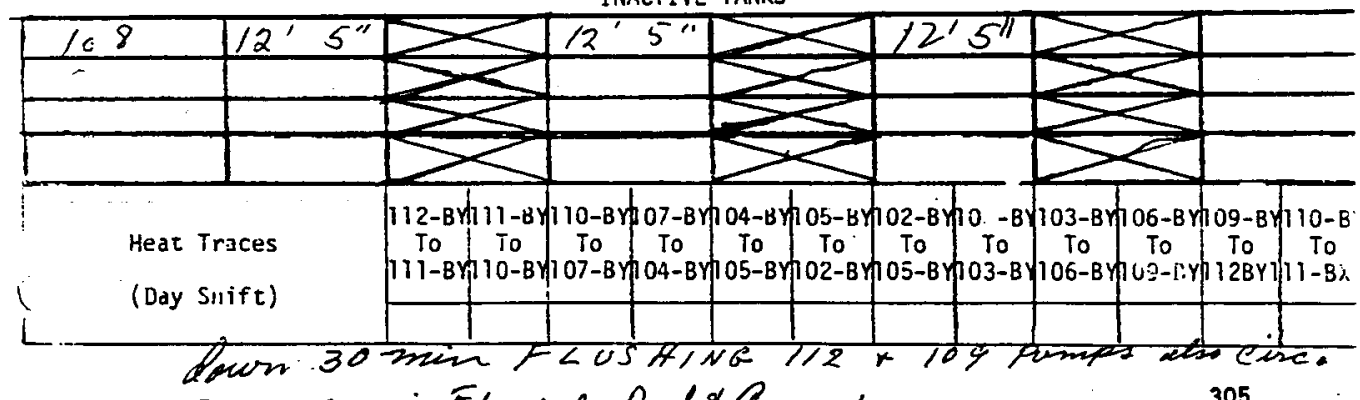

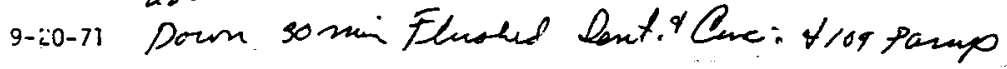

305 


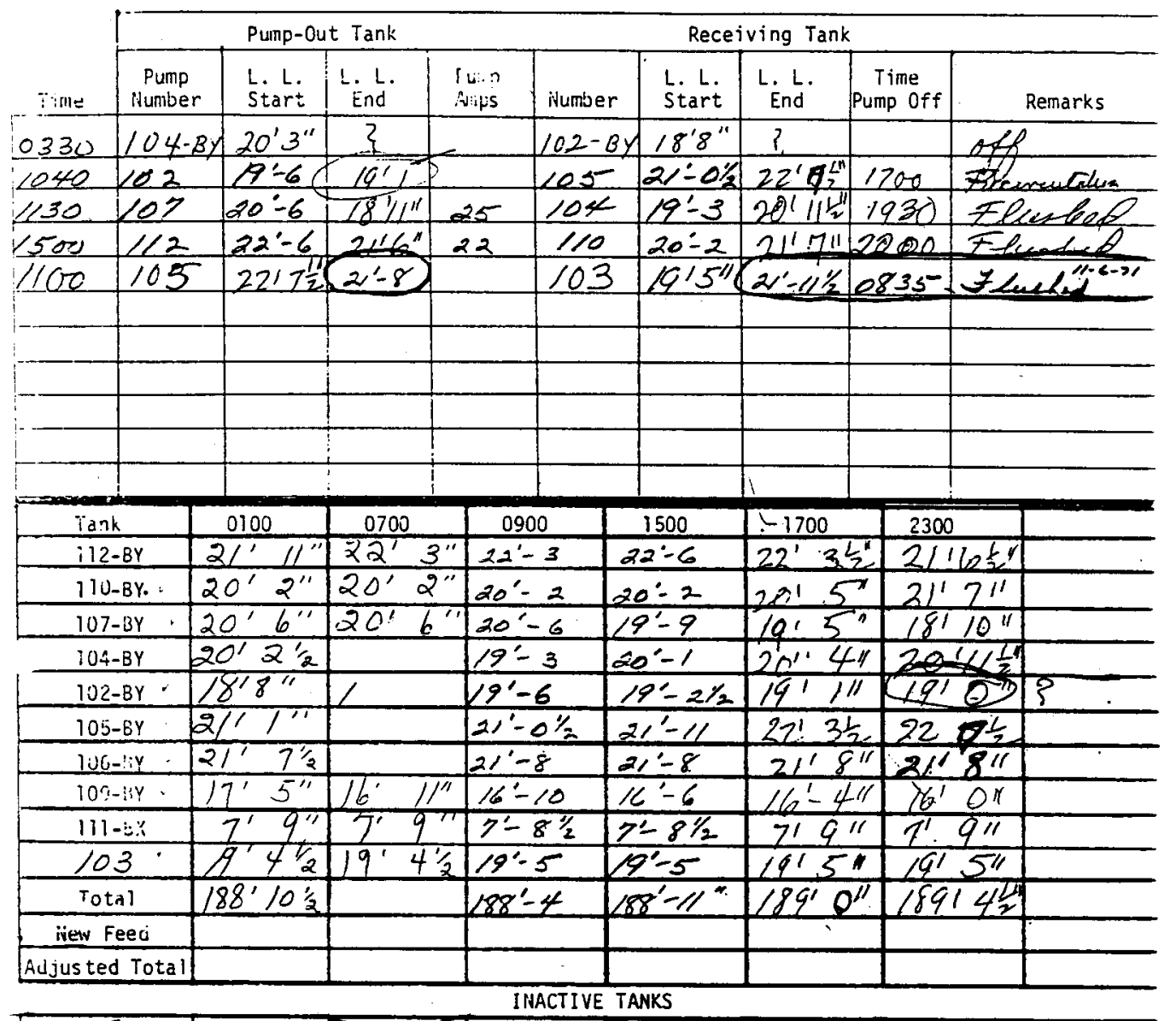

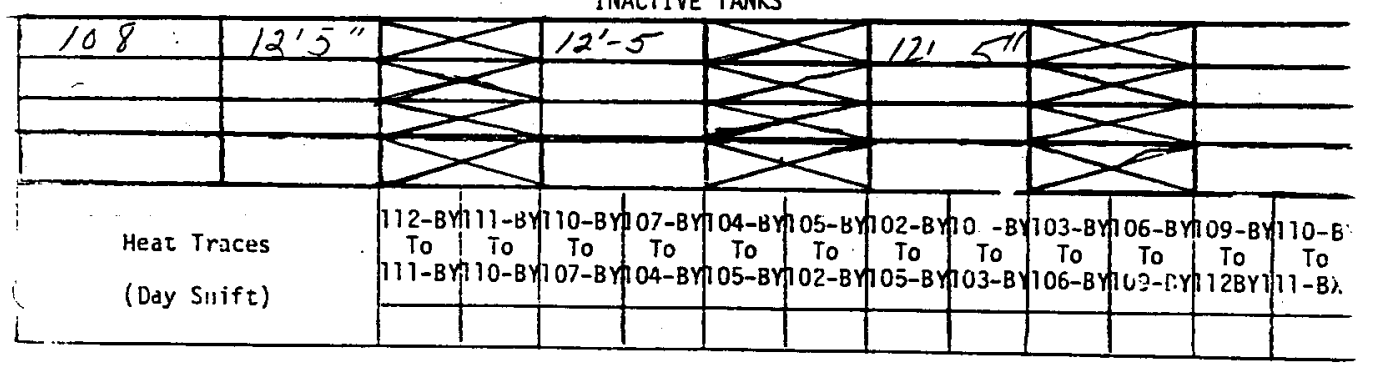




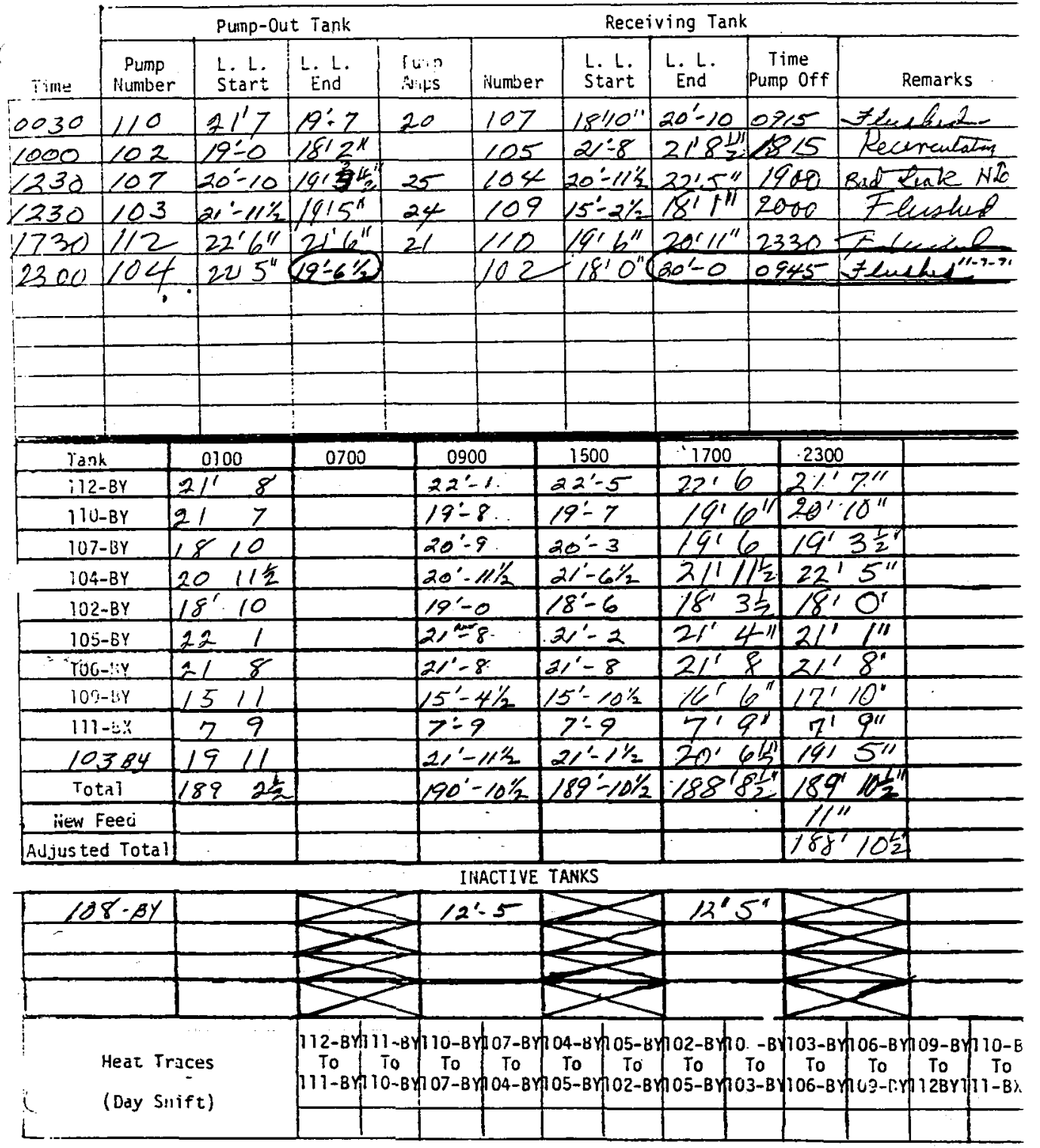




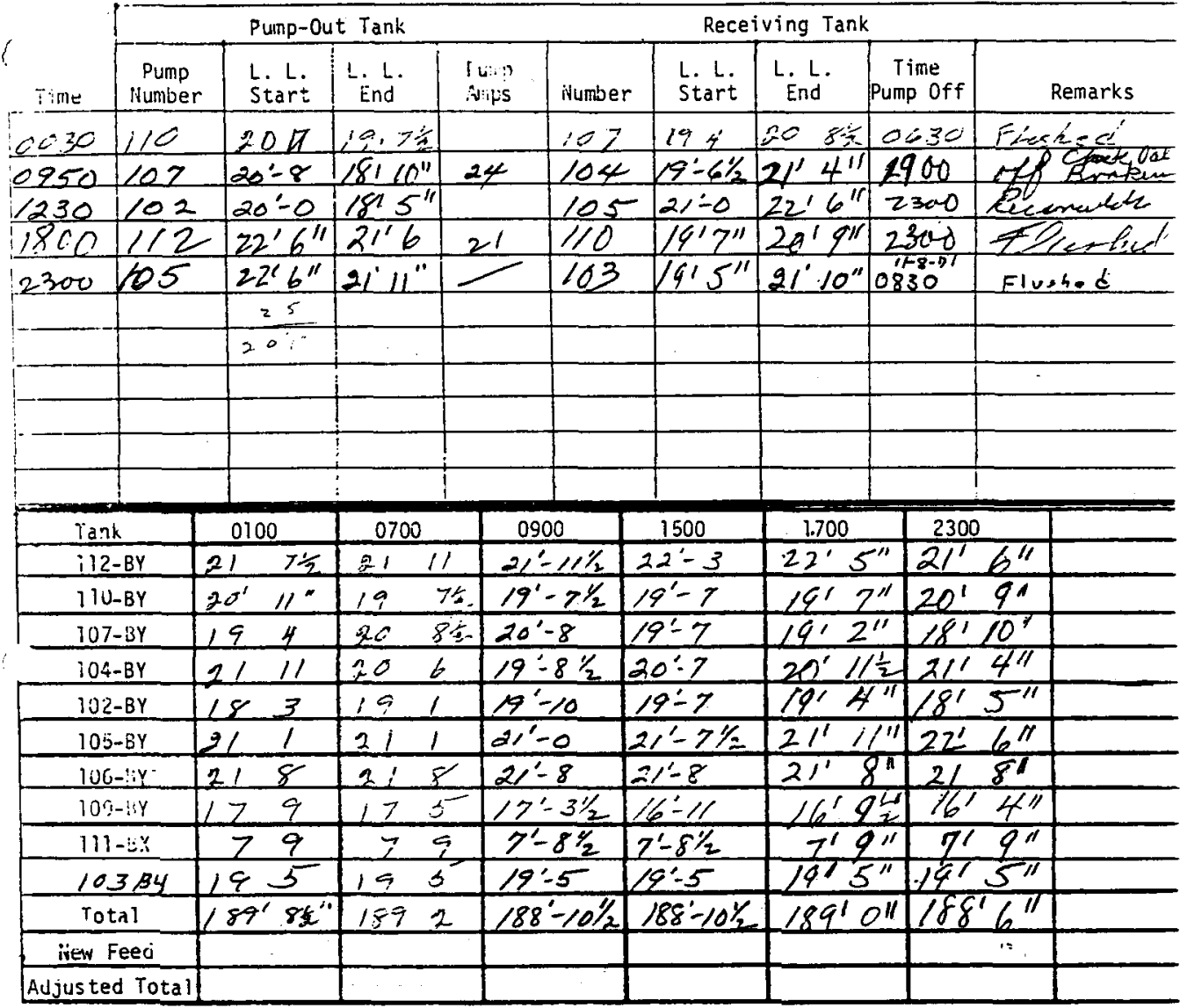

INACTIVE TANKS

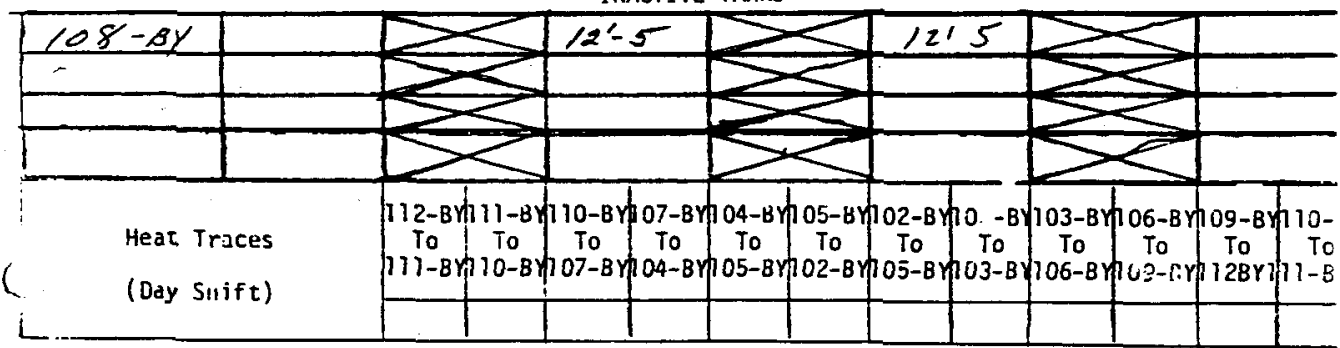




\begin{tabular}{|c|c|c|c|c|c|c|c|c|c|}
\hline \multirow[b]{2}{*}{ Tme } & \multicolumn{4}{|c|}{ Pump-Out Tank } & \multicolumn{4}{|c|}{ Receiving Tank } & \multirow[b]{2}{*}{ Remarks } \\
\hline & $\begin{array}{l}\text { Pump } \\
\text { Number }\end{array}$ & $\begin{array}{l}\text { L. L. } \\
\text { Start }\end{array}$ & $\begin{array}{l}\text { L. L. } \\
\text { End }\end{array}$ & 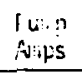 & iNumber & $\begin{array}{l}\text { L. L. } \\
\text { Start }\end{array}$ & $\begin{array}{l}\text { L. L. } \\
\text { End }\end{array}$ & $\begin{array}{c}\text { Time } \\
\text { Pump off }\end{array}$ & \\
\hline 0045 & 110 & $20^{\prime} 9 \ln$ & $18^{\prime} 6^{\prime \prime}$ & & $107^{\circ}$ & $15^{\prime \prime} 11$ & $20^{\prime} \frac{1}{2}$ & 1300 & Floshed \\
\hline $1 / 30$ & 104 & $21^{\prime} 4 \frac{6}{2}$ & 191811 & & 102 & $18^{\prime} 8^{\prime \prime \prime}$ & $20^{\prime} / 1$ & 1900 & Illakkal \\
\hline 1145 & 103 & $21^{\prime} 10^{\prime \prime}$ & $19^{\prime} 6 "$ & & 109 & $15^{\prime} 10^{\prime \prime}$ & $17^{i} 0^{11}$ & 1830 & Eludeno \\
\hline 1900 & 102 & $20^{\prime} 111$ & $18^{\prime} 0^{\prime \prime}$ & & 105 & $21^{\prime} 10^{\prime \prime}$ & $22^{1} 4 \frac{1}{2}$ & 1645 & Pecincubtam \\
\hline 2335 & 112 & $225 \frac{1}{2}$ & $916^{\prime \prime}$ & & 110 & $1915 \frac{31}{4}$ & $20^{\prime \prime \prime \prime}$ & 0830 & Flushed \\
\hline & & & & & & & & & \\
\hline & & & & & & & & & \\
\hline & & & & & & & & & \\
\hline & & & & & & & & & \\
\hline & & & & & & & & & \\
\hline & & & & & & & & & \\
\hline
\end{tabular}

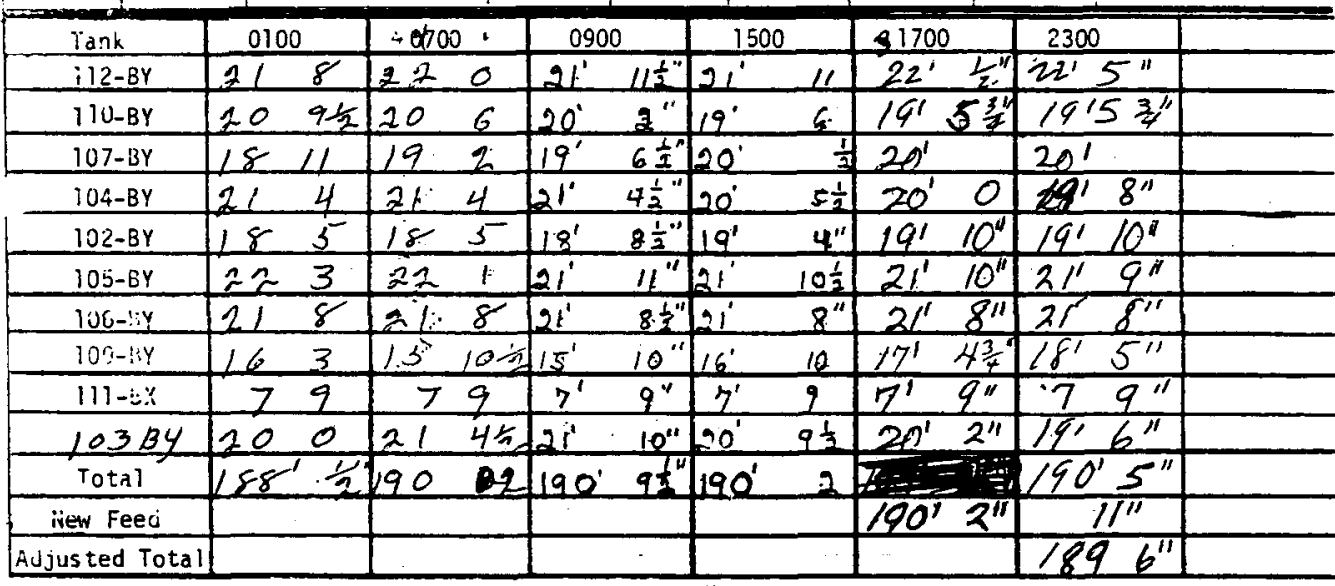

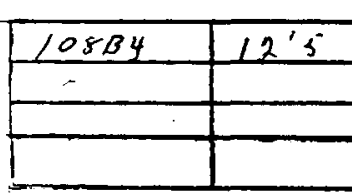

Heat Traces

(Day Siift)

\section{INACTIVE TANKS}

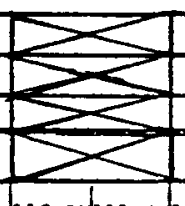

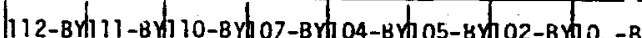

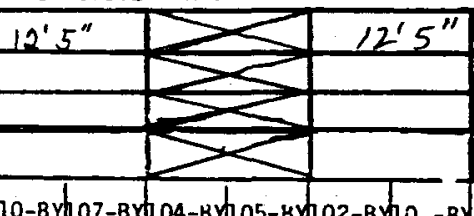

To To To 11-81 10-B का $07-8$

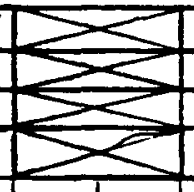

$109-B$ 作

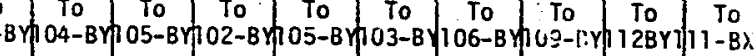




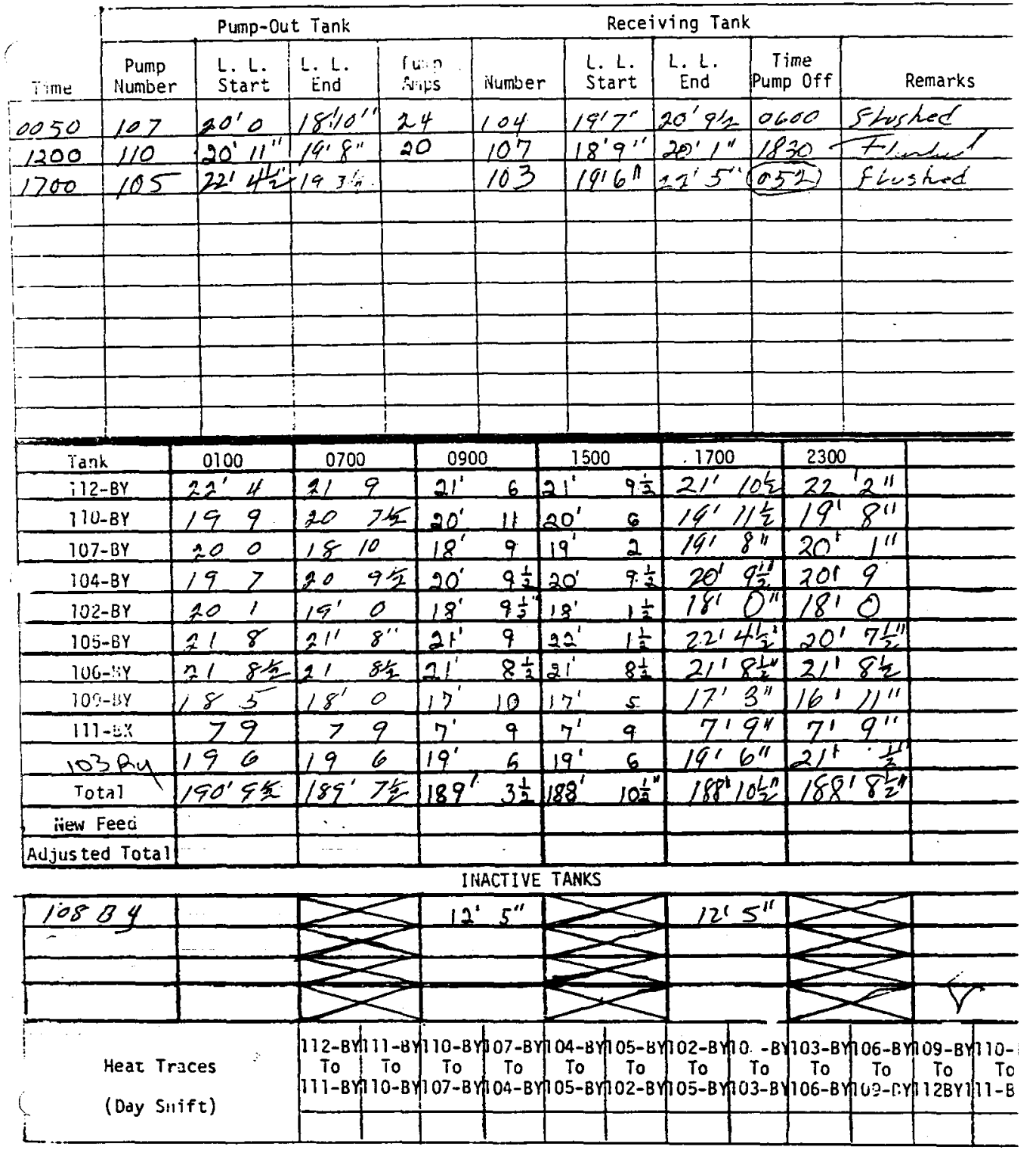




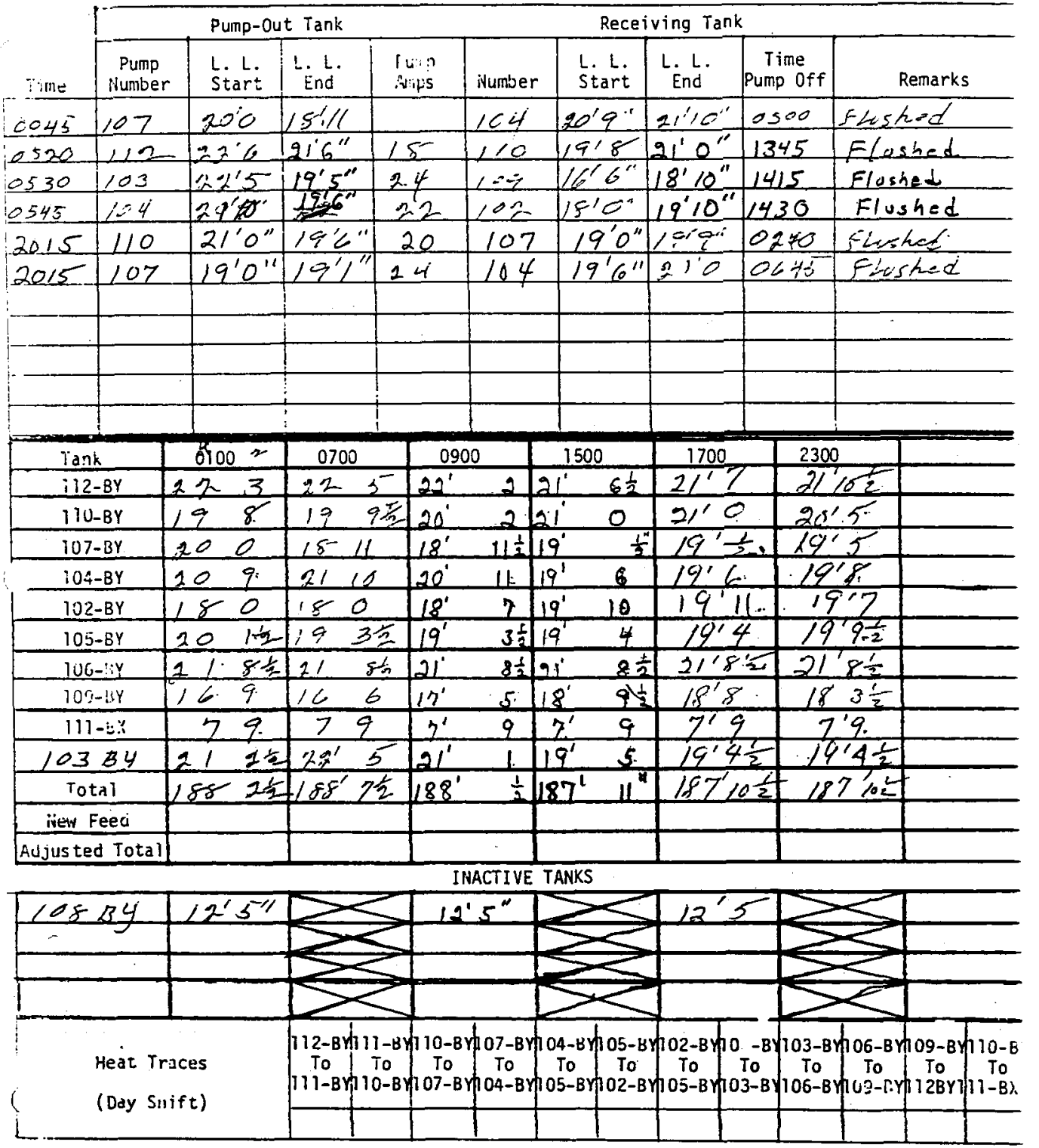




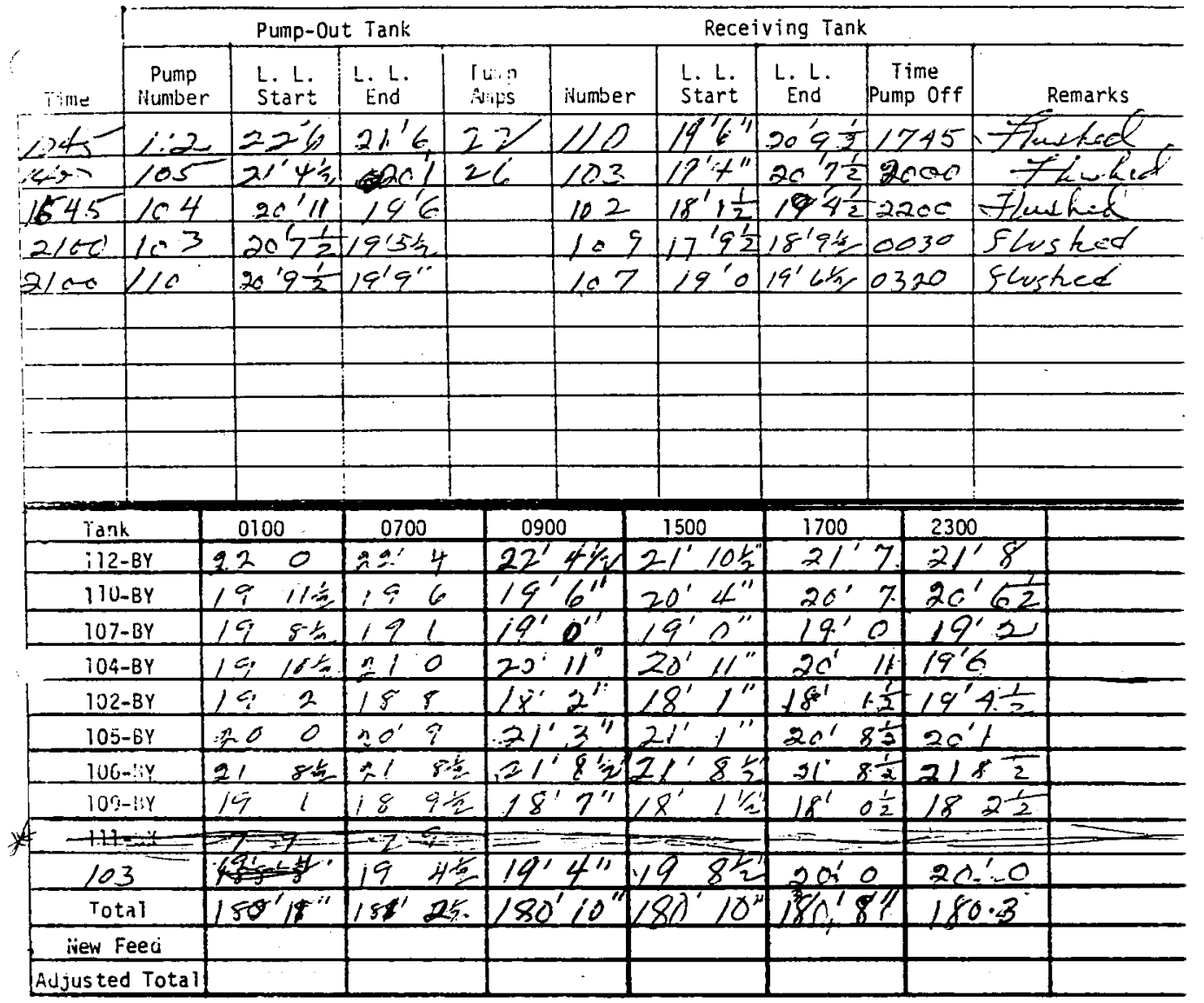

\section{IHACTIVE TANKS}
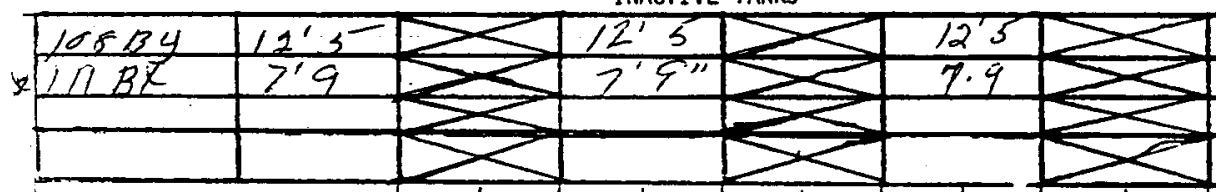

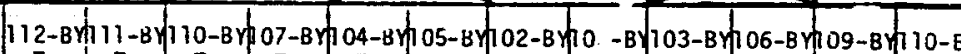

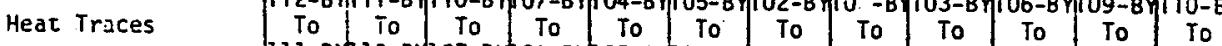

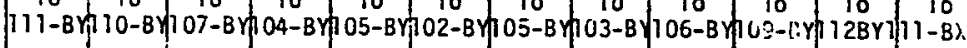

\section{(Day Sijift)}

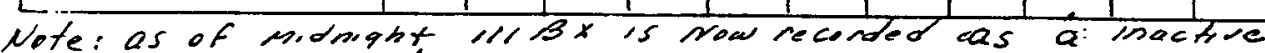
9-¿0-71 Tant. Ret for ap. 


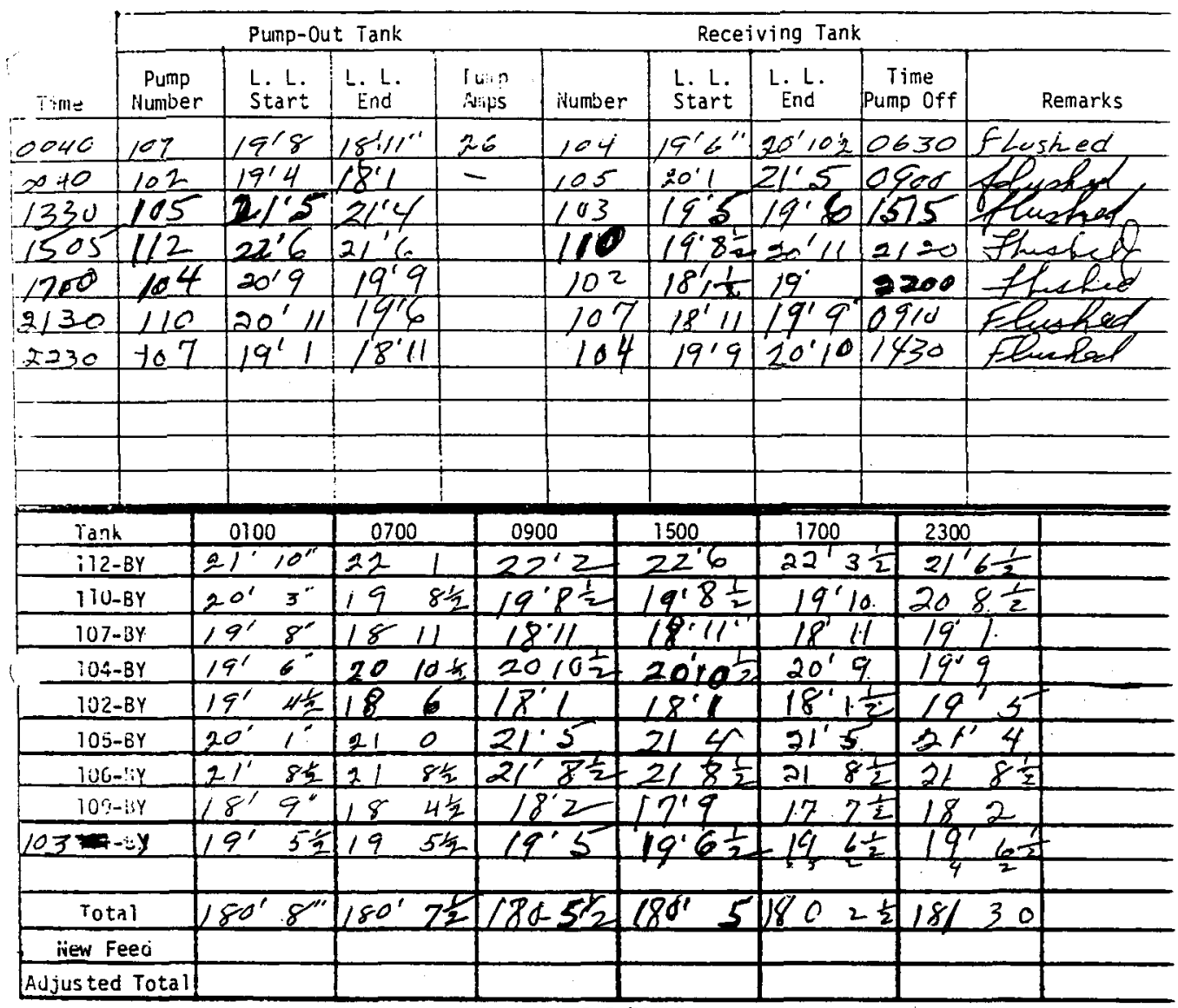

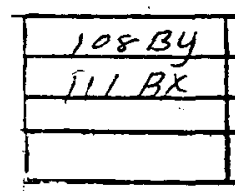

Heat Traces

(Day Suift)

\section{IHACT IVE TANKS}

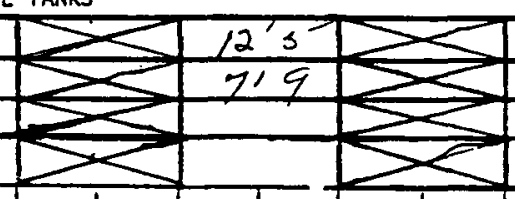

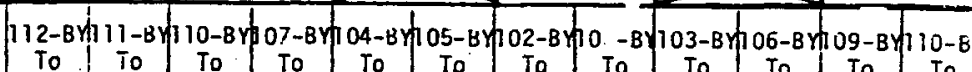

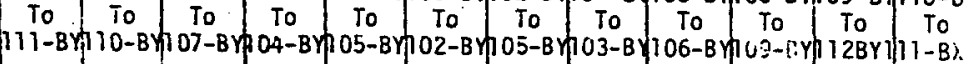


Date $\angle x+2-2$

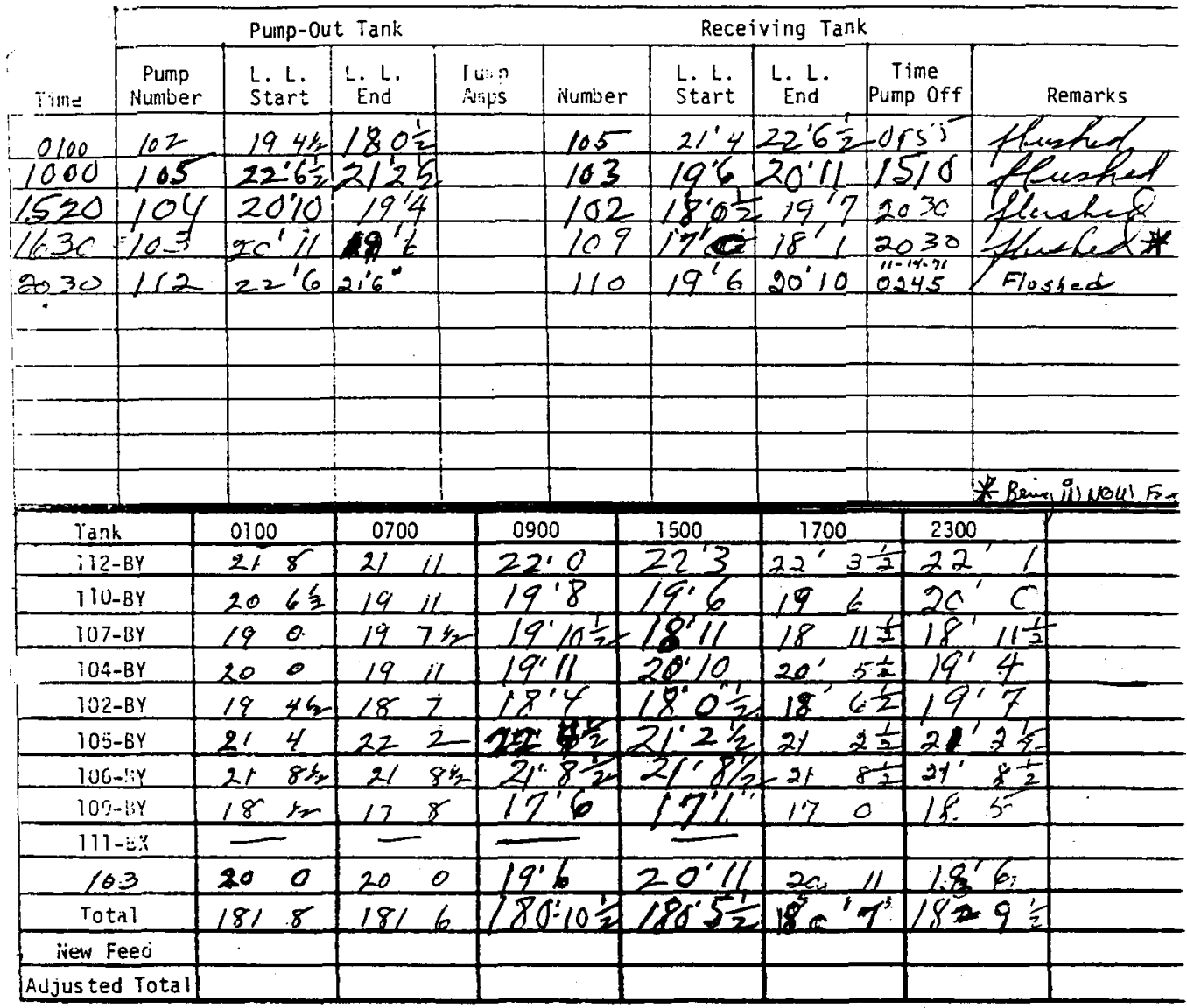

INACTIVE TANKS

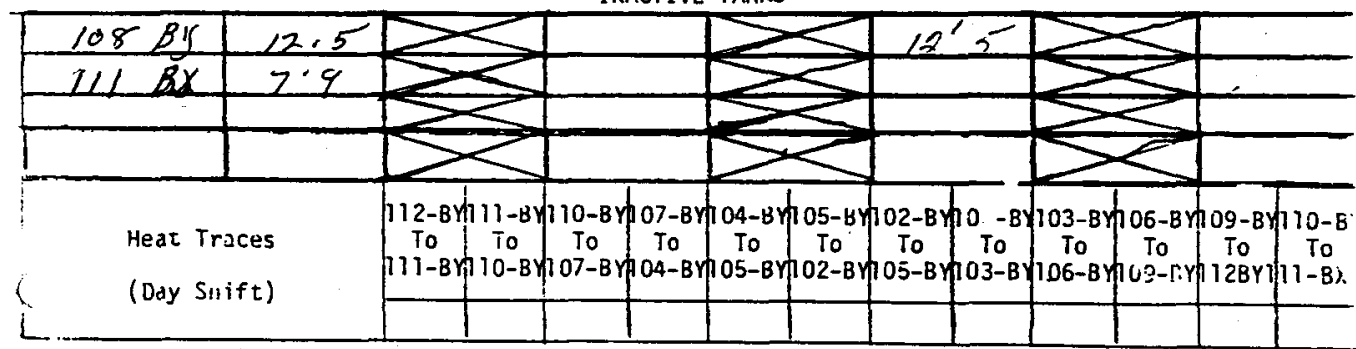




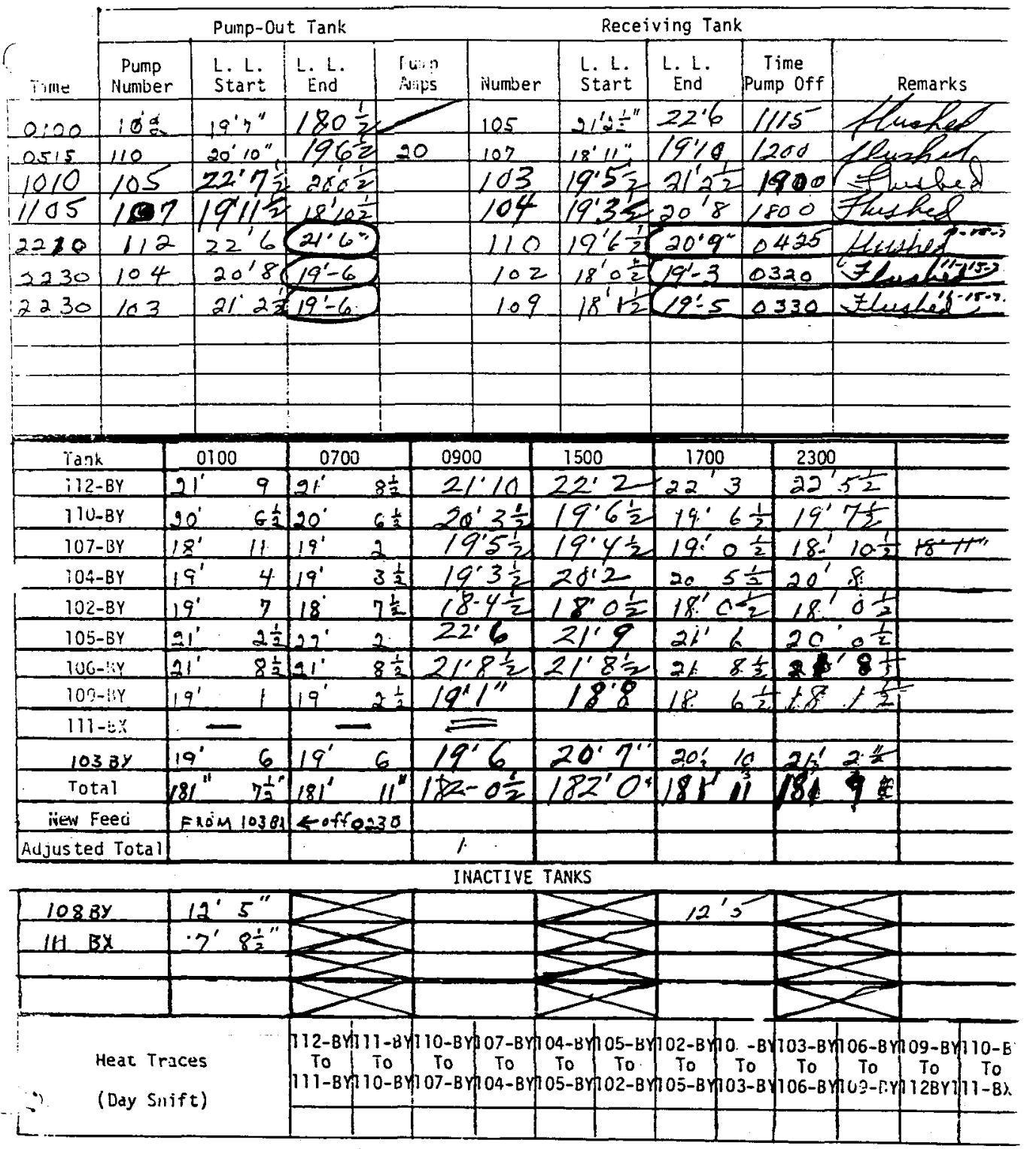




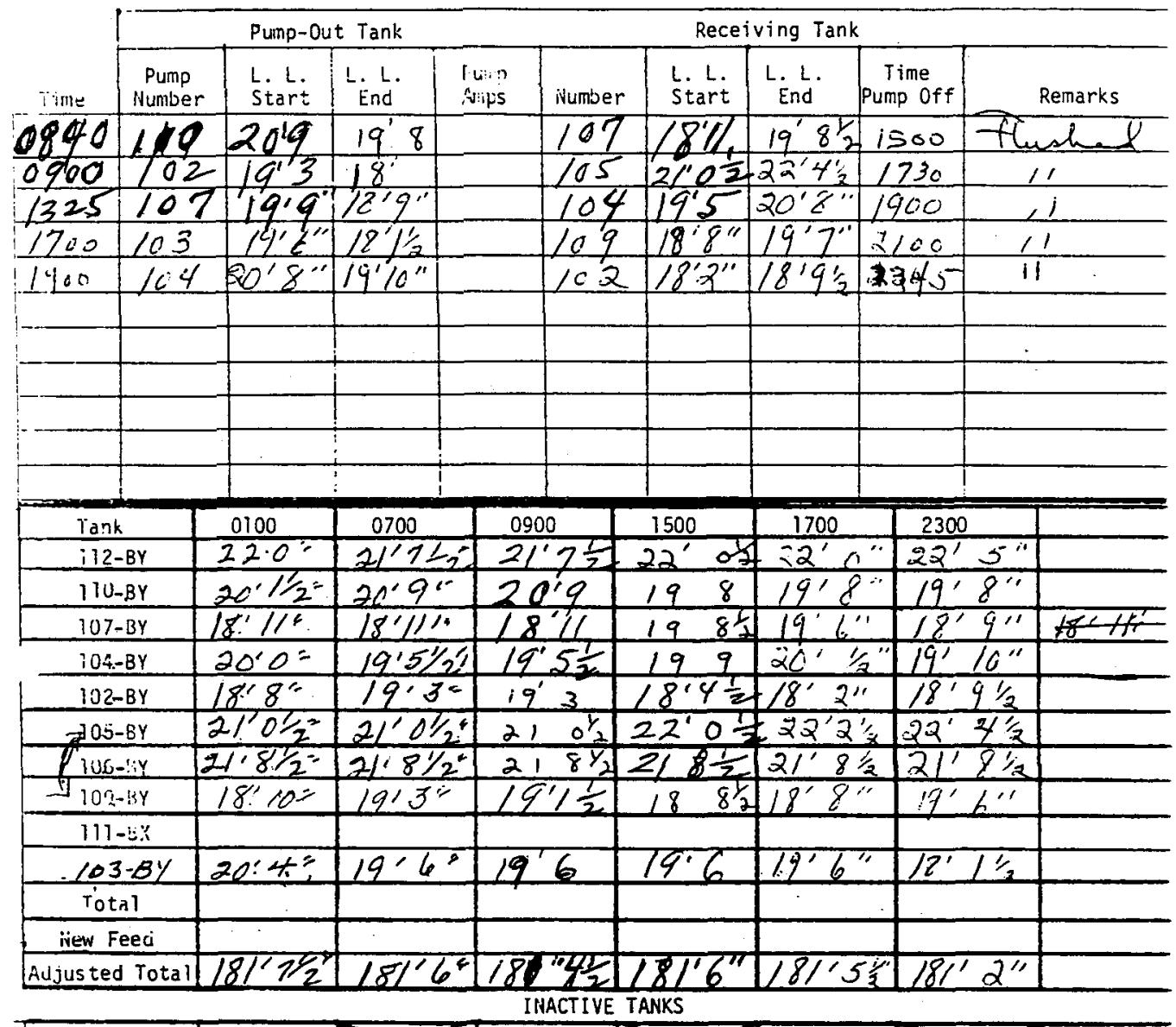

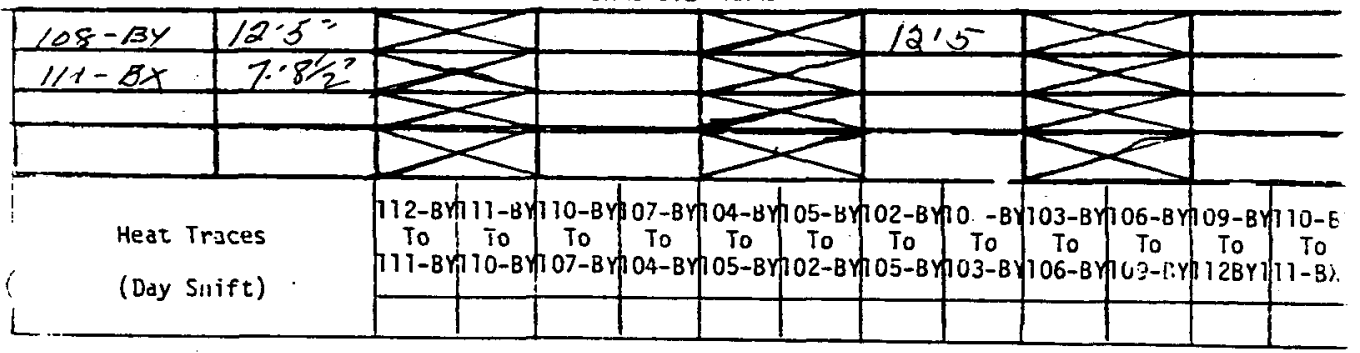




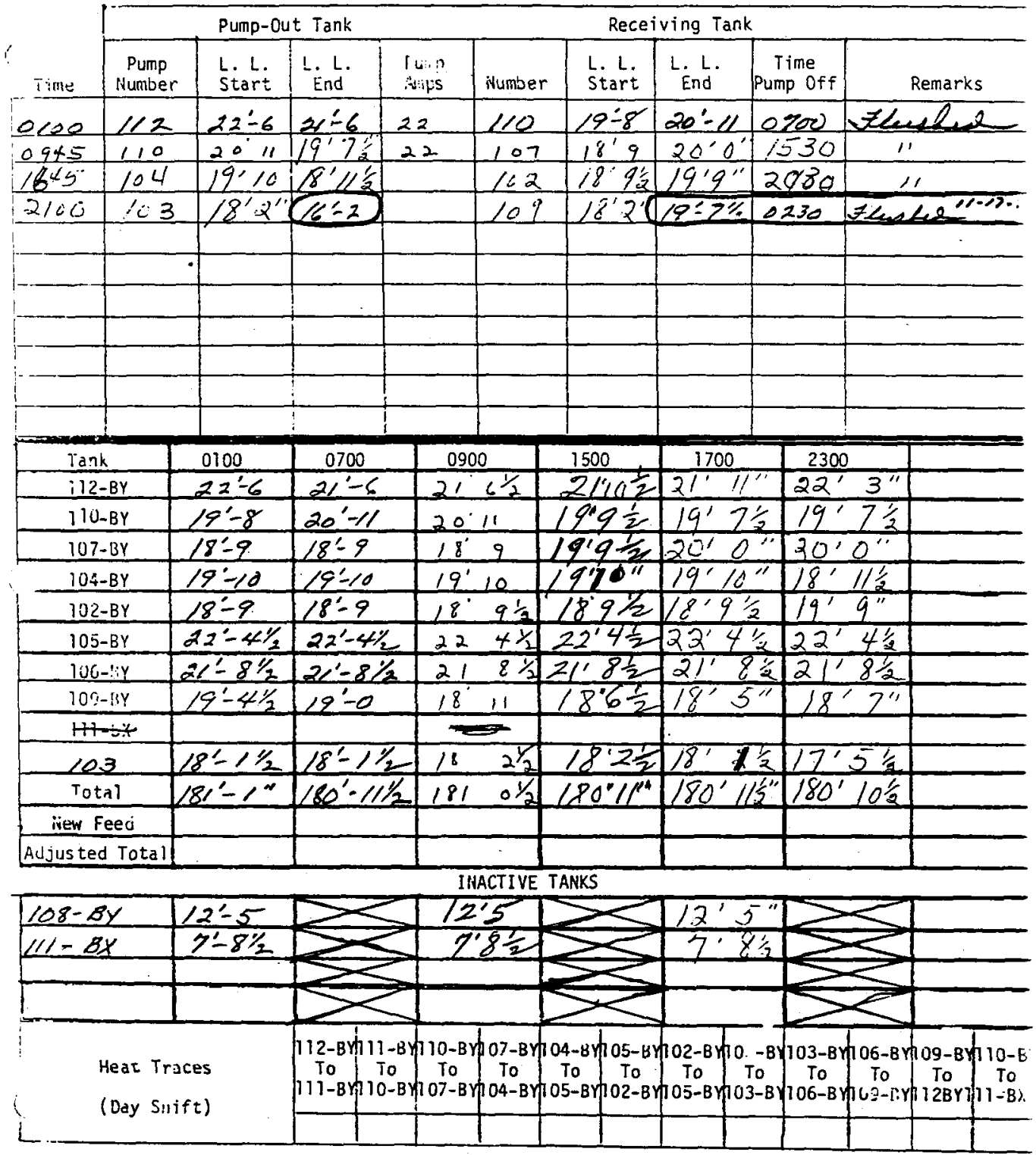




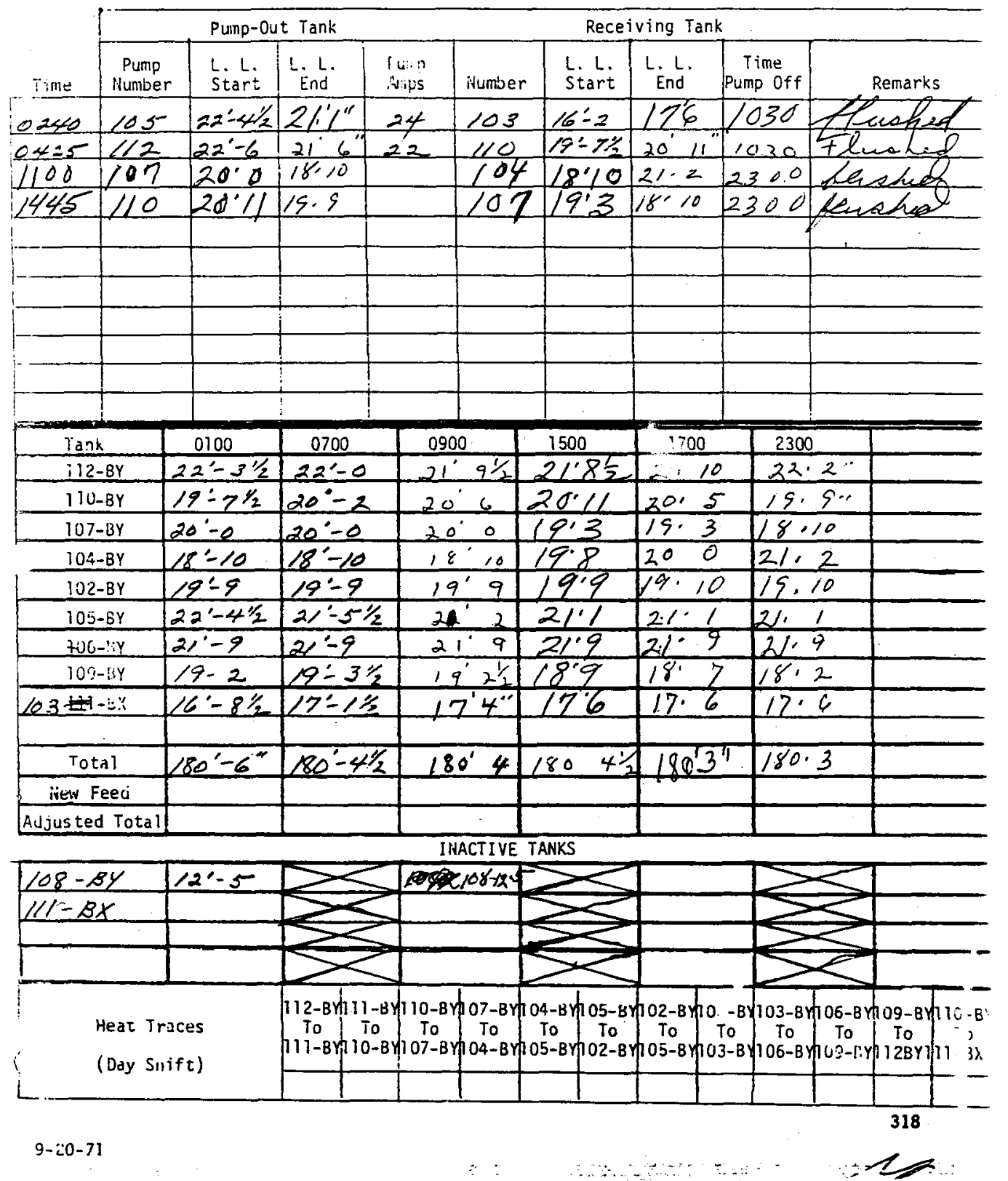




\section{ITS-2 IHVENTORY AND PUMPING \\ Date $1 /-18-31$}

\begin{tabular}{|c|c|c|c|c|c|c|c|c|c|}
\hline \multirow[b]{2}{*}{ Uime } & \multicolumn{4}{|c|}{ Puinp-Out Tank } & \multicolumn{4}{|c|}{ Receiving Tank } & \multirow[b]{2}{*}{ Remarks } \\
\hline & $\begin{array}{l}\text { Pump } \\
\text { Number }\end{array}$ & $\begin{array}{l}\text { L. L. } \\
\text { Start }\end{array}$ & E. $L$. & $\begin{array}{l}\text { lwi? } \\
\text { nips }\end{array}$ & ivumber & $\begin{array}{l}\text { L. L. } \\
\text { Start }\end{array}$ & $\begin{array}{l}\text { L. L. } \\
\text { End }\end{array}$ & $\begin{array}{c}\text { Time } \\
\text { Pump Off }\end{array}$ & \\
\hline 0200 & 102 & $19^{\prime}-9^{\prime \prime}$ & $18^{\prime} 6^{\prime \prime}$ & & 105 & $21-1$ & $226 \frac{13}{21}$ & 1500 & $\Rightarrow \equiv$ Recur \\
\hline 060 & 112 & $22^{\prime}-6$ & $21^{\prime} 6^{\prime \prime}$ & 20 & 110 & $19 \div 9$ & $2011^{\prime \prime}$ & 2330 & Teneto \\
\hline 1400 & 110 & 20111 & 19,7 & 20 & 107 & $18^{\prime} 10^{\prime \prime}$ & $202 \ldots$ & 2230 & Thunes \\
\hline & & & & & & & & & \\
\hline & & & & & & & & & . \\
\hline & & & & & & & & & \\
\hline & & & & & & & & & \\
\hline & & & & & & & & & \\
\hline & & & & & & & & & \\
\hline & & & & & & & & & \\
\hline & & & & & & & & & \\
\hline
\end{tabular}

\begin{tabular}{|c|c|c|c|c|c|c|c|}
\hline Tank & 0100 & 0700 & 0900 & .1500 & 1700 & 2300 & \\
\hline i $12-B Y$ & $22-31 / 2$ & $22^{\prime} 5^{\prime \prime}$ & $22^{\prime} 0$ & $21^{\prime} 7 \frac{1}{2}$ & $21^{\prime \prime}$ & 221 & \\
\hline $110-B Y$ & $19: 9$ & $19^{\prime} 11^{\prime \prime}$ & $20^{\prime} \kappa^{\prime \prime}$ & $20^{\prime} / 1^{\prime \prime}$ & $20^{\prime} 5^{\prime \prime}$ & 19:7: & \\
\hline $107-B Y$ & $18-10$ & $18^{\circ} 10^{\circ}$ & $18^{\prime} 10$ & $18^{\prime} 10^{\prime \prime}$ & $19^{\circ} \cdot 4^{\prime \prime}$ & $30 \cdot 2.1$ & \\
\hline $\mathrm{O} 04-\mathrm{BY}$ & $2 r \div 3$ & $21^{\prime} 3^{\prime \prime}$ & $2 t^{\prime} 2^{\prime \prime}$ & $21^{\prime} 2^{\prime \prime}$ & $21^{\circ} 2^{\prime \prime}$ & $2 / \div 20^{\circ}$ & \\
\hline 102-BY & $19^{\prime}-9 \frac{1}{2}$ & $19^{\prime} 3^{\prime \prime}$ & $\angle G^{\prime} 1^{\prime \prime}$ & $18^{\prime} 6^{\prime \prime}$ & $18^{\prime} 6^{\prime \prime}$ & $18 \cdot 6$ & \\
\hline $105-6 Y$ & $21 \div 1$ & $21^{\prime} 8 \frac{1}{2}$ & $21 / 05$ & 22162 & $22^{\prime} 62^{\prime \prime}$ & $21: 61 / 2$ & \\
\hline $100-5 y$ & $21: 9$ & $21^{\prime} 9^{\prime \prime}$ & $269^{\prime \prime}$ & $21^{\prime} \quad 9 \prime$ & $21^{\prime} 9^{\prime \prime}$ & $21: 9$ & \\
\hline $109-5 y$ & $18-0$ & $17^{\prime} 7^{\prime \prime}$ & $17^{\prime} 5{ }^{\prime \prime}$ & $17^{\prime} 1^{\prime \prime}$ & $17^{\prime}$ & $1.7 \cdot 6$. & \\
\hline $103 \pm+21$ & $17-5 / 2$ & $17^{\prime} 5 k_{2}^{\prime \prime}$ & $17^{\prime} 5 \frac{2}{2}$ & 171 & $17^{\prime}$ & $-17: 512$ & \\
\hline Total & $180^{\circ}-2 / 2$ & $180^{\prime} 2^{\prime \prime}$ & $179^{1} 7^{1 / 4}$ & 1791102 & $179^{\prime} 9^{\prime \prime}$ & 是 & \\
\hline irew Feed & & & & & & & \\
\hline Aujusted Total & & & & & & & \\
\hline
\end{tabular}

INACTIVE TANKS

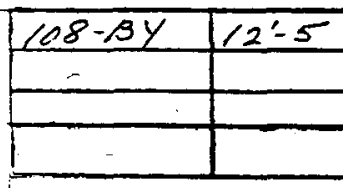

Heat Traces

(Day Snift)

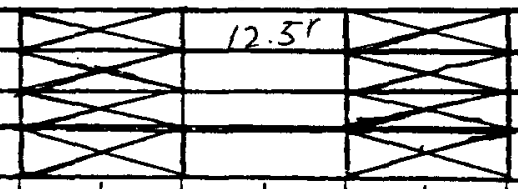

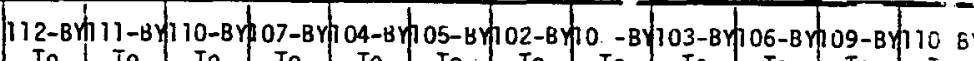

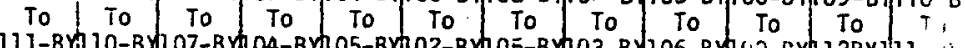

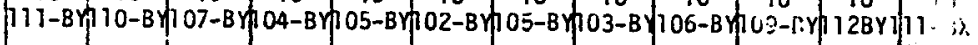
L.

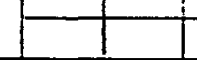




\begin{tabular}{|c|c|c|c|c|c|c|c|c|c|}
\hline \multirow[b]{2}{*}{ Tinle } & \multicolumn{4}{|c|}{ Pump-Out Tank } & \multicolumn{4}{|c|}{ Receiving Tank } & \multirow[b]{2}{*}{ Remarks } \\
\hline & $\begin{array}{l}\text { Pump } \\
\text { Number }\end{array}$ & $\begin{array}{l}\text { L. L. } \\
\text { Start }\end{array}$ & $\begin{array}{l}\text { L. L. } \\
\text { End }\end{array}$ & $\begin{array}{l}\text { fuin } \\
\text { Nitus }\end{array}$ & ivumber & $\begin{array}{l}\text { L. L. } \\
\text { Start }\end{array}$ & L. L. & $\begin{array}{c}\text { Time } \\
\text { Pump off }\end{array}$ & \\
\hline 0400 & 104 & $20^{\prime}-2$ & $19^{\prime} 0^{\prime \prime}$ & 244 & 1044 & $2 \div-2$ & $22^{\prime} 3^{\prime \prime}$ & & \\
\hline 0600 & 103 & $17^{\prime}-5 \%$ & $15^{\prime} 1^{\prime \prime}$ & 25 & 109 & $17^{\prime}-0^{1 / 2}$ & $18^{\prime} 10^{\prime \prime}$ & 1500 & \\
\hline 0960 & $1 / 2$ & $22^{\prime} 6^{\prime \prime}$ & $21 \cdot 6$ & 14 & 110 & $19^{\prime} 7^{\prime \prime}$ & $20 \cdot 11$ & 19.30 & \\
\hline 1300 & 105 & $22^{\prime} 6^{\prime \prime}$ & 20211 & 19 & 103 & $15^{\prime} 1^{\prime \prime}$ & 17.5 & 0100 & 7 lustied \\
\hline & & & & & & & & & \\
\hline & & & & & & & & & \\
\hline & & & & & & & & & \\
\hline & & & & & & & & & \\
\hline & & & & & & & & & \\
\hline & & & & & & & & & \\
\hline & & & & & & & & & \\
\hline
\end{tabular}

\begin{tabular}{|c|c|c|c|c|c|c|c|}
\hline Tank & 0100 & 0700 & 0900 & 1500 & 1700 & 2300 & \\
\hline$i 12-B Y$ & $22^{\prime} 2^{\prime \prime}$ & 22.5 & $22^{\prime} 6^{\prime \prime}$ & $22^{\prime} a^{\prime \prime}$ & $21-9 \cdot 1$ & $21-8$ & \\
\hline IIU-BY & $19^{\prime} 7^{\prime}$ & $19^{\prime 7}$ & $19^{\prime} 7^{\prime \prime}$ & $20^{\prime} 3^{i k}$ & 20.6 & $20-11$ & \\
\hline $107-B Y$ & $20^{\prime} 2 " 1$ & 1417 & $19^{\prime} 4^{\prime \prime}$ & $19^{\circ} 0^{\prime \prime}$ & 19.0 & $19-0$ & \\
\hline $104-B Y$ & $21^{\prime} 2^{\prime \prime}$ & $21^{\prime} \sin ^{\prime \prime}$ & $22^{\prime} 8^{1 / 2}$ & $2 \alpha^{\prime} 3^{\prime \prime}$ & $22 \cdot 3$ & $22-3$ & \\
\hline $102-B Y$ & $18^{\prime} 6$ & 186 & $18^{\prime} 6^{\prime \prime}$ & $18^{\prime} 61 / 2$ & $18 \cdot 6$ & $1.8 \cdot 6$ & \\
\hline $105-B Y$ & $22.6 \div 2$ & $224 \%$ & $2: 2^{\prime} 6^{\prime \prime}$ & $22^{\prime} 1 \frac{1}{2}$ & 21.8 & $20 \cdot 11$ & \\
\hline $106-1: y$ & $21^{\prime} 5^{\prime \prime}$ & $21+9=$ & $21^{\prime} 9^{\prime \prime}$ & $\left.\bar{x}\right|^{\prime} q \cdots$ & $21 \cdot 4$ & $21-9$ & \\
\hline $105-14 y$ & $17^{\prime} 4 \frac{1}{2}$ & $17^{\prime} 4^{\prime \prime}$ & $17^{\prime} 8^{\prime \prime}$ & 18.9. & $18 \cdot 8$ & $18-5$ & \\
\hline $103=8 y$ & $17^{\prime} 5 \frac{1}{2}$ & $17^{\prime} 1^{\prime \prime}$ & $16^{\prime} 6^{\prime \prime}$ & $15^{\prime} 6^{4}$ & $16 \cdot 6$ & 16.9 & \\
\hline Total & $180^{\prime} 8^{\prime} \frac{1}{2}$ & $180^{\prime} \mathrm{b}$ & $180^{\prime} 7^{\prime \prime}$ & 1. & $180 \cdot 7=$ & 180.51 & \\
\hline iiew Feeu & & & & & & & \\
\hline Adjus ted Total & & & & & & & \\
\hline
\end{tabular}

\section{IHACTIVE TANKS}

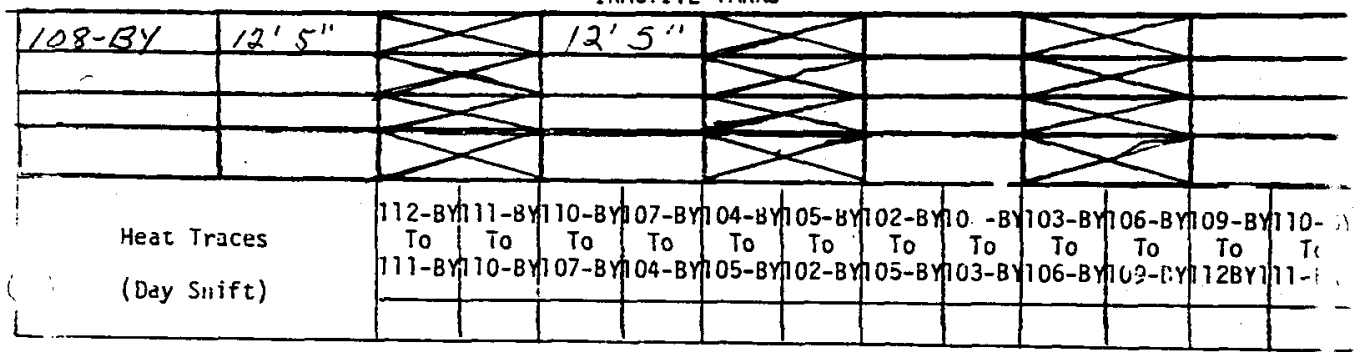




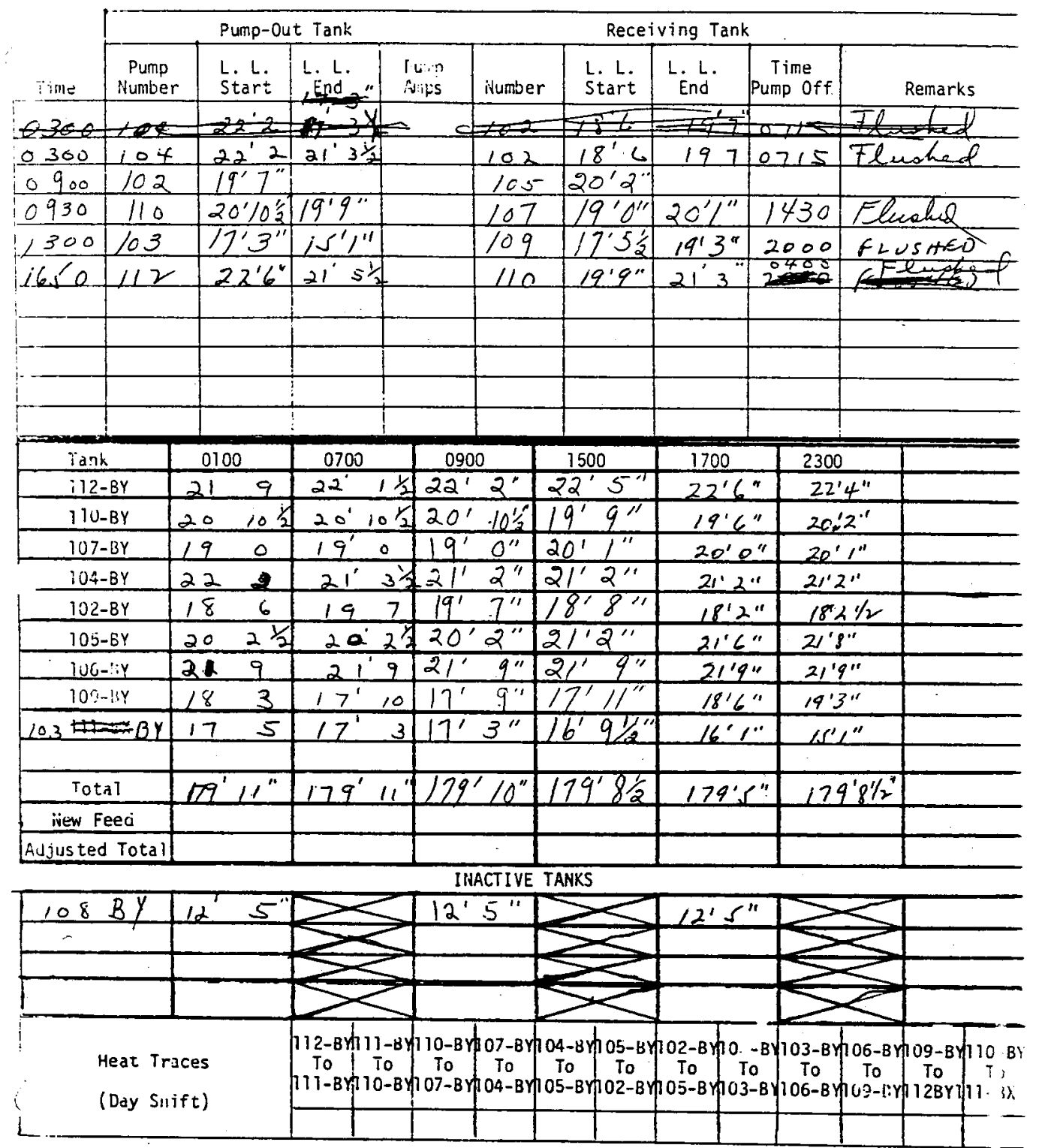




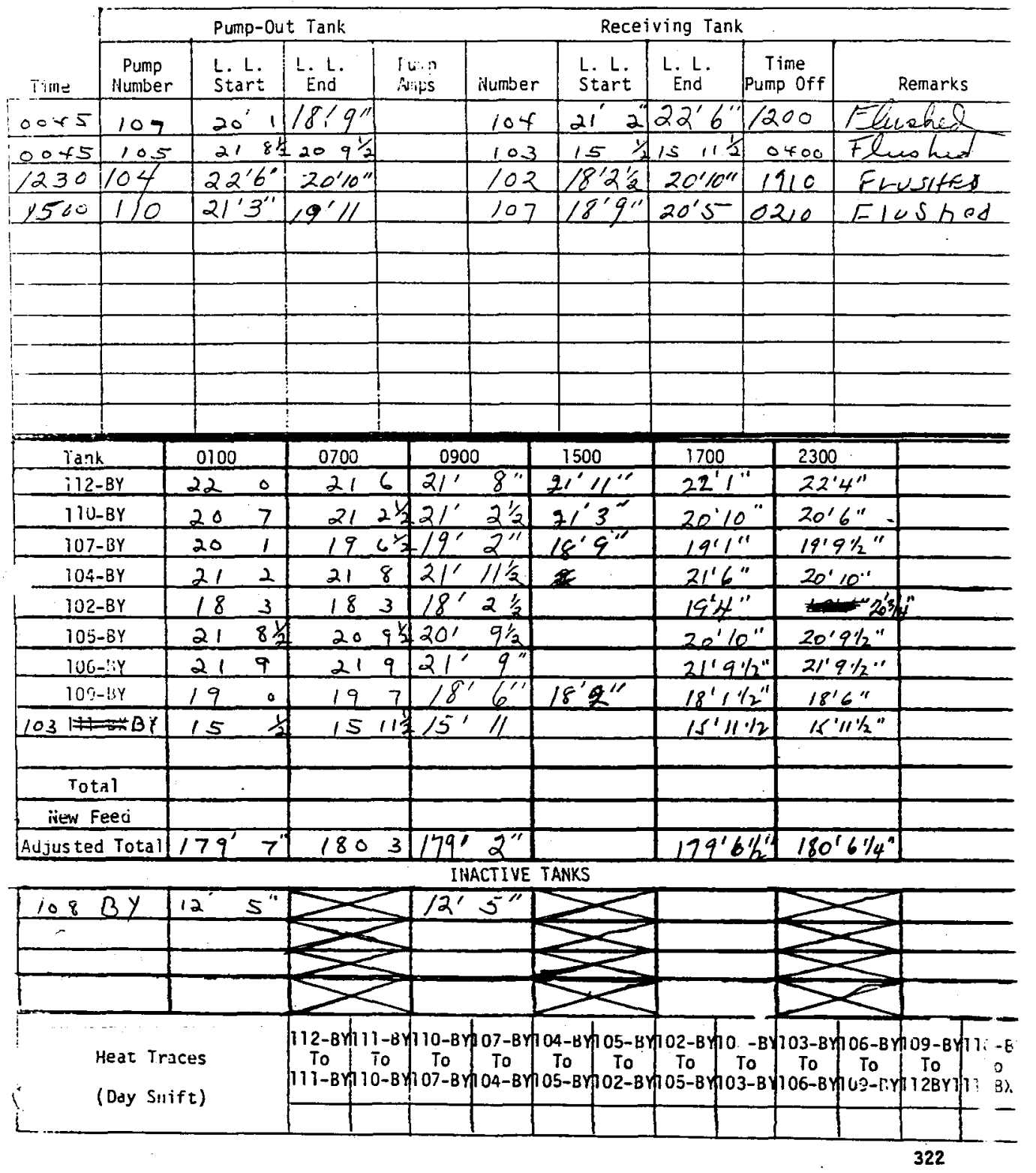




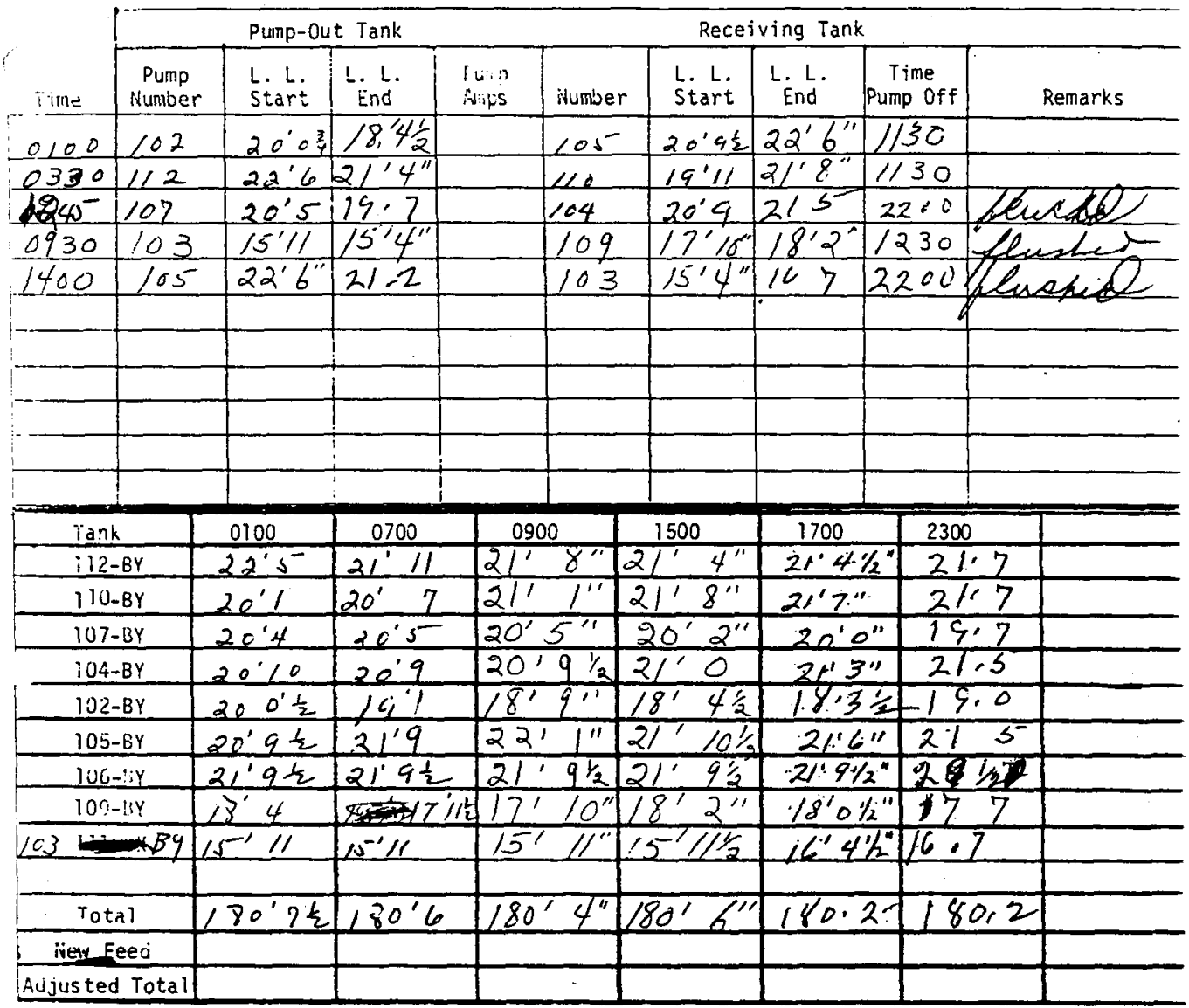

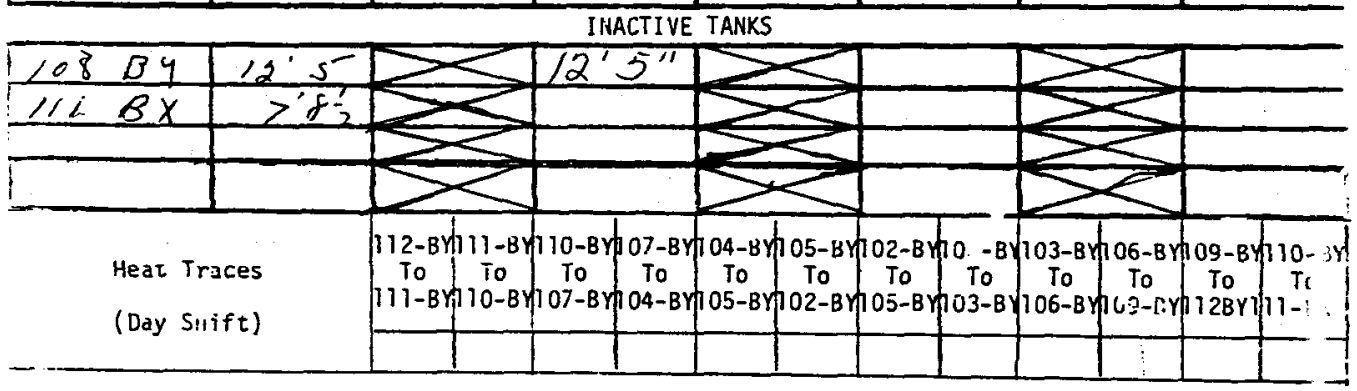




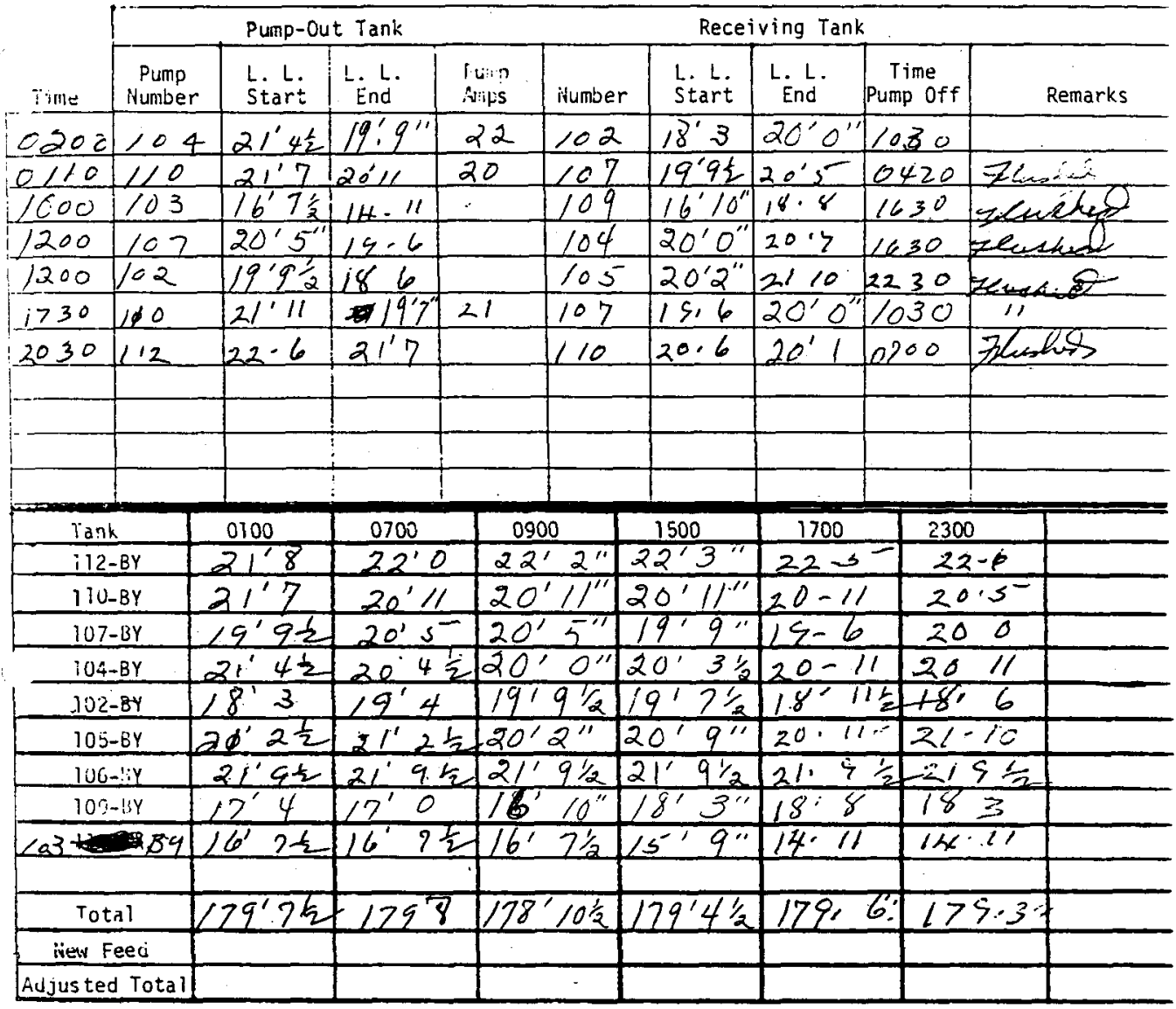

\section{INACTIVE TANKS}

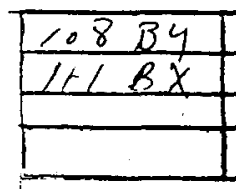

Heat Traces

i (Day Silift)

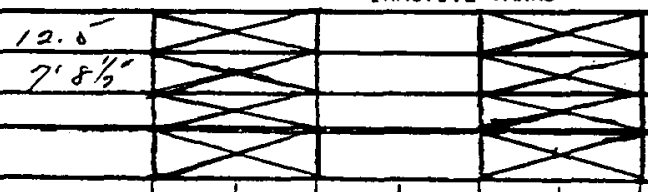

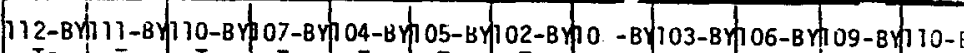

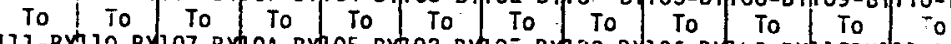

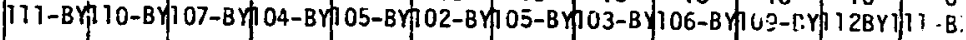




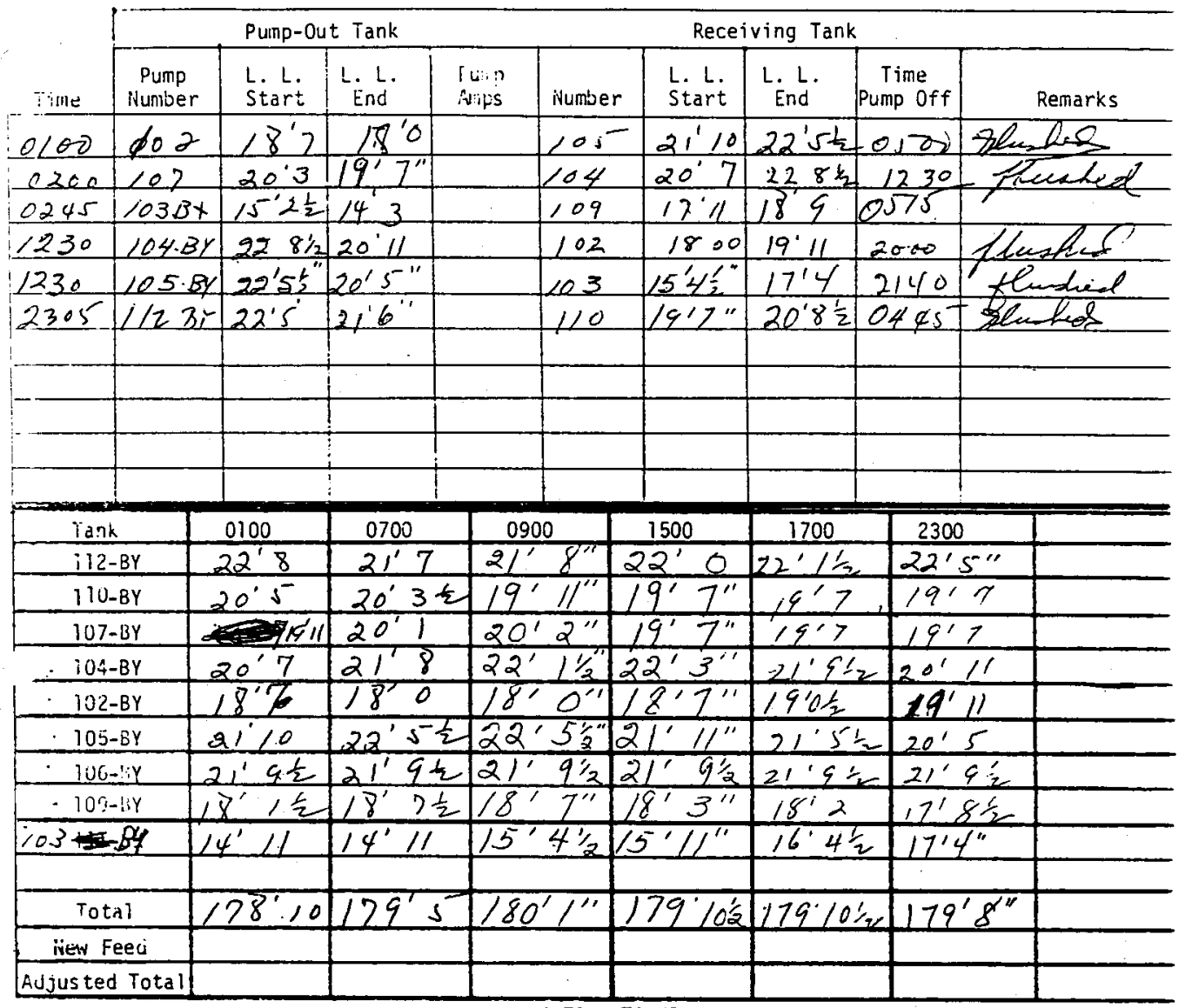

\section{INACTIVE TANKS}

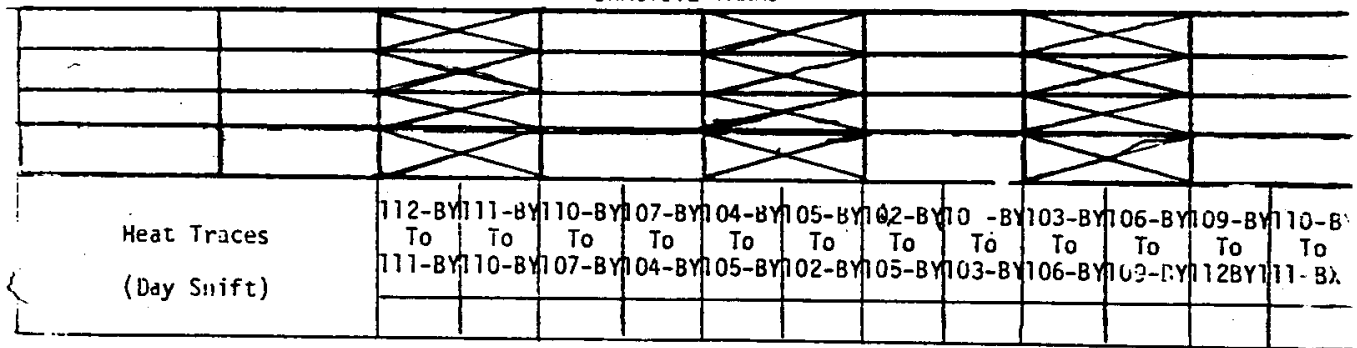




IIS-2 IAVENTORY AND PUMPING Cate $11-2 \not 5-7$

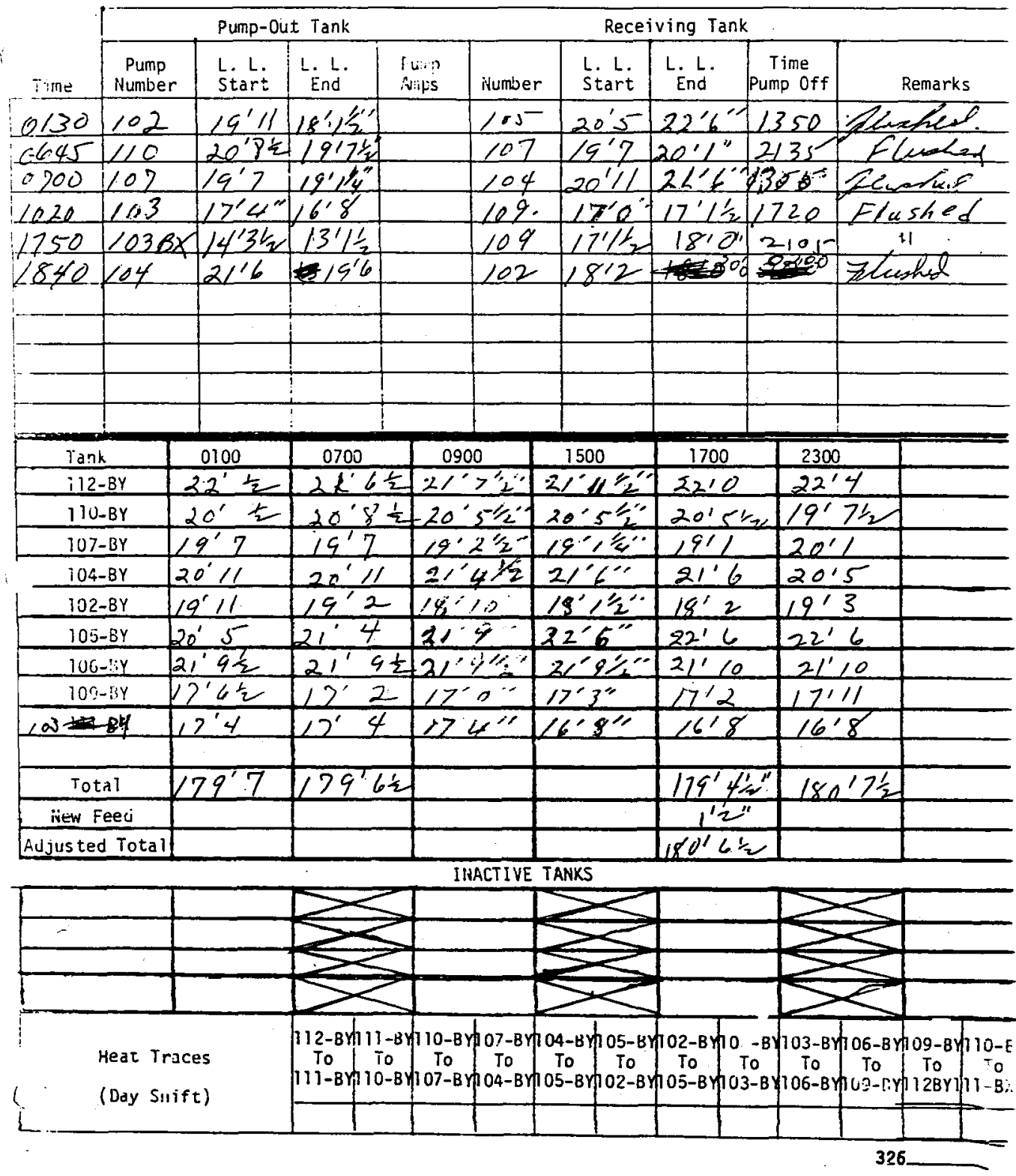




\section{ITS-2 IHIVENTORY AND PUMPING \\ Cate $|1-26-7|$}

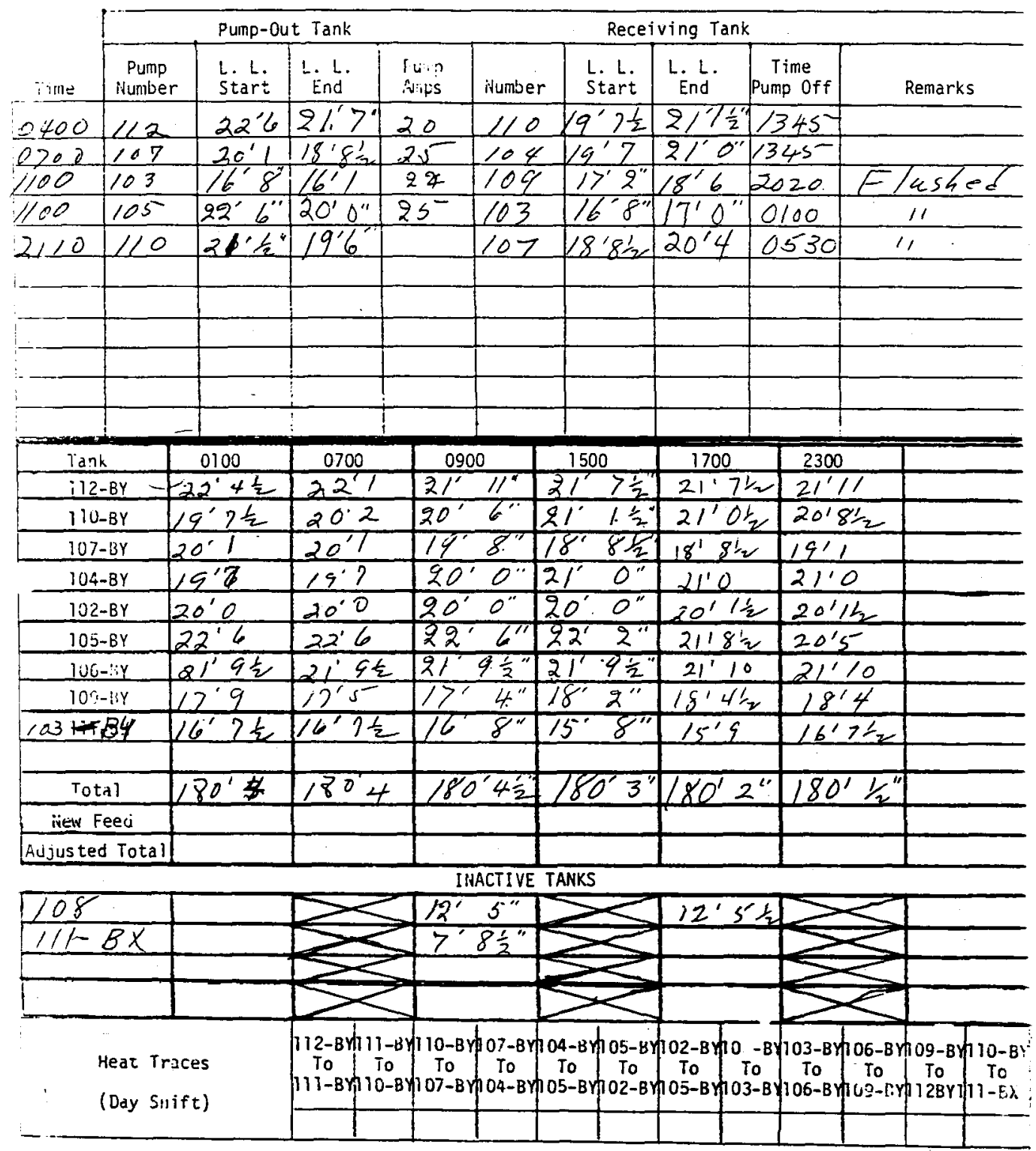


IIS-2 IHVENTORY AND PUMPING Date $11-27-71$

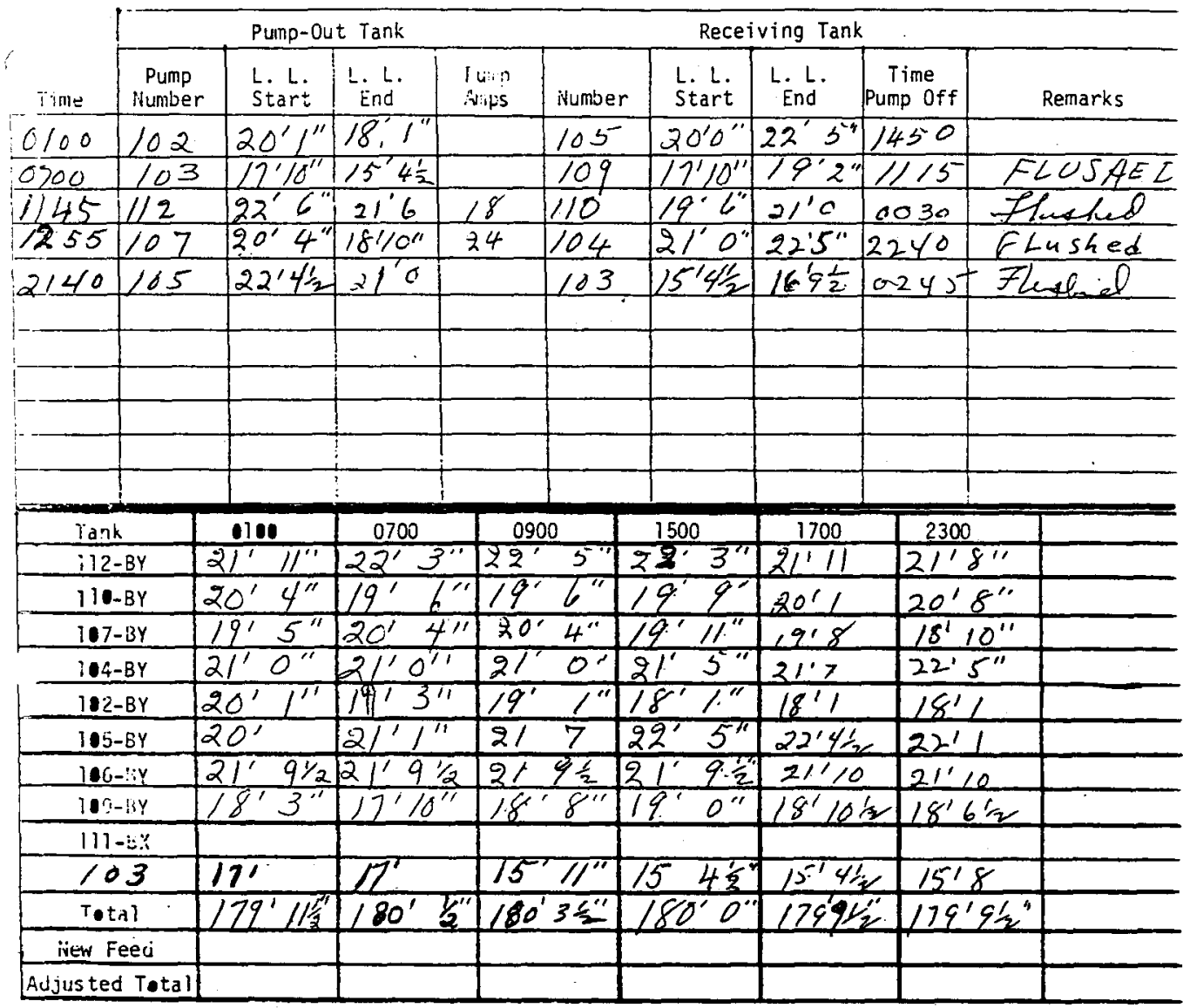

INACTIVE TANKS

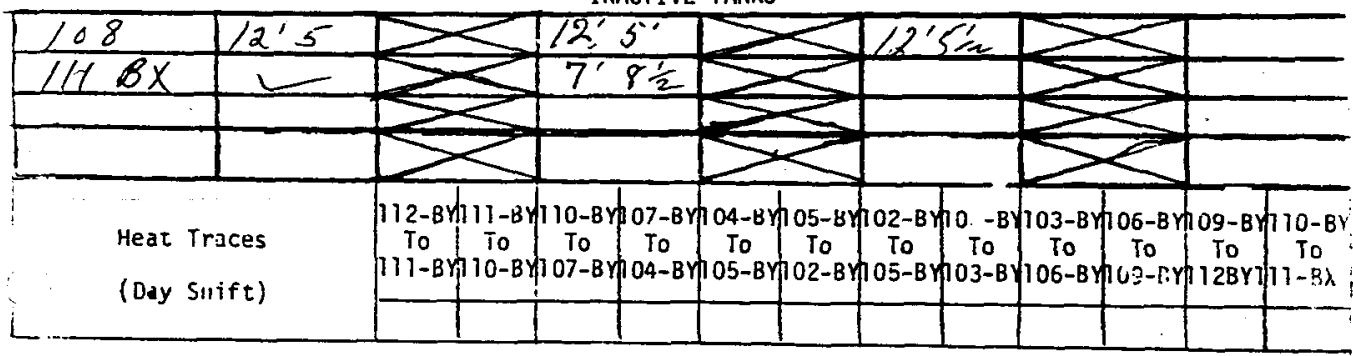




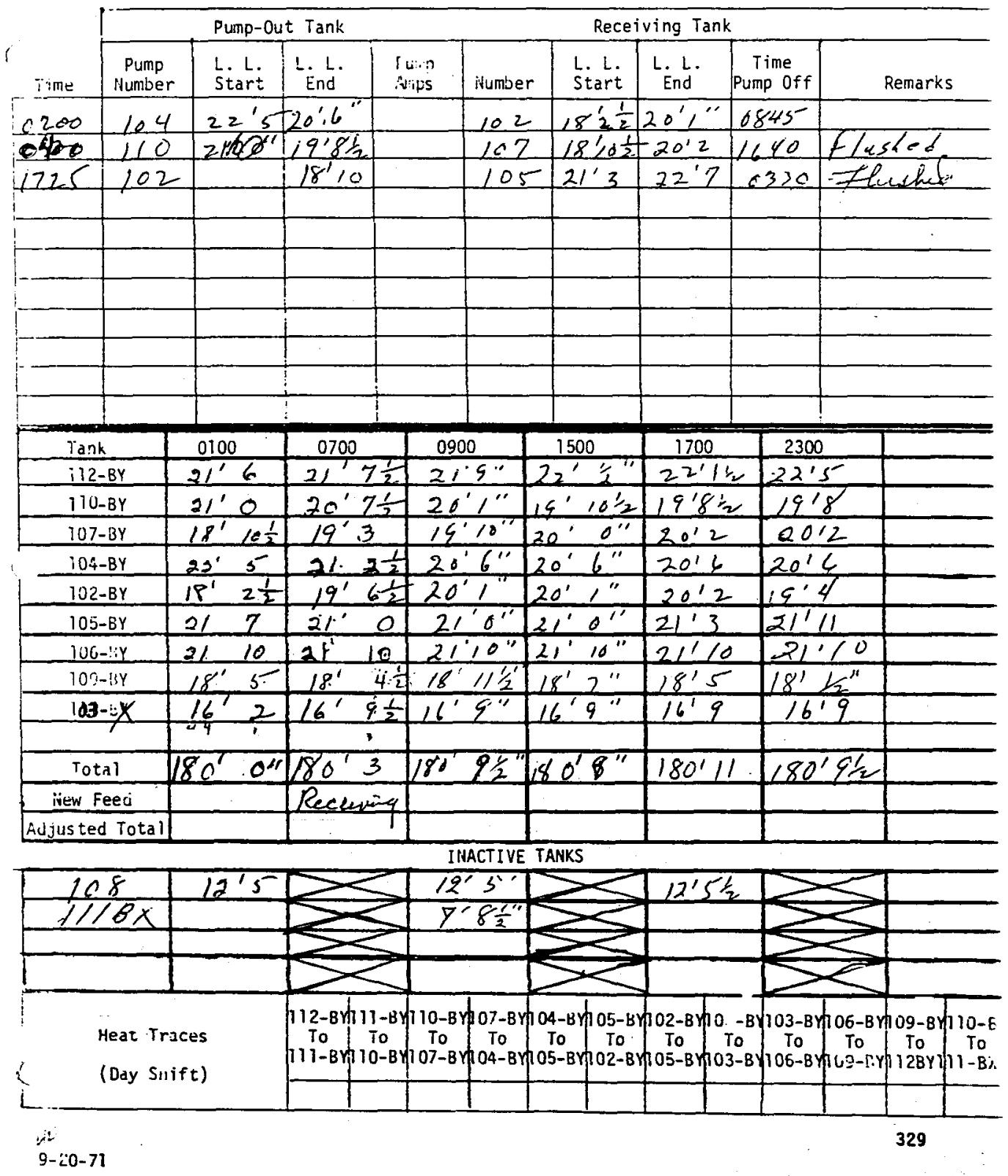




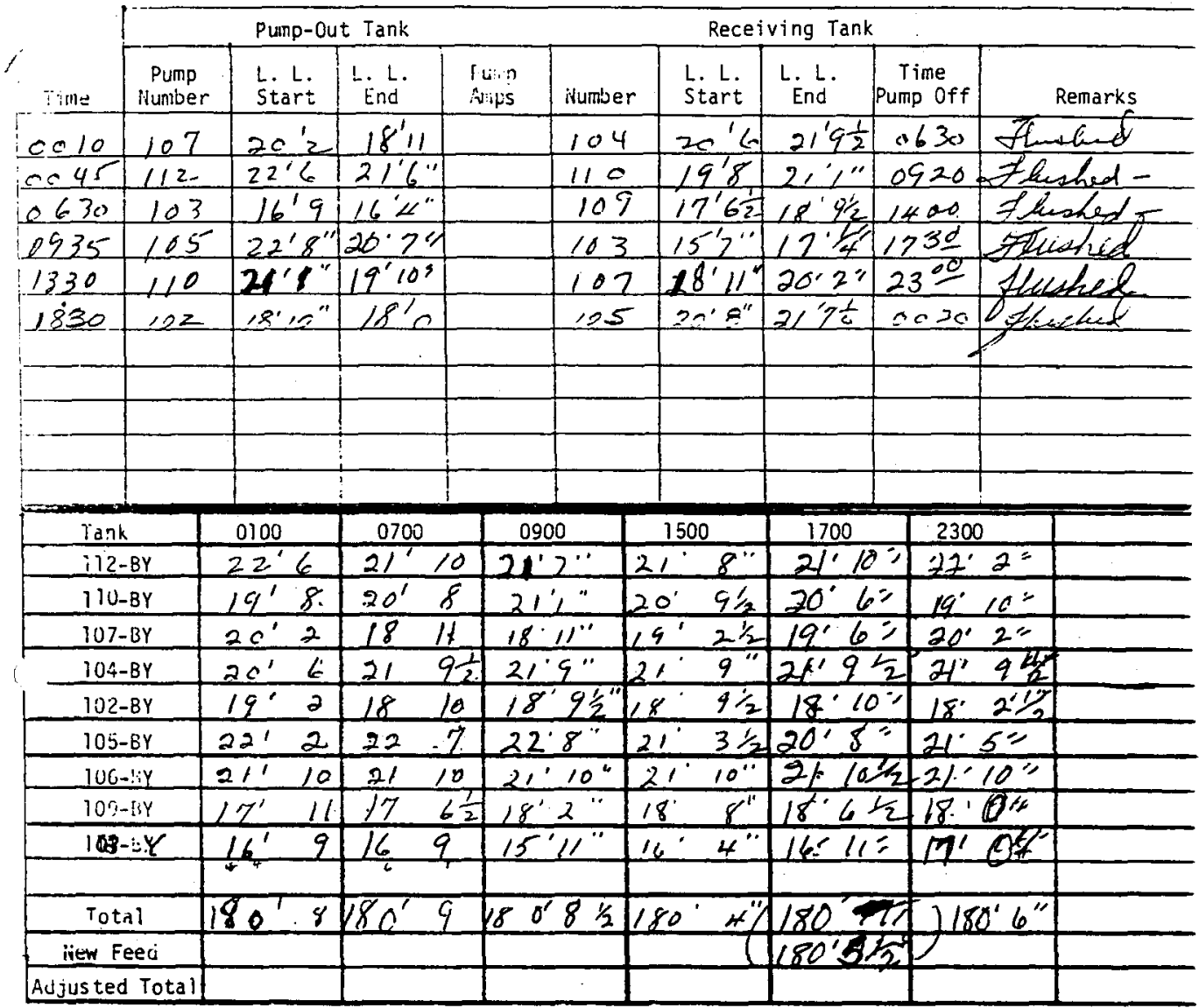

\section{IRACTIVE TANKS}

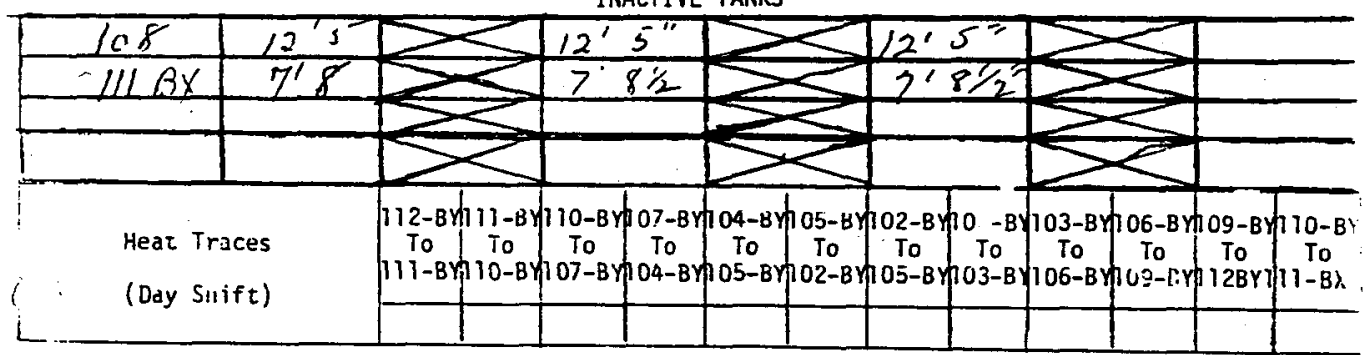




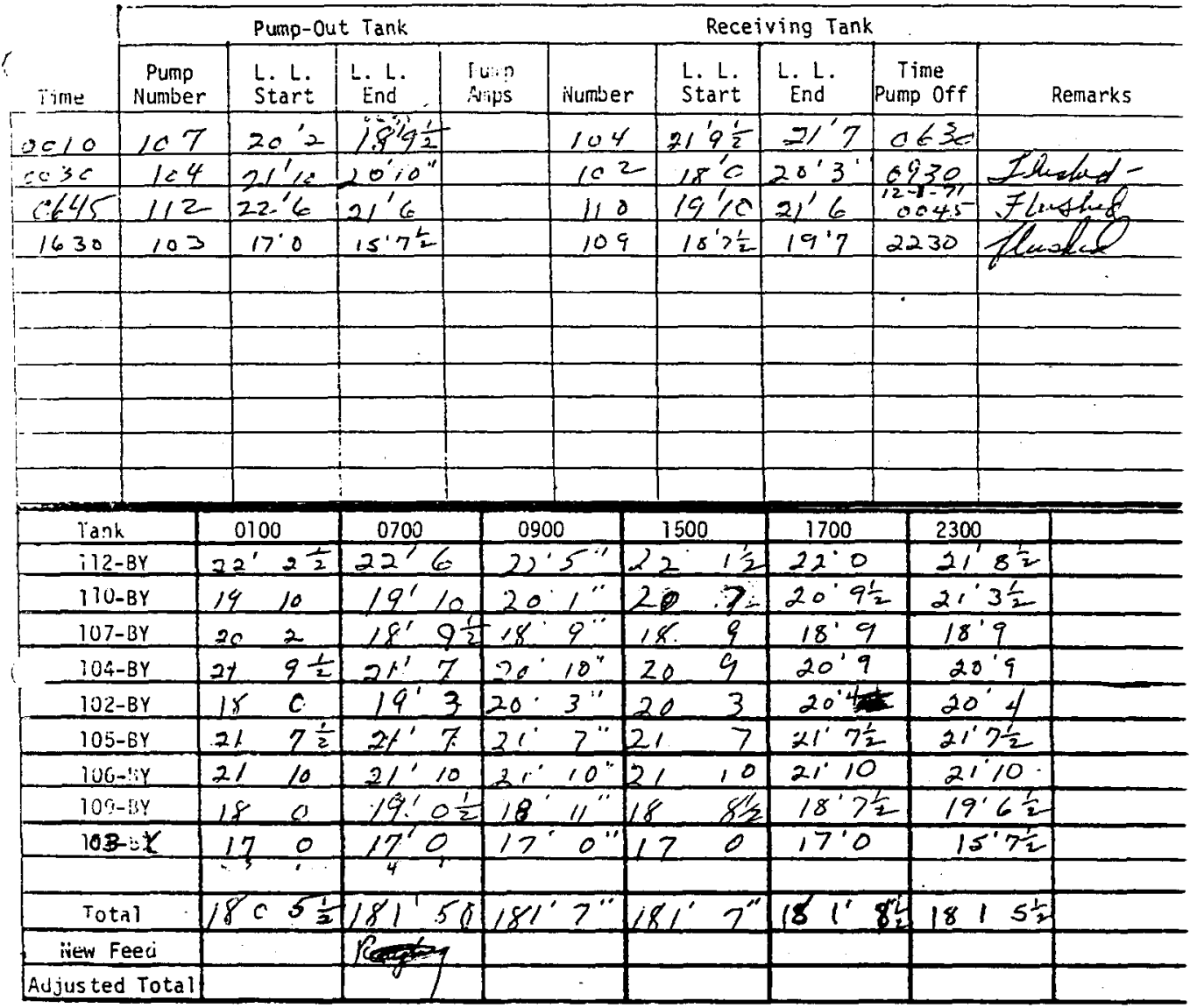

INACTIVE TANKS

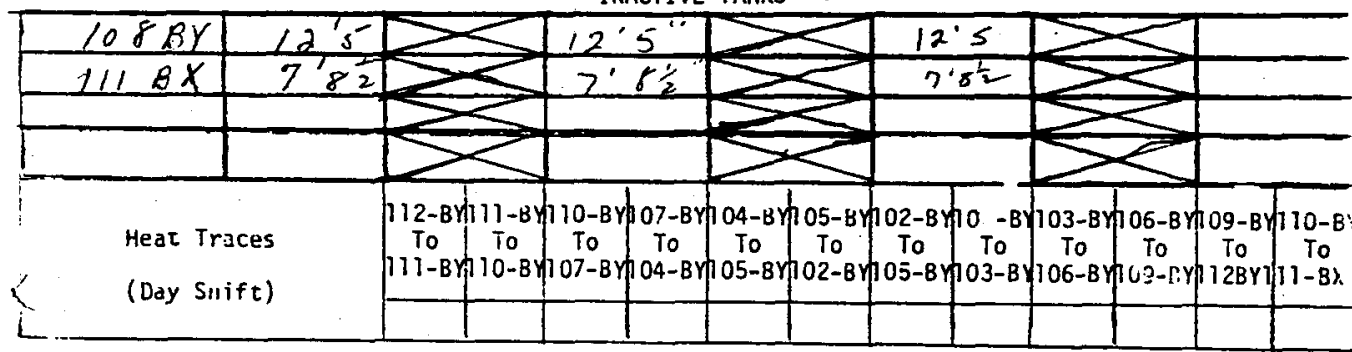




\begin{tabular}{|c|c|c|c|c|c|c|c|c|c|}
\hline \multirow{2}{*}{ Time } & \multicolumn{4}{|c|}{ Punp-Out Tank } & \multicolumn{4}{|c|}{ Receiving Tank } & \multirow[b]{2}{*}{ Remarks } \\
\hline & $\begin{array}{l}\text { Pump } \\
\text { Nunber }\end{array}$ & $\begin{array}{l}\text { L. L. } \\
\text { Start }\end{array}$ & $\begin{array}{l}\text { L. L. } \\
\text { End }\end{array}$ & 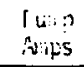 & Humber & $\begin{array}{l}\text { L. L. } \\
\text { start }\end{array}$ & L. L. & $\begin{array}{c}\text { Time } \\
\text { Pump off }\end{array}$ & \\
\hline 315 & $1 / 0$ & $21^{\prime} 6$ & $19^{\prime}, 9^{\prime \prime}$ & 19 & 107 & $\angle \delta^{\prime} \delta$ & $20^{\prime} \mathrm{C}^{\prime \prime}$ & 1330 & \\
\hline$\leqslant 200$ & 10.5 & $21^{\prime} 7$ & $19^{\prime} 4^{\prime \prime}$ & 25 & 103 & $15^{-1} 7 \frac{1}{2}$ & $-17^{\prime} 5^{\prime \prime}$ & 1330 & \\
\hline & 162 & $22^{\prime} 4^{\prime \prime}$ & $18^{\prime} 1$ & & 105 & $19^{\prime} 9^{\prime \prime}$ & $2216 \frac{1}{2}$ & $0<30$ & $t \operatorname{lok} d$ \\
\hline 1900 & 107 & $205 / 2$ & $18 \%$ & 25 & 104 & $208 \%$ & $22^{\prime} 5^{\prime \prime}$ & 0300 & FLUSHED \\
\hline & 112 & $22^{\prime} 6 "$ & & & DT & 1940 & & & $\cdot$ \\
\hline & & & & & & & & & \\
\hline & & & & & & & & & \\
\hline & & & & & & & & & \\
\hline & & & & & & & & & \\
\hline & & & & & & & & & \\
\hline & & & & & & & & & \\
\hline
\end{tabular}

\begin{tabular}{|c|c|c|c|c|c|c|c|}
\hline Tank & 0100 & 0700 & 0900 & 1500 & 1700 & 2300 & \\
\hline i12-BY & $21^{\prime} \leq$ & $21^{\prime} 8 \frac{1}{5}$ & $21^{\circ} 10^{\prime \prime}$ & $222^{\circ}$ & $23^{\prime} 0^{\prime \prime}$ & 22 & \\
\hline $110-B Y$ & 2166 & $21^{\prime} 0 \frac{1}{2}$ & $20^{\prime} \quad 8^{\prime \prime}$ & $19^{\circ} 9^{\circ}$ & $19^{\prime} 10$ & $19 \quad 10$ & \\
\hline $107-B Y$ & $18^{\prime} 9$ & $19^{\prime} 3$ & $19^{\prime} 8$ & $206^{\prime \prime}$ & $20^{\circ} 51 / 2$ & 197 & \\
\hline$\$ 04-B Y$ & $.20^{\prime} \quad 8 \frac{1}{2}$ & $208 \frac{1}{2}$ & $20^{\prime} 8 \frac{1}{2} "$ & $20^{\prime} E^{\prime} / \varepsilon^{\prime}$ & $20 \% 8 \%$ & 217 & \\
\hline $102-B Y$ & $20^{\prime} 4^{\prime \prime}$ & $2 n^{\prime} 4^{\prime \prime}$ & $20^{\prime} 4^{\prime \prime}$ & $22^{\prime} 4^{\prime \prime}$ & 2072 & 191 & $20^{\prime}$ \\
\hline $305-6 y$ & $21^{\prime} 7$ & $21^{\prime} 7$ & $21^{\prime} 2 \frac{1}{2}$ & $19^{\circ} 9^{\circ}$ & $\frac{3}{30}$ & 214 & $11^{\prime \prime}$ \\
\hline $100-3 y$ & $21^{\prime} \quad 10 \frac{1}{2}$ & $26^{\prime} 10 \frac{1}{2}$ & $21^{\prime} 10 \frac{1}{2}$ & 210.10 & $21 \quad 10 \%$ & $2110 \%$ & $=5^{\prime}$ \\
\hline $30 r_{-13 y}$ & $19^{\prime} 5$ & $190 \frac{1}{2}$ & $18^{\prime} 11^{\prime \prime}$ & $28 \times 2 " 4$ & 672 & 181. & \\
\hline IOB:V & $1572 \frac{7}{2}$ & $\angle 5 \quad 7 \frac{2}{2}$ & $16^{\prime} 0^{\prime \prime}$ & $17^{\prime} \mathrm{s}^{\prime \prime}$ & 17 & 175 & \\
\hline & & & & & & & \\
\hline Total & $181^{\prime} \quad 3 \frac{1}{2}$ & 1812 & $181^{\prime} 2 \frac{1}{2}$ & $181^{\prime} 7^{\prime \prime}$ & $8 / 2$ & $1812 \%$ & \\
\hline iiew Feed & & & & & & - & \\
\hline Aujus ted Totai & & & & & & & \\
\hline
\end{tabular}

INACTIVE TANKS

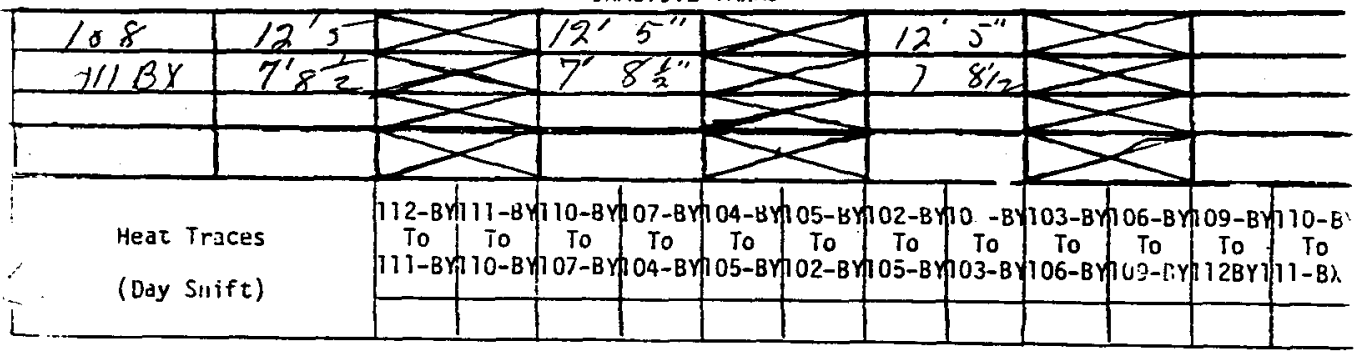




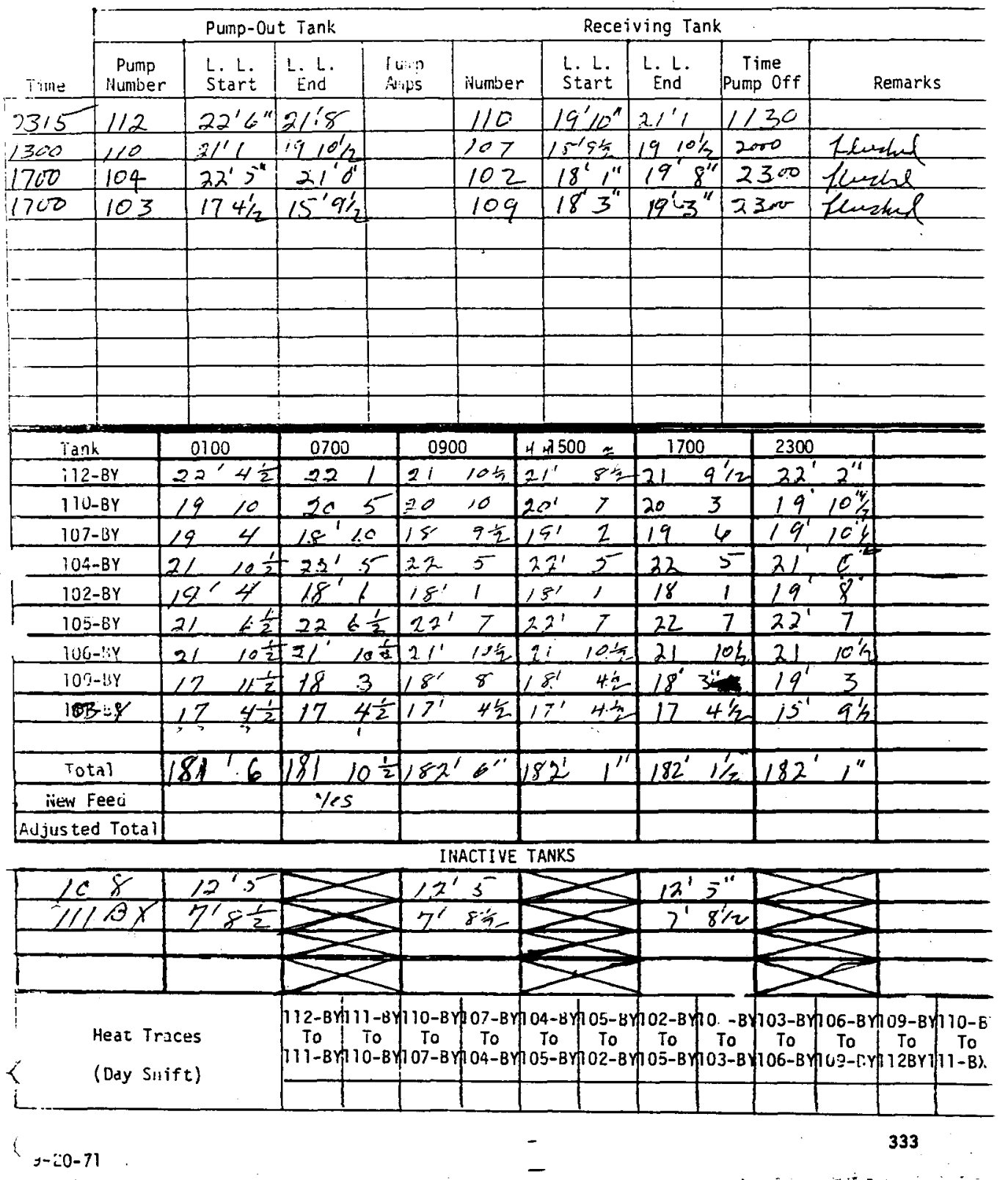




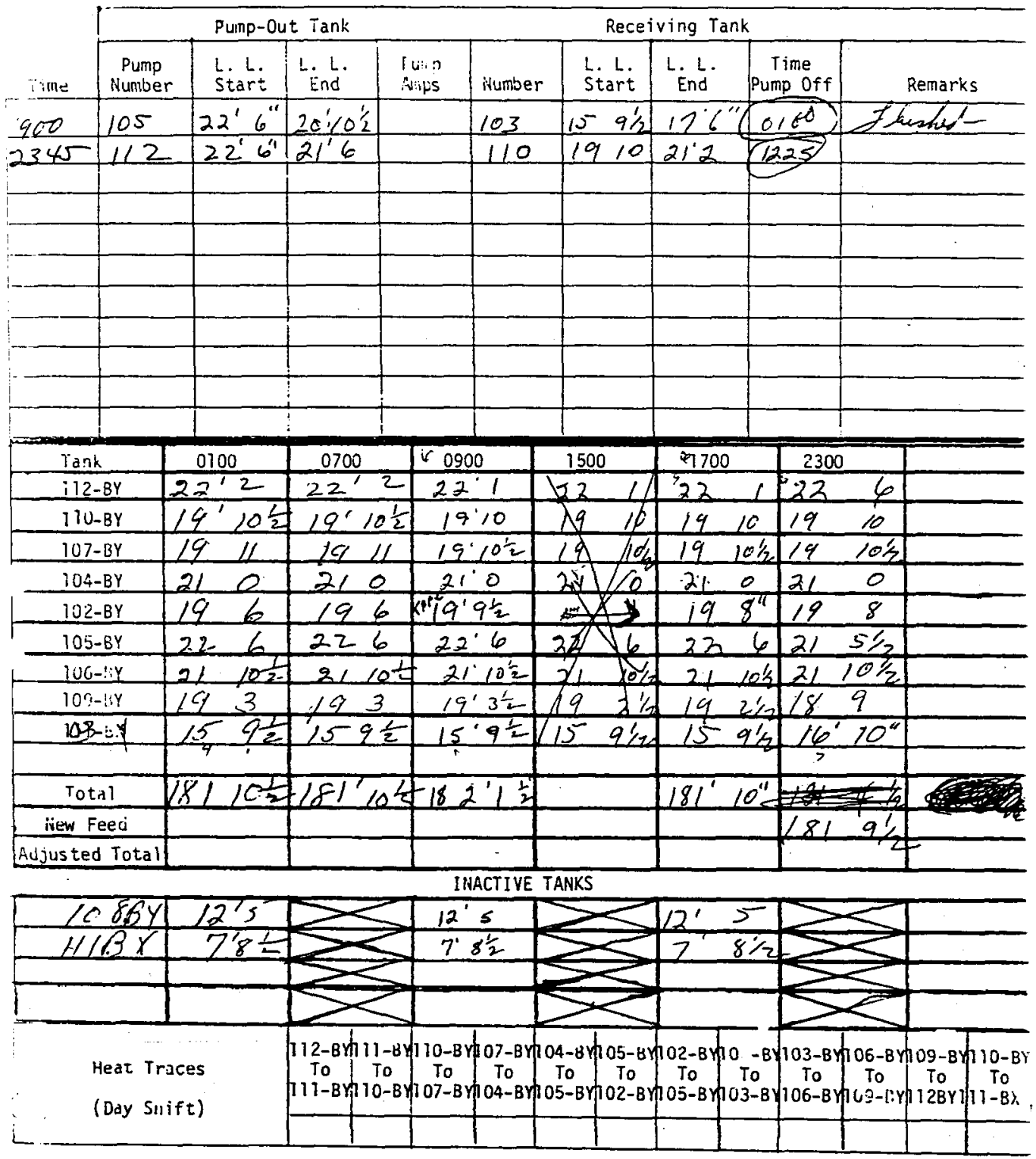




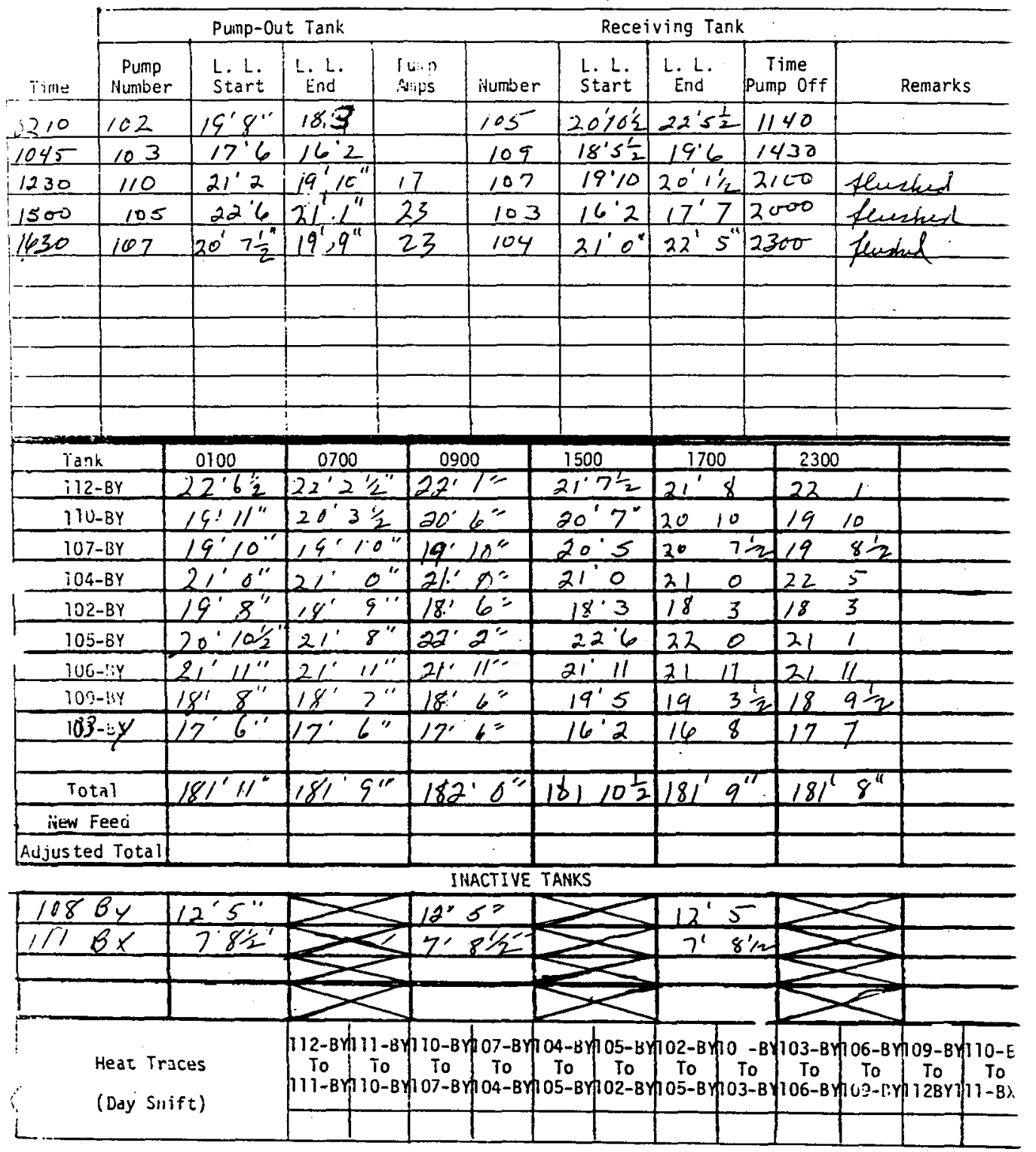


ITS-2 IIIVENTORY ANU PUMPING Date $12-5 \cdot 21$

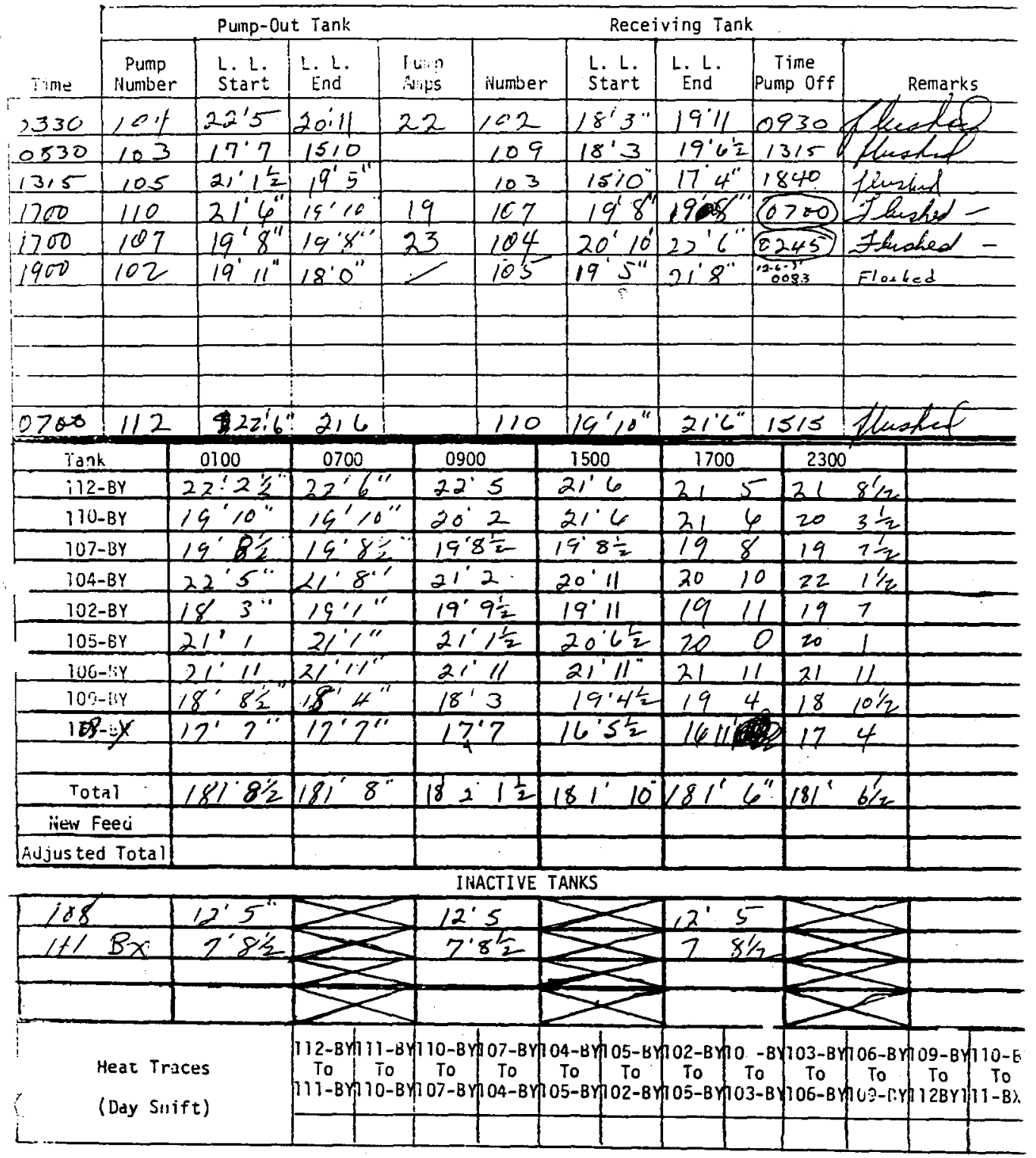




\section{ITS-2 IIIVENTORY ANU PUMPING $\quad$ Cate $12-6-71$}

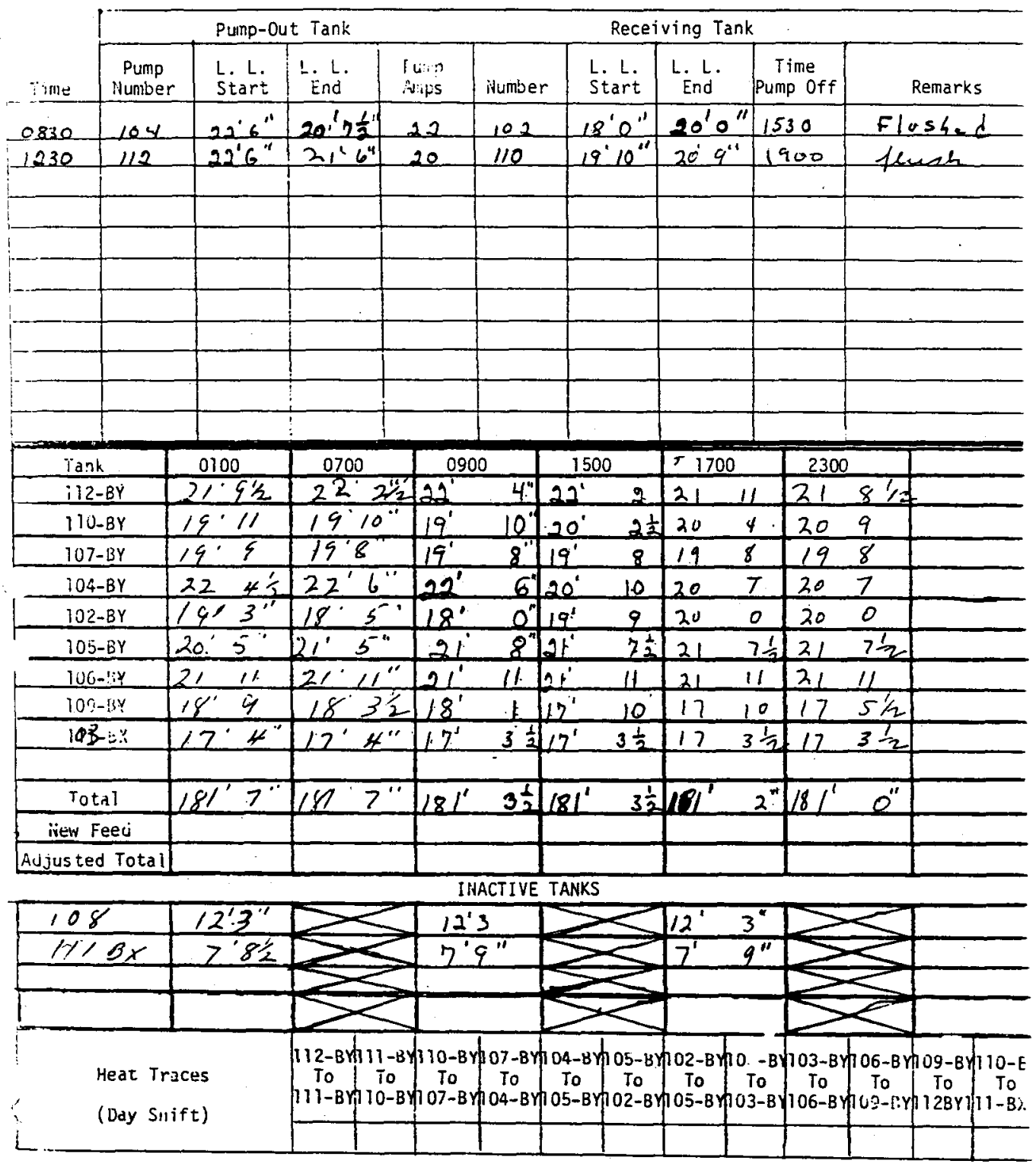




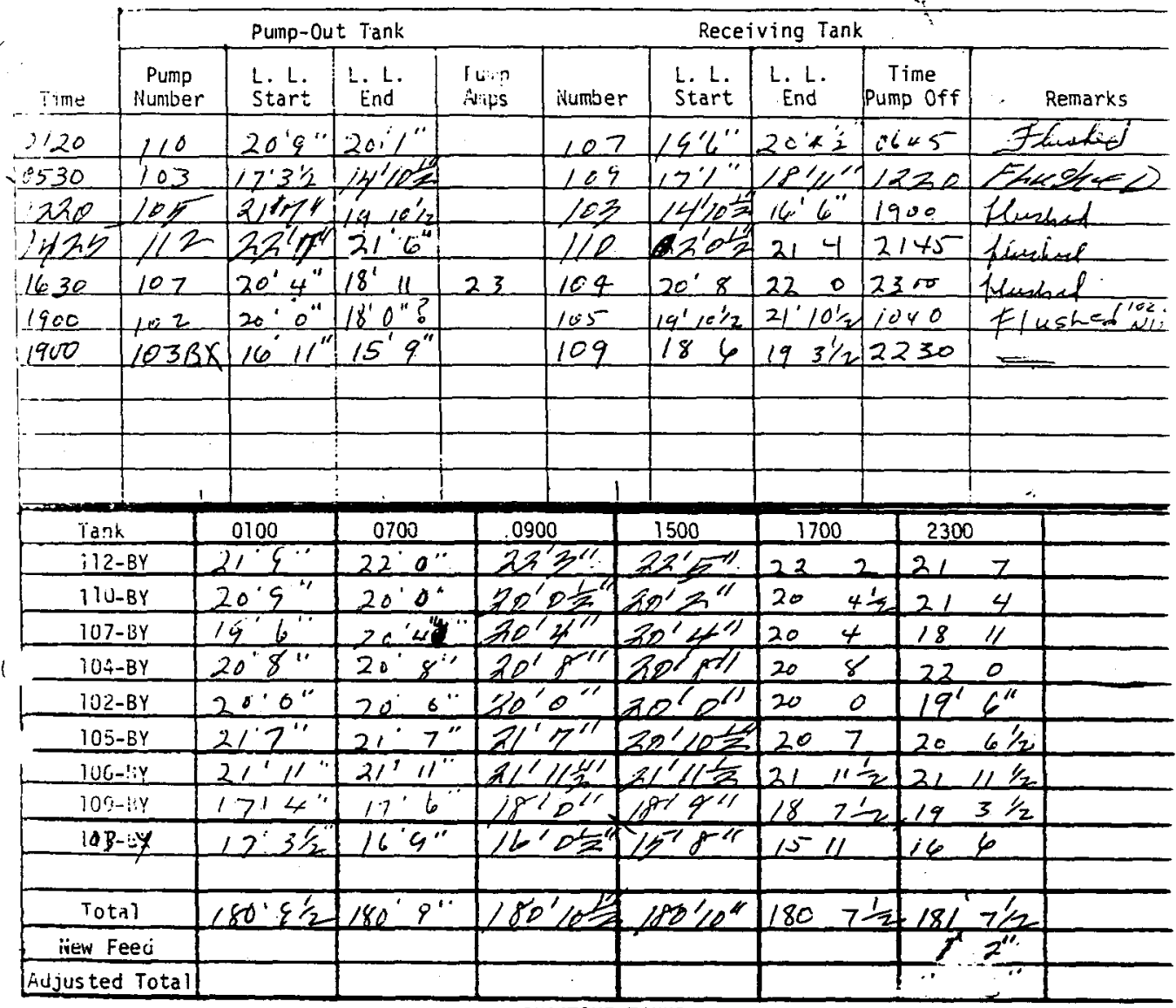

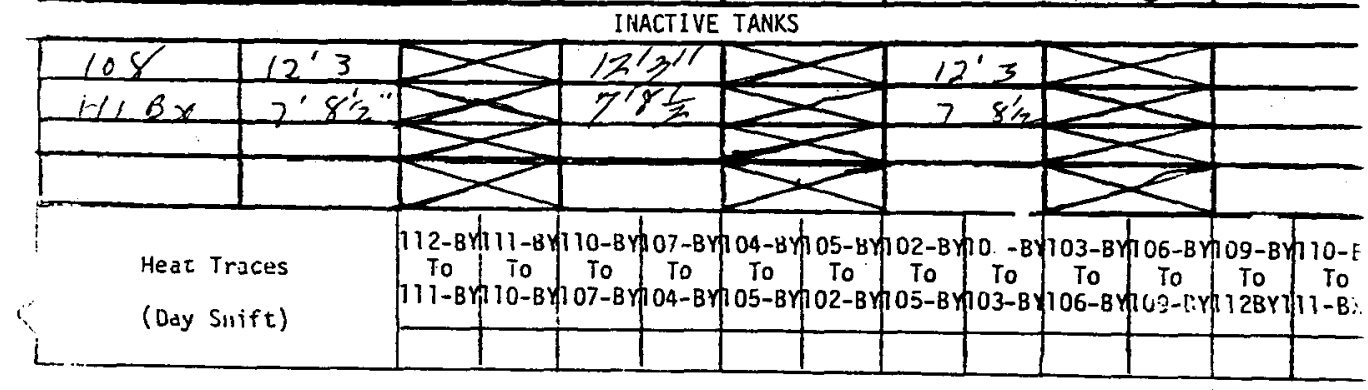

INACTIVE TANKS

Heat Traces

(Day Silift)

(1)

$\frac{0700}{22^{\circ} 0^{\prime \prime}}$ 


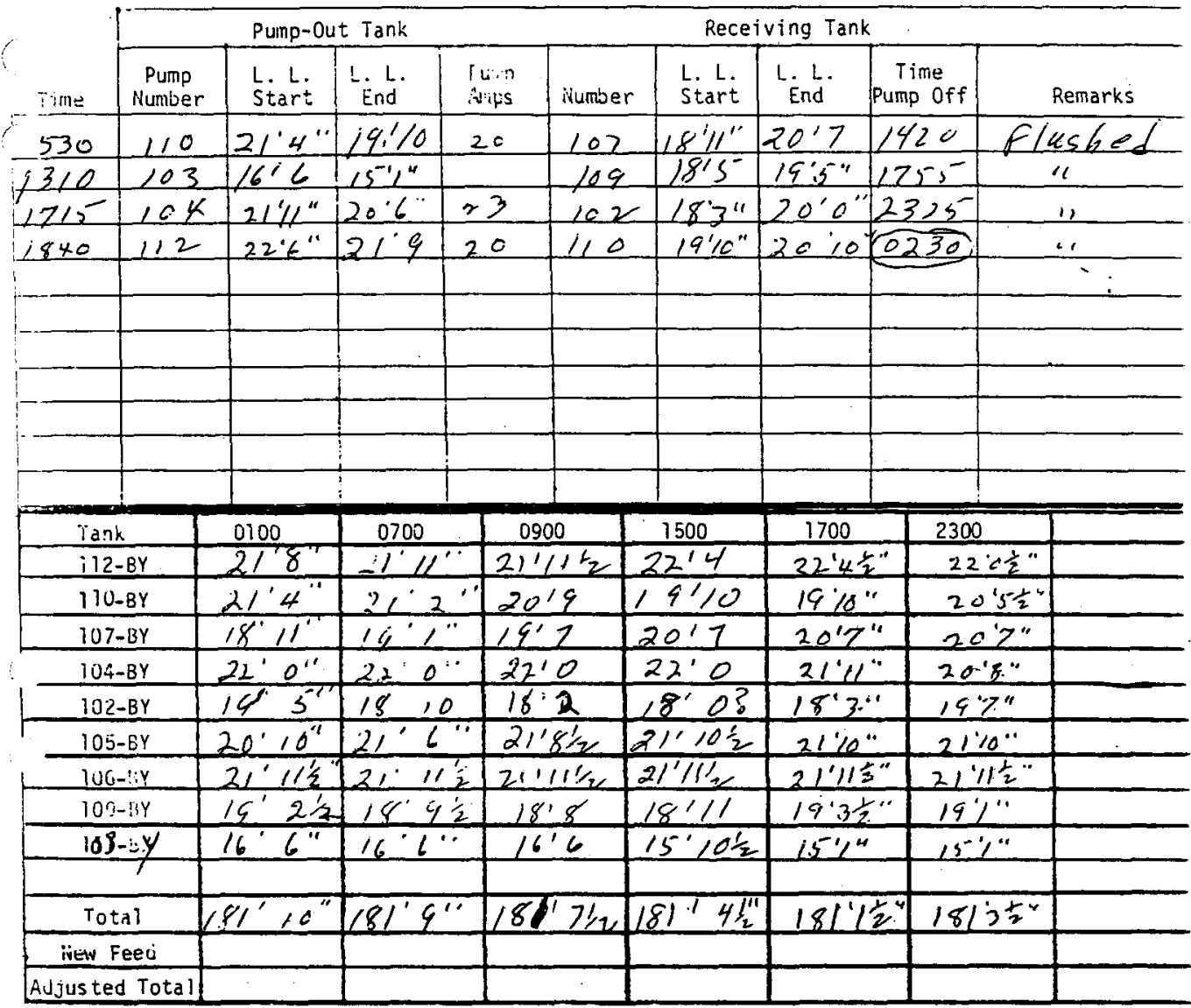

INACTIVE TANKS

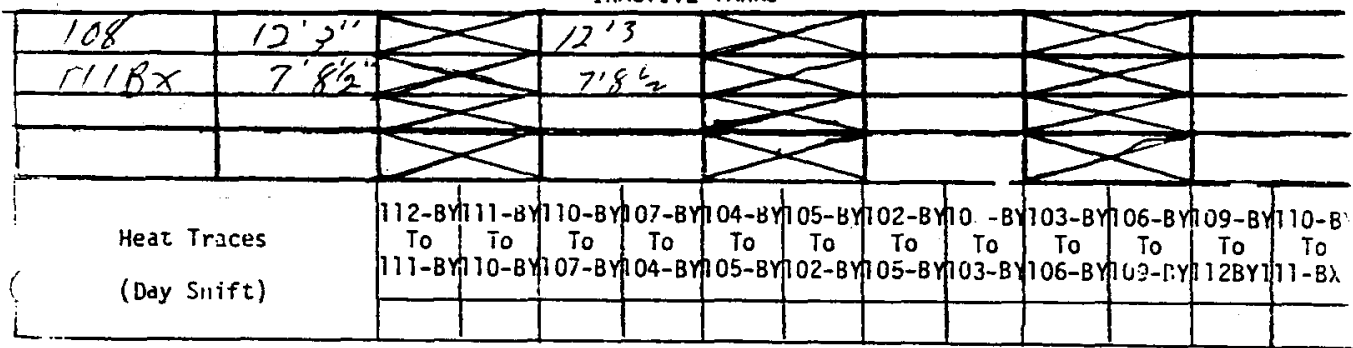




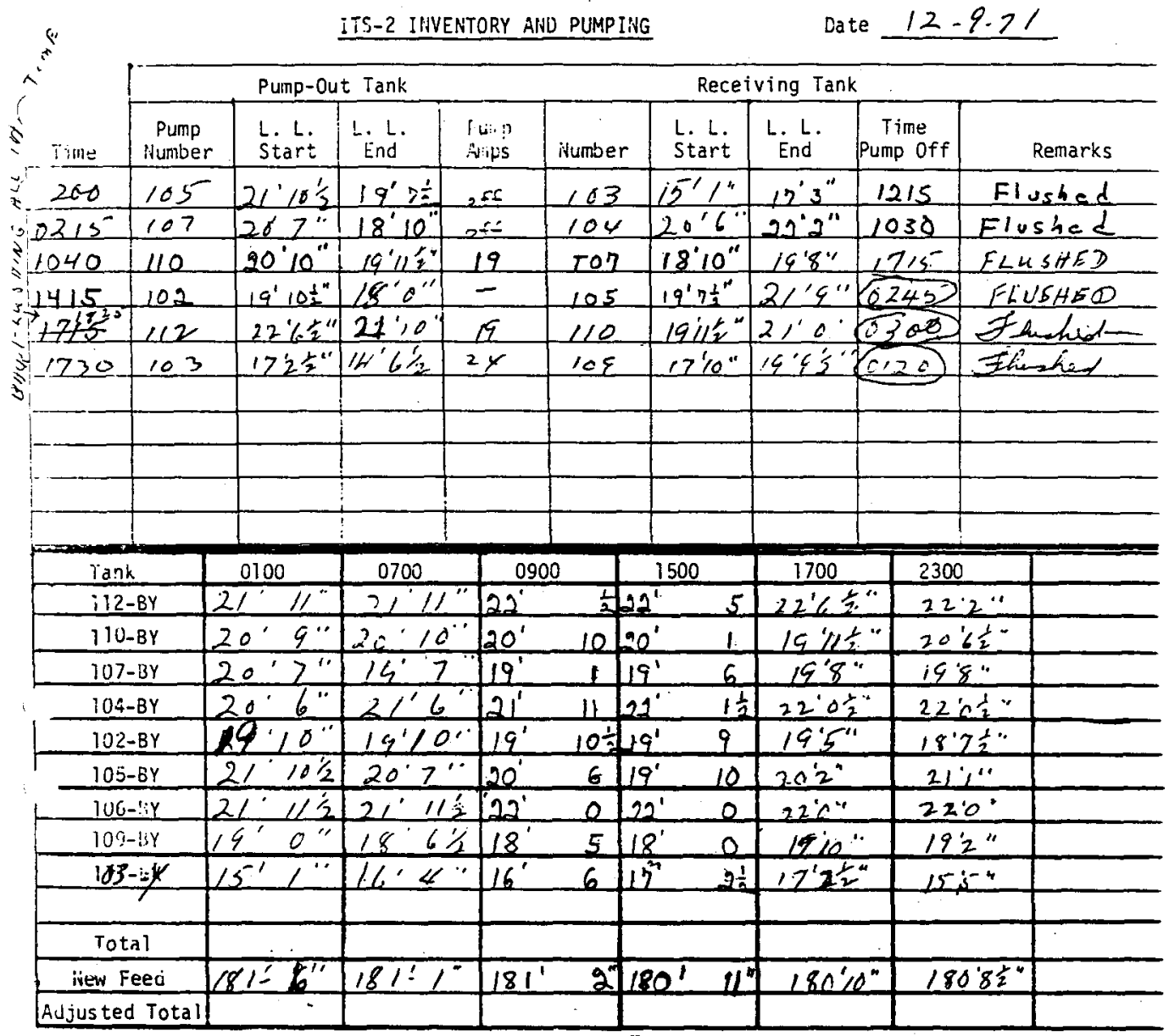

INACTIVE TANKS

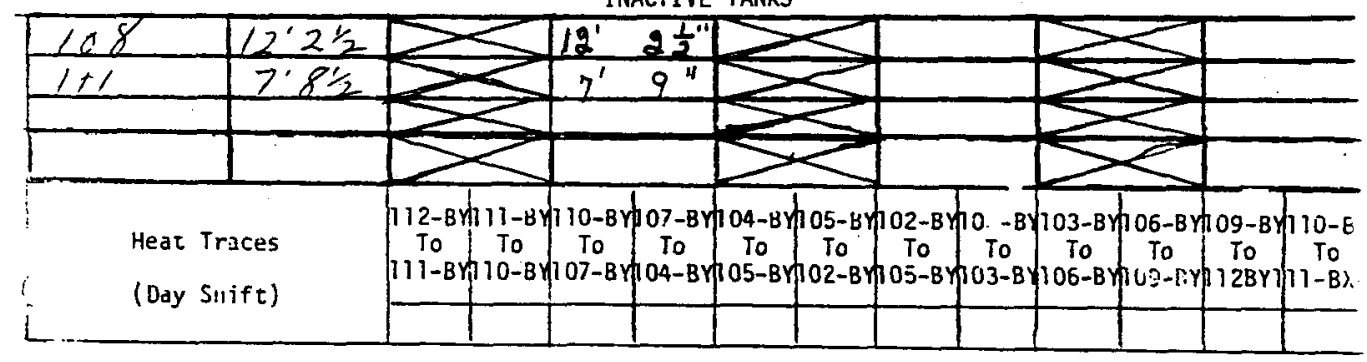




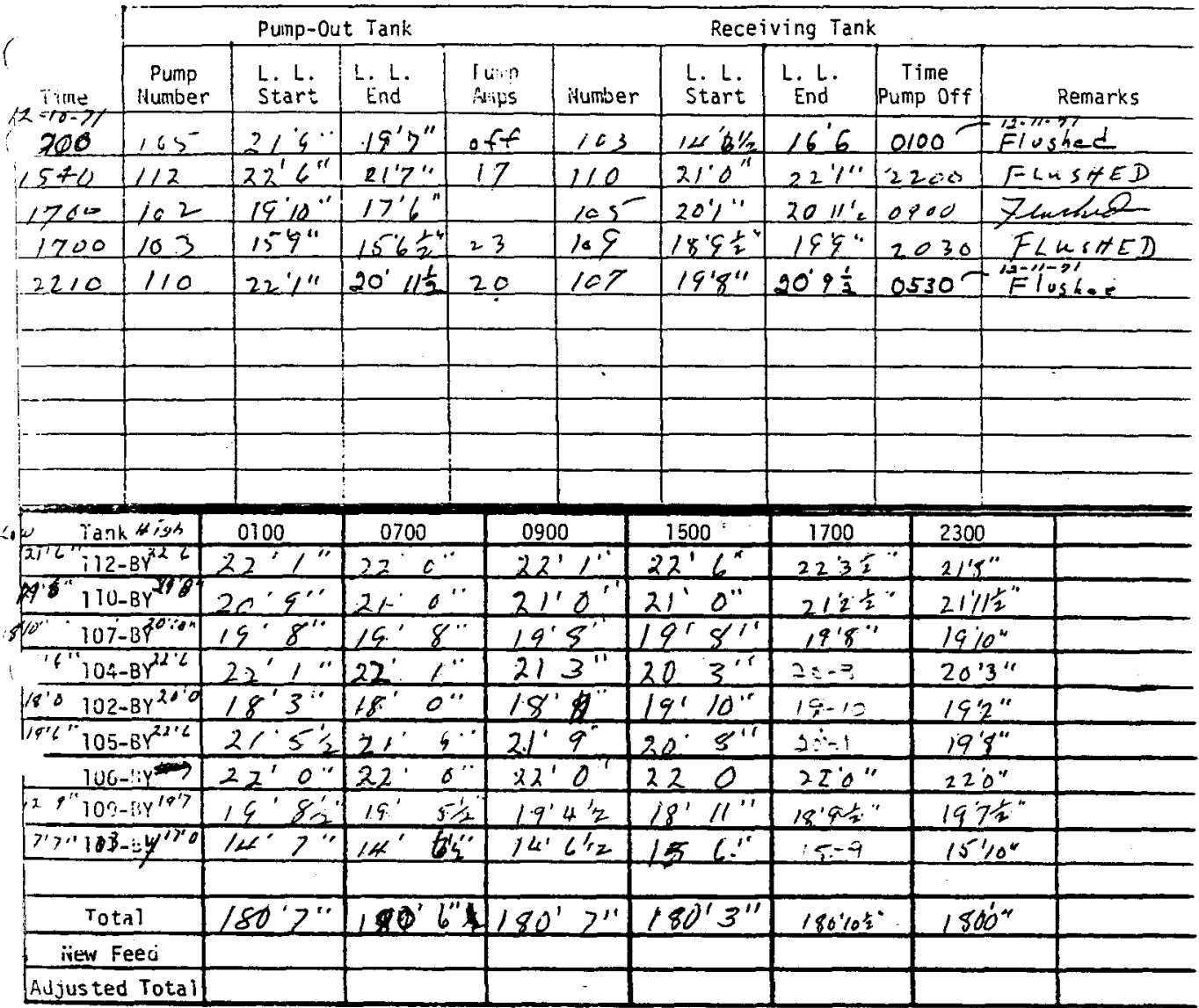

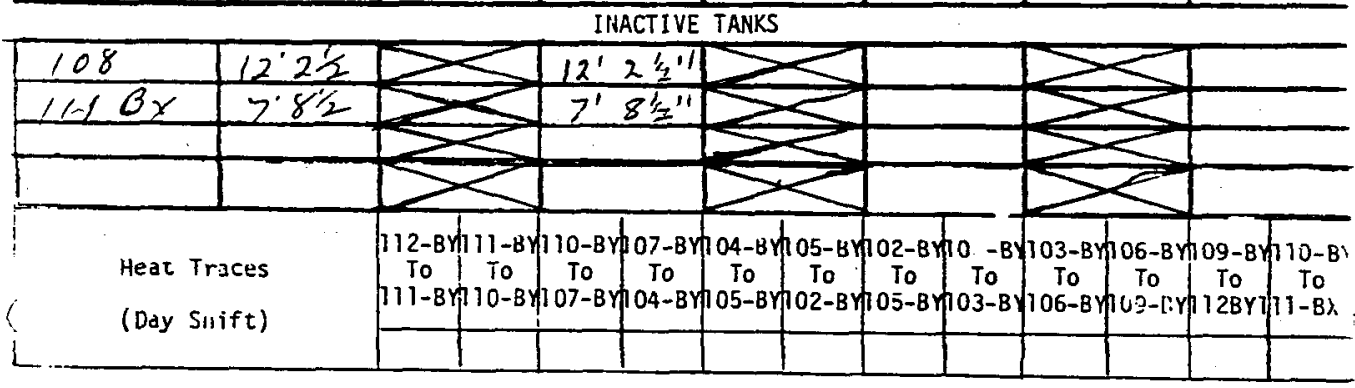




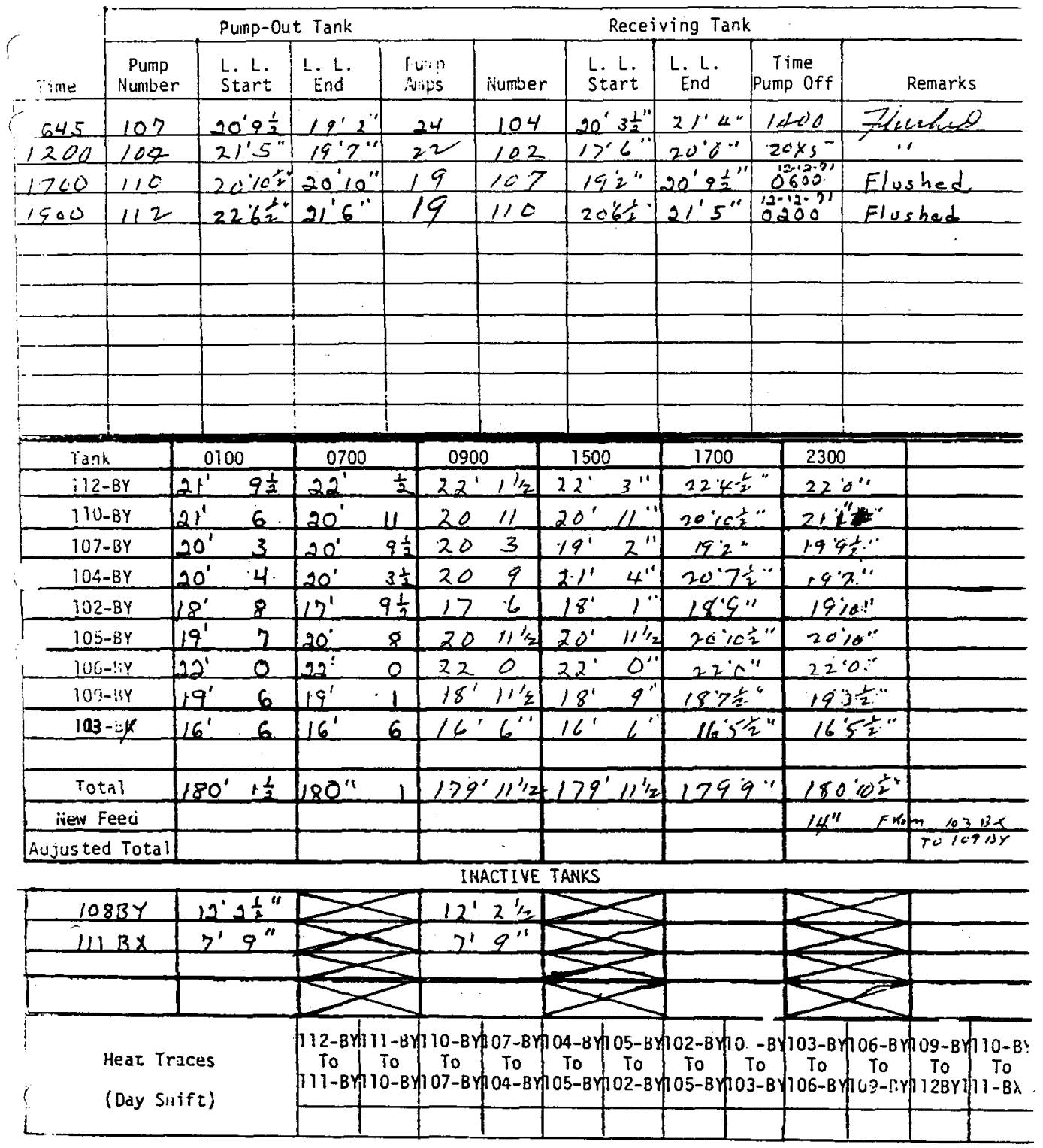




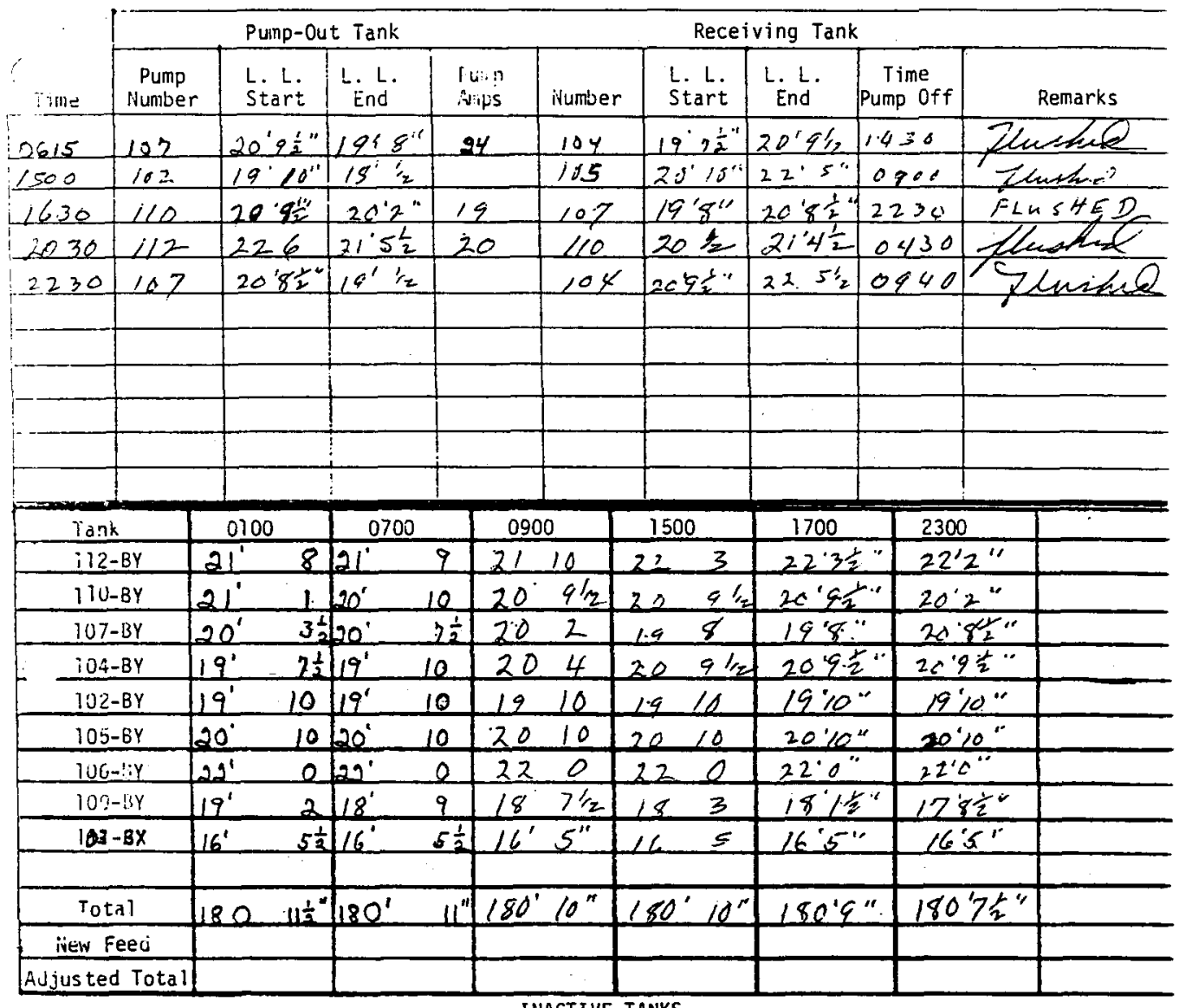

INACTIVE TANKS

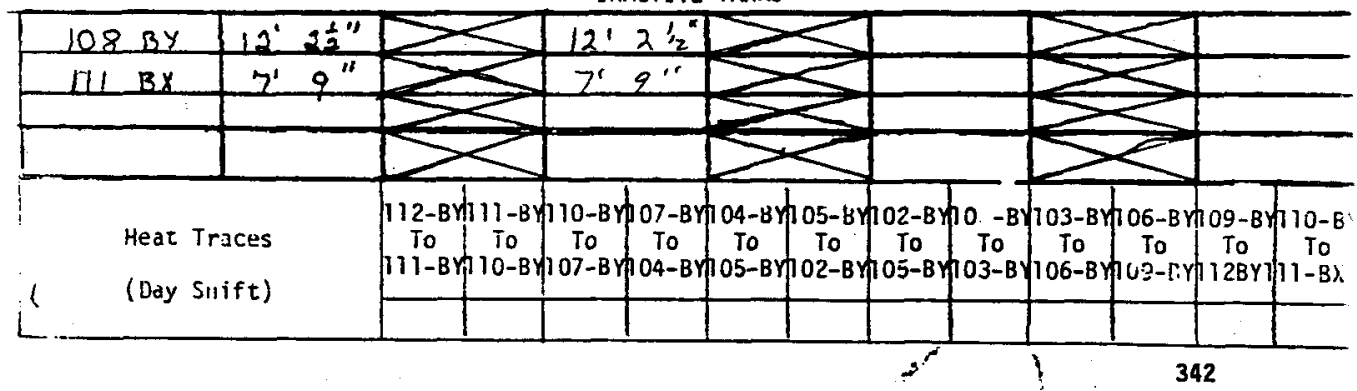




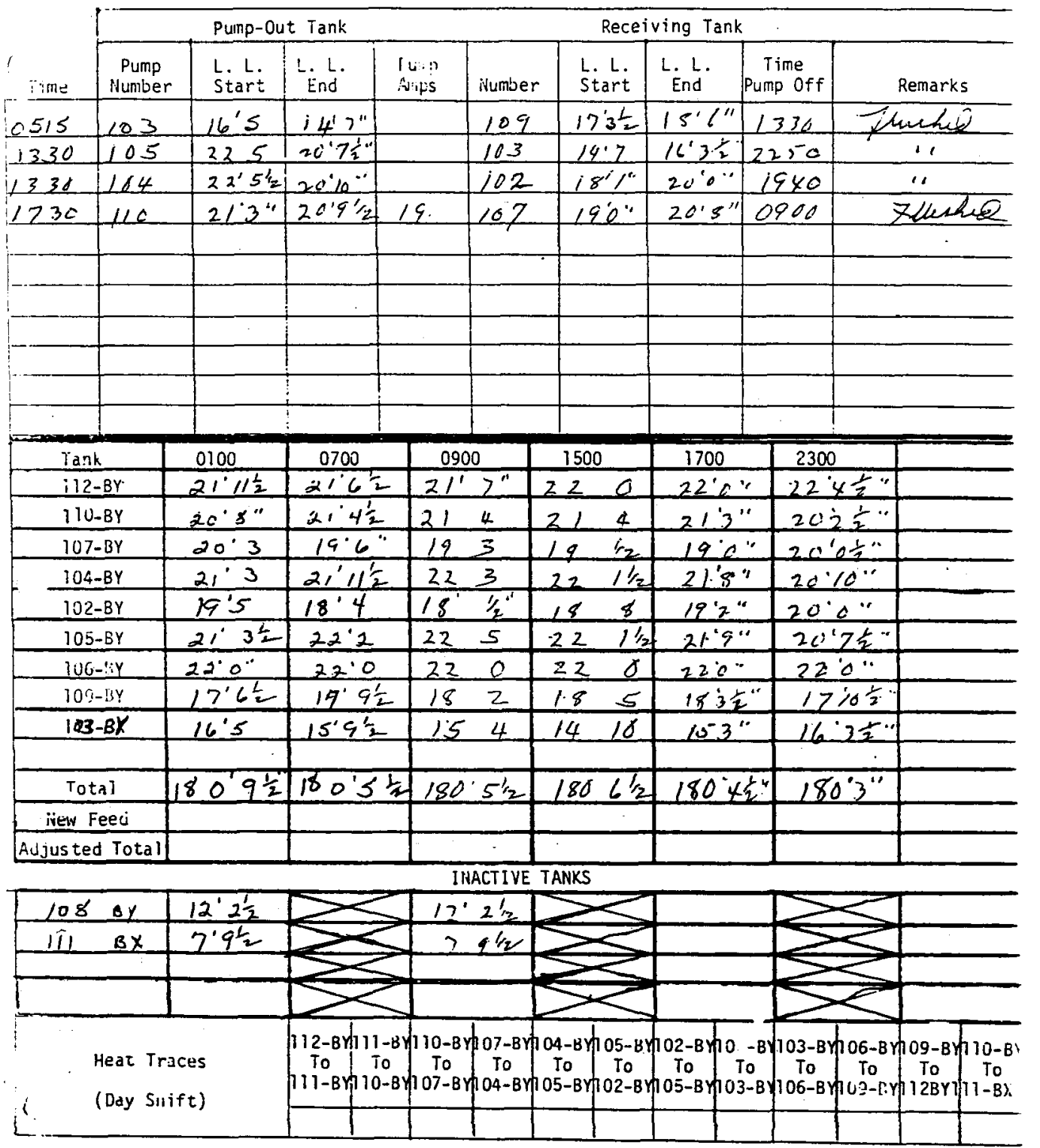




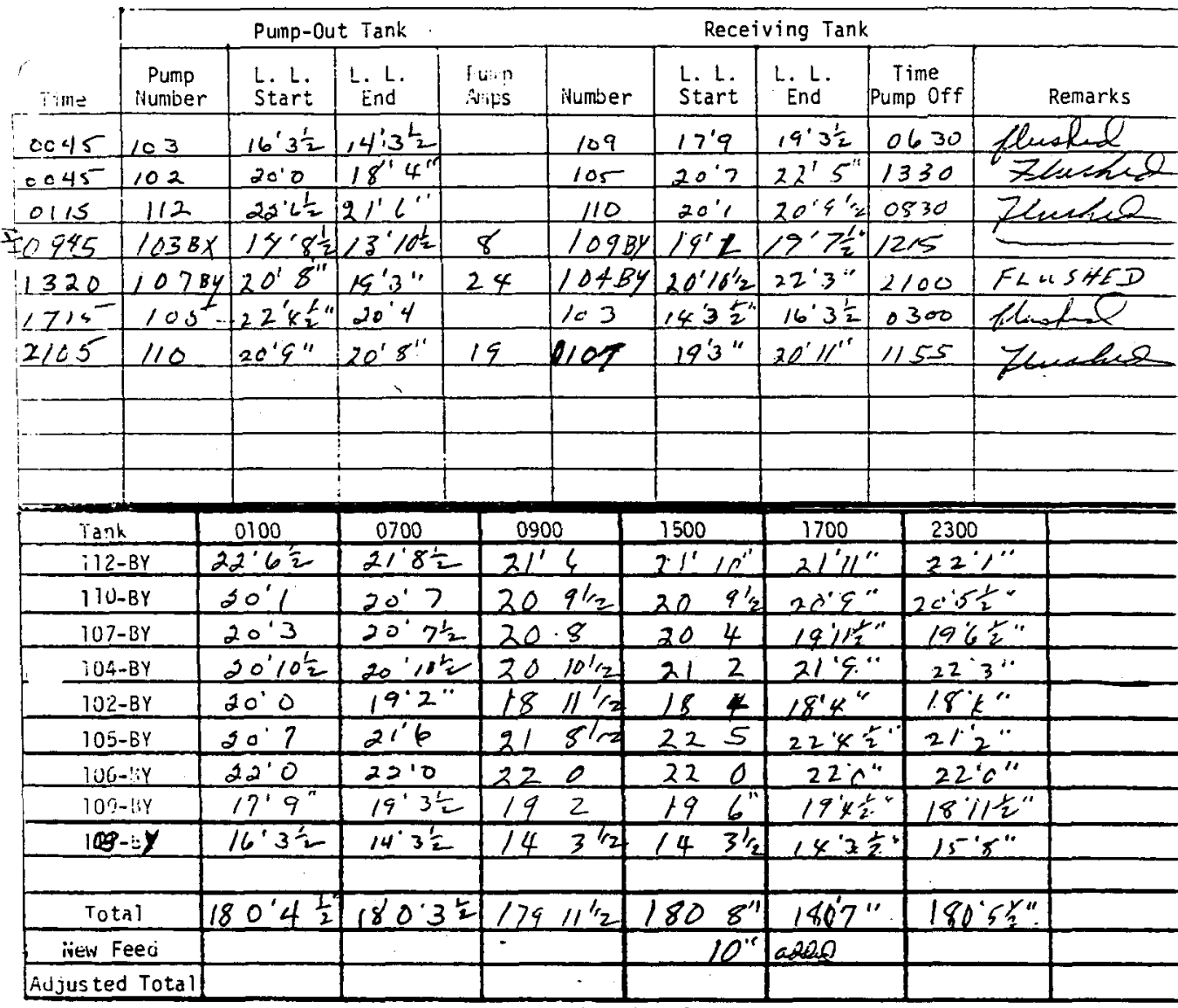

\section{INACTIVE TANKS}

\begin{tabular}{|l|l|}
\hline 108 & $12^{\prime} 2 \frac{1}{2}$ \\
\hline 61 & $7^{\prime} 9 \frac{1}{2}$ \\
\hline & \\
\hline
\end{tabular}

Heat Traces

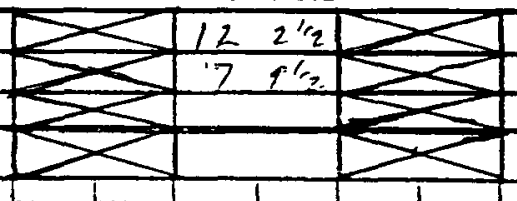

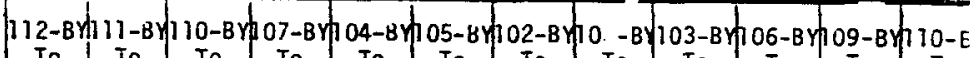

(Day Stift)

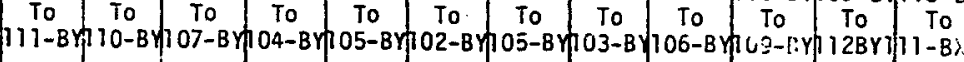




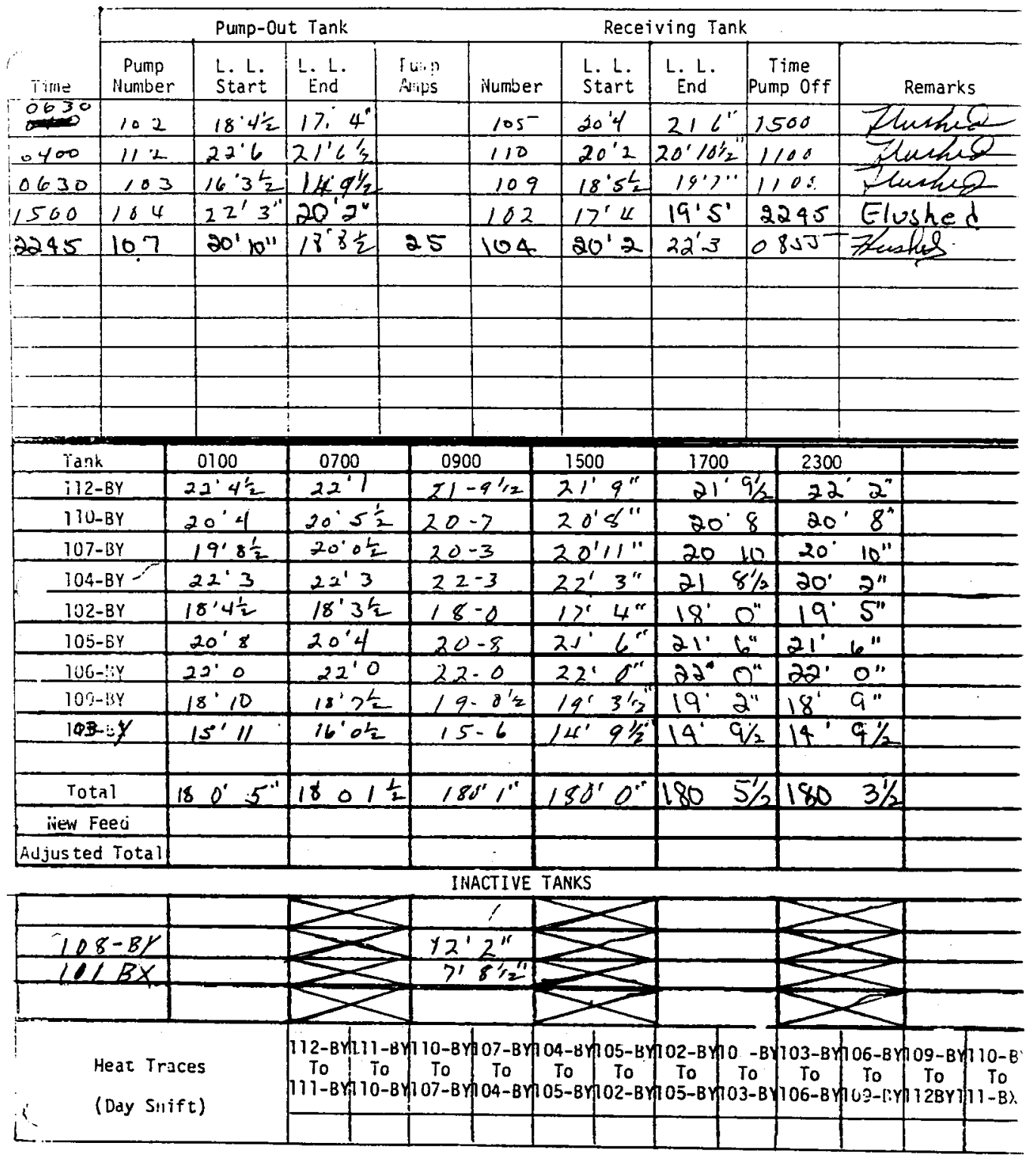




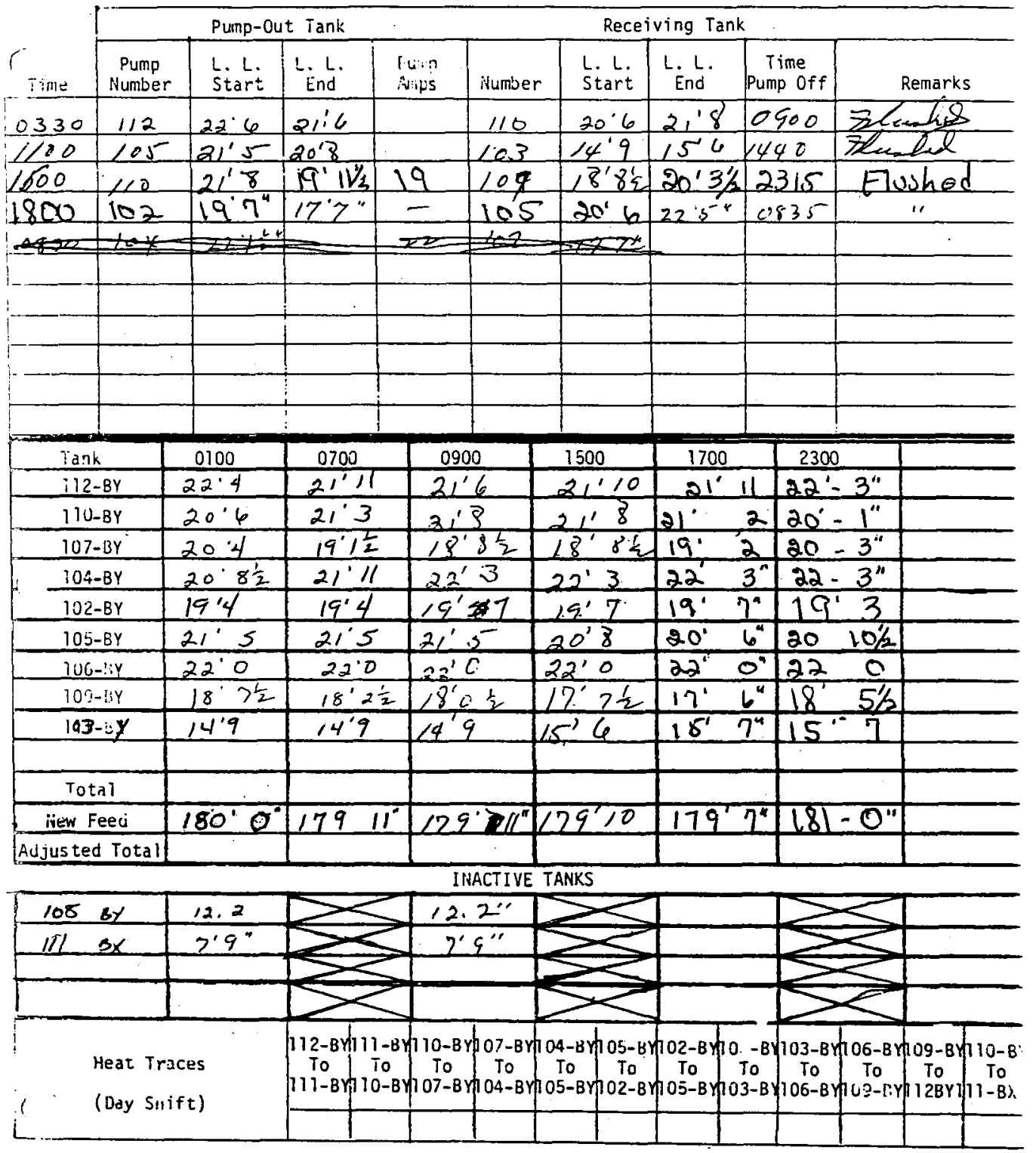




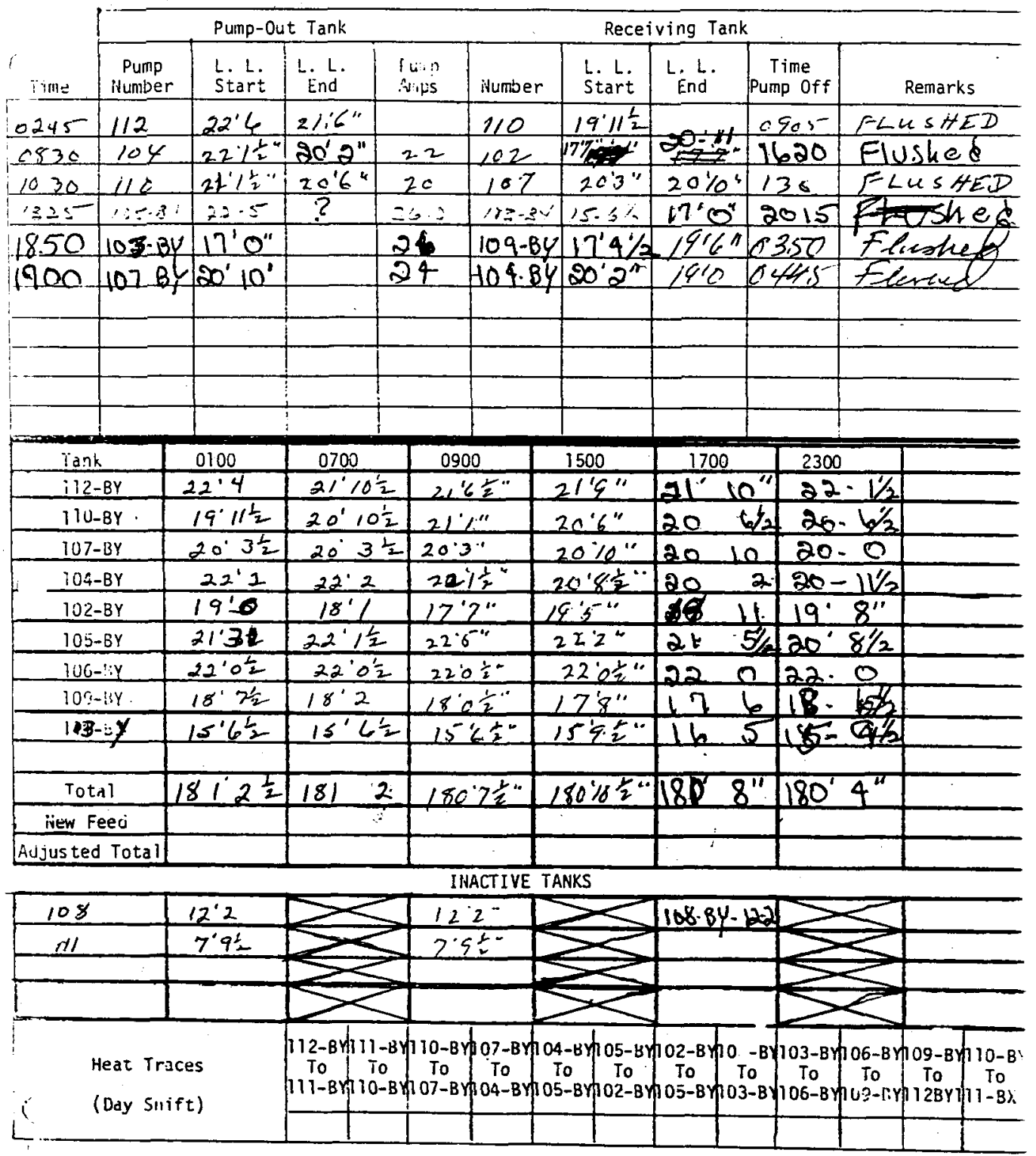




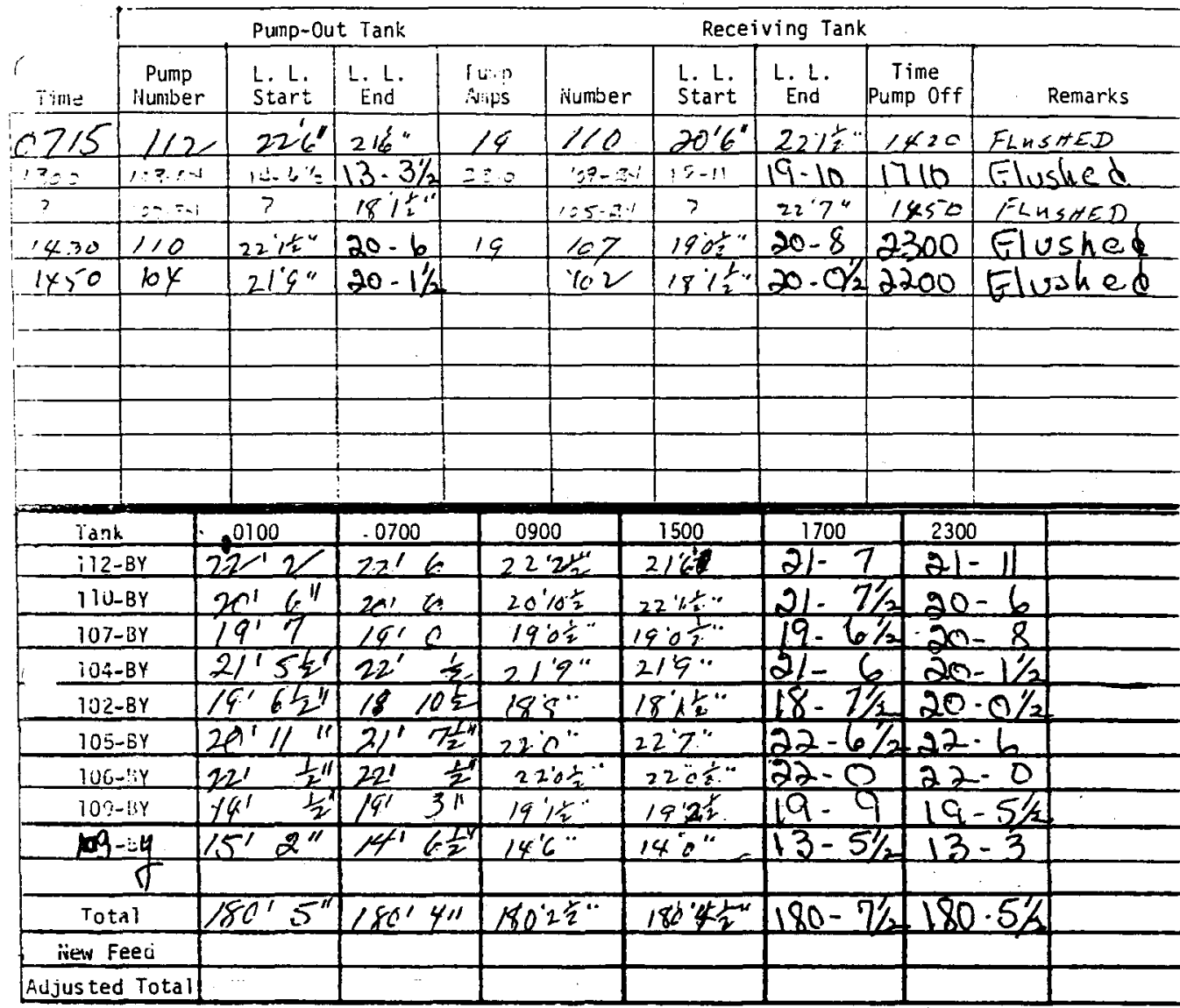

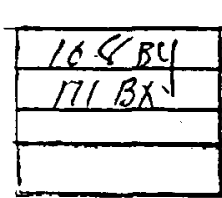

Heat Traces

( ) (Day Silift)

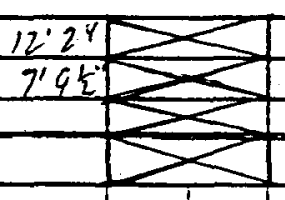

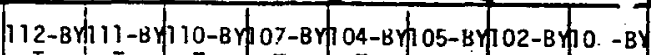
INACTIVE TANKS

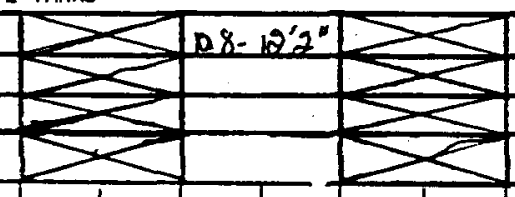

To To To To To

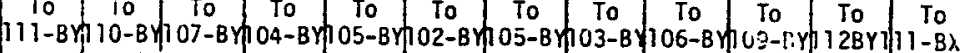

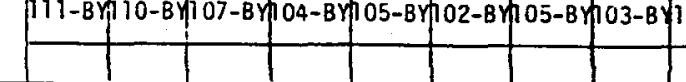


WHC-SD-WM-DP-209, Rev. 0

ITS-2 IINVENTORY AINU PUMPING

wate $12-14.7$

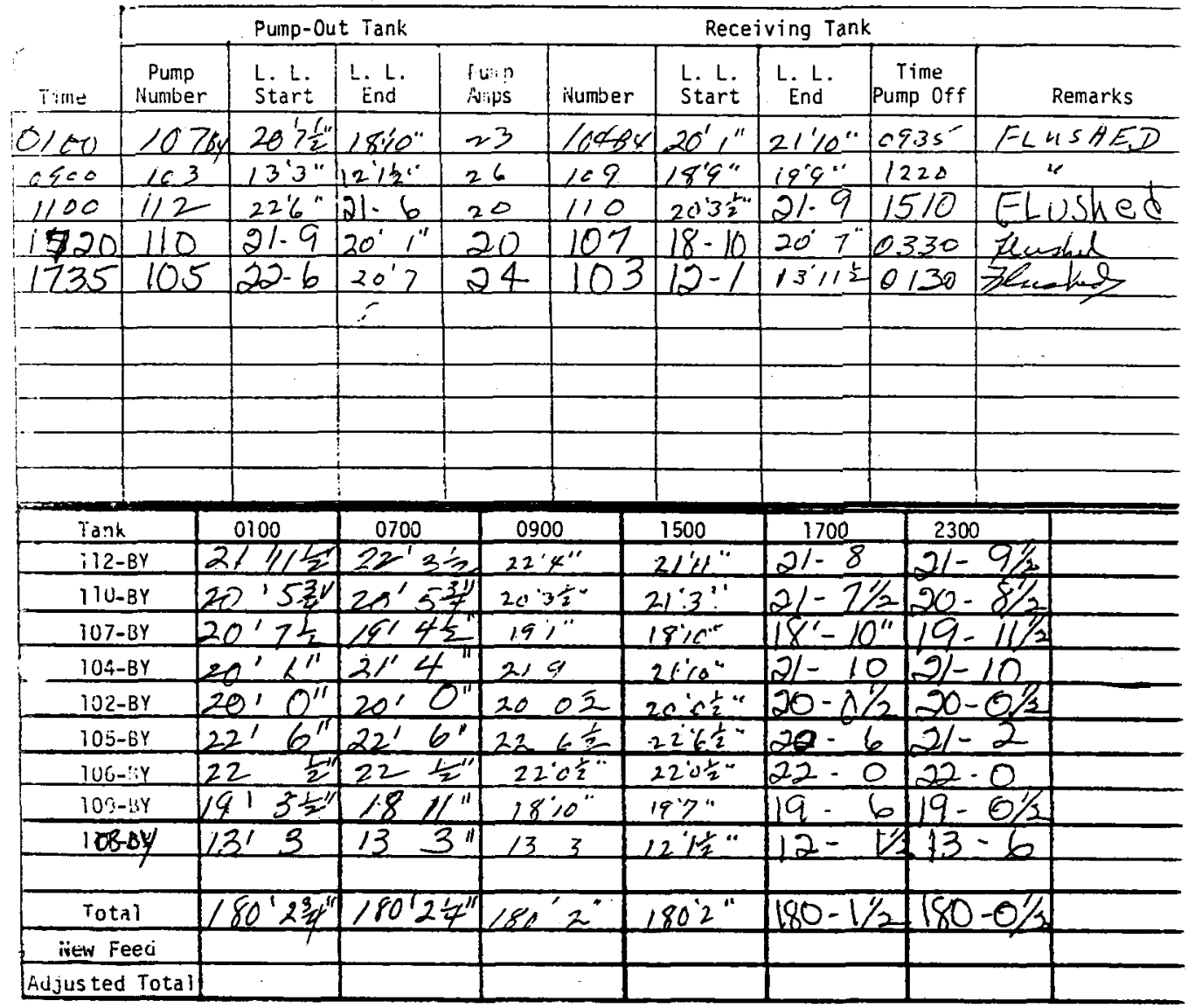

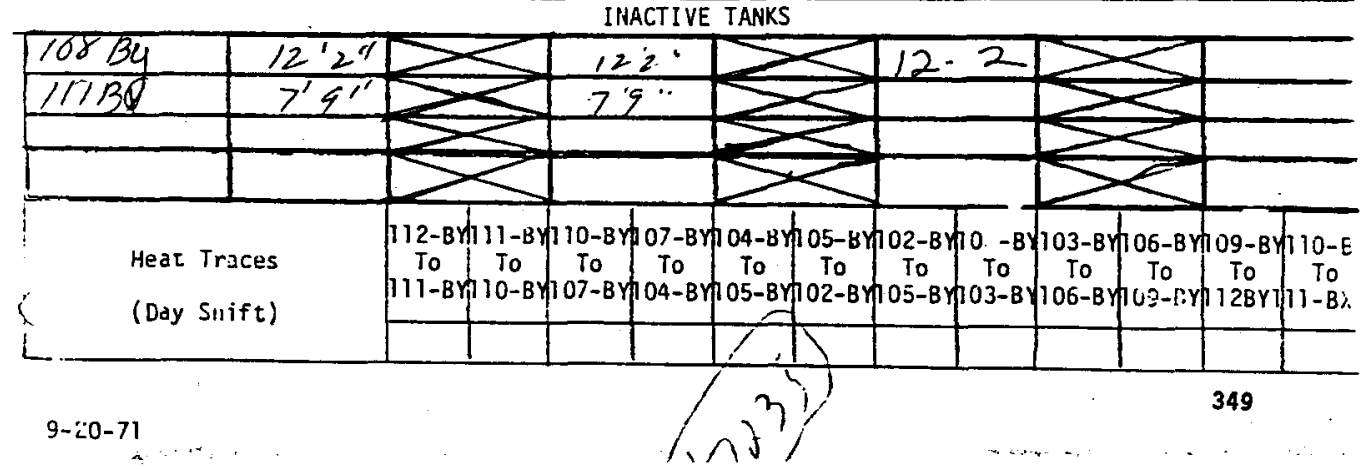




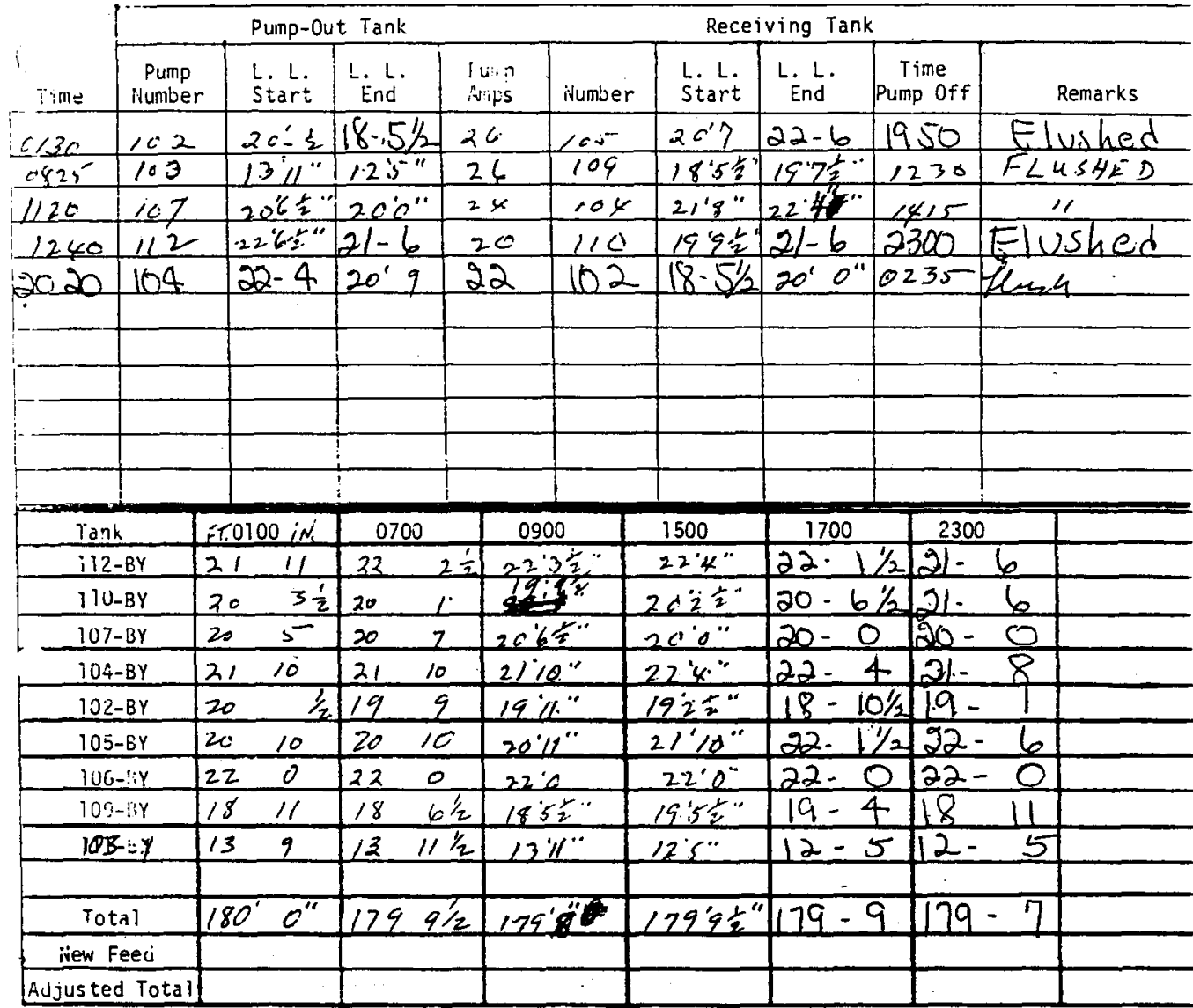

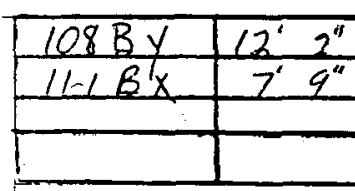

Heat Traces

(Day Suift)

\section{INACTIVE TANKS}

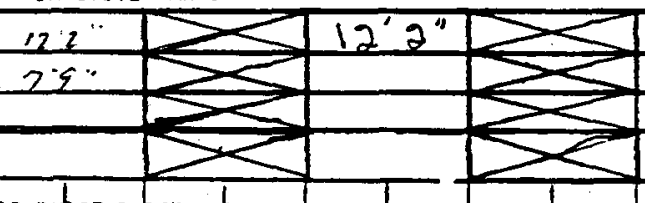

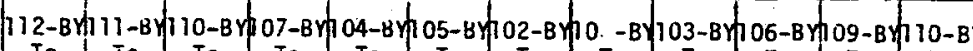

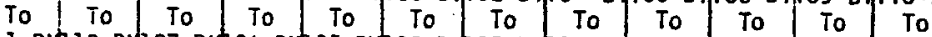

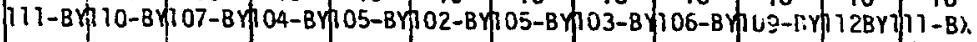




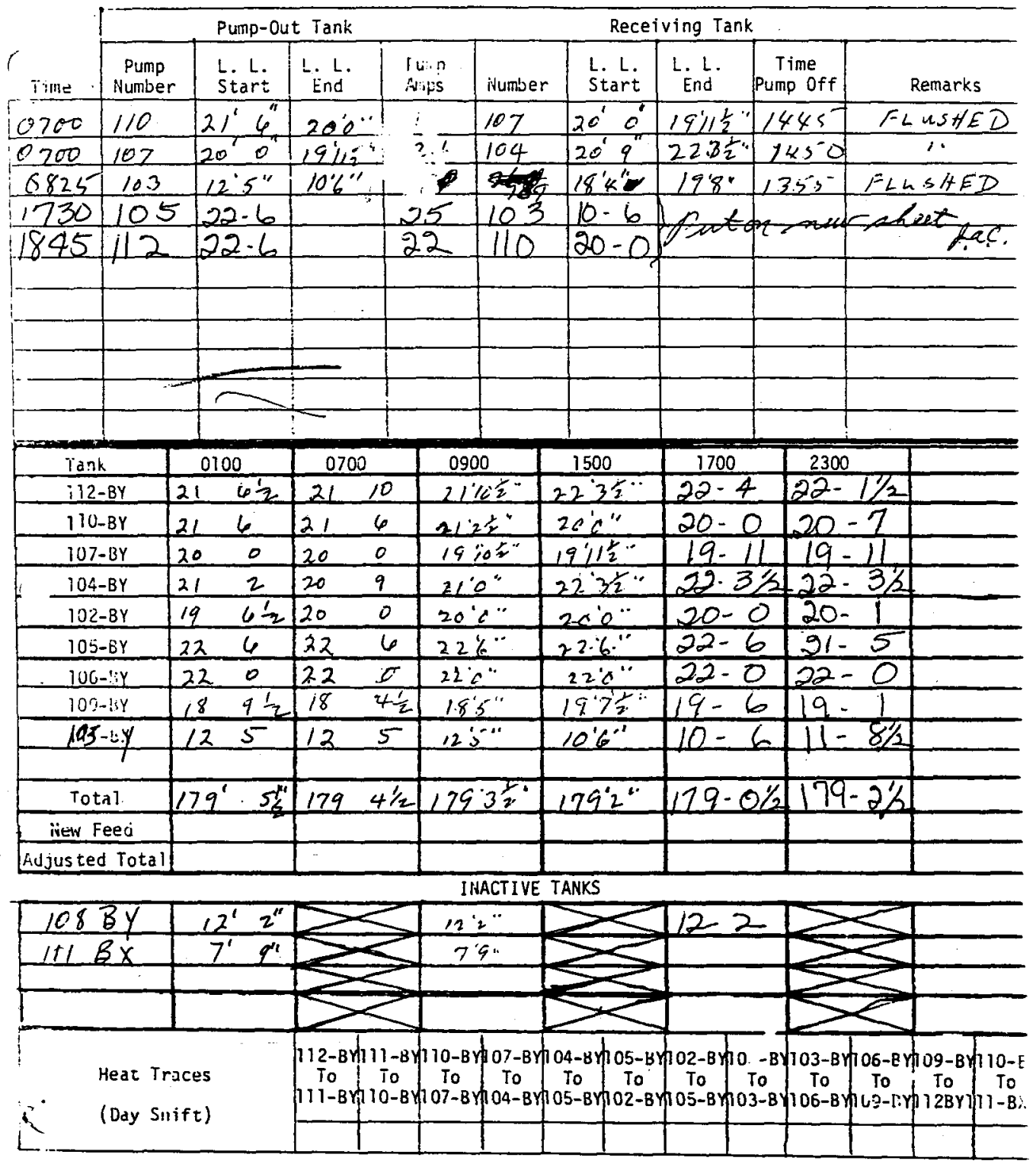




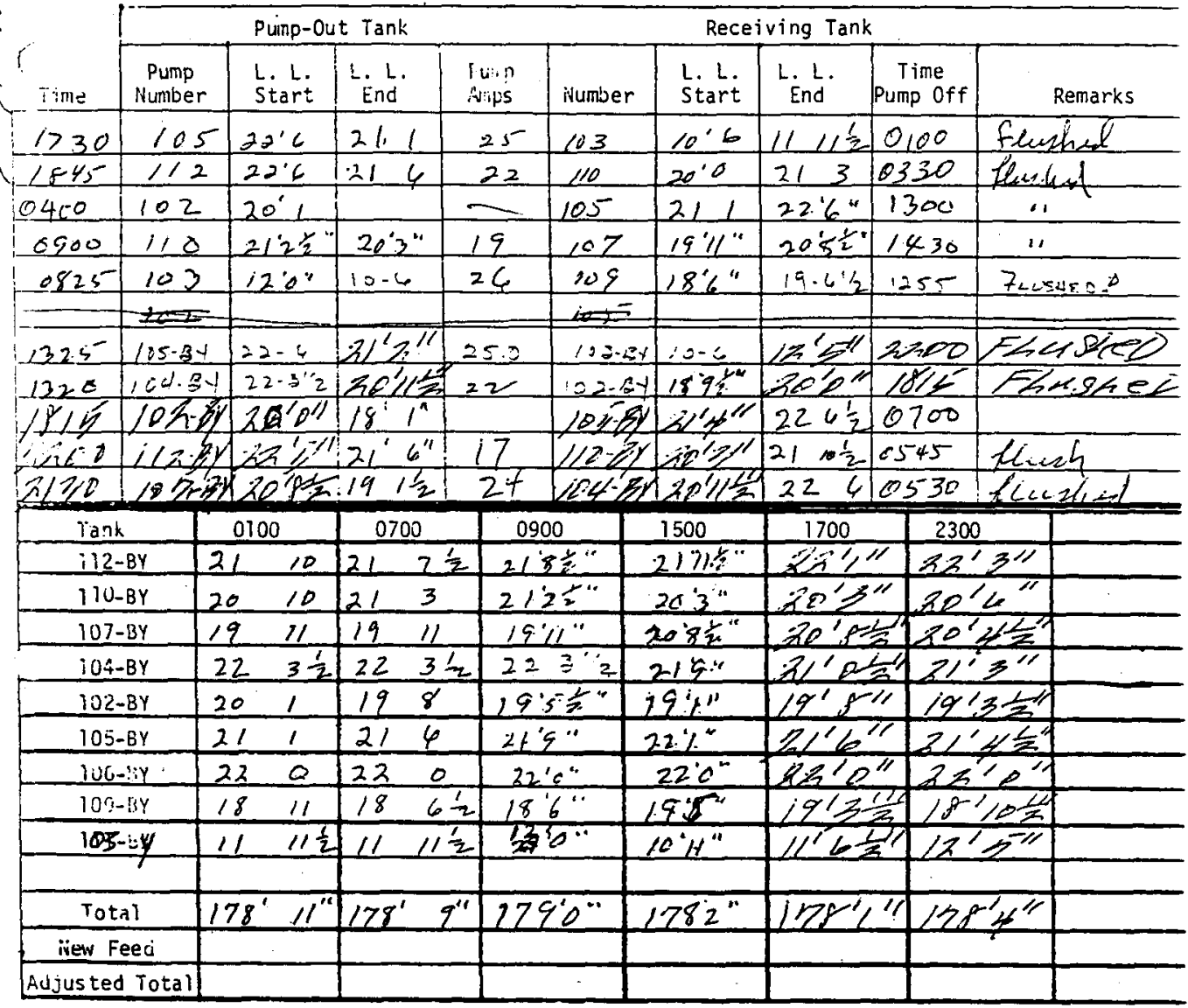

\section{INACTIVE TANKS}

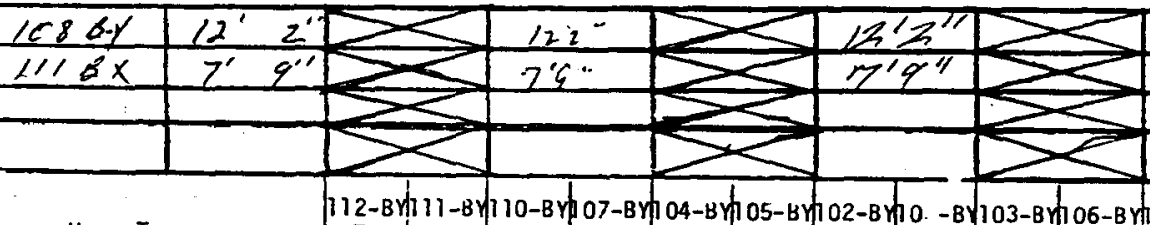

Heat Traces

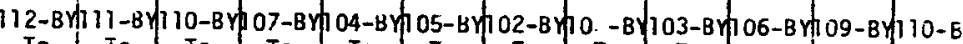

(Day Suift).

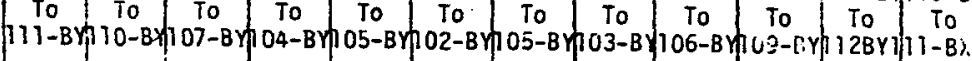


ITS-2 IIVENTORY AIND PUMPING Date $12.23-71$

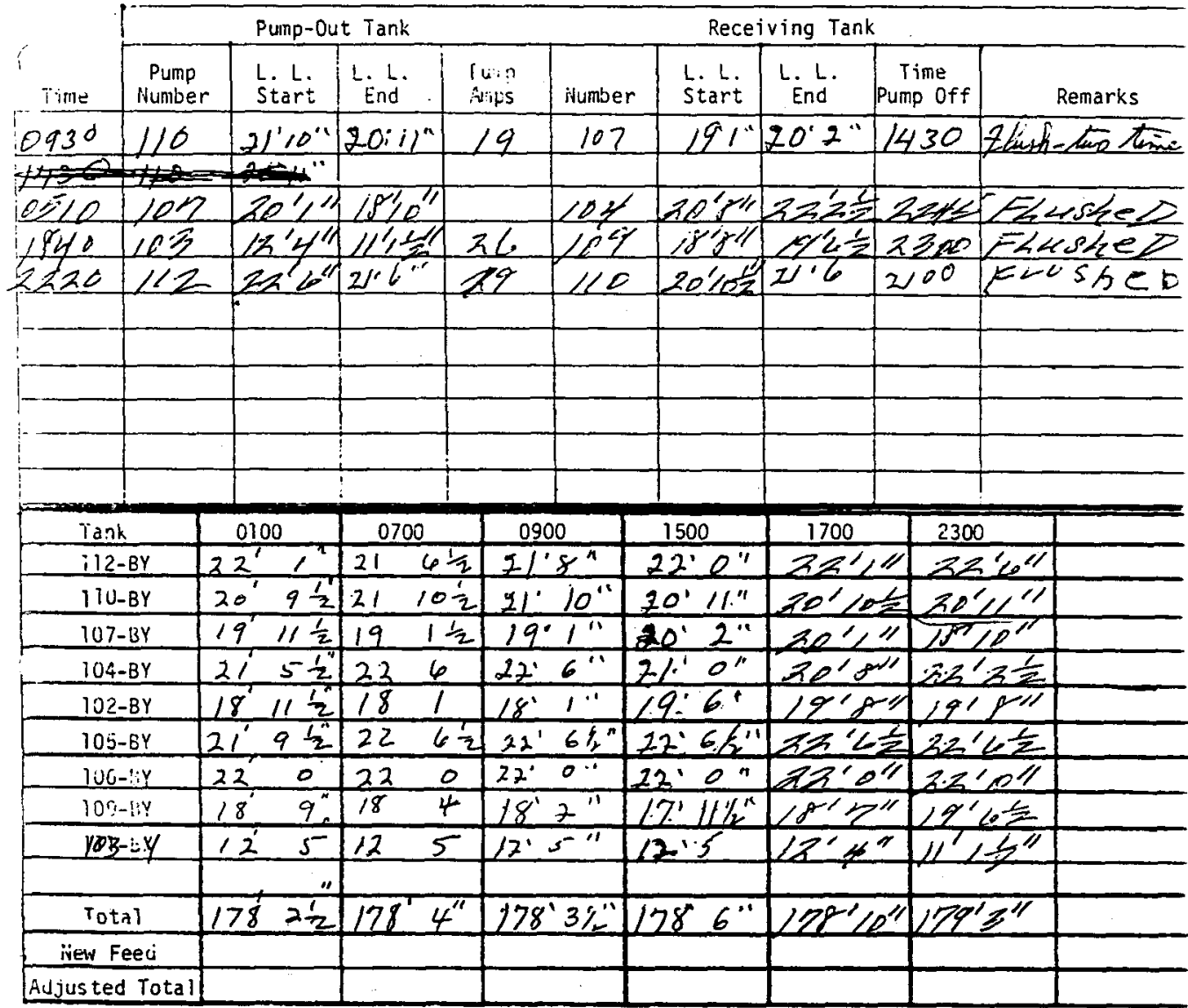

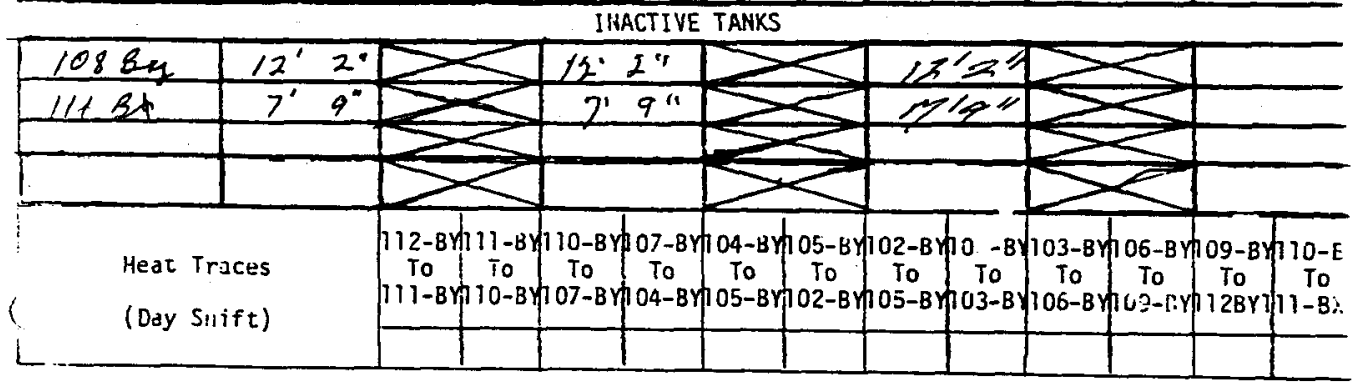

Heat Traces End
Oi i
$181 / 2$
218

WHC-so-Lar-DP-209, Rev. O

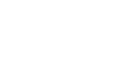




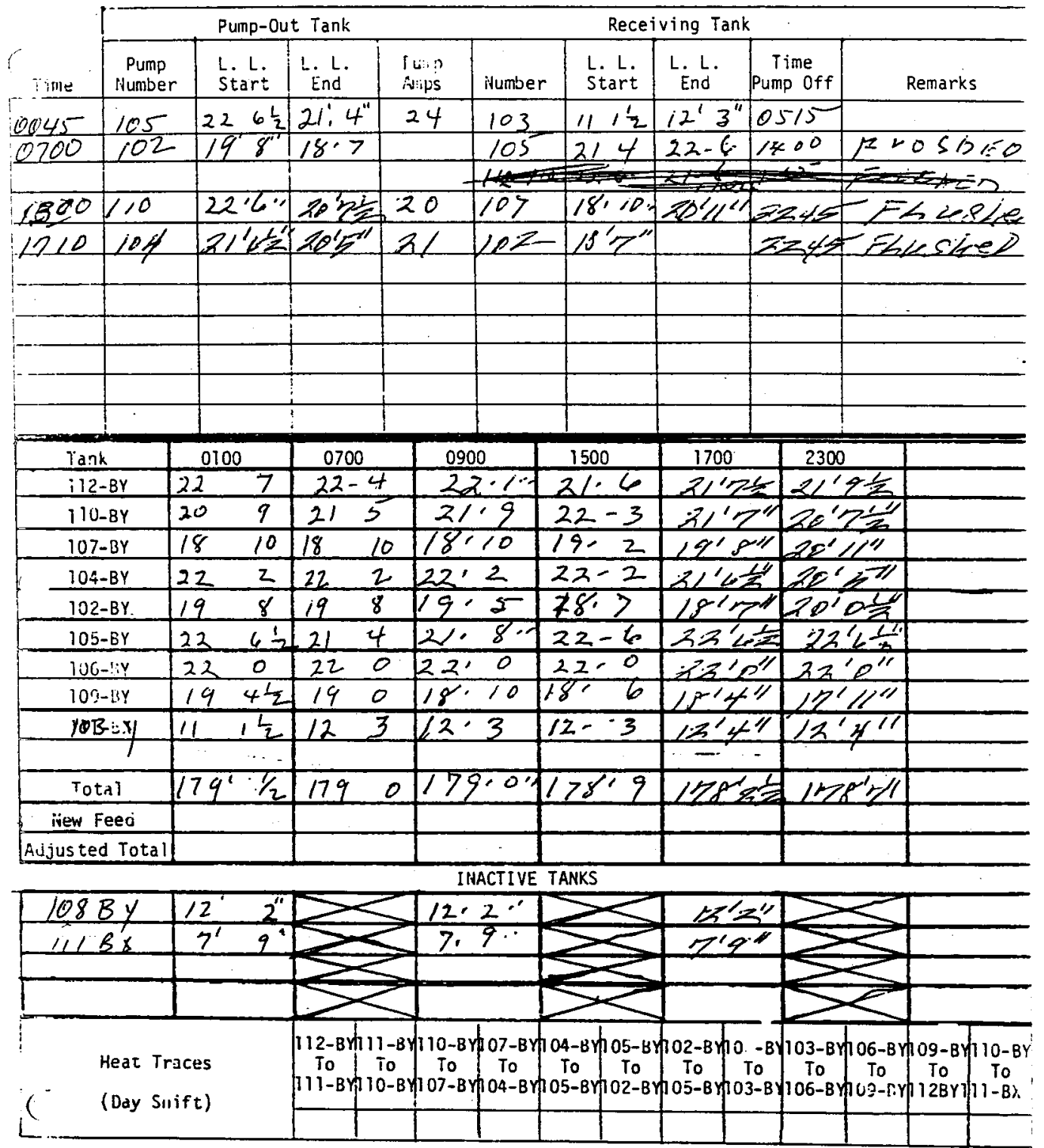




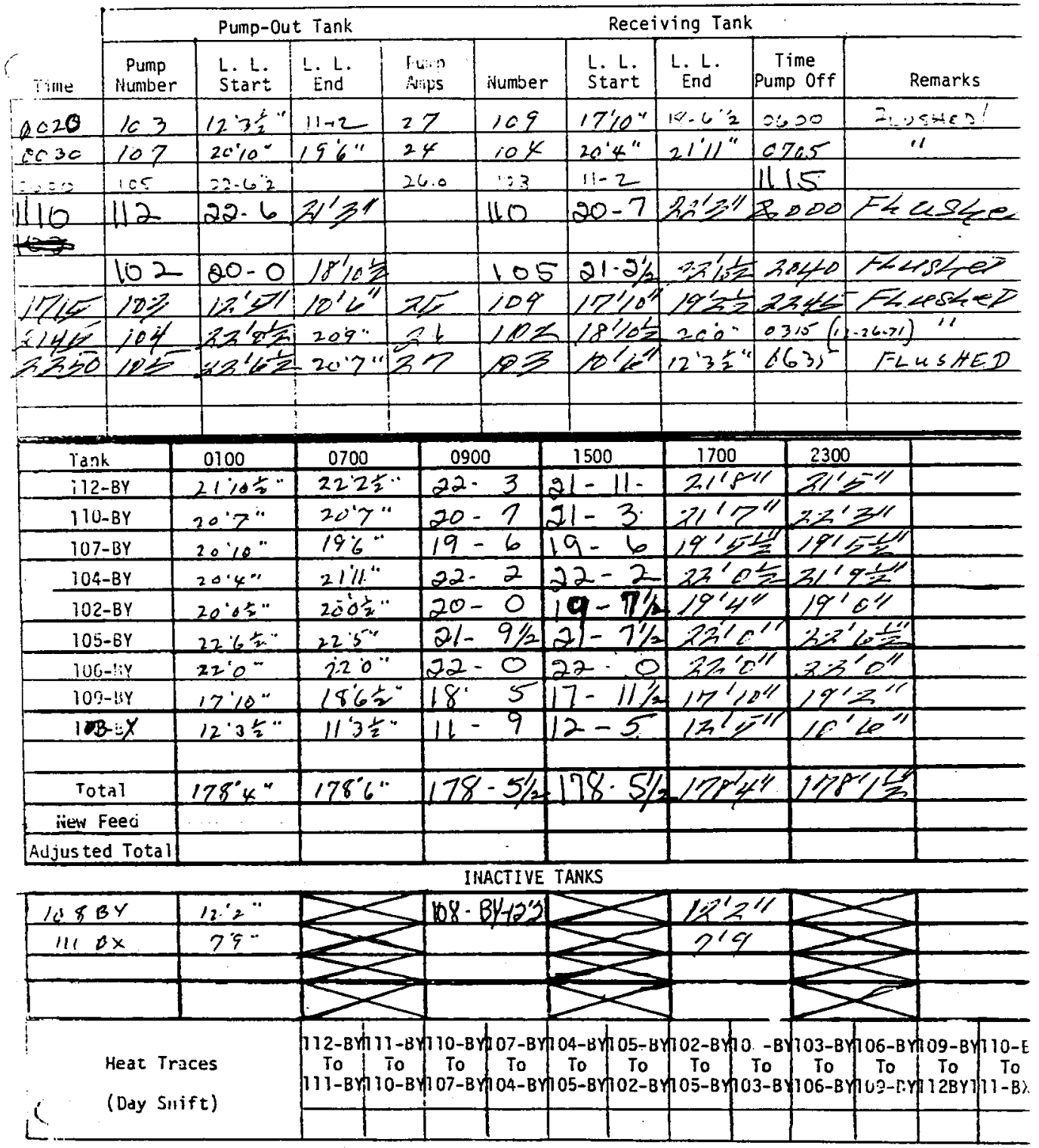




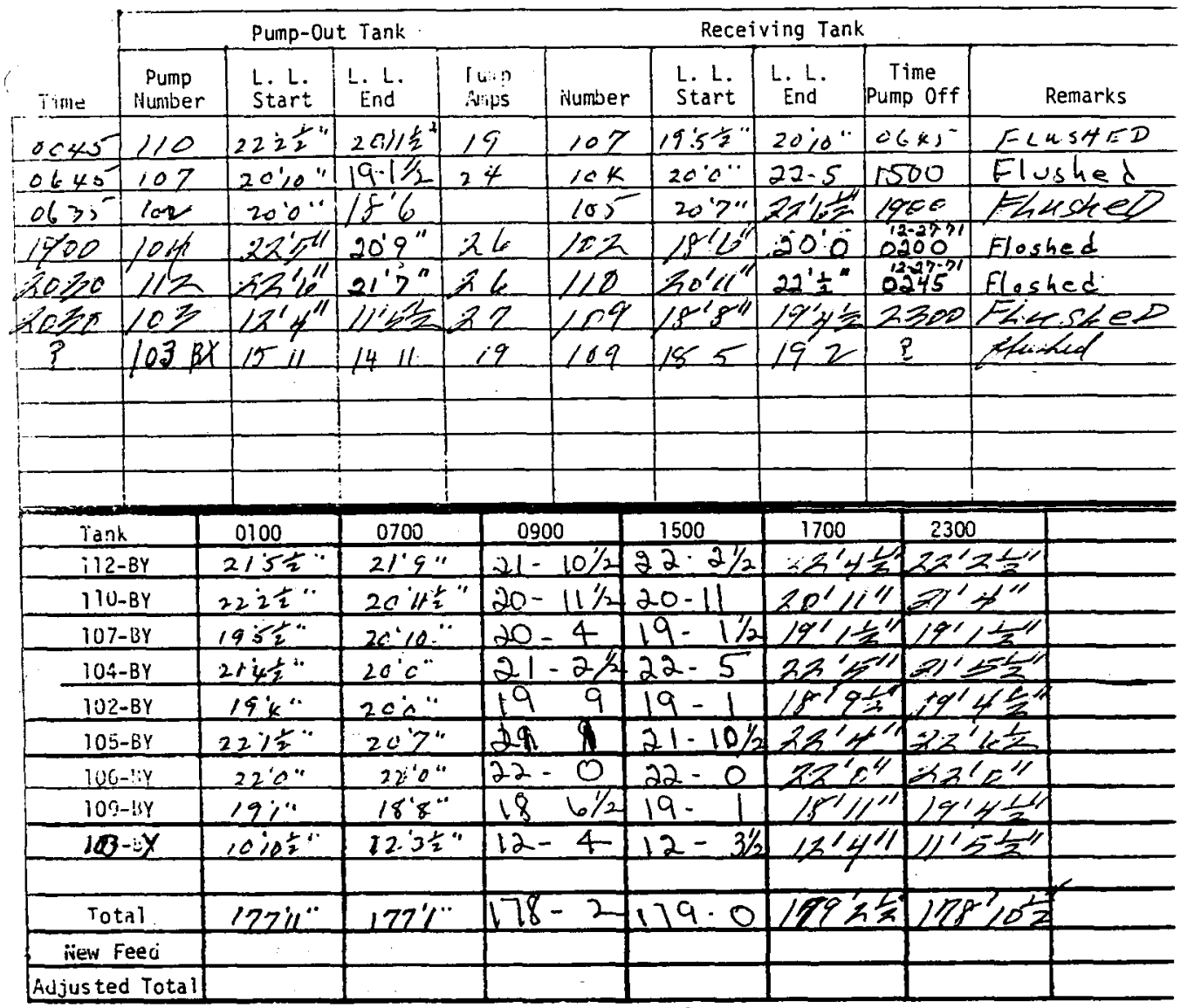

\section{INACTIVE TANKS}

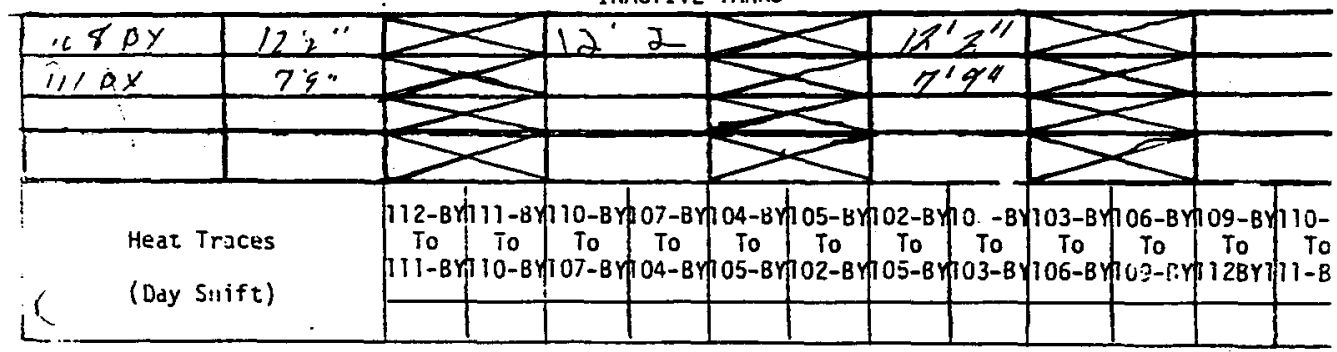




\begin{tabular}{|c|c|c|c|c|c|c|c|c|c|}
\hline & \multicolumn{4}{|c|}{ Punp-Out Tank } & \multicolumn{4}{|c|}{ Receiving Tank } & \multirow[b]{2}{*}{ Remarks } \\
\hline inte & $\begin{array}{l}\text { Pump } \\
\text { Number }\end{array}$ & $\begin{array}{l}\text { L. L. } \\
\text { Start }\end{array}$ & E. L. & $\begin{array}{l}\text { Plinn } \\
\text { ntips }\end{array}$ & itumber & $\begin{array}{l}\text { L. L. } \\
\text { Start }\end{array}$ & $\begin{array}{l}\text { L. L. } \\
\text { End }\end{array}$ & $\begin{array}{c}\text { Time } \\
\text { Pump 0ff }\end{array}$ & \\
\hline 0050 & : ৩5ーシ! & $z=\cdot \because z$ & $21^{\prime} 6^{\prime \prime}$ & 26.0 & $\therefore 3 \cdot 9$ & $11-5$ & $19^{\prime} 5^{\prime \prime}$ & 0510 & Flushied \\
\hline 0300 & 110 & $22^{1} \frac{1}{2}^{\prime \prime}$ & $20-4$ & 20 & 107 & $19^{\prime}, \frac{1}{3}, 1$ & $20-7$ & $10^{\circ 0}$ & Flushed \\
\hline 1800 & 112 & $22^{\prime}+\frac{1}{2}$ & - & - & 110 & $20^{\circ}+\frac{1}{2}$ & - & - & - \\
\hline 1800 & 107 & $20^{\circ} 8$ & $18^{\prime} 10^{\prime \prime}$ & $0 \neq F$ & 104 & $20^{\prime} 9$ & $22: 3=$ & 0250 & $F / 2$ \\
\hline 1800 & 103 & $12 \cdot 5$ & $10^{\circ} 8 \frac{1}{2}$ & & 109 & $18^{\prime} 3$ & $19^{\prime} 6$ & 2300 & Heaph \\
\hline & & & & & & & & & . \\
\hline & & & & & & & & & \\
\hline & & & & & & & & & \\
\hline & & & & & & & & & \\
\hline & & & & & & & & & \\
\hline
\end{tabular}

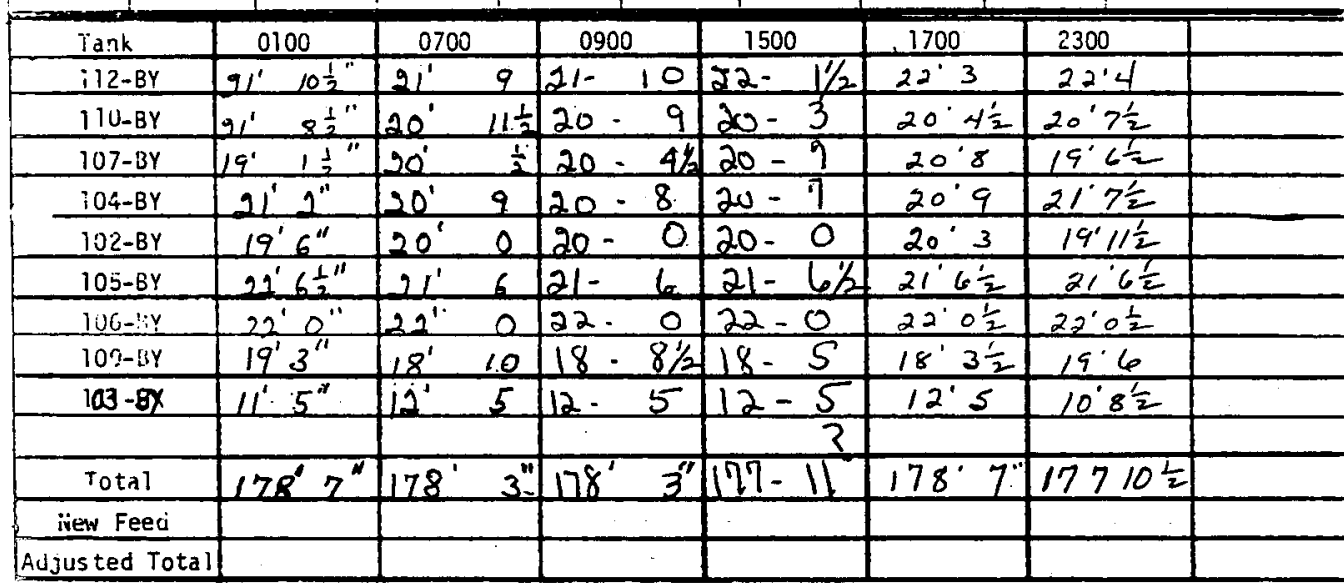

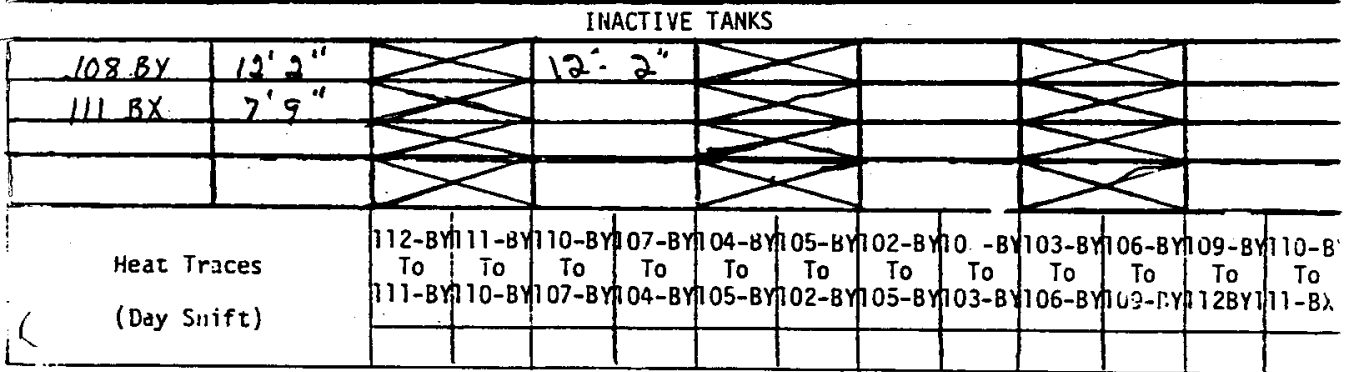




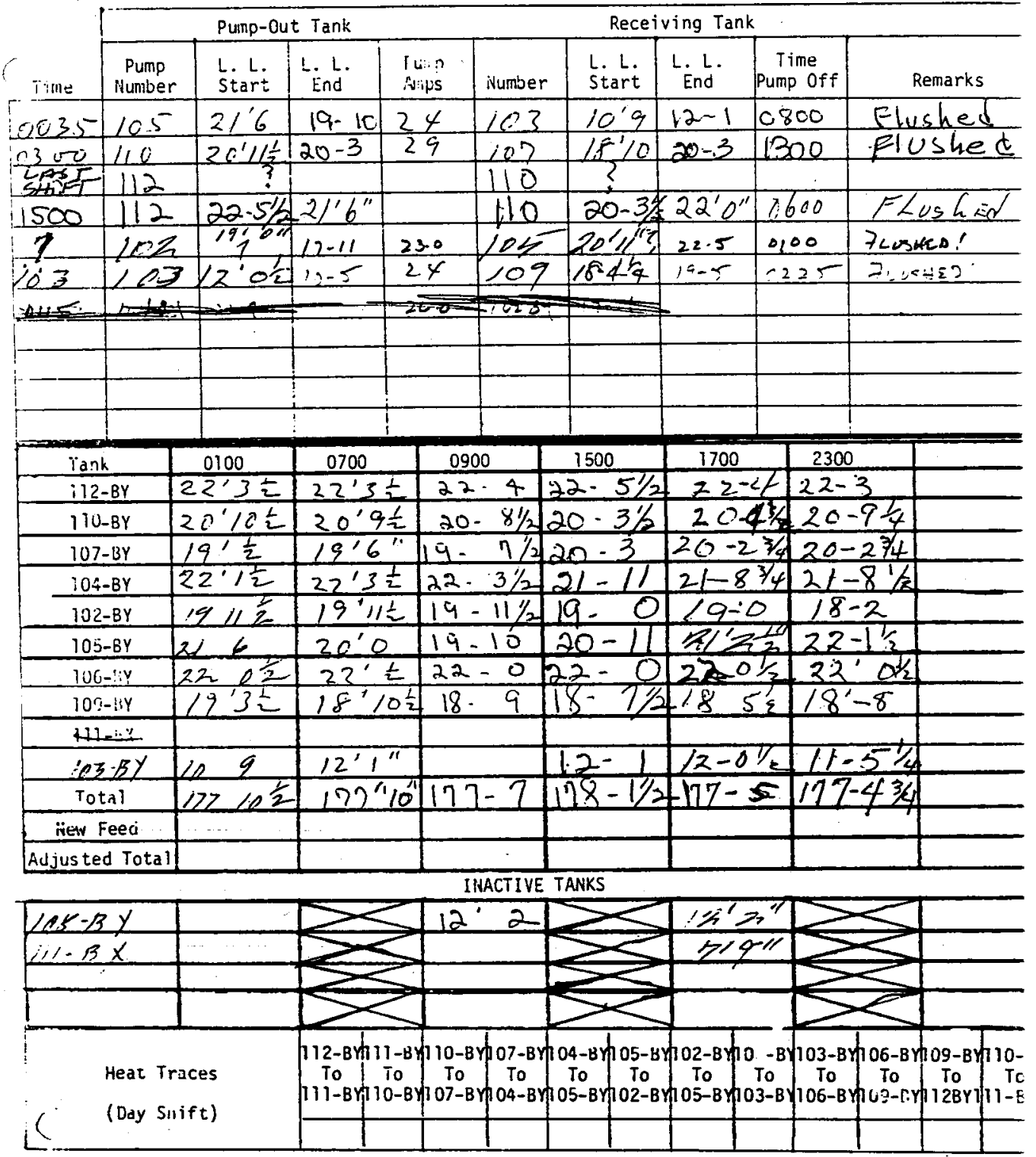




\begin{tabular}{|c|c|c|c|c|c|c|c|c|c|}
\hline \multirow[b]{2}{*}{$\therefore$ Time } & \multicolumn{4}{|c|}{ Puinp-Out Tank } & \multicolumn{4}{|c|}{ Receiving Tank } & \multirow[b]{2}{*}{ Remarks } \\
\hline & $\begin{array}{l}\text { Pump } \\
\text { Number }\end{array}$ & $\begin{array}{l}\text { L. L. } \\
\text { Start }\end{array}$ & L. L. & $\begin{array}{l}\text { I win? } \\
\text { Aits }\end{array}$ & isumber & $\begin{array}{l}\text { L. L. } \\
\text { Start }\end{array}$ & L. L. & $\begin{array}{c}\text { Time } \\
\text { Pump off }\end{array}$ & \\
\hline 0115 & 104 & $21^{\prime} 9^{\prime \prime}$ & 19,7 & 26.0 & 102 & $1 y^{\prime} / 1^{\prime \prime}$ & 20.000 & & FLSin \\
\hline$\therefore 3-$ & $9 \div$ & 22.5 & $19 \cdot 8$ & $2 \%$ & $\because 3$ & $\therefore=$ & $12: 7$ & & FLISGED \\
\hline 1.300 & 107 & $20 \cdot 2$ & $19^{\prime} 0$ & 25 & 104 & $19 \cdot 7$ & $20^{\prime} 8$ & & (12crades \\
\hline 1330 & 102 & 20.0 & & & 105 & 19.8 & 0400 & & Eluahed \\
\hline 2120 & $1 / C$ & $21^{\prime} 10$ & 20.3 & 19 & 107 & $19^{\prime} 0$ & $21-3$ & 103 & Elushed \\
\hline & & & & & & & & & \\
\hline & & & & & & & & & \\
\hline & & & & & & & & & \\
\hline & & & & & & & & & \\
\hline & & & & & & & & & \\
\hline
\end{tabular}

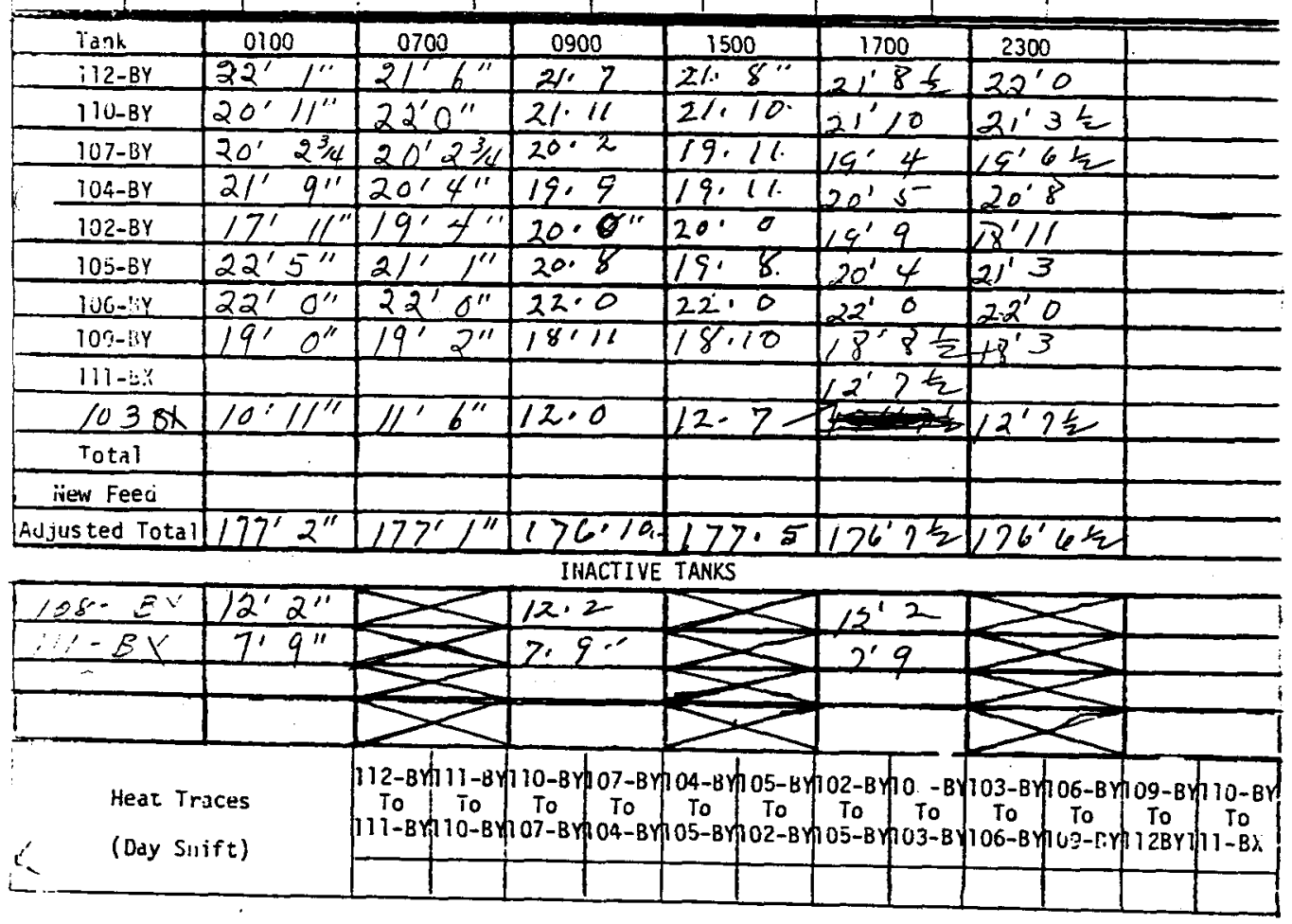




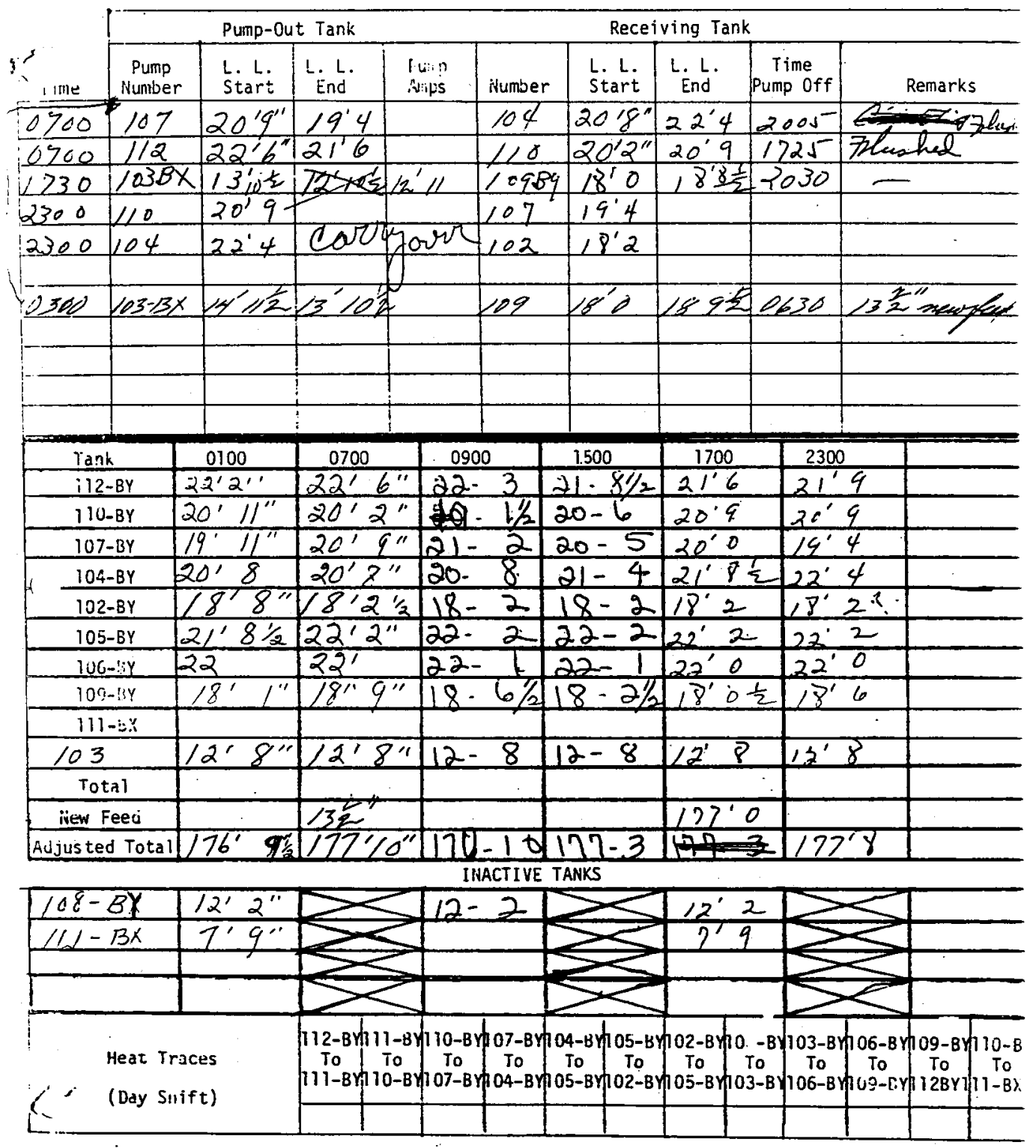




\begin{tabular}{|c|c|c|c|c|c|c|}
\hline \multicolumn{7}{|c|}{ DISTRIBUTION SHEET } \\
\hline To & \multirow{2}{*}{\multicolumn{4}{|c|}{$\begin{array}{l}\text { From } \\
\text { Remote System and Sensor } \\
\text { Applications }\end{array}$}} & \multicolumn{2}{|c|}{ Page 1 of 1} \\
\hline $\begin{array}{l}\text { Remote System and Sensor } \\
\text { Applications }\end{array}$ & & & & & \multicolumn{2}{|c|}{ Date $7 / 29 / 96$} \\
\hline \multicolumn{5}{|c|}{ Project TitleN Work Order } & \multicolumn{2}{|c|}{ EDT No. 617410} \\
\hline Sensor/Analytical Technology & jects & & & & \multicolumn{2}{|c|}{ ECN No. } \\
\hline Name & & MSIN & $\begin{array}{l}\text { Text } \\
\text { With All } \\
\text { Attach. }\end{array}$ & Text Only & $\begin{array}{l}\text { Attach./ } \\
\text { Appendix } \\
\text { Only }\end{array}$ & $\begin{array}{l}\text { EDT/ECN } \\
\text { Only }\end{array}$ \\
\hline $\begin{array}{l}\text { H. Babad } \\
\text { W. B. Barton } \\
\text { C. H. Brevick } \\
\text { R. J. Cash } \\
\text { M. D. Crippen } \\
\text { D. R. Dickinson } \\
\text { D. W. Jeppson } \\
\text { N. W. Kirch } \\
\text { D. J. McCain } \\
\text { J. E. Meacham } \\
\text { F. R. Reich } \\
\text { D. A. Reynolds } \\
\text { Central Files (original +1) }\end{array}$ & & $\begin{array}{l}S 7-14 \\
R 2-11 \\
S 3-10 \\
S 7-14 \\
L 5-31 \\
L 5-31 \\
L 5-31 \\
R 2-11 \\
R 2-12 \\
S 7-14 \\
L 5-55 \\
R 2-11 \\
\text { A } 3-89\end{array}$ & $\begin{array}{l}x \\
x \\
x \\
x \\
x \\
x \\
x \\
x \\
x \\
x \\
x \\
x \\
x\end{array}$ & & & \\
\hline
\end{tabular}

